Florida International University FIU Digital Commons

$11-12-2008$

\title{
Seismic Performance of Hybrid Fiber Reinforced Polymer-Concrete Pier Frame Systems
}

Bin Li

Florida International University

DOI: $10.25148 /$ etd.FI10022528

Follow this and additional works at: https://digitalcommons.fiu.edu/etd

\section{Recommended Citation}

Li, Bin, "Seismic Performance of Hybrid Fiber Reinforced Polymer-Concrete Pier Frame Systems" (2008). FIU Electronic Theses and Dissertations. 195.

https://digitalcommons.fiu.edu/etd/195

This work is brought to you for free and open access by the University Graduate School at FIU Digital Commons. It has been accepted for inclusion in FIU Electronic Theses and Dissertations by an authorized administrator of FIU Digital Commons. For more information, please contact dcc@fiu.edu. 


\section{FLORIDA INTERNATIONAL UNIVERSITY}

Miami, Florida

\section{SEISMIC PERFORMANCE OF HYBRID FIBER REINFORCED POLYMER-CONCRETE PIER FRAME SYSTEMS}

A dissertation submitted in partial fulfillment of the requirements for the degree of DOCTOR OF PHILOSOPHY

in CIVIL ENGINEERING

by

Bin Li

2008 
To: Interim Dean Amir Mirmiran

College of Engineering and Computing

This dissertation, written by Bin Li, and entitled Seismic Performance of Hybrid Fiber Reinforced Polymer-Concrete Pier Frame Systems, having been approved in respect to style and intellectual content, is referred to you for judgment.

We have read this dissertation and recommend that it be approved.

\section{Ton-Lo Wang}

Nakin Suksawang

\begin{tabular}{c}
\hline Yimin Zhu \\
\hline Amir Mirmiran, Major Professor
\end{tabular}

Date of Defense: November 12, 2008

The dissertation of Bin Li is approved.

\begin{tabular}{r} 
Interim Dean Amir Mirmiran \\
College of Engineering and Computing \\
\hline Dean George Walker \\
University Graduate School
\end{tabular}

Florida International University, 2008 
C) Copyright 2008 by Bin Li

All rights reserved. 


\section{ACKNOWLEDGMENTS}

This dissertation could not have been completed without the guidance and support of the following people:

Firstly, I would especially like to thank my academic advisor, Dr. Amir Mirmiran, for his patience, hard work, and commitment in developing my lab work and research skills throughout the research program.

I am also grateful for having an exceptional doctoral committee, and wish to thank Dr. Ton-Lo Wang, Dr. Yimin Zhu and Dr. Nakin Suksawang for reviewing my dissertation and putting forward valuable opinions and suggestions.

Finally, my deepest gratitude goes to my family, my father, and my sister, for their love, support and encouragement throughout my life. This dissertation is dedicated to them. 


\section{ABSTRACT OF THE DISSERTATION \\ SEISMIC PERFORMANCE OF HYBRID FIBER REINFORCED POLYMER- CONCRETE PIER FRAME SYSTEMS}

by

\section{Bin Li}

Florida International University, 2008

Miami, Florida

\section{Professor Amir Mirmiran, Major Professor}

As an alternative to transverse spiral or hoop steel reinforcement, fiber reinforced polymers (FRPs) were introduced to the construction industry in the 1980's. The concept of concrete-filled FRP tube (CFFT) has raised great interest amongst researchers in the last decade. FRP tube can act as a pour form, protective jacket, and shear and flexural reinforcement for concrete. However, seismic performance of CFFT bridge substructure has not yet been fully investigated.

Experimental work in this study included four two-column bent tests, several component tests and coupon tests. Four 1/6-scale bridge pier frames, consisting of a control reinforced concrete frame (RCF), glass FRP-concrete frame (GFF), carbon FRPconcrete frame (CFF), and hybrid glass/carbon FRP-concrete frame (HFF) were tested under reverse cyclic lateral loading with constant axial loads. Specimen GFF did not show any sign of cracking at a drift ratio as high as $15 \%$ with considerable loading capacity, whereas Specimen CFF showed that lowest ductility with similar load capacity as in Specimen GFF. FRP-concrete columns and pier cap beams were then cut from the pier frame specimens, and were tested again in three point flexure under monotonic 
loading with no axial load. The tests indicated that bonding between FRP and concrete and yielding of steel both affect the flexural strength and ductility of the components. The coupon tests were carried out to establish the tensile strength and elastic modulus of each FRP tube and the FRP mold for the pier cap beam in the two principle directions of loading.

A nonlinear analytical model was developed to predict the load-deflection responses of the pier frames. The model was validated against test results. Subsequently, a parametric study was conducted with variables such as frame height to span ratio, steel reinforcement ratio, FRP tube thickness, axial force, and compressive strength of concrete. A typical bridge was also simulated under three different ground acceleration records and damping ratios. Based on the analytical damage index, the RCF bridge was most severely damaged, whereas the GFF bridge only suffered minor repairable damages. Damping ratio was shown to have a pronounced effect on FRP-concrete bridges, just the same as in conventional bridges.

This research was part of a multi-university project, which is founded by the National Science Foundation (NSF) - Network for Earthquake Engineering Simulation Research (NEESR) program. 


\section{TABLE OF CONTENTS}

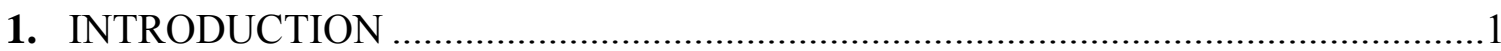

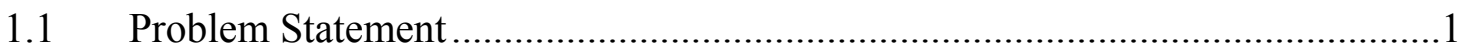

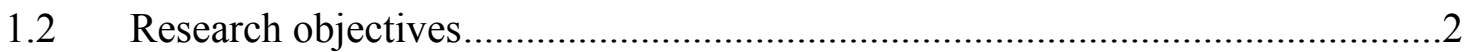

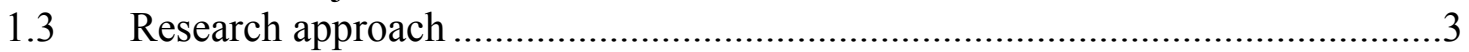

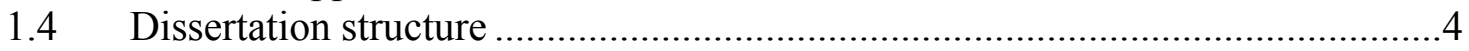

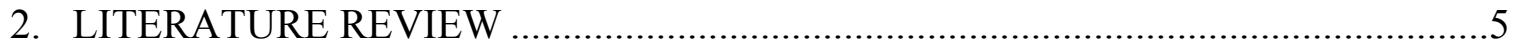

2.1 Flexural Behavior of Concrete-Filled FRP Tubes ...........................................

2.2 Axial-Flexural Behavior of Concrete-Filled FRP Tubes ......................................6

2.3 Seismic Performance of Concrete-Filled FRP Tubes ...........................................

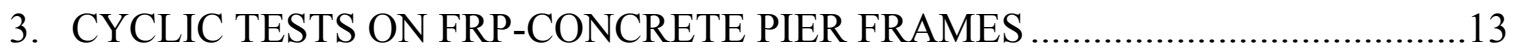

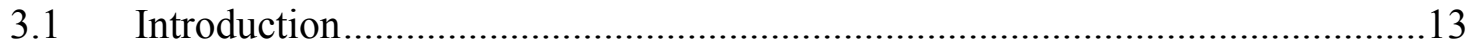

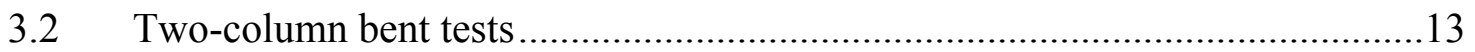

3.2.1 Test Matrix and Specimen Preparation.......................................................13

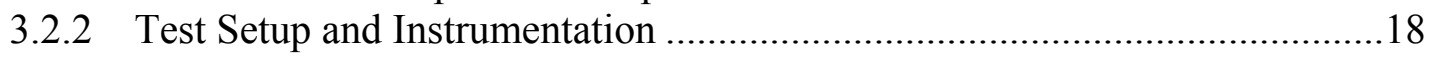

3.2.3 Test Procedure and Observations...............................................................20

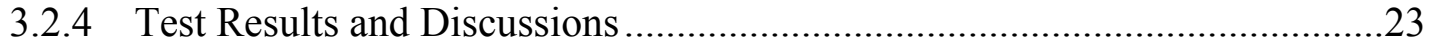

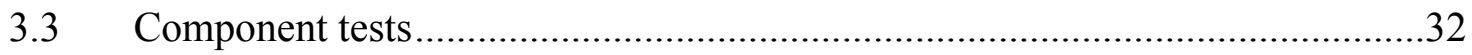

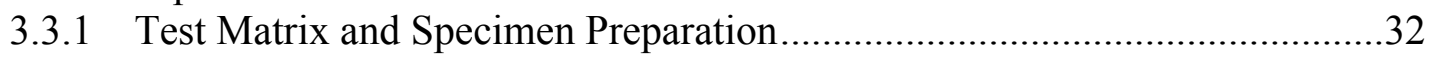

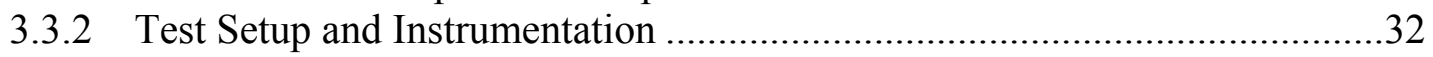

3.3.3 Test Procedure and Observations.................................................................33

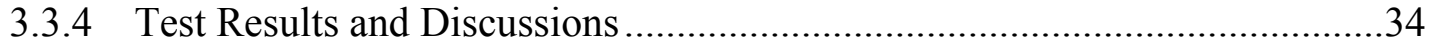

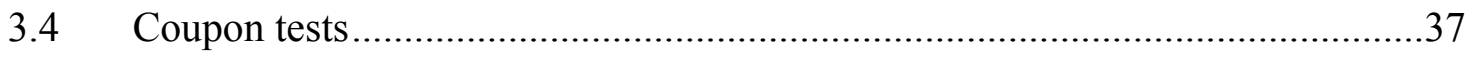

3.4.1 Test Matrix and Specimen Preparation.........................................................37

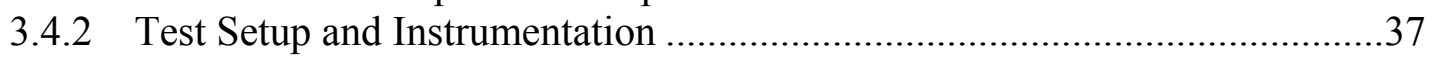

3.4.3 Test Procedure and Observations..................................................................38

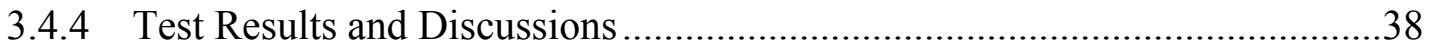

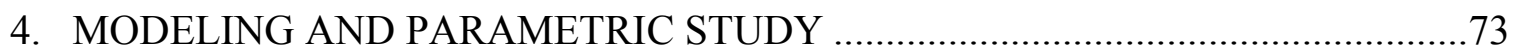

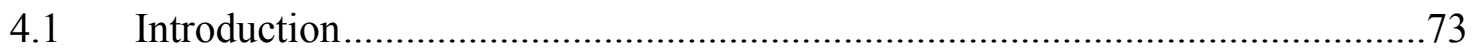

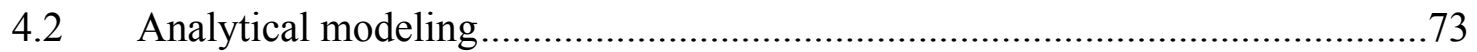

4.2.1 General Features of OpenSees Program .......................................................73

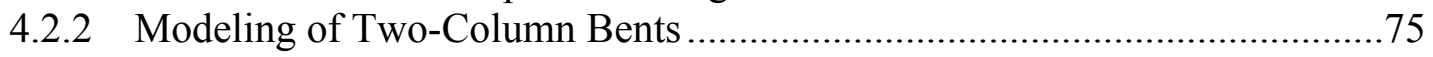

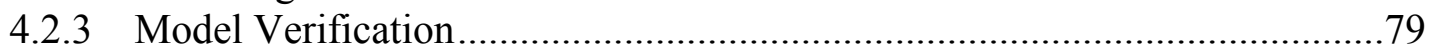

4.3 Parametric study of frp-concrete pier frame system ..........................................80

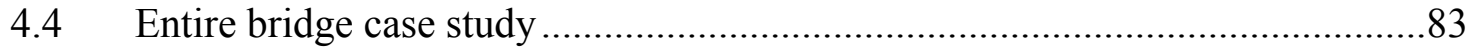


5. CONCLUSIONS AND DESIGN RECOMMENDATIONS.

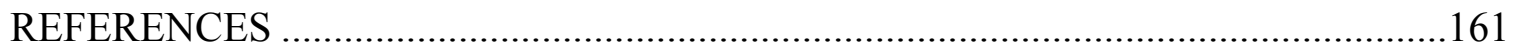

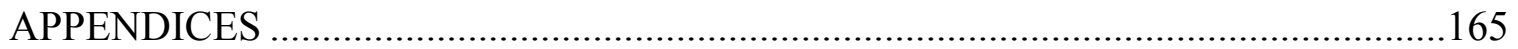

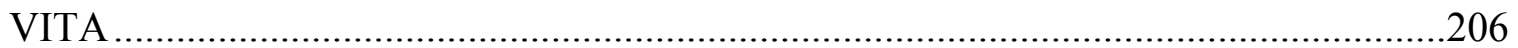




\section{LIST OF TABLES}

TABLE

PAGE

Table 3.1 Test Matrix of FRP-Concrete Pier Frame Specimens .................................. 39

Table 3.2 Design Parameters for Pier Frame Specimens.............................................. 39

Table 3.3 Response Measures for Pier Frame Specimens ......................................... 40

Table 3.4 Test Matrix of Pier Cap Beams and CFFT Columns................................... 40

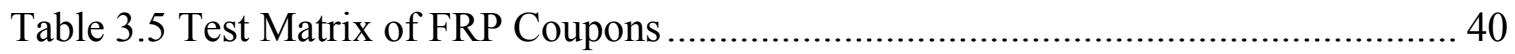

Table 4.1 Manufacturer Data for Carbon and Glass FRP in Specimen HFF.................. 89

Table 4.2 Peak Load Standard Deviation and RMSN Error of Simulation ..................... 89

Table 4.3 Cumulative Dissipated Energy Standard Deviation and RMSN Error of

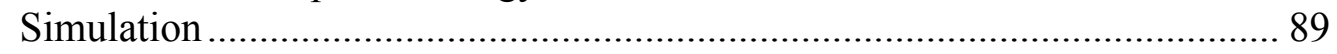

Table 4.4 Case Study for Hybrid FRP-Concrete Pier Frame Systems .......................... 89

Table 4.5 Parametric Study Results for Specimen GFF .......................................... 90

Table 4.6 Parametric Study Results for Specimen HFF ......................................... 90

Table 4.7 Parametric Study Results for Specimen CFF …..................................... 91

Table 4.8 The First Three Natural Frequency of All types of Bridges ......................... 91

Table 4.9 Peak Frequencies of Three Earthquake Ground Acceleration........................ 91 


\section{LIST OF FIGURES}

FIGURE

PAGE

Fig. 3.1 Schematics of Pier Frame Specimens......................................................... 41

Fig. 3.2 Construction of Steel Reinforcement Cage for FRP Columns ......................... 41

Fig. 3.3 Completed Steel Cage for FRP Columns ................................................. 41

Fig. 3.4 Steel Reinforcement Details for Pier Cap Beams........................................ 42

Fig. 3.5 Steel Reinforcement Details for RC Footings ......................................... 42

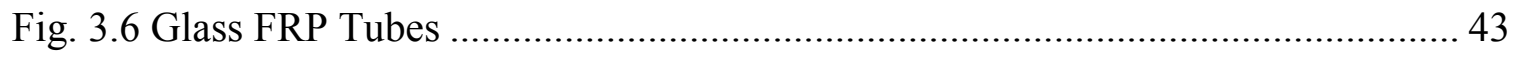

Fig. 3.7 Preparing a Carbon FRP Tube ..................................................................... 43

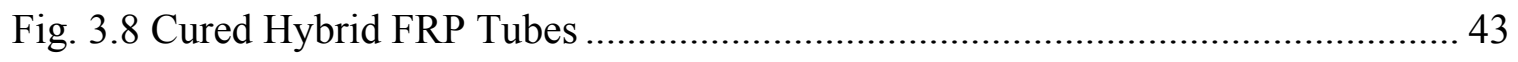

Fig. 3.9 Cured Carbon FRP Tubes........................................................................ 43

Fig. 3.10 Embedment of FRP Tubes........................................................................ 44

Fig. 3.11 Slots and Holes in Glass FRP Tube........................................................ 44

Fig. 3.12 Wooden Mold for FRP Pier Cap Beam ..................................................... 44

Fig. 3.13 Cured FRP Pier Cap Forms …............................................................... 44

Fig. 3.14 Welding Anchorage Bars in Column Heads............................................... 45

Fig. 3.15 Epoxy Placement in Transition Zone ...................................................... 45

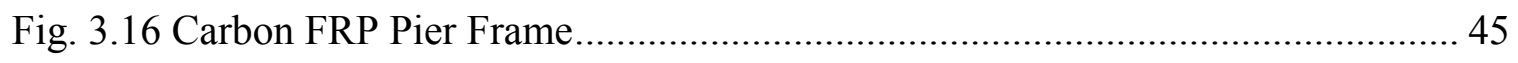

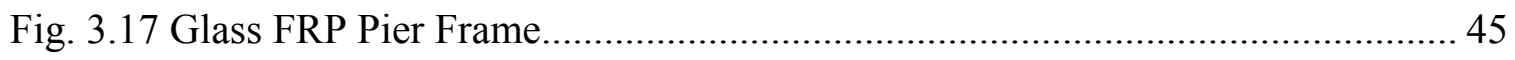

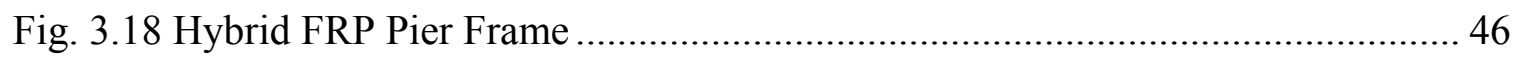

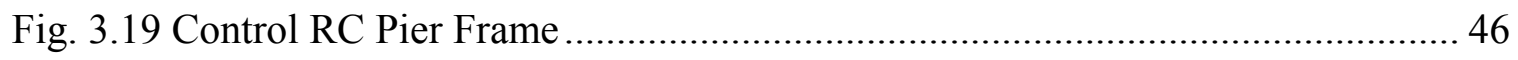

Fig. 3.20 Grinded Edge of Pier Cap Beam .......................................................... 46

Fig. 3.21 FRP Wrapping at Joints for Pier Cap Beam ............................................. 46

Fig. 3.22 Plan View of Test Layout for Two-Column Bent Specimens.......................... 47 


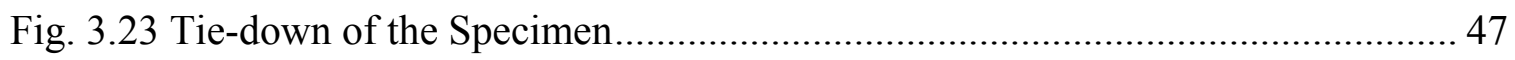

Fig. 3.24 Steel Tie Beam for Lateral Reaction .................................................................. 47

Fig. 3.25 Pier Cap Beam Connection With the Actuator.................................................... 48

Fig. 3.26 Completed Test Layout of Two Column Bent Specimens ................................ 48

Fig. 3.27 Steel Spreader Beam with Hydraulic Jacks ..................................................... 48

Fig. 3.28 Hand Pump with Pressure Relief Valve .......................................................... 48

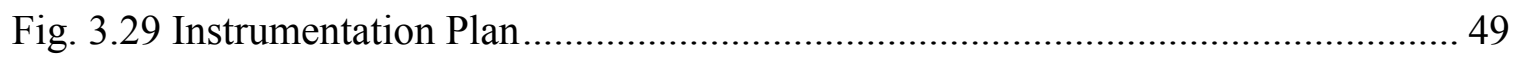

Fig. 3.30 Reverse Cyclic Loading Regime ……………….......................................... 49

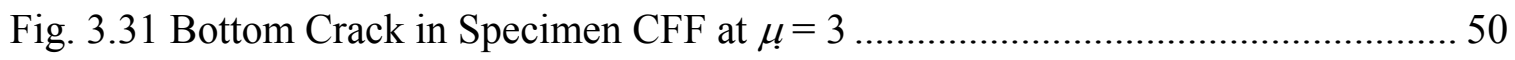

Fig. 3.32 Vertical Crack at Column Top in Specimen CFF at $\mu=7 \ldots \ldots \ldots \ldots \ldots \ldots \ldots \ldots \ldots \ldots \ldots . . . . .50$

Fig. 3.33 Crack in Specimen HFF Enlarged to Middle of the Column Base Section at

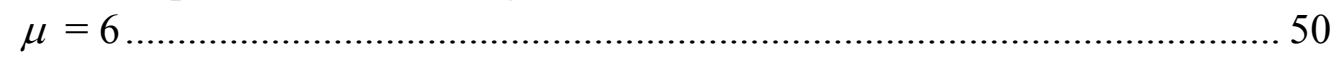

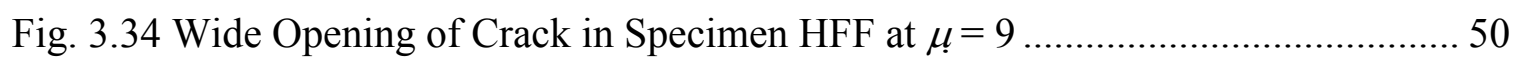

Fig. 3.35 Cracks in Pier Cap Beam of Specimen HFF ................................................. 51

Fig. 3.36 Cracks in Epoxy Transition Zone of Specimen GFF ...................................... 51

Fig. 3.37 Separation between FRP tube and Pier Cap Beam in Specimen HFF at $\mu=$ 9

Fig. 3.38 Slippage of Column Base in Specimen GFF at $\mu=10 \ldots \ldots \ldots \ldots \ldots \ldots \ldots \ldots \ldots \ldots \ldots \ldots \ldots . . . . . . \ldots 1$

Fig. 3.39 Cracks in the Plastic Hinge Zone and Chamfer in Specimen RCF .................. 52

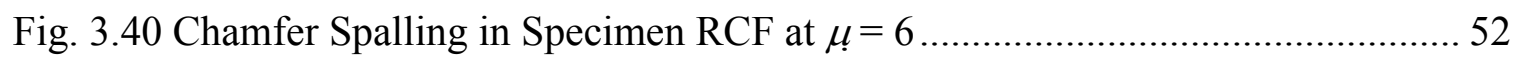

Fig. 3.41 Lateral Deflection of Specimen GFF at $\mu=10$ with Axial Load...................... 52

Fig. 3.42 Lateral Deflection of Specimen GFF at $\mu=25$ without Axial Load................. 52

Fig. 3.43 Load-Deflection Hysteretic Response of Specimen RCF ………………….... 53

Fig. 3.44 Load-Deflection Hysteretic Response of Specimen HFF.................................. 53

Fig. 3.45 Load-Deflections Hysteretic Response of Specimen CFF ............................... 54 
Fig. 3.46 Load-Deflection Hysteretic Response of Specimen GFF................................. 54

Fig. 3.47 Load-Deflection Envelope Curves of Pier Frame Specimens ........................... 55

Fig. 3.48 Residual Displacements at Zero Load after Each First Push Cycle .................. 55

Fig. 3.49 Residual Load at Zero Displacement after Each First Push Cycle.................... 56

Fig. 3.50 Monotonic Load-Deflection Responses After Removal of Axial Load ............ 56

Fig. 3.51 Normalized Moments Versus Total Reinforcement Strength Index ................. 57

Fig. 3.52 Slippage of East Column Head in Specimen GFF .......................................... 57

Fig. 3.53 Cumulative Dissipated Energy Versus Drift Ratio for All Specimens ............. 58

Fig. 3.54 Comparisons of Deflected Shapes of West Columns of All Specimens at

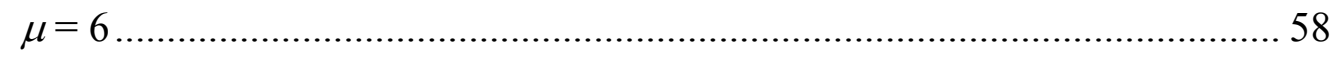

Fig. 3.55 Deflected Shapes of East Columns of All Specimens at $\mu=6 \ldots \ldots \ldots \ldots \ldots \ldots \ldots \ldots \ldots . . .59$

Fig. 3.56 Longitudinal Strain Profiles Along West Column of Specimen GFF in Push .. 59

Fig. 3.57 Longitudinal Strain Profiles Along West Column of Specimen GFF in Pull ... 60

Fig. 3.58 Longitudinal Strain Profiles Along East Column of Specimen GFF in Push ... 60

Fig. 3.59 Longitudinal Strain Profiles Along East Column of Specimen GFF in Pull.... 61

Fig. 3.60 Curvature Profiles Along West Column of Specimen GFF in Push and Pull ... 61

Fig. 3.61 Curvature Profiles Along East Column of Specimen GFF in Push and Pull .... 62

Fig. 3.62 Plastic Hinge Length Ratio Versus Ductility for Specimen GFF..................... 62

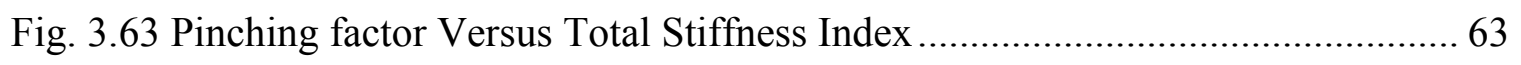

Fig. 3.64 Rotations of Pier Cap Beam-Column Joints...................................................... 63

Fig. 3.65 Hoop Strains at the Base of West Columns in All Specimens ........................... 64

Fig. 3.66 Hoop Strains at the Base of East Columns in All Specimens ........................... 64

Fig. 3.67 Mid-Span Strains of Pier Cap Beams in All Specimens .................................... 65

Fig. 3.68 Mid-Span Deflection of Pier Cap Beams in Specimen GFF ............................ 65

Fig. 3.69 Mid-Span Deflection Envelopes of Pier Cap Beams in All Specimens ............ 66 
Fig. 3.70 Overview of the Setup for Pier Cap Beam Test ............................................... 66

Fig. 3.71 Pier Cap Beam Tested Upside Down .................................................................. 66

Fig. 3.72 Instrumentation Plan for Pier Cap Beam Tests ................................................ 67

Fig. 3.73 Specially Designed Semi-Circular Steel Support............................................. 67

Fig. 3.74 Overview of the Setup for CFFT Column Test ................................................ 67

Fig. 3.75 Instrumentation Plan for CFFT Column Tests .................................................. 67

Fig. 3.76 Cracks in Concrete in Pier Cap Beam ……………………............................. 68

Fig. 3.77 FRP Cracks at the Bottom of Pier Cap Beam Tested Upside Down................. 68

Fig. 3.78 Longitudinal Crack in Carbon CFFT Column................................................. 68

Fig. 3.79 Cracks at Mid-Span of Hybrid CFFT Column ................................................. 68

Fig. 3.80 Large Deformability of Glass CFFT Colum ……………………………....... 69

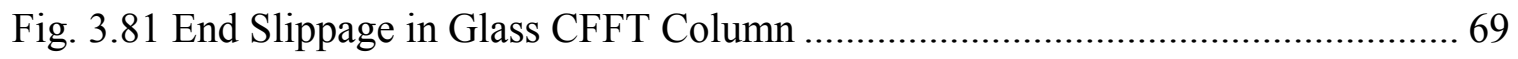

Fig. 3.82 Residual Load-Deflection Responses of Pier Cap Beams................................. 69

Fig. 3.83 Residual Load-Strain Responses of Pier Cap Beams ....................................... 70

Fig. 3.84 End Rotations of Pier Cap Beams …………………………….................. 70

Fig. 3.85 Residual Load-Deflection Responses of CFFT Columns................................... 71

Fig. 3.86 Residual Load-Strain Responses of CFFT Columns ......................................... 71

Fig. 3.87 Stress-Strain Response of FRP Coupons ......................................................... 72

Fig. 4.1 Hybrid FRP-Concrete Pier Frame Model........................................................... 92

Fig. 4.2 Illustration of Sectional Analysis Strip Method ................................................ 92

Fig. 4.3 Typical Hysteretic Stress-Strain Model for Concrete (OpenSees 2006) ............. 93

Fig. 4.4 Typical Hysteric Stress-Strain Model for Steel Reinforcement (OpenSees

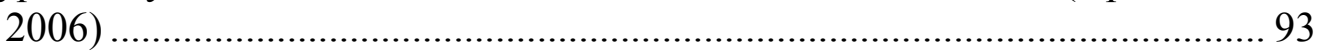

Fig. 4.5 Typical Stress-Slip Curve of Bond Slip Model (OpenSees 2006) …………...... 94

Fig. 4.6 Hysteretic Bar Stress-Slip Model in (OpenSees 2006) .................................... 94 
Fig. 4.7 Hysteretic Cyclic Bar Stress-Slip Model (OpenSees 2006) 95

Fig. 4.8 Hysteretic Stress-Strain Response of Glass FRP Tube in Longitudinal Direction 95

Fig. 4.9 Stress-Strain Response of Carbon and Hybrid FRP Tubes in Longitudinal Direction

Fig. 4.10 Simulation of Hysteretic Lateral Load-Deflection Response of Specimen $\mathrm{RCF}$ 96

Fig. 4.11 Simulation of Hysteretic Lateral Load-Deflection Response of Specimen CFF

Fig. 4.12 Simulation of Hysteretic Lateral Load-Deflection Response of Specimen GFF

Fig. 4.13 Simulation of Hysteretic Lateral Load-Deflection Response of Specimen $\mathrm{HFF}$ 98

Fig. 4.14 Effect of H/L Ratio on Hysteretic Response of Frame GFF 98

Fig. 4.15 Effect of Concrete Compressive Strength on Hysteretic Response of Frame GFF

Fig. 4.16 Effect of Axial Load Ratio on Hysteretic Response of Frame GFF. 99

Fig. 4.17 Effect of Steel Reinforcement Ratio on Hysteretic Response of Frame GFF . 100

Fig. 4.18 Effect of D/t Ratio on Hysteretic Response of Frame GFF.......................... 100

Fig. 4.19 Effect of H/L Ratio on Response Envelope of Frame GFF 101

Fig. 4.20 Effect of Concrete Compressive Strength on Response Envelope of Frame GFF 101

Fig. 4.21 Effect of Axial Load Ratio on Response Envelope of Frame GFF 102

Fig. 4.22 Effect of Steel Reinforcement Ratio on Response Envelope of Frame GFF .. 102 Fig. 4.23 Effect of D/t Ratio on Response Envelope of Frame GFF ........................... 103

Fig. 4.24 Effect of H/L Ratio on Cumulative Energy of Frame GFF .......................... 103

Fig. 4.25 Effect of Concrete Compressive Strength on Cumulative Energy of Frame GFF 104

Fig. 4.26 Effect of Axial Load Ratio on Cumulative Energy of Frame GFF 104 
Fig. 4.27 Effect of Steel Reinforcement Ratio on Cumulative Energy of Frame GFF... 105

Fig. 4.28 Effect of D/t Ratio on Cumulative Energy of Frame GFF ............................ 105

Fig. 4.29 Effect of H/L Ratio on Hysteretic Response of Frame HFF ......................... 106

Fig. 4.30 Effect of Concrete Compressive Strength on Hysteretic Response of Frame

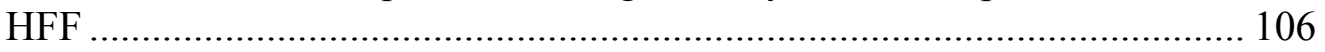

Fig. 4.31 Effect of Axial Load Ratio on Hysteretic Response of Frame HFF............... 107

Fig. 4.32 Effect of Steel Reinforcement Ratio on Hysteretic Response of Frame HFF . 107

Fig. 4.33 Effect of D/t Ratio on Hysteretic Response of Frame HFF............................ 108

Fig. 4.34 Effect of H/L Ratio on Response Envelope of Frame HFF........................... 108

Fig. 4.35 Effect of Concrete Compressive Strength on Response Envelope of Frame HFF 109

Fig. 4.36 Effect of Axial Load Ratio on Response Envelope of Frame HFF 109

Fig. 4.37 Effect of Steel Reinforcement Ratio on Response Envelope of Frame HFF .. 110

Fig. 4.38 Effect of D/t Ratio on Response Envelope of Frame HFF ............................ 110

Fig. 4.39 Effect of H/L Ratio on Cumulative Energy of Frame HFF .......................... 111

Fig. 4.40 Effect of Concrete Compressive Strength on Cumulative Energy of Frame HFF

Fig. 4.41 Effect of Axial Load Ratio on Cumulative Energy of Frame HFF ............... 112

Fig. 4.42 Effect of Steel Reinforcement Ratio on Cumulative Energy of Frame HFF... 112

Fig. 4.43 Effect of D/t Ratio on Cumulative Energy of Frame HFF ........................... 113

Fig. 4.44 Effect of H/L Ratio on Hysteretic Response of Frame CFF.......................... 113

Fig. 4.45 Effect of Concrete Compressive Strength on Hysteretic Response of Frame $\mathrm{CFF}$ 114

Fig. 4.46 Effect of Axial Load Ratio on Hysteretic Response of Frame CFF ............... 114

Fig. 4.47 Effect of Steel Reinforcement Ratio on Hysteretic Response of Frame CFF . 115

Fig. 4.48 Effect of D/t Ratio on Hysteretic Response of Frame CFF .......................... 115 
Fig. 4.49 Effect of H/L Ratio on Response Envelope of Frame CFF 116

Fig. 4.50 Effect of Concrete Compressive Strength on Response Envelope of Frame $\mathrm{CFF}$ 116

Fig. 4.51 Effect of Axial Load Ratio on Response Envelope of Frame CFF 117

Fig. 4.52 Effect of Steel Reinforcement Ratio on Response Envelope of Frame CFF... 117

Fig. 4.53 Effect of D/t Ratio on Response Envelope of Frame CFF ........................... 118

Fig. 4.54 Effect of H/L Ratio on Cumulative Energy of Frame CFF .......................... 118

Fig. 4.55 Effect of Concrete Compressive Strength on Cumulative Energy of Frame $\mathrm{CFF}$ 119

Fig. 4.56 Effect of Axial Load Ratio on Cumulative Energy of Frame CFF 119

Fig. 4.57 Effect of Steel Reinforcement Ratio on Cumulative Energy of Frame CFF... 120

Fig. 4.58 Effect of D/t Ratio on Cumulative Energy of Frame CFF ............................ 120

Fig. 4.59 Effect of H/L Ratio on Maximum Load of All Frames............................... 121

Fig. 4.60 Effect of D/t Ratio on Maximum Load of All Frames .................................. 121

Fig. 4.61 Effect of Axial Load Ratio on Maximum Load of All Frames ...................... 122

Fig. 4.62 Effect of Steel Reinforcement Ratio on Maximum Load of All Frames....... 122

Fig. 4.63 Effect of Concrete Compressive Strength on Maximum Load of All Frames 123

Fig. 4.64 Effect of H/L Ratio on Ductility Factor of All Frames ................................ 123

Fig. 4.65 Effect of D/t Ratio on Ductility Factor of All Frames.................................. 124

Fig. 4.66 Effect of Axial Load Ratio on Ductility Factor of All Frames........................ 124

Fig. 4.67 Effect of Steel Reinforcement Ratio on Ductility Factor of All Frames ........ 125

Fig. 4.68 Effect of Concrete Compressive Strength on Ductility Factor of All Frames. 125

Fig. 4.69 Effect of H/L Ratio on Pinching Factor of All Frames ................................ 126

Fig. 4.70 Effect of D/t Ratio on Pinching Factor of All Frames................................... 126

Fig. 4.71 Effect of Axial Load Ratio on Pinching Factor of All Frames...................... 127 
Fig. 4.72 Effect of Steel Reinforcement Ratio on Pinching Factor of All Frames ........ 127

Fig. 4.73 Effect of Concrete Compressive Strength on Pinching Factor of All Frames. 128

Fig. 4.74 Effect of H/L Ratio on Maximum Drift Ratio of All Frames........................ 128

Fig. 4.75 Effect of D/t Ratio on Maximum Drift Ratio of All Frames .......................... 129

Fig. 4.76 Effect of Axial Load Ratio on Maximum Drift Ratio of All Frames ............. 129

Fig. 4.77 Effect of Steel Reinforcement Ratio on Maximum Drift Ratio of All Frames ....................................................................................... 130

Fig. 4.78 Effect of Concrete Compressive Strength on Maximum Drift Ratio of All Frames..... 130

Fig. 4.79 Effect of H/L Ratio on Maximum Cumulative Energy of All Frames........... 131

Fig. 4.80 Effect of D/t Ratio on Maximum Cumulative Energy of All Frames ............ 131

Fig. 4.81 Effect of Axial Load Ratio on Maximum Cumulative Energy of All Frames 132

Fig. 4.82 Effect of Steel Reinforcement Ratio on Maximum Cumulative Energy of

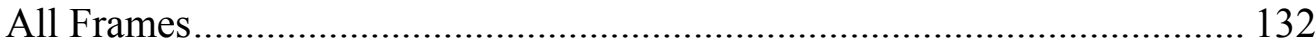

Fig. 4.83 Effect of Compressive Concrete Strength on Maximum Cumulative Energy

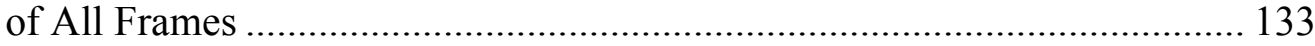

Fig. 4.84 Pinching Factor Versus FRP and Total Stiffness Index ............................. 133

Fig. 4.85 Normalized Moment Versus FRP and Total Strength Index........................ 134

Fig. 4.86 Normalized Moment Versus Steel and Total Strength Index........................ 134

Fig. 4.87 Schematics of Bridge Case Study (a) Plan View, (b) Elevation .................... 135

Fig. 4.88 Cross Section of Bridge Superstructure...................................................... 135

Fig. 4.89 Finite Element Model for Entire Bridge Structure .................................... 136

Fig. 4.90 Tabas (1978) Longitudinal Ground Acceleration Record ............................. 136

Fig. 4.91 Tabas (1978) Transverse Ground Acceleration Record .............................. 137

Fig. 4.92 Llollelo (1985) Ground Acceleration Record........................................... 137

Fig. 4.93 Sylmar (1971) Ground Acceleration Record............................................ 138 
Fig. 4.94 Normalized Drift Ratio at Each Pier under Sylmar Earthquake at $\xi=0.03 .138$

Fig. 4.95 Normalized Drift Ratio at Each Pier under Tabas Earthquake at $\xi=0.03 \ldots 139$

Fig. 4.96 Normalized Drift Ratio at Each Pier under Llolleo Earthquake at $\xi=0.03 .139$

Fig. 4.97 Normalized Drift Ratio at Each Pier under Sylmar Earthquake at $\xi=0.05 .140$

Fig. 4.98 Normalized Drift Ratio at Each Pier under Tabas Earthquake at $\xi=0.05$.. 140

Fig. 4.99 Normalized Drift Ratio at Each Pier under Llolleo Earthquake at $\xi=0.05 .141$

Fig. 4.100 Normalized Drift Ratio at Each Pier under Sylmar Earthquake at $\xi=$ 0.08 .

Fig. 4.101 Normalized Drift Ratio at Each Pier under Tabas Earthquake at $\xi=0.08 .142$

Fig. 4.102 Normalized Drift Ratio at Each Pier under Llolleo Earthquake at $\xi=0.08142$

Fig. 4.103 Effect of Damping Ratio on Performance of RCF Bridge .......................... 143

Fig. 4.104 Effect of Damping Ratio on Performance of CFF Bridge.......................... 143

Fig. 4.105 Effect of Damping Ratio on Performance of GFF Bridge........................... 144

Fig. 4.106 Effect of Damping Ratio on Performance of HFF Bridge.......................... 144

Fig. 4.107 Effect of Damping Ratio on Normalized Drift Ratio of Pier 2 for All Bridges under Tabas (1978) Earthquake.................................................. 145

Fig. 4.108 Effect of Damping Ratio on Normalized Drift Ratio of Pier 2 for All Bridges under Sylmar (1971) Earthquake............................................... 145

Fig. 4.109 Effect of Damping Ratio on Normalized Drift Ratio of Pier 2 for All Bridges under Llollello (1985) Earthquake ............................................ 146

Fig. 4.110 Damage Index at Each Pier under Sylmar Earthquake at $\xi=0.03 \ldots \ldots \ldots \ldots . .146$

Fig. 4.111 Damage Index at Each Pier under Tabas Earthquake at $\xi=0.03 \ldots \ldots \ldots \ldots . . .147$

Fig. 4.112 Damage Index at Each Pier under Llollelo Earthquake at $\xi=0.03 \ldots \ldots \ldots \ldots . .147$

Fig. 4.113 Damage Index at Each Pier under Sylmar Earthquake at $\xi=0.05 \ldots \ldots \ldots \ldots . . .148$

Fig. 4.114 Damage Index at Each Pier under Tabas Earthquake at $\xi=0.05 \ldots \ldots \ldots \ldots \ldots . .148$ 
Fig. 4.115 Damage Index at Each Pier under Llolleo Earthquake at $\xi=0.05 \ldots \ldots \ldots \ldots . . .149$

Fig. 4.116 Damage Index at Each Pier under Sylmar Earthquake at $\xi=0.08 \ldots \ldots \ldots \ldots . . .149$

Fig. 4.117 Damage Index at Each Pier under Tabas Earthquake at $\xi=0.08 \ldots \ldots \ldots \ldots \ldots . . .150$

Fig. 4.118 Damage Index at Each Pier under Llolleo Earthquake at $\xi=0.08 \ldots \ldots \ldots \ldots . . .150$

Fig. 4.119 Effect of Damping Ratio on Damage Index of Pier 2 in All Bridges under Tabas (1978) Earthquake ..................................................................... 151

Fig. 4.120 Effect of Damping Ratio on Damage Index of Pier 2 in All Bridges under Sylmar (1971) Earthquake ..................................................................... 151

Fig. 4.121 Effect of Damping Ratio on Damage Index of Pier 2 in All Bridges under Llolleo (1985) Earthquake .................................................................... 152

Fig. 5.1 Stress-Strain Response of Steel Hoop or Spiral Confined Concrete Based on

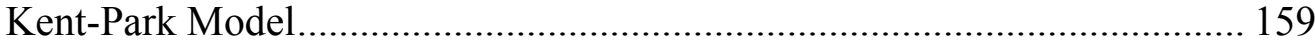

Fig. 5.2 Stress-Strain Response of FRP Confined Concrete Based on Samaan Model.. 159

Fig. 5.3 t/D Versus $\rho_{v}$ of Proposed Design Method for All Types of FRP Tubes ........ 160 


\section{INTRODUCTION}

\subsection{PROBLEM STATEMENT}

Reinforced concrete (RC) accounts for a large portion of the infrastructure construction projects. Because confinement of concrete significantly improves its strength and ductility, spiral or hoop reinforcing steel is commonly used as transverse reinforcement in typical RC columns.

As an alternative to transverse spiral or hoop steel reinforcement, fiber reinforced polymers (FRPs) were introduced to the construction industry in the early 1980's. Numerous studies have since focused on the use of FRPs, and the results show that using FRPs instead of steel hoop or spiral is quite an effective way to increase the strength and ductility of RC columns. The concept of concrete-filled FRP tube (CFFT) has further raised great interest amongst researchers and practitioners in the last decade. FRP tube can act as a pour form, protective jacket, and shear and flexural reinforcement for concrete. Extensive studies in the past decade have shown good performance of CFFTs under axial compression, and have led to numerous confinement models for FRPconfined concrete. Other studies have focused on the flexural and axial-flexural behavior of CFFT beam-columns, their long-term performance under sustained loads, and their field applications (Fam et al. 2003). CFFTs have so far been used for several bridges and field applications in Virginia, California, and Florida (Zhu et al. 2006).

Seible et al. (1996) studied the feasibility of carbon CFFT columns under simulated seismic actions. They concluded that CFFTs without any starter steel bars 
would fail prematurely under combined compressive and inter-laminar shear stresses. On the other hand, when carbon tube was augmented with sufficient number of starter bars, ductility of the column was increased significantly. A number of more recent studies (as cited in Zhu et al. 2006) have provided evidence as to the advantages of glass over carbon in providing additional ductility for the column. This may be attributed to the lower modulus of elasticity of glass FRP, as compared to carbon. Most recently, Zhu et al. (2006) also showed construction feasibility and superior performance of cast-in-place or precast CFFT columns with reinforced or prestressed connection to the footing.

This research is part of a multi-university project, which is funded by the National Science Foundation (NSF)-Network for Earthquake Engineering Simulation Research (NEESR) program. The project aims at better understanding of CFFT pier frames under seismic loading by testing large-scale specimens and developing appropriate analytical tool to improve their design.

\subsection{RESEARCH OBJECTIVES}

The main goal of this study was to investigate a new generation of bridge substructure to resist seismic loads. The following objectives were therefore established for the proposed experimental and analytical development:

(1) Compare the performance of pier frame systems using glass, carbon, or hybrid (i.e., glass and carbon) FRP tubes under reverse cyclic lateral loading and constant axial load; and

(2) Develop analytical tools and conduct parametric study for the FRP-concrete pier frames and the entire bridge to help improve their design. 


\subsection{RESEARCH APPROACH}

In order to achieve the above stated research objectives, an experimental and analytical work plan was developed. The experimental program consisted of the following components:

(1) Design, build, and test to failure comparable pier frame specimens with glass, carbon, or hybrid FRP materials under constant axial loads and reverse cyclic lateral loads; and compare their responses with that of the control RC pier frame (RCF);

(2) Test residual strength of individual columns and pier cap beams of the three FRPconcrete pier frames using three-point flexure in monotonic loading; and

(3) Perform coupon tests to obtain material properties for the three different FRP tubes and FRP-concrete pier cap beam in two principal directions of loading.

The analytical program focused on developing predictive models for the FRPconcrete pier frames and the entire bridge, and consisted of the following components:

(1) Develop Open System for Earthquake Engineering Simulation (OpenSees) models to analyze the FRP-concrete pier frame system, using the Kent-Park concrete model (Taucel et al. 1991), as modified by Scott et al. (1982);

(2) Parametric study of FRP-concrete pier frames, with different frame spans and heights, thickness of the FRP tubes, reinforcement ratios of conventional steel, concrete compressive strengths and levels of axial load as variables; and

(3) Conduct seismic analysis of the entire bridge made of CFFT substructure, using historical earthquake input and different damping ratios. 


\subsection{DISSERTATION STRUCTURE}

This dissertation consists of five chapters. Chapter 1, this chapter, includes background, research objectives, and research approach. Chapter 2 provides a synthesis of literature related to the CFFT systems under flexural, axial-flexural and seismic loading. Chapter 3 summarizes the experimental program, whereas Chapter 4 presents the analytical work including frame simulation, parametric study and the seismic analysis of the entire bridge. Chapter 5 is devoted to summaries, conclusions, and suggestions for future research. 


\section{CHAPTER 2}

\section{LITERATURE REVIEW}

\subsection{FLEXURAL BEHAVIOR OF CONCRETE-FILLED FRP TUBES}

Concrete-filled FRP tubes (CFFTs) combine the FRP tube with concrete core for use as flexural members in civil infrastructure applications. Tests have been conducted by different research groups focusing on the strength and ductility of CFFTs.

Large-scale four-point bending tests were carried out by Davol et al. (2001) on two 13.5 in. diameter carbon FRP shells filled with concrete. Both beams failed in compression, but the shell with additional hoop fibers failed at a much higher strain, implying the effect of confinement. A model was proposed by Davol et al. (2001) to adequately characterize the behavior of CFFT members in flexure.

Lam et al. (2002) conducted a theoretical study on reinforced concrete (RC) circular members wrapped with FRP sheets using available confinement models from the literature. The analytical results showed almost no correlation between confinement level and flexural strength, while ductility was improved significantly as a result of higher confinement levels.

More recently, Cole (2006) tested seven (7) columns with different types of confinement (either FRP tubes or steel spirals) and different longitudinal bars (either steel or FRP bars). The specimens had an outsider diameter of $8.67 \mathrm{in}$. and a length of $8.1 \mathrm{ft}$. Test results showed that steel spiral improved column ductility slightly but not the

flexural strength, as compared to the columns without any spirals. However, the glass 
FRP tube, which consisted of eight layers with fibers oriented in both the longitudinal and hoop directions, improved the flexural strength by $69 \%$, and provided a much greater ductility, as compared to the columns with steel spirals.

\subsection{AXIAL-FLEXURAL BEHAVIOR OF CONCRETE-FILLED FRP TUBES}

Mirmiran et al. (2000) tested a total of sixteen (16) $9.17 \mathrm{ft}$ long CFFT beamcolumns with two types of FRP tubes. Type I tubes had 40 layers, with a symmetric layup consisting of $0^{\circ}$ and $\pm 45^{\circ}$ lamina, and were considered as over-reinforced specimens. Type II tubes had an asymmetric layup consisting of $\pm 55^{\circ}$ lamina, and were considered as under-reinforced specimens. Each specimen was first subjected to a constant axial load, before lateral loads were applied in four-point bending. Two of the specimens were loaded in pure bending without any axial load. From test results, it was concluded that over-reinforced specimens showed little P- $\Delta$ effect and high strength and stiffness, whereas the under-reinforced specimens had lower strength and stiffness with much larger P- $\Delta$ effect due to their larger deformability. Most Type I tubes failed in compression, whereas Type II tubes failed in tension. For both types of the tubes, limited slippage was noted due to their free end conditions. Further analysis revealed FRPconcrete columns to be superior to similar conventional prestressed concrete columns of the same size.

Fan et al. (2000) studied the seismic performance of carbon FRP-wrapped (hoop direction) concrete columns under cyclic loading. Test results indicated that the FRP tube did not increase column strength, but greatly enhanced its hysteretic response up to a displacement ductility of 10. Zhuo et al. (2001) tested bridge columns wrapped with glass 
FRP under shake-table ground motion. They showed that FRP wrapped in hoop direction may significantly increase the ductility. Taking advantage of the off-axis behavior of FRP, Yuan et al. (2002) proposed concrete-filled GFRP tube with $\pm 45^{\circ}$ fiber orientation and without any internal steel reinforcement. They reported that the FRP tube enhanced the strength of concrete by 2.5 times. Coupon tests of FRP tube itself indicated a bilinear response with a distinct yield point similar to the findings of Shao (2003). In Japan, Yamakawa et al. (2001) reported similar observations on the seismic performance of square RC columns that were confined with aramid FRP (AFRP) tubes.

Fam et al. (2003) tested ten (10) CFFT specimens, and used the test results to propose a new moment-thrust interaction diagram. Two types of glass FRP tubes were used in the project with similar wall thickness but different laminate structures. Type A tubes had almost equal fiber percentage, oriented at $3^{\circ}$ and $88^{\circ}$ with respect to the longitudinal direction, while Type B tubes had $70 \%$ of the fibers oriented in $\pm 34^{\circ}$ and $30 \%$ at $80^{\circ}$ with respect to the longitudinal direction. Type A tubes had higher effective elastic modulus in the hoop direction and lower Poisson's ratio, as compared to Type B tubes. Fam et al. (2003) proposed a variable confinement model with reduced confinement level as a function of the eccentricity of the axial load. Layer-by-layer approach was used for the integration process within the cross section. The predicted values matched experimental results rather well. Conclusions were drawn that the shape of the interaction curves of the CFFT columns was similar to that of the conventional RC columns, and that the laminate structure of FRP tubes significantly affected the interaction diagram. For Type B tubes, increasing the amount of fibers in both the axial and hoop directions increased the axial strength. However, for Type A tubes, the axial 
strength was improved mainly due to the increase of fibers in the axial direction. This implied that proper level of hoop confinement may effectively contribute to the axial strength of CFFTs.

\subsection{SEISMIC PERFORMANCE OF CONCRETE-FILLED FRP TUBES}

FRPs have been widely used to retrofit and repair bridge girders and pier columns in recent years, particularly in seismic region. This has encouraged the industry to consider using CFFTs in new bridge construction in seismic regions. As an example, this concept was applied in Route 40 Bridge in Virginia (Fam et al. 2003). However, this relatively new field has been investigated only by a limited number of researchers.

Seible et al. (1996) tested two columns made of concrete-filled carbon FRP tubes, both with $12.3 \mathrm{ft}$ height and $2 \mathrm{ft}$ inside diameter, under simulated seismic actions. The specimen with internal steel reinforcement at the column-footing joint performed superior to the conventional RC column, whereas the specimen without any internal steel reinforcement failed prematurely due to the combined compressive and inter-laminar shear stresses with very little energy dissipation capacity. They concluded that CFFT without any internal steel reinforcement may not be appropriate for use in seismic regions, and that internal reinforcement would be necessary to develop plastic hinge in the system. However, this study was limited to carbon FRP with specific fiber architecture.

Shao (2003) tested six (6) concrete-filled glass FRP tubes to investigate the ductility of CFFTs, and the usage of the internal mild steel reinforcement. Two types of FRP tubes were used; similar to the ones reported by Mirmiran et al. (2000), and discussed earlier in Section 2.2. For each type of tubes, three different steel reinforcement 
ratios $(0 \%, 1.7 \%$, and $2.5 \%)$ were used. Specimens were tested under constant axial loading and reverse cyclic lateral loading. Test results showed that the thin tube (Type II) without any internal steel failed in tension, while exhibiting much more ductile response than the thick one (Type I), which failed in a brittle compression mode even with internal steel reinforcement. This finding showed the importance of fiber architecture. The usage of internal steel reinforcement improved the ductility of both types of FRP tubes, however the effects were more pronounced with the off-axis FRP tubes (Type II). Moreover, the study showed that internal steel in excess of $2 \%$ of the concrete core area may lead to premature crushing failure of the tube. The coupon tests of the thick tube (Type I) showed a linear stress-strain response, whereas that of the thin tube (Type II) exhibited a nonlinear stress-strain response due to the off-axis fiber orientation. A stressstrain model was then developed for each FRP laminate according to their respective coupon tests.

Zhu (2004) conducted a study to investigate the performance of CFFT columns with different connections to RC footings under axial and lateral loadings. Three different types of connection were developed; cast-in-place reinforced, precast reinforced with grouting, and precast post-tensioned without grouting. The test results showed that the various CFFT-RC joints performed quite similarly, as long as the FRP tube was properly embedded into the footing. The sufficient embedment length of FRP tube was considered to be equal to the outside diameter of the tube. A minimum thickness of FRP tube, or a maximum diameter-to-thickness ratio, was found necessary to achieve composite action in the longitudinal direction. 
Ozbakkaloglu (2006) tested four (4) CFFT columns under reverse cyclic lateral loading. One of the specimens had a cantilever span of $3.94 \mathrm{ft}$, while the other three had similar spans of $6.56 \mathrm{ft}$. High-strength concrete (HSC) and normal-strength concrete (NSC) were both used in this study. Test results indicated that shear effect dominated the performance of the short span CFFT column as it failed at a drift ratio of only $4 \%$, while longer columns failed at drift ratios above $10 \%$. For both short and long columns, the presence of carbon FRP tubes significantly improved the strength. As for the ductility, it was observed that (a) inelastic deformability of HSC may be increased by up to $12 \%$ lateral drift ratio with FRP tubes, (b) column deformability decreases with increasing the axial load, and (c) under the same level of applied axial load, HSC column could develop higher lateral drift capacity than NSC column with the same confinement level. Three models were used to predict the column strength. Popovics (1973) model, which accounted for the unconfined HSC produced lower strength values than those observed, while steel-confined concrete model (Razvi and Saatcioglu 1999) overestimated the strength. The FRP-confined model proposed by Saafi et al. (1999) matched the test results rather well. Plastic hinge lengths played important roles on column drift capacity. The lengths were determined based on the transverse strain gages at different heights of the column. The plastic hinge factor was defined as the ratio of the length of yielded region to the column cross-sectional dimension. Test results showed that short column with HSC had the similar plastic hinge factor of 1.0 as that of long column with NSC. Another long column with higher HSC and more FRP layers had a lower plastic hinge factor of 1.20 , as compared with 1.35 for the long column with lower strength HSC and less FRP layers. 
Zhu et al. (2006) used his experimental database (Zhu 2004) to carry out a case study analysis of bridge substructures. The push-over analysis results showed that CFFT frames had much more deformation capacity than RC frames, because of the effectiveness of the FRP tube in extending the plastic hinge zone and developing better energy dissipation characteristics. For a measured earthquake record of $7.5 \sim 7.9$ on the Richter scale, imposed simultaneously in both the longitudinal and transverse directions of the bridge, the analysis showed the RC substructure to reach its ultimate drift capacity, whereas CFFT substructure reached only $28 \%$ of its ultimate drift capacity. The study further showed the superior performance of the CFFT and its capacity to withstand a major earthquake with 2500-year return period, while its conventional RC counterpart would suffer an irreparable damage.

Most recently, Shi et al. (2007) conducted a series of cyclic tests of five CFFT columns and one control RC column to investigate the flexure-dominant versus sheardominant conditions. Five CFFTs used two carbon, one glass, one hybrid, and one offshelf glass FRP tubes. All specimens were 60-in. long (short columns) except for one carbon CFFT, which was 96-in. long (long column). All specimens were tested under constant axial and reverse cyclic lateral loads. Test results showed that all CFFTs outperformed RC column in strength and ductility. The study also showed that combined effects of high moment and high shear on seismic behavior of CFFT columns are insignificant.

Wu et al. (2007) tested four carbon FRP-wrapped circular RC columns and one control RC column under constant axial and reverse cyclic lateral loads to simulate 
seismic action. The columns had diameters of $14 \mathrm{in}$. and were $39.4 \mathrm{in.}$ long. Test results showed that failure modes of the columns changed from shear to flexure, when the layers of carbon FRP increased. The performance of CFFT columns also improved with the increase of FRP layers. 


\section{CHAPTER 3}

\section{CYCLIC TESTS ON FRP-CONCRETE PIER FRAMES}

\subsection{INTRODUCTION}

Experimental work included two-column bent tests, component tests and coupon tests. Four 1/6-scale bridge pier frames, consisting of control reinforced concrete pier frame $(\mathrm{RCF})$, glass FRP-concrete pier frame (GFF), carbon FRP-concrete pier frame (CFF), and hybrid FRP-concrete pier frame (HFF) were tested under reverse cyclic lateral loading with constant axial loads. Most notably, Specimen GFF did not show any sign of cracking at a drift ratio of about $15 \%$ with considerable loading capacity, whereas Specimen CFF showed that lowest ductility with similar load capacity as in Specimen GFF. FRP-concrete columns and pier cap beams were then cut from the tested pier frame specimens, and were tested again in three point flexure under monotonic loading with no axial load. The tests indicated that bonding between FRP and concrete may significantly affect the flexural behavior of the components, and that yielding of steel plays an important role on the flexural strength and ductility of each component. The coupon tests were carried out to establish the tensile strength and elastic modulus of each FRP tube and the FRP mold for the pier cap beam in the two principle directions of loading.

\subsection{TWO-COLUMN BENT TESTS}

\subsubsection{Test Matrix and Specimen Preparation}

The test matrix for the two-column bents included three FRP-concrete pier frames

(FCPF) using different FRP tubes made of glass, carbon, or hybrid (i.e., glass and carbon) 
FRP and one control RC frame, as shown in Table 3.1. The height of the columns was 61 in. and the frame span was 50 in. The pier columns and the pier cap beams in all four specimens were cast from a single batch of concrete with a 28-day measured compressive strength of 6.39 ksi. All footings were cast earlier using another batch of concrete with a 28-day measured compression strength of $4.3 \mathrm{ksi}$. Figure 3.1 sketches the dimensions of the pier frame.

All eight pier frame columns had the same longitudinal reinforcement of eight (8) No. 3 steel bars of Grade $60 \mathrm{ksi}$ along the entire length of the columns, with adequate embedment of $20 \mathrm{in}$. into the footings. The RC columns additionally included a $0.192 \mathrm{in}$. diameter steel wire spiral reinforcement of Grade $60 \mathrm{ksi}$ with 7 in. outside diameter placed at a pitch of 1.25 in. FRP pier frame columns had no transverse steel reinforcement, except for five hoops of 7 in. diameter steel wires placed at a spacing of 10 in., only to hold the longitudinal reinforcement cage in position. A clear cover of 0.5 in. for the steel reinforcement was maintained in all columns. Figure 3.2 shows how the steel reinforcement cages of the FRP columns were prepared. Figure 3.3 shows the completed steel reinforcement cage for FRP columns.

The steel reinforcement in the pier cap beams was four (4) No. 5 bars at the top, and two (2) No. 5 with two (2) No. 6 bars at the bottom. Figure 3.4 shows the detailing of steel reinforcement in the pier cap beams.

All specimens had the same size RC footing, designed specifically overreinforced to avoid any base failure. Figure 3.5 shows the dimensions and the steel reinforcing details in the footings. 
The column formwork for Specimen RCF included two (2) 8 in. diameter sonotubes, whereas for other specimens, FRP tubes were used as the formwork.

One type of the FRP tubes was off-the-shelf product made by filament winding of $\pm 55^{\circ}$ E-glass fibers and epoxy resin, with a nominal outside diameter of 8.64 in. and a wall thickness of 0.14 in. (Figure 3.6).

The other two types of FRP tubes were prepared in the Structures and Construction Lab (SCL) at the Florida International University (FIU), by wrapping resinimpregnated FRP fabrics (Figure 3.7) around sonotubes of the same diameter as that used for the control RC frame. Sikadur 300 epoxy made by Sika Corporation was used as the resin for all the laboratory-made FRP tubes. The hybrid (Figure 3.8) and carbon FRP tubes (Figures 3.9) both consisted of 6 in. overlaps in the hoop direction and 12 in. overlap in the longitudinal direction. In the hybrid FRP tubes, two layers of unidirectional carbon FRP sheets in the longitudinal direction were alternately wrapped with three layers of unidirectional glass FRP sheets in the hoop direction. The unidirectional carbon and glass FRP sheets used were SikaWrap Hex 103C and SikaWrap Hex 100G, respectively, made by Sika Corporation. The carbon FRP tubes were made with two layers of 3TEX bi-directional carbon fiber sheets.

The FRP strength index $\omega_{\text {str }}$ and stiffness index $\omega_{\text {sti }}$ are hereby, defined as: $\omega_{s t r}=f_{F R P} \rho_{F R P} / f^{\prime}{ }_{c}$ or $\omega_{s t r}=f_{F R P} \rho_{F R P} / f^{\prime}{ }_{c u}$, and $\omega_{s t i}=E_{F R P} \rho_{F R P} / E_{c}$, where $f_{F R P}$ and $f^{\prime}{ }_{c}$ are the tensile strength of FRP and the compressive strength of unconfined concrete, respectively; $f^{\prime}{ }_{c u}$ is the compressive strength of confined concrete, after the model by Kent-Park (Taucel et al, 1991), as modified by Scott et al. model (1982) for 
steel-confined concrete in RCF, and Samaan et al. (1998) model for FRP-confined concrete; $\rho_{F R P}$ is the FRP reinforcement ratio in the column; defined as $4 \mathrm{t} / \mathrm{D}$; where $\mathrm{t}$ is the thickness of FRP tube and D is the outside diameter of FRP tube; and $E_{F R P}$ and $E_{c}$ are the moduli of elasticity of FRP and concrete, respectively. These parameters in both hoop and longitudinal directions are listed in Table 3.2 for all four specimens. The total reinforcement strength or stiffness index represents the sum of the respective FRP index and steel index.

The FRP tubes were all embedded 12 in. into the footings (Figure 3.10) to provide sufficient development length for the force transfer, whereas the embedment into the column heads was only 5 in. A few holes and slots were cut out from the FRP tubes to facilitate the pier cap reinforcement in the embedded areas of the column heads (Figure 3.11).

The pier cap beams for the FRP pier frames consisted of FRP formwork made with bi-directional glass fabric. The FRP mold or bath tub was made by wrapping epoxyimpregnated bi-directional glass fabric (GFRP) around a specially built wooden form (Figure 3.12). The finished pier cap beam mold is shown in Figure 3.13.

In order to provide adequate embedment of the longitudinal bars of the columns into the pier cap beam, one piece of No. 3 bar was welded to every two longitudinal bars of the columns by a professional welder, as shown in Figure 3.14. Effective embedment of this method was verified during the tests, especially for Specimen GFF in which the chamfer was extensively damaged, but the columns and the pier cap beam still worked together quite well without any apparent load drop or separation. 
The transition area between the rectangular cross section of the pier cap beam and the circular section of the columns was filled with epoxy prior to casting of concrete. Few No. 3 bars were also placed in the chamfer to improve its rigidity. Figure 3.15 shows the transition zone and the epoxy placement.

The ready-mixed concrete was ordered with a slump of 6 in. Concrete casting was scheduled in two stages. The first casting was carried out for all four footings on April 13, 2007. The second batch for all columns and pier cap beam was cast on May 23, 2007. Adequate numbers of cylinders were prepared to measure the compressive strength of each batch of concrete. The 28-day compressive strengths for the two batches measured as average of at least 3 cylinders were $4.30 \mathrm{ksi}$ and $6.39 \mathrm{ksi}$, respectively. Figures 3.16 through 3.19 show the four specimens after demolding.

The top of the FRP pier cap beam formwork was left open as in a bath tub for casting concrete. After concrete hardened, the top was grinded to provide a smooth and level surface, and the edges were also rounded to avoid sharp corners, as shown in Figures 3.20. After preparing the surfaces and edges of the pier cap beams, two layers of bi-directional glass FRP sheets were wrapped around them. It may be of interest to mention that glass FRP sheets were cut according to the shape of the pier cap beam. The middle region of the beam was wrapped thoroughly, whereas at the column joints, the wraps were U-shaped. Figure 3.21 shows the FRP wrapping of the middle part and the joints. 


\subsubsection{Test Setup and Instrumentation}

The specimens were tested vertically, as shown in Figure 3.22. Each specimen was tied down with 16 threaded rods through two steel beams, as shown in Figure 3.23. All threaded rods were 1 in. diameter Grade B-7 with a yield strength of 105 ksi. Moreover, four threaded rods tied the footing with the steel reaction frame through a steel tie beam (Figure 3.24). The pier cap beam was connected to the actuator using a steel shoe and four threaded rods outside of the pier cap beam section (Figure 3.25). The completed test setup is shown in Figure 3.26. Two large concrete blocks further restrained the footing against any unintended rotation in the horizontal plane. One of the blocks is seen in front of the specimen in Figure 3.26.

Each specimen was subjected to an external post-tensioning force to simulate the dead load acting on the frame, for a total of 25.7 kips corresponding to $0.04 \mathrm{f}_{\mathrm{c}} \mathrm{A}_{\mathrm{g}}$, where $\mathrm{f}^{\prime}{ }_{\mathrm{c}}$ is the 28-day compressive strength of concrete at $6.39 \mathrm{ksi}$, and $\mathrm{A}_{\mathrm{g}}$ is the gross cross sectional area of the two column together. Using two $1 \mathrm{in.} \mathrm{diameter} \mathrm{threaded} \mathrm{rods} \mathrm{through}$ the pier cap beam and the footing, post-tensioning was carried out using two interconnected hydraulic jacks controlled by a single hand pump. The axial load was transferred as concentrated loads at the beam-column joints using a steel spreader beam positioned at the top of the pier cap beam, as shown in Figure 3.27. The axial load was maintained constant during the experiments using a pressure relief valve, as shown in Figure 3.28, when the load was exceeding its target value, and by further pumping when

the load was dropping below its target value. Further measurements of the axial load showed that the loading system was quite effective, as the axial load fluctuation was limited to less than $\pm 2.5 \%$ of its target value. 
Each specimen was instrumented with 8 string pots, 8 linear potentiometers, 26 strain gages, and 2 inclinometers. The instrumentation plan is shown in Figure 3.29.

Seven string pots with a range of $12 \mathrm{in}$. were placed on the two sides of each specimen to monitor the lateral displacements of the frame at different locations. An additional string pot was set at the mid-span of the pier cap beam to monitor its vertical deflection.

Slippage of the FRP tube at column base and column head were monitored using eight 1.5 in. range potentiometers, with one placed on each side of each column at each end, i.e., top and bottom. Two inclinometers were placed at the beam-column joints to monitor their rotations.

Four strain gages were pre-attached to the longitudinal steel bars in the column at each side before concrete casting. Sixteen strain gages were placed along the two columns in the longitudinal direction, and two strain gages were placed at the bottom of each column in the hoop direction. Finally, two strain gages were placed at the top and bottom of the pier cap beam in the mid span.

Two high-speed data acquisition systems, Vishay 6000 and Megadac 3415, were used simultaneously to provide for the 46 channels required in these tests. In order to synchronize the data from the two different data acquisition systems, the signals from the actuator displacement and load were split as T-connections and were connected to both data acquisition systems. Recording frequency was set at $1 \mathrm{~Hz}$ for both data acquisition systems. 


\subsubsection{Test Procedure and Observations}

Each specimen was first subjected to the external post-tensioning force to simulate the dead load acting on the frame. Subsequently, a reverse cyclic lateral load was applied in displacement control in a number of incremental steps. Frame drift was applied in terms of displacement ductility $\mu$, which is the ratio of the imposed displacement to a reference displacement. The reference displacement corresponded to the first yielding of the internal steel reinforcement in the control $\mathrm{RC}$ frame, which was measured as $0.36 \mathrm{in}$.

All specimens were tested at a constant loading rate of $0.36 \mathrm{in} . / \mathrm{min}$. At each level of ductility, two full cycles of reverse lateral loading were applied. The cyclic loading regime is shown in Figure 3.30. Loading for each specimen continued until either a load drop of approximately $20 \%$ of the maximum achieved load or an obvious irreparable damage was noted. Subsequently, the axial load was removed for safety reasons, and the specimen was further loaded monotonically to failure in the lateral direction.

\section{Cracks in FRP Tubes}

Specimen GFF remained intact without any noticeable matrix cracking in the FRP tubes. On the other hand, the FRP tubes in Specimens HFF and CFF cracked with a sudden and loud noise and a noticeable load drop.

In Specimen CFF, popping sound was heard at $\mu=2$. Fibers cracked in tension at the top and bottom of the columns. Cracks extended further at $\mu=3$ (Figure 3.31) and widened significantly to the middle of the section during $\mu=4,5$, and 6 . At the first push cycle of $\mu=7$, a loud sound was heard and a vertical crack appeared (Figure 3.32) at the 
top of one column, causing a noticeable load drop just before the test was terminated. When the axial loading was removed, the specimen was loaded again monotonically through $\mu=7$. The vertical crack extended significantly and the test was stopped.

In Specimen HFF, cracks were first observed at $\mu=3$. Cracks then began to enlarge at $\mu=5$. Following on to $\mu=6$ and 7 , cracks extended to the middle of the column base section with a loud popping sound and a sudden load drop (Figures 3.33). At

$\mu=9$, which was the last cycle of the test, the crack opened wide enough for the concrete core to be visible from the outside (Figures 3.34).

\section{Cracks in Pier Cap Beams}

No crack was noted in Specimen CFF, whereas cracks were visible in Specimen HFF (Figure 3.35) at $\mu=8$ around the pier cap beam-column connection, and in Specimen GFF at $\mu=3$ in the epoxy transition zone (Figure 3.36).

\section{Separation and Slippage at Column Base and Column Head}

In Specimen CFF, no large separation or slippage was observed at either end of the columns. In Specimen HFF, noticeable slippage was observed only at beam-column joints, but not at column base (Figure 3.37).

In Specimen GFF, significant slippage and separation at both ends of the columns were noted after $\mu=5$ (Figures 3.38). The separation and slippage at beam-column joints were larger than those at column base. However, the separation and slippage seemingly did not cause any disturbance in the load transfer mechanism between the columns and the footing, or between the columns and the pier cap beam. 


\section{Failure Modes}

Specimen RCF demonstrated typical cracking and failure mode of conventional reinforced concrete specimens. Visible cracks were observed at $\mu=1 \sim 2$. Major flexural cracks occurred at $\mu=3 \sim 4$, mostly concentrated at the top and bottom of the columns, as shown in Figure 3.39, along with some $45^{\circ}$ shear cracks. The pier cap beam-column connection was the weakest part of the specimen, where a large number of cracks were observed. The lateral load did not increase after $\mu=3$, and began to drop after $\mu=6$ when the test stopped. After removing the axial load, the specimen was loaded monotonically up to $\mu=10$. Concrete cover at the chamfer and column-footing connections spalled, revealing the steel spirals (Figure 3.40).

The failure of Specimen CFF was caused by the vertical crack at the top of one column, as mentioned before, at $\mu=7$ with a noticeable load drop.

Specimen HFF was loaded up to $\mu=9$, when the load dropped significantly. Horizontal cracks had already opened widely during $\mu=6 \sim 7$ with a large popping sound. Specimen GFF exhibited a superior performance as it was loaded up to $\mu=10$ (Figure 3.41). No load drop was noted, and the FRP tubes remained intact with no cracks. After removing the axial load, the specimen was loaded monotonically up to $\mu=25$ or a drift ratio of $15 \%$, which was almost the stroke limitation of the actuator. Even then, the lateral load remained constant, although the pier cap beam-column connections were severely damaged (Figure 3.42). 


\subsubsection{Test Results and Discussions}

\section{Load-Deflection Response}

Figures 3.43 through 3.46 show the hysteretic load-deflection responses of the four tested specimens. The deflection is shown both as the absolute lateral displacement $\delta$ and the normalized drift ratio $\delta / L$, where $L$ is the frame height of $61 \mathrm{in}$. from the top of $\mathrm{RC}$ footing to the mid-depth of pier cap beam. The hysteretic loops in the figures are marked with their respective ductility factor $\mu$.

The hysteretic response of Specimen HFF (Figure 3.44) shows that the load capacity of the column continued to increase until $\mu=6$. Then, the load dropped by $8.9 \%$ at $\mu=7$, when the cracks extended significantly, and by $20 \%$ when the specimen was being pushed at $\mu=9$. Specimen HFF had the maximum load capacity of $53 \mathrm{kips}$, the highest among all four specimens.

Specimen CFF had a load capacity of about 36 kips, similar to that of Specimen GFF. The load dropped by about $25 \%$ in the first cycle of $\mu=7$, when the vertical crack appeared with a loud popping noise, after which the test was stopped.

The performance of Specimen GFF was quite impressive and certainly superior to all others tested in this study. The load continued to increase, and did not drop even at $\mu$ $=10$, which was the maximum preset limit for the cyclic loading test due to safety reasons in the presence of axial load. However, the load was quite steady after $\mu=8$ at about 37 kips. No cracks were noticed on the GFRP tubes throughout the test.

The peak loads for all specimens are listed in Table 3.3. 


\section{Load-Deflection Envelop Curves}

Figure 3.47 shows a comparison of the load-deflection envelope curves for all four specimens. Specimen HFF demonstrated the highest load capacity and initial stiffness with considerable ductility. Specimens GFF and CFF exhibited similar performances to each other before $\mu=7$. Subsequently, Specimen CFF developed a large vertical crack and a major load drop, whereas Specimen GFF showed no cracks in the FRP tubes and continued to stiffen until $\mu=10$. The initial stiffness of all specimens is listed in Table 3.3 .

Figures 3.48 and 3.49 show the comparisons of the residual displacements and residual loads, respectively, for all FRP specimens at different ductility factors in the push direction.

Residual displacements varied between a minimum of $1.6 \%$ of column height for Specimen HFF and a maximum of $2.1 \%$ of column height for Specimen GFF. Specimen CFF had the largest residual displacement at zero load, whereas, Specimen HFF exhibited the smallest after $\mu=4$. Residual displacement for Specimen GFF and Specimen HFF were quite similar for the most part.

Specimen CFF also had the largest residual load at neutral position, whereas Specimen GFF exhibited the smallest but quite close to that of Specimen HFF. In terms of their respective ultimate load, the residual load for Specimens CFF, GFF, and HFF were $22 \%, 12 \%$, and $18 \%$, respectively.

All specimens were tested monotonically after the cyclic loading was completed, and the axial load was removed. Figure 3.50 shows the monotonic load-deflection responses of the four specimens after removing the axial load. Specimens GFF and CFF 
had the largest and smallest residual ductility, respectively. In Specimen GFF, the load did not drop even at $\mu=25$ with a $15 \%$ drift ratio, and yet no cracks were noticeable in the glass FRP tubes.

\section{Effect of Strength Index}

Figure 3.51 shows the relationship between sectional moments and strength index. The moments are normalized as $\mathrm{M} / \mathrm{M}_{\mathrm{RCF}}$, where $\mathrm{M}$ is the moment of each specimen at the column base, and $\mathrm{M}_{\mathrm{RCF}}$ is the moment for Specimen RCF at its column base. The moments include both the primary moment from the lateral load and the secondary moment from the P- $\Delta$ effect of the axial load. Total strength index is the sum of the FRP strength index and the steel strength index using both confined and unconfined concrete compressive strength. The confined strength index considers the hoop effect of FRP tubes and steel spirals. A natural logarithmic trend is defined for each case with their respective goodness of the fit $R^{2}$.

\section{Slippage at Column Base and Column Head}

As explained earlier, a total of 8 potentiometers were placed to monitor slippage, one at the each end of each column and at each side of the section. Each potentiometer was labeled with three letters, for instance WTE. The first letter donates the position of the column, i.e., $\mathrm{W}$ for the west column and the E for east column. The second letter donates the position of the column end, i.e., $\mathrm{T}$ for top and B for bottom. The last letter donates the side of the potentiometer at the column end, i.e., $\mathrm{W}$ for the west side and $\mathrm{E}$ for the east side. Therefore, WTE refers to the potentiometer at the east side, at the top end of the west column, as was shown earlier in Figure 3.29. 
Due to the malfunction of some of the potentiometers, some data were not recorded properly and could not be recovered. Also, some potentiometers detached from the specimens during the tests at larger displacements. The recorded data is shown in Table 3.3.

Figure 3.52 shows typical slippage patterns for ETW and ETE in Specimen GFF. Generally, the slippage on the tension side was always larger than that on the compression side. Specimen GFF showed the largest slippage at about 0.4 in. Slippage in Specimen CFF was about 0.31 in. Specimen RCF had the smallest slippage of 0.13 in.

\section{Energy Dissipation}

Figure 3.53 shows the cumulative dissipated energy in all four tested specimens versus their respective drift ratios. For the same drift ratio, Specimen HFF showed the highest cumulative dissipated energy and dissipation rate, i.e., slope of the curve. Specimens GFF and CFF showed quite similar levels of dissipated energy and dissipation rate to each other. Specimen RCF displayed the worst performance. The cumulative energy data is summarized in Table 3.3.

\section{Deflected shapes of Pier Frames}

For comparisons, the deflected shapes of all four specimens at $\mu=6$ are plotted in Figures 3.54 and 3.55 for the west and east columns, respectively. Due to limited accuracy of the string pots, the top points in Figure 3.54 did not match exactly as expected. However, it may be concluded that Specimens RCF and HFF had the largest and smallest deformations, respectively. 


\section{Longitudinal Strain Profiles and Spatial Distribution of Curvatures}

In Specimen RCF, the readings from the strain gages at top of the columns were visibly affected by cracks at or near their locations. The patterns of the strain profiles for Specimens CFF and HFF were quite similar perhaps because of the cracks in the hoop direction at both ends of the columns in those specimens.

The most reliable strain profile data was that of Specimen GFF (Figures 3.56 through 3.59), which did not show any crack in FRP tubes throughout the test. At a height of $20 \mathrm{in.} \mathrm{(1/3} \mathrm{column} \mathrm{length),} \mathrm{the} \mathrm{readings} \mathrm{were} \mathrm{close} \mathrm{to} \mathrm{zero} \mathrm{similar} \mathrm{to} \mathrm{that} \mathrm{of}$ Specimen RCF. The readings for the strain gages at column base were the largest. Some of the readings for the bottom strain gages decreased in the last several cycles, primarily because of the damage to the strain gages at large deflections. The data from the strain gages at the heights of $10 \mathrm{in.}$ and $40 \mathrm{in.}$ were quite similar, indicating that the inflection point was approximately at a height of $25 \mathrm{in.}$

Figures 3.60 and 3.61 show the spatial distribution of curvatures along the east and west columns of Specimen GFF, respectively.

\section{Plastic Hinge Length}

As mentioned earlier, the strain gage data for Specimen GFF was most reliable. Therefore, plastic hinge length for Specimen GFF is plotted at each ductility level in Figure 3.62. The plastic hinge length increased substantially after $\mu=2$, when the steel reinforcement at the column base yielded extensively. The plastic hinge length reached almost $1 / 3$ of the column length in the last cycle. The plastic hinge length of Specimen RCF could not be drawn exactly due to the cracks on the concrete surface. However the 
strain gauge readings at $10 \mathrm{in}$. from the column base remained less than the steel yielding strain. Therefore, a general conclusion could be drawn that the plastic hinge length in Specimen RCF is less than 10 in. which is $1 / 6$ of the column length, or half the plastic hinge length in Specimen GFF. This attribute of FRP tubes in extending the plastic hinge length of RC column, as also demonstrated by Zhu et al. (2006), has a pronounced effect on the performance of bent columns under seismic action.

\section{Pinching Effect}

Pinching factor is defined as the ratio of the maximum width of the hysteretic load-deflection response and its minimum width at the origin (Shao 2005). Table 3.3 lists the pinching factors for all four tested specimens. All specimens had similar pinching factors, except for Specimen HFF with a much greater value by about $50 \%$ over that of the others. The primary reason for the pinching effect is the slippage between the yielded steel reinforcement and the surrounding concrete during the opening and closing of concrete cracks under cyclic loading. Normally, pinching effect depends mainly on the internal steel reinforcement rather than the FRP tube. However, this data shows that certain type of FRP tube may influence the pinching effect. Figure 3.63 shows the pinching factors versus stiffness index, which accounts for the sum of FRP stiffness index and steel stiffness index. A linear relationship with a high goodness of the fit implies that steel together with FRP affects the pinching.

\section{Rotations of the Pier Cap Beam-Column Joints}

Figure 3.64 shows the joint rotations of the pier cap beams in all specimens at different levels of ductility displacement. Counterclockwise rotation was considered 
positive. Data was generated from two inclinometers at the two pier cap beam-column joints on the south face of each specimen. The largest joint rotations for all specimens are listed in Table 3.3. Specimens RCF, CFF, and HFF had similar small rotations of about maximum $0.6^{\circ}$ clockwise in push, and the same amount but counterclockwise in pull. Specimen GFF had the largest rotation readings of about $0.9^{\circ}$ in the same directions as the others in push and pull. For all specimens, the two readings from the inclinometers were quite close, implying the rigidity of the pier cap beam.

\section{Hoop Strains at Column Base}

Figures 3.65 and 3.66 show the strain profile in the hoop direction at column base for all specimens at different levers of displacement ductility. On each side of the column in the plane of loading, there was one strain gage attached in the hoop direction.

All readings from the strain gages were below 0.002 except for Specimen GFF which had the largest transverse strain of 0.008 in tension and 0.004 in compression. For Specimens HFF and CFF, the readings were quite similar. This may be attributed to the activation of confinement in Specimen GFF, contrary to the others.

\section{Mid-Span Strains and Mid-Span Deflections of Pier Cap Beam}

Figure 3.67 shows the top and bottom strains at the mid-span of the pier cap beam for different levels of displacement ductility. All readings were rather small, which confirmed that the mid-span was the inflection point of the pier cap beam. The strain profiles for all specimens were quite similar, which showed the behaviors of the pier cap beams at their mid-span did not change significantly during the tests for different types of FRP columns. 
The mid-span deflections were generally negligible. Specimen RCF had the lowest mid-span deflection at about 0.1 in. Specimens HFF and CFF had their mid-span deflections at about 0.25-0.3 in., whereas Specimen GFF had the largest mid-span deflection at about 0.4 in. Figure 3.68 shows the response of vertical deflection versus lateral deflection for Specimen GFF.

For comparison, the mid-span deflections of all specimens at different levels of displacement ductility are plotted in Figure 3.69. Specimens CFF and GFF had almost the same vertical deflections at different levels of $\mu$, while Specimen HFF showed the smallest vertical deflection.

\section{Concluding Remarks}

The following conclusions may be drawn from the cyclic tests of the four pier frame specimens:

1. FRP tubes increased load capacity of pier frames significantly, as compared to the conventional RC pier frame.

2. Specimen HFF demonstrated the highest load capacity and initial stiffness among the four specimens.

3. Specimen GFF showed the highest ductility with considerable load capacity, and as well the largest hoop strains at the column base among the four specimens. The specimen did not show any crack even at a drift ratio of $15 \%$.

4. Specimen CFF had the least ductility among the FRP-concrete pier frames with a vertical crack developing after the earlier cracks in the hoop direction enlarged.

5. Total strength index using the compressive strength of confined concrete showed a good correlation to the normalized moment of the pier frames. 
6. The pinching behavior in pier frames is affected significantly by the internal steel reinforcement as well as the type of FRP tube.

7. Pier cap beams worked as rigid beams with small rotations and an inflection point at mid-span.

8. The tests showed the adequacy of 12 in. (1.5D) and 5 in. (0.625D) embedments of the FRP tubes in the footing and the cap beam, respectively, the latter with welding, where $\mathrm{D}$ is the diameter of the column.

9. Specimens HFF and GFF had the least residual deflections at zero loading (for different ductility factors) and the least residual forces at neutral positions. Specimen CFF exhibited largest residual forces at neutral positions and the largest residual deflections at zero loading.

10. At the same drift ratio, Specimen HFF had the highest cumulative dissipated energy and dissipation rate. Specimen GFF performed almost the same as Specimen CFF, before the cracks in the latter enlarged and the vertical crack appeared.

11. The deflected shapes of the columns were not linear throughout their lengths. The inflection points were generally around the height of 25 in. The plastic hinge length of Specimen GFF was twice as large as that of Specimen RCF, at about 1/3 of the column length.

12. The slippage and separation of FRP tube at column base was the largest in Specimen GFF, as compared with those of the other FRP-concrete pier frame specimens. 


\subsection{COMPONENT TESTS}

\subsubsection{Test Matrix and Specimen Preparation}

The test matrix for the component tests included three FRP-concrete pier cap beam using bi-directional glass FRP and six FRP-concrete columns with carbon, glass, and hybrid FRP tubes. All nine specimens were cut from the FRP-concrete pier frame specimens discussed in Section 3.2. The test matrix is shown in Table 3.4. The six CFFT columns were cut between the bottom of the chamfer and the top surface of the footing. The CFFT columns were 44 in. long with approximately 8.5 in. outside diameter. The pier cap beam had a span of $72 \mathrm{in.} \mathrm{and} \mathrm{a} \mathrm{square} \mathrm{cross} \mathrm{section} \mathrm{of} 10 \mathrm{in.} \times 10 \mathrm{in}$. All nine specimens were cast from a single batch of concrete with a 28-day measured compressive strength of $6.39 \mathrm{ksi}$, as discussed earlier in Section 3.2.

\subsubsection{Test Setup and Instrumentation}

Each pier cap beam was placed on a strong RC footing and was leveled onto a formed and cast mortar-based support. Two neoprene pads were placed at the mid-span with a steel plate on top. One of the pier cap beam was turned upside down to test its negative flexural strength. Figures 3.70 and 3.71 show the two test setups.

Each pier cap beam specimen was instrumented with one 12 in. range string pot at mid-span, two (2) strain gages at mid-span (top and bottom), and two (2) inclinometers at mid-height of the beam-column joints, as shown in Figure 3.72.

The CFFT columns were placed on a strong RC footing with two semi-circular steel supports (Figure 3.73). Hydrostone was placed between the support and the 
specimen to improve contact surface. A similar semi-circular steel was used as loading plate for the actuator. An overview of the test setup is shown in Figure 3.74.

Instrumentation for the CFFT columns included a 12 in. range string pot at midspan, two (2) string pots at the two ends of the specimen to monitor support displacements due to the deformation of hydrostone, and one strain gauge at the midspan, as shown in Figure 3.75.

\subsubsection{Test Procedure and Observations}

Each specimen was subjected to a monotonic load at mid-span applied in displacement control at a rate of $0.008-0.01 \mathrm{in} . / \mathrm{min}$ for pier cap beams and $0.03-0.07$ in./min for CFFT columns.

The first pier cap beam specimen test was stopped when the beam overturned sideways due to instability of the support and the loading mechanism. The FRP form was then removed to verify the condition of the concrete inside (Figure 3.76). Some flexural cracks appeared at the top and bottom. In the second pier cap beam test, the top FRP wrap showed cracks, but the bottom was still intact. The third and last pier cap beam was tested upside down. It showed a steady increase in load until failure, with a sudden load drop accompanied by a loud noise, and some cracks at the tension face of the specimen (Figure 3.77).

The carbon CFFT columns developed longitudinal cracks along the column. In the first carbon CFFT column, the crack that had developed in the pier frame tests extended along the length of the column at a load of 30 kips. The second carbon CFFT column failed in a similar manner with a large crack along the column but at twice the 
capacity of the first column (Figure 3.78). The slippage of concrete core relative to the FRP tube was found negligible.

The hybrid CFFT columns had the highest load capacity of all CFFT columns at about 140 kips. The first hybrid column failed with a huge sound and appearance of major cracks at the bottom of the column at mid-span (Figure 3.79). The second hybrid column specimen reached about the same load before the actuator lost its stability and the test was stopped. No visible cracks, however, were found at the bottom, while large slippage was noted at both ends.

The glass CFFT columns showed an impressive performance due to their large deflections (Figure 3.80). Both columns reached a load of 70 kips. Noise could be heard throughout the test as cracks began to appear along the filament angle at the bottom of the specimens at mid-span. A large noise was heard as the small cracks joined to make a large crack, leading to a significant load drop and test stoppage. Similar to the hybrid CFFT columns, large visible slippage was noted at both ends (Figure 3.81).

\subsubsection{Test Results and Discussions}

\section{Pier Cap Beams}

Figure 3.82 shows the residual load-deflection responses of the three FRPconcrete pier cap beam specimens. The first two specimens (1 and 2) showed almost identical ealsto-plastic response. For the third specimen tested upside down, the response was generally nonlinear with a sudden load drop due to the cracks in the FRP on tension side of the test setup. The difference between this specimen and the other two was in the bond between FRP and concrete. In the first two specimens, the FRP sheet at the bottom 
was not bonded to concrete, whereas in the third specimen, the FRP sheet was bonded with epoxy to the concrete at the top, which was in tension when tested upside down.

Figures 3.83 shows the residual load-strain responses of the three pier cap beams. The strains are those measured at the top and bottom at mid-span. In the first two specimens, yielding plateau was initiated at a strain between 0.0022 and 0.0028 . For the

third specimen tested upside down, the response was nonlinear, and the load dropped suddenly when the strain reached 0.009 with the FRP sheet cracking and with the loss of composite action between FRP and concrete. It should be noted that in the first pier cap specimen, the strain gauge on the compression side was affected by the two neoprene pads that had somehow restrained the deformation of the FRP sheets during the testing, and therefore, showed an abnormal tensile stress on the top.

Figure 3.84 shows the end rotations versus mid-span deflections of the three tested pier cap beams. In all specimens, clockwise rotations were considered positive. The end rotations were clearly very small, and did not exceed $1.20^{\circ}$ for the first two specimens and $1.50^{\circ}$ for the third specimen tested upside down.

\section{CFFT Columns}

The residual load-deflection responses of the three types of CFFT columns are shown together in Figure 3.85. For each column type, two columns were tested.

The hybrid CFFT column showed a bilinear response with a small transition zone in between. The carbon CFFT column showed a linear response up to $30 \mathrm{kips}$, but afterwards followed a nonlinear response to failure. The glass CFFT column had a linear load-deflection response up to a load of about $30 \mathrm{kips}$, followed by a softening nonlinear 
response with a distinct and prolonged yield plateau. Both glass CFFT columns had a similar load capacity of 70 kips.

The carbon and glass CFFT columns had the highest and lowest initial stiffness, respectively. On the other hand, the carbon and glass CFFT columns had the least and the most residual deflections, respectively.

Figure 3.86 shows the residual load-strain responses of the three types of CFFT columns together. The carbon CFFT column showed a bilinear response transitioning around a strain of 0.0015 . This was lower than the other two types of CFFT columns due to the per-damaged state of the carbon FRP tube. For hybrid CFFT columns, a similar bilinear response was noted with a transition strain of $0.002-0.003$, corresponding to the yielding of steel. The ultimate strain in the column reached 0.0075, when FRP cracked. For glass CFFT columns, an elasto-plastic response was noted with a transition strain of 0.003, a yield plateau and some strain hardening near the failure.

\section{Concluding Remarks}

The following conclusions may be drawn from the monotonic loading of the pier cap beams and CFFT columns cut from the already tested pier frame specimens:

1 The interface bond between FRP and concrete affects the flexural behavior of pier cap beams. When concrete is cast against FRP with no epoxy, yielding of steel would play an important role in the flexural strength and ductility of the beam, and therefore, the response follows that of steel reinforcement.

2 Glass CFFT columns performed very well with quite large deflections and tensile strains at mid-span, as compared with the other two types of CFFT columns. Hybrid CFFT columns had the largest load capacity and a bilinear load-deflection response. 
Carbon CFFT columns had the least load capacity, tensile strain, mid-span deflection, and ductility; perhaps due to a large horizontal crack that was developed in the pier frame tests. On the other hand, larger slippage was noted in glass and hybrid CFFT columns, as opposed to the carbon CFFT columns.

\subsection{COUPON TESTS}

\subsubsection{Test Matrix and Specimen Preparation}

Tension tests were conducted on 34 FRP coupons for the carbon and hybrid CFFT columns, and the pier cap beam. Test matrix for FRP coupons is shown in Table 3.5, where each coupon specimen is labeled with 5 letters. The first there letters denote the specimen type, while the fourth letter indicates the FRP direction, i.e., H represents the hoop direction and $\mathrm{L}$ specifies the longitudinal direction. The last letter is a number that identifieds the number of layers of the coupon specimen in Roman numerals. All coupons were tested according to the ASTM standard D3039. A detailed coupon test for the glass CFFT column was carried out in an earlier study (Shao 2004), and its results were adopted here.

\subsubsection{Test Setup and Instrumentation}

The coupon samples were prepared in the laboratory using the same procedure as that used for the large-scale specimens. The coupons were mounted in the grips of the mechanical testing machine, and were monotonically loaded in tension while recording the load. Strain gage was attached at the mid-point (see inset of Figure 3.87) with Vishay 6000 data acquisition system used for recording. 


\subsubsection{Test Procedure and Observations}

A total of 34 coupons were prepared and tested, as shown in Table 3.5. All coupons showed a brittle failure mode with mid-point rupture and a sudden loud noise. The inset of Figure 3.87 shows the failure mode of the carbon FRP coupon.

\subsubsection{Test Results and Discussions}

Figure 3.87 shows the average measured stress-strain responses for the all coupon specimens and the inset shows the typical failure mode of the carbon coupon test. All coupons showed a linear elastic response. The carbon coupon had an ultimate strength of $17 \mathrm{ksi}$ and an ultimate strain of 0.0065 in the longitudinal direction, and $51.9 \mathrm{ksi}$ and 0.0096 in the hoop direction. The hybrid coupon had an ultimate strength of $36.9 \mathrm{ksi}$ and an ultimate strain of 0.0075 in longitudinal direction, and $26.1 \mathrm{ksi}$ and 0.011 in the hoop direction. The FRP pier cap beam had the largest ultimate strains of 0.012 and 0.013 in the longitudinal and hoop directions, respectively. The ultimate strengths were similar in both directions for the FRP pier cap beam at $28 \mathrm{ksi}$. 
Table 3.1 Test Matrix of FRP-Concrete Pier Frame Specimens

\begin{tabular}{|c|c|c|c|c|c|c|}
\hline $\begin{array}{c}\text { Specimen } \\
\text { Name }\end{array}$ & $\begin{array}{c}\text { Frame } \\
\text { Span } \\
\text { (in.) }\end{array}$ & $\begin{array}{c}\text { Frame } \\
\text { Height } \\
\text { (in.) }\end{array}$ & $\begin{array}{c}\text { Column } \\
\text { Diameter } \\
\text { (in.) }\end{array}$ & $\begin{array}{c}\mathbf{f}_{\mathbf{c}} \\
\text { (ksi) }\end{array}$ & $\begin{array}{c}\text { Longitudinal } \\
\text { FRP }\end{array}$ & $\begin{array}{c}\text { Transverse } \\
\text { FRP }\end{array}$ \\
\hline RCF & 50.0 & 61.0 & 8.0 & 6.39 & None \\
\hline CFF & 50.0 & 61.0 & 8.4 & 6.39 & 2 Layers of Bi-directional Carbon \\
\hline GFF & 50.0 & 61.0 & 8.6 & 6.39 & \multicolumn{2}{|c|}{ $\pm 0.55^{\circ}$ E-Glass } \\
\hline HFF & 50.0 & 61.0 & 8.7 & 6.39 & $\begin{array}{c}\text { 2 Layers of } \\
\text { Carbon }\end{array}$ & $\begin{array}{c}\text { 3 Layers of } \\
\text { Uni-directional } \\
\text { Glass }\end{array}$ \\
\hline
\end{tabular}

Table 3.2 Design Parameters for Pier Frame Specimens

\begin{tabular}{|c|c|c|c|c|c|c|c|}
\hline \multirow{2}{*}{$\begin{array}{c}\text { Specimen } \\
\text { Name }\end{array}$} & \multicolumn{2}{|c|}{$\begin{array}{c}\text { Steel Reinforcement } \\
\text { Ratio }\end{array}$} & \multirow{2}{*}{$\begin{array}{c}\text { Confinement } \\
\text { Effectiveness } \\
\mathbf{f}_{\mathbf{c u}} / \mathbf{f}^{\prime}{ }^{*}\end{array}$} & \multicolumn{2}{|c|}{$\begin{array}{l}\text { Total Reinforcement } \\
\text { Strength Index ** }\end{array}$} & \multicolumn{2}{|c|}{$\begin{array}{l}\text { Total Reinforcement } \\
\text { Stiffness Index }\end{array}$} \\
\hline & Longitud. & Hoop & & Longitud. & Hoop & Longitud. & Hoop \\
\hline RCF & $1.75 \%$ & $1.24 \%$ & 1.13 & - & - & - & - \\
\hline CFF & $1.58 \%$ & - & 1.54 & $\begin{array}{c}0.118 \\
(0.076)\end{array}$ & $\begin{array}{c}0.317 \\
(0.205)\end{array}$ & 0.050 & 0.028 \\
\hline GFF & $1.50 \%$ & - & 1.59 & $\begin{array}{c}0.234 \\
(0.148)\end{array}$ & $\begin{array}{c}0.346 \\
(0.219)\end{array}$ & 0.028 & 0.023 \\
\hline HFF & $1.45 \%$ & - & 2.04 & $\begin{array}{c}0.693 \\
(0.340)\end{array}$ & $\begin{array}{c}0.479 \\
(0.235)\end{array}$ & 0.066 & 0.140 \\
\hline
\end{tabular}

* Based on Kent-Park model, as modified by Scott et al.(1982) for steel-confined concrete, and Samaan et al. (1998) model for FRP-confined concrete.

** Numbers in parenthesis are based on compressive strength of confined concrete. 
Table 3.3 Response Measures for Pier Frame Specimens

\begin{tabular}{|c|c|c|c|c|c|c|}
\hline $\begin{array}{c}\text { Specimen } \\
\text { Name }\end{array}$ & $\begin{array}{c}\text { Initial } \\
\text { Stiffness } \\
\text { (kip/in.) }\end{array}$ & $\begin{array}{c}\text { Peak } \\
\text { Load } \\
\text { (kips) }\end{array}$ & $\begin{array}{c}\text { Max. } \\
\text { Joint } \\
\text { Rotation }\end{array}$ & $\begin{array}{c}\text { Max. } \\
\text { Slippage } \\
\text { (in.) }\end{array}$ & $\begin{array}{c}\text { Cumulative } \\
\text { Energy } \\
\text { (kip-in.) }\end{array}$ & $\begin{array}{c}\text { Pinching } \\
\text { Factor }\end{array}$ \\
\hline RCF & 30.9 & 18.5 & $0.28^{\circ}$ & 0.13 & 32.7 & 1.86 \\
\hline CFF & 41.7 & 36.0 & $0.37^{\circ}$ & 0.32 & 65.2 & 2.00 \\
\hline GFF & 46.9 & 37.6 & $0.57^{\circ}$ & 0.40 & 109.7 & 1.83 \\
\hline HFF & 56.6 & 53.3 & $0.90^{\circ}$ & $0.11^{*}$ & 131.3 & 3.00 \\
\hline
\end{tabular}

*Recording was abrupted before the conclusion of the test.

Table 3.4 Test Matrix of Pier Cap Beams and CFFT Columns

\begin{tabular}{|c|c|c|c|c|c|}
\hline Specimen Name & $\begin{array}{c}\text { Flexural } \\
\text { Span } \\
\text { (in.) }\end{array}$ & $\begin{array}{c}\text { Cross } \\
\text { Section }\end{array}$ & $\begin{array}{c}\mathbf{f} \text { 'c } \\
\text { (ksi) }\end{array}$ & $\begin{array}{c}\text { Longitudinal } \\
\text { FRP }\end{array}$ & Transverse FRP \\
\hline Pier Cap Beam & 72.0 & 10 in. $\times 10$ in. & 6.39 & \multicolumn{2}{|c|}{ Bi-directional Glass } \\
\hline $\begin{array}{c}\text { Glass CFFT } \\
\text { Column }\end{array}$ & 44.0 & $\begin{array}{c}8.6 \text { in. } \\
\text { Diameter }\end{array}$ & 6.39 & \multicolumn{2}{|c|}{17 Layers of $\pm 55^{\circ}$ E-Glass } \\
\hline $\begin{array}{c}\text { Carbon CFFT } \\
\text { Column }\end{array}$ & 44.0 & $\begin{array}{c}8.4 \text { in. } \\
\text { Diameter }\end{array}$ & 6.39 & \multicolumn{2}{|c|}{ 2 Layers of Bi-directional Carbon } \\
\hline $\begin{array}{c}\text { Hybrid CFFT } \\
\text { Column }\end{array}$ & 44.0 & $\begin{array}{c}8.7 \text { in. } \\
\text { Diameter }\end{array}$ & 6.39 & $\begin{array}{c}\text { 2 Layers of Uni- } \\
\text { directional } \\
\text { Carbon }\end{array}$ & $\begin{array}{c}\text { 3 Layers of Uni- } \\
\text { directional Glass }\end{array}$ \\
\hline
\end{tabular}

Table 3.5 Test Matrix of FRP Coupons

\begin{tabular}{|c|c|c|c|}
\hline Specimen Name & \multirow{2}{*}{ Manufacturer } & Architecture & $\begin{array}{c}\text { Number of } \\
\text { Specimens* }\end{array}$ \\
\hline CFF(H)-II & \multirow{2}{*}{ 3TEX P3W-C1059 } & Hoop = Warp & $8(5)$ \\
\cline { 3 - 4 } & & Longitudinal = Fillet & $5(5)$ \\
\hline PCB(L)-II & \multirow{2}{*}{ 3TEX P3W-GII } & Hoop = Warp & $5(3)$ \\
\hline PCB(L)-III & & Longitudinal = Fillet & $8(8)$ \\
\hline HFF(L)-V & \multirow{2}{*}{$\begin{array}{c}\text { SikaWrapHex103C } \\
\text { SikaWrapHex100G }\end{array}$} & G 90/C 0/G 90 /C0 /G 90 & $4(4)$ \\
\cline { 4 - 5 } \cline { 3 - 4 } & HFF(H)-V & G 0/C 90/G 0/C 90/G 0 & $4(4)$ \\
\hline
\end{tabular}

* Numbers in parenthesis show number of specimens instrumented with strain gauge. 


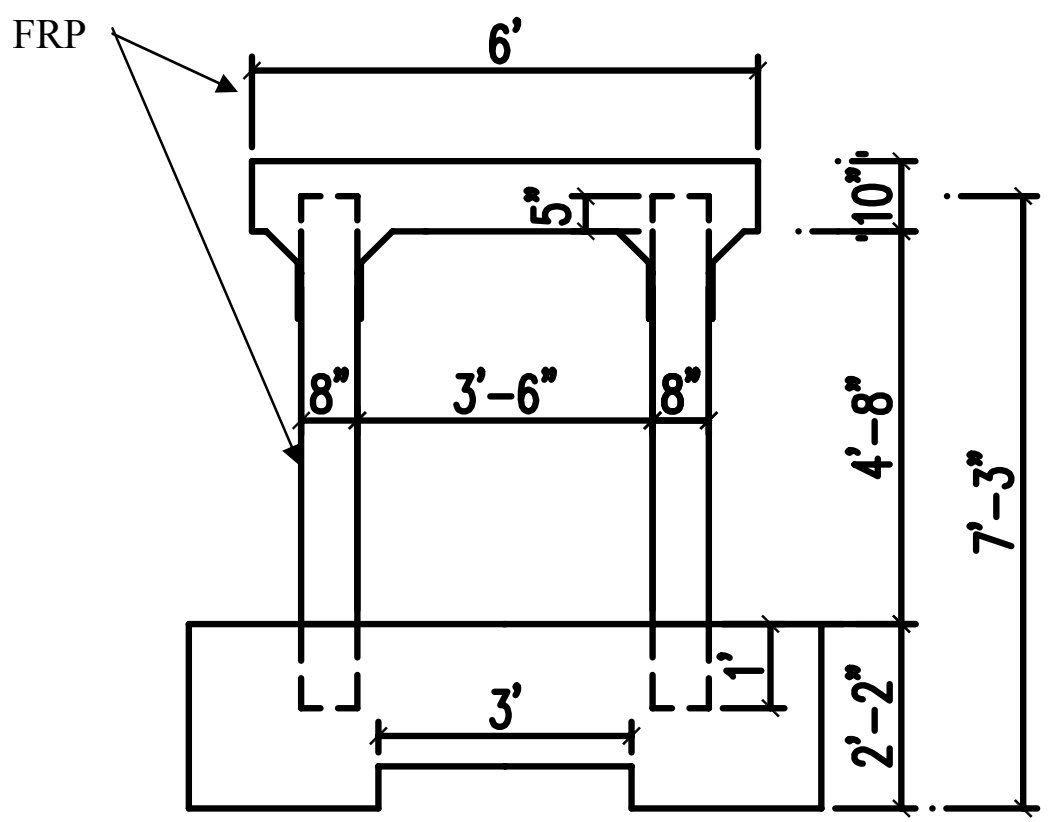

Fig. 3.1 Schematics of Pier Frame Specimens

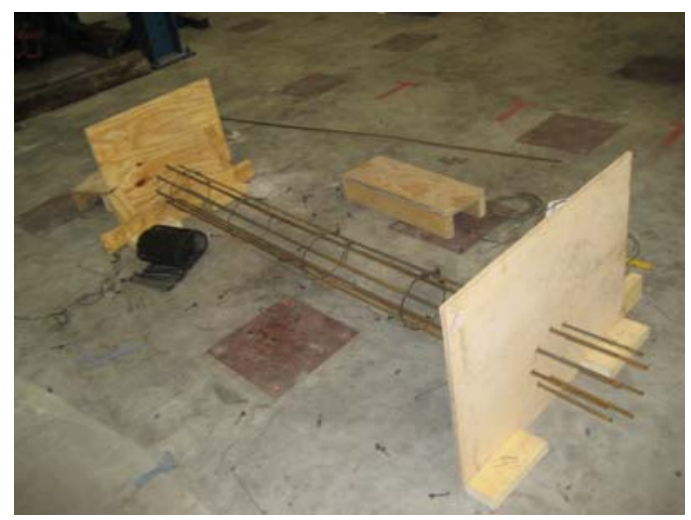

Fig. 3.2 Construction of Steel Reinforcement Cage for FRP Columns

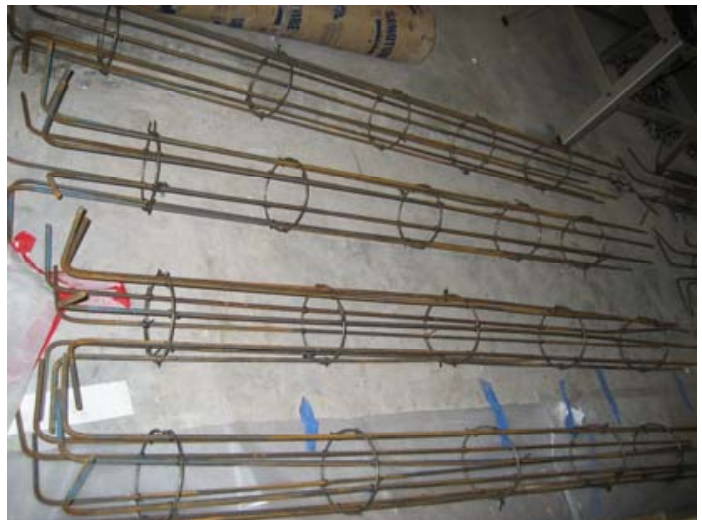

Fig. 3.3 Completed Steel Cage for FRP Columns 

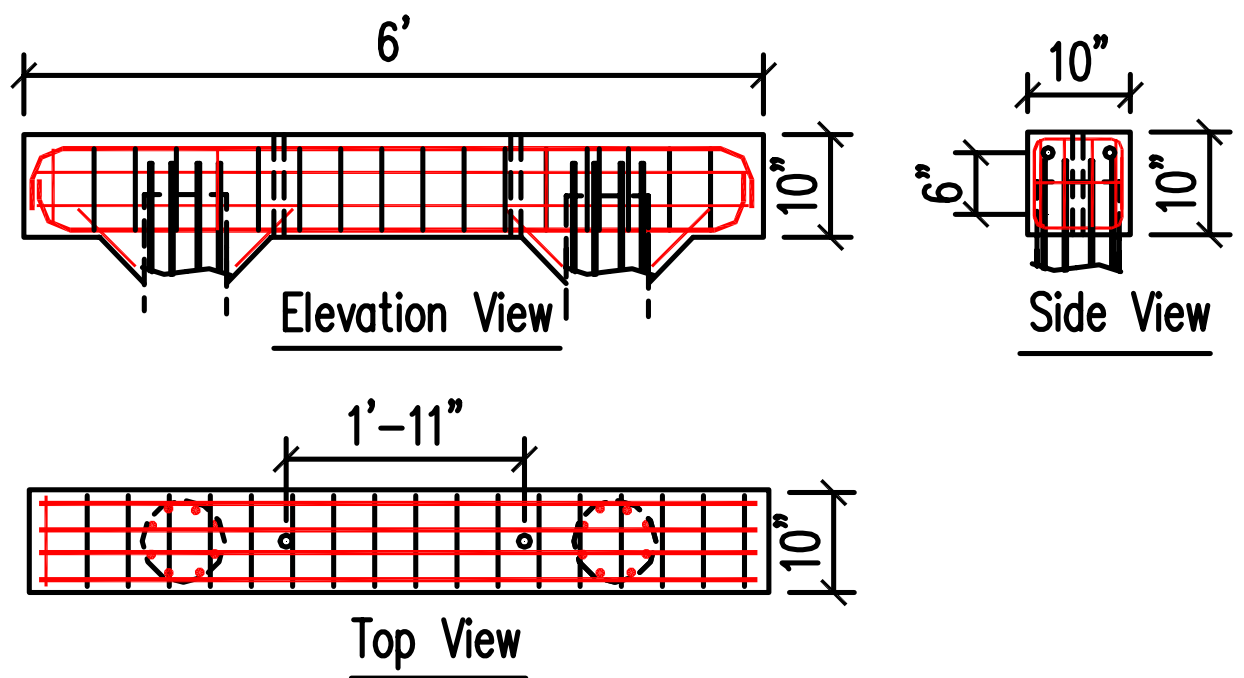

Fig. 3.4 Steel Reinforcement Details for Pier Cap Beams
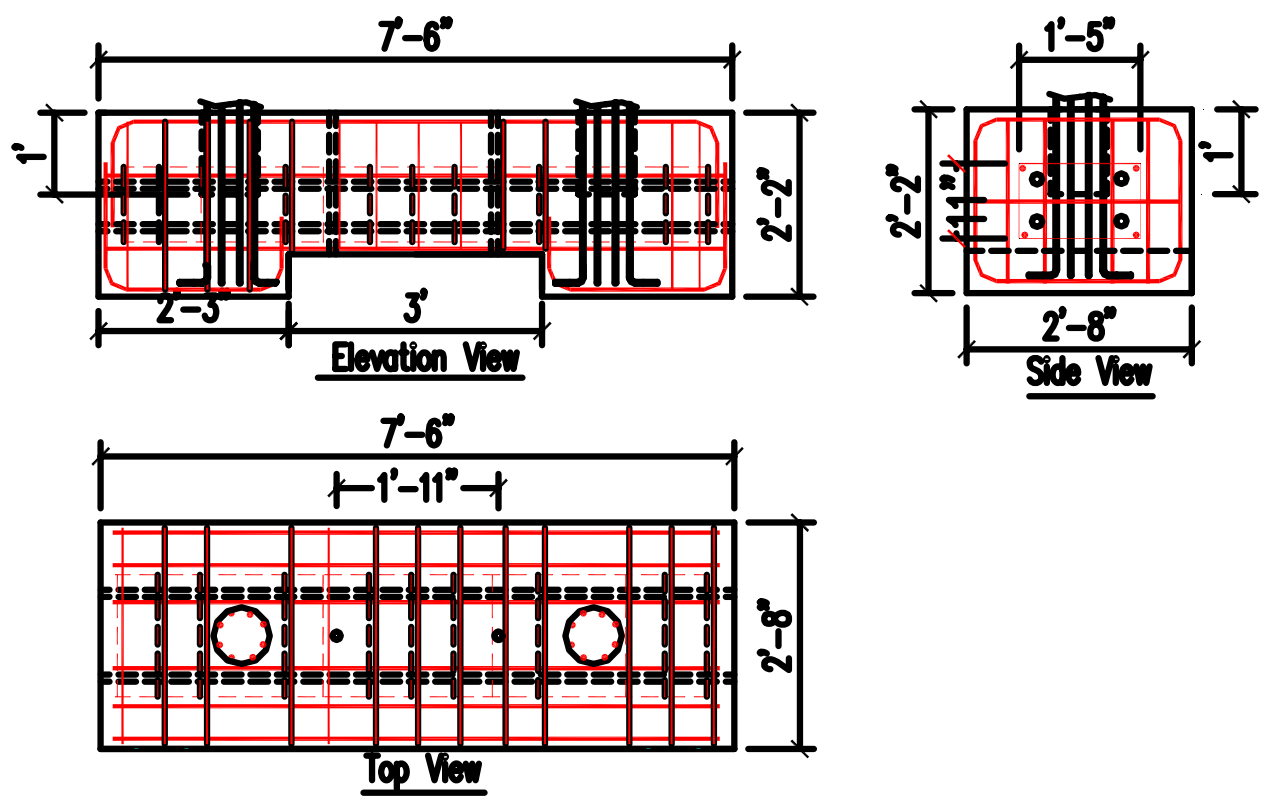

Fig. 3.5 Steel Reinforcement Details for RC Footings 


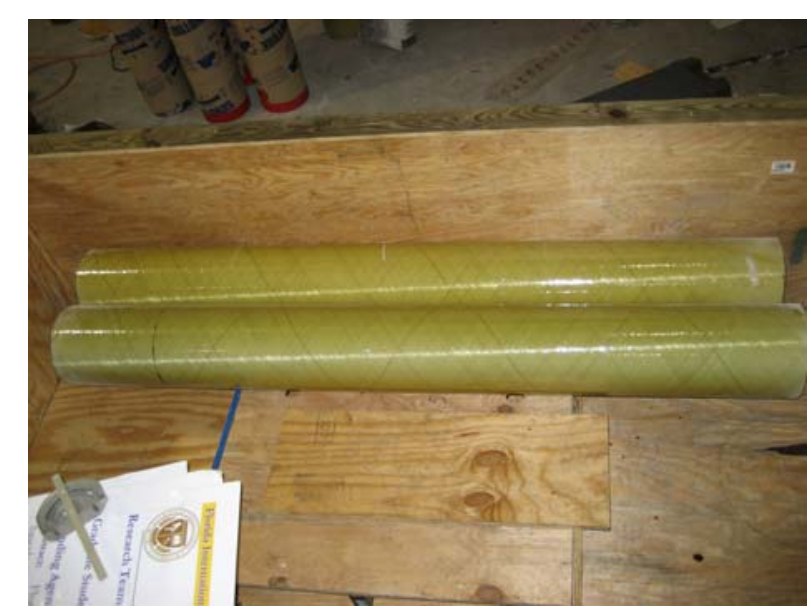

Fig. 3.6 Glass FRP Tubes

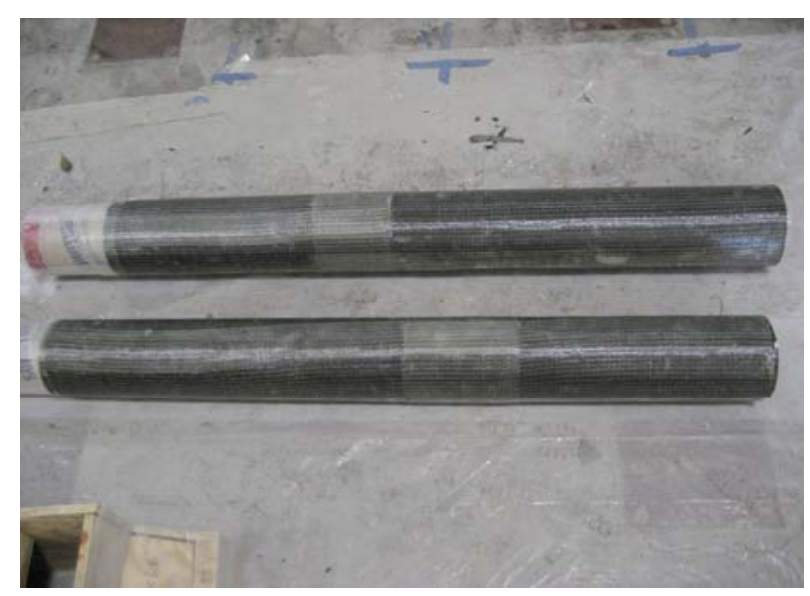

Fig. 3.8 Cured Hybrid FRP Tubes

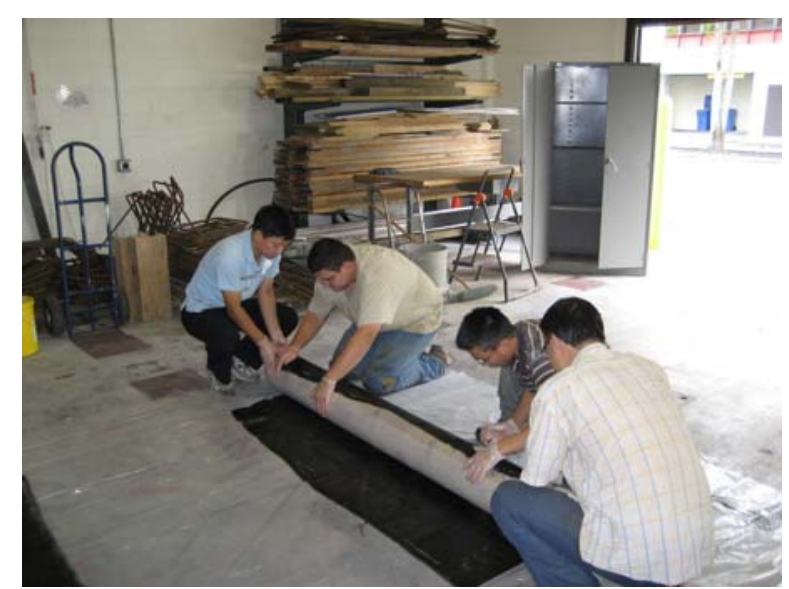

Fig. 3.7 Preparing a Carbon FRP Tube

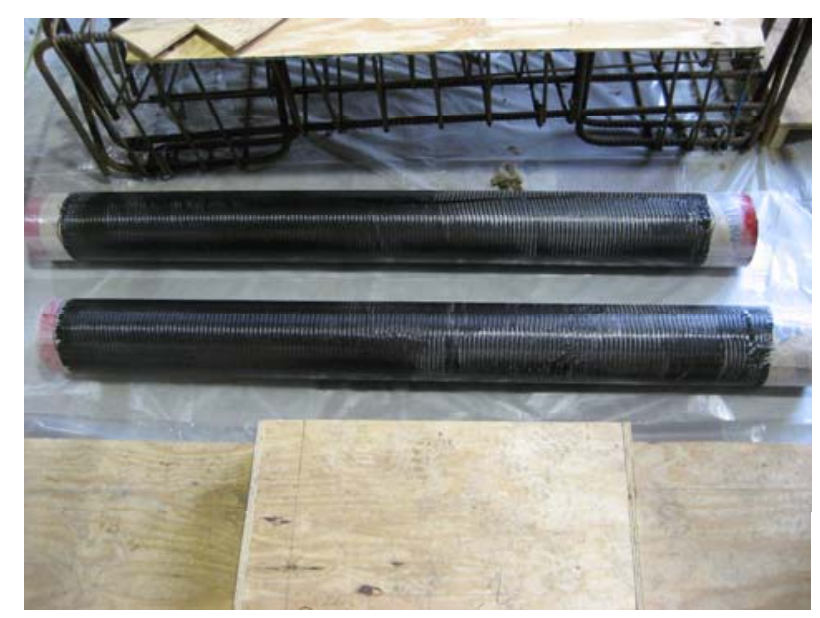

Fig. 3.9 Cured Carbon FRP Tubes 


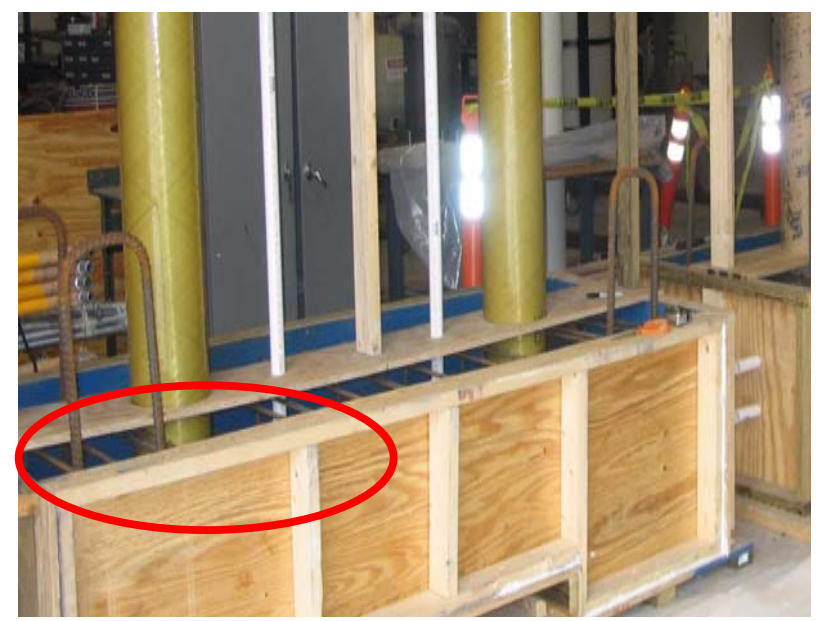

Fig. 3.10 Embedment of FRP Tubes

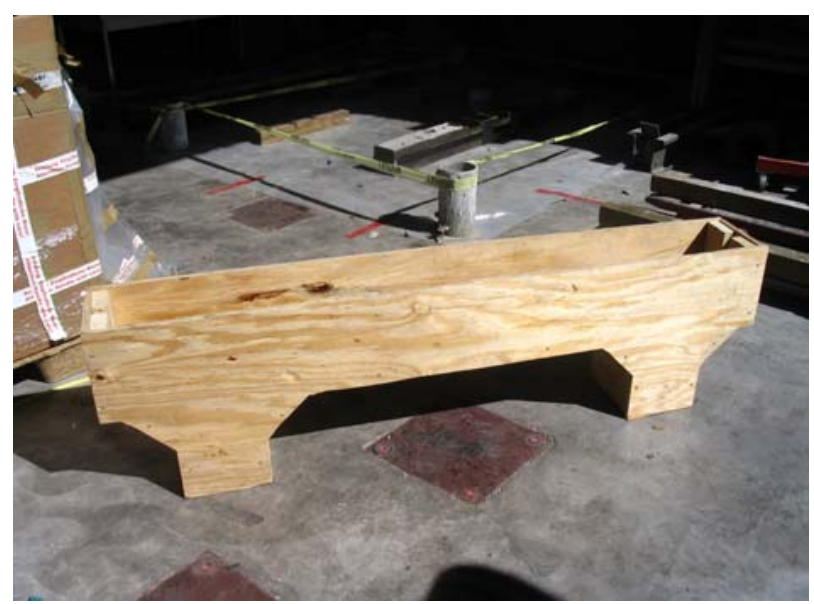

Fig. 3.12 Wooden Mold for FRP Pier Cap Beam

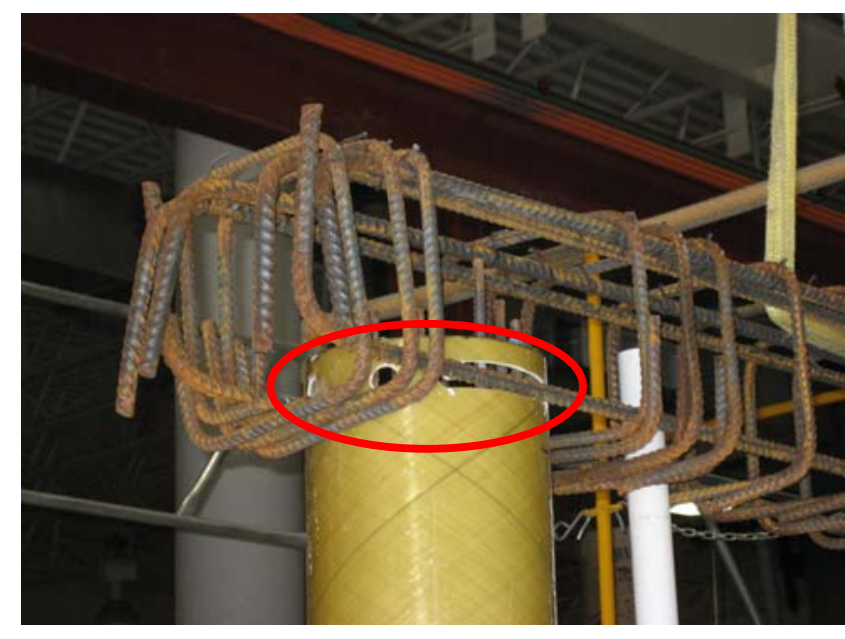

Fig. 3.11 Slots and Holes in Glass FRP Tube

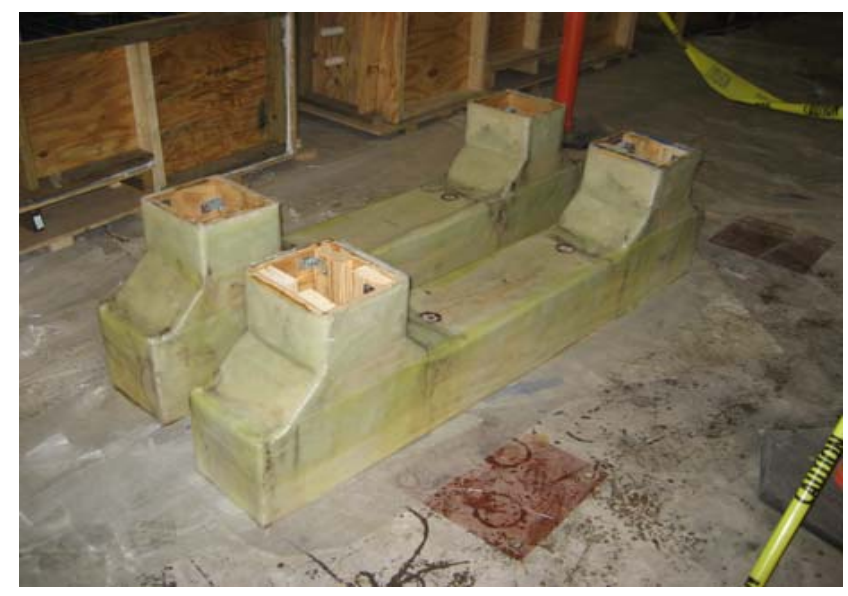

Fig. 3.13 Cured FRP Pier Cap Forms 


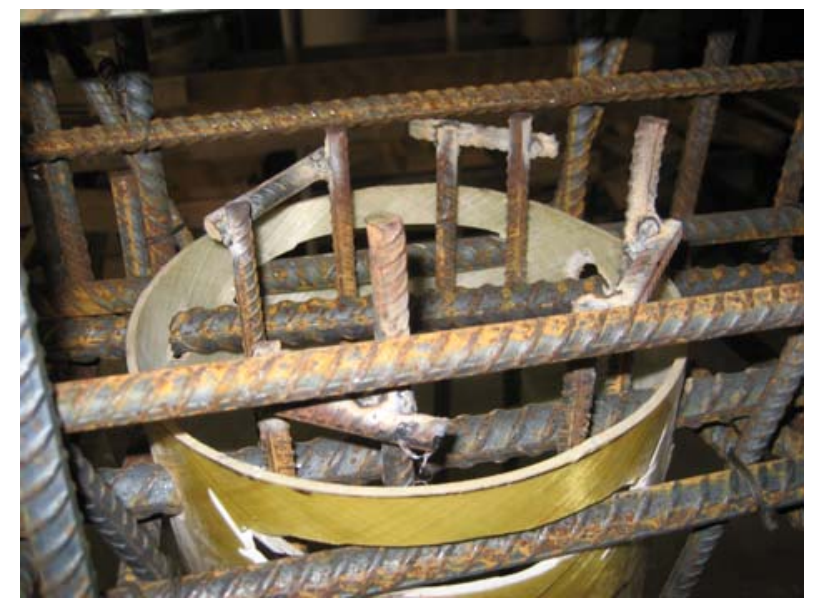

Fig. 3.14 Welding Anchorage Bars in Column Heads

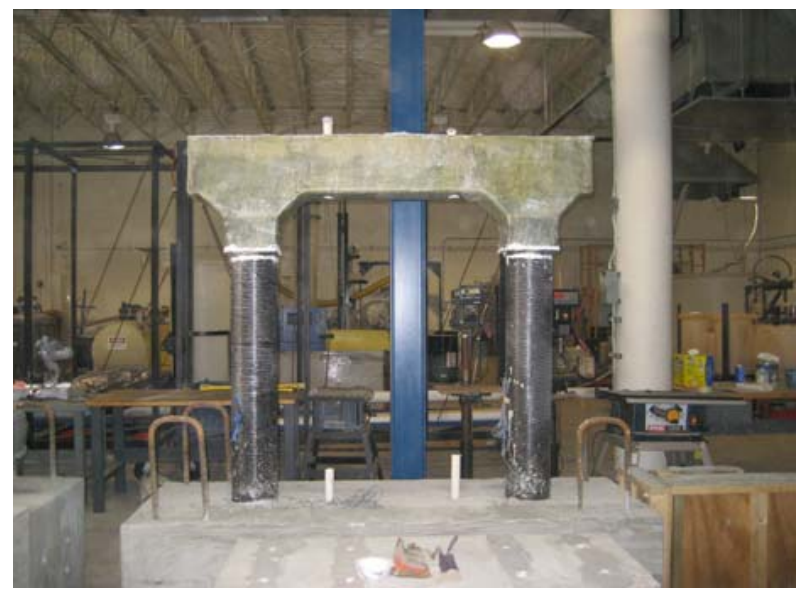

Fig. 3.16 Carbon FRP Pier Frame

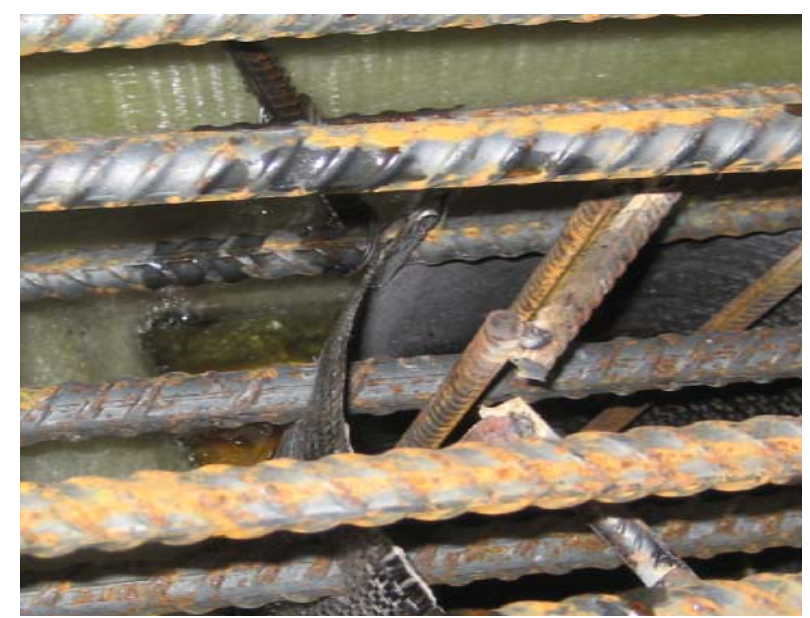

Fig. 3.15 Epoxy Placement in Transition Zone

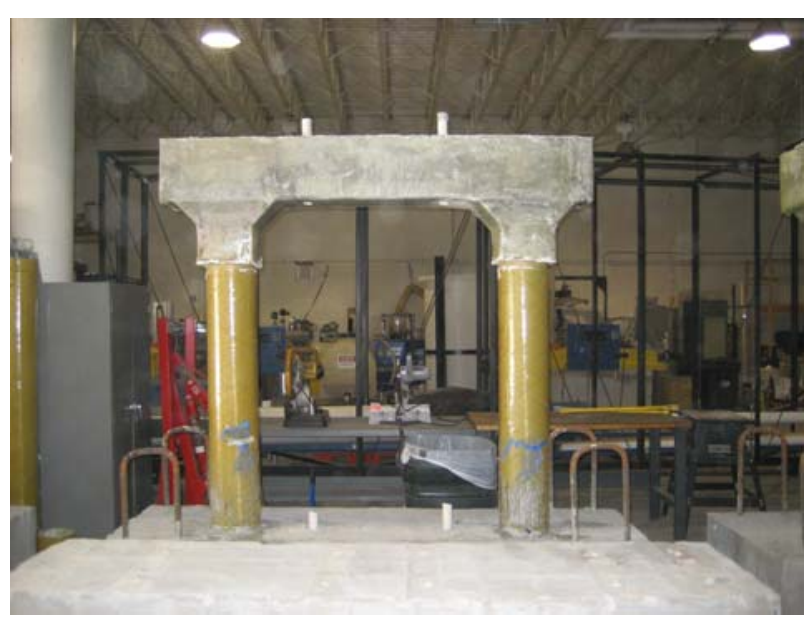

Fig. 3.17 Glass FRP Pier Frame 


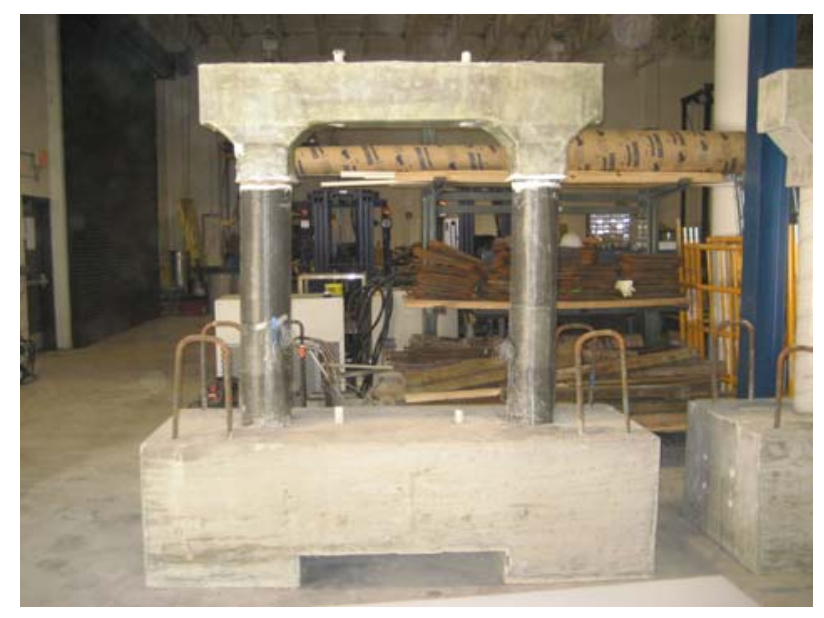

Fig. 3.18 Hybrid FRP Pier Frame

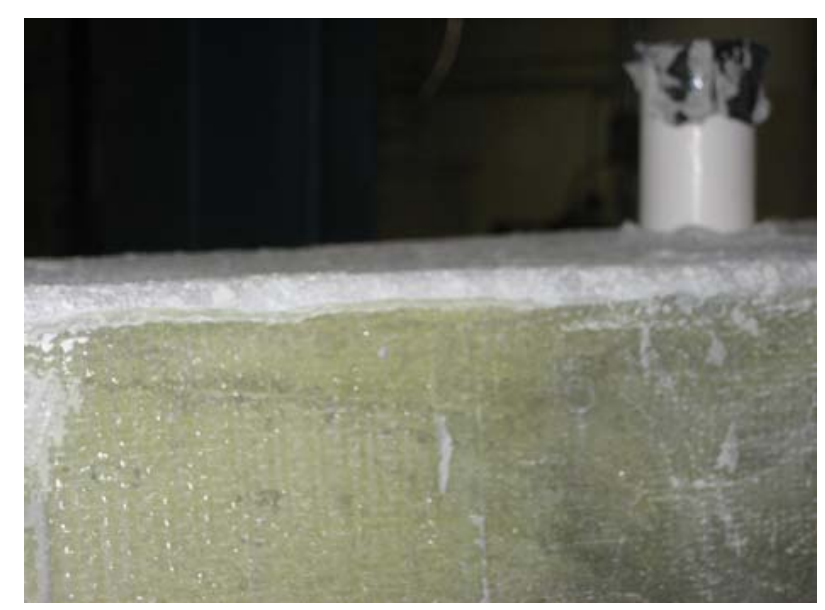

Fig. 3.20 Grinded Edge of Pier Cap Beam

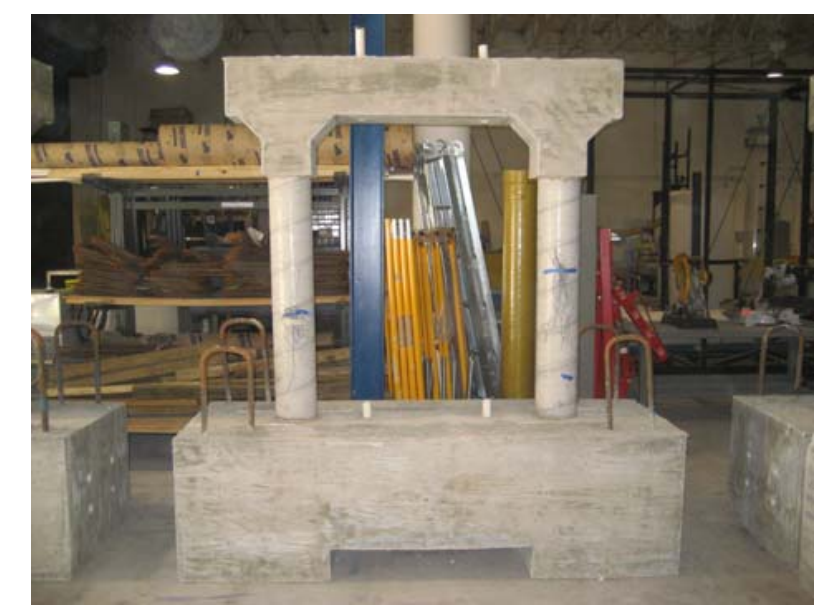

Fig. 3.19 Control RC Pier Frame

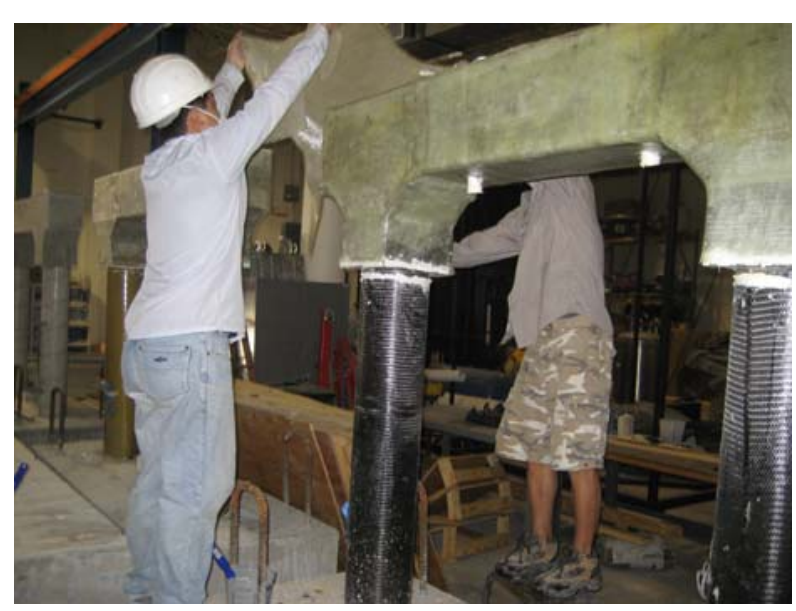

Fig. 3.21 FRP Wrapping at Joints for Pier Cap Beam 


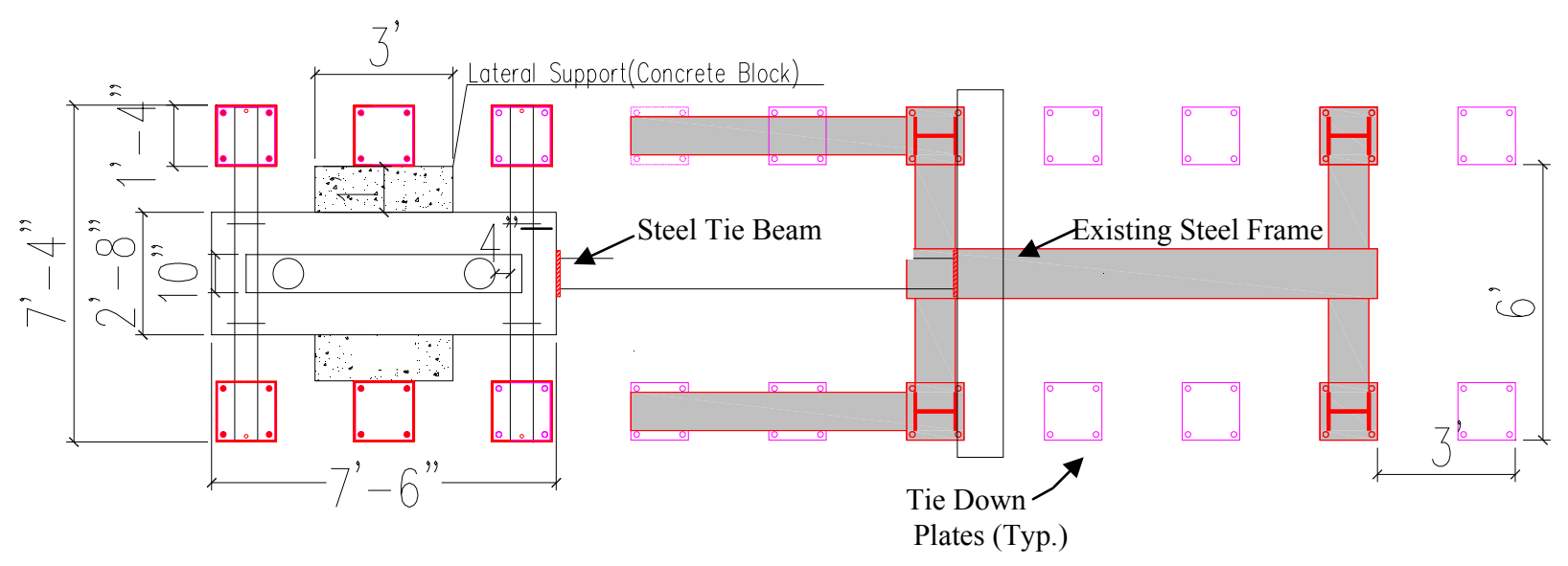

Fig. 3.22 Plan View of Test Layout for Two-Column Bent Specimens

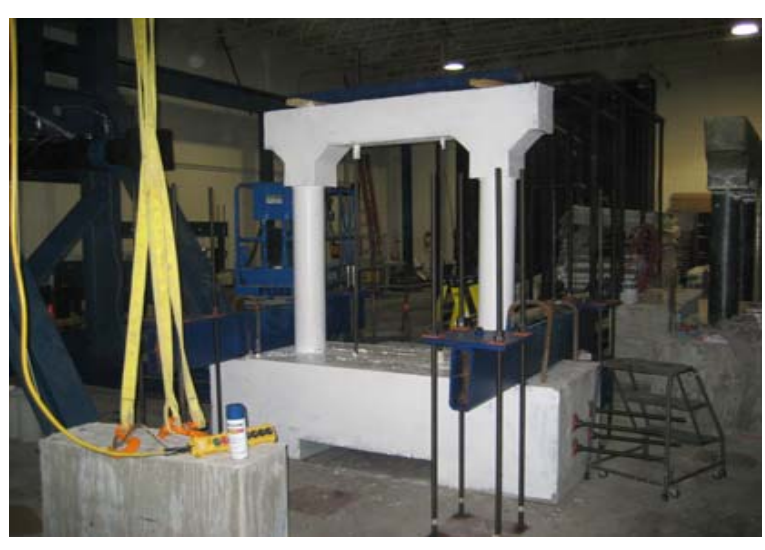

Fig. 3.23 Tie-down of the Specimen

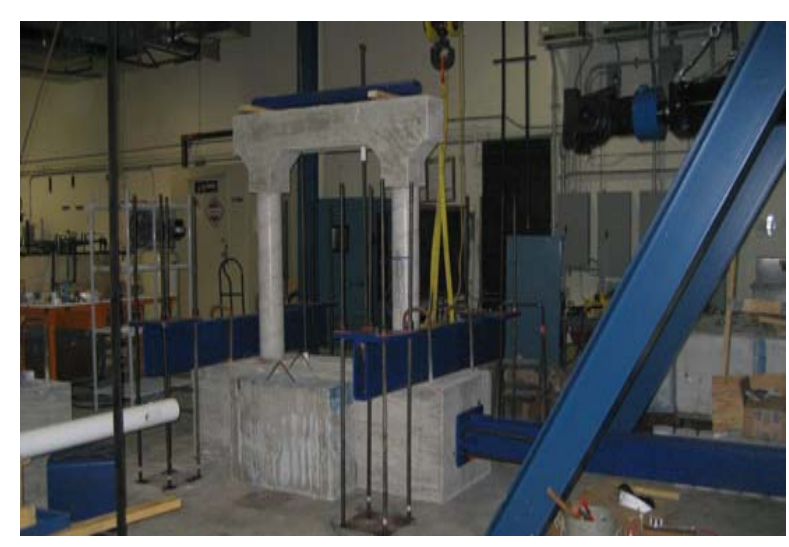

Fig. 3.24 Steel Tie Beam for Lateral Reaction 


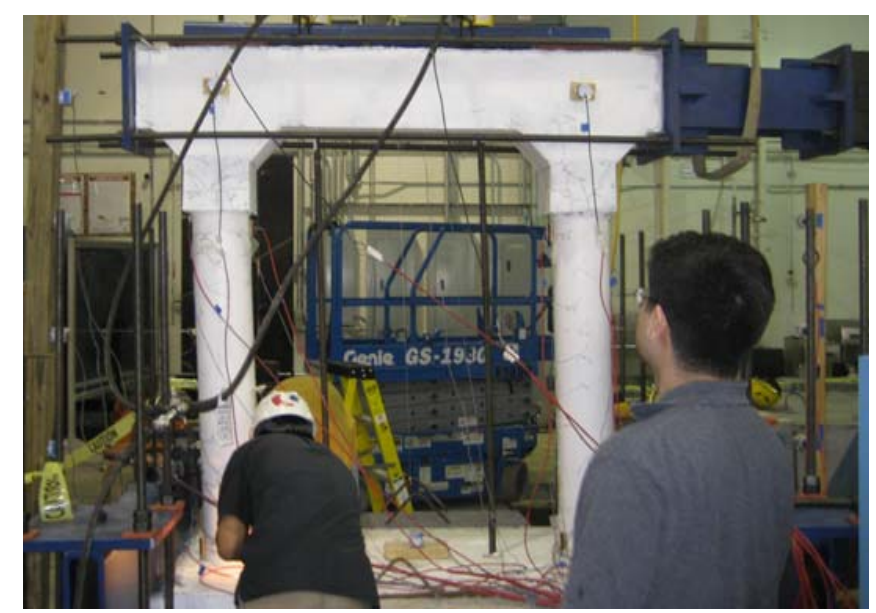

Fig. 3.25 Pier Cap Beam Connection With the Actuator

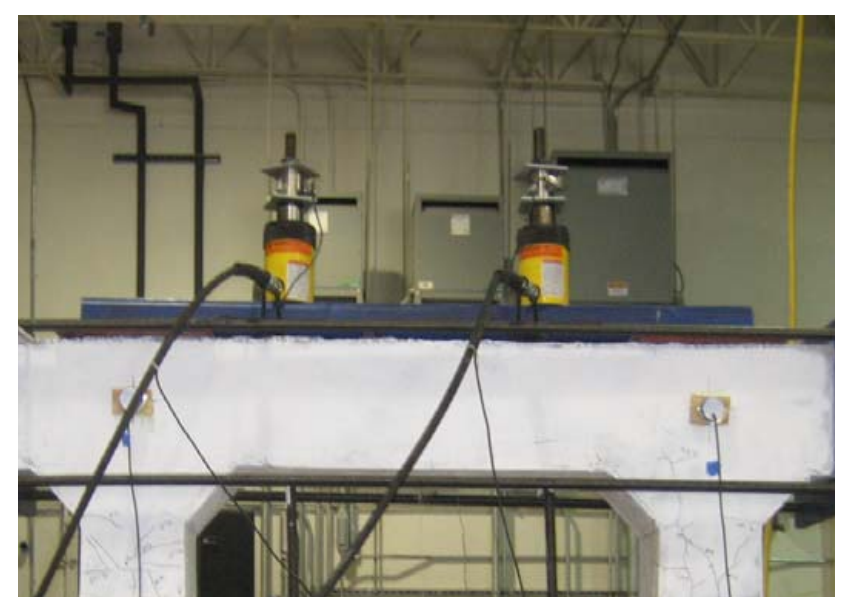

Fig. 3.27 Steel Spreader Beam with Hydraulic Jacks

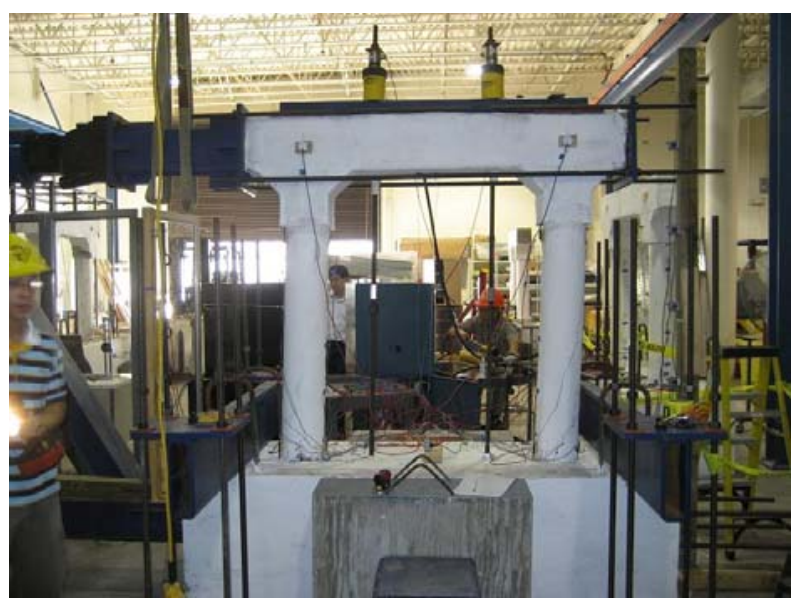

Fig. 3.26 Completed Test Layout of Two Column Bent Specimens

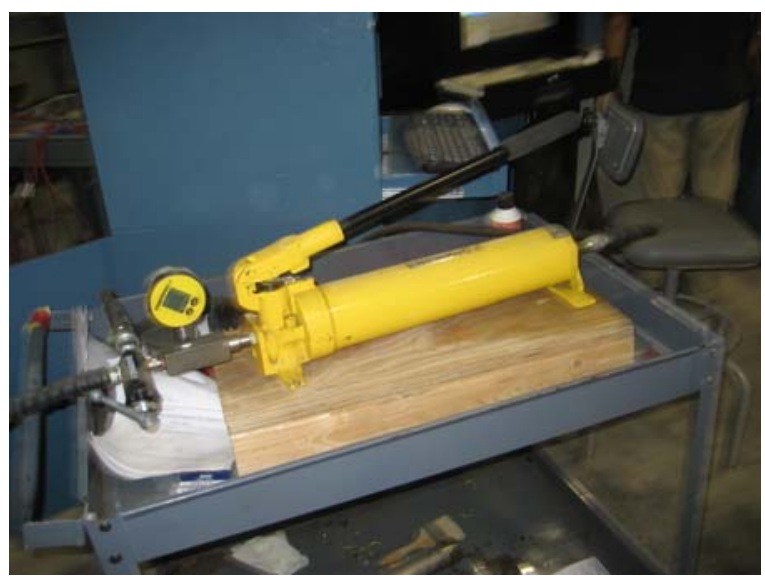

Fig. 3.28 Hand Pump with Pressure Relief Valve 
C) Copyright 2008 by Bin Li

All rights reserved. 


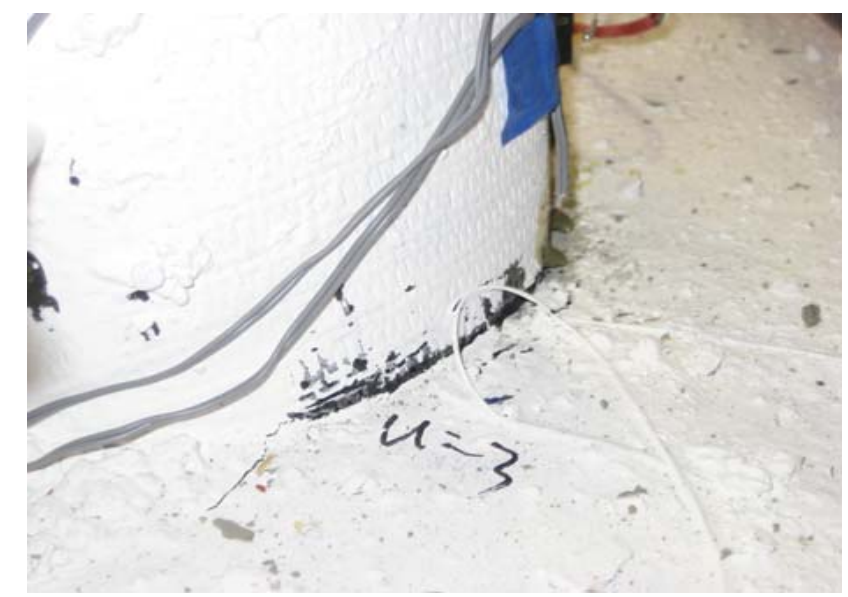

Fig. 3.31 Bottom Crack in Specimen CFF at $\mu=3$

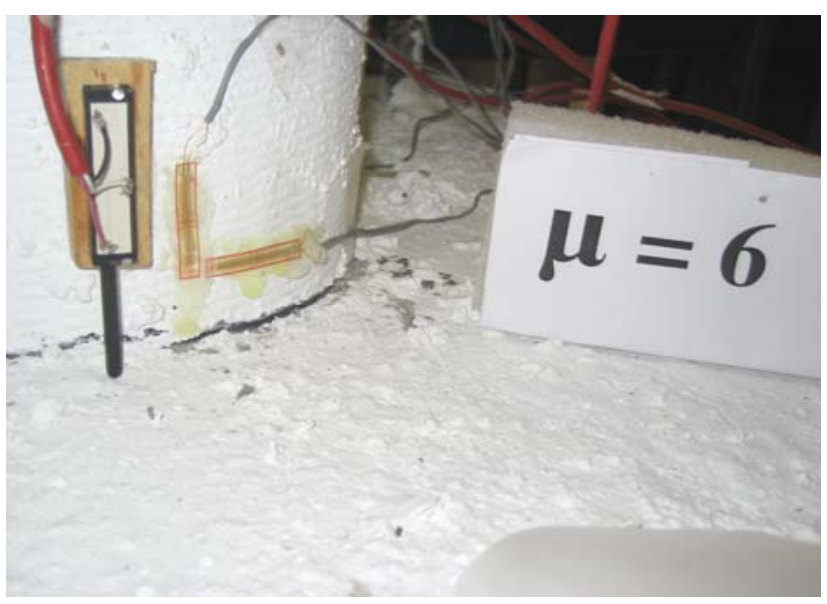

Fig. 3.33 Crack in Specimen HFF Enlarged to Middle of the Column Base Section at $\mu=6$

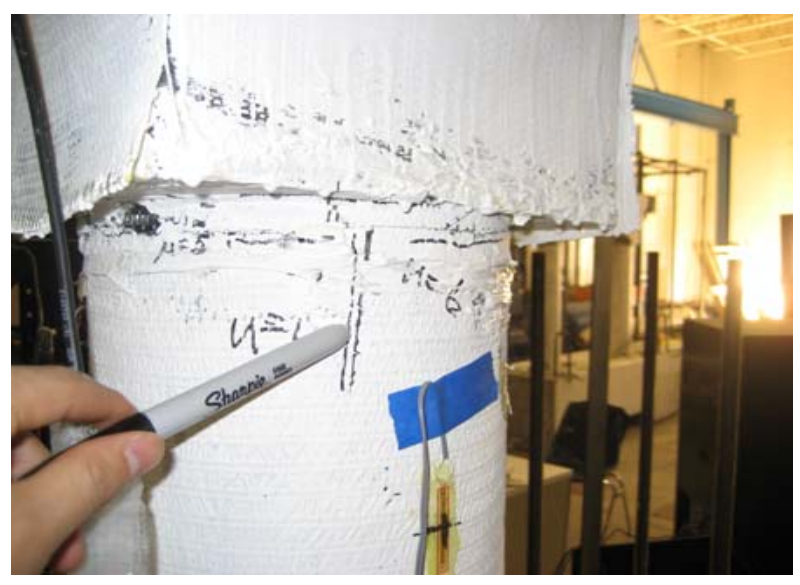

Fig. 3.32 Vertical Crack at Column Top in Specimen CFF at $\mu=7$

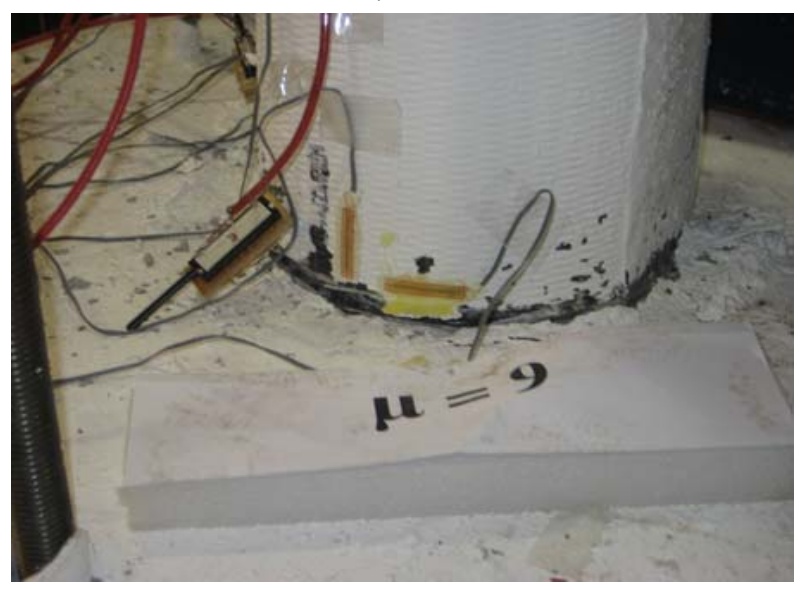

Fig. 3.34 Wide Opening of Crack in Specimen HFF at $\mu=$ 9 


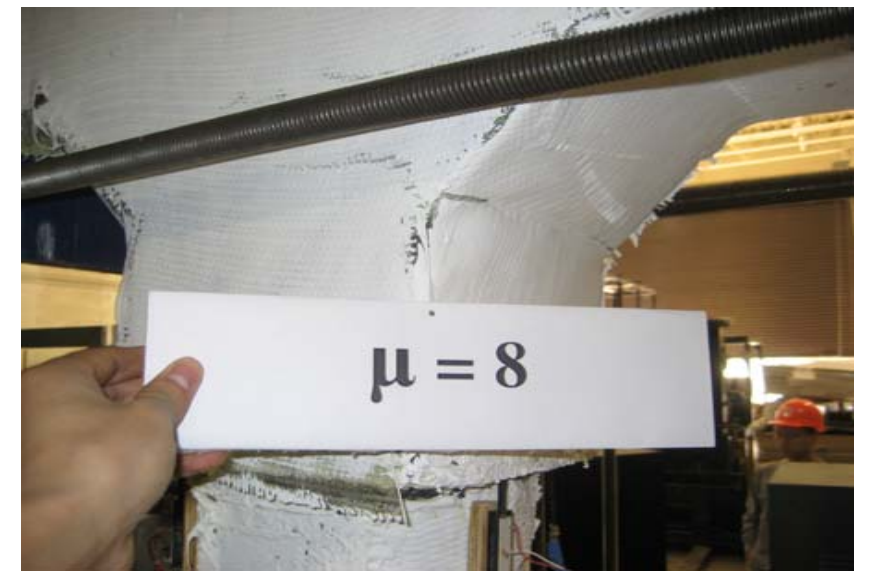

Fig. 3.35 Cracks in Pier Cap Beam of Specimen HFF

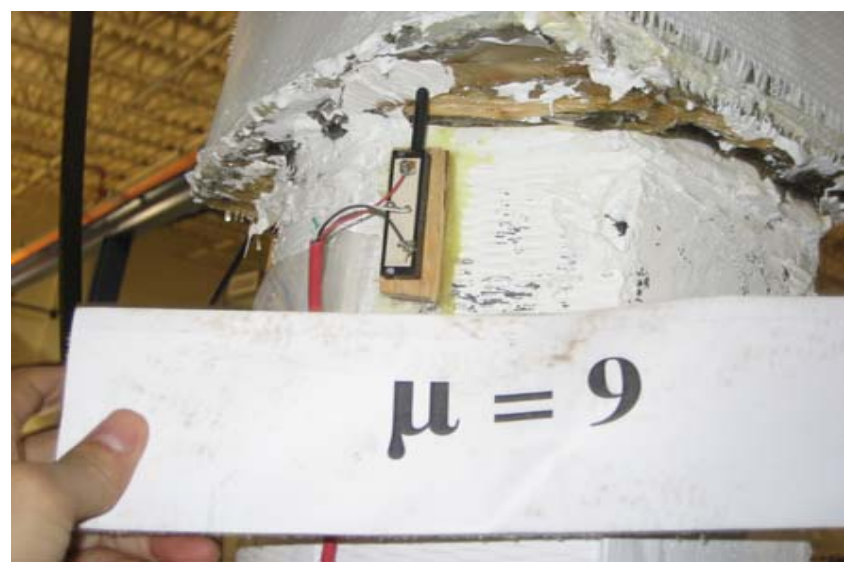

Fig. 3.37 Separation between FRP tube and Pier Cap Beam in Specimen HFF at $\mu=9$

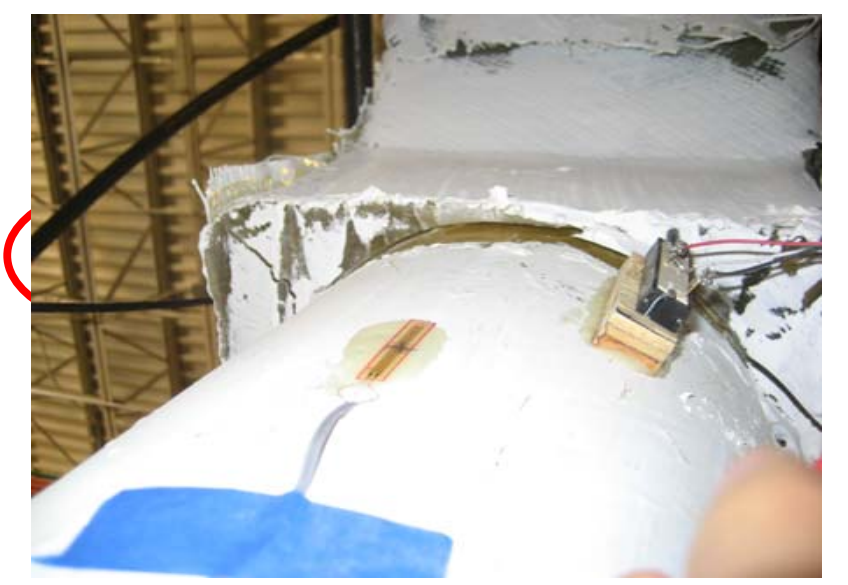

Fig. 3.36 Cracks in Epoxy Transition Zone of Specimen GFF

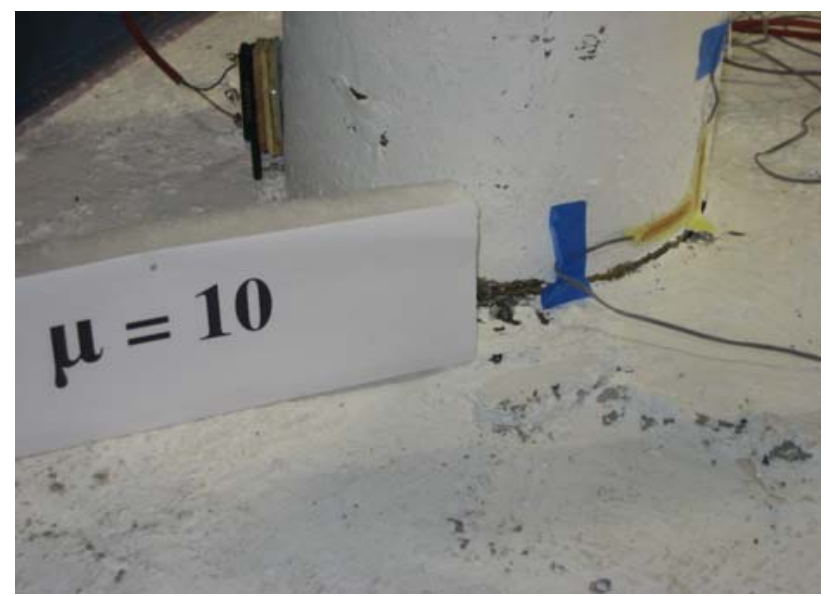

Fig. 3.38 Slippage of Column Base in Specimen GFF at $\mu=10$ 


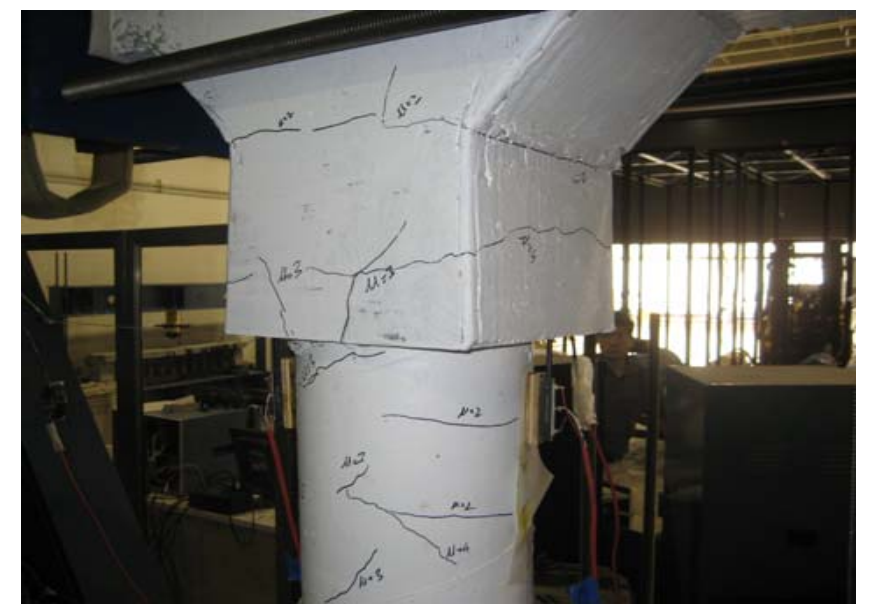

Fig. 3.39 Cracks in the Plastic Hinge Zone and Chamfer in Specimen RCF

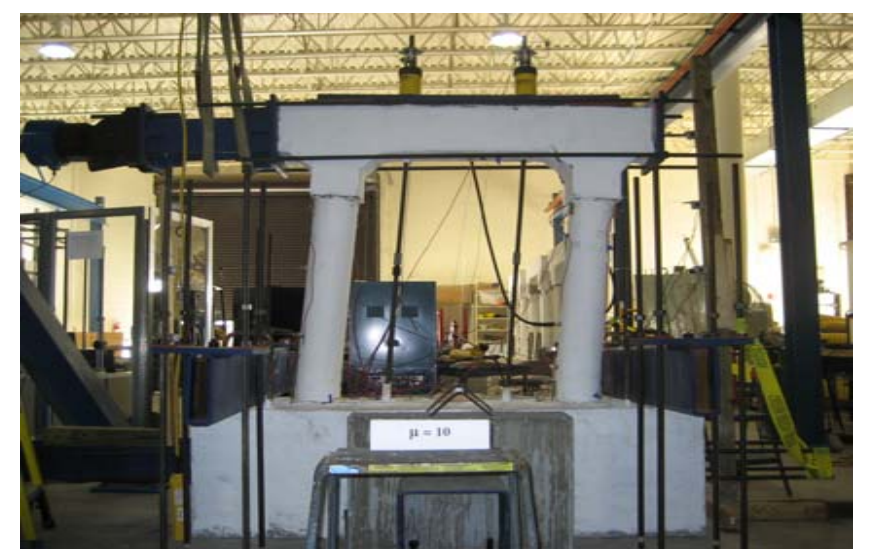

Fig. 3.41 Lateral Deflection of Specimen GFF at $\mu=10$ with Axial Load

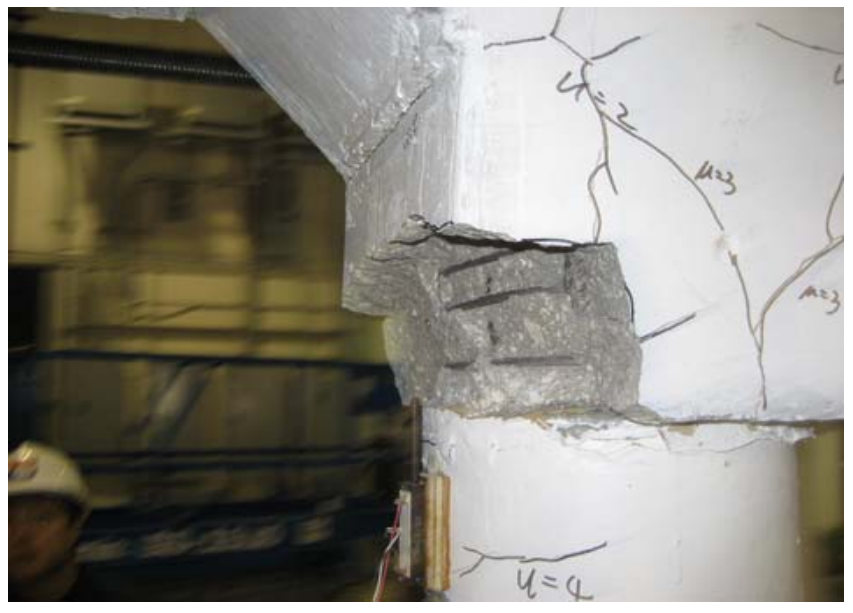

Fig. 3.40 Chamfer Spalling in Specimen RCF at $\mu=6$

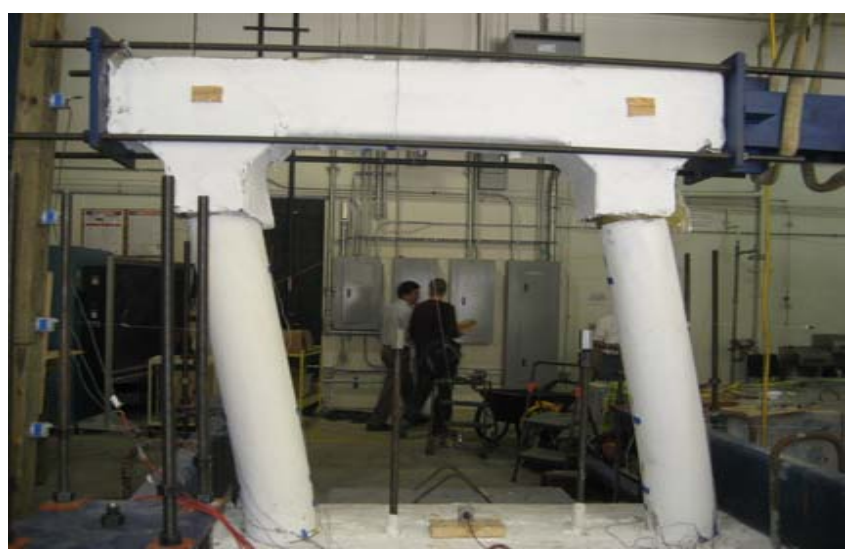

Fig. 3.42 Lateral Deflection of Specimen GFF at $\mu=25$ without Axial Load 


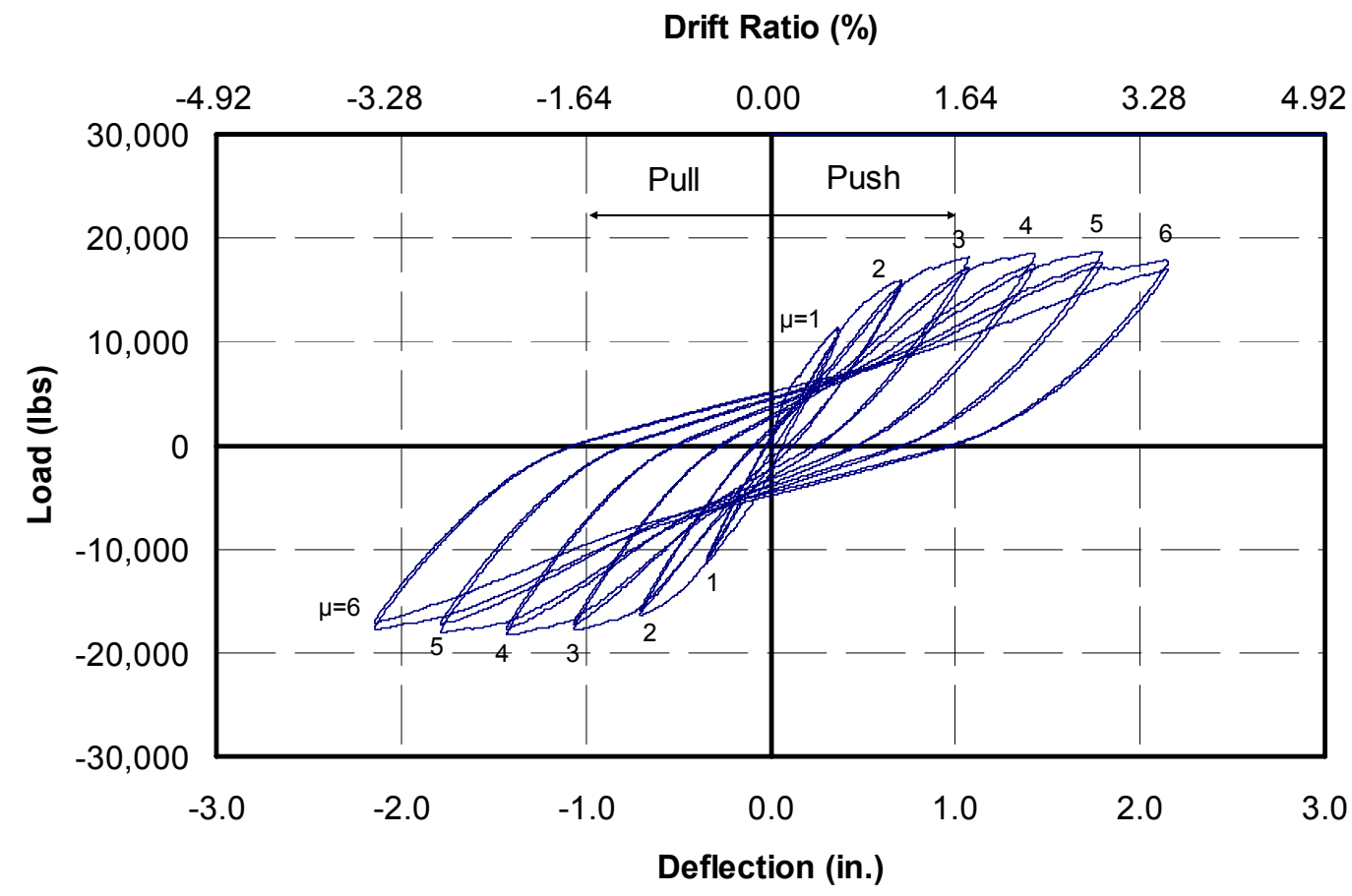

Fig. 3.43 Load-Deflection Hysteretic Response of Specimen RCF

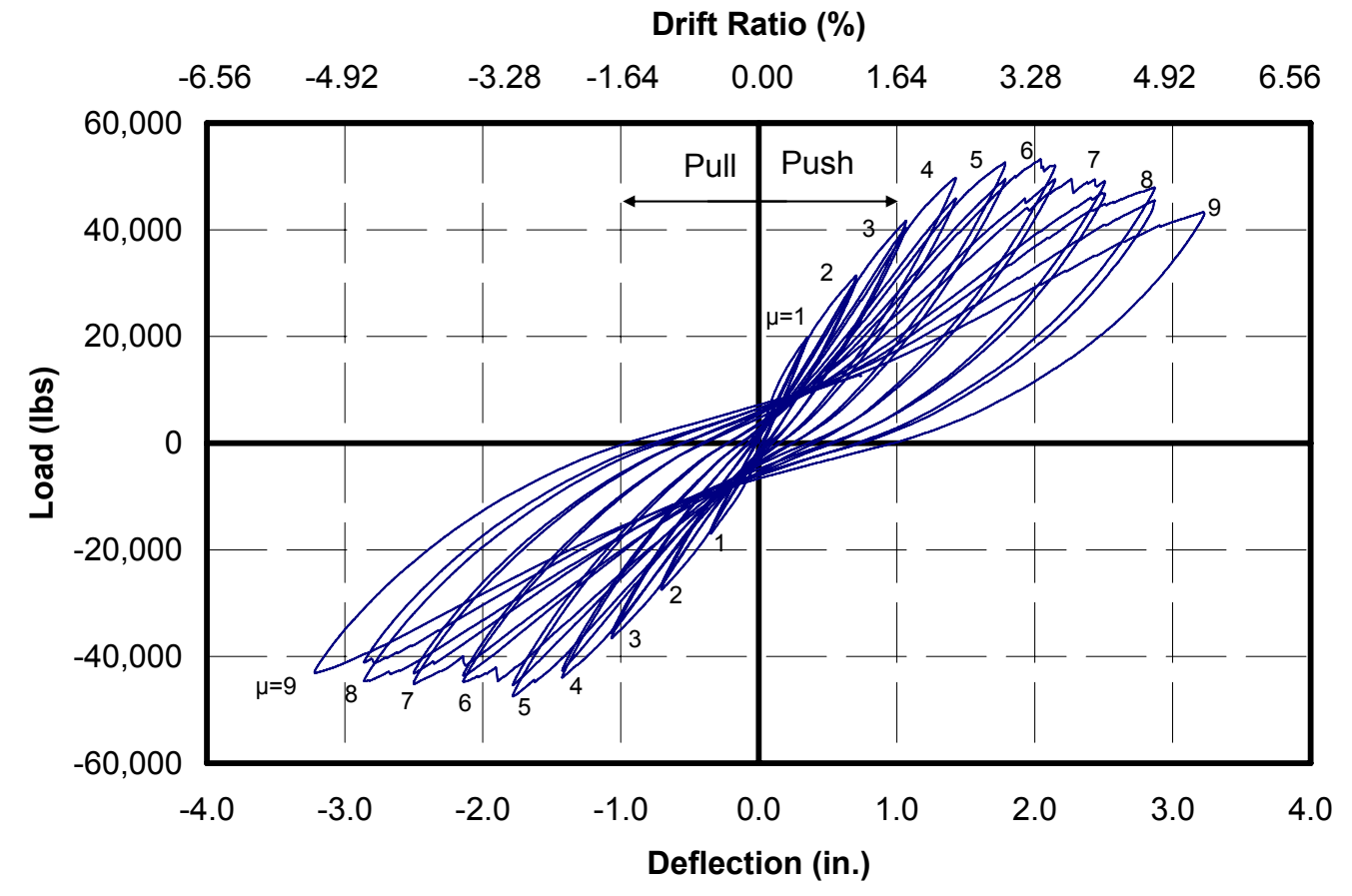

Fig. 3.44 Load-Deflection Hysteretic Response of Specimen HFF 


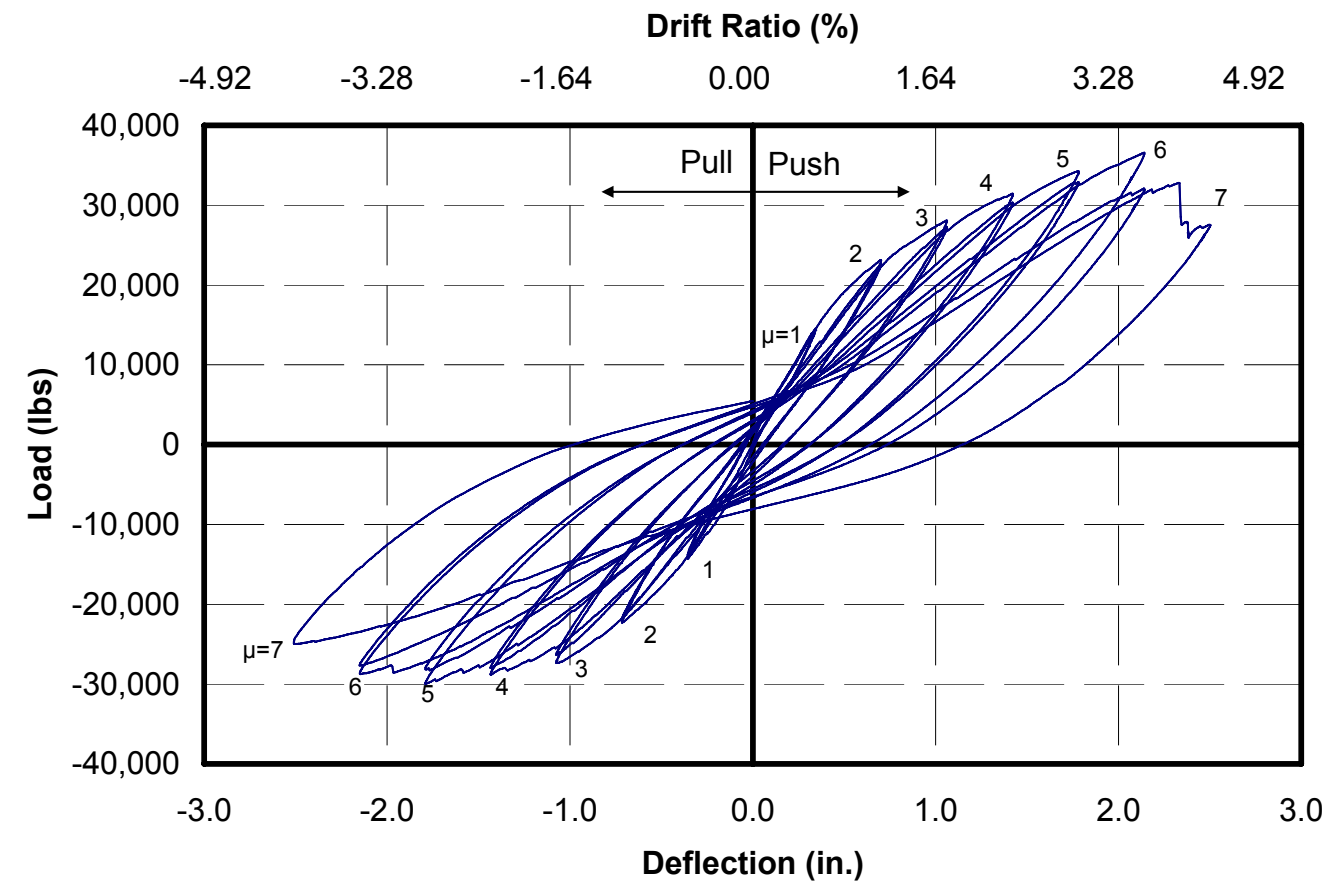

Fig. 3.45 Load-Deflections Hysteretic Response of Specimen CFF

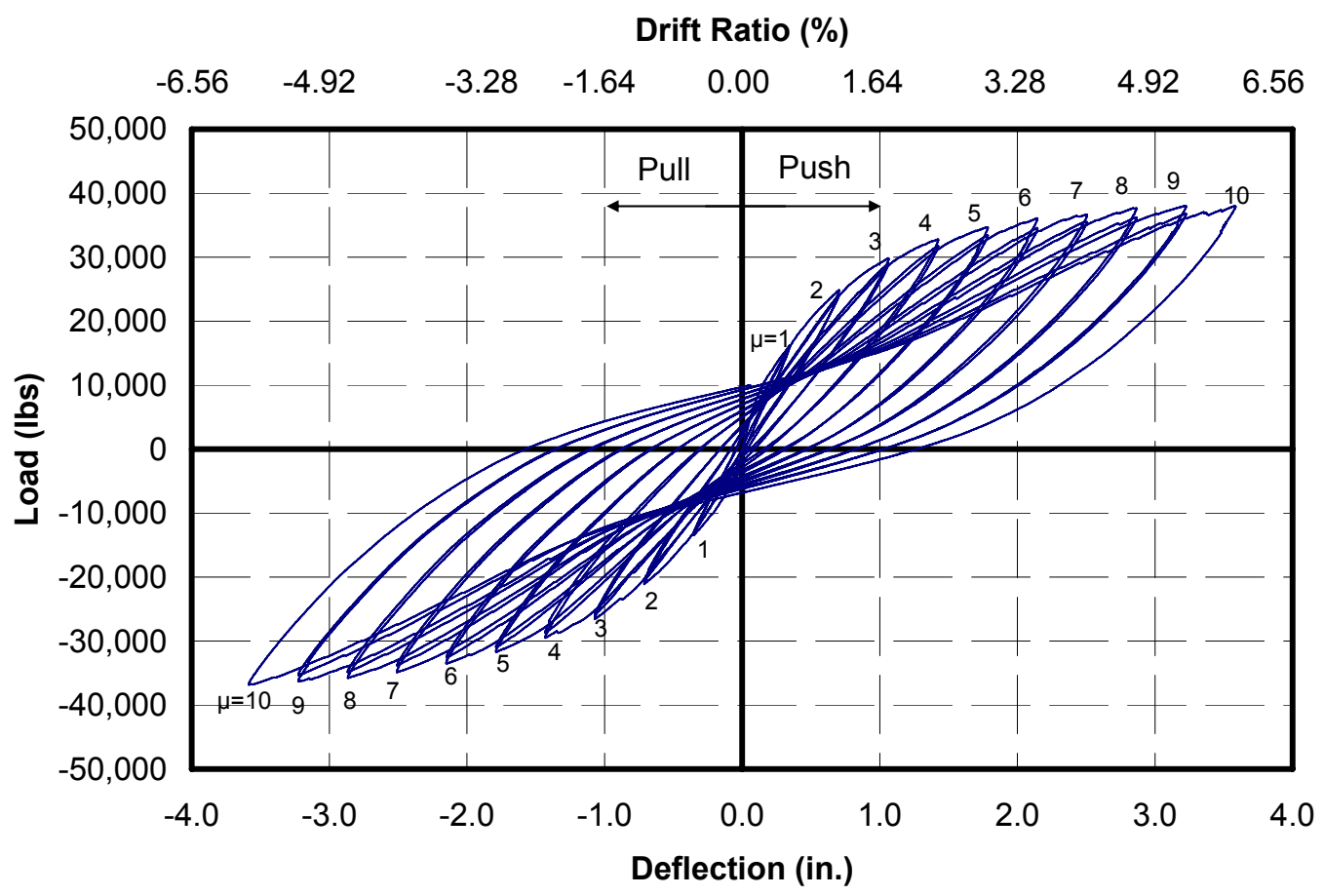

Fig. 3.46 Load-Deflection Hysteretic Response of Specimen GFF 


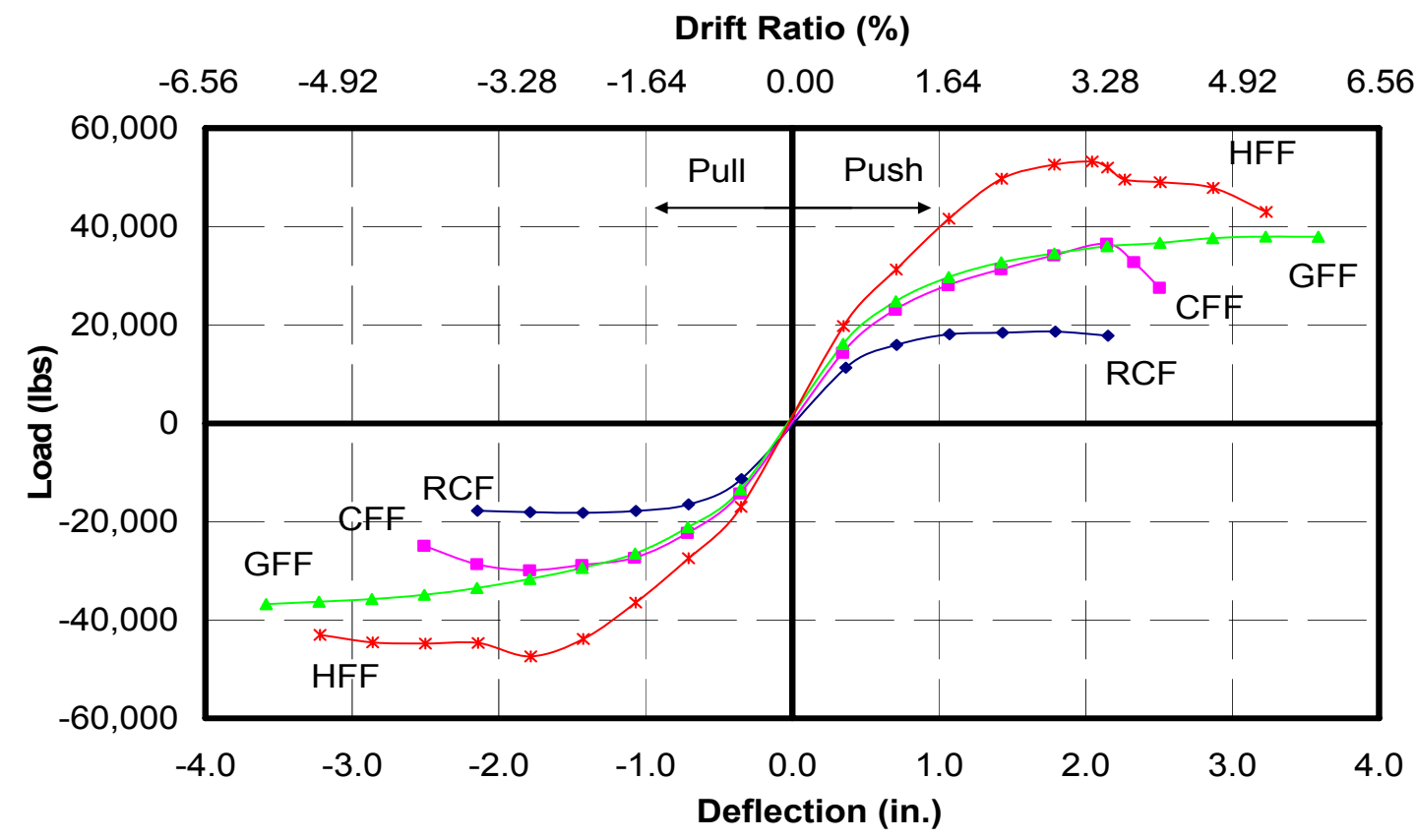

Fig. 3.47 Load-Deflection Envelope Curves of Pier Frame Specimens

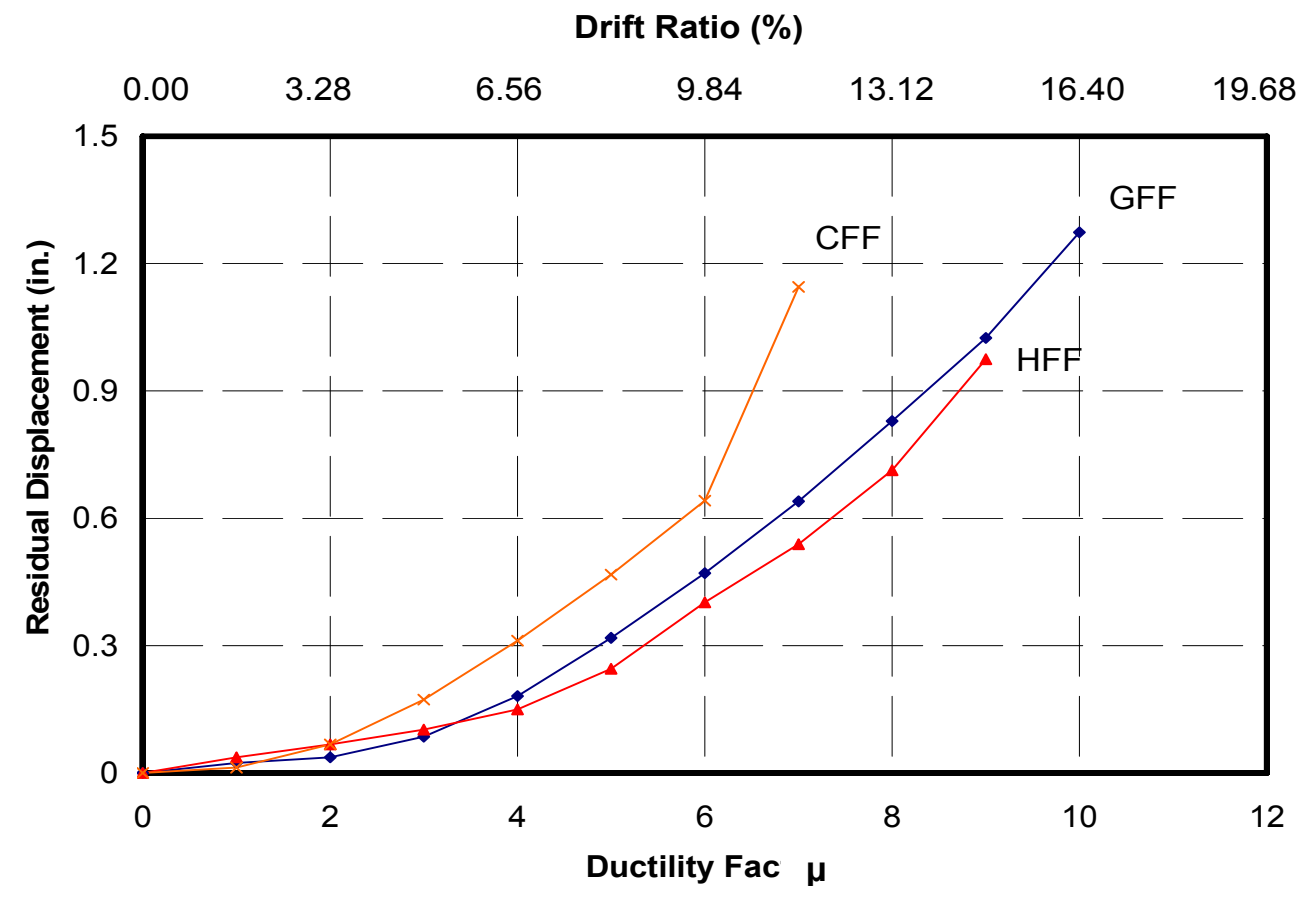

Fig. 3.48 Residual Displacements at Zero Load after Each First Push Cycle 


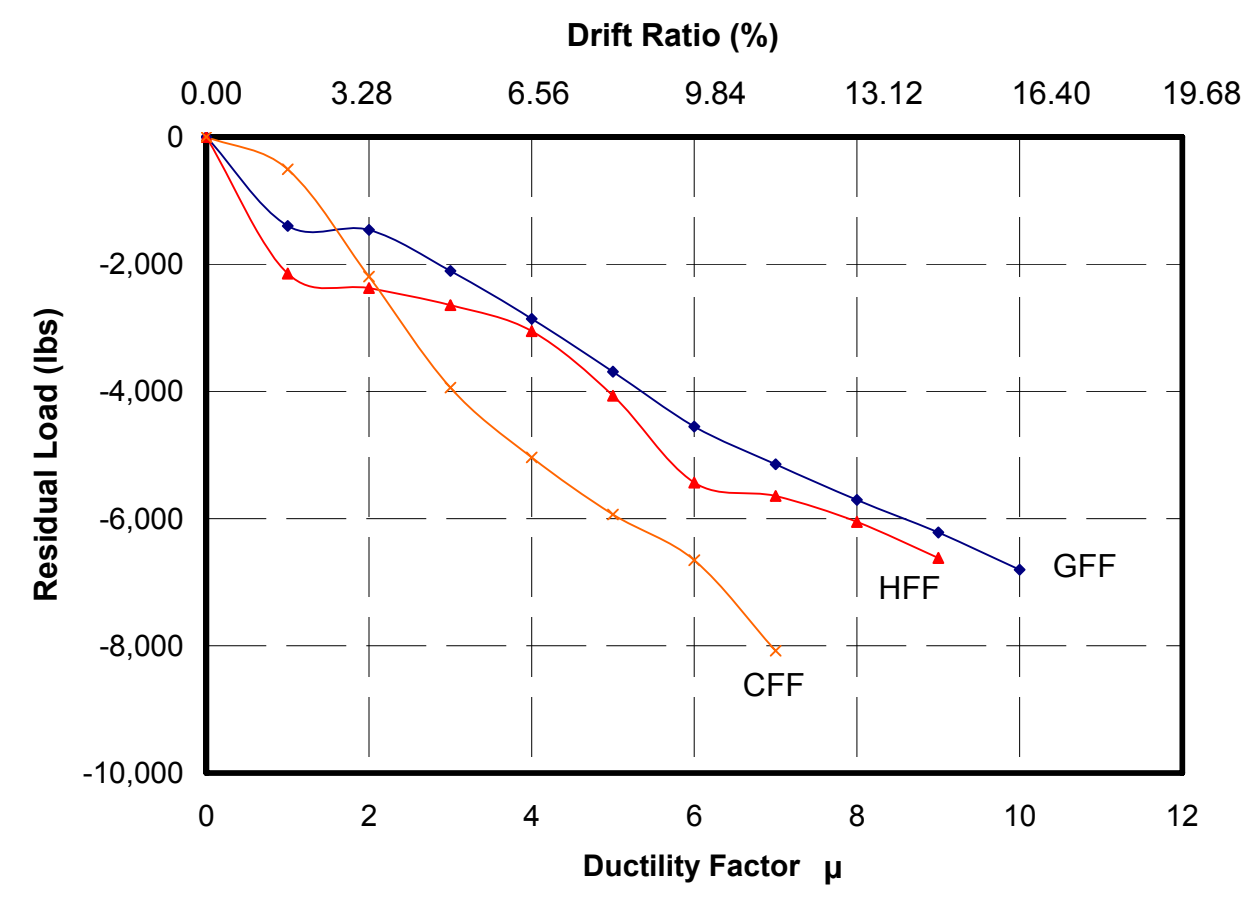

Fig. 3.49 Residual Load at Zero Displacement after Each First Push Cycle

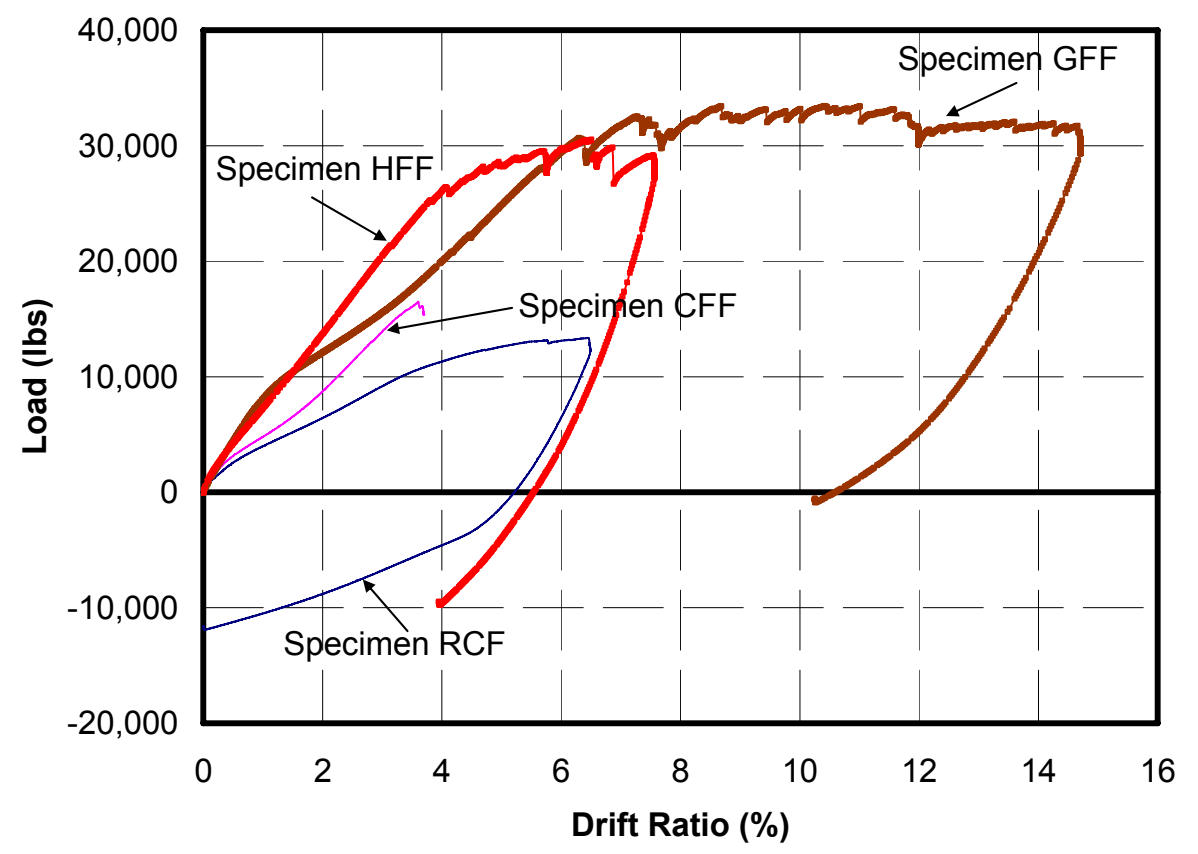

Fig. 3.50 Monotonic Load-Deflection Responses After Removal of Axial Load 


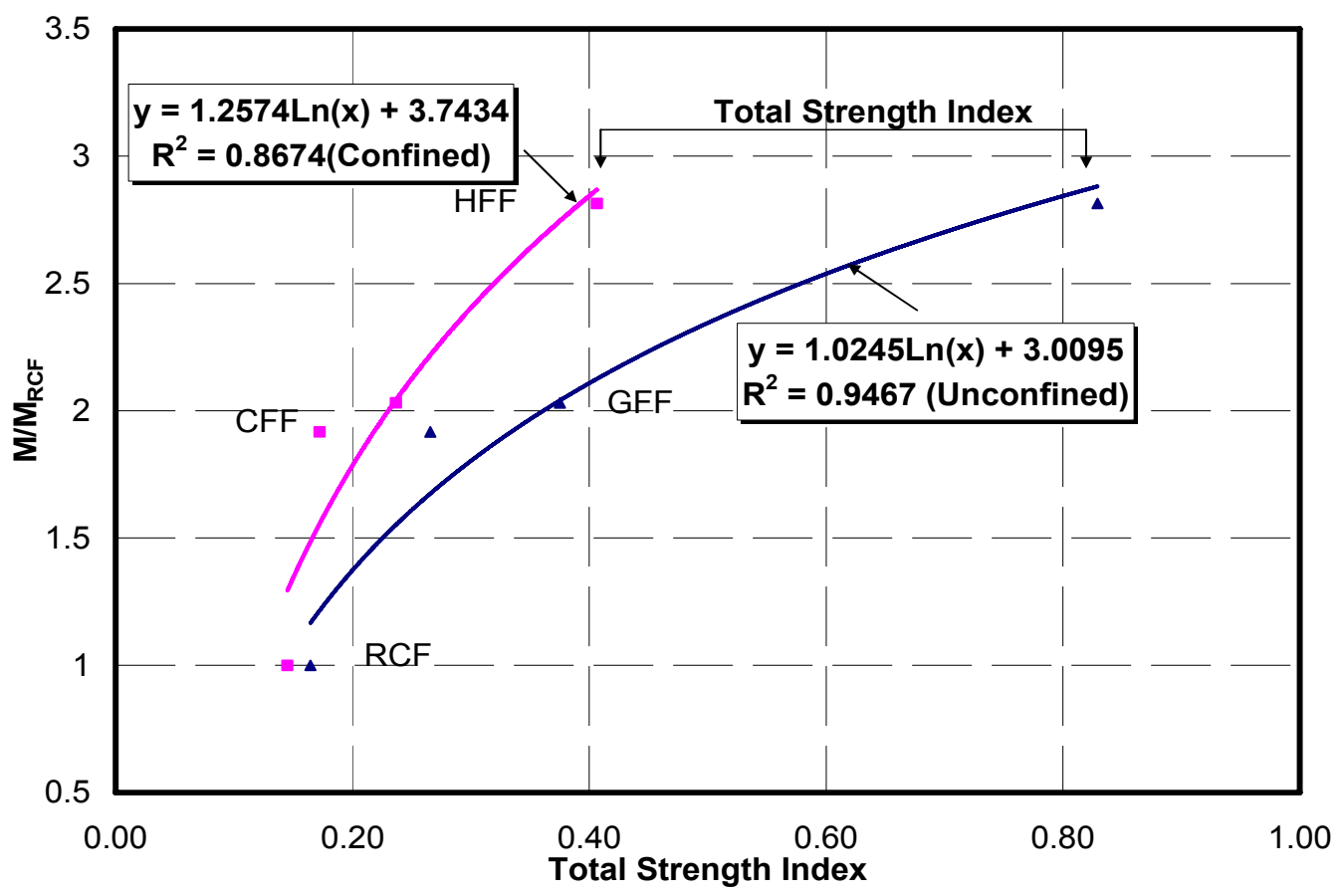

Fig. 3.51 Normalized Moments Versus Total Reinforcement Strength Index

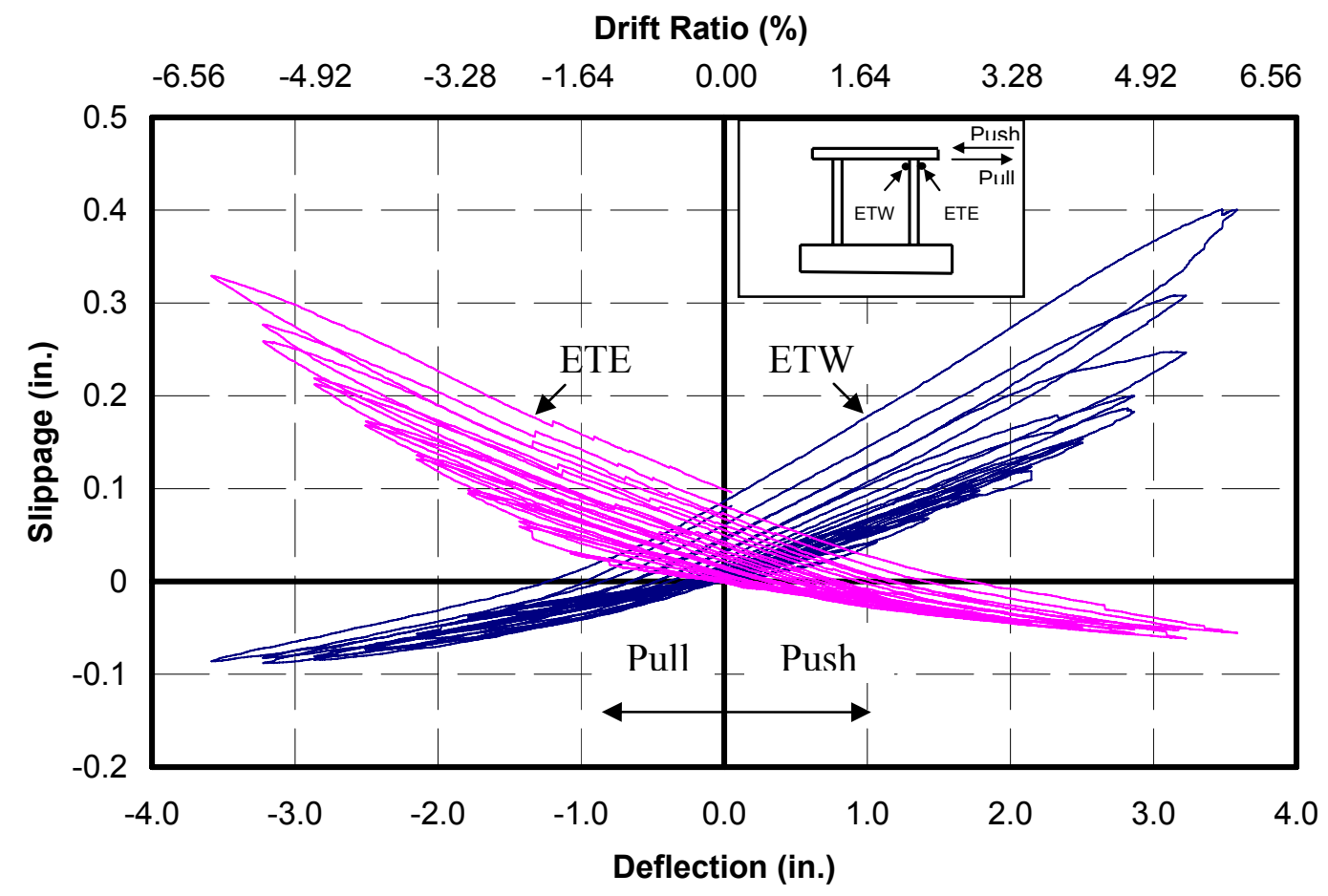

Fig. 3.52 Slippage of East Column Head in Specimen GFF 


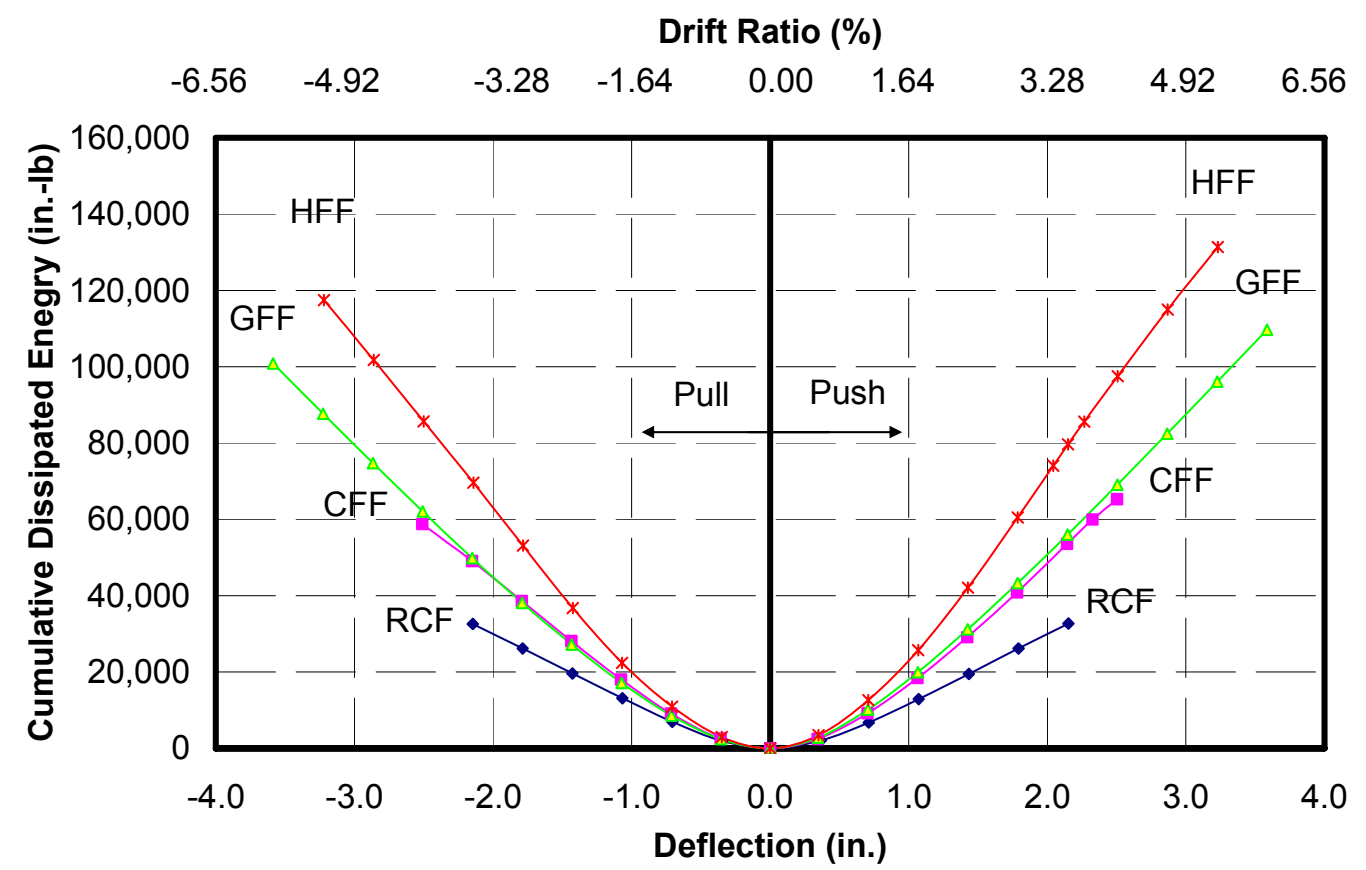

Fig. 3.53 Cumulative Dissipated Energy Versus Drift Ratio for All Specimens

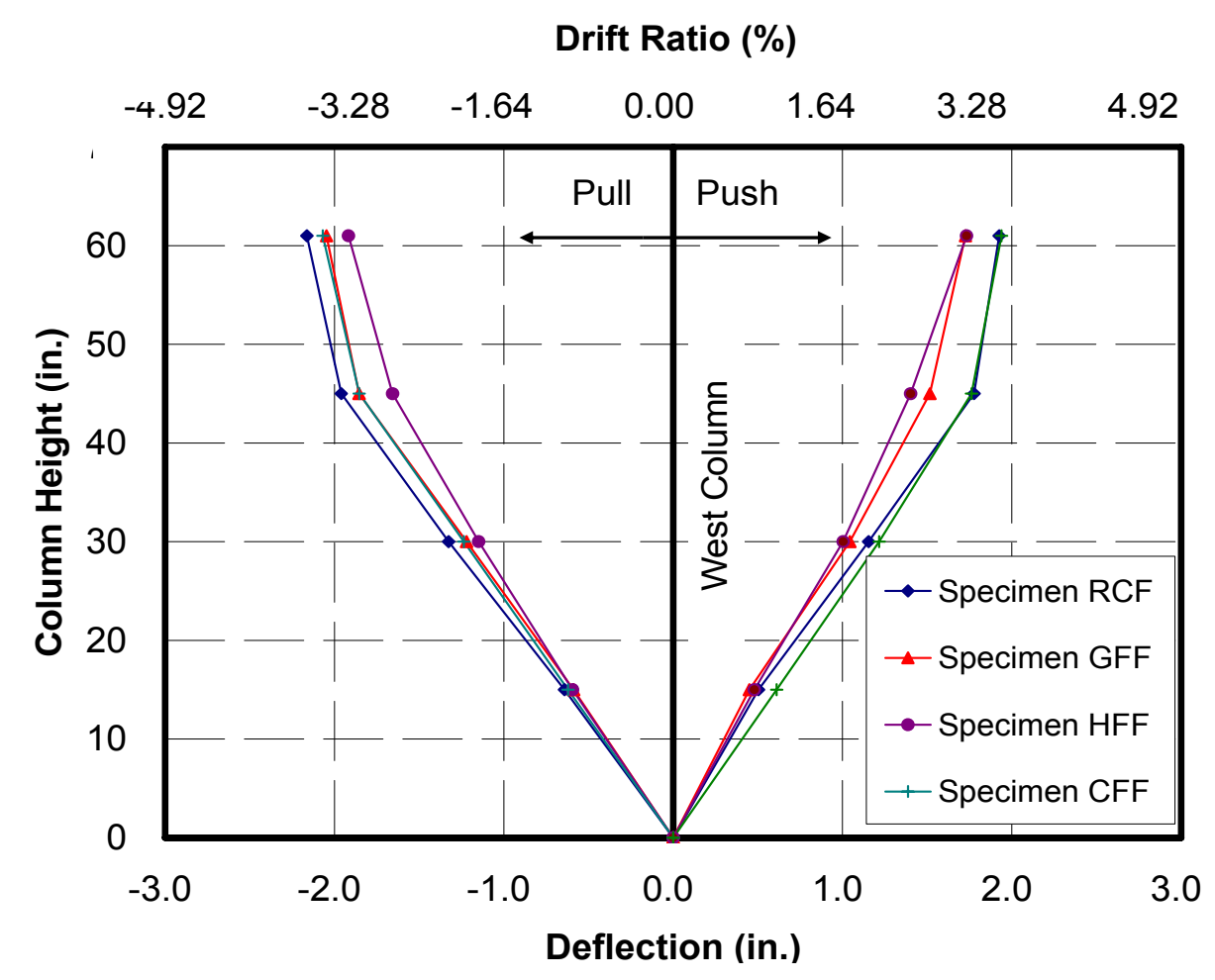

Fig. 3.54 Comparisons of Deflected Shapes of West Columns of All Specimens at $\mu=$ 


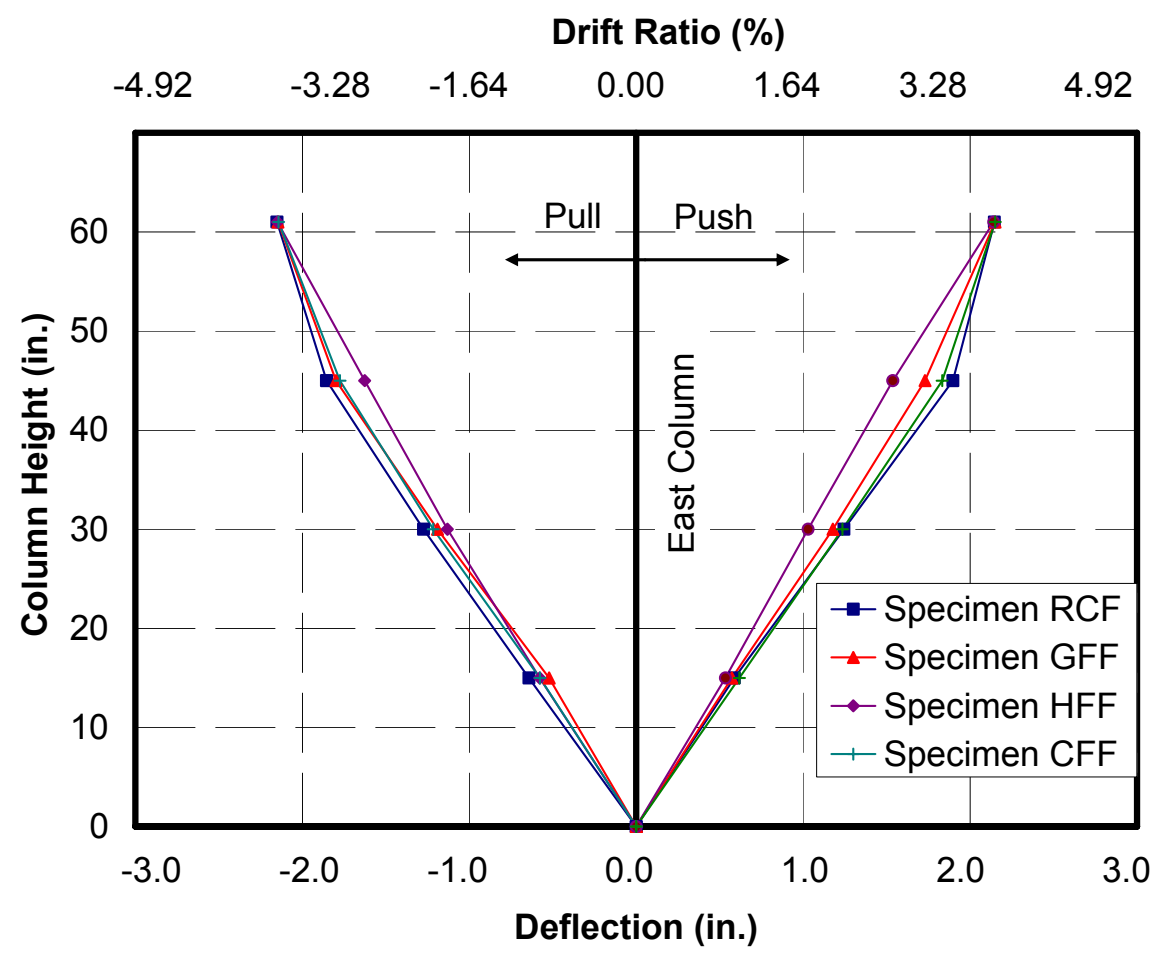

Fig. 3.55 Deflected Shapes of East Columns of All Specimens at $\mu=6$

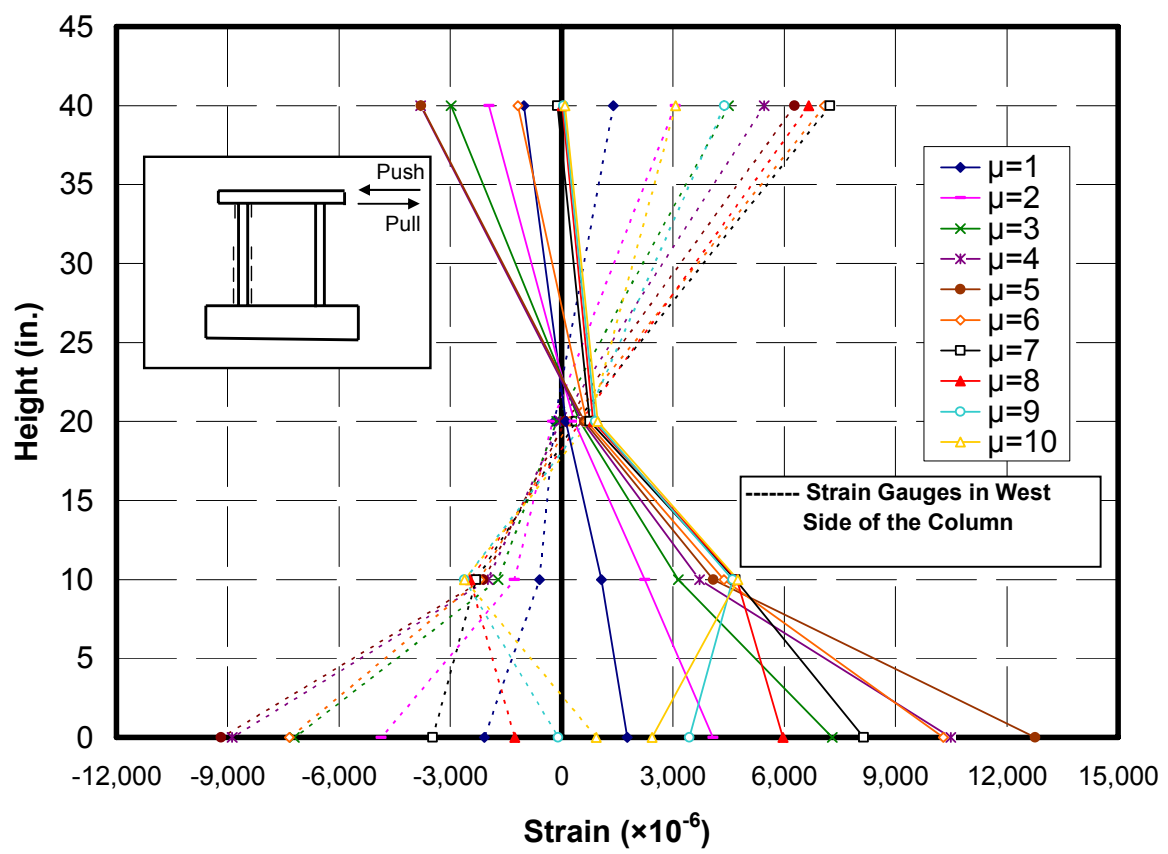

Fig. 3.56 Longitudinal Strain Profiles Along West Column of Specimen GFF in Push 


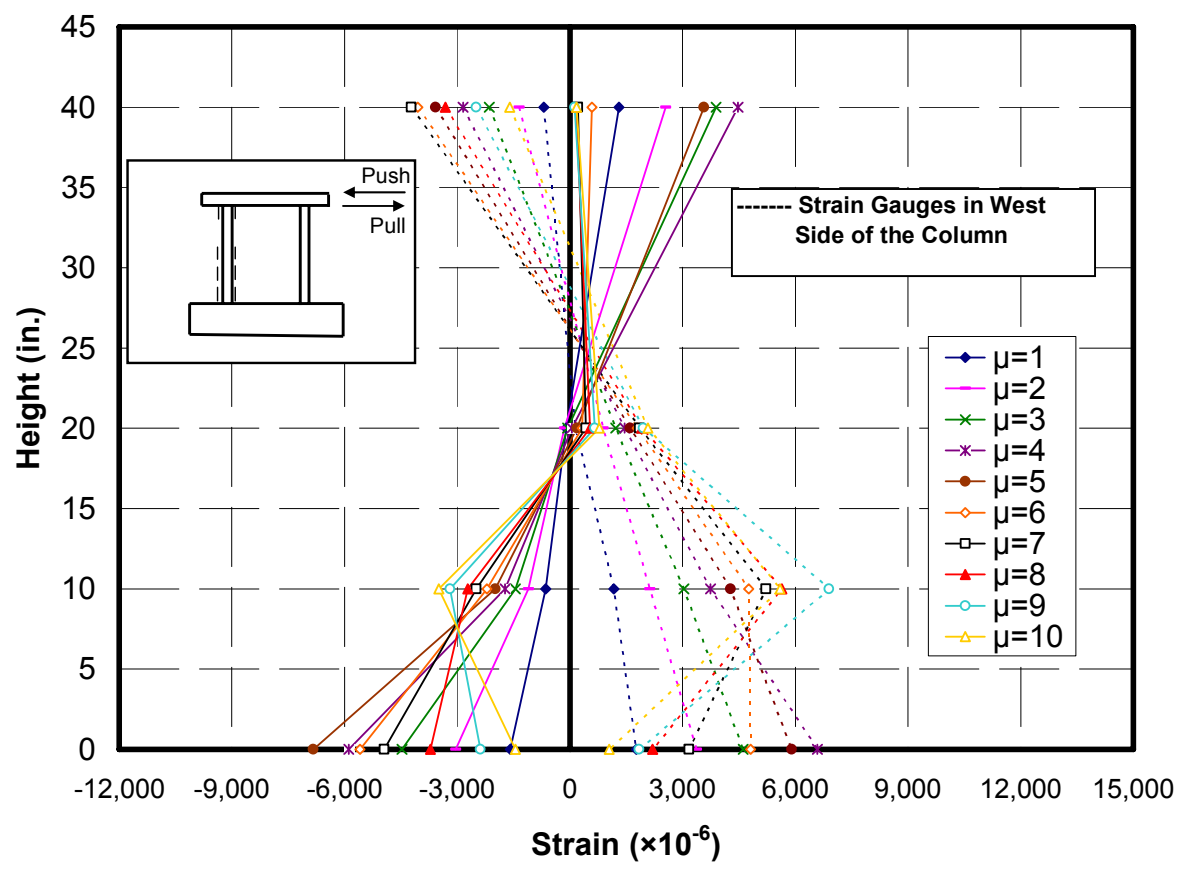

Fig. 3.57 Longitudinal Strain Profiles Along West Column of Specimen GFF in Pull

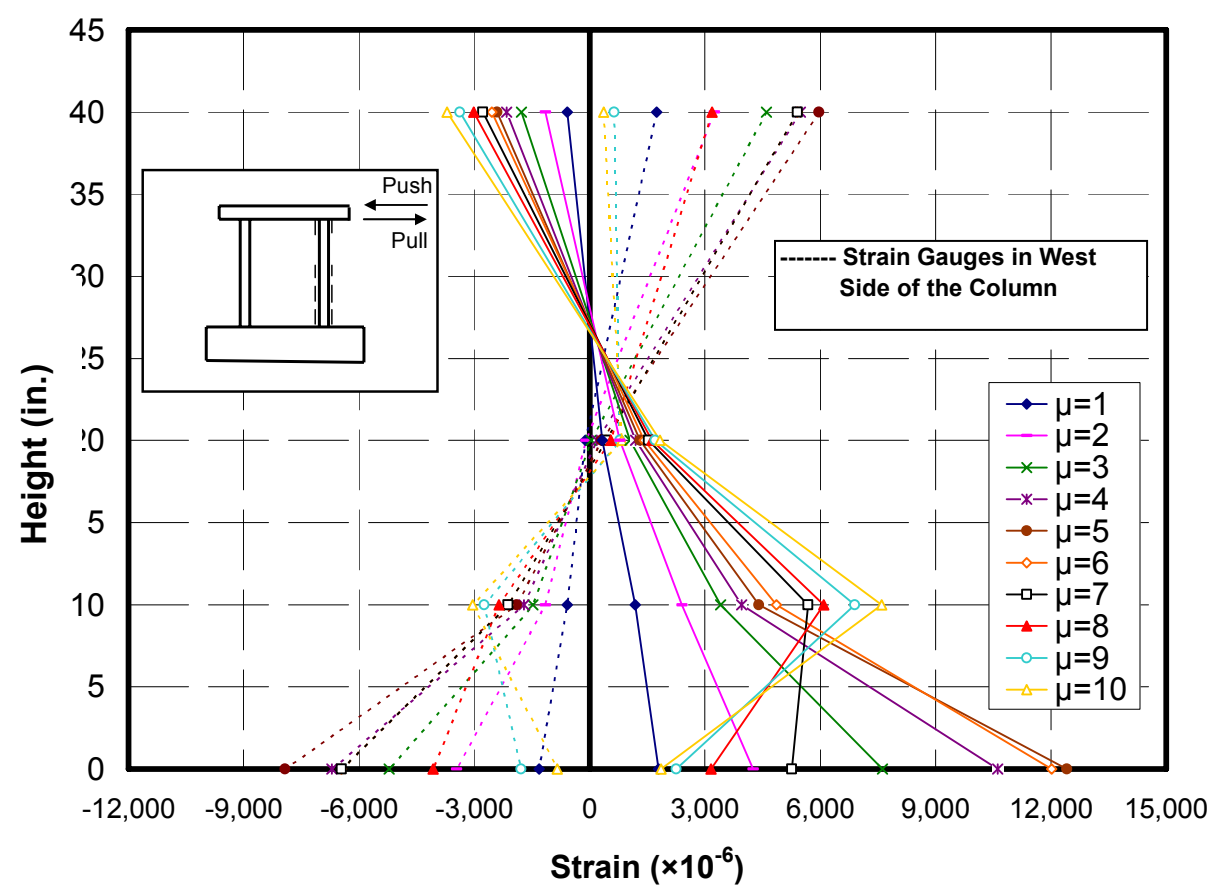

Fig. 3.58 Longitudinal Strain Profiles Along East Column of Specimen GFF in Push 


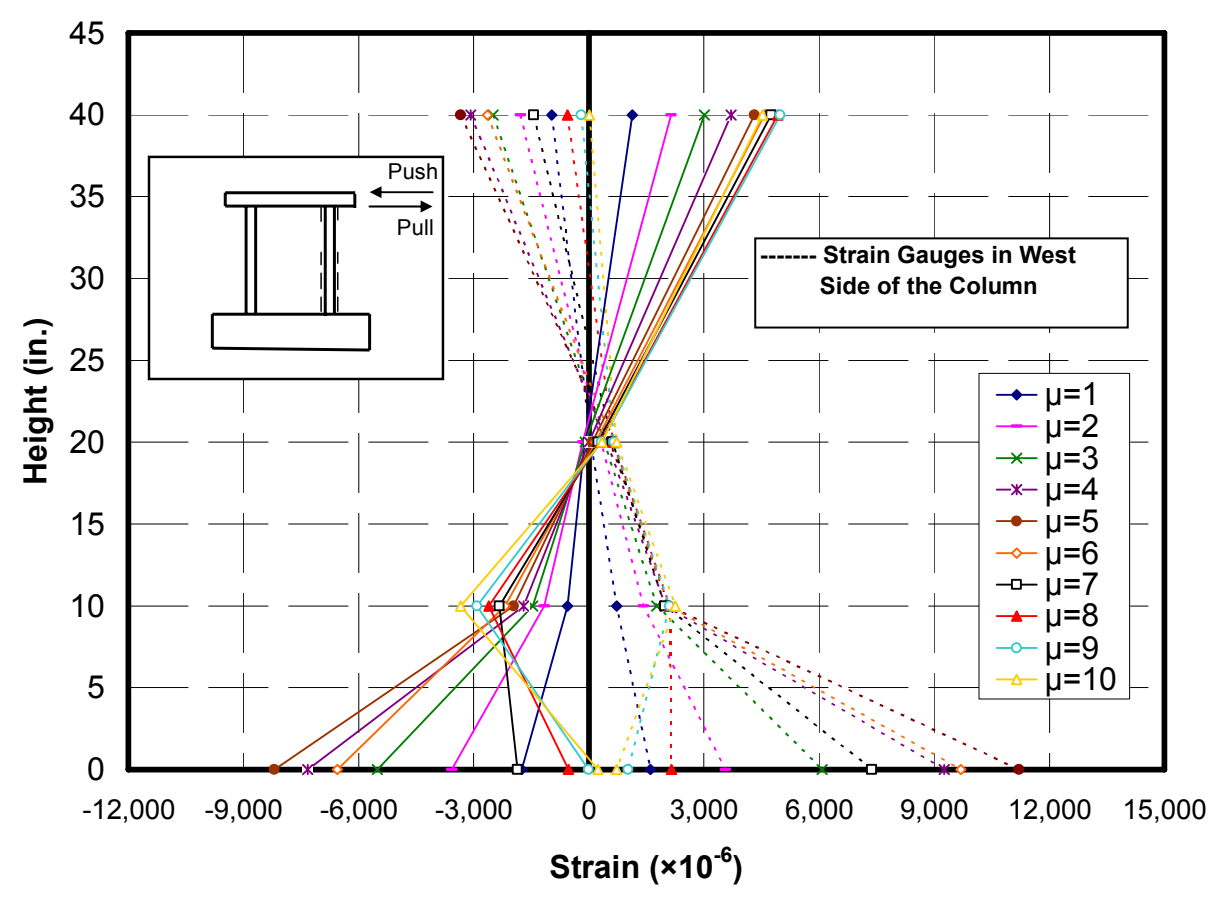

Fig. 3.59 Longitudinal Strain Profiles Along East Column of Specimen GFF in Pull

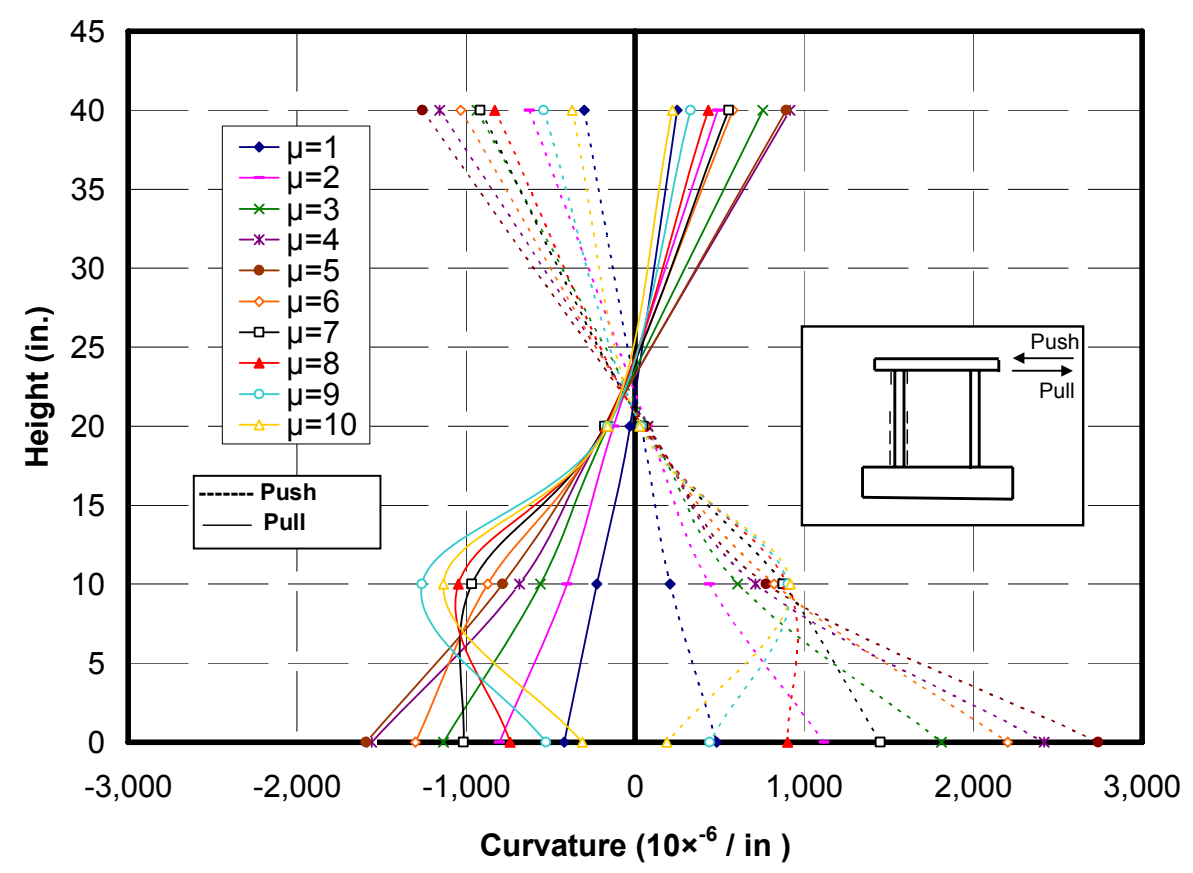

Fig. 3.60 Curvature Profiles Along West Column of Specimen GFF in Push and Pull 


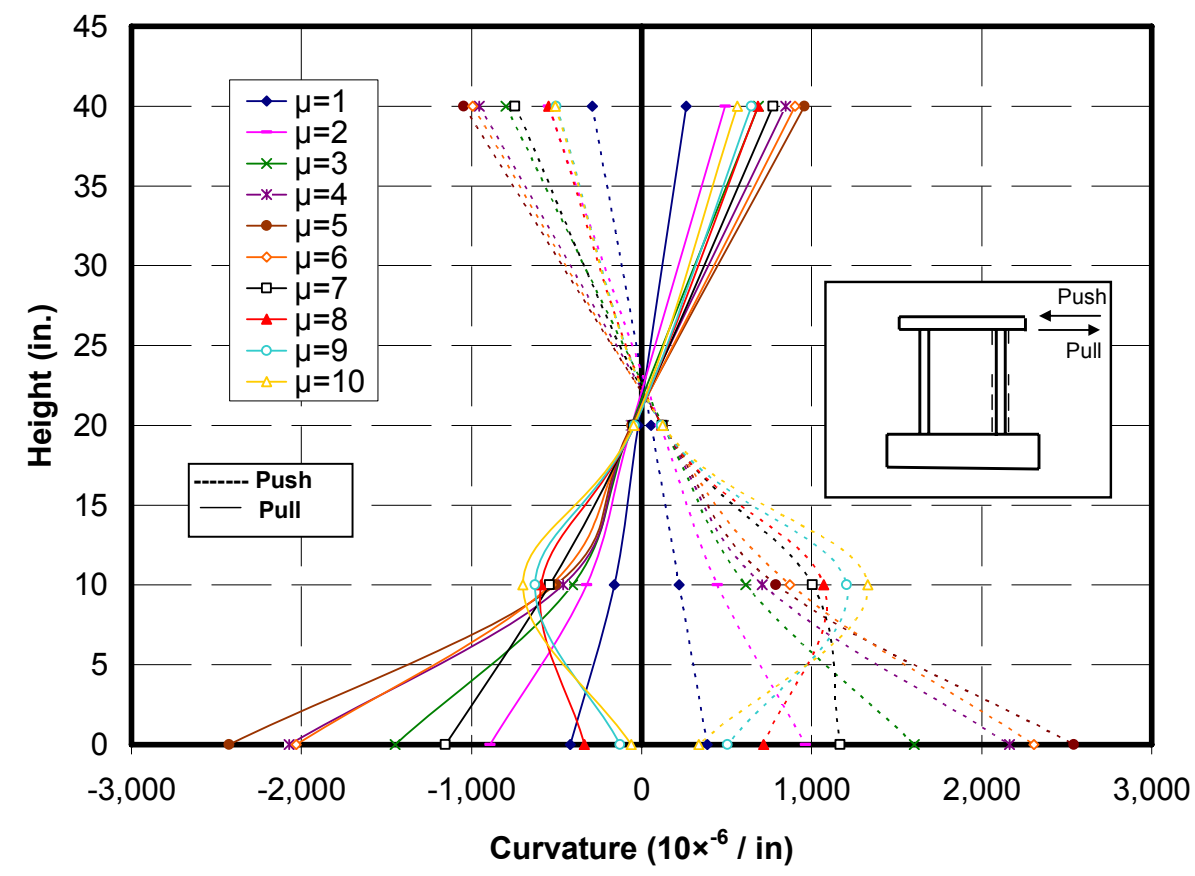

Fig. 3.61 Curvature Profiles Along East Column of Specimen GFF in Push and Pull

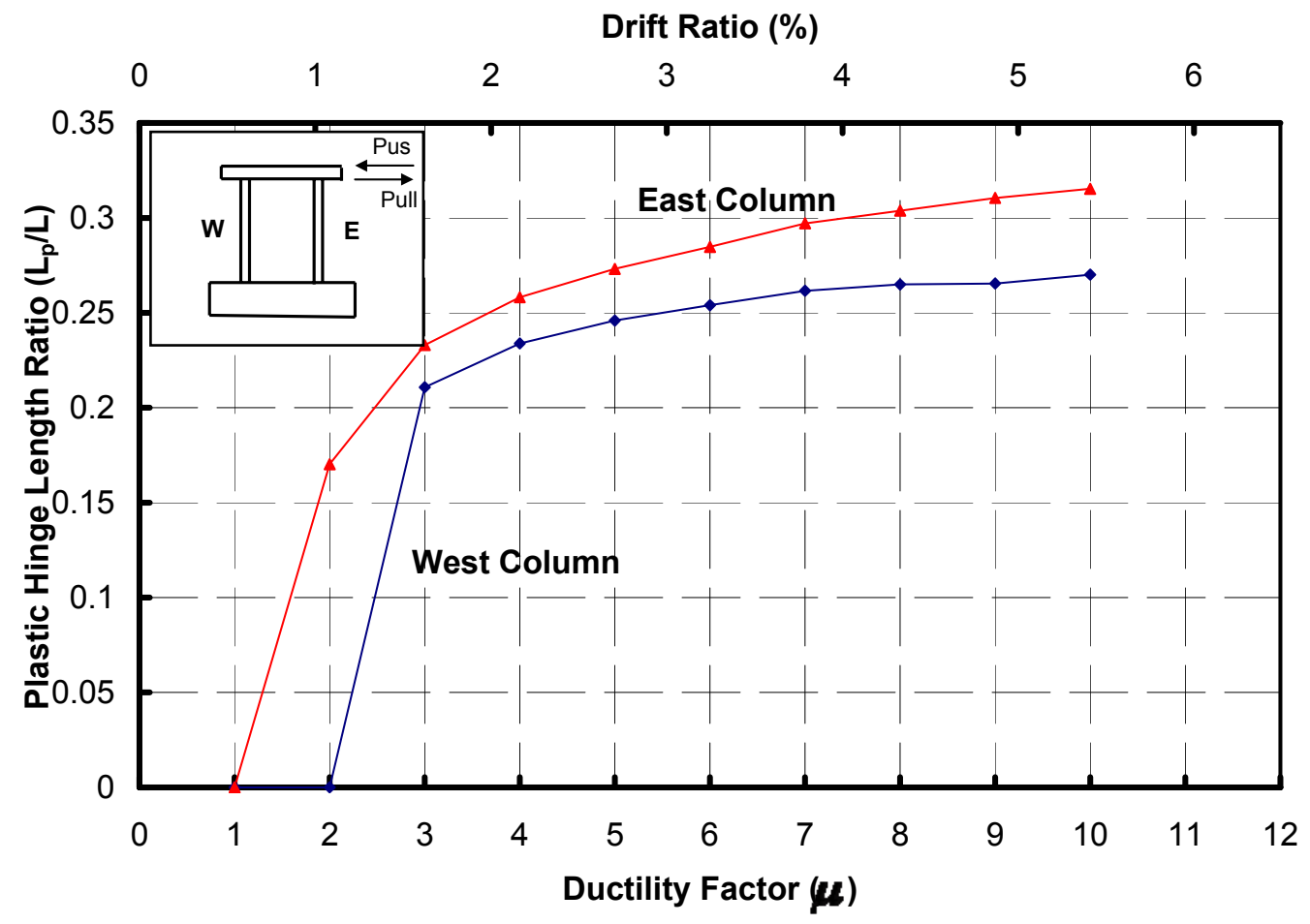

Fig. 3.62 Plastic Hinge Length Ratio Versus Ductility for Specimen GFF 


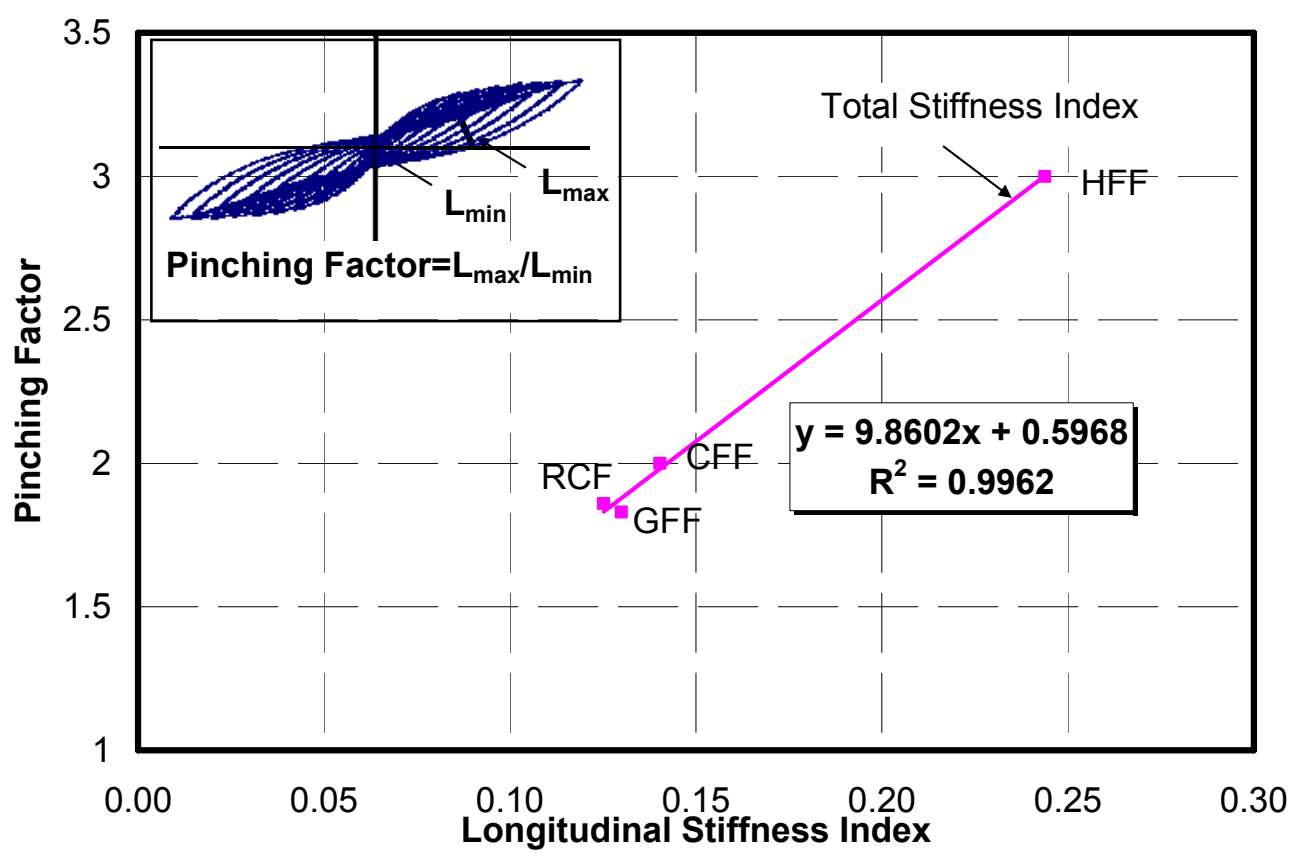

Fig. 3.63 Pinching factor Versus Total Stiffness Index

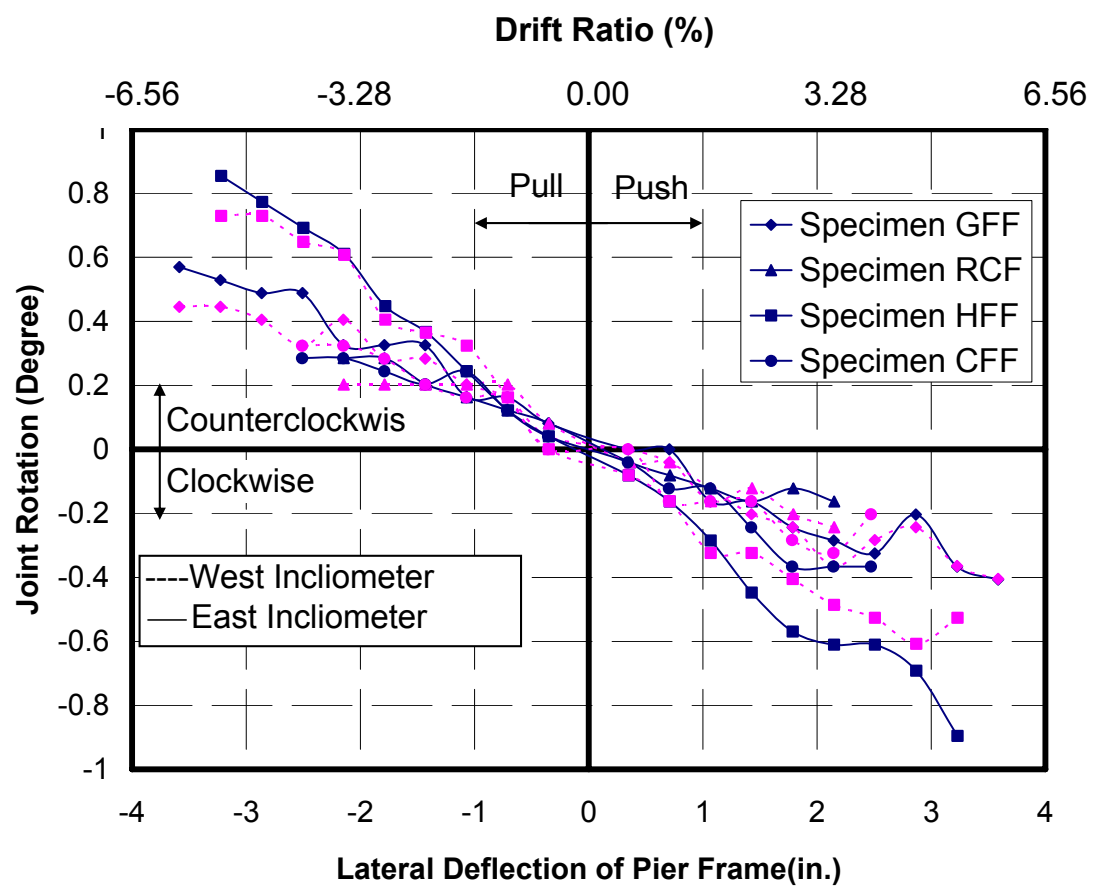

Fig. 3.64 Rotations of Pier Cap Beam-Column Joints 


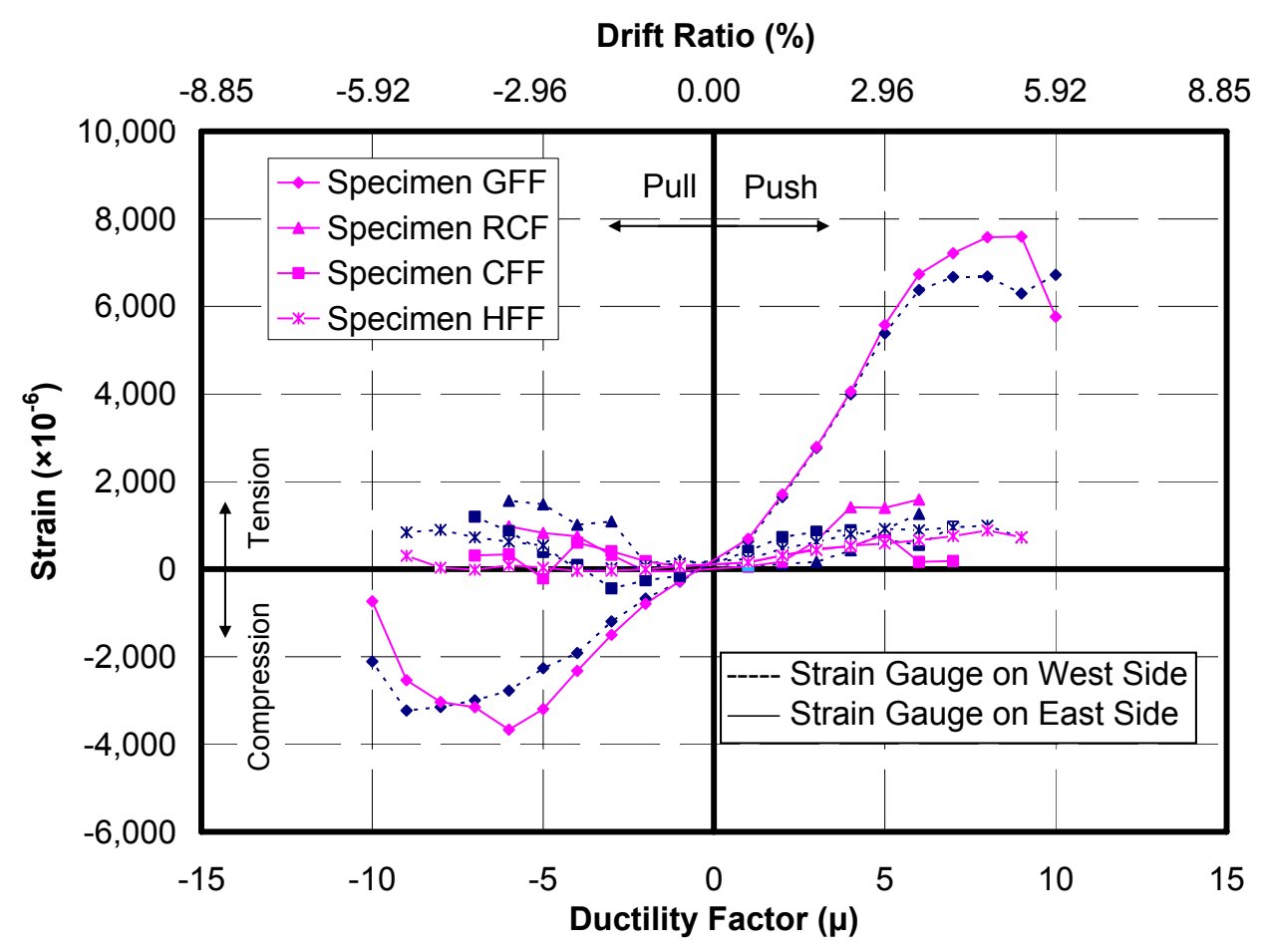

Fig. 3.65 Hoop Strains at the Base of West Columns in All Specimens

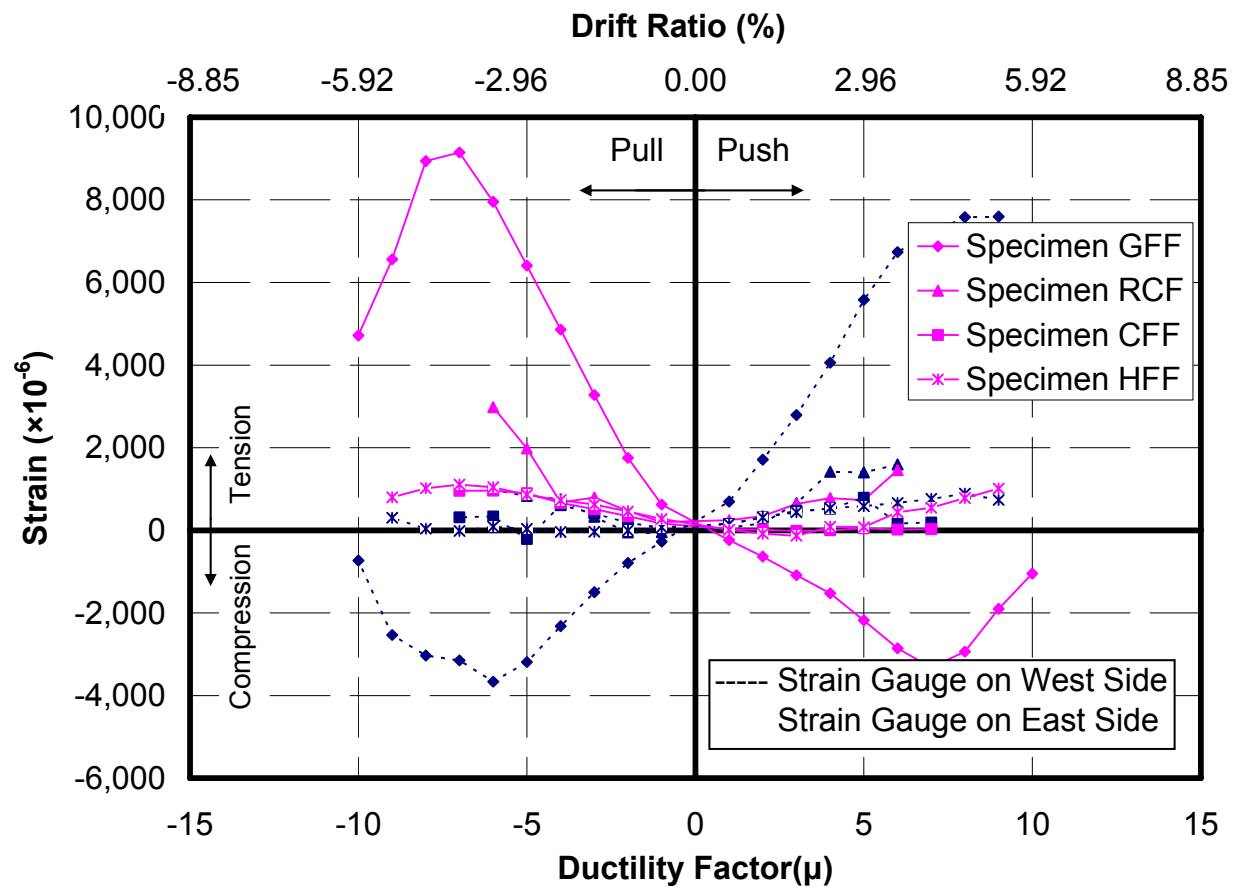

Fig. 3.66 Hoop Strains at the Base of East Columns in All Specimens 


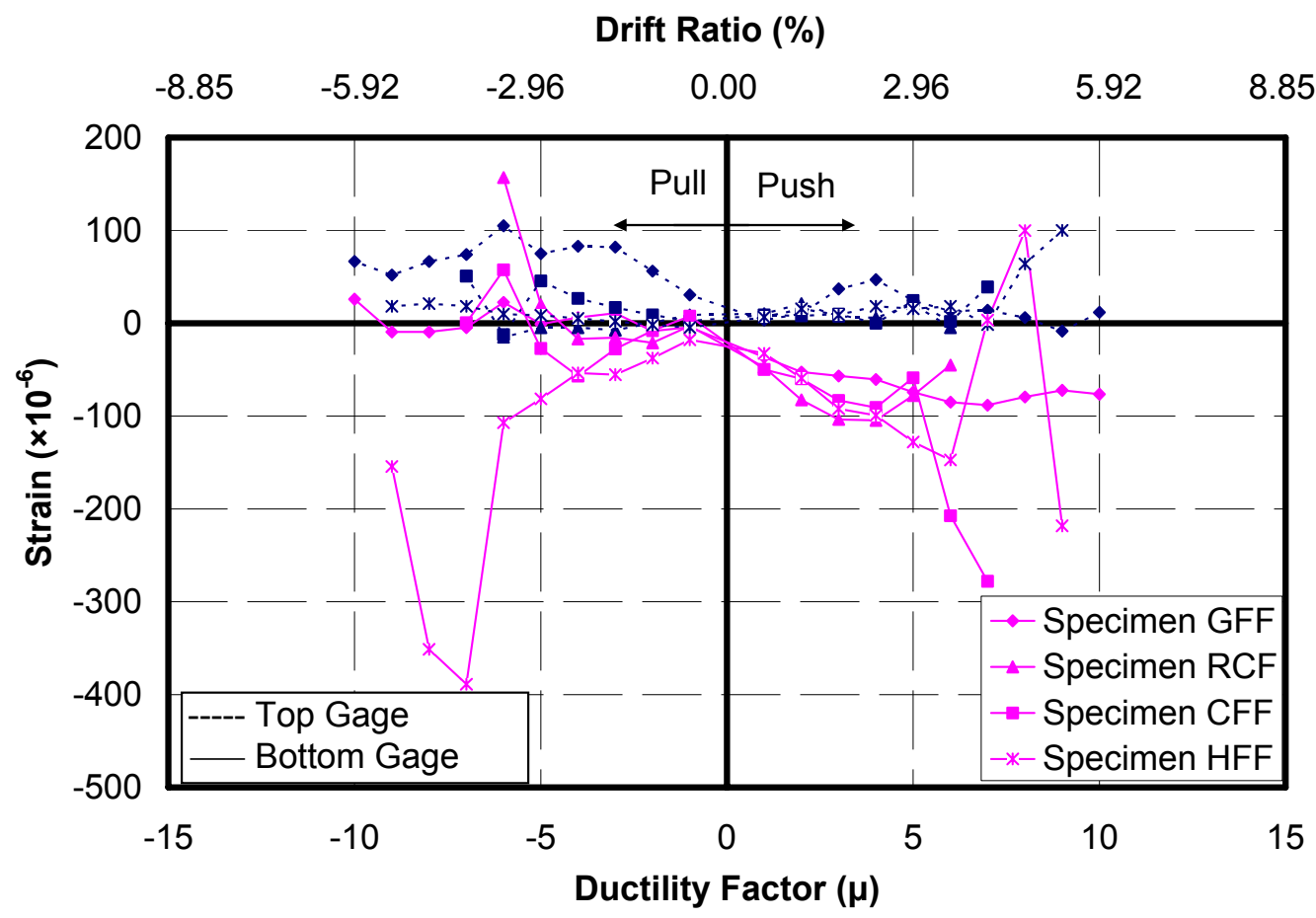

Fig. 3.67 Mid-Span Strains of Pier Cap Beams in All Specimens

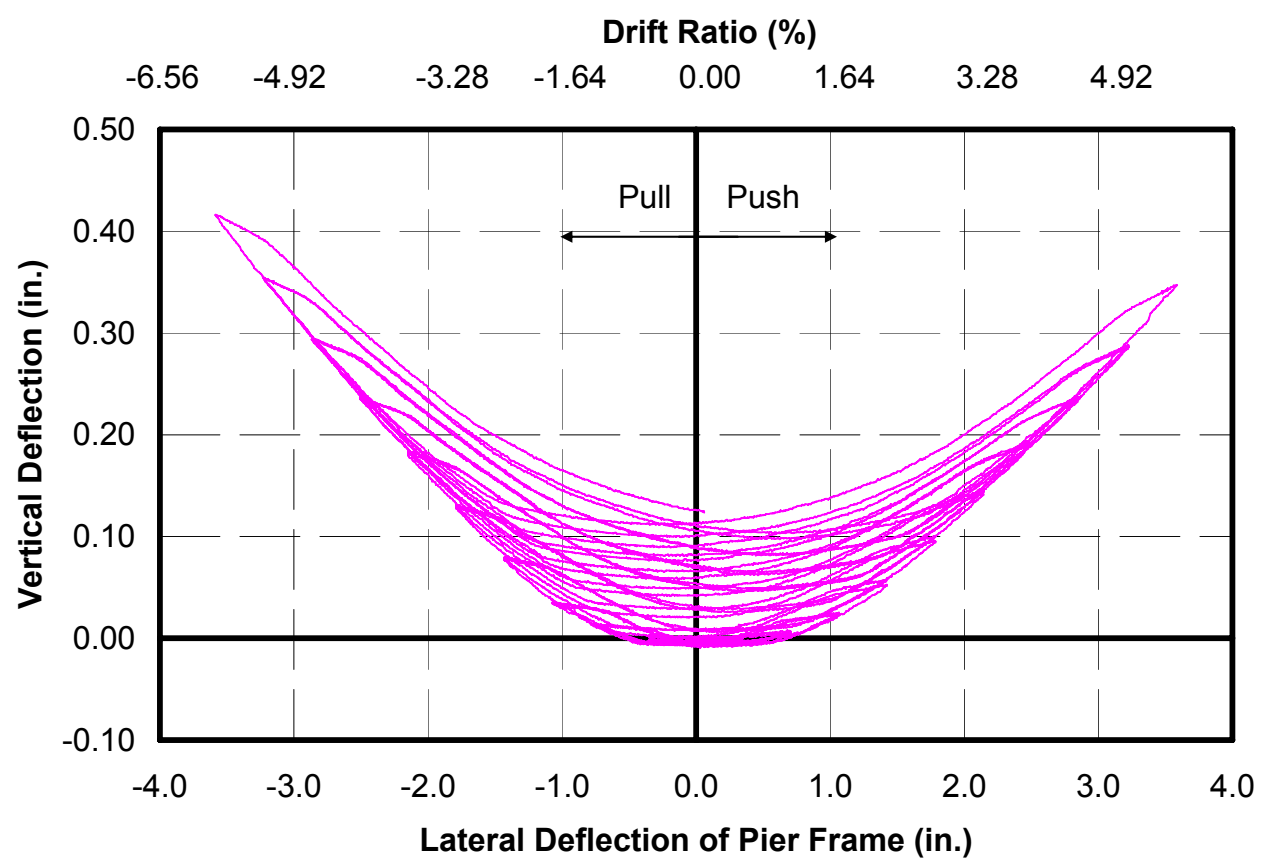

Fig. 3.68 Mid-Span Deflection of Pier Cap Beams in Specimen GFF 


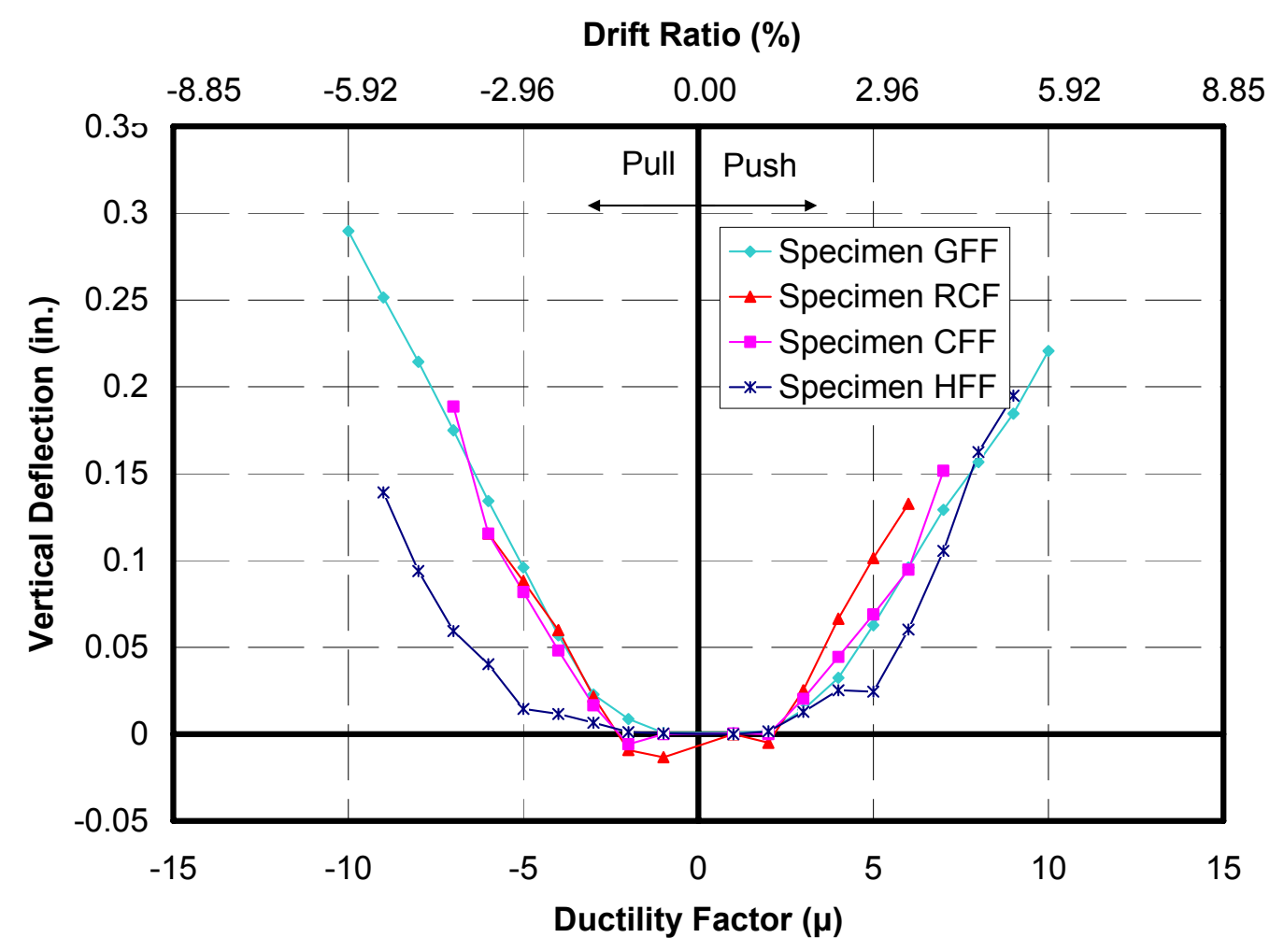

Fig. 3.69 Mid-Span Deflection Envelopes of Pier Cap Beams in All Specimens

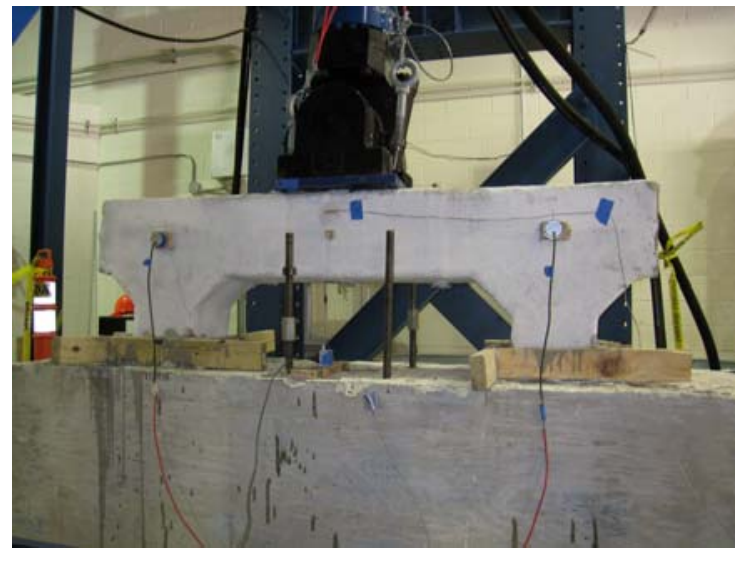

Fig. 3.70 Overview of the Setup for Pier Cap Beam Test

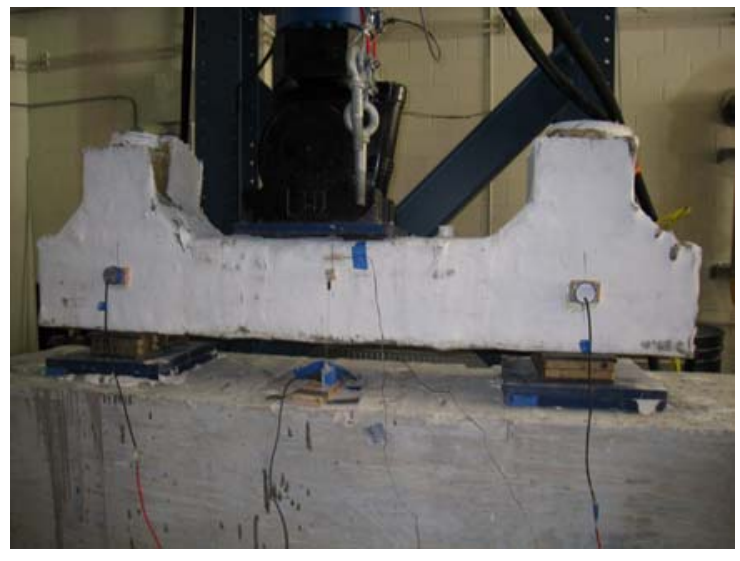

Fig. 3.71 Pier Cap Beam Tested Upside Down 


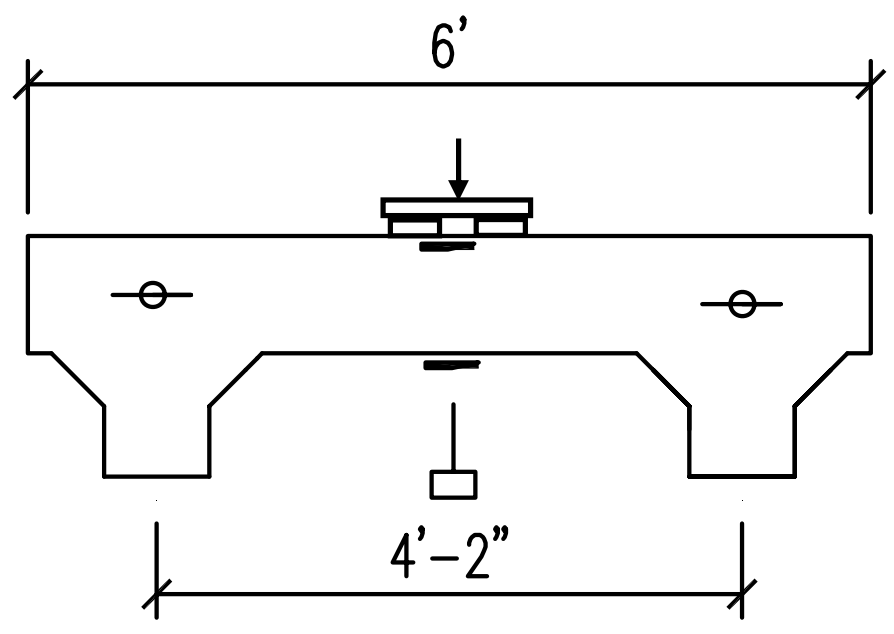

\begin{tabular}{|l|l|}
\hline Legend & Name \\
\hline$O-$ & Inclinometer \\
\hline$\square-$ & String Pot \\
\hline- & Strain Gauge \\
\hline
\end{tabular}

Fig. 3.72 Instrumentation Plan for Pier Cap Beam Tests
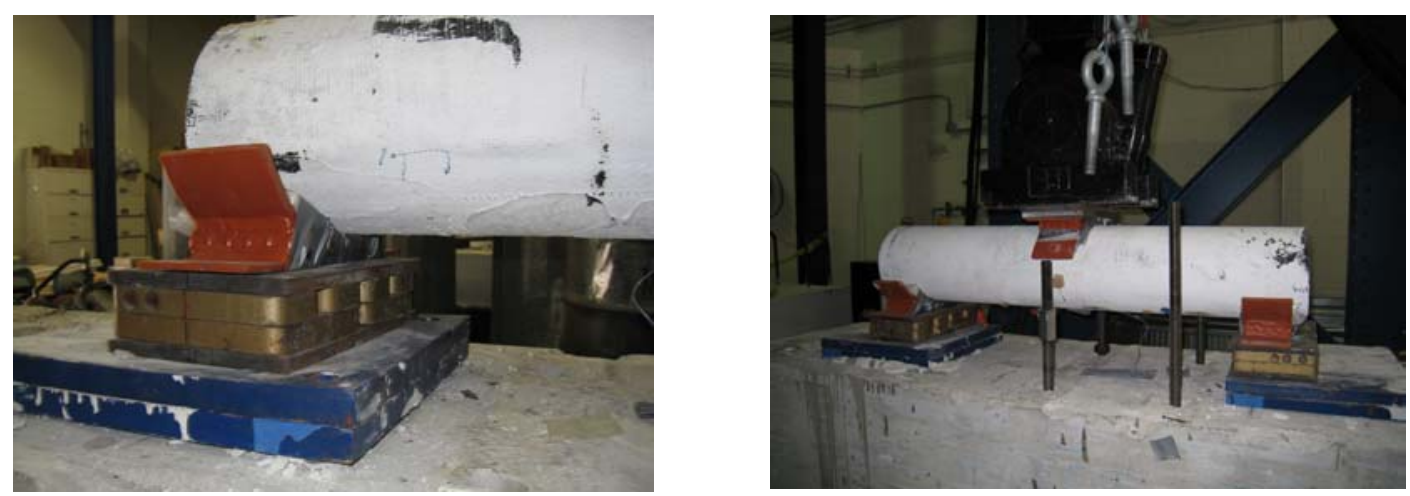

Fig. 3.73 Specially Designed Semi-Circular Steel Support

Fig. 3.74 Overview of the Setup for CFFT Column Test

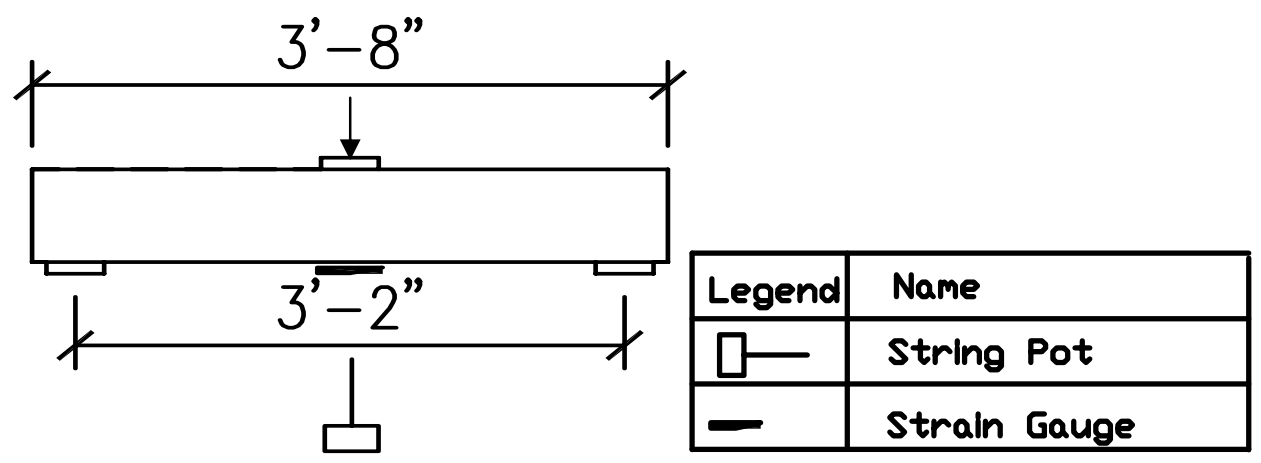

Fig. 3.75 Instrumentation Plan for CFFT Column Tests 


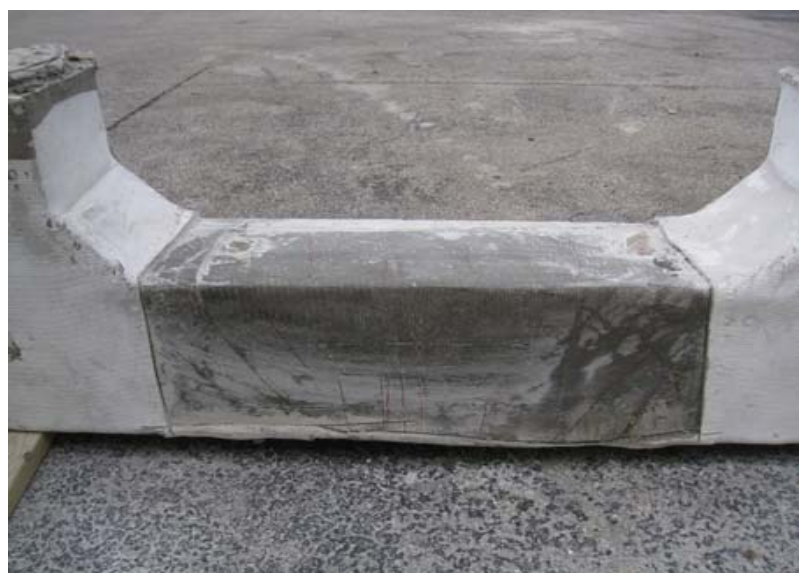

Fig. 3.76 Cracks in Concrete in Pier Cap Beam

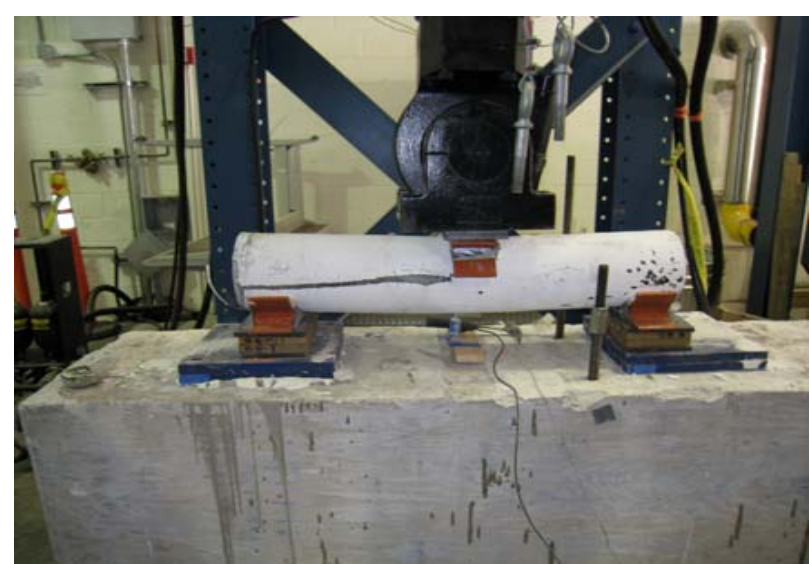

Fig. 3.78 Longitudinal Crack in Carbon CFFT Column

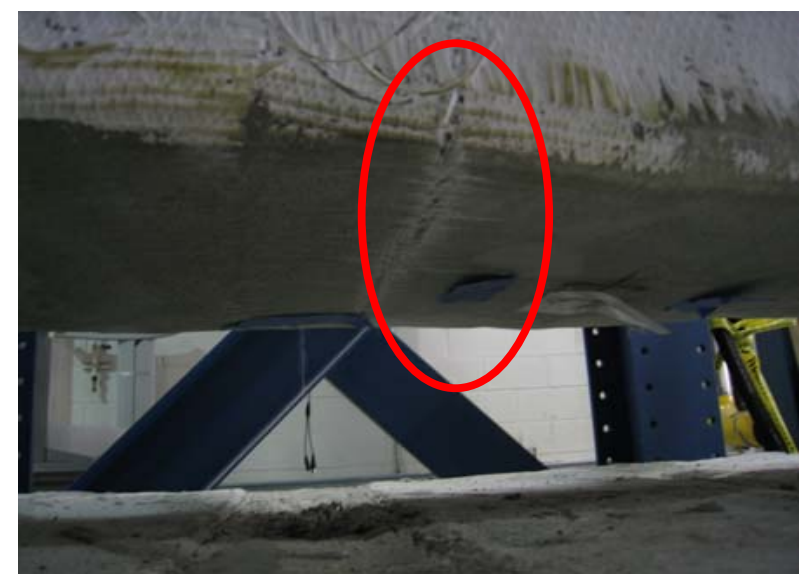

Fig. 3.77 FRP Cracks at the Bottom of Pier Cap Beam Tested Upside Down

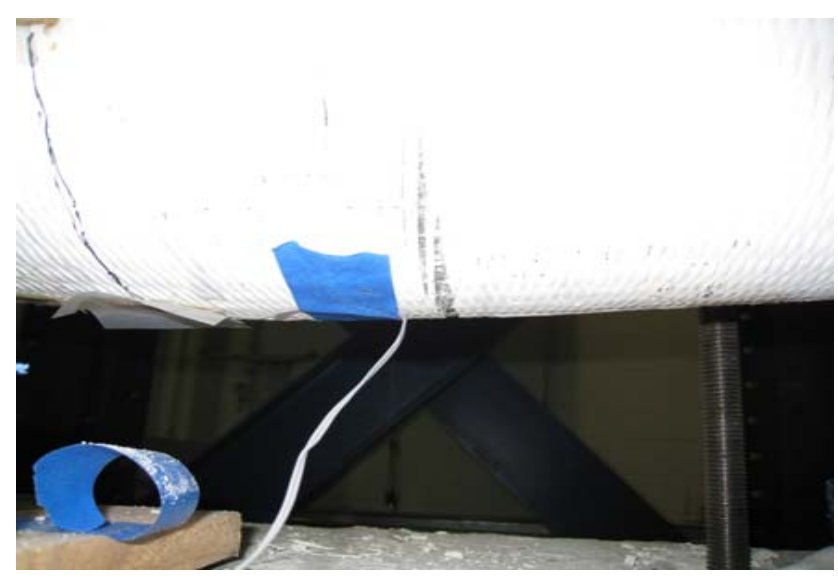

Fig. 3.79 Cracks at Mid-Span of Hybrid CFFT Column 


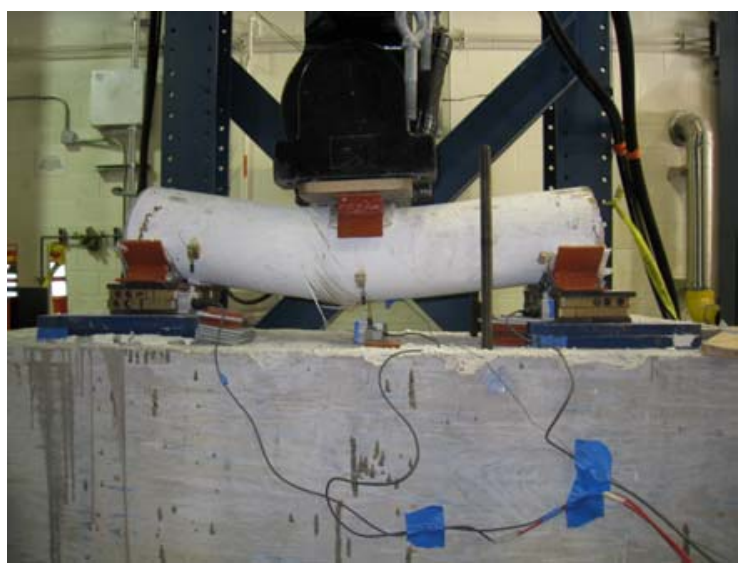

Fig. 3.80 Large Deformability of Glass CFFT Colum

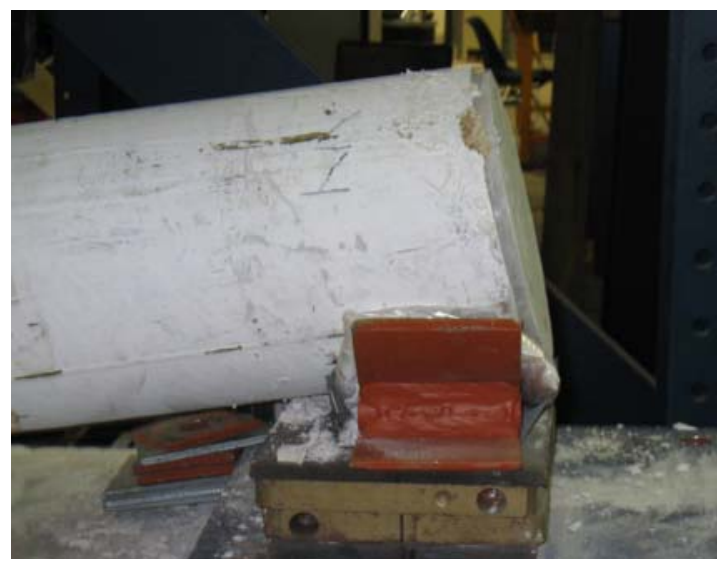

Fig. 3.81 End Slippage in Glass CFFT Column

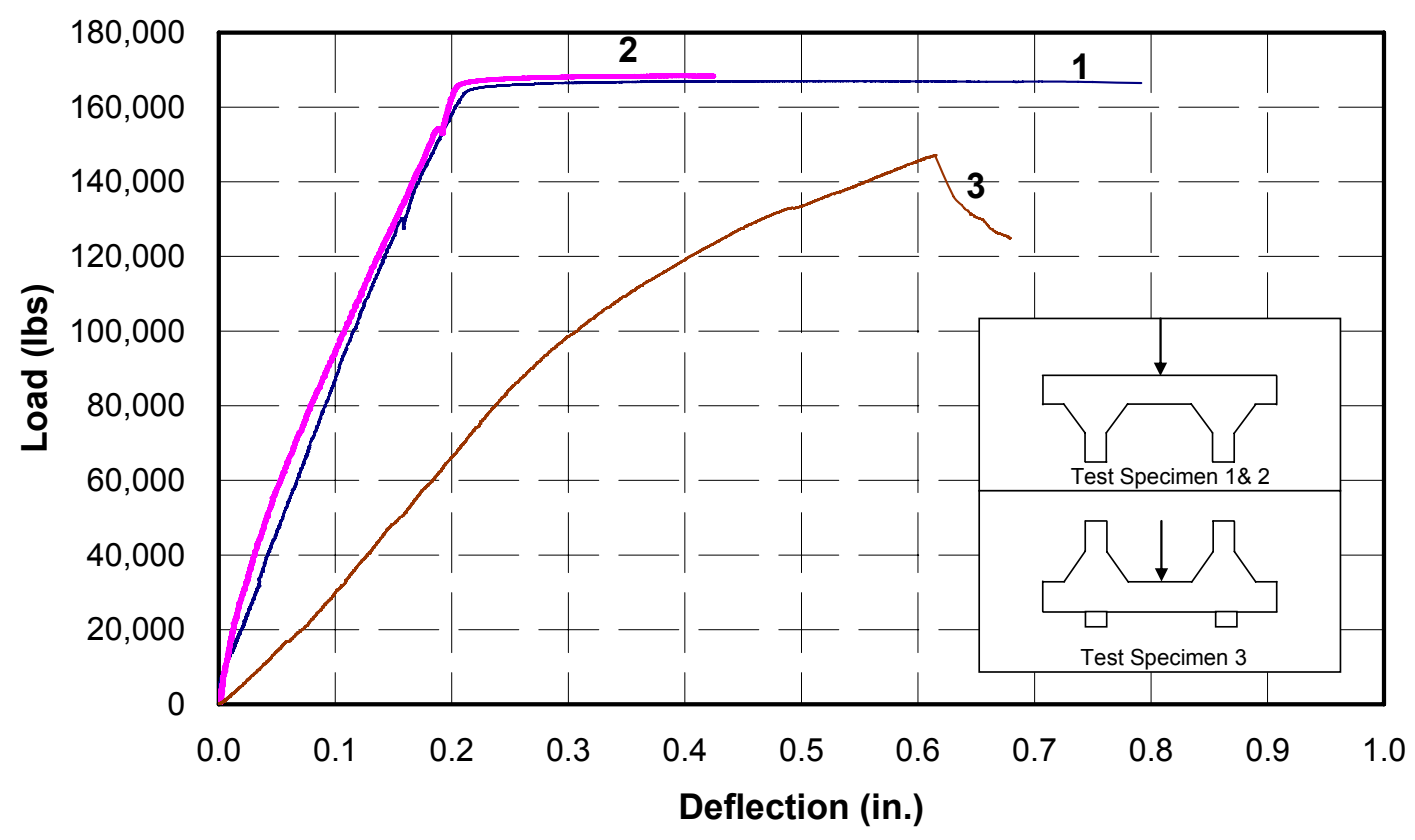

Fig. 3.82 Residual Load-Deflection Responses of Pier Cap Beams 


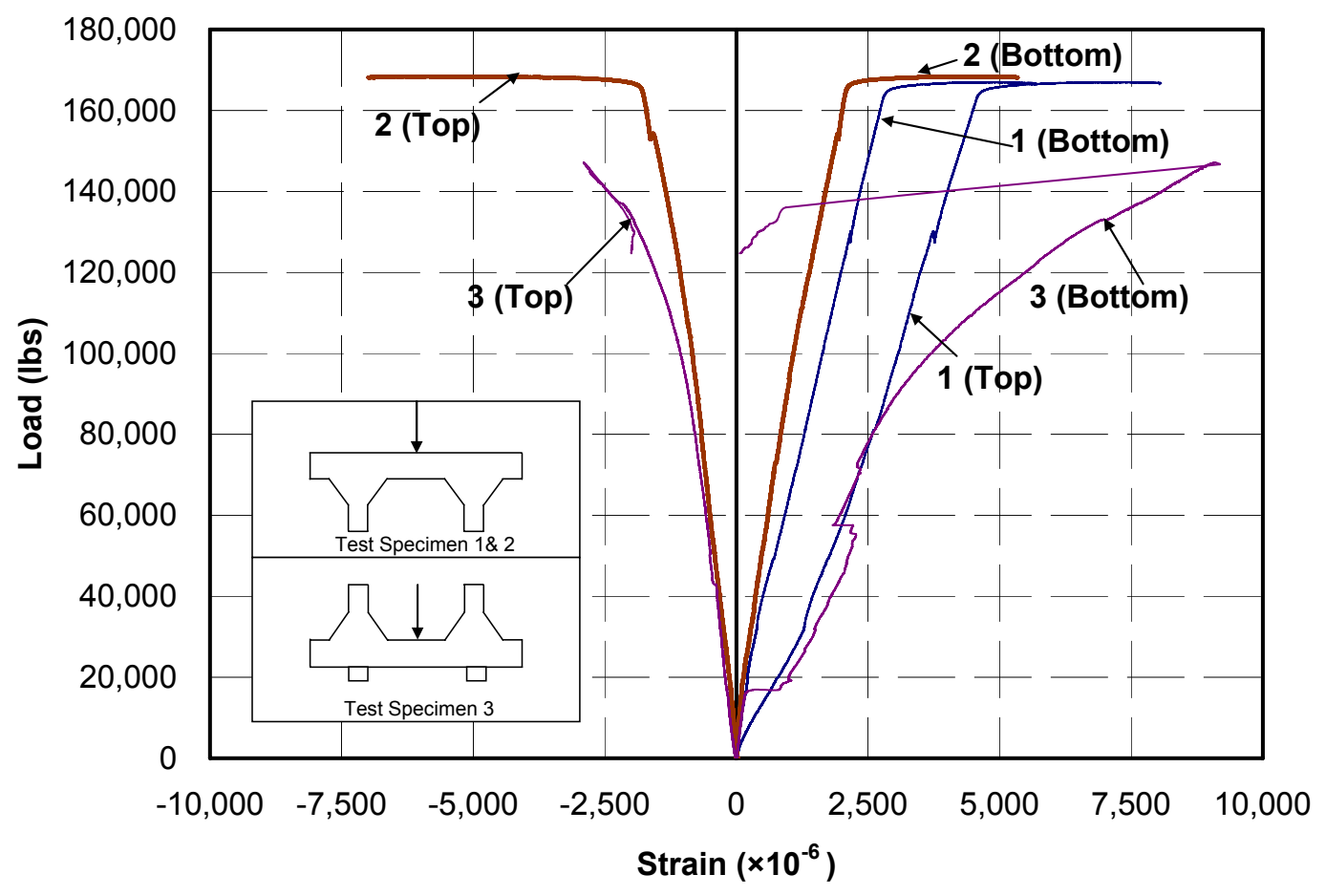

Fig. 3.83 Residual Load-Strain Responses of Pier Cap Beams

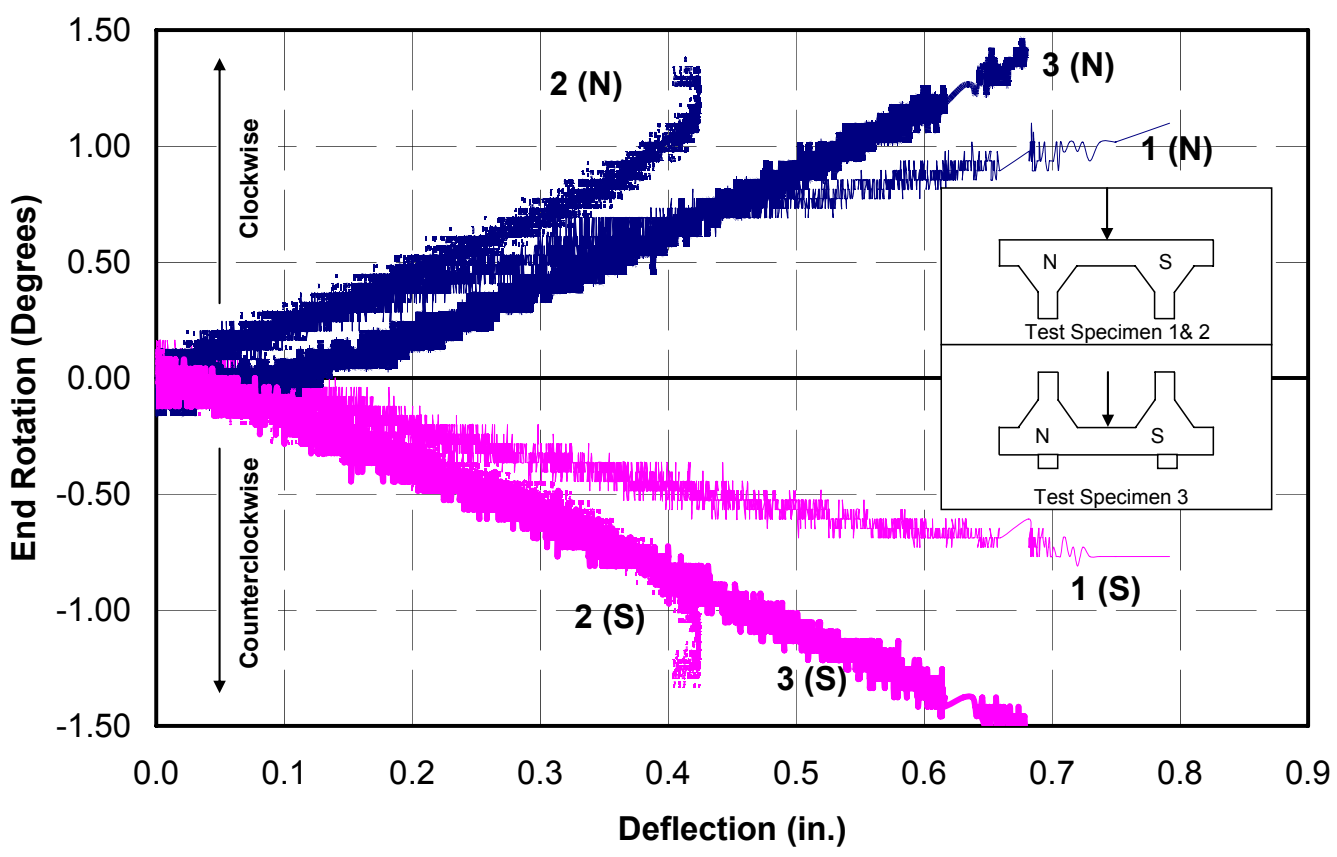

Fig. 3.84 End Rotations of Pier Cap Beams 


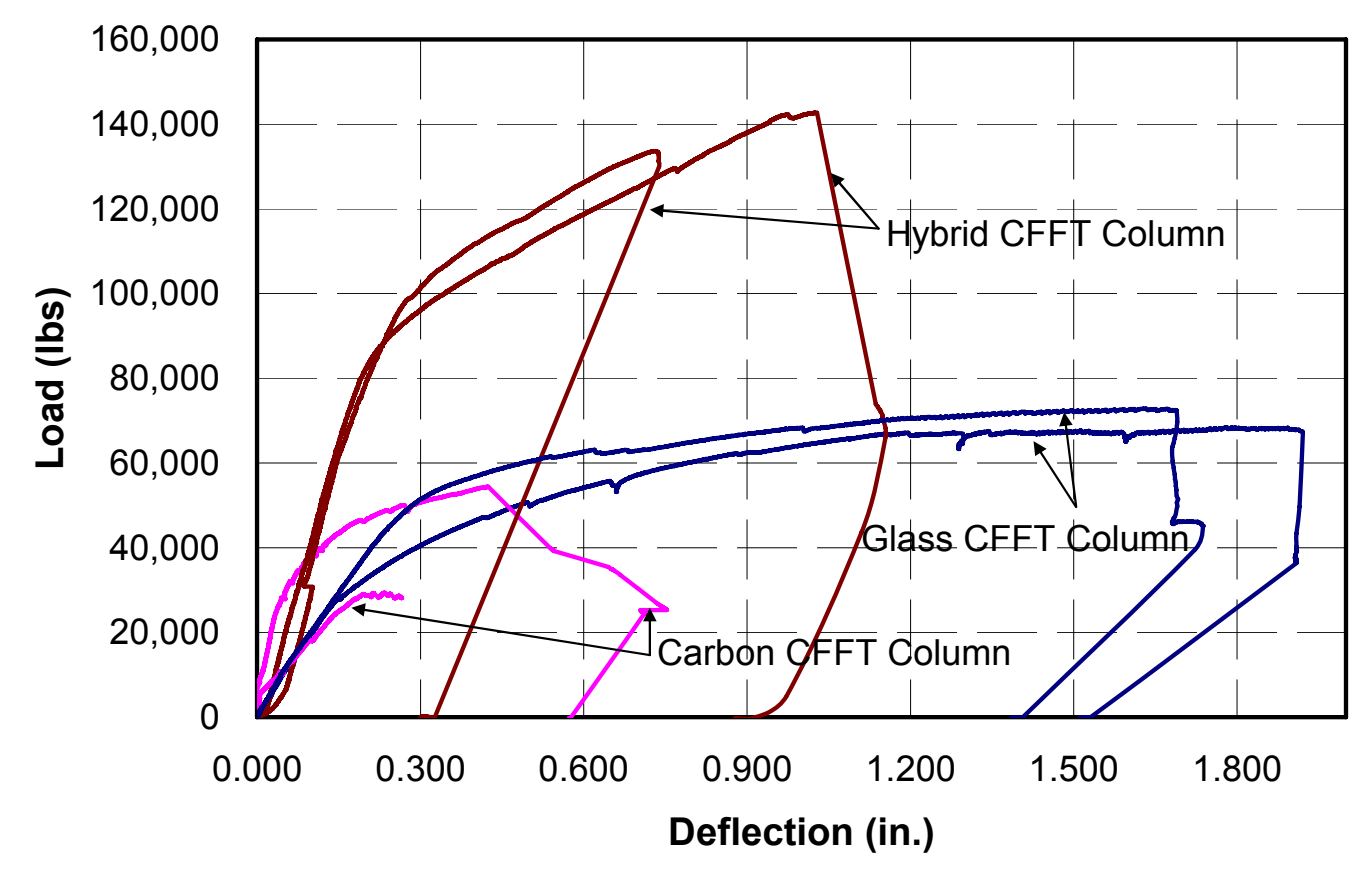

Fig. 3.85 Residual Load-Deflection Responses of CFFT Columns

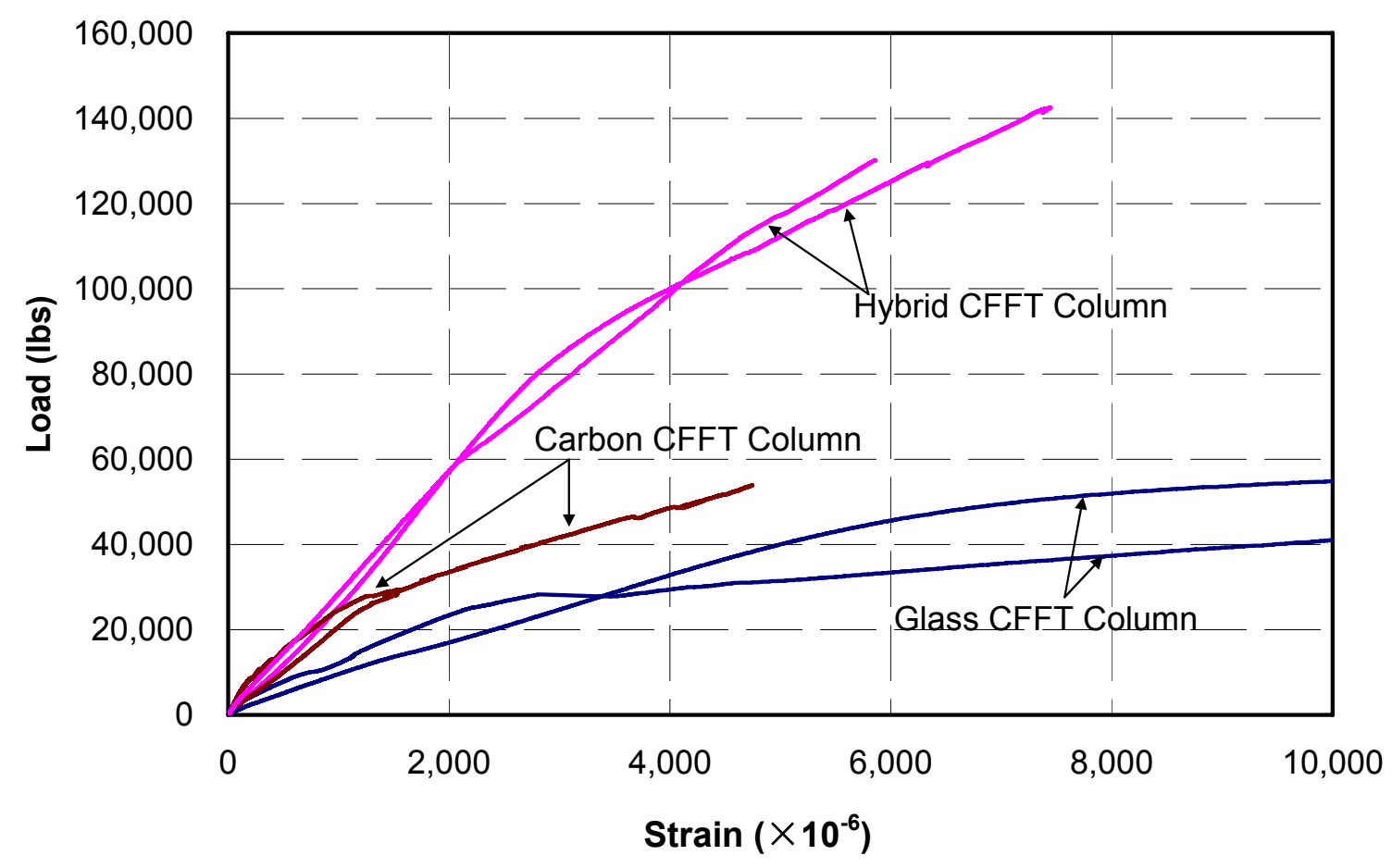

Fig. 3.86 Residual Load-Strain Responses of CFFT Columns 


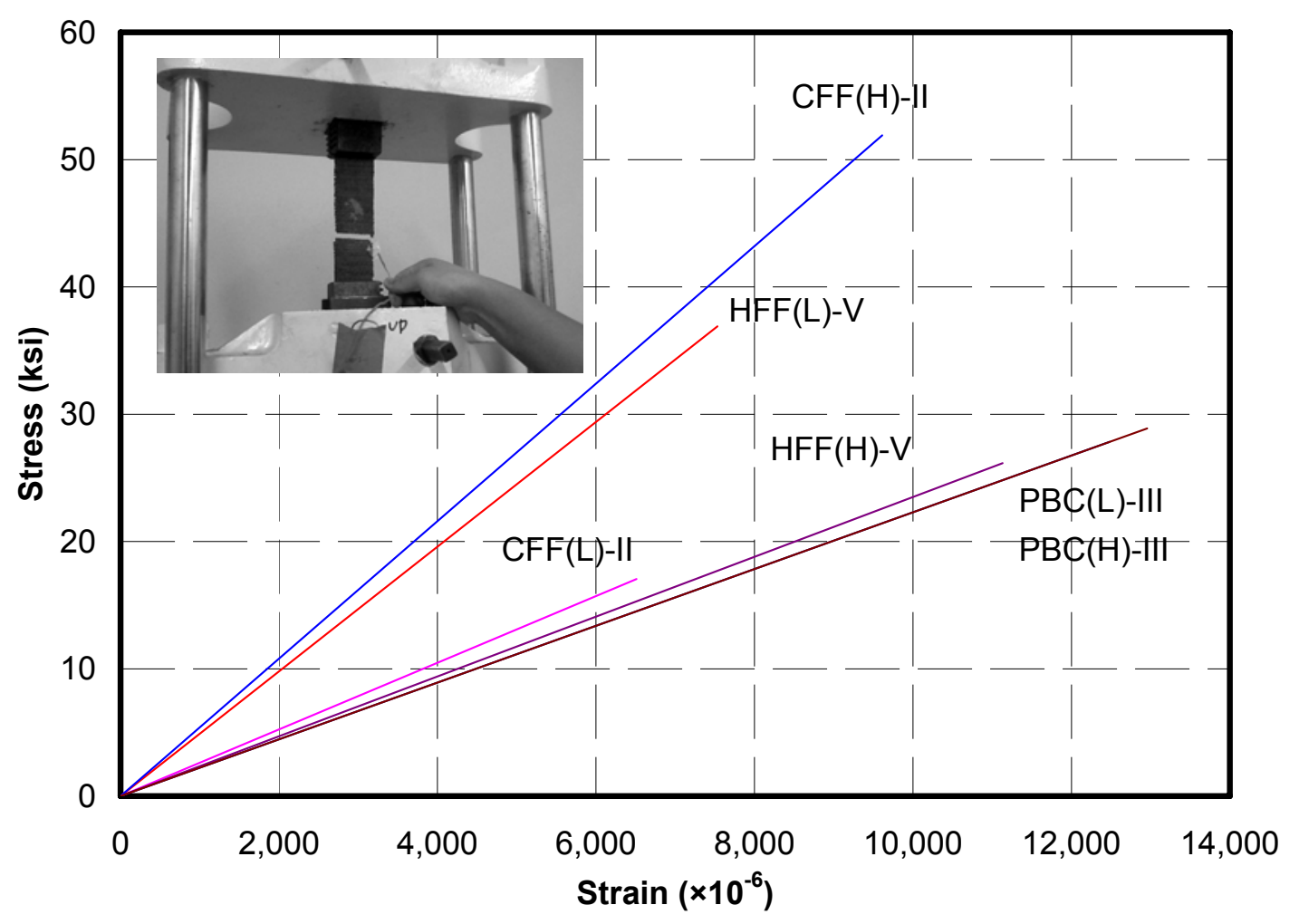

Fig. 3.87 Stress-Strain Response of FRP Coupons 


\section{CHAPTER 4}

\section{MODELING AND PARAMETRIC STUDY}

\subsection{INTRODUCTION}

In this chapter, analytical modeling of the hybrid FRP-concrete pier frame system is presented using an open source object-oriented nonlinear structural analysis software, Open System for Earthquake Engineering Simulation (OpenSees 2006). A parametric study was performed and a typical bridge case was analyzed under three historical ground acceleration records. Different damping ratios were chosen in the sensitivity analysis of the entire bridge.

\subsection{ANALYTICAL MODELING}

\subsubsection{General Features of OpenSees Program}

The OpenSees program was developed as an open source code at the University of California, Berkeley (Opensees.berkeley.edu/index.html), primarily to support a wide range of simulation applications in earthquake engineering. It also contains static analysis features with both load and displacement control schemes. It includes pre-processing, static or dynamic analysis, and post-processing. Pre-processing involves creating a model with defined elements, nodes, load patterns and constraints. Different types of elements are available in the program; including beam-column, zero-length, truss, and quad; all of which are three-dimensional, except for the two-dimensional quad element. The beam-

column element can be used with a linear elastic material, concentrated plasticity integrated over specified hinge lengths, or a distributed plasticity integrated along the 
entire element length. The zero-length elements use multiple uniaxial materials to model the element force-deformation relation. The truss element can model material nonlinearity, either by uniaxial material stress-strain integrated over the cross-sectional area, or through force-deformation relationships at the cross-section. Finally, quad is a twodimensional bilinear isoperimetric element. A number of material models are available for uniaxial systems, including linear elastic; elastic-perfectly-plastic; bilinear model with combined linear isotropic and kinematic hardening; bilinear steel model with linear kinematic and exponential isotropic hardening; concrete model with Kent-Park envelope and degraded linear unloading/reloading rules, bond-slip model to construct a uniaxial material object for capturing strain penetration effect at the column-to-footing, columnto-bridge bent caps, and wall-to-footing intersections; and a trilinear hysteretic backbone with pinching, damage, and degraded unloading stiffness. The program allows discretizing the cross-section with a number of fibers that collectively define the sectional response. Available fiber models associate with a uniaxial material and enforce the Bernouli beam assumptions for the axial and flexural loading.

Two types of analysis are possible with the OpenSees program: simple static nonlinear analysis or a transient nonlinear analysis. The analysis object is composed of several component objects, which define how the analysis is performed. They include linear equation solvers, eigenvalue solvers, numbering of degrees of freedom, static and transient integrators, and algorithms for solution of linear and nonlinear problems, convergence tests, and constraints. The program also has a post-processing feature, which allows monitoring of any element or node parameter during the analysis. The open source 
feature of the program enables researchers to add new material models to the database by themselves, greatly enhancing its capabilities.

\subsubsection{Modeling of Two-Column Bents}

Modeling of the pier frames follows some basic assumptions; (a) plane sections, including both FRP and concrete, remain plane after bending (b) buckling of the FRP tubes is ignored; and (c) slippage of the FRP tubes is neglected due to their adequate embedment in the footing and column head. The nonlinear beam-column element option of the program was chosen for modeling. The finite element mesh is shown in Figure 4.1.

The columns were fixed at the footing; and the pier cap beam was simulated as a rigid beam due to its small deformations recorded in the tests. The column capital that was embedded in the pier cap beam was simulated as a rigid link, because of additional confinement effects expected from the adjoining members and because of the complex localized deformations at the joint. At both beam-column and column-footing joints, zero-length section elements were used. In OpenSees, the zero-length section elements are assumed to have unit length, such that the element deformations (i.e., elongations and rotations) would be equal to the section deformation (i.e., axial strain and curvature). Both the typical reinforced concrete (RC) column section and the FRP-concrete column section are discretized into a number of integration strips or layers. For each section and each layer, material models are adopted from the constitutive models. The material interactions within the section (e.g., confinement effects) are considered at the materials level, and not in the sectional analysis. The discretization for the FRP-concrete column section is shown in Figure 4.2. The concept is also applicable to RC columns, by simply removing the FRP elements. 
As for the material modeling, Kent-Park concrete model (see Taucel et al. 1991) was used for concrete confinement. It has three strain regions, as:

$$
\begin{aligned}
& \varepsilon_{c} \leq \varepsilon_{0}: \quad \sigma_{c}=K \frac{f_{c}^{\prime}}{0.145}\left[2\left(\frac{\varepsilon_{c}}{\varepsilon_{0}}\right)-\left(\frac{\varepsilon_{c}}{\varepsilon_{0}}\right)^{2}\right] \\
& \varepsilon_{0} \leq \varepsilon_{c} \leq \varepsilon_{u}: \quad \sigma_{c}=K \frac{f_{c}^{\prime}}{0.145}\left[1-Z\left(\varepsilon_{c}-\varepsilon_{0}\right)\right] \geq 0.2 K \frac{f_{c}^{\prime}}{0.145} \\
& \varepsilon_{c} \geq \varepsilon_{u}: \quad \sigma_{c}=0.2 K \frac{f_{c}^{\prime}}{0.145}
\end{aligned}
$$

where $\varepsilon_{0}$ is concrete strain at peak stress, given by

$$
\varepsilon_{0}=0.002 K
$$

and $\mathrm{K}$ is a factor accounting for the strength increase due to confinement, given by

$$
K=1+\frac{\rho_{s} f_{y h}}{f_{c}^{\prime}}
$$

and $\mathrm{Z}$ is the strain-softening slope, given by

$$
Z=\frac{0.5}{\frac{3+2 f_{c}^{\prime}}{1000 f_{c}^{\prime}-1000}+0.75 \rho_{s} \sqrt{\frac{h^{\prime}}{s_{h}}}-0.002 K}
$$

and $\mathrm{f}_{\mathrm{c}}$ is the compressive strength of unconfined concrete in ksi, $\mathrm{f}_{\mathrm{yh}}$ is the yield strength of stirrups in ksi, $\rho_{s}$ is the volumetric ratio of the hoop reinforcement to the concrete core, $\mathrm{h}^{\prime}$ is the width of concrete core measured to the outside of the stirrups, and $\mathrm{s}_{\mathrm{h}}$ is the center to center spacing of the stirrups or hoops. 
In the case of concrete confined with stirrups or ties, Scott et al. (1982) suggested that $\varepsilon_{u}$ be determined conservatively as

$$
\varepsilon_{u}=0.004+0.9 \rho_{s}\left(f_{y h} / 43.5\right)
$$

To account for crushing of concrete cover, the strength in the cover layer is reduced to $0.3 \mathrm{f}^{\prime}$, once the compressive strain exceeds the value of $\varepsilon_{u}$, which in this study was taken as 0.006 .

Hysteretic response of concrete core in the Kent-Park model is simulated with the following rules:

1. Unloading from a point on the envelope curve takes place along a straight line connecting the unloading point $\varepsilon_{r}$ to the plastic strain $\varepsilon_{p}$ on the strain axis given as

$$
\frac{\varepsilon_{p}}{\varepsilon_{0}}=0.145\left(\frac{\varepsilon_{r}}{\varepsilon_{0}}\right)^{2}+0.13\left(\frac{\varepsilon_{r}}{\varepsilon_{0}}\right) \quad \text { for }\left(\frac{\varepsilon_{c}}{\varepsilon_{0}}\right)<2
$$

2. In order to ensure that modulus of elasticity for unloading always remains positive, the following relation governs at higher levels of compressive strains:

$$
\frac{\varepsilon_{p}}{\varepsilon_{0}}=0.707\left(\frac{\varepsilon_{r}}{\varepsilon_{0}}-2\right)+0.834 \quad \text { for }\left(\frac{\varepsilon_{c}}{\varepsilon_{0}}\right) \geq 2
$$

3. Tensile resistance of concrete is neglected. Stress in concrete is assumed to be zero for strains less than the plastic strain; and

4. Reloading follows the previous unloading path.

The shape of a typical hysteretic stress-strain response of concrete is shown in Figure 4.3. 
The steel reinforcement was modeled using a uniaxial bilinear stress-strain model with isotropic hardening, described by a non-linear evolution equation. The second slope of the curve was chosen as 0.035 to account for the hardening phase. Figure 4.4 shows the stress-strain model for the steel reinforcement.

The material model for the steel reinforcement in the zero-length section element represents the bar slippage instead of strain for a given bar stress. The uniaxial material model Bond_SP01 developed in OpenSees especially for steel fibers in the zero-length section elements was adopted here. Figure 4.5 shows the typical stress-slip curve that follows

$S_{y}($ in. $)=0.1\left(\frac{d_{b}(\text { in. }) F_{y}(p s i)}{4000 \sqrt{f_{c}^{\prime}(p s i)}}(2 \times \alpha+1)^{1 / \alpha}+0.013(\right.$ in. $)$

where $d_{b}$ is rebar diameter, $F_{y}$ is the yield strength of the steel reinforcement, $f^{\prime}{ }_{c}$ is the compressive strength of unconfined concrete in joints, and $\alpha$ is the parameter used in the local bond-slip relation and can be taken as 0.4 (Zhao 2007), and

$S_{u}=(30 \sim 40) S_{y}$

and $b$ in Figure 4.5 is the initial hardening ratio in the monotonic bar stress-slip response (0.3 0.5) (see Figure 4.6), and $\mathrm{R}$ is the pinching factor for the cyclic bar stress-slip response (0.5 1.0) (see Figure 4.7).

The glass FRP material is modeled using an orthotropic linear elastic model in this study, with the hoop tensile modulus and strength taken as 1,460 ksi and $34 \mathrm{ksi}$, respectively. Based on earlier coupon tests (Shao and Mirmiran 2004), the stress-strain curve for the glass FRP tube in the longitudinal direction is defined as: 
$\sigma=(\varepsilon / A B S(\varepsilon)) \times(-3.08+\sqrt{9.49+13,423 \times A B S(\varepsilon)}) \quad A B S(\varepsilon) \leq 0.05$

The above relationship was modeled in the OpenSees program as a tri-linear hysteretic material, without any pinching or damage effects. An empirical value of 0.3 was selected for parameter $\beta$ to simulate the softening effect of the material upon unloading. Figure 4.8 shows the hysteretic stress-strain response output for the FRP tube in the longitudinal direction.

The coupon test results of carbon and hybrid FRP tubes are shown in Figure 4.9. For carbon FRP, linear elastic models were adopted, as shown in Figure 4.9. Due to lack of any manufacturer's data, the coupon test results were used with 2 layers of carbon FRP. For the hybrid tubes, because the glass and carbon FRP sheets have different tensile strengths and ultimate strains in the hoop and longitudinal directions; considering 5 different layers is a better way than just regarding it as a single hybrid FRP layer. In the simulation of Specimen HFF, manufacturer's coupon test data was adopted for both glass and carbon FRP. Table 4.1 lists the manufacturer's data for the carbon and glass FRP in Specimen HFF.

\subsubsection{Model Verification}

The elements and material models mentioned above were used in the OpenSees program. Figures 4.10 through 4.13 show comparisons of the test data with the model predictions for Specimens RCF, CFF, GFF, and HFF, respectively.

In each figure, the thick solid line represents the experimental data, while the thin line indicates the model prediction. In general, very good agreement is noted for 
Specimens RCF, GFF, and HFF, while the simulation results are reasonably close for Specimen CFF.

The measured peak load at each ductility level was compared with the simulation results. The standard deviation and root mean square normalized (RMSN) error for all specimens are summarized in Table 4.2. The experimental cumulative dissipated energy at each ductility level was also compared with the simulation results. The standard deviation and RMSN error for all specimens are listed in Table 4.3.

\subsection{PARAMETRIC STUDY OF FRP-CONCRETE PIER FRAME SYSTEM}

A parametric study was carried out for the pseudo-static push-over analysis of the frame with five different variables, including frame height/span ratio, concrete compressive strength, level of axial loads, reinforcement ratio of conventional steel, and thickness of the FRP tube. The frames tested in the laboratory were used as the prototypes for the parametric study.

The frame height/span $(\mathrm{H} / \mathrm{L})$ ratio was chosen between 0.8 to 1.6 , where $\mathrm{H}$ is the frame height and $\mathrm{L}$ is the frame span. The range is similar to that used in common practice for H/D between 5 and 10 (Zhu 2004), where D is the diameter of the column.

Concrete compressive strength $\left(\mathrm{f}_{\mathrm{c}}\right)$waschosenas 4 , 5, and 6 ksi that are most commonly used in bridge construction.

The ratio of axial force $(\mathrm{P})$ versus column capacity $\left(\mathrm{P}_{\mathrm{o}}=\mathrm{f}_{\mathrm{c}} \mathrm{A}_{\mathrm{g}}\right.$, where $\mathrm{f}_{\mathrm{c}}$ is the 28 days concrete compressive strength and $\mathrm{A}_{\mathrm{g}}$ is the gross cross-sectional area of the column) also selected as 5\%, 10\%, and 20\%. 
Steel reinforcement ratio $\left(\rho_{s}\right)$ varied from $1 \%$ to $3 \%$, as suggested by Shao (2003) since excessive internal steel reinforcement ratio may lead to premature crushing failure of the FRP tube.

As for the FRP thickness, the $\mathrm{D} / \mathrm{t}$ ratio was chosen as 25,50 , and 100 ; where $\mathrm{D}$ is the outside diameter of the column and $t$ is the thickness of the FRP tube. However, carbon and hybrid FRP tubes were limited by the layers of the FRP sheets, and therefore, their $\mathrm{D} / \mathrm{t}$ ratios were determined by the integer number of FRP layers. In this study, 2,3 , and 4 layers of carbon FRP sheets were chosen, with D/t ratios of 99.5, 66.3, and 49.7, respectively. For the hybrid FRP, 3, 5, and 7 layers of FRP sheets were chosen, with D/t ratios of $68.7,41.3$, and 29.5 , respectively.

Table 4.4 lists all variables used in this study for Frames GFF, HFF, and CFF.

Figures 4.14 through 4.18 show the parametric study results for the hysteretic loaddeflection response of Frame GFF. Figures 4.19 through 4.23 show load-deflection envelopes, and Figures 4.24 through 4.28 show the cumulative energy capacity for the same frame.

Figures 4.29 through 4.33 show the parametric study results for the hysteretic loaddeflection response of Frame HFF. Figures 4.34 through 4.38 show load-deflection envelopes, and Figures 4.39 through 4.43 show the cumulative energy capacity for the same frame.

Figures 4.44 through 4.48 show the parametric study results for the hysteretic loaddeflection responses of Frame CFF. Figures 4.49 through 4.53 show load-deflection envelopes, and Figures 4.54 through 4.58 show the cumulative energy capacity for the same frame. 
It is quite clear that the frame height/span ratio (H/L), FRP thickness (D/t), and steel reinforcement ratio $\left(\rho_{s}\right)$ are three parameters that affect the performance of the frame the most. On the other hand, concrete compressive strength $\left(\mathrm{f}_{\mathrm{c}}{ }_{\mathrm{c}}\right)$ is the parameter with least influence on the frame performance.

Figures 4.59 through 4.63 show the effects of selected parameters on the maximum loads of all frames. Figures 4.64 through 4.68 show the effects of selected parameters on ductilities of all frames. Figures 4.69 through 4.73 show the effects of selected parameters on pinching factors of all frames. Figures 4.74 through 4.78 show the effects of selected parameters on maximum drift ratios of all frames. Figures 4.79 through 4.83 show the effects of selected parameters on cumulative energy of all frames.

It is clear that the frame height/span ratio $(\mathrm{H} / \mathrm{L})$ affects the maximum load, ductility, drift ratio, and cumulative energy, but not the pinching effect. The FRP thickness $(\mathrm{D} / \mathrm{t})$ and steel reinforcement ratio $\left(\rho_{s}\right)$ also affect these response measures quite significantly. As for the pinching factor, FRP thickness $(\mathrm{D} / \mathrm{t})$ and steel reinforcement ratio $\left(\rho_{s}\right)$ played the most important roles together. A proper design of frame height/span ratio $(\mathrm{H} / \mathrm{L})$ ratio is perhaps one of the most important considerations, after which FRP thickness $(\mathrm{D} / \mathrm{t})$ and steel reinforcement ratio $\left(\rho_{s}\right)$ need to be designed for. Compressive strength of concrete has the least effect on FRP-concrete frames, This may be attributed to the fact that for weaker concrete, the same FRP tube will result in a higher confinement ratio $\left(\mathrm{f}^{\prime}{ }_{\mathrm{cu}} / \mathrm{f}_{\mathrm{c}}{ }_{\mathrm{c}}\right)$, where $\mathrm{f}_{\mathrm{cu}}$ is confined strength of concrete and $\mathrm{f}_{\mathrm{c}}{ }_{\mathrm{c}}$ is unconfined strength of concrete.

In addition to the load-deflection hysteretic response, load-deflection envelopes, and cumulative energy capacity; other response measures including maximum strength, 
ductility, and pinching factors summarized and listed in Tables 4.5 through Table 4.7 for Frames GFF, HFF, and CFF, respectively. The ductility and pinching factors were defined earlier in Chapter 2. The results show that the ductility and pinching factors are mostly affected by FRP thickness $(\mathrm{D} / \mathrm{t})$ and steel reinforcement ratio $\left(\rho_{s}\right)$. Figure 4.84 confirms the findings of the experimental work that pinching factor correlates well with the total reinforcement index of the column with a high goodness of the fit. Figures 4.85 and 4.86 show the effect of the reinforcement strength index (including FRP and steel) versus the normalized flexural strength for all three types of FRP-concrete frame systems, where $\mathrm{M}_{\mathrm{RCF}}$ is the flexural strength of conventional $\mathrm{RC}$ frame at the column base. The moments include both the primary moment from the lateral load and the secondary moment from the P- $\Delta$ effect of the axial load. The confined and unconfined compressive strengths of concrete were both used for comparison. The results confirm the correlation between the reinforcement index and the ultimate load capacity of the frame systems.

\subsection{ENTIRE BRIDGE CASE STUDY}

A typical 4-span bridge was selected as a case study for FRP-concrete substructure. The two end spans and the two intermediate spans were 98 and $116 \mathrm{ft}$, respectively. The three pier frames were $22.5,30.5$, and $26.5 \mathrm{ft}$ high, respectively. The plan and elevation of the bridge is shown in Figure 4.87. The bridge superstructure consists of a concrete box girder with three cells supported by to the pier cap beams, as shown in Figure 4.88. The analytical model for the entire bridge is shown in Figure 4.89. The pier cap beam integrated with the box girder was simulated as a rigid beam in the model. Different type of piers, namely, RCF, GFF, CFF, and HFF, were used for the 
bridge bents with the same concrete core diameter of $4 \mathrm{ft}$. The concrete compressive strength was selected as $5 \mathrm{ksi}$ since the parametric study had shown it to have little or no influence on the performance of FRP-concrete frames. In each column, twenty No. 10 bars were evenly distributed around the column with a reinforcement ratio of $1.4 \%$. All steel reinforcement was of Grade $60 \mathrm{ksi}$. The transverse steel was No. 6 spiral of Grade $60 \mathrm{ksi}$ at 3.25 in. pitch for the RC columns only. The FRP tube thicknesses were 0.858 , 0.551 , and 1.614 in for the GFF, CFF and HFF, respectively.

All structures have their own natural frequencies which greatly affect the dynamic behavior of structures. The fundamental natural frequencies of a structure are dependent on the structural characteristics, such as stiffness, geometric configuration, mass distribution, etc. Table 4-8 shows the results of first three fundamental natural frequencies for all types of bridges in this study. The lowest frequency is larger than the natural frequency of the wind which is normally about $1 \mathrm{~Hz}$ and the therefore, earthquake may be the more critical dynamic loading for such structures.

Three types of seismic loading (Figures 4.90 through 4.93) were applied onto the bridge, including Tabas (Iran 1978), Sylmar (California 1971), and Llollelo (Chile 1985). Of the selected quakes, Tabas had the highest ground acceleration with an extended period of shake and two orthogonal input directions. Sylmar spanned a much shorter time period, while Llollelo had an apparent higher frequency and higher energy input. The peak frequencies of all three selected quakes are listed in Table 4-9.

For RC structures, the damping ratio of 0.05 (Kovacs 1998 and Park et al. 1987) is generally considered acceptable. In this study, the damping ratio was varied within the range of $0.03-0.08$ for single columns and two-column pier frames. For FRP-concrete 
structures, the damping contribution of FRP materials to the entire structure is determined using the following equation, based on the proportionality of sectional stiffness:

$\xi_{F R P-R C}=\xi_{R C}+\frac{E_{F R P}}{E_{c}} \rho_{F R P} \xi_{F R P}$

where $\xi_{F R P-R C}$ is the damping ratio of FRP-RC structure; $\xi_{R C}$ is the damping ratio of RC structure, which is considered within the range of $0.03 \sim 0.08 ; E_{F R P}$ is the tensile modulus of FRP laminate; $E_{c}$ is the elastic modulus of concrete, $\rho$ is the volumetric ratio of FRP tube with respect to concrete core; and $\xi_{F R P}$ is the damping ratio of FRP materials, which is considered to be in the range of 0.005 0.01 (Tanimoto 2007).

The elastic modulus of concrete $\left(E_{c}\right)$ is defined by the following equation:

$E_{c}=57,000 \sqrt{f_{c}^{\prime}}[\mathrm{psi}]$

where $f_{c}^{\prime}$ is the compressive strength of confined concrete.

The FRP volumetric ratio $\left(\rho_{F R P}\right)$ is given by the following equation:

$\rho=\frac{4 t}{D}$

where $\mathrm{t}$ is the FRP thickness and $\mathrm{D}$ is the outside diameter of the column.

According to Equation (4.13), the damping ratio of FRP-RC structure turns out to be in the range of 0.0301 to 0.0802 . Compared with the damping ratio of $\mathrm{RC}$ structure, the damping contribution of FRP materials is less than $1 \%$. Therefore, the damping effects from the FRP materials were considered negligible in this study. Three different damping ratios were chosen between 0.03 and 0.08 for both the RC and the FRP-RC structures, to study the effect of damping on seismic performance of the bridge.

The ground accelerations records for Tabas (1978) earthquake were imposed simultaneously in the longitudinal and transverse directions of the bridge. However, the 
bridge superstructure exhibited a very rigid response in the longitudinal direction. Therefore, in this study, only the transverse responses of the bridge under the three seismic loadings are considered and compared. Because the pier frames had different heights, lateral deflections of the bents were normalized with respect to the maximum drift capacity of each pier frame. The maximum drift capacity of each pier frame was determined using the push-over analysis of the individual frame, as discussed in the previous section. Figures 4.94 through 4.102 show the maximum normalized lateral drift ratio $\left(\delta / \delta_{u}\right)$ for each type of bridge under different ground accelerations and damping ratios, where $\delta$ is the experienced drift ratio and $\delta_{u}$ is the maximum drift ratio from the push-over analysis. It may be concluded that the RCF bridge has the highest normalized drift ratio, and the GFF bridge the least. The damping ratio clearly has an impact on the performance of the bridges, as shown in Figures 4.103 through 4.106. In these figures, the maximum deflection of each bent under each type of seismic loading was normalized by its maximum deflection for the damping ratio of $0.08\left(\delta / \delta_{\zeta=0.08}\right)$. The results show that the maximum drift ratio could increase by $20 \%-60 \%$, when damping ratio was reduced from 0.08 to 0.03 . This implies that installing a dynamic damping device on key members may effectively enhance the performance of concrete or FRP-concrete bridge substructures.

Figures 4.107 through 4.109 show the effect of damping ratio on normalized drift ratio of the center pier (Pier 2) under different earthquakes for all bridges. Similar conclusion can be made for each type of bridge that the increase of damping ratio could reduce the maximum normalized drift ratio and enhance the seismic performance of the bridge. 
In order to better quantify the state of damage after an earthquake, a damage index was calculated as suggested by Park and Ang (1985) and implemented by Williams and Sexsmith (1995). The index consists of a simple linear combination of normalized deformation and energy absorption, as given by

$D=\frac{\delta_{m}}{\delta_{u}}+\beta \frac{\int d E}{F_{y} \delta_{u}}$

where $\delta_{m}$ is the maximum deformation experienced, $\delta_{u}$ is the ultimate deformation under monotonic loading, $\mathrm{F}_{\mathrm{y}}$ is the calculated yield strength, $\mathrm{dE}$ is the increment of dissipated energy, and

$\beta=\left(-0.447+0.73 l / d+0.24 n_{0}+0.314 \rho_{s}\right) \cdot 0.7 \rho_{w}$

where $l / d$ is the ratio of shear span to beam (or column) depth, $\mathrm{n}_{\mathrm{o}}$ is the normalized axial force, $\rho_{w}$ is the reinforcement ratio for lateral confining steel, and $\rho_{s}$ is the reinforcement ratio for the main, i.e., longitudinal steel.

The above damage index consists of two parts. The first part is a simple pseudostatic displacement measure which does not account for the cumulative damage. The second term accounts for the cumulative energy. The advantages of this model are its simplicity and the fact that it has been calibrated against a significant number of observed damages from seismic events, including some instances of shear and bond failure. Park and Ang (1985) suggested the use of $\mathrm{D}=0.4$ as a threshold value between repairable and irreparable damage, while the same authors later suggested a more detailed classification (Park et al. 1987), as below:

$D<0.1$ No damage or localized minor cracking; 
$0.1 \leq D<0.25$ Minor damage with light cracking throughout;

$0.25 \leq D<0.4$ Moderate damage with severe cracking, and localized spalling;

$0.4 \leq D<0.8$ Severe damage with concrete crushing and exposed reinforcement;

and

$D \geq 0.8$ Collapse.

Figures 4.110 through Figure 4.118 show the normalized damage index for the four types of bridges under different seismic loading and damping ratios. The RCF bridge had the most severe damage under all three earthquakes, while the GFF bridge only showed minor damage or no damage. The performance of the CFF and HFF bridges were in between but much better than the RCF bridge.

One can conclude that the GFF bridge distinguishes itself among the four types of bridges studied here, for its low damage index when subjected to three different major earthquake records.

Figures 4.119 through Figure 4.121 show the effect of damping ratio on damage index of the center pier (Pier 2) under different earthquakes for all bridges. Similar conclusion can be drawn that the increase of damping ratio could mitigate the severity of the damages incurred by a bridge under major earthquakes. 
Table 4.1 Manufacturer Data for Carbon and Glass FRP in Specimen HFF

\begin{tabular}{|c|c|c|c|c|}
\hline \multirow{2}{*}{ Fiber Direction } & $\begin{array}{c}\text { FRP } \\
\text { Material }\end{array}$ & Product Name & $\begin{array}{c}\text { Tensile } \\
\text { Strength (ksi) }\end{array}$ & $\begin{array}{c}\text { Ultimate Strain } \\
\text { (in./in.) }\end{array}$ \\
\hline \multirow{2}{*}{ Hoop } & Carbon & SikaWrapHex103C & 3.5 & 0.0045 \\
\cline { 2 - 5 } & Glass & SikaWrapHex100G & 88.8 & 0.0245 \\
\hline \multirow{2}{*}{ Longitudinal } & Carbon & SikaWrapHex103C & 123.2 & 0.0112 \\
\cline { 2 - 5 } & Glass & SikaWrapHex100G & 4.4 & 0.0046 \\
\hline
\end{tabular}

Table 4.2 Peak Load Standard Deviation and RMSN Error of Simulation

\begin{tabular}{|c|c|c|c|}
\hline Specimen & Up to $\mu$ & $\begin{array}{c}\text { Standard Deviation } \\
\text { (kips) }\end{array}$ & RMSN Error \\
\hline RCF & \pm 6 & 0.850 & $1.30 \%$ \\
\hline HFF & \pm 9 & 7.021 & $4.37 \%$ \\
\hline CFF & \pm 7 & 7.067 & $6.77 \%$ \\
\hline GFF & \pm 10 & 2.731 & $5.14 \%$ \\
\hline
\end{tabular}

Table 4.3 Cumulative Dissipated Energy Standard Deviation and RMSN Error of Simulation

\begin{tabular}{|c|c|c|c|}
\hline Specimen & Up to $\mu$ & $\begin{array}{c}\text { Standard Deviation } \\
\text { (kips) }\end{array}$ & RMSN Error \\
\hline RCF & \pm 6 & 0.226 & $0.34 \%$ \\
\hline HFF & \pm 9 & 4.408 & $1.87 \%$ \\
\hline CFF & \pm 7 & 7.443 & $6.19 \%$ \\
\hline GFF & \pm 10 & 2.731 & $3.56 \%$ \\
\hline
\end{tabular}

Table 4.4 Case Study for Hybrid FRP-Concrete Pier Frame Systems

\begin{tabular}{|c|c|c|c|c|c|c|c|}
\hline \multirow{2}{*}{ Parameters } & \multirow{2}{*}{$\mathbf{H} / \mathbf{L}$} & \multirow{2}{*}{$f^{\prime}{ }_{c}(\mathbf{k s i})$} & \multirow{2}{*}{ P/Po } & \multirow{2}{*}{$\begin{array}{c}\text { Steel } \\
\text { Ratio } \\
\left(\rho_{s}\right)\end{array}$} & \multicolumn{3}{|c|}{$D / t$} \\
\hline & & & & & GFF & HFF & CFF \\
\hline \multirow{3}{*}{ Values } & 0.8 & 4 & $5 \%$ & $1 \%$ & 25 & $\begin{array}{c}31.2 \\
\text { (7 layers) }\end{array}$ & $\begin{array}{c}48.7 \\
\text { (4 layers) }\end{array}$ \\
\hline & 1.2 & 5 & $10 \%$ & $2 \%$ & 50 & $\begin{array}{c}43.3 \\
\text { (5 layers) }\end{array}$ & $\begin{array}{c}64.3 \\
\text { (3 layers) }\end{array}$ \\
\hline & 1.6 & 6 & $20 \%$ & $3 \%$ & 100 & $\begin{array}{c}70.8 \\
\text { (3 layers) }\end{array}$ & $\begin{array}{c}95.4 \\
\text { (2 layers) }\end{array}$ \\
\hline
\end{tabular}


Table 4.5 Parametric Study Results for Specimen GFF

\begin{tabular}{|c|c|c|c|c|}
\hline \multirow{3}{*}{ Parameters } & Value & $\mathbf{P}_{\max }(\mathbf{k i p s})$ & $\begin{array}{c}\text { Ductility } \\
(\boldsymbol{\mu})\end{array}$ & Pinching Factor \\
\hline \multirow{3}{*}{$\mathrm{H} / \mathrm{L}$} & 0.8 & 80.0 & 8.9 & 1.3 \\
\cline { 2 - 5 } & 1.2 & 50.3 & 20.7 & 1.5 \\
\cline { 2 - 5 } & 1.6 & 36.7 & 37.3 & 1.6 \\
\hline \multirow{3}{*}{$f^{\prime}{ }_{c}(\mathrm{ksi})$} & 4 & 48.7 & 22.0 & 1.5 \\
\cline { 2 - 5 } & 5 & 50.0 & 22.9 & 1.5 \\
\hline \multirow{3}{*}{$\mathrm{P} / \mathrm{P}_{\mathrm{o}}$} & 6 & 50.2 & 22.5 & 1.5 \\
\cline { 2 - 5 } & $5 \%$ & 51.7 & 22.9 & 1.6 \\
\cline { 2 - 5 } & $10 \%$ & 54.7 & 24.2 & 1.8 \\
\hline \multirow{3}{*}{ Steel Ratio $\left(\rho_{s}\right)$} & $20 \%$ & 63.3 & 27.6 & 2.0 \\
\cline { 2 - 5 } & $1 \%$ & 42.9 & 21.0 & 1.7 \\
\cline { 2 - 5 } & $2 \%$ & 56.1 & 23.6 & 1.6 \\
\hline \multirow{3}{*}{$\mathrm{D} / \mathrm{t}$} & $2 \%$ & 69.1 & 26.3 & 1.5 \\
\cline { 2 - 5 } & 50 & 81.3 & 24.9 & 1.4 \\
\cline { 2 - 5 } & 100 & 54.2 & 22.0 & 1.3 \\
\hline
\end{tabular}

Table 4.6 Parametric Study Results for Specimen HFF

\begin{tabular}{|c|c|c|c|c|}
\hline \multirow{2}{*}{ Parameters } & Value & $\mathbf{P}_{\max }(\mathbf{k i p s})$ & $\begin{array}{c}\text { Ductility } \\
(\boldsymbol{\mu})\end{array}$ & Pinching Factor \\
\hline \multirow{3}{*}{$\mathrm{H} / \mathrm{L}$} & 0.8 & 76.1 & 3.3 & 3.4 \\
\cline { 2 - 5 } & 1.2 & 47.0 & 7.7 & 3.4 \\
\cline { 2 - 5 } & 1.6 & 35.9 & 14.4 & 3.2 \\
\hline \multirow{3}{*}{$f^{\prime}{ }_{c}(\mathrm{ksi})$} & 4 & 45.7 & 8.1 & 3.0 \\
\cline { 2 - 5 } & 5 & 46.4 & 8.0 & 3.6 \\
\hline \multirow{3}{*}{$\mathrm{P} / \mathrm{P}_{\mathrm{o}}$} & 6 & 47.1 & 7.8 & 3.7 \\
\cline { 2 - 5 } & $10 \%$ & 47.9 & 7.8 & 3.6 \\
\cline { 2 - 5 } & $20 \%$ & 50.5 & 8.0 & 4.3 \\
\hline \multirow{3}{*}{ Steel Ratio $\left(\rho_{s}\right)$} & $1 \%$ & 55.3 & 8.5 & 4.2 \\
\cline { 2 - 5 } & $2 \%$ & 43.4 & 5.9 & 3.6 \\
\cline { 2 - 5 } & $3 \%$ & 49.0 & 7.6 & 2.9 \\
\hline \multirow{3}{*}{$\mathrm{D} / \mathrm{t}$} & $31.2(7$ layers $)$ & 65.0 & 8.5 & 4.7 \\
\cline { 2 - 5 } & $43.3(5$ layers $)$ & 47.3 & 70.3 & 3.2 \\
\cline { 2 - 5 } & $70.8(3$ layers $)$ & 32.6 & 5.1 & 1.9 \\
\hline
\end{tabular}


Table 4.7 Parametric Study Results for Specimen CFF

\begin{tabular}{|c|c|c|c|c|}
\hline \multirow{3}{*}{ Parameters } & Value & $\mathbf{P}_{\max }(\mathbf{k i p s})$ & $\begin{array}{c}\text { Ductility } \\
(\boldsymbol{\mu})\end{array}$ & Pinching Factor \\
\hline \multirow{3}{*}{$\mathrm{H} / \mathrm{L}$} & 0.8 & 34.4 & 2.3 & 1.9 \\
\cline { 2 - 5 } & 1.2 & 22.3 & 4.7 & 1.9 \\
\cline { 2 - 5 } & 1.6 & 16.1 & 7.0 & 1.7 \\
\hline \multirow{3}{*}{$f^{\prime}{ }_{c}(\mathrm{ksi})$} & 4 & 21.3 & 5.9 & 2.0 \\
\cline { 2 - 5 } & 5 & 21.5 & 5.9 & 2.0 \\
\cline { 2 - 5 } & 6 & 21.8 & 5.9 & 2.0 \\
\cline { 2 - 5 } $\mathrm{P} / \mathrm{P}_{\mathrm{o}}$ & $5 \%$ & 22.6 & 5.4 & 2.1 \\
\cline { 2 - 5 } & $10 \%$ & 25.6 & 5.6 & 2.5 \\
\hline \multirow{3}{*}{ Steel Ratio $\left(\rho_{s}\right)$} & $20 \%$ & 30.1 & 5.8 & 2.7 \\
\cline { 2 - 5 } & $1 \%$ & 17.9 & 4.2 & 2.3 \\
\cline { 2 - 5 } & $2 \%$ & 23.8 & 5.1 & 1.3 \\
\hline \multirow{3}{*}{$\mathrm{D} / \mathrm{t}$} & $48.7(4$ layers $)$ & 28.7 & 5.9 & 2.7 \\
\cline { 2 - 5 } & & & 7.6 & 2.3 \\
\cline { 2 - 5 } & $64.3(3$ layers $)$ & 25.3 & 6.4 & 2.1 \\
\cline { 2 - 5 } & $95.4(2$ layers $)$ & 21.7 & 5.4 & \\
\hline
\end{tabular}

Table 4.8 The First Three Natural Frequency of All types of Bridges

\begin{tabular}{|c|c|c|c|}
\hline & f1 (Hz) & f2 (Hz) & f3 (Hz) \\
\hline RCF Bridge & 2.83 & 11.00 & 23.73 \\
\hline HFF Bridge & 2.12 & 10.22 & 26.11 \\
\hline CFF Bridge & 1.61 & 9.44 & 23.41 \\
\hline GFF Bridge & 1.54 & 9.35 & 23.09 \\
\hline
\end{tabular}

Table 4.9 Peak Frequencies of Three Earthquake Ground Acceleration

\begin{tabular}{|c|c|}
\hline & Peak frequency (Hz) \\
\hline Tabas (1978) & 1.33 \\
\hline Sylmar (1971) & 0.70 \\
\hline LLollelo (1985) & 1.90 \\
\hline
\end{tabular}




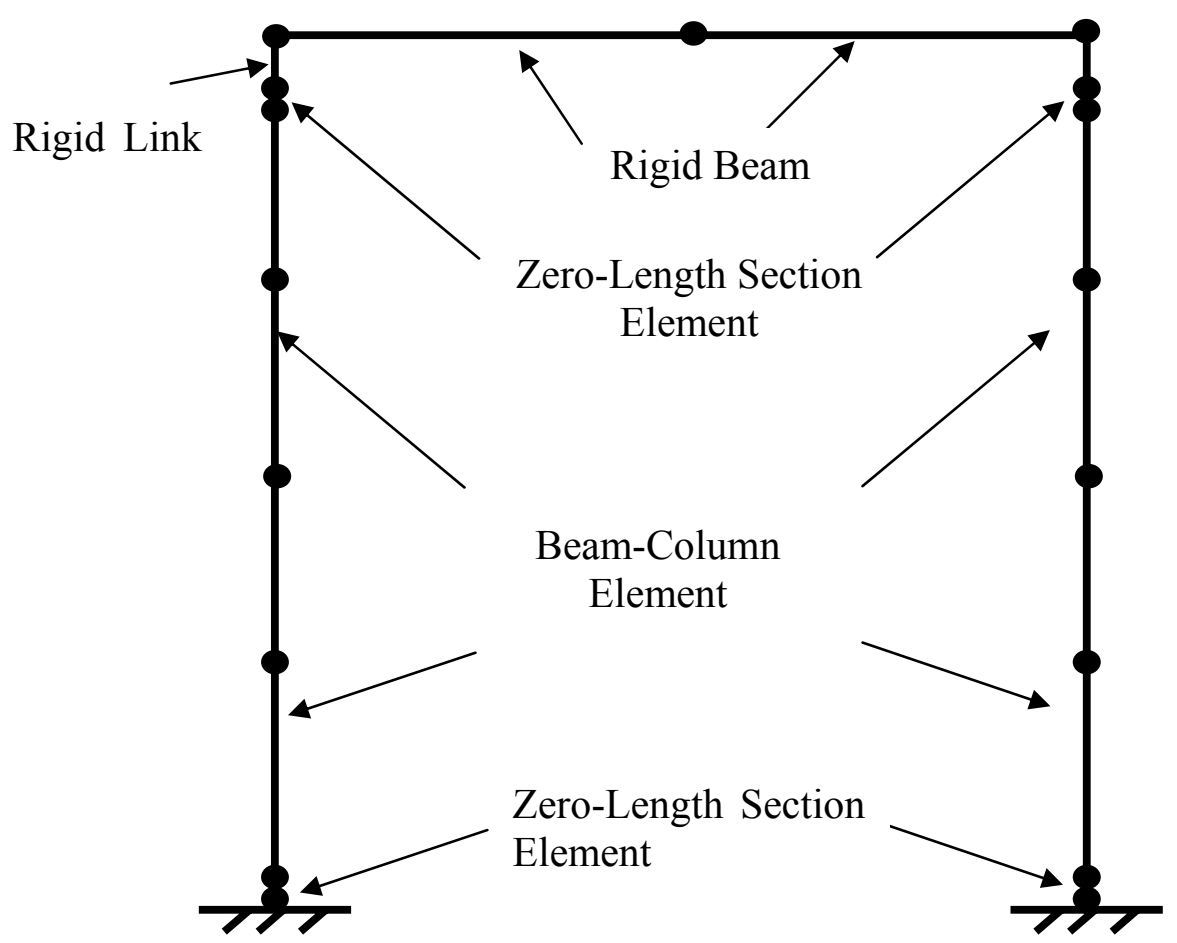

Fig. 4.1 Hybrid FRP-Concrete Pier Frame Model

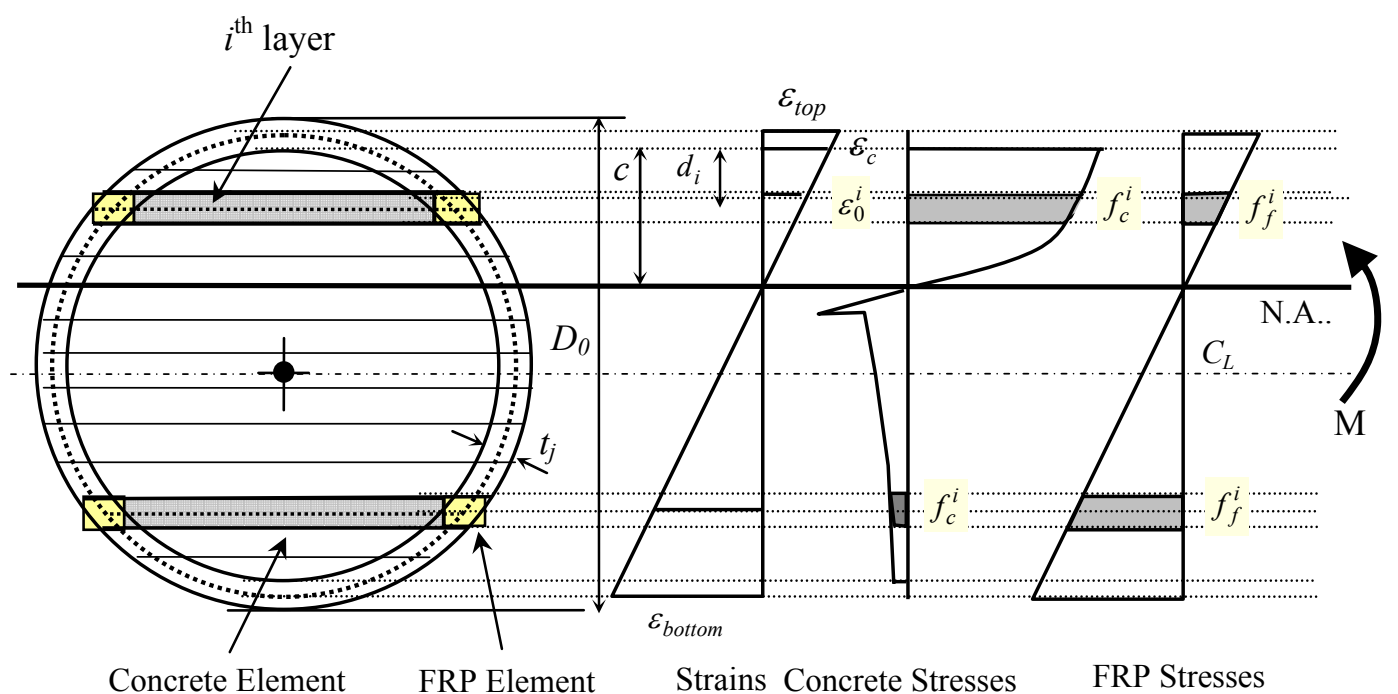

Fig. 4.2 Illustration of Sectional Analysis Strip Method 


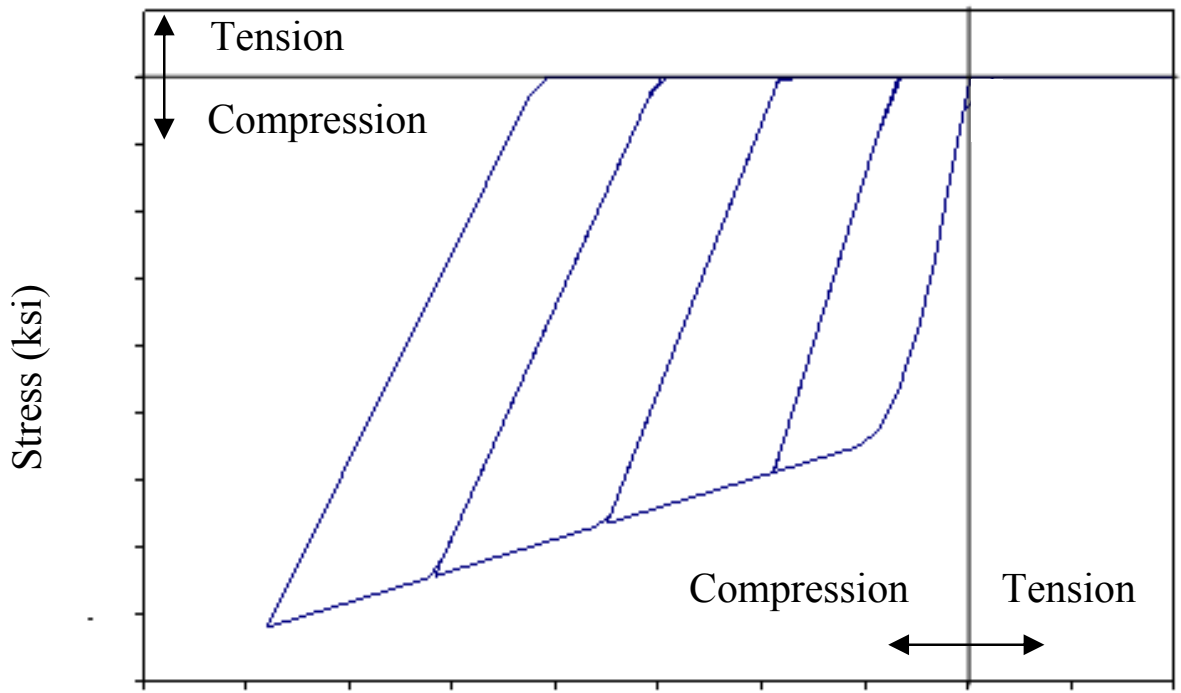

Strain (in./in.)

Fig. 4.3 Typical Hysteretic Stress-Strain Model for Concrete (OpenSees 2006)

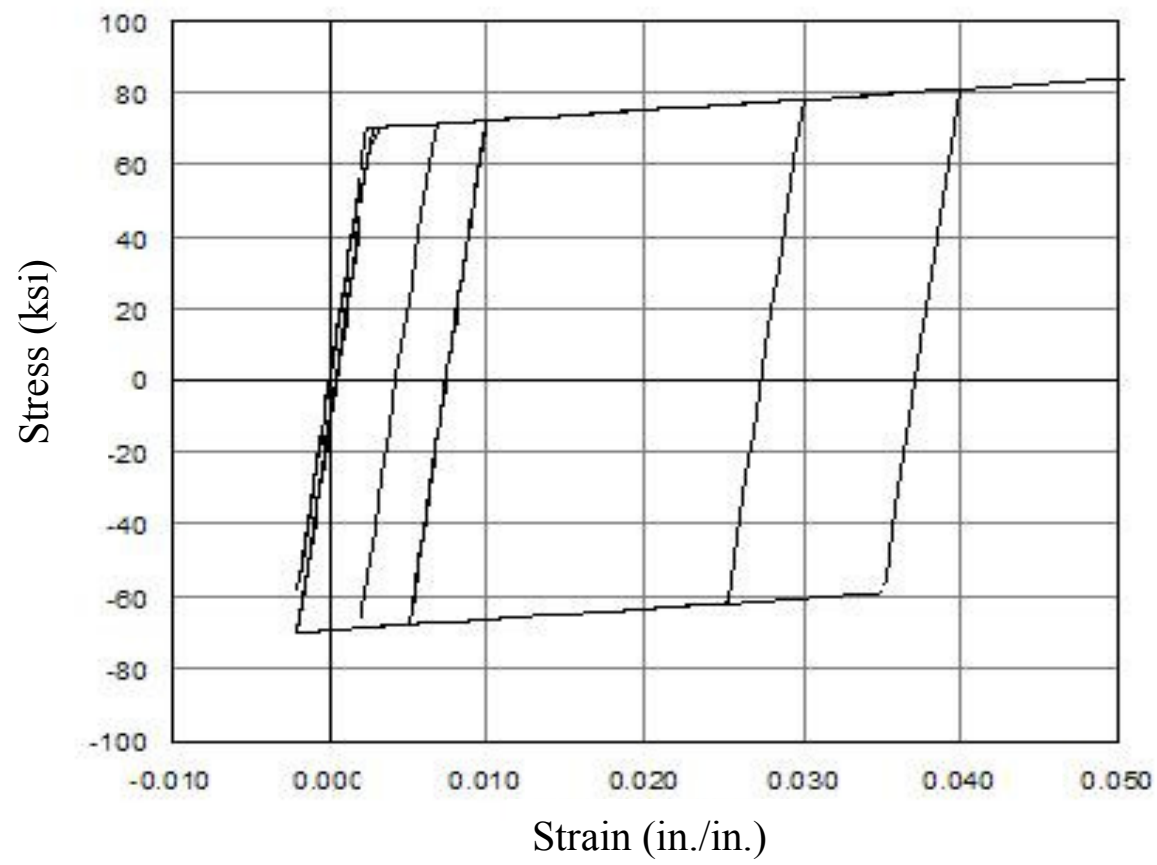

Fig. 4.4 Typical Hysteric Stress-Strain Model for Steel Reinforcement (OpenSees 2006) 


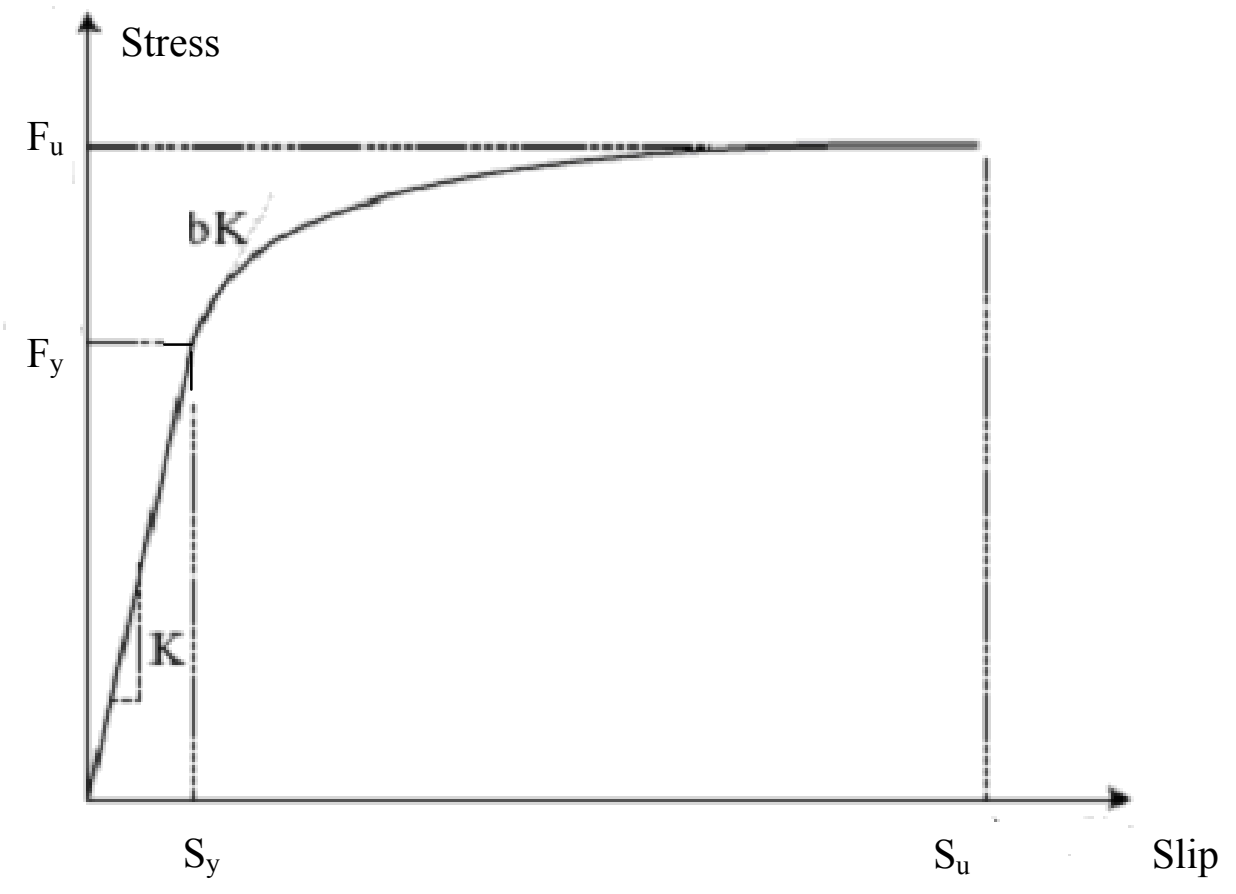

Fig. 4.5 Typical Stress-Slip Curve of Bond Slip Model (OpenSees 2006)

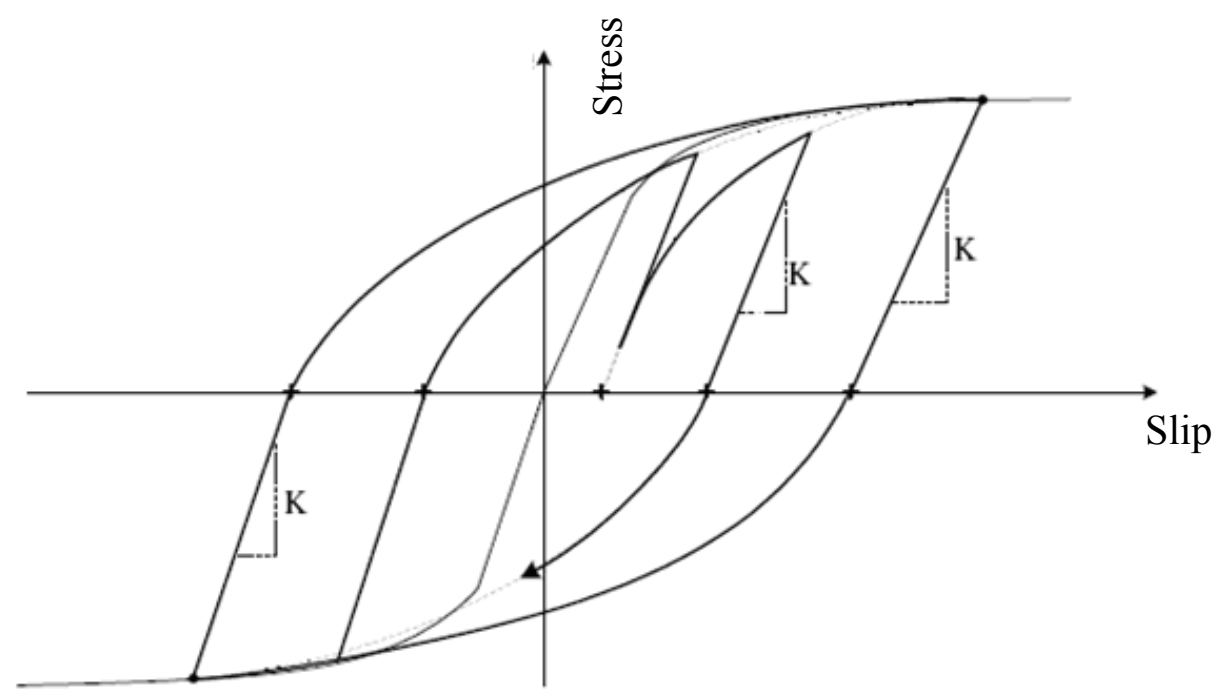

Fig. 4.6 Hysteretic Bar Stress-Slip Model in (OpenSees 2006) 


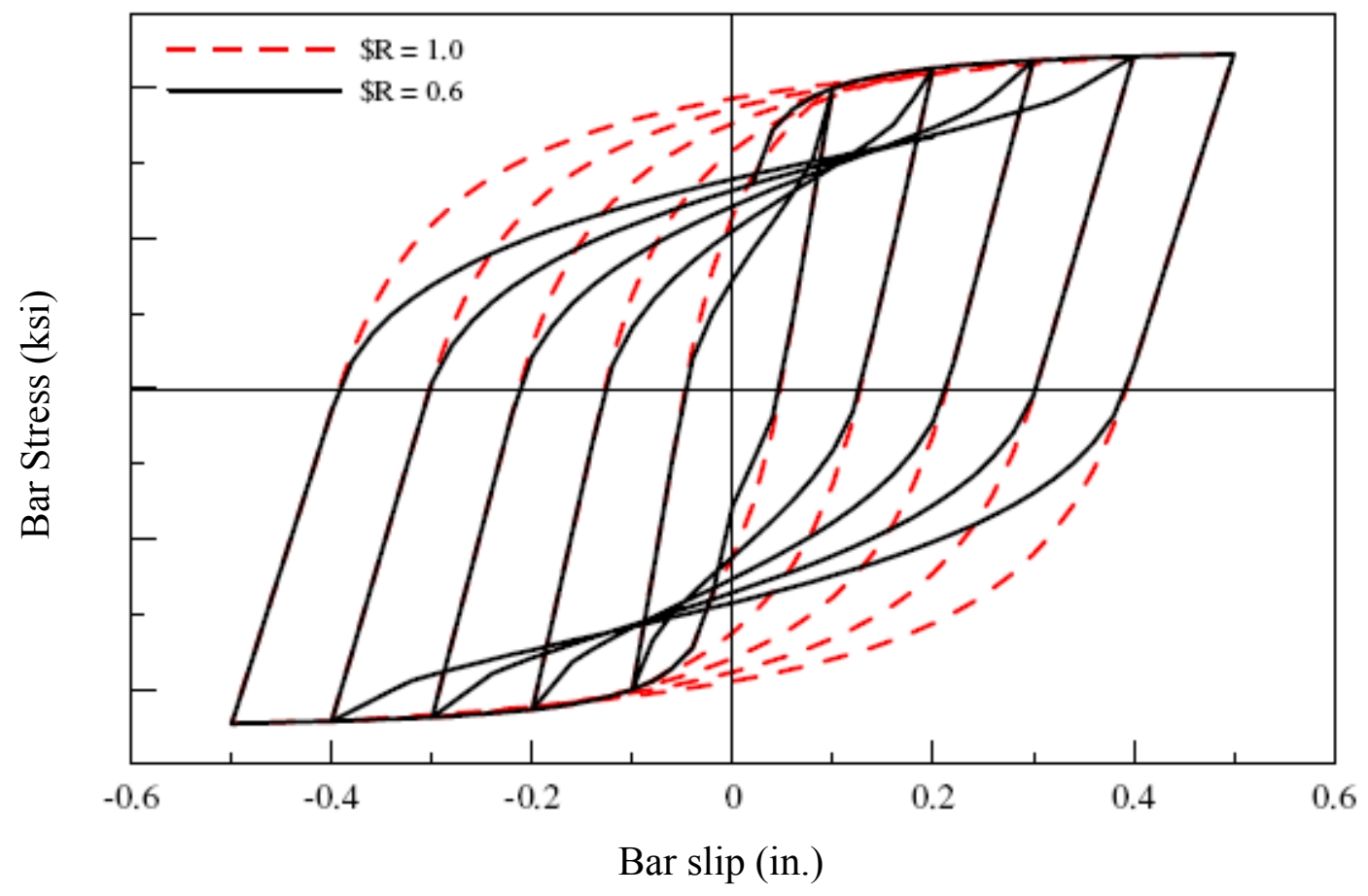

Fig. 4.7 Hysteretic Cyclic Bar Stress-Slip Model (OpenSees 2006)

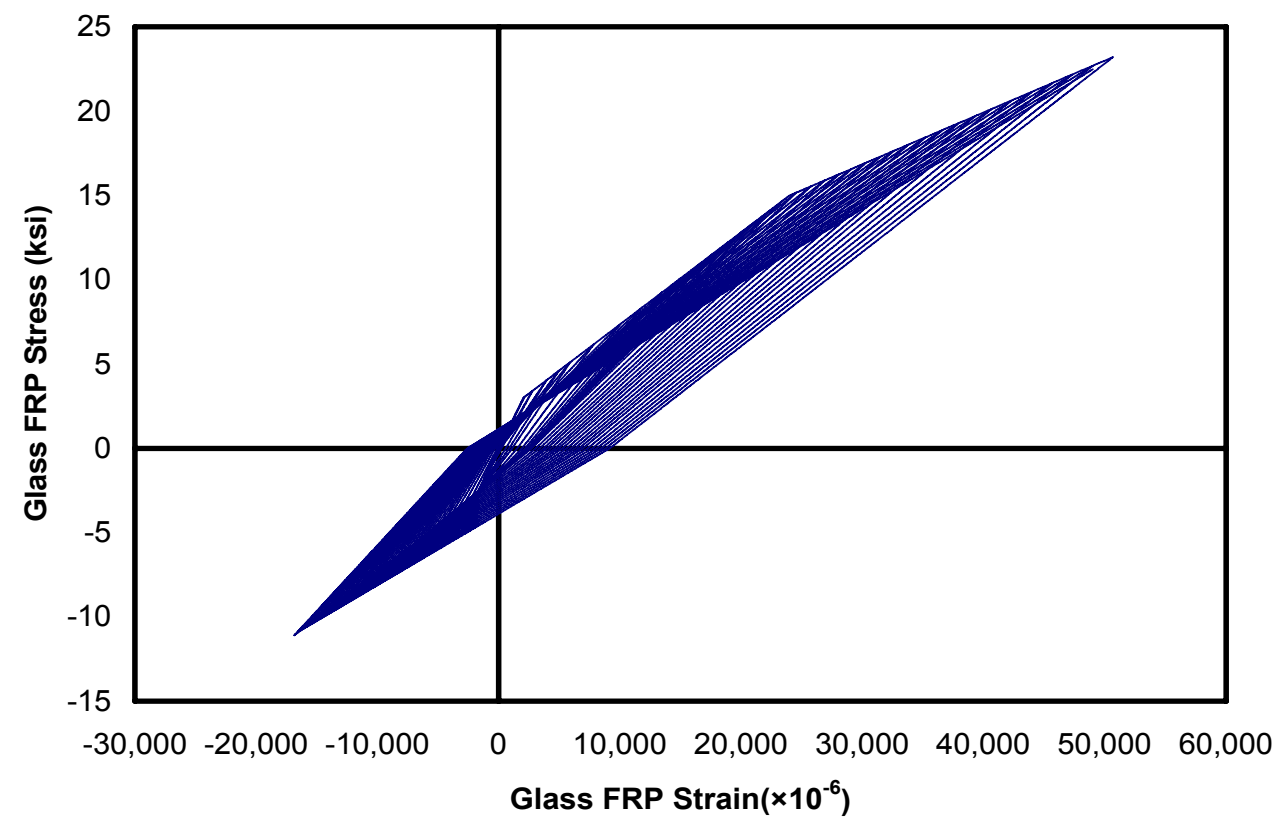

Fig. 4.8 Hysteretic Stress-Strain Response of Glass FRP Tube in Longitudinal Direction 


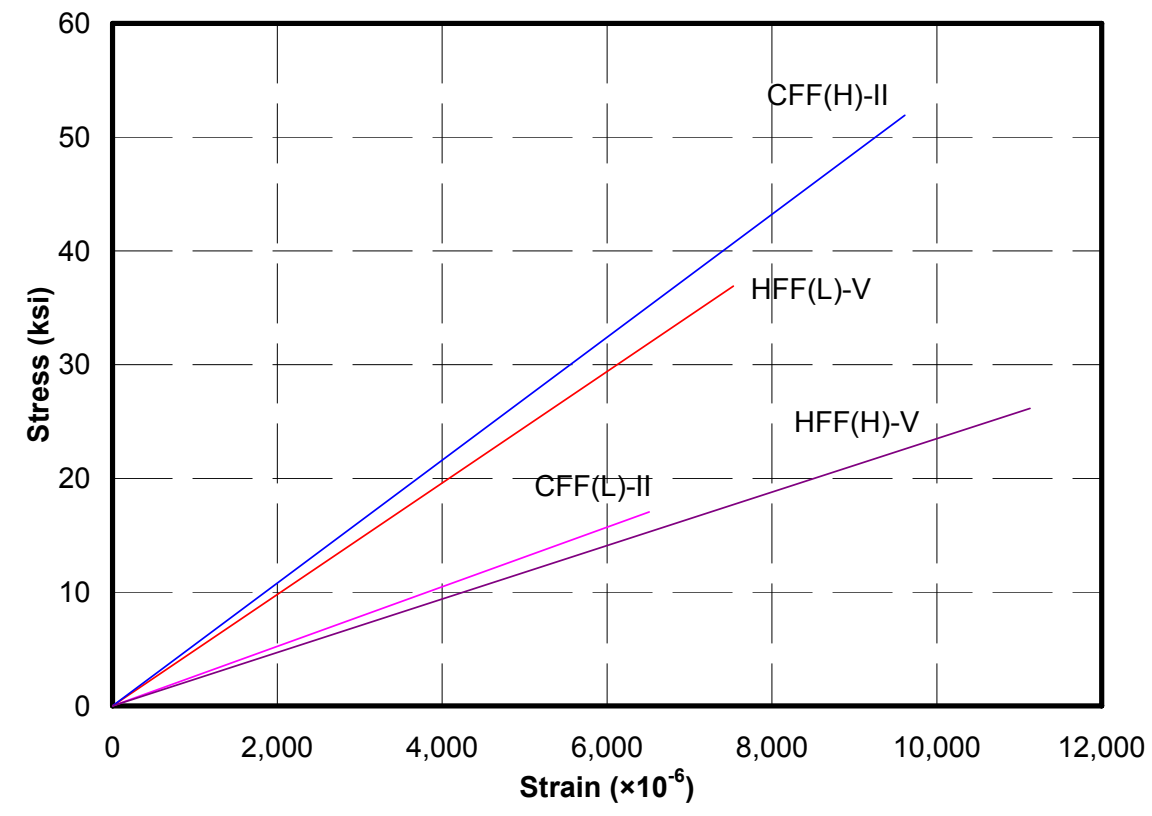

Fig. 4.9 Stress-Strain Response of Carbon and Hybrid FRP Tubes in Longitudinal Direction

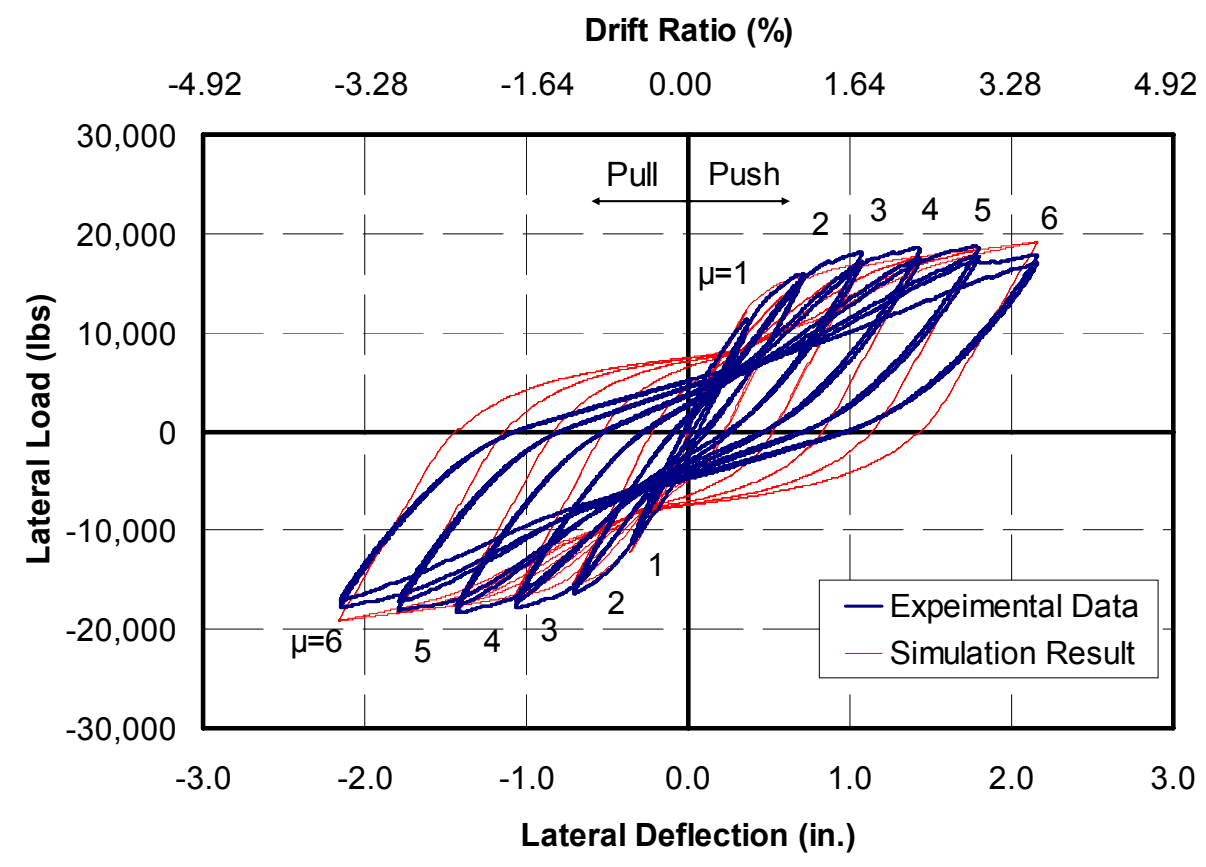

Fig. 4.10 Simulation of Hysteretic Lateral Load-Deflection Response of Specimen $\mathrm{RCF}$ 


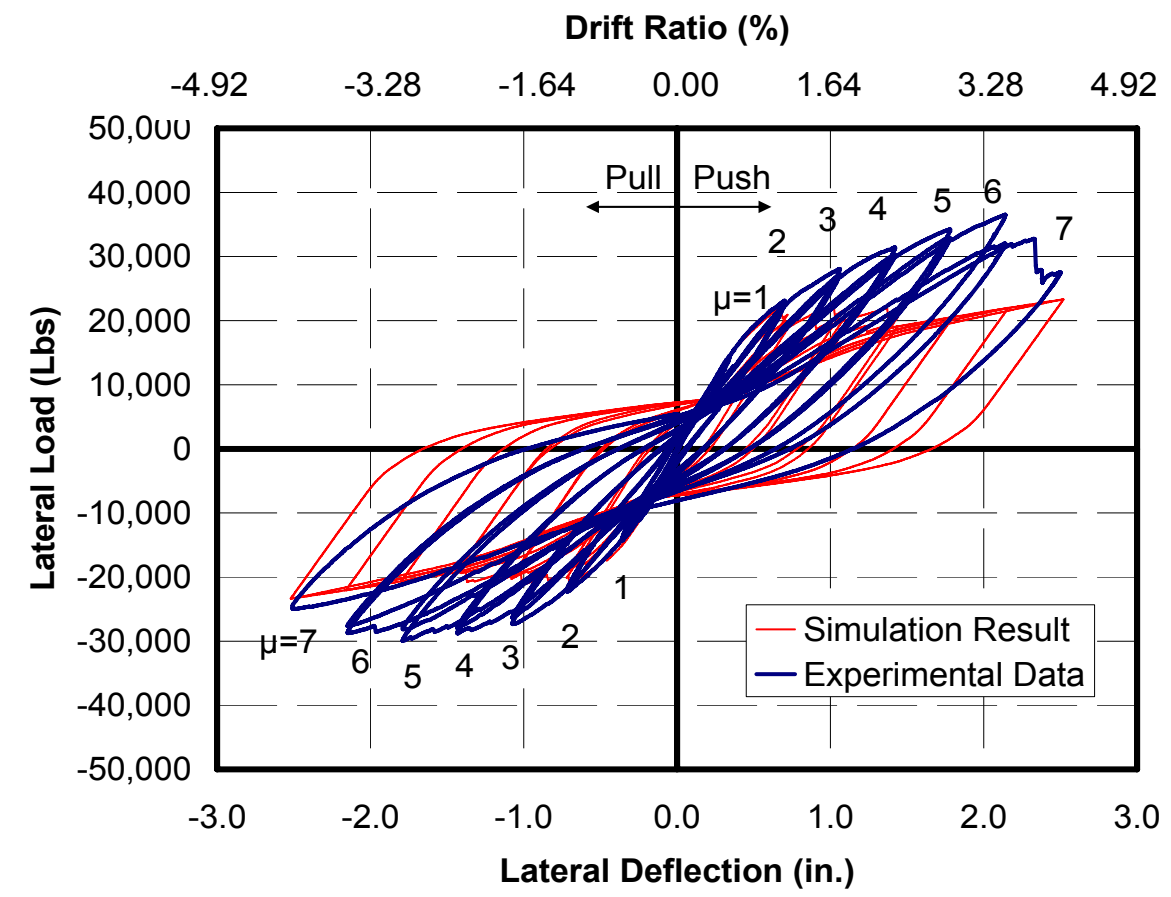

Fig. 4.11 Simulation of Hysteretic Lateral Load-Deflection Response of Specimen CFF

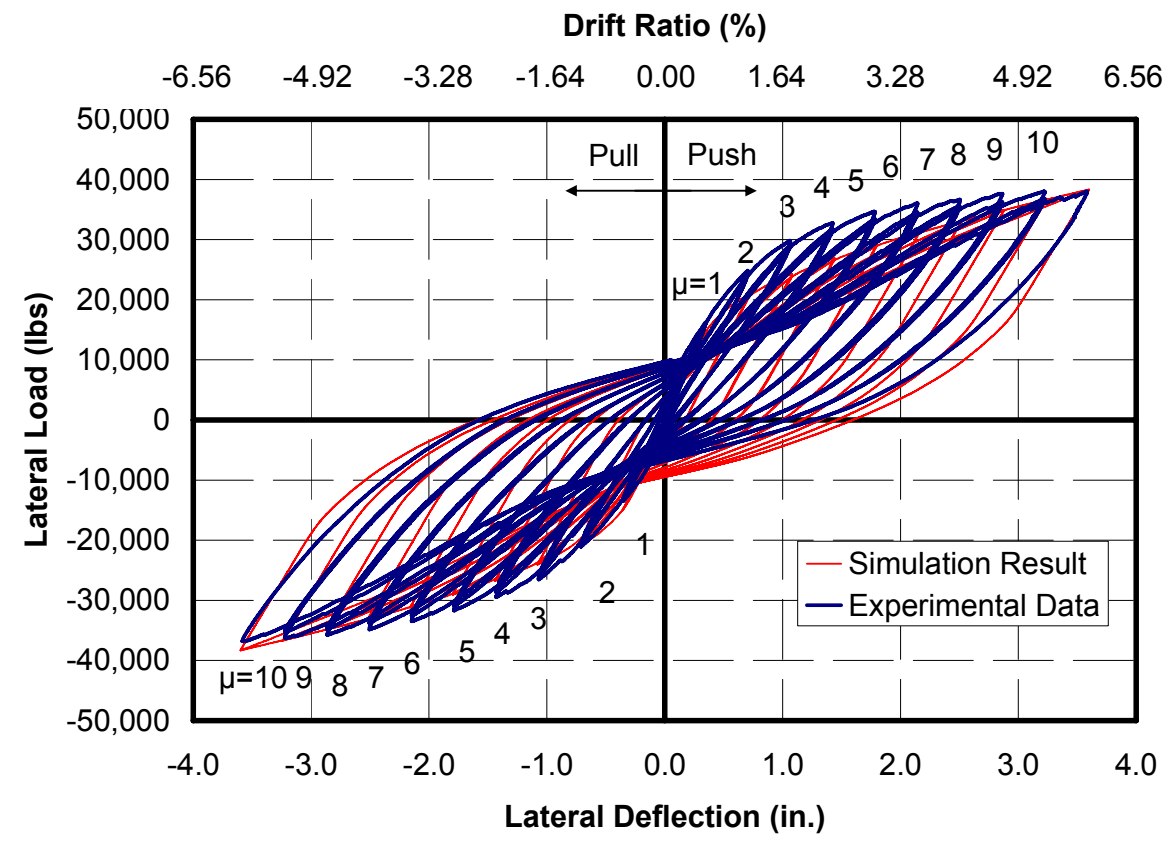

Fig. 4.12 Simulation of Hysteretic Lateral Load-Deflection Response of Specimen GFF 


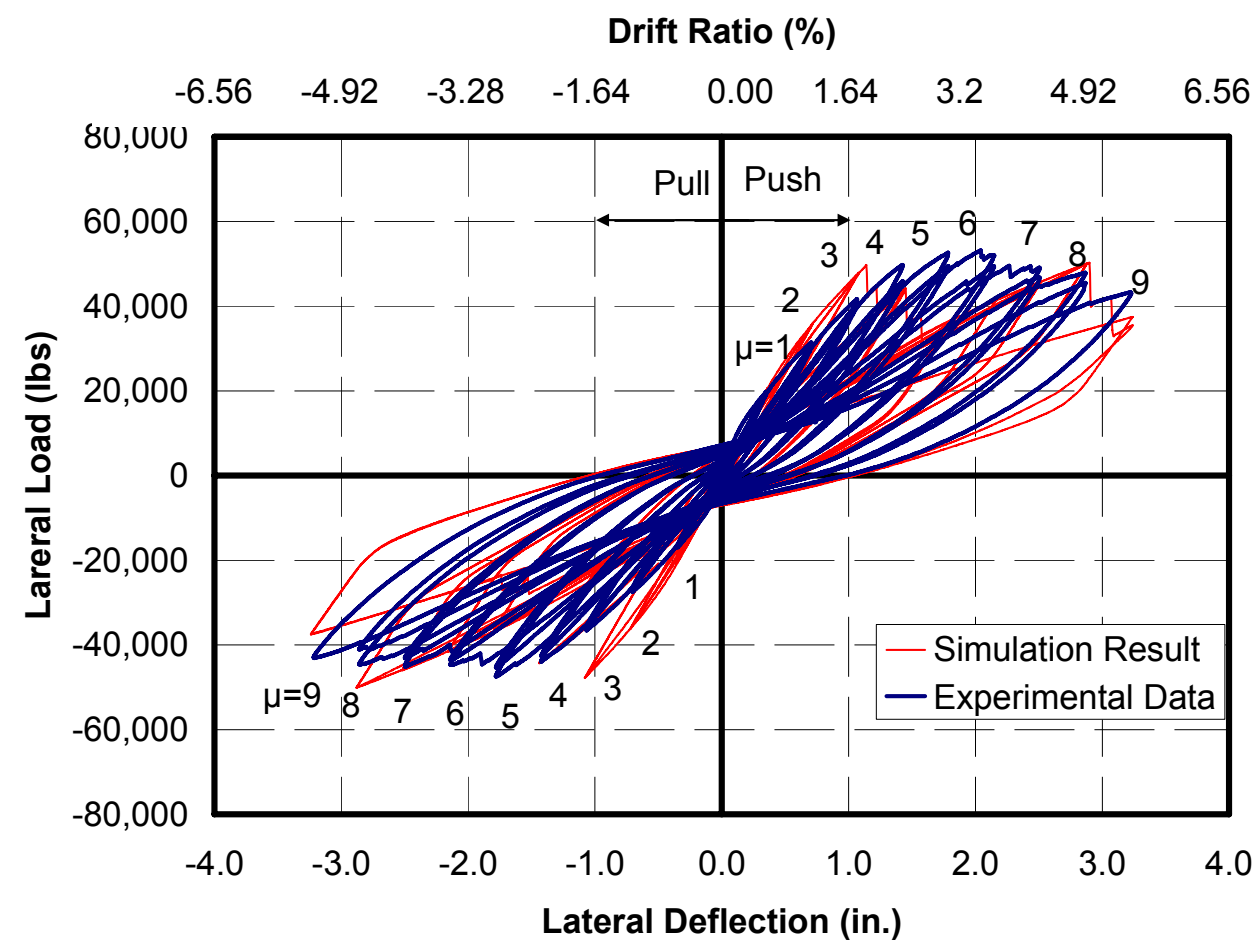

Fig. 4.13 Simulation of Hysteretic Lateral Load-Deflection Response of Specimen HFF

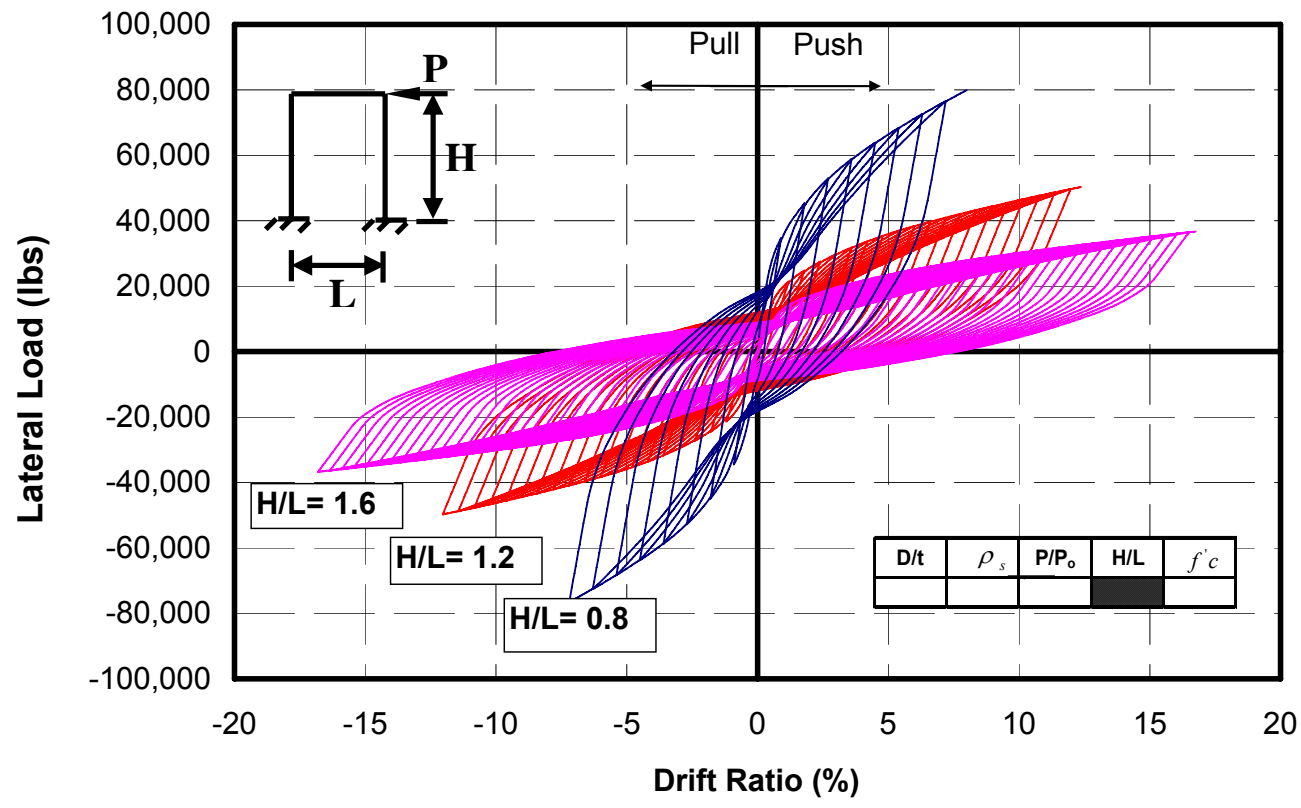

Fig. 4.14 Effect of H/L Ratio on Hysteretic Response of Frame GFF 


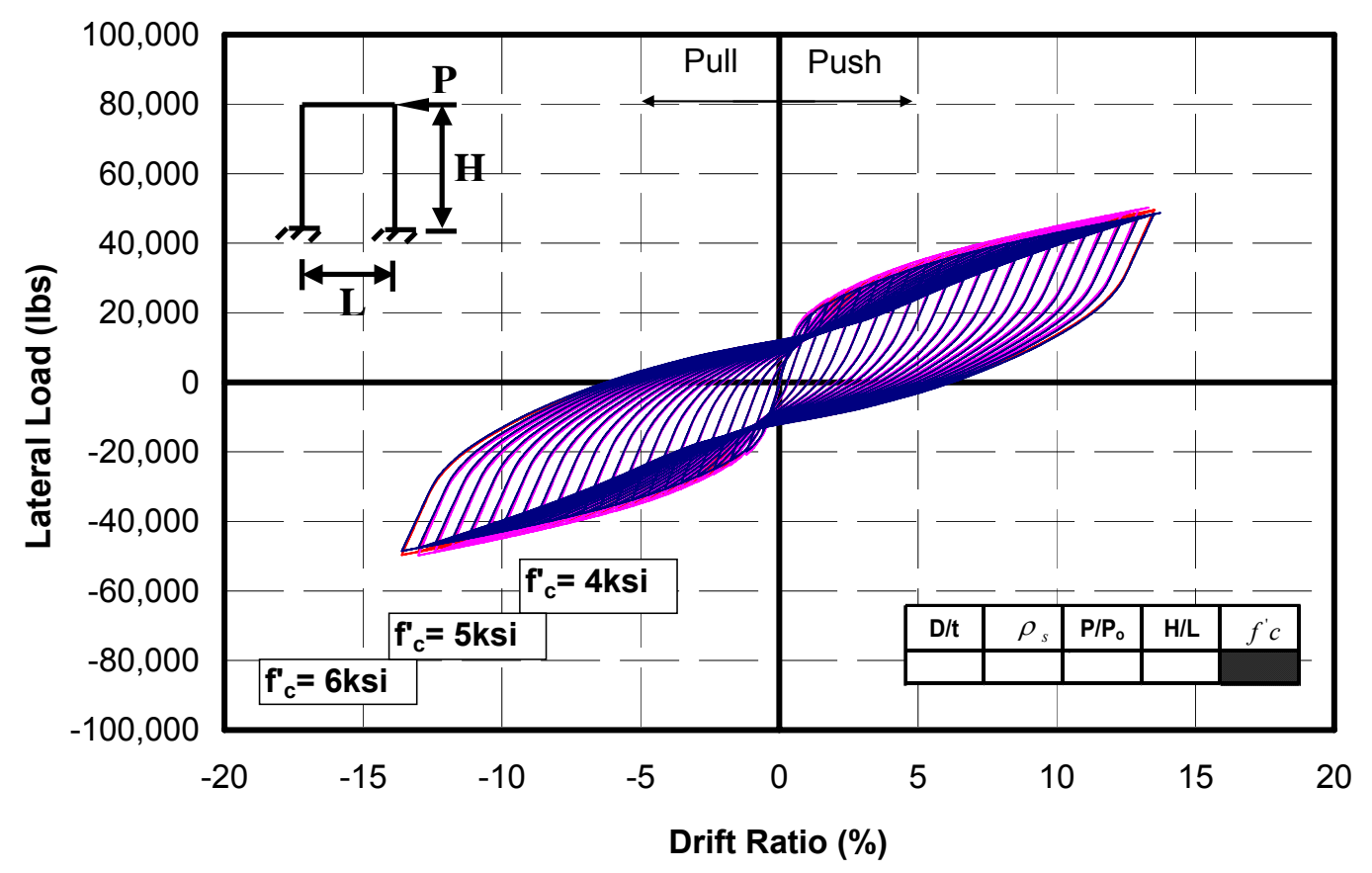

Fig. 4.15 Effect of Concrete Compressive Strength on Hysteretic Response of Frame GFF

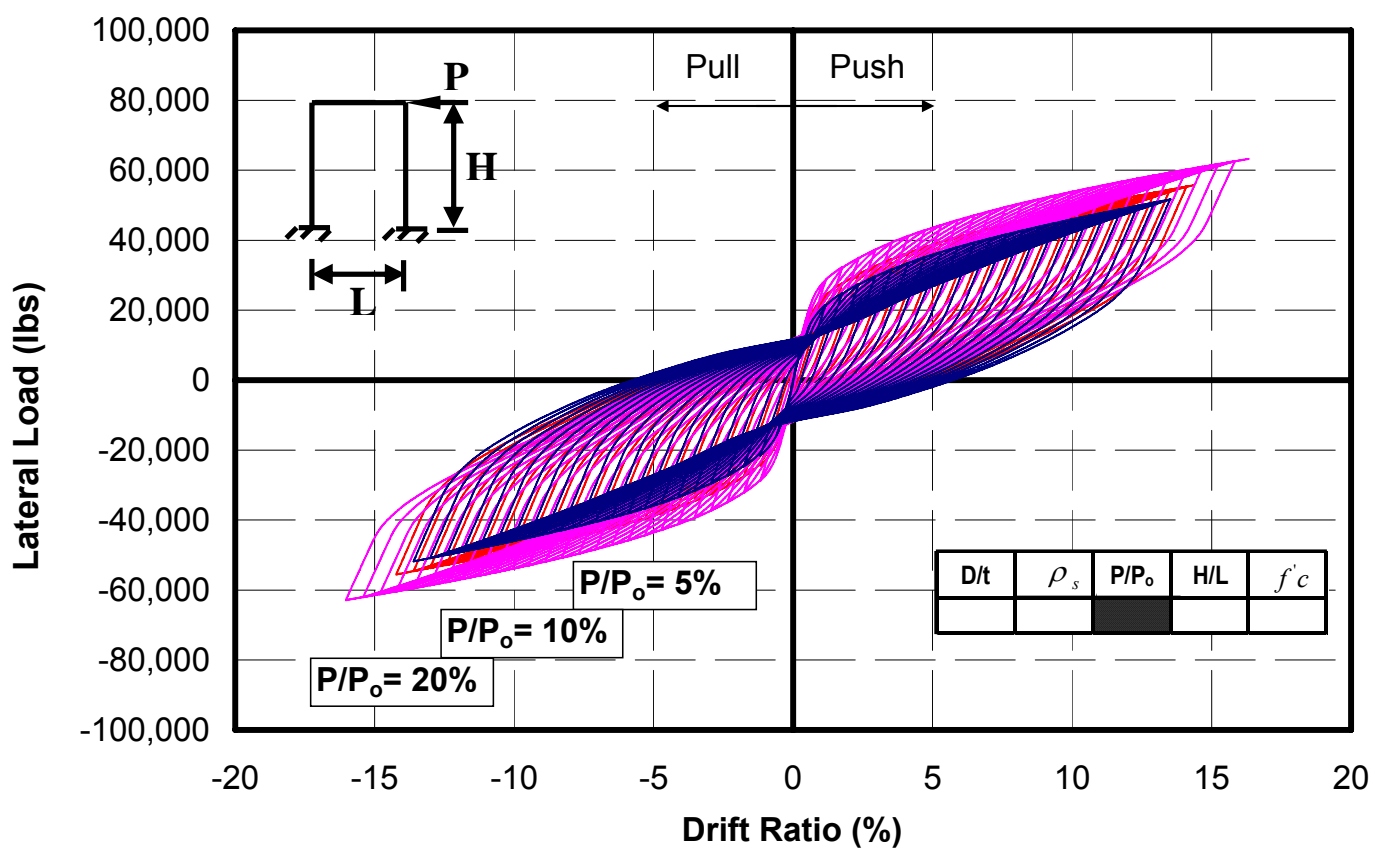

Fig. 4.16 Effect of Axial Load Ratio on Hysteretic Response of Frame GFF 


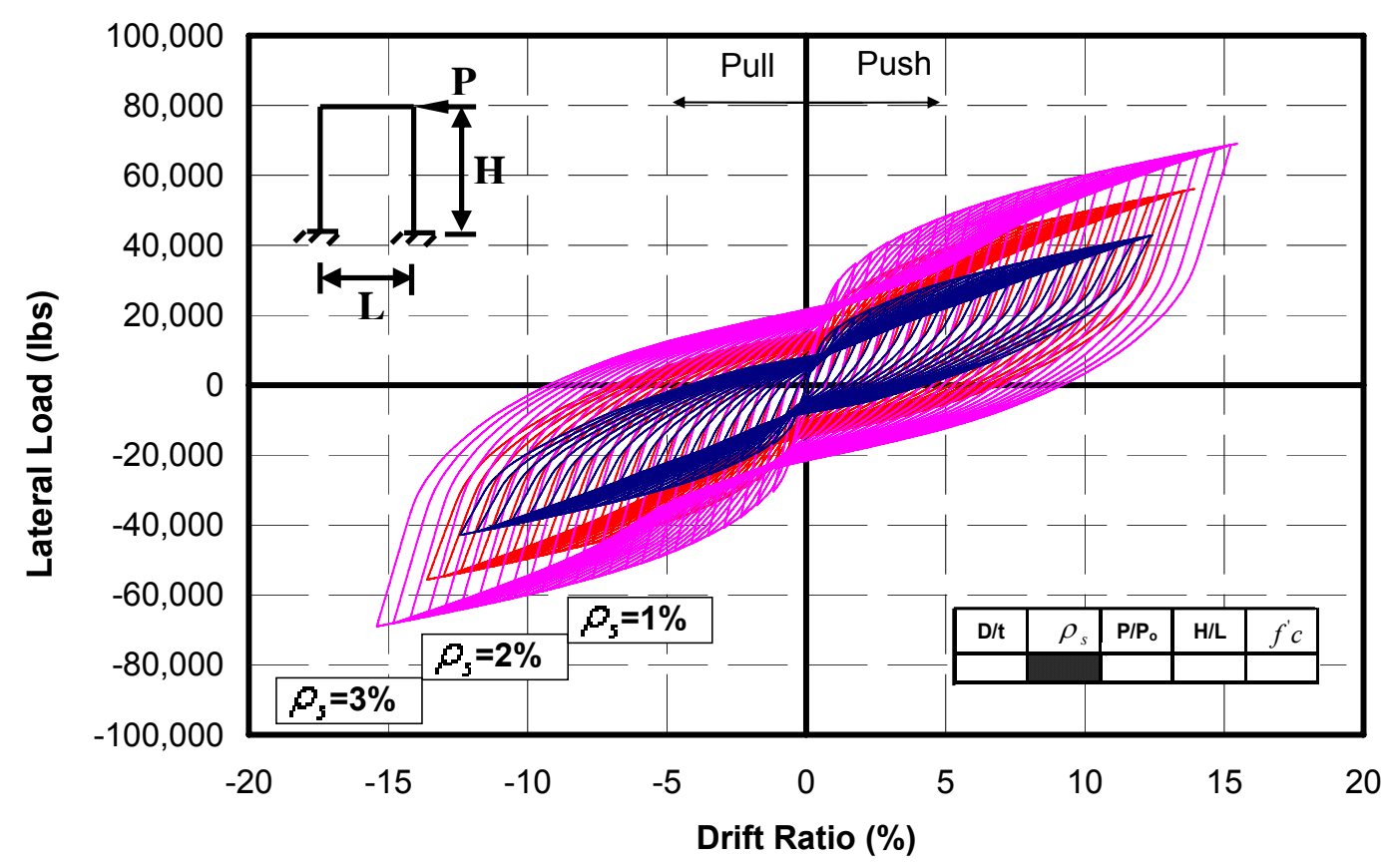

Fig. 4.17 Effect of Steel Reinforcement Ratio on Hysteretic Response of Frame GFF

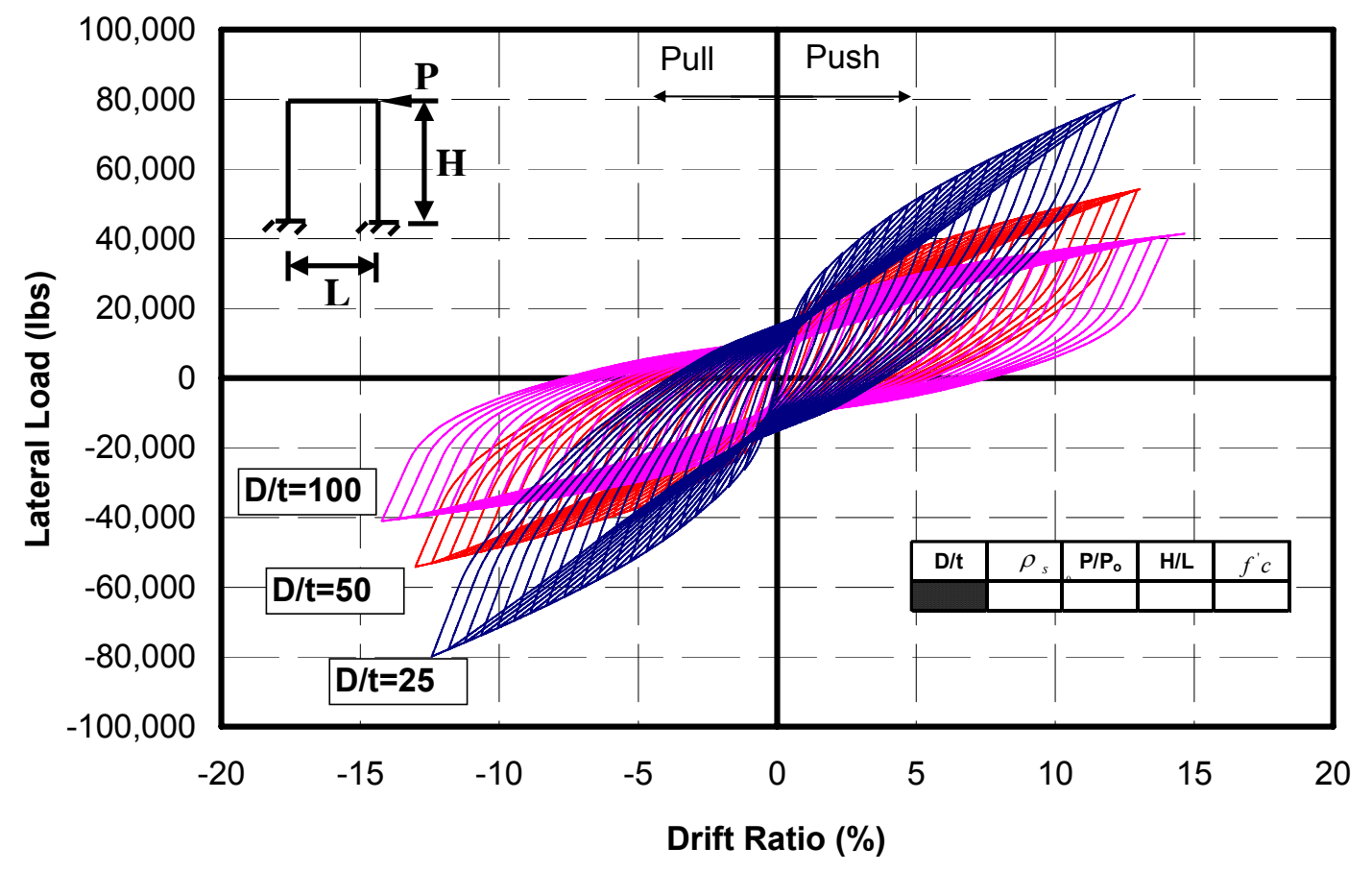

Fig. 4.18 Effect of D/t Ratio on Hysteretic Response of Frame GFF 


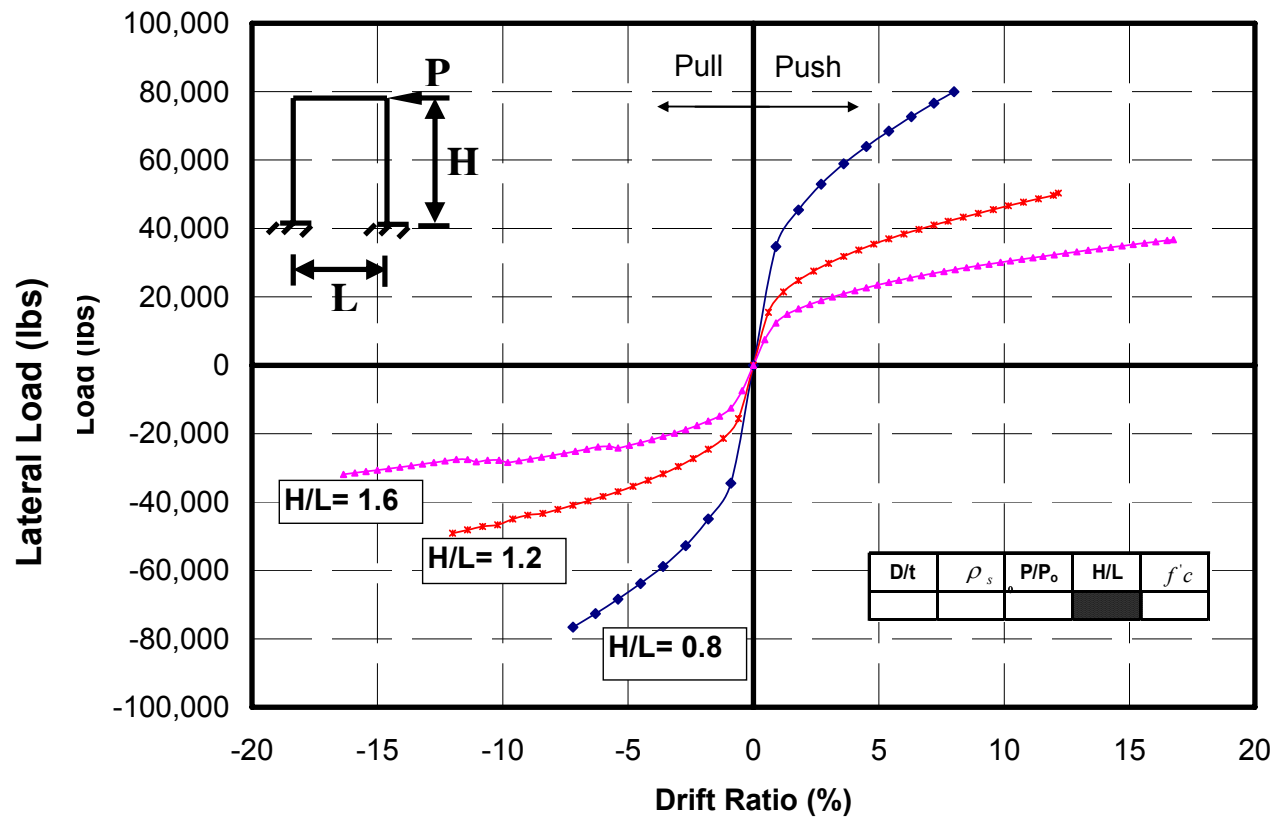

Fig. 4.19 Effect of H/L Ratio on Response Envelope of Frame GFF

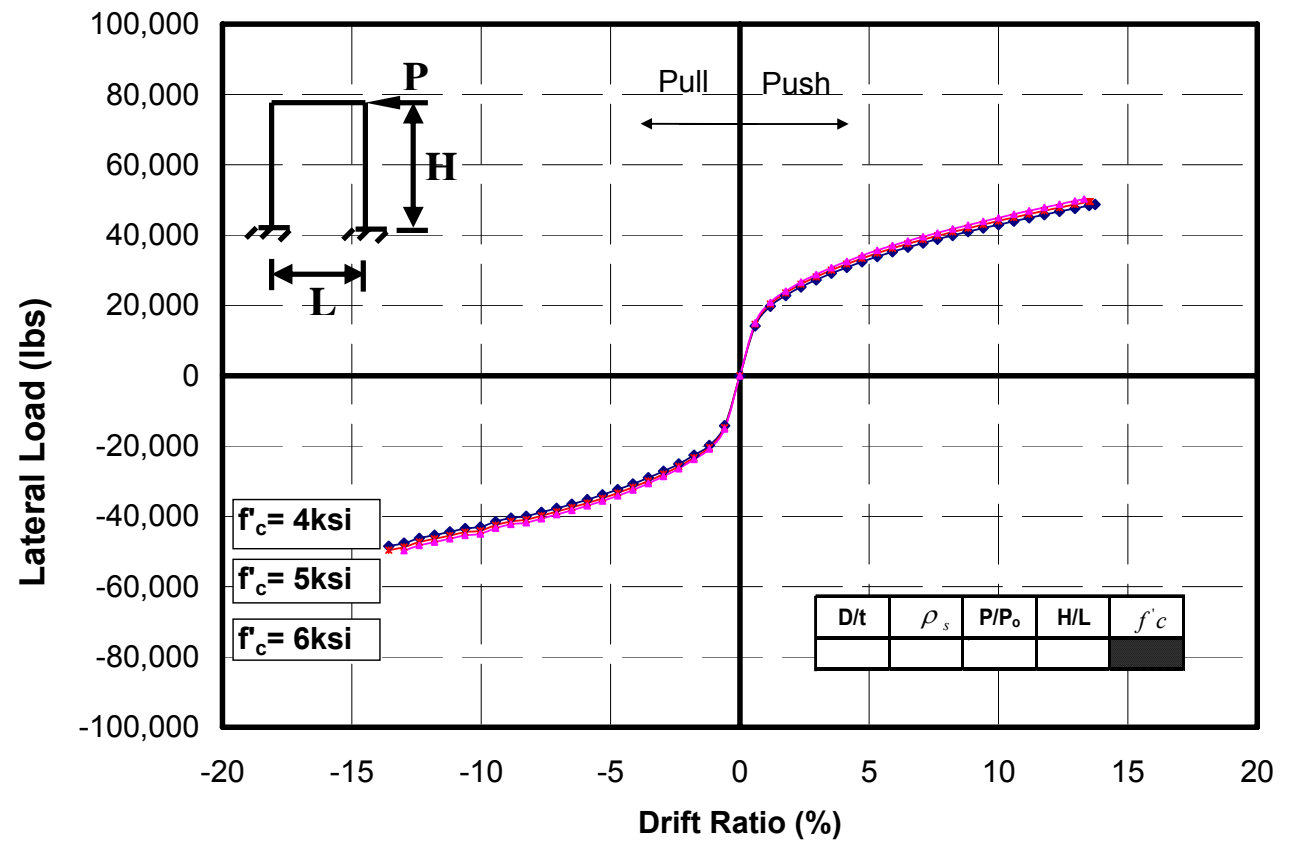

Fig. 4.20 Effect of Concrete Compressive Strength on Response Envelope of Frame GFF 


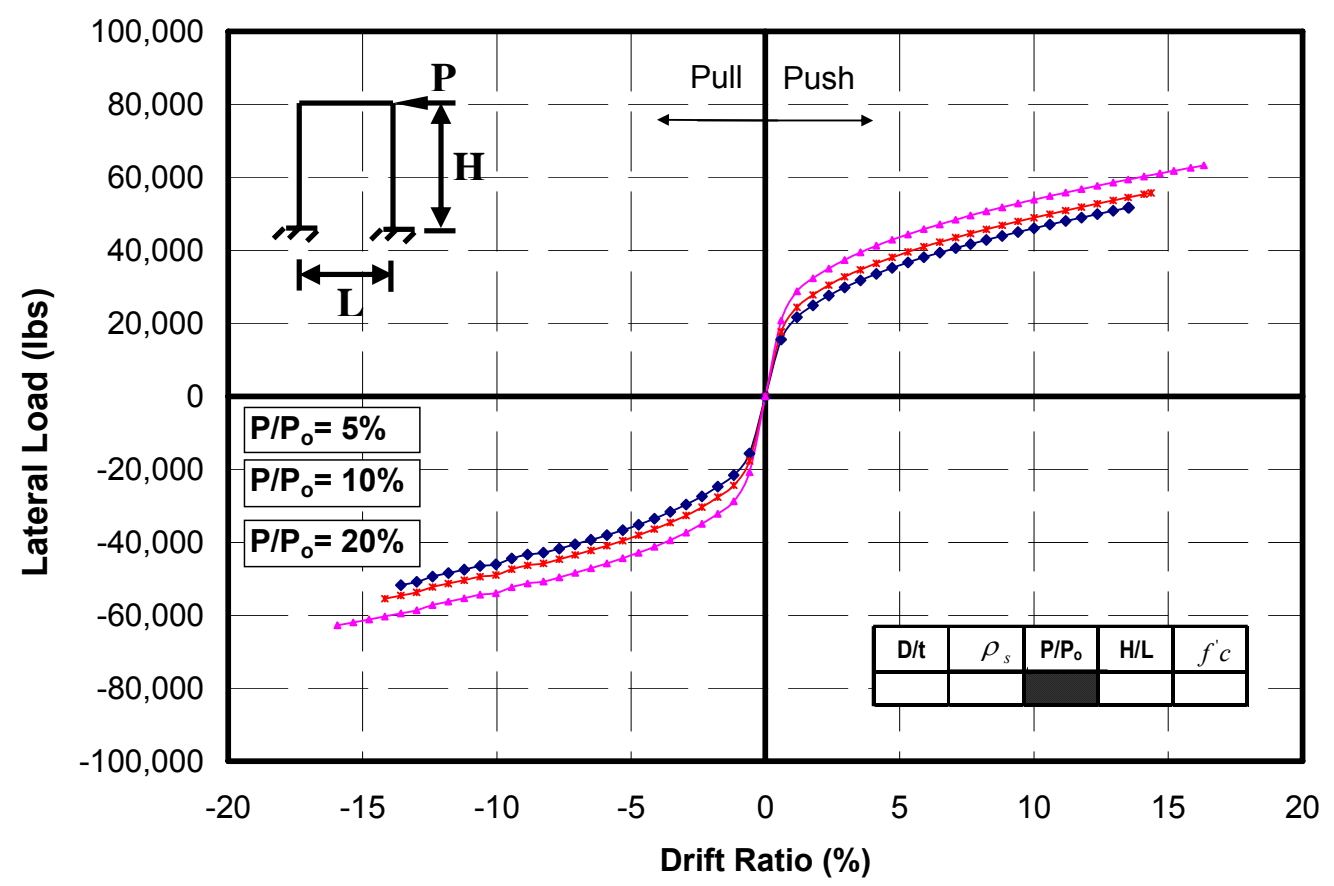

Fig. 4.21 Effect of Axial Load Ratio on Response Envelope of Frame GFF

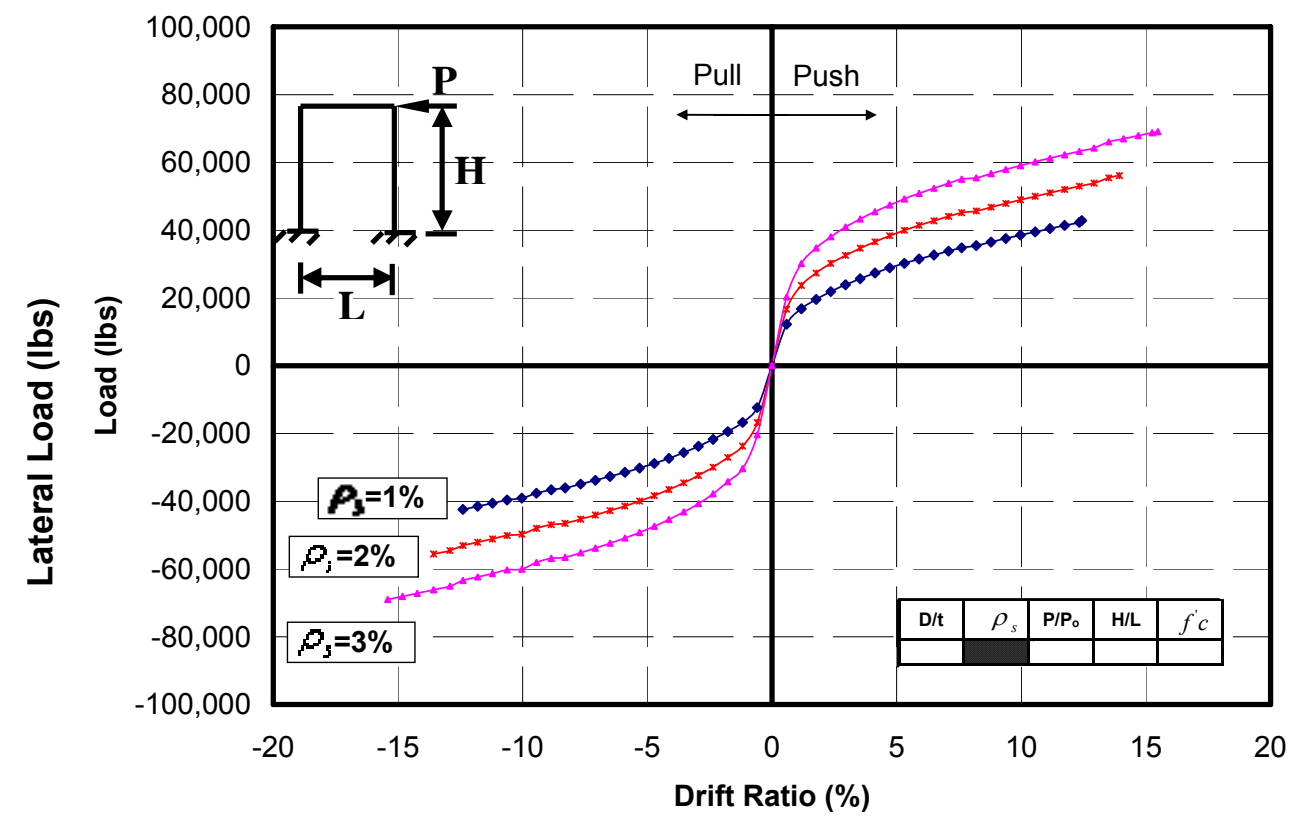

Fig. 4.22 Effect of Steel Reinforcement Ratio on Response Envelope of Frame GFF 


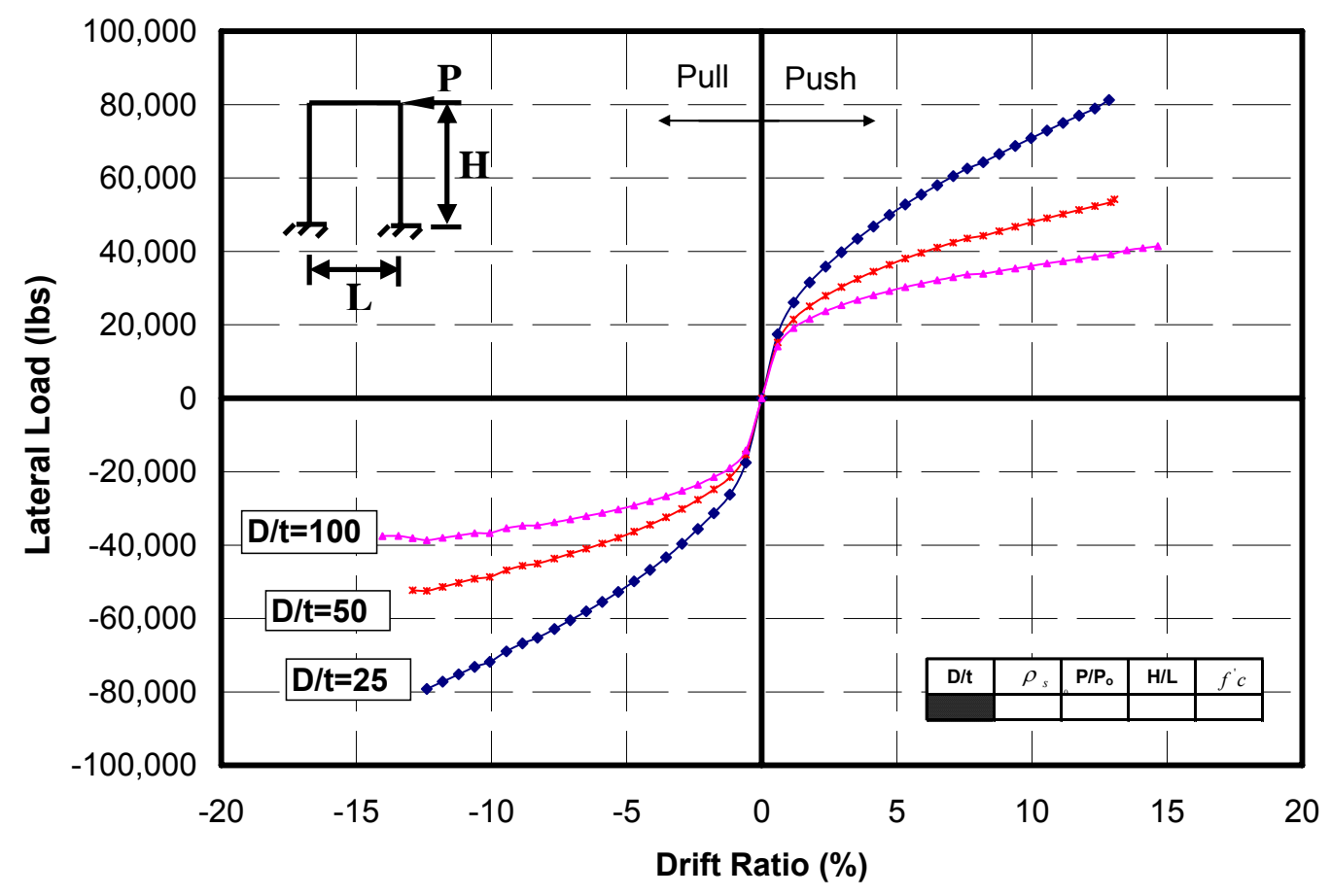

Fig. 4.23 Effect of D/t Ratio on Response Envelope of Frame GFF

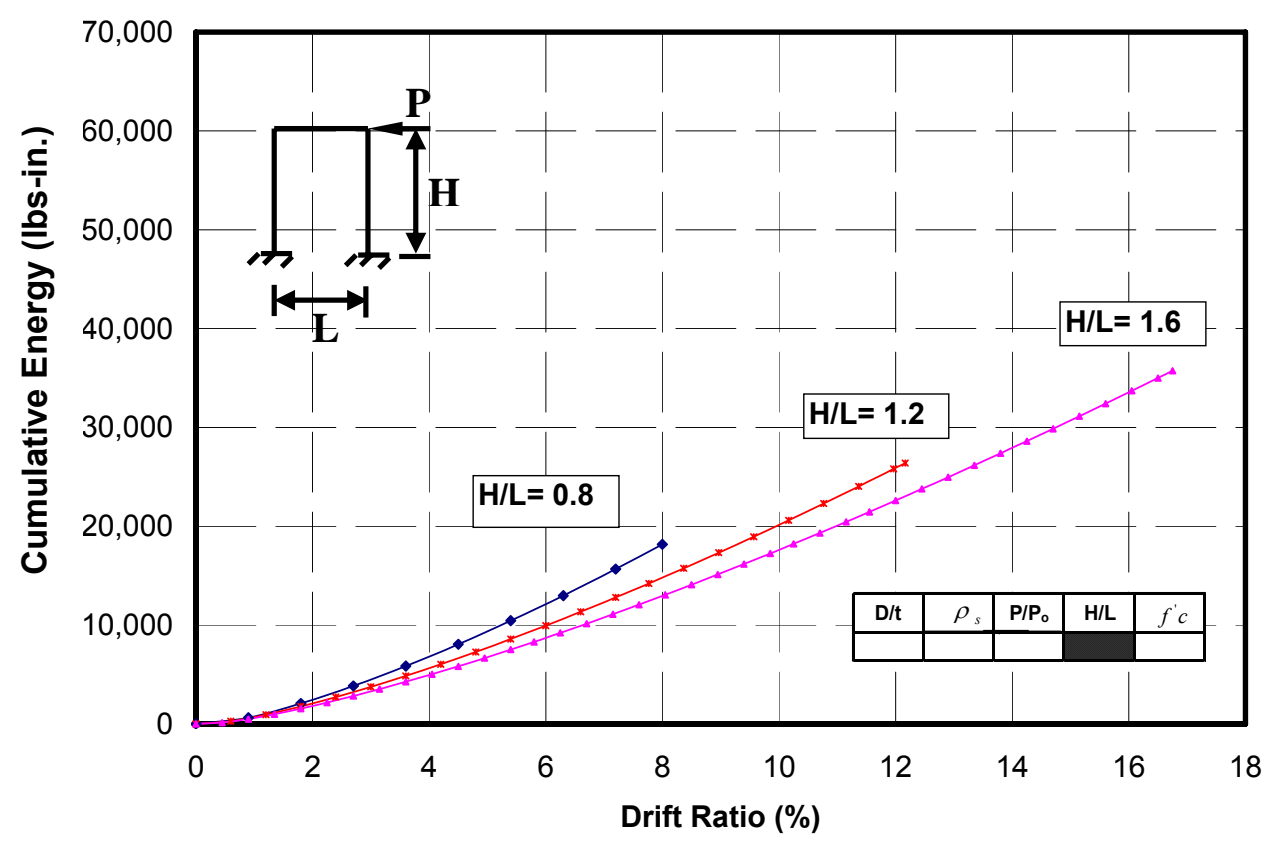

Fig. 4.24 Effect of H/L Ratio on Cumulative Energy of Frame GFF 


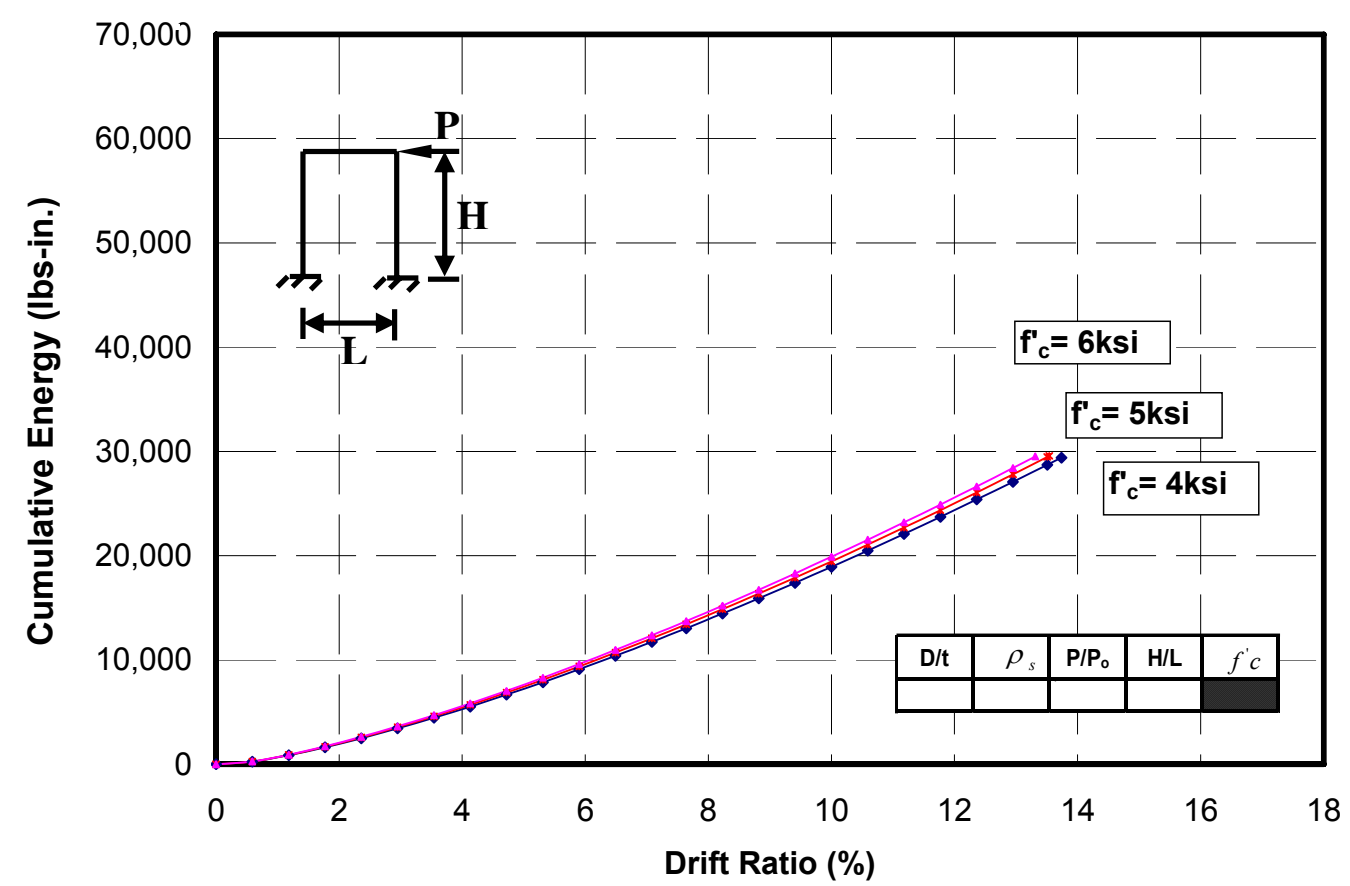

Fig. 4.25 Effect of Concrete Compressive Strength on Cumulative Energy of Frame GFF

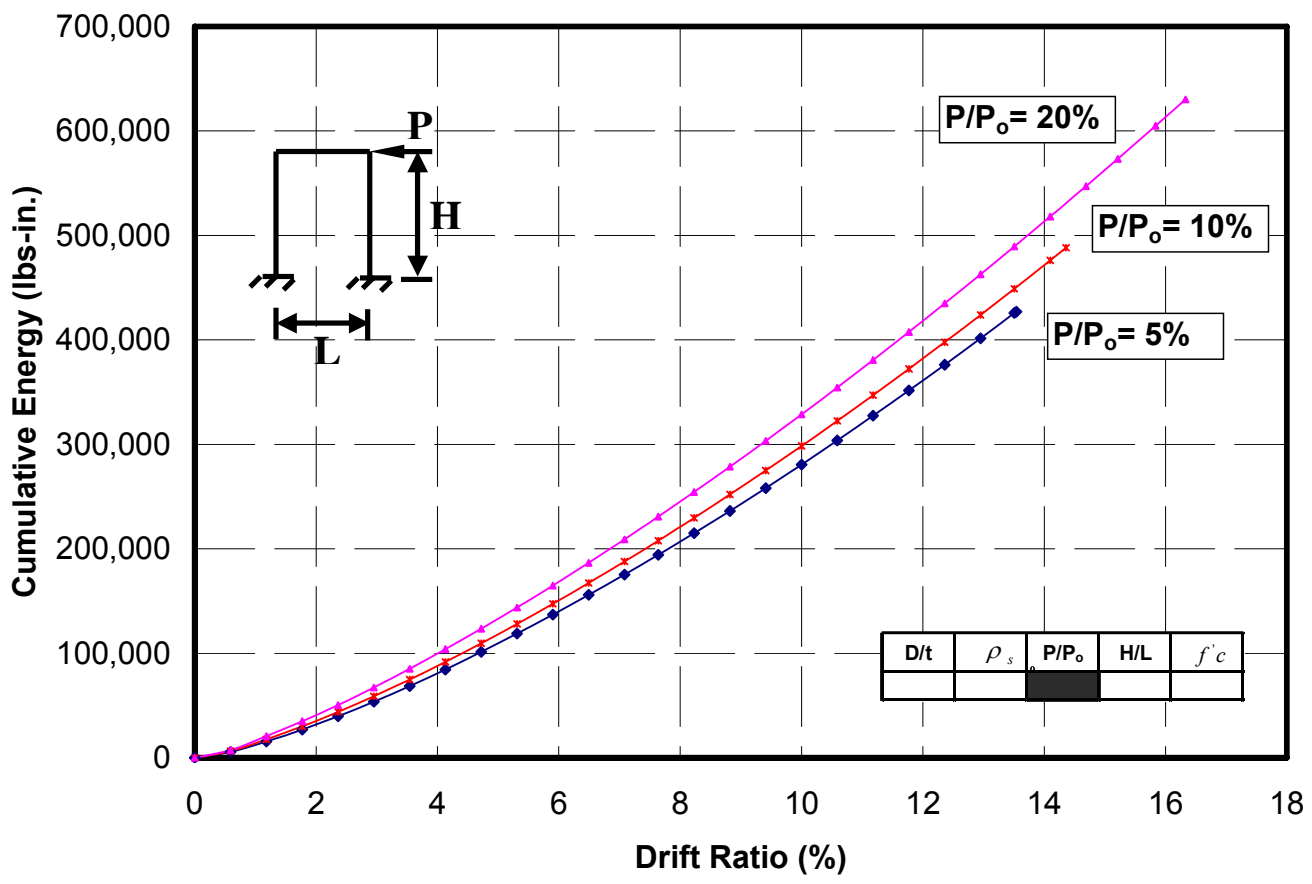

Fig. 4.26 Effect of Axial Load Ratio on Cumulative Energy of Frame GFF 


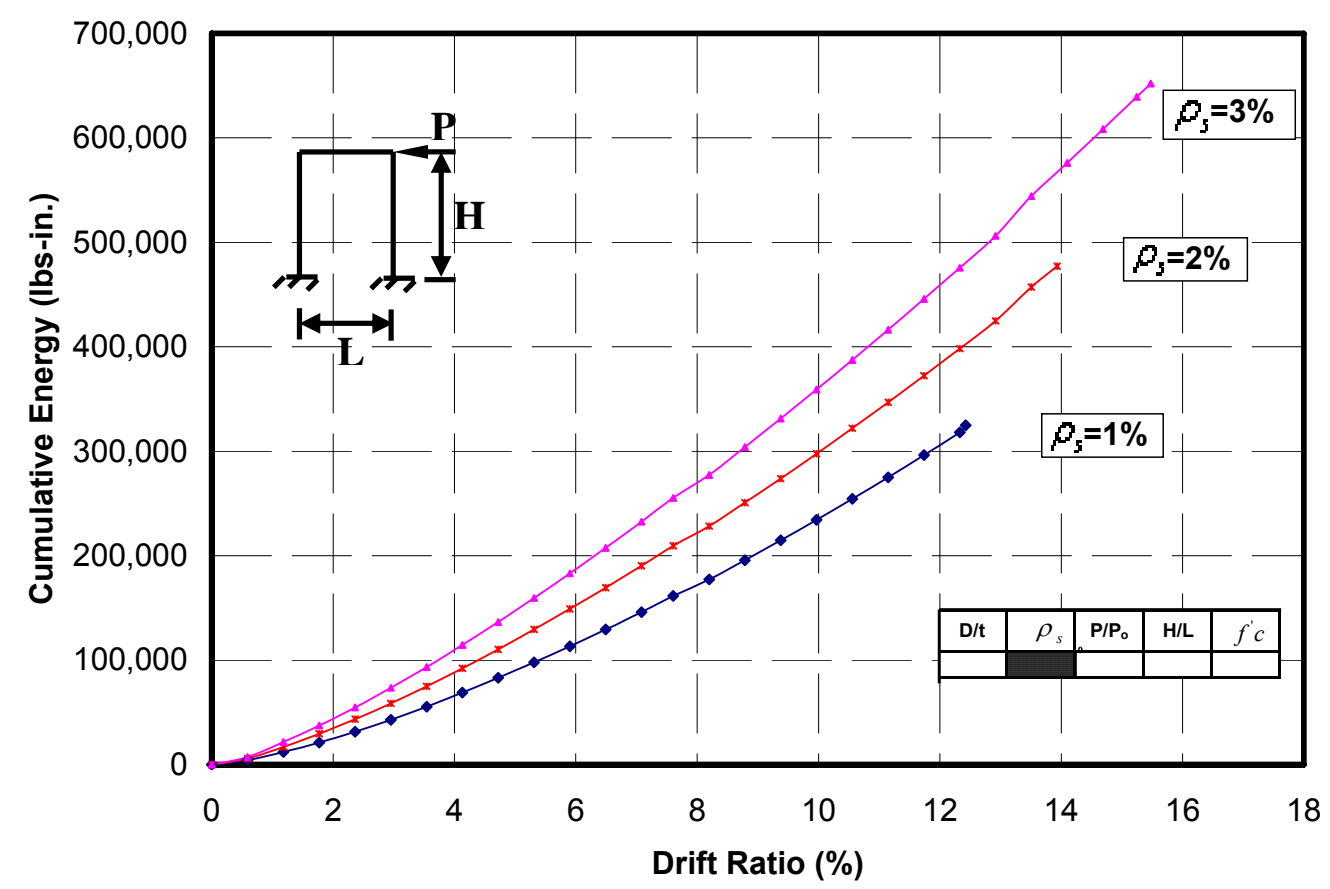

Fig. 4.27 Effect of Steel Reinforcement Ratio on Cumulative Energy of Frame GFF

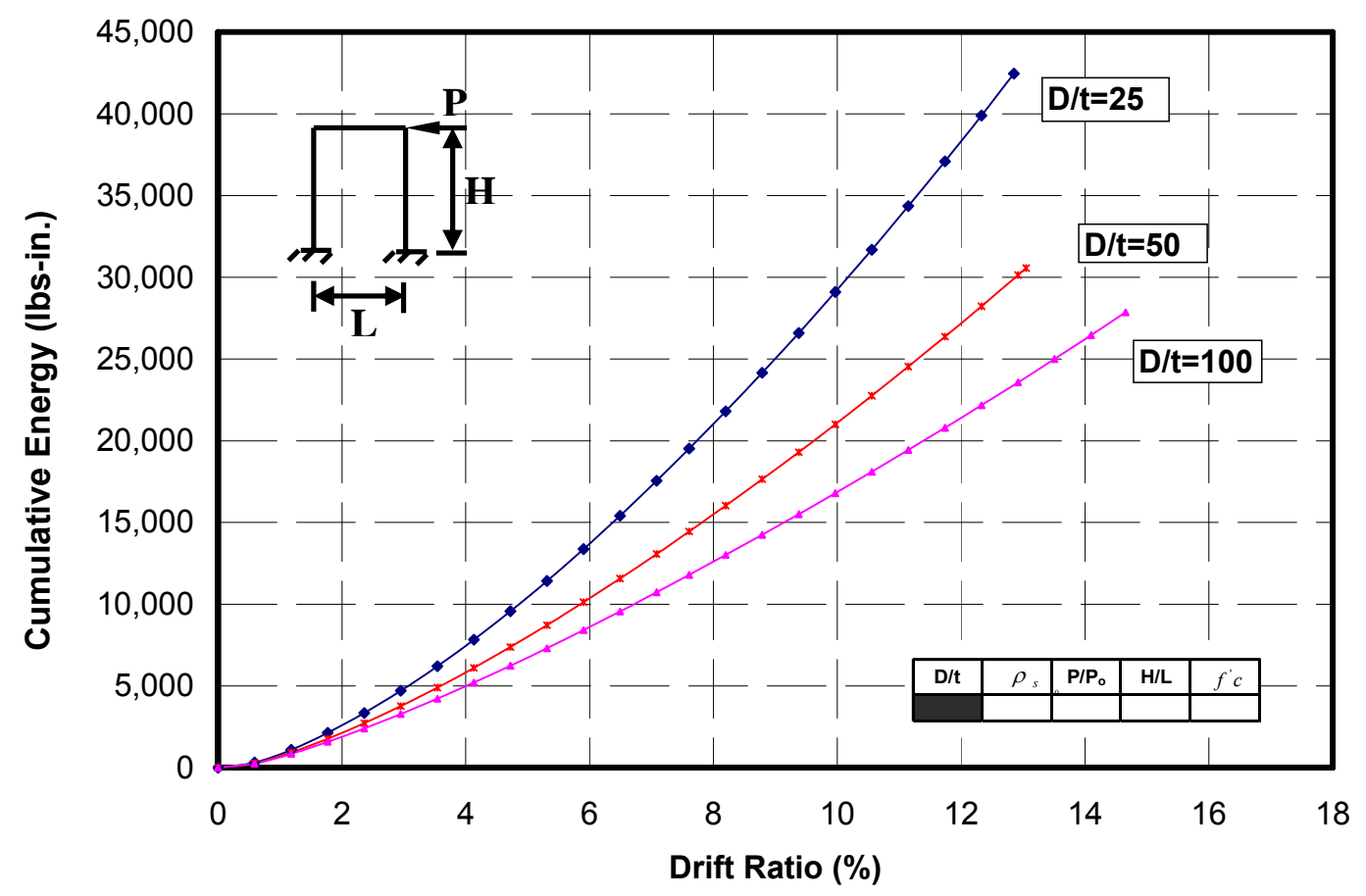

Fig. 4.28 Effect of D/t Ratio on Cumulative Energy of Frame GFF 


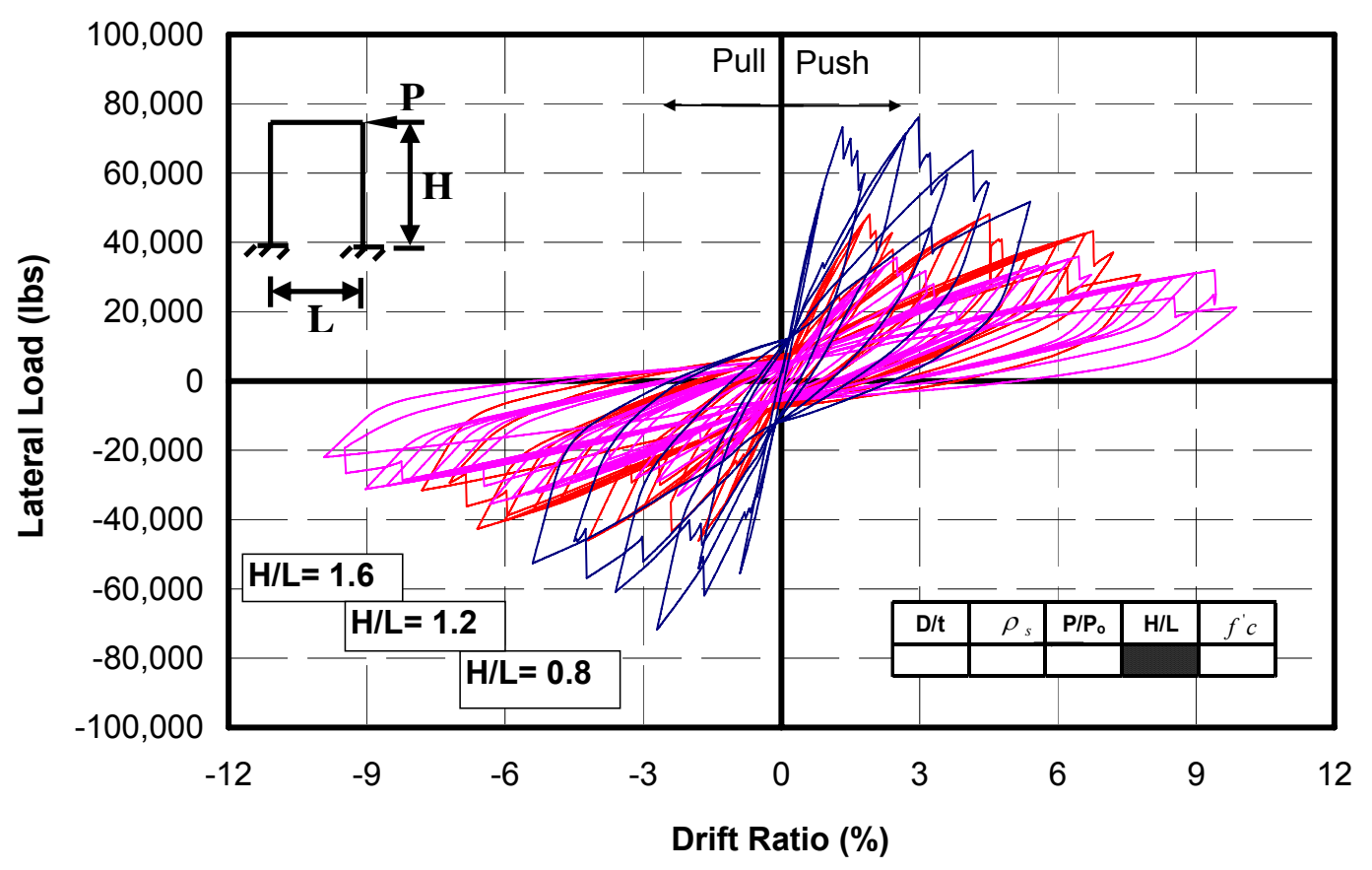

Fig. 4.29 Effect of H/L Ratio on Hysteretic Response of Frame HFF

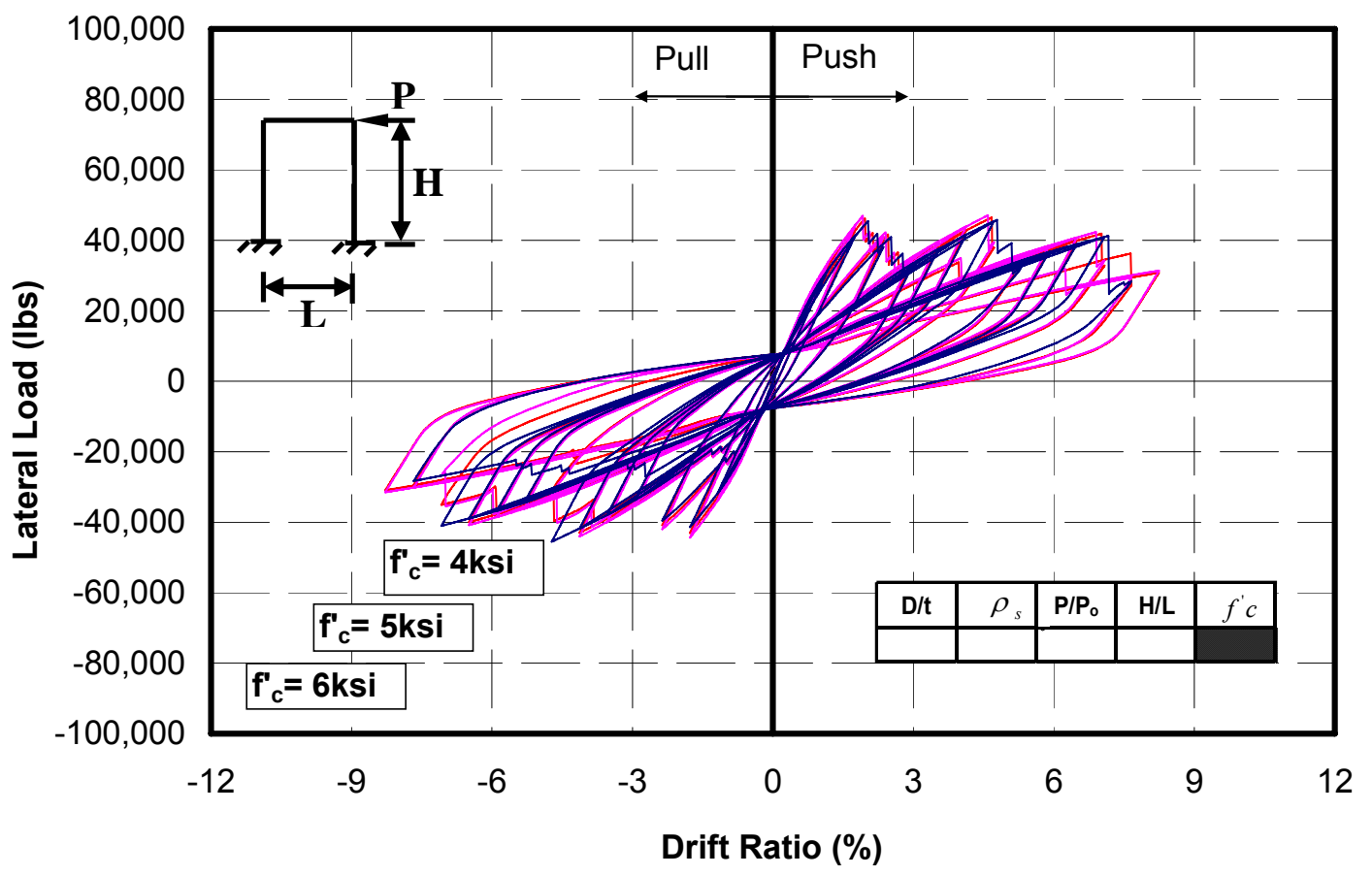

Fig. 4.30 Effect of Concrete Compressive Strength on Hysteretic Response of Frame HFF 


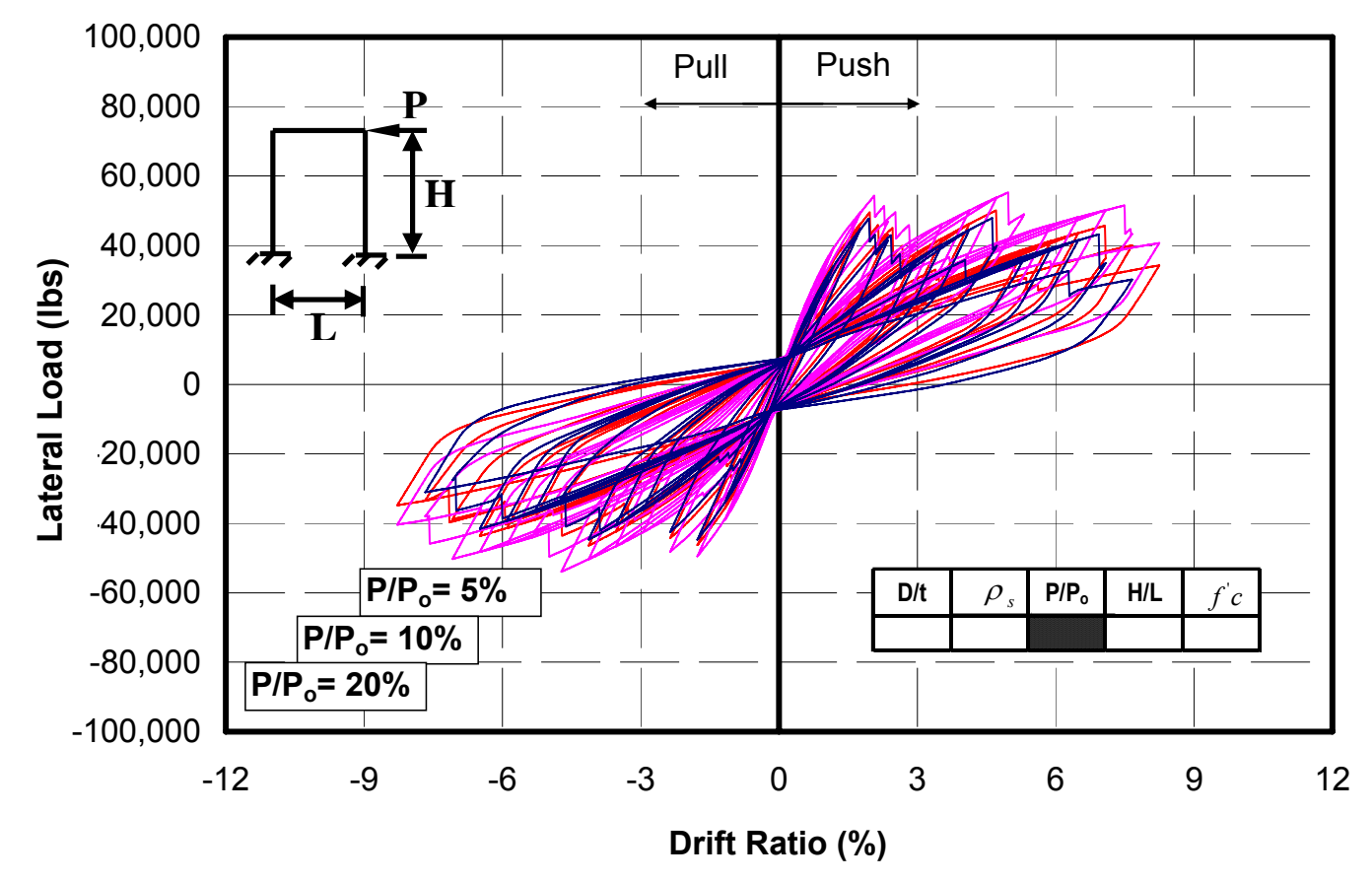

Fig. 4.31 Effect of Axial Load Ratio on Hysteretic Response of Frame HFF

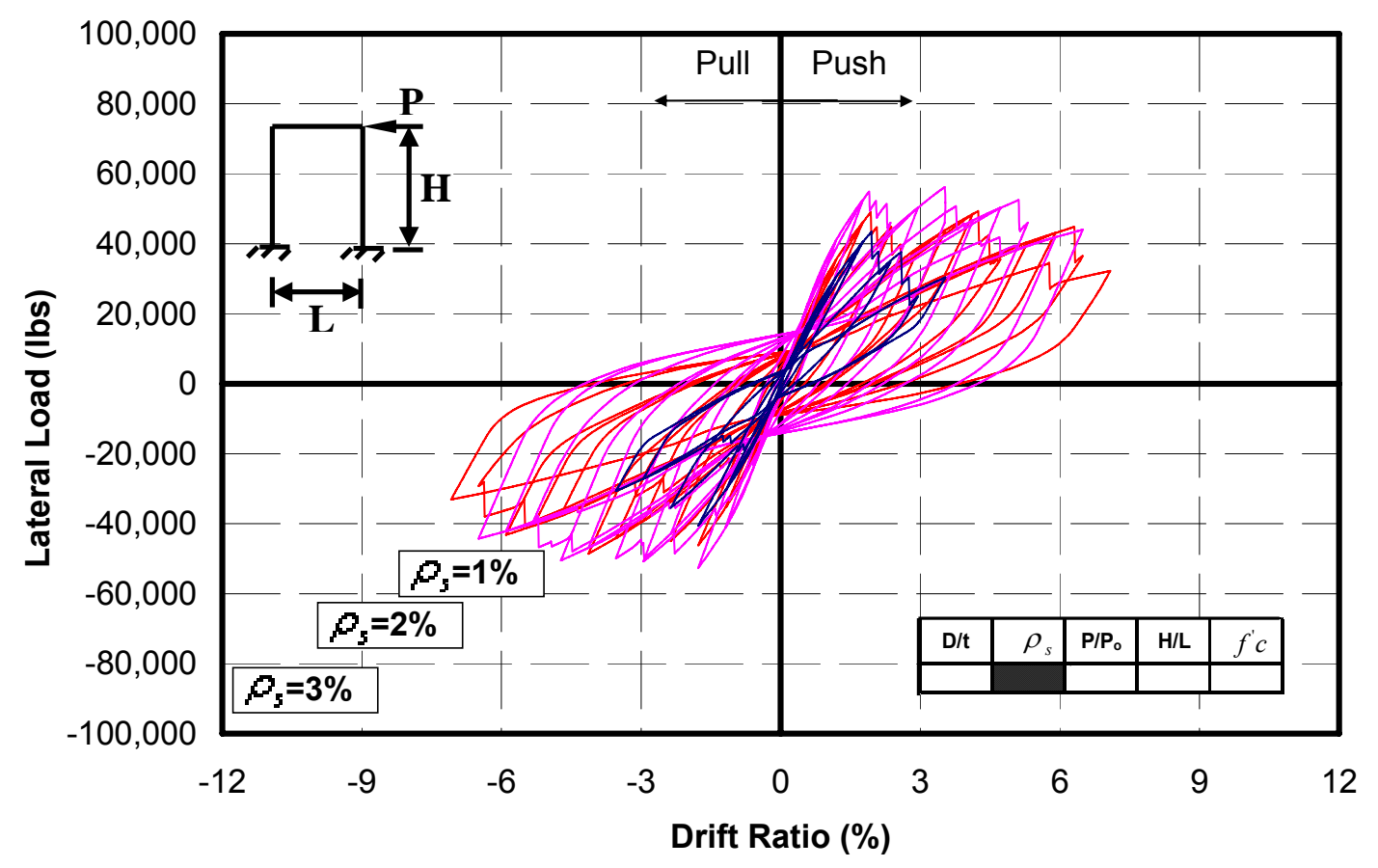

Fig. 4.32 Effect of Steel Reinforcement Ratio on Hysteretic Response of Frame HFF 


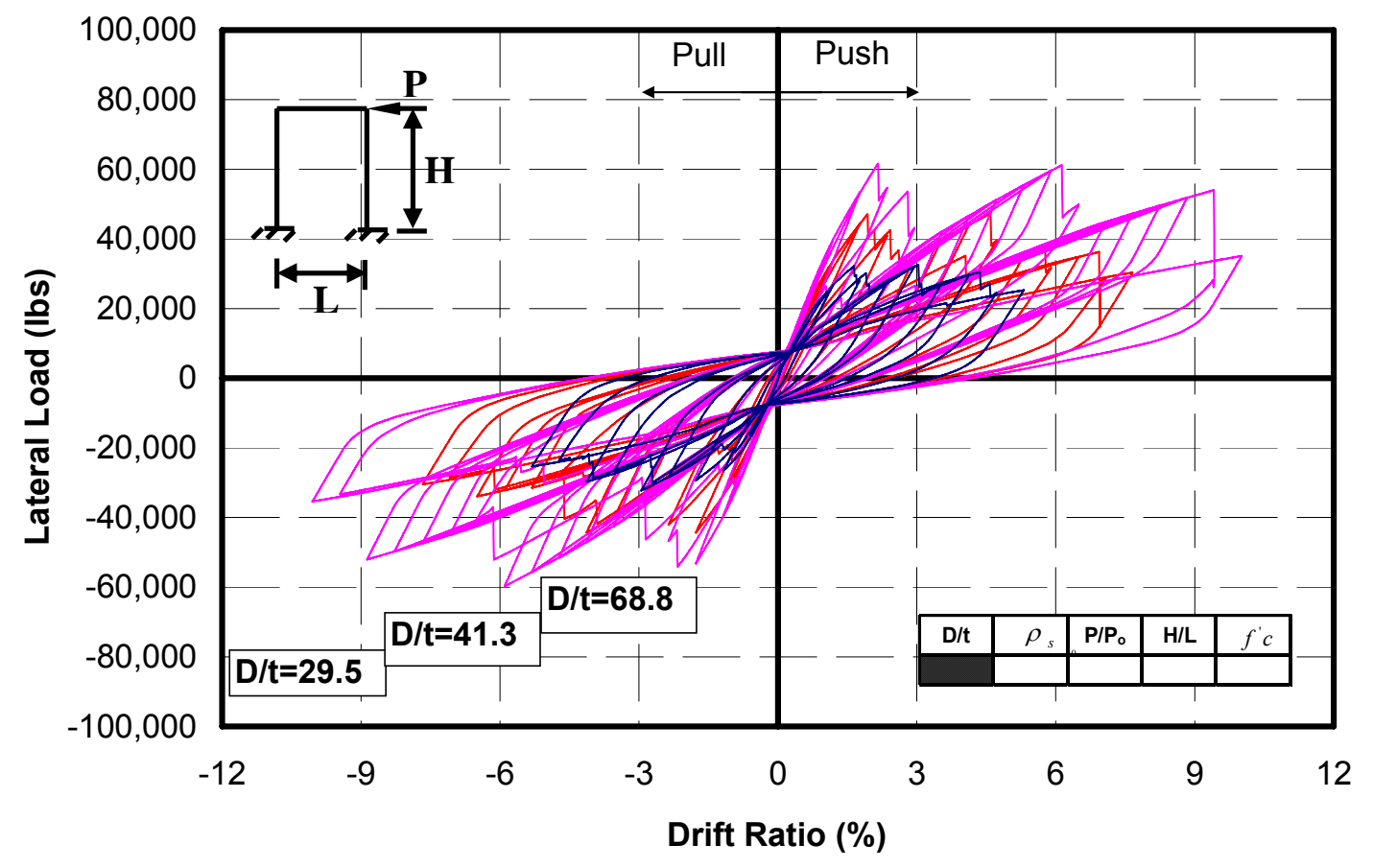

Fig. 4.33 Effect of D/t Ratio on Hysteretic Response of Frame HFF

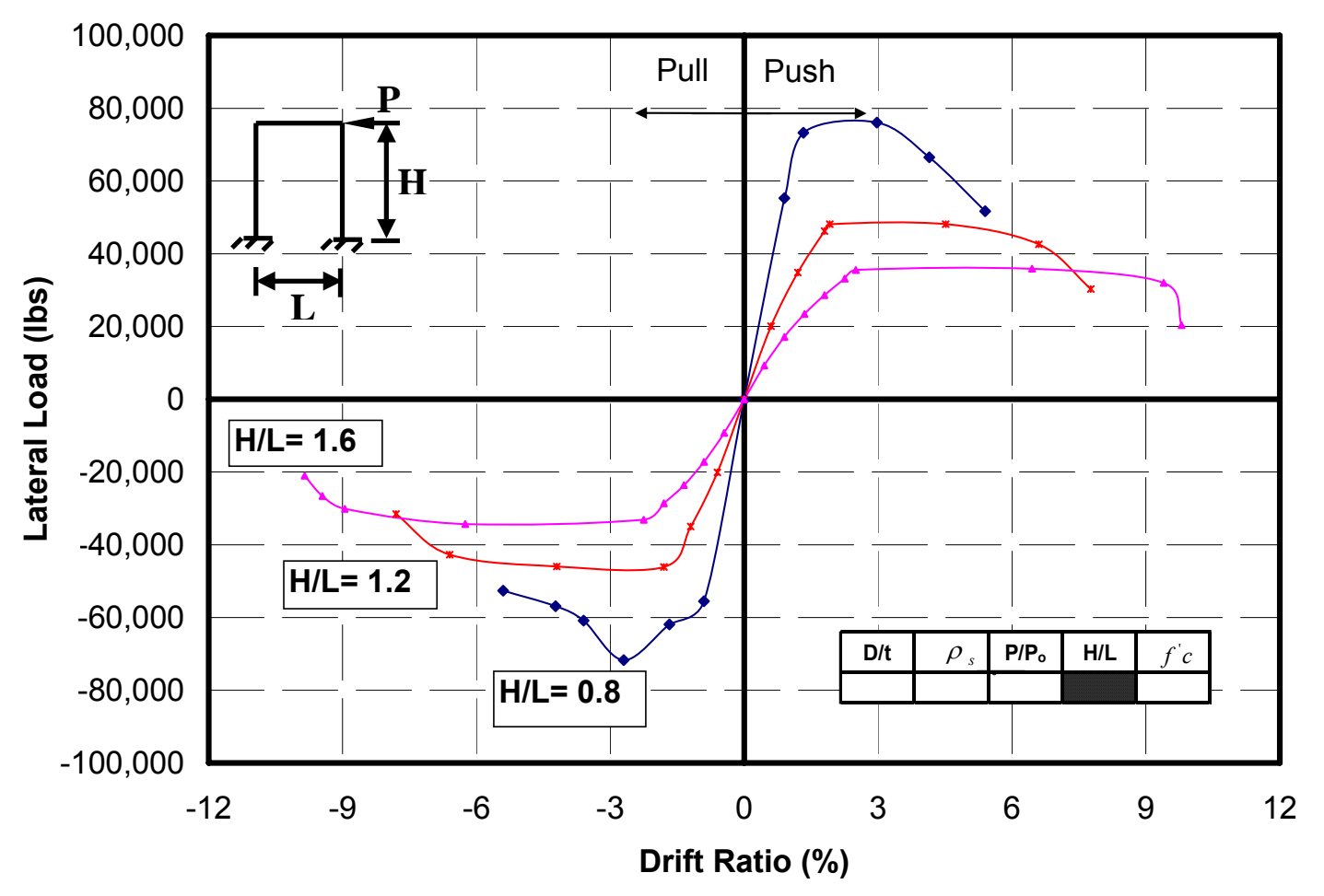

Fig. 4.34 Effect of H/L Ratio on Response Envelope of Frame HFF 


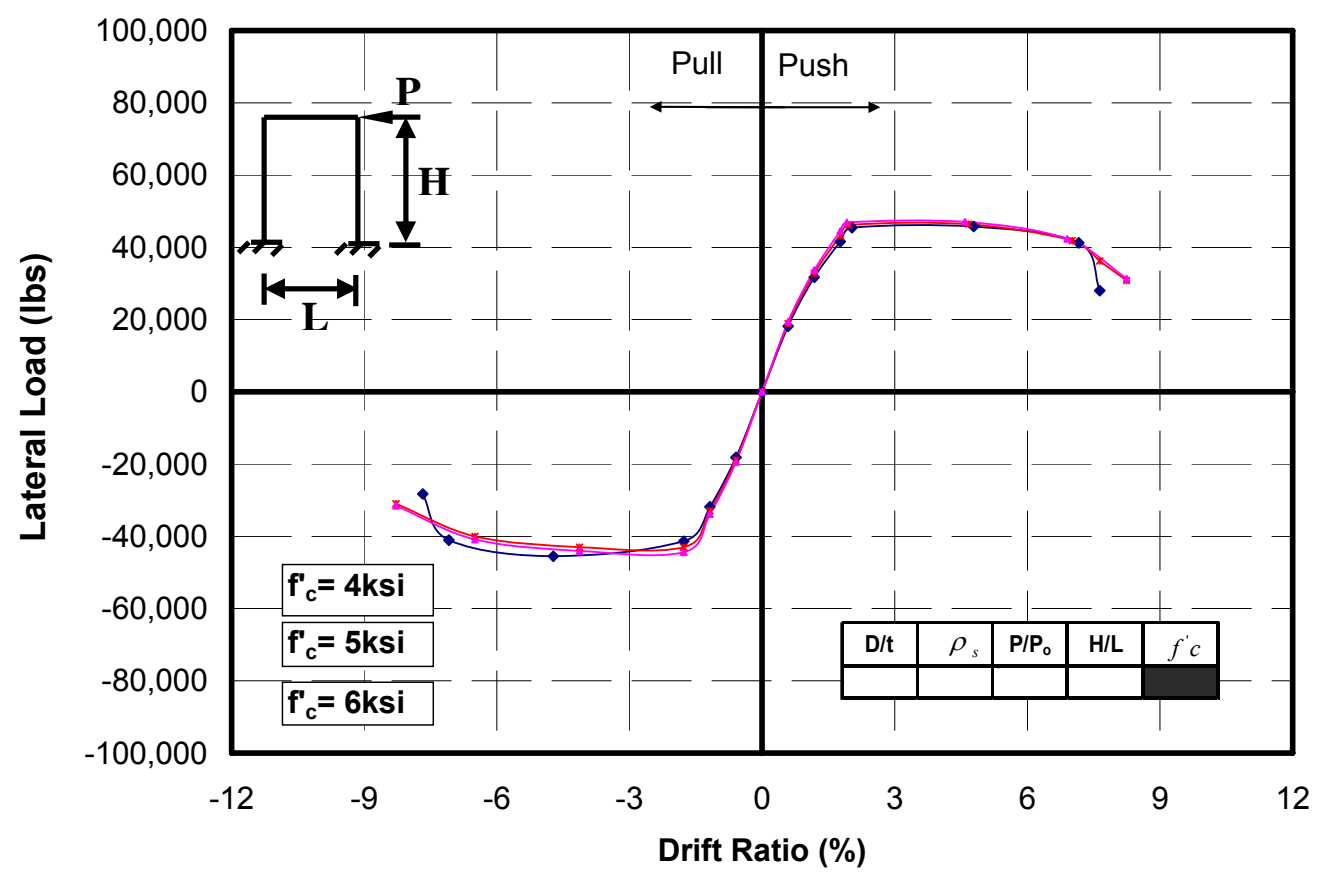

Fig. 4.35 Effect of Concrete Compressive Strength on Response Envelope of Frame HFF

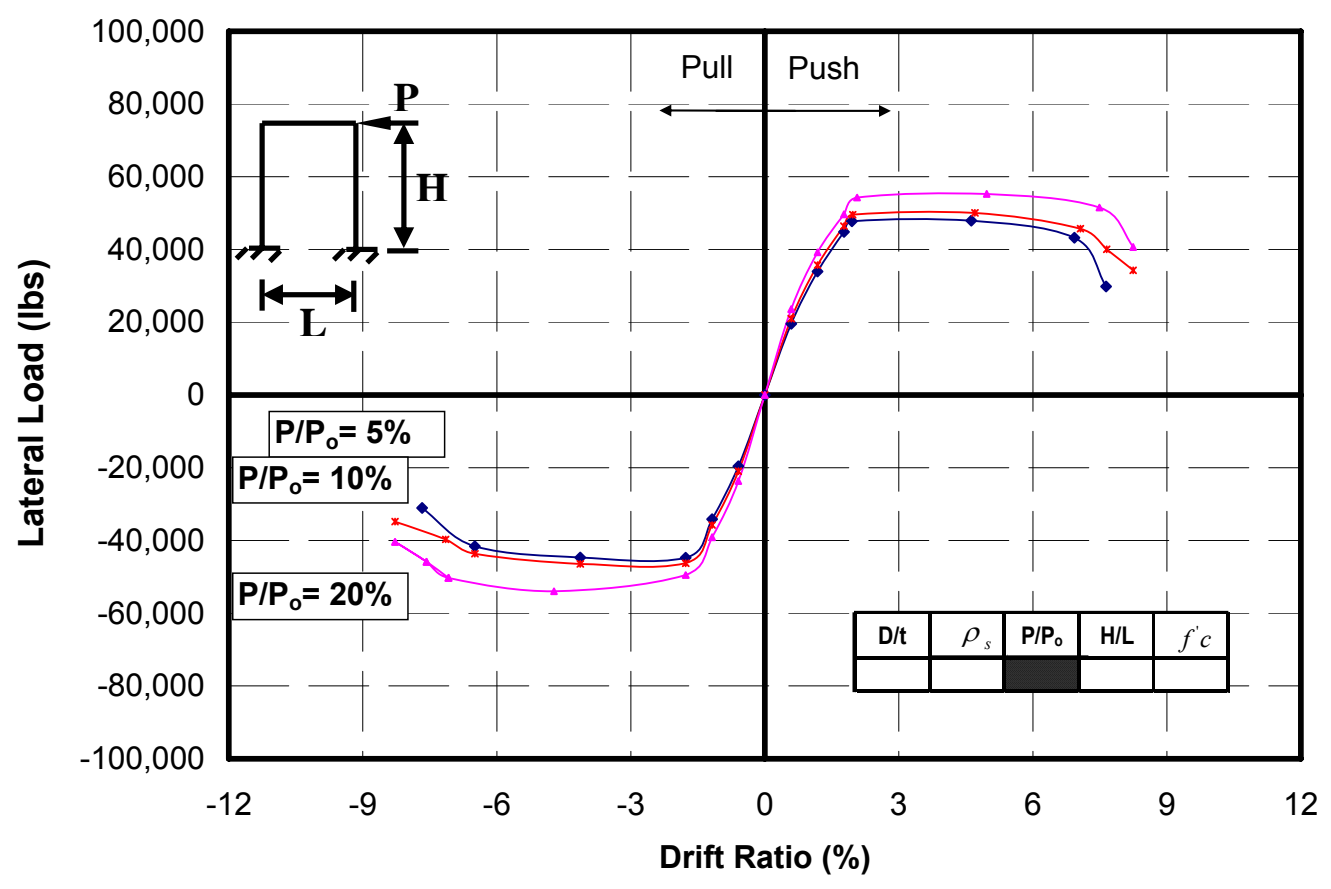

Fig. 4.36 Effect of Axial Load Ratio on Response Envelope of Frame HFF 


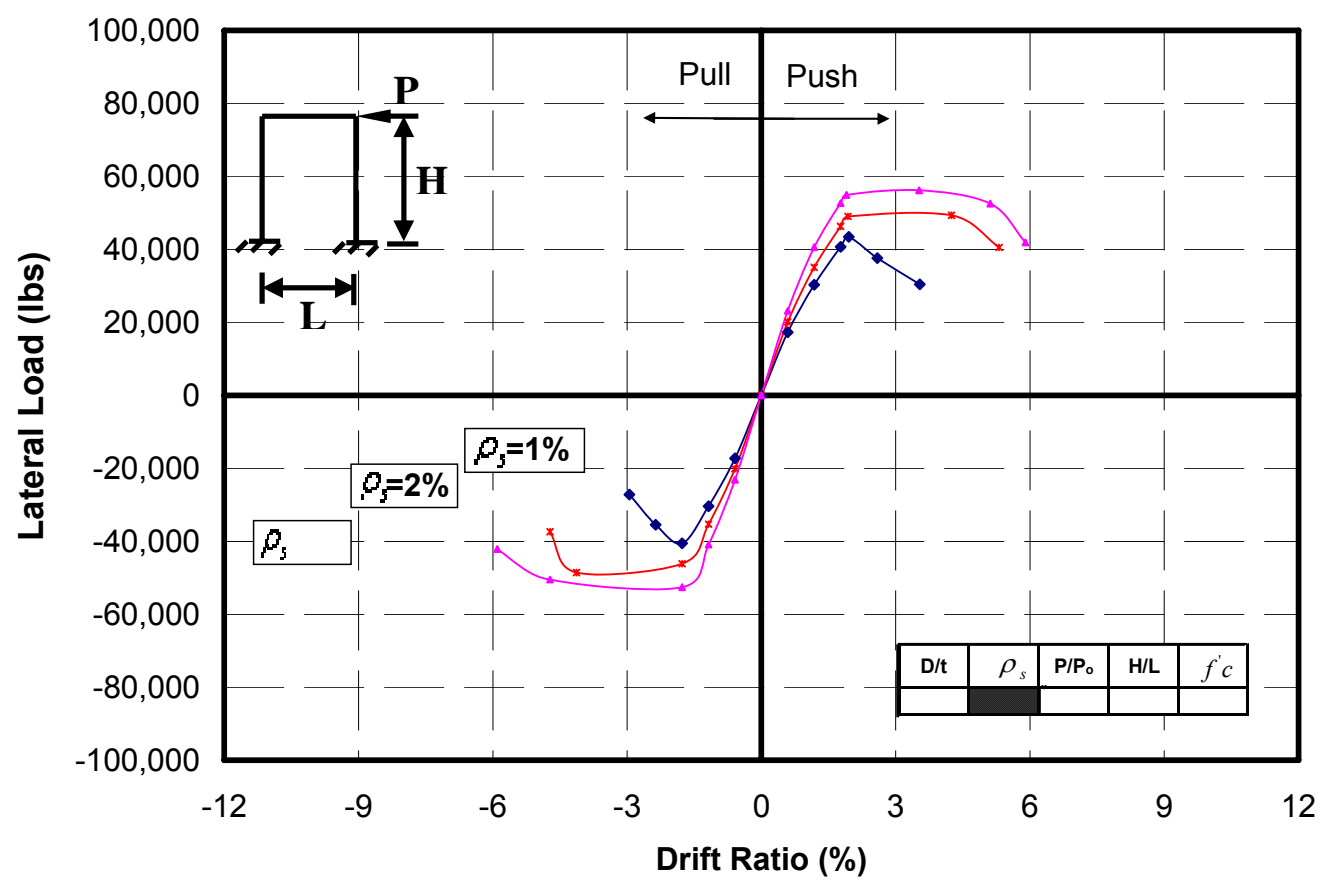

Fig. 4.37 Effect of Steel Reinforcement Ratio on Response Envelope of Frame HFF

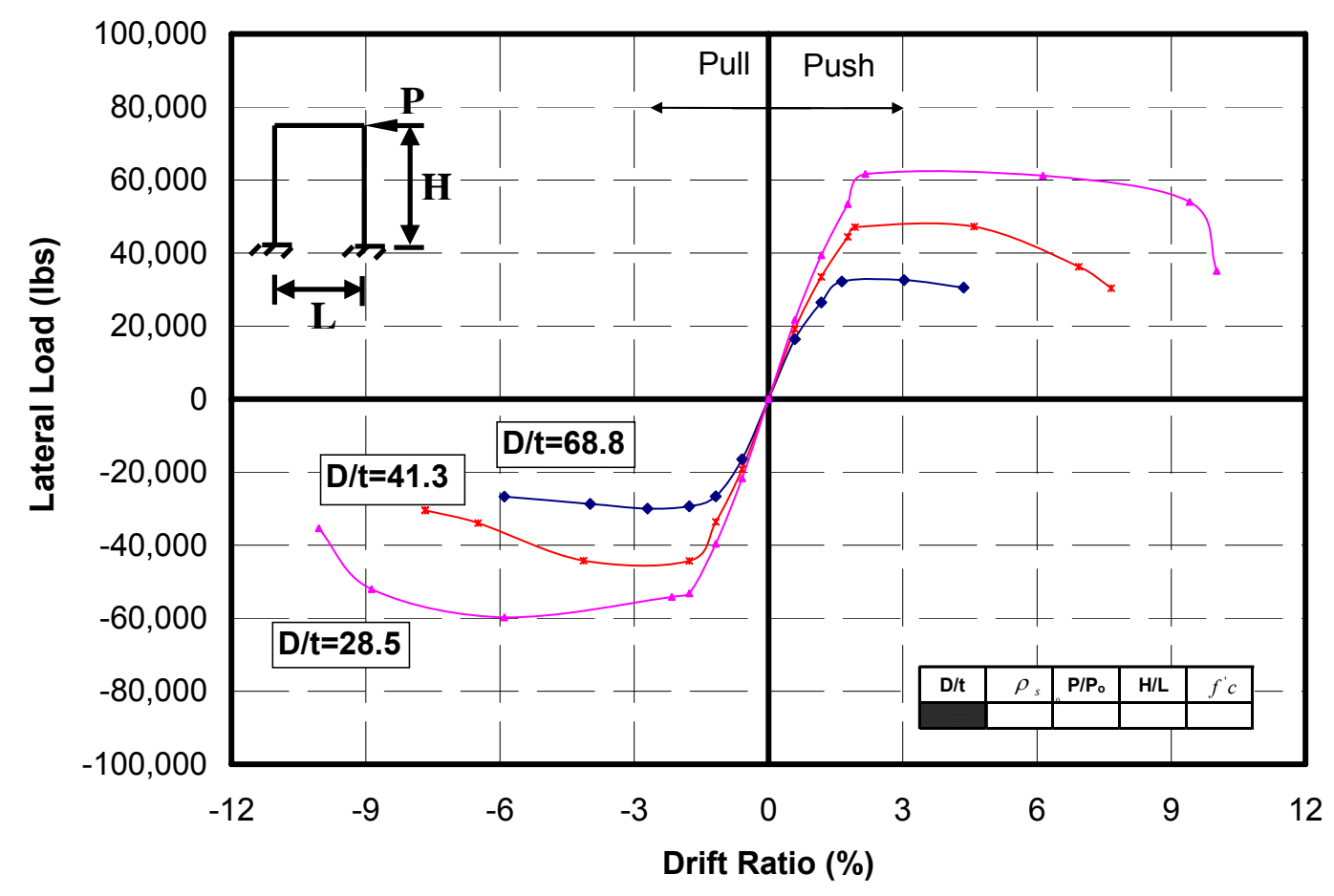

Fig. 4.38 Effect of D/t Ratio on Response Envelope of Frame HFF 


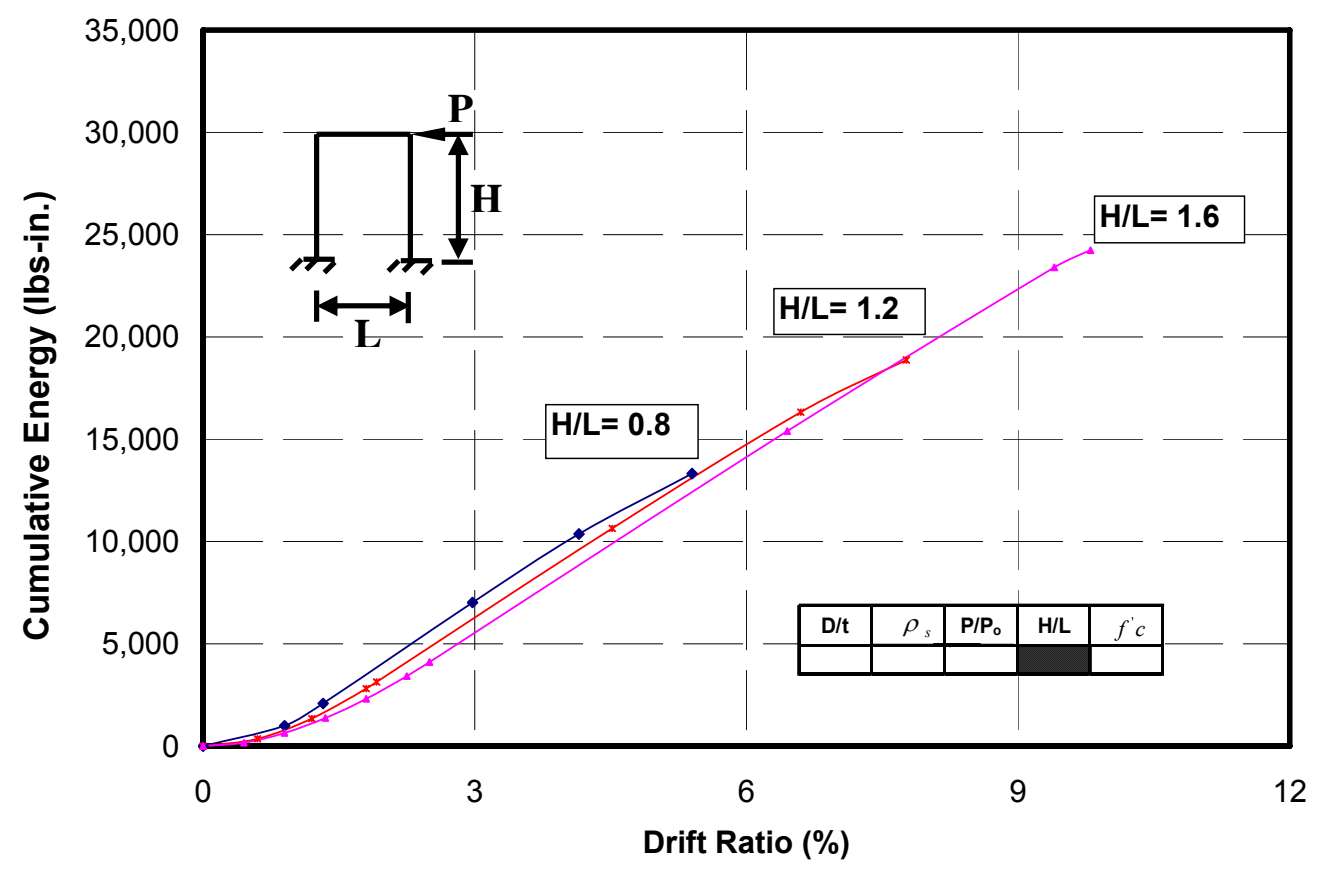

Fig. 4.39 Effect of H/L Ratio on Cumulative Energy of Frame HFF

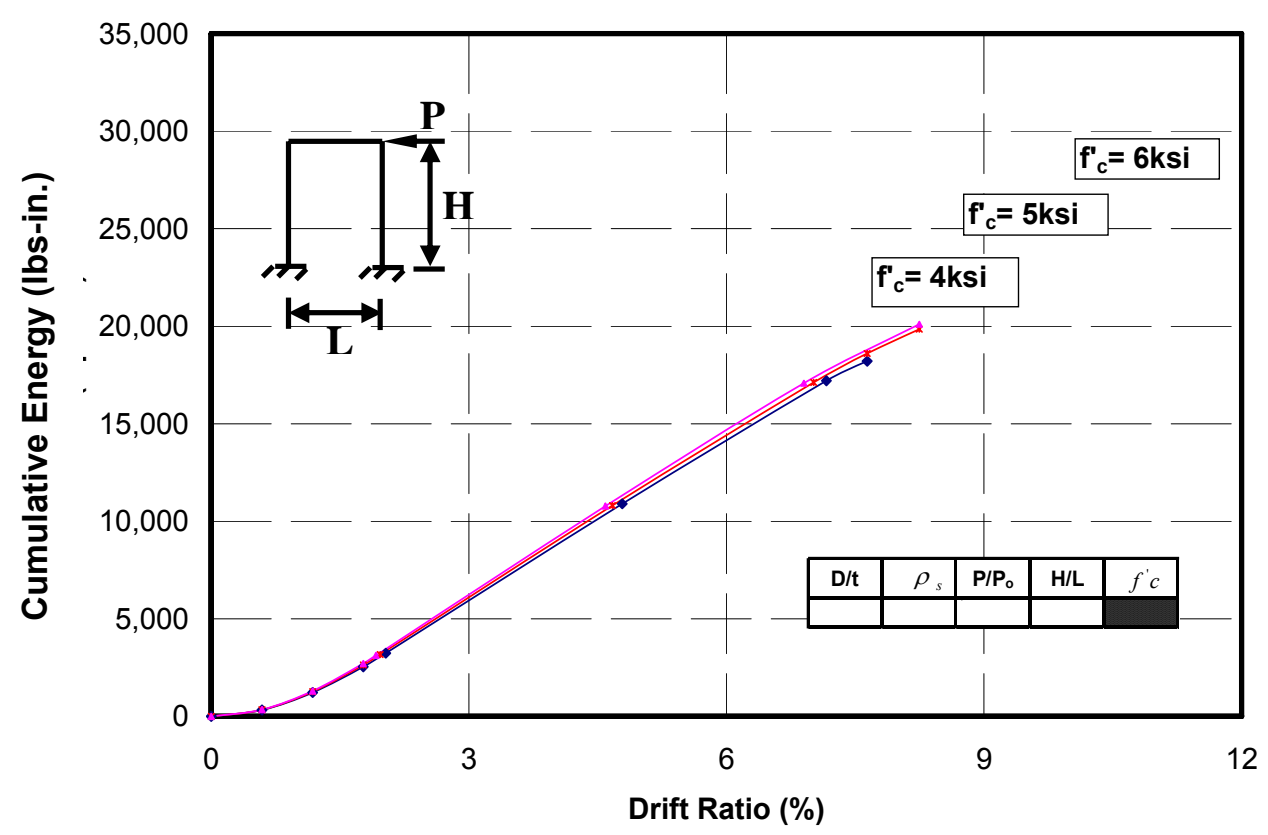

Fig. 4.40 Effect of Concrete Compressive Strength on Cumulative Energy of Frame HFF 


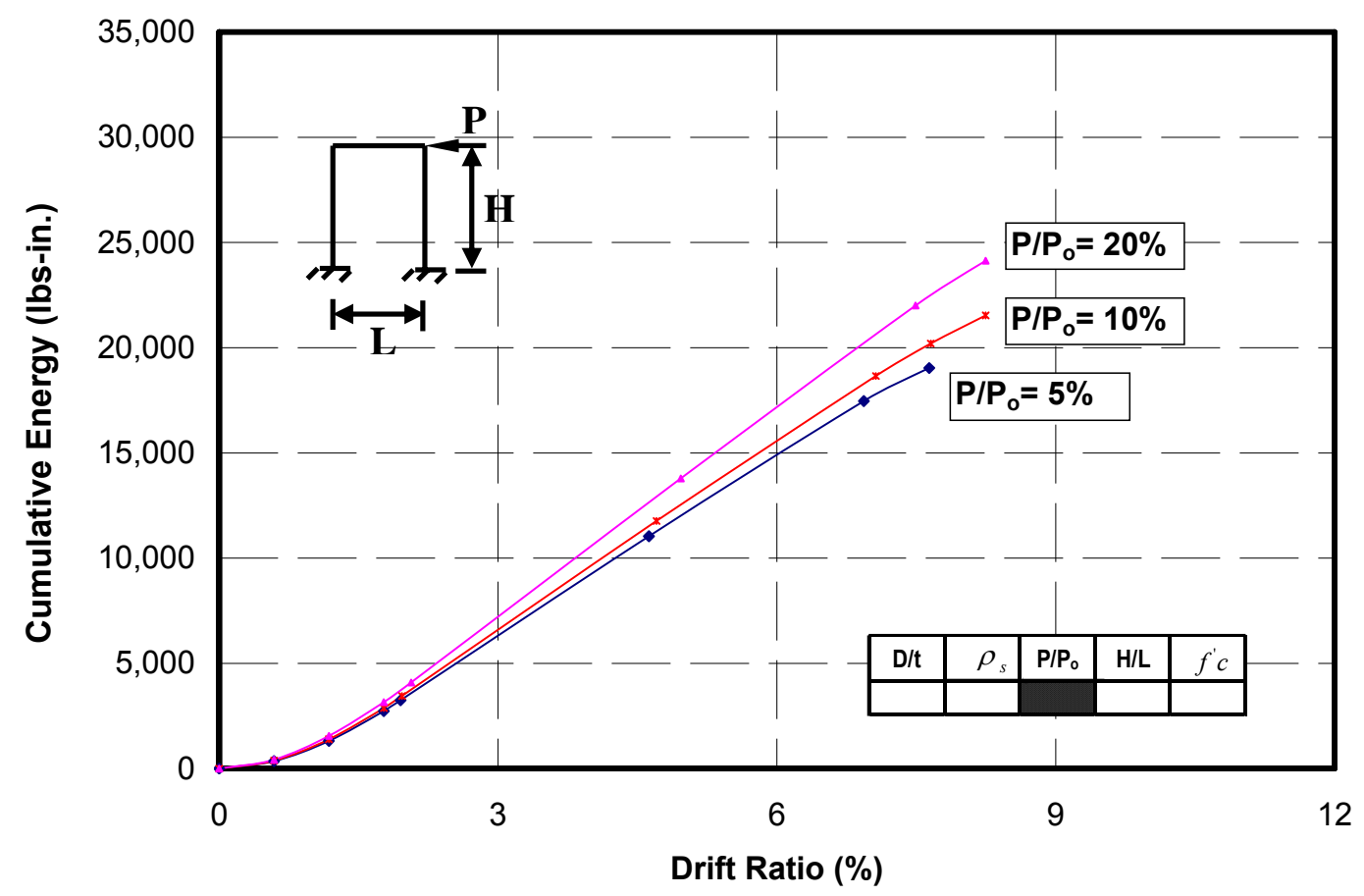

Fig. 4.41 Effect of Axial Load Ratio on Cumulative Energy of Frame HFF

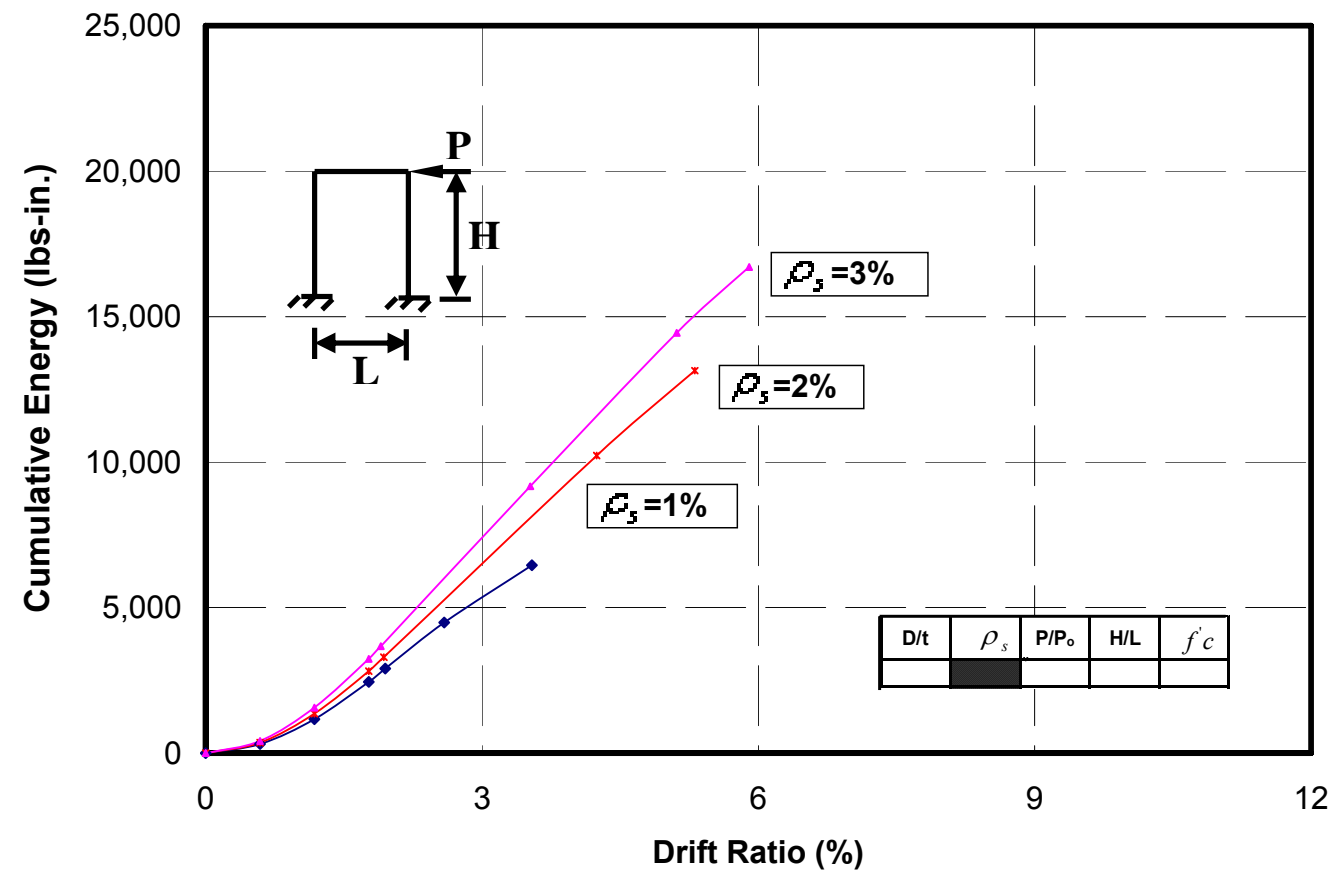

Fig. 4.42 Effect of Steel Reinforcement Ratio on Cumulative Energy of Frame HFF 


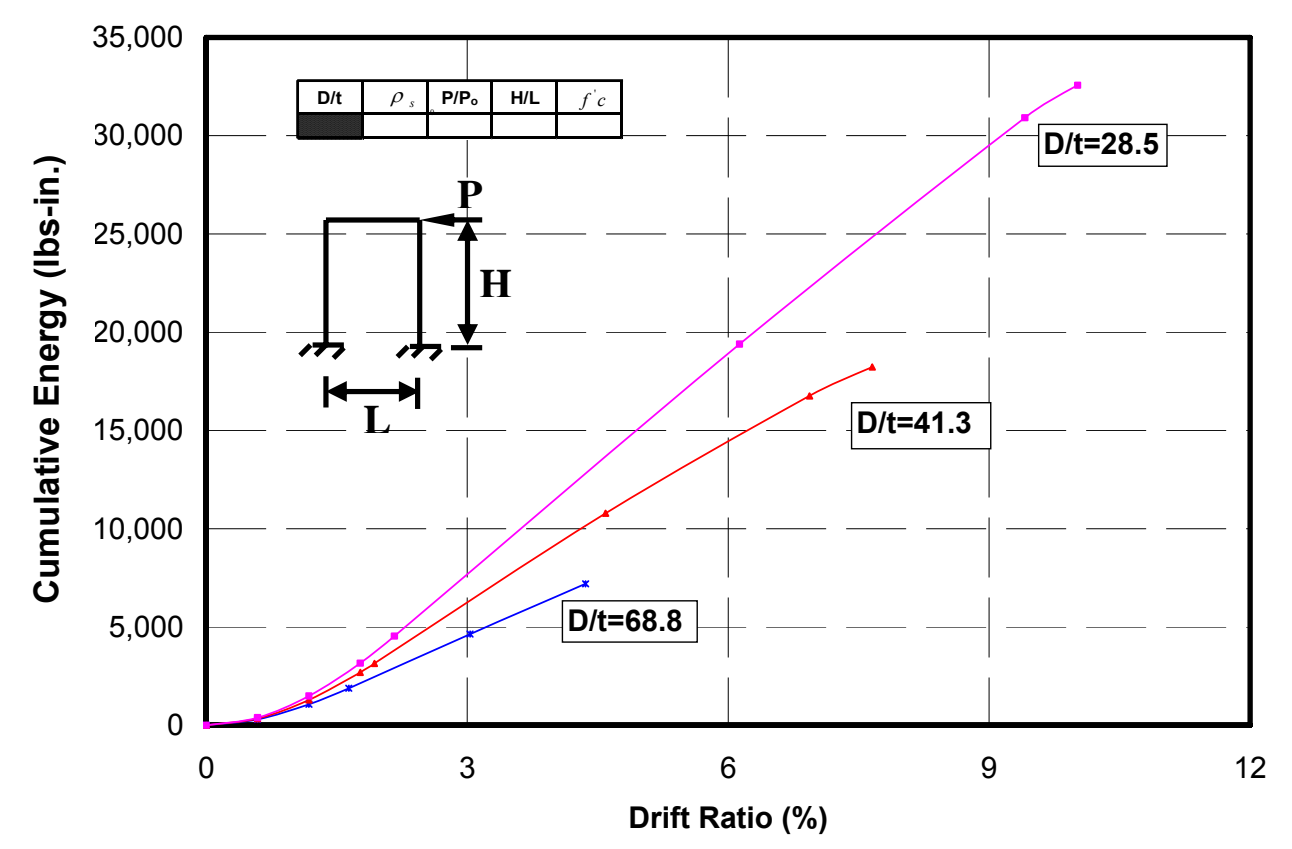

Fig. 4.43 Effect of D/t Ratio on Cumulative Energy of Frame HFF

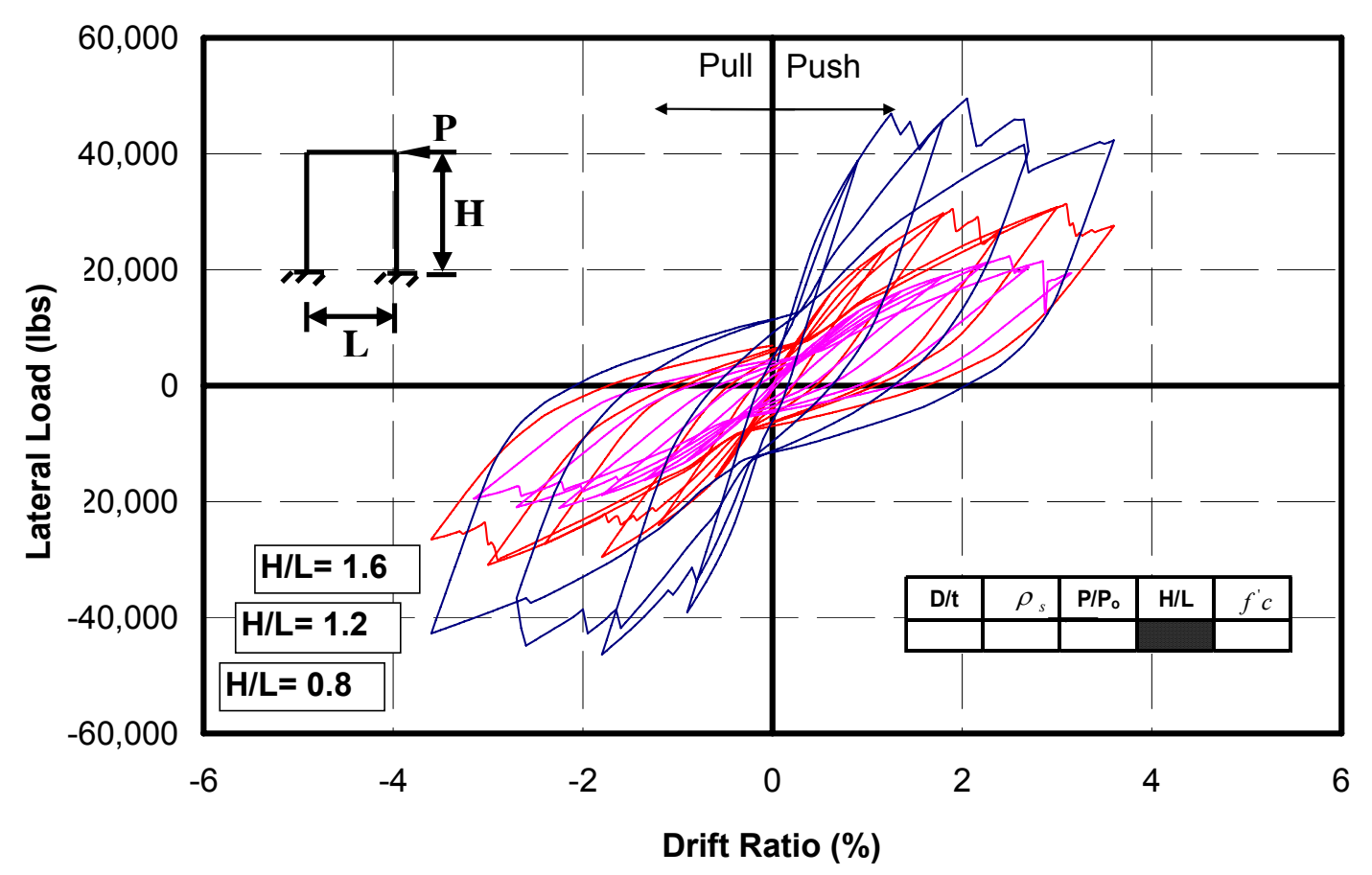

Fig. 4.44 Effect of H/L Ratio on Hysteretic Response of Frame CFF 


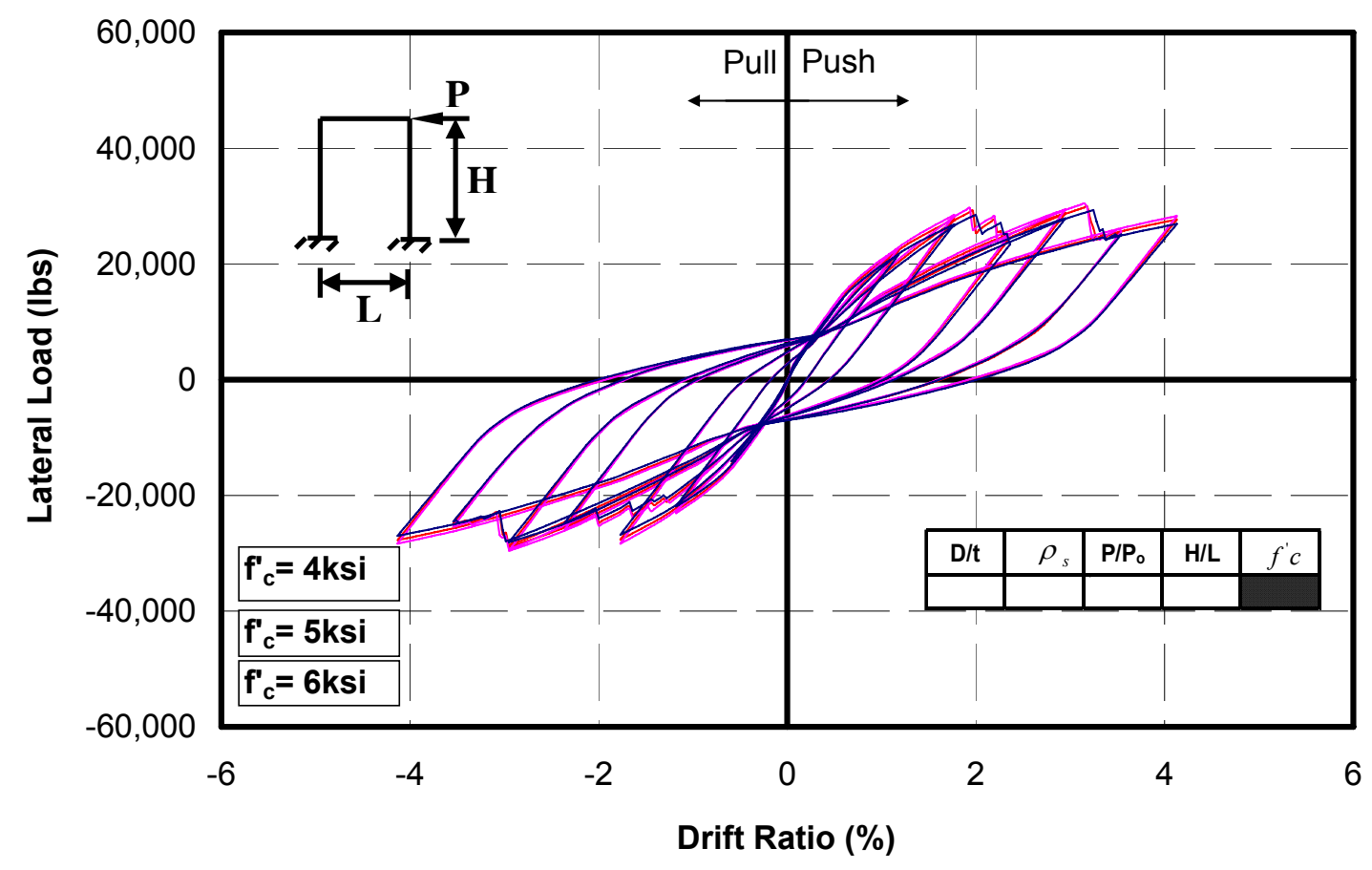

Fig. 4.45 Effect of Concrete Compressive Strength on Hysteretic Response of Frame $\mathrm{CFF}$

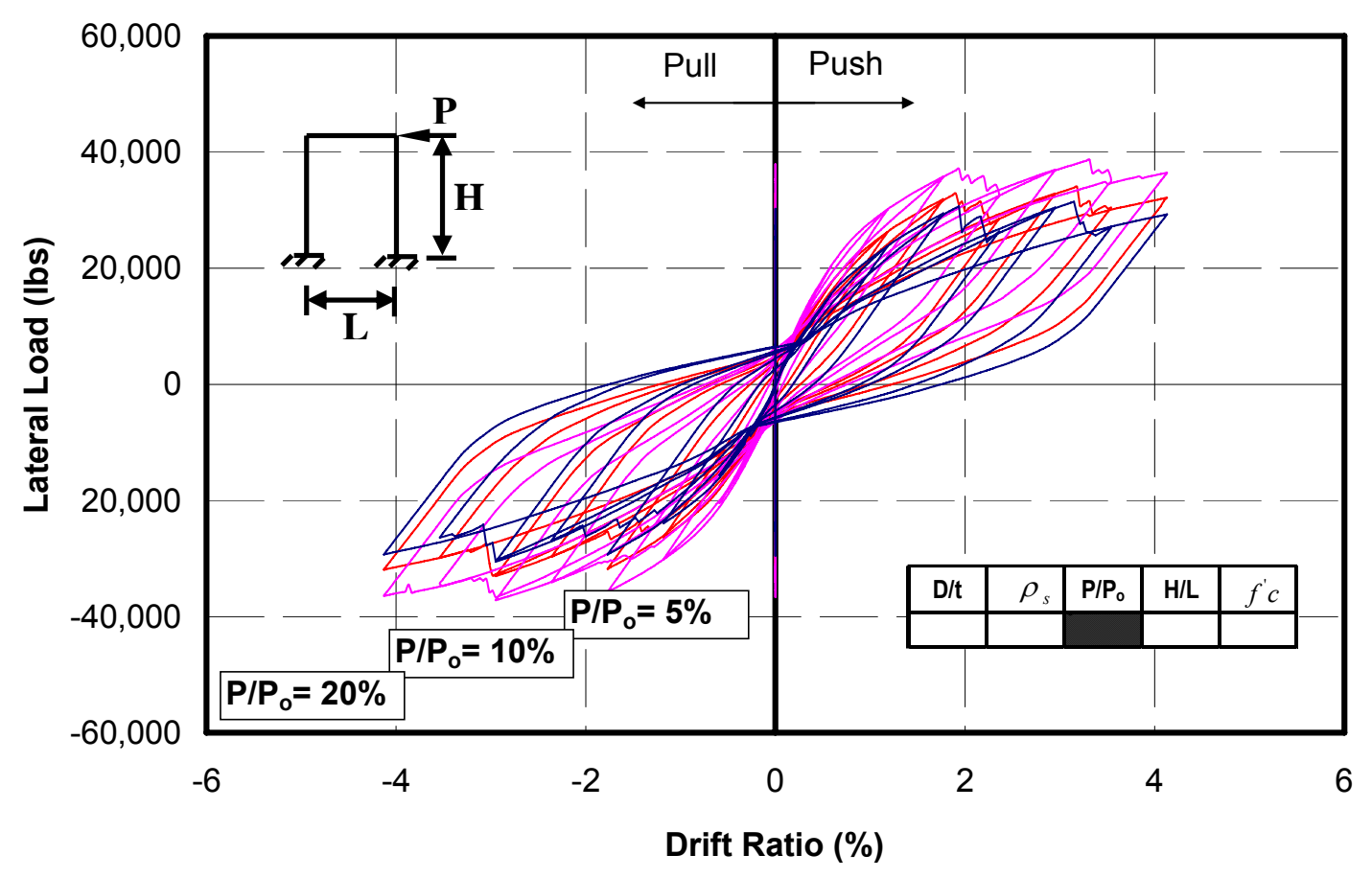

Fig. 4.46 Effect of Axial Load Ratio on Hysteretic Response of Frame CFF 


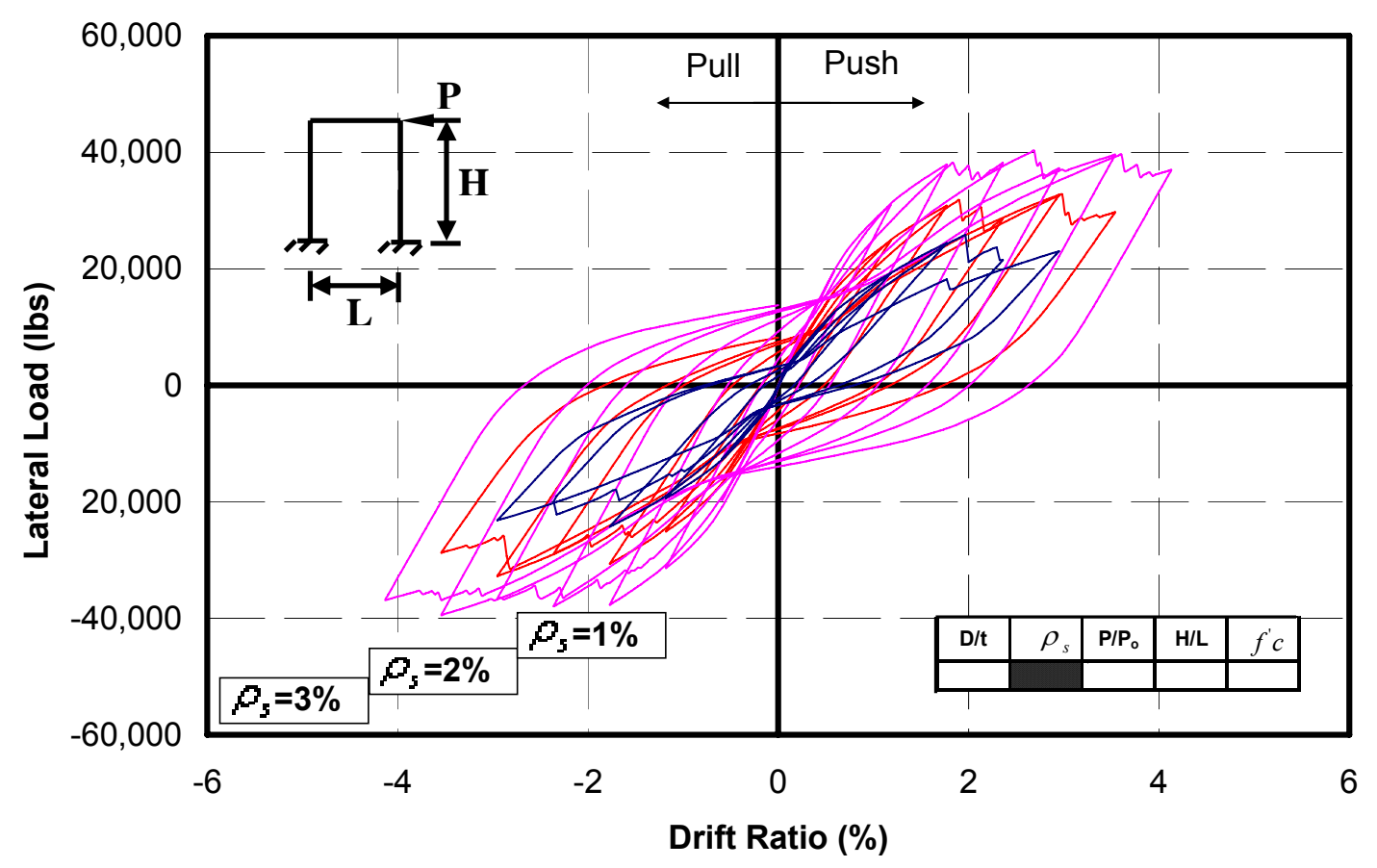

Fig. 4.47 Effect of Steel Reinforcement Ratio on Hysteretic Response of Frame CFF

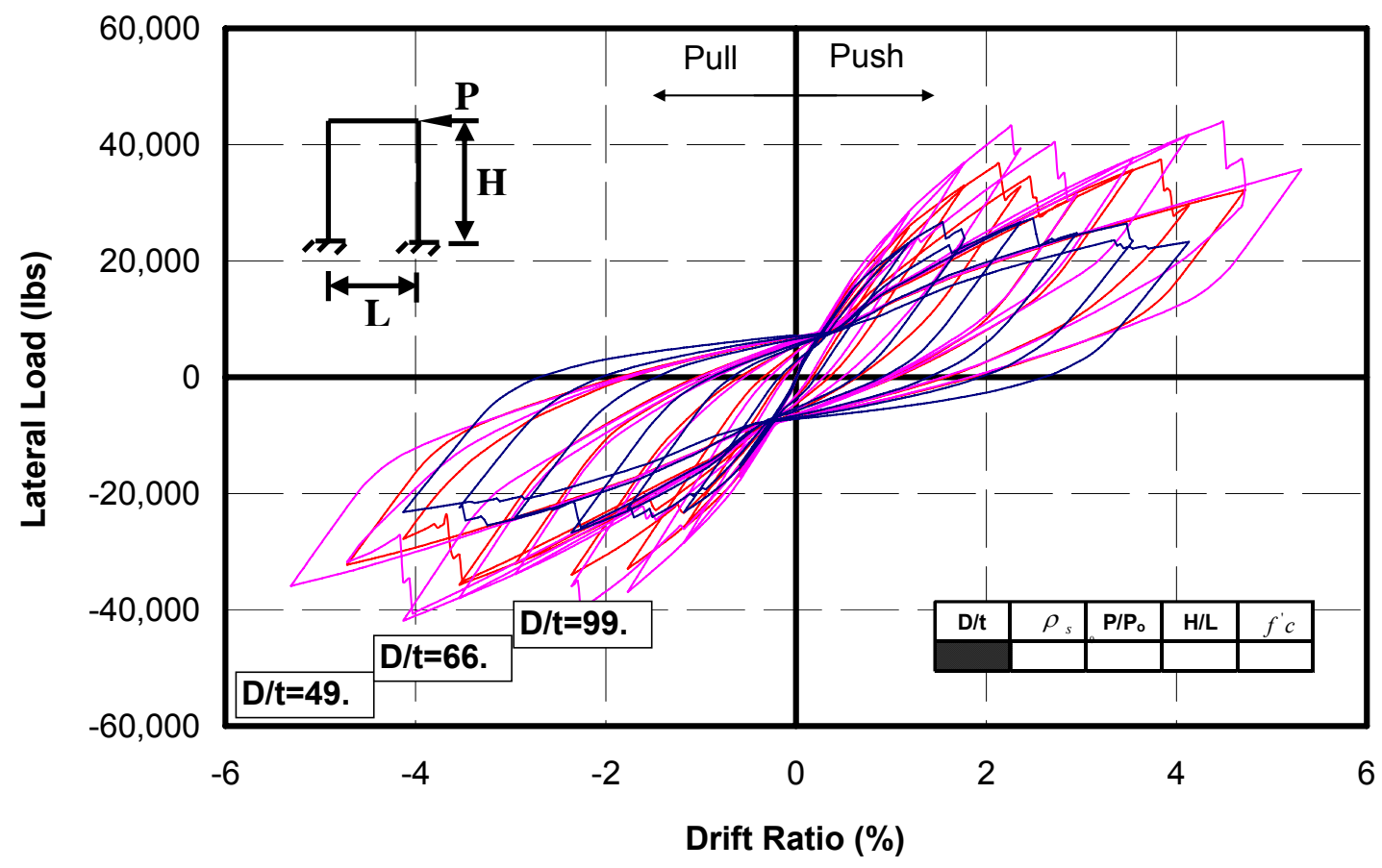

Fig. 4.48 Effect of D/t Ratio on Hysteretic Response of Frame CFF 


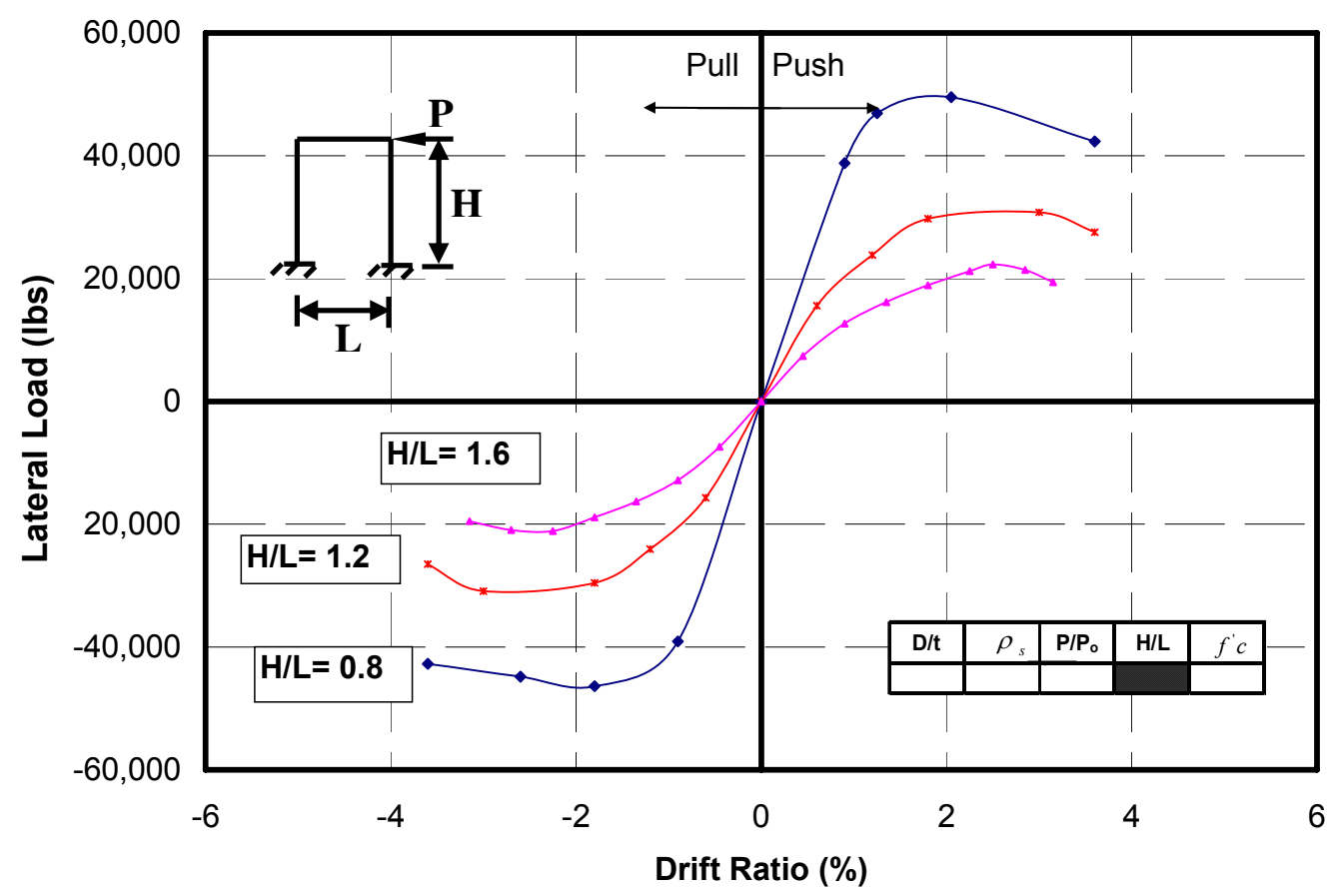

Fig. 4.49 Effect of H/L Ratio on Response Envelope of Frame CFF

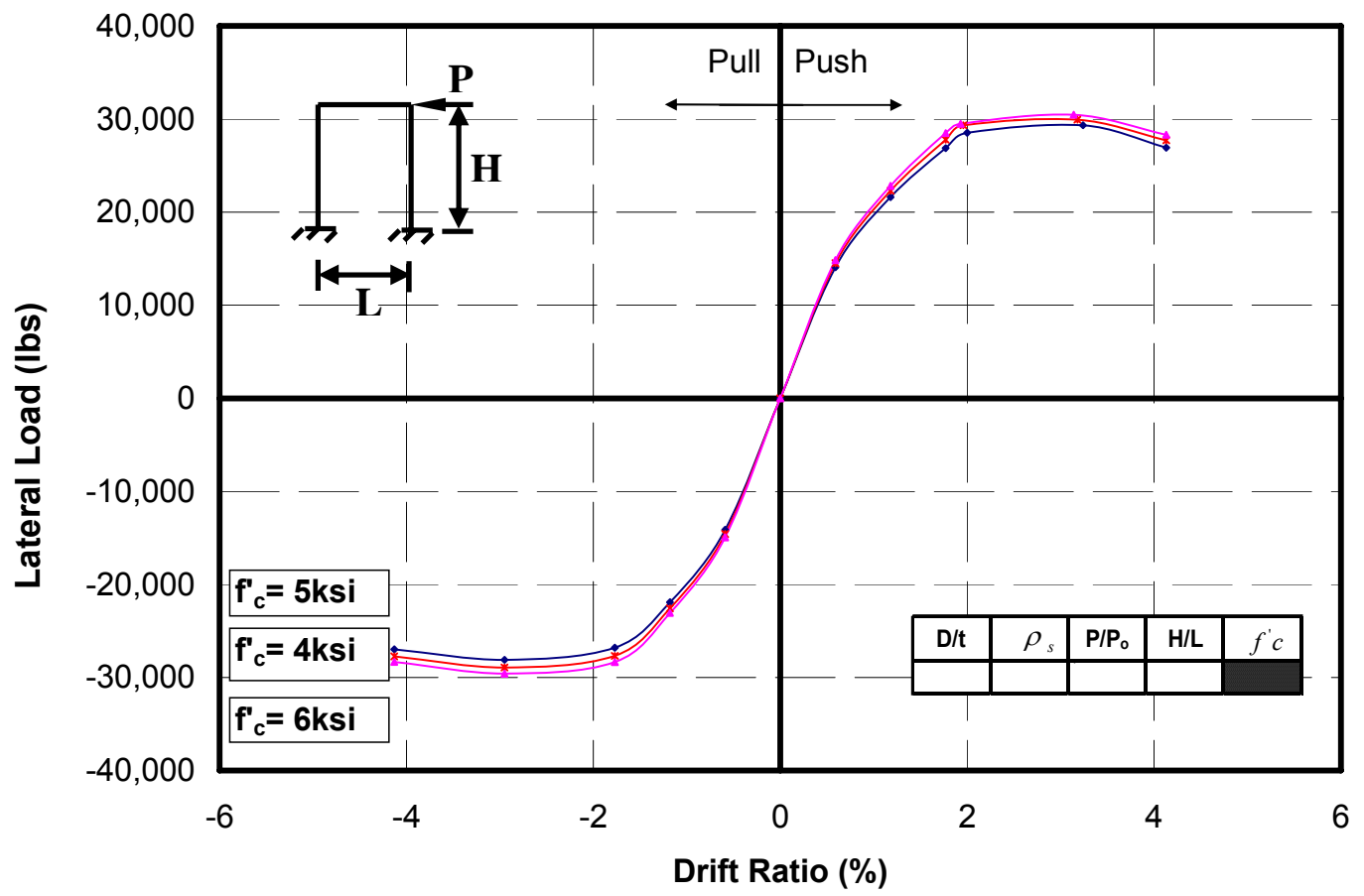

Fig. 4.50 Effect of Concrete Compressive Strength on Response Envelope of Frame $\mathrm{CFF}$ 


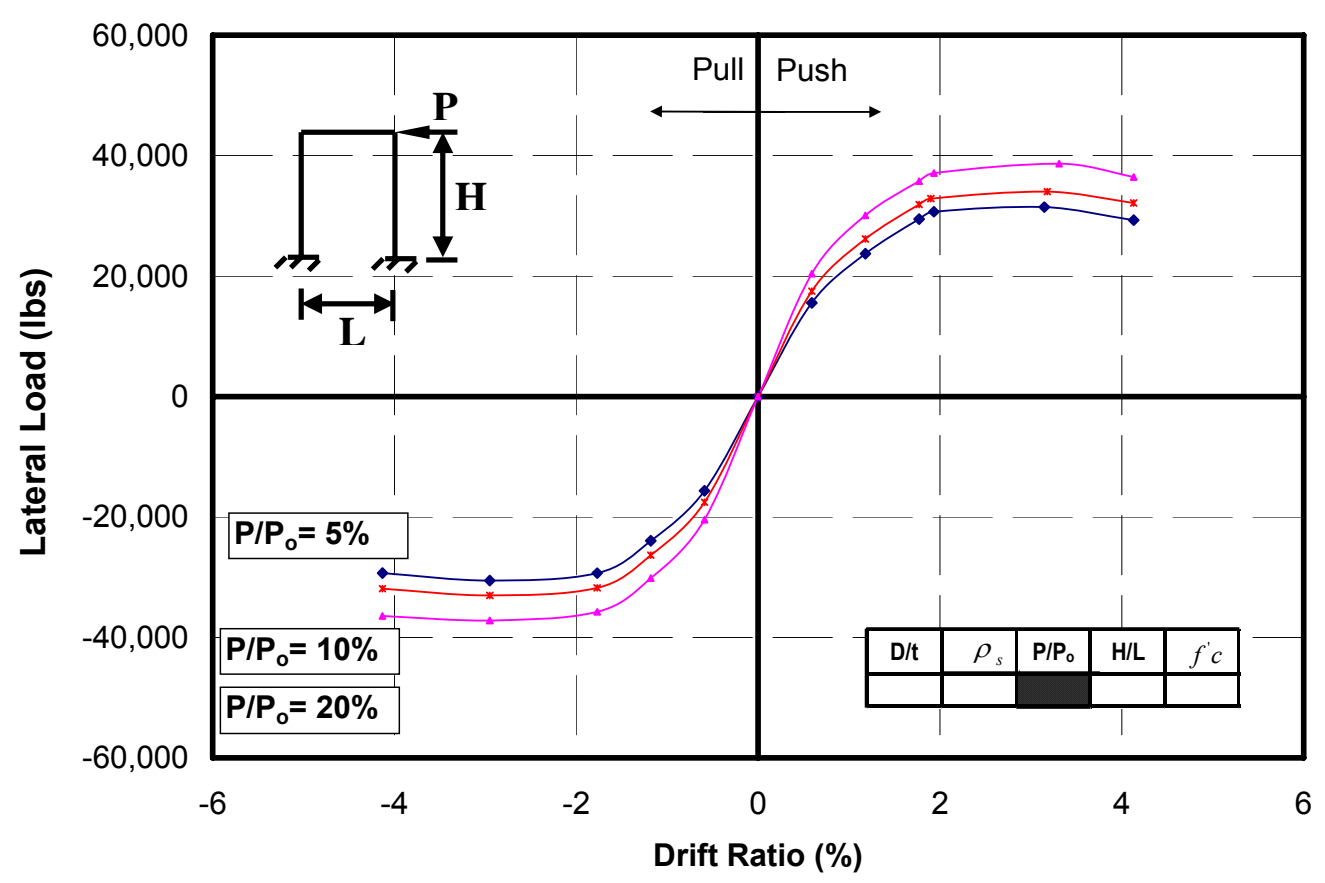

Fig. 4.51 Effect of Axial Load Ratio on Response Envelope of Frame CFF

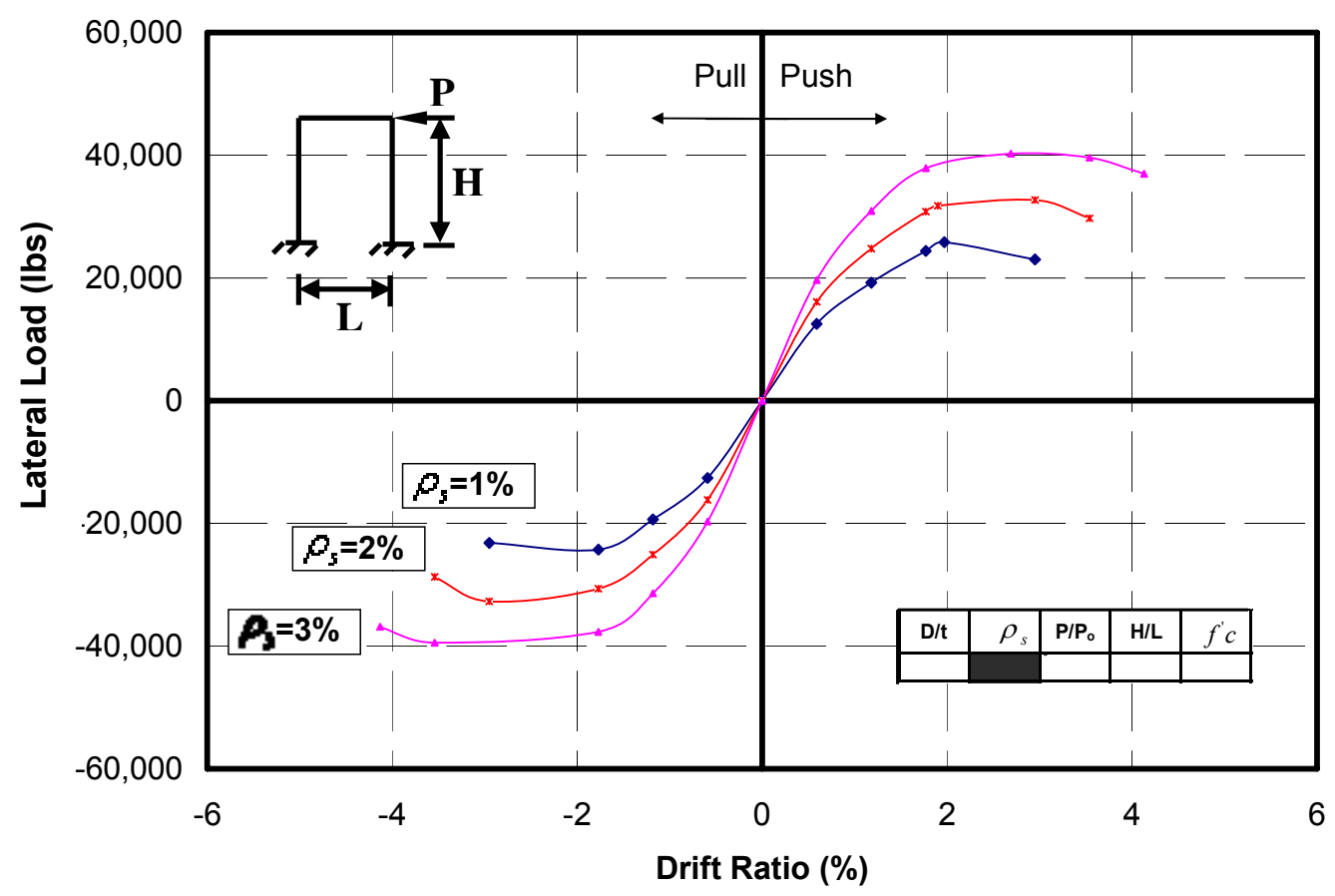

Fig. 4.52 Effect of Steel Reinforcement Ratio on Response Envelope of Frame CFF 


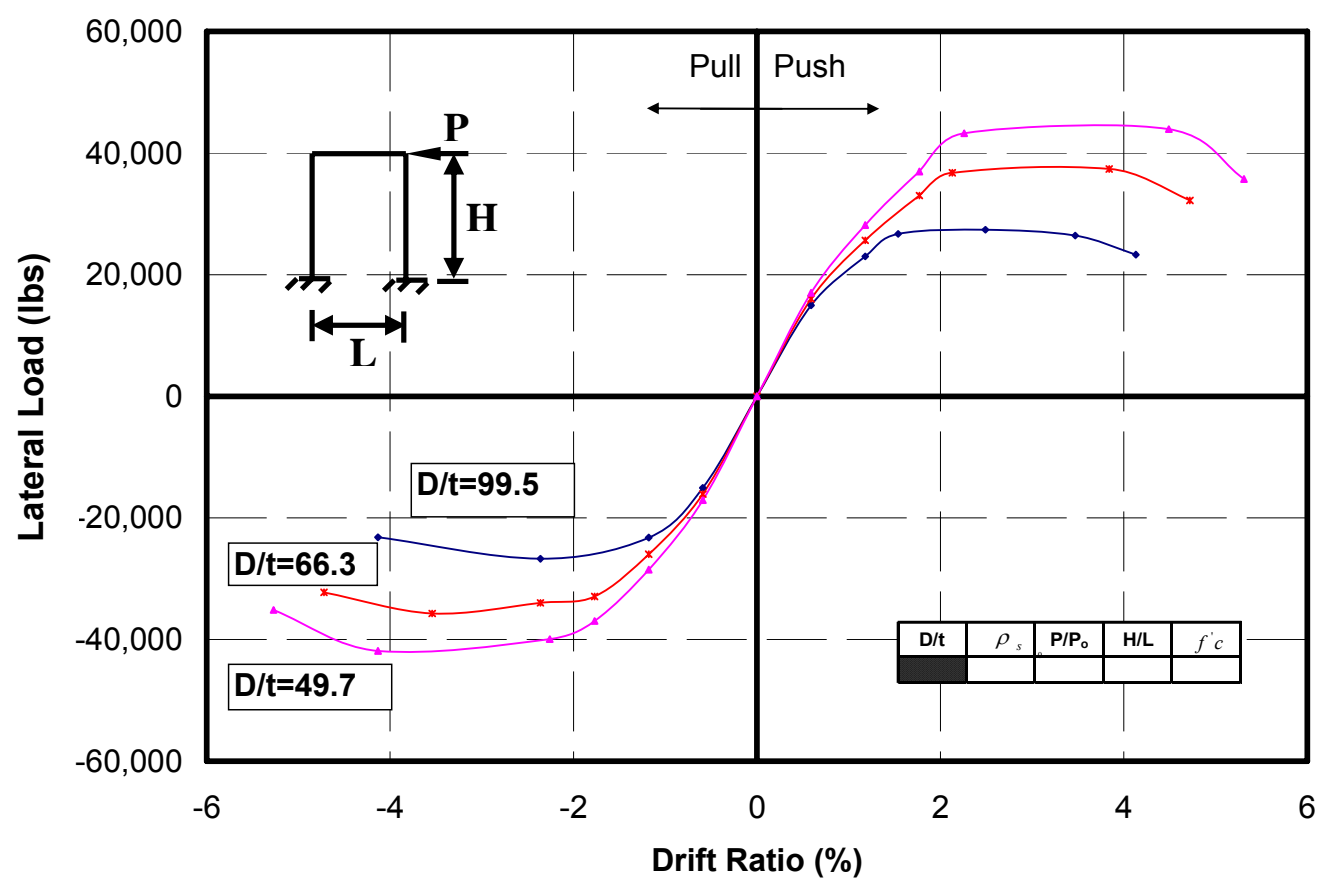

Fig. 4.53 Effect of D/t Ratio on Response Envelope of Frame CFF

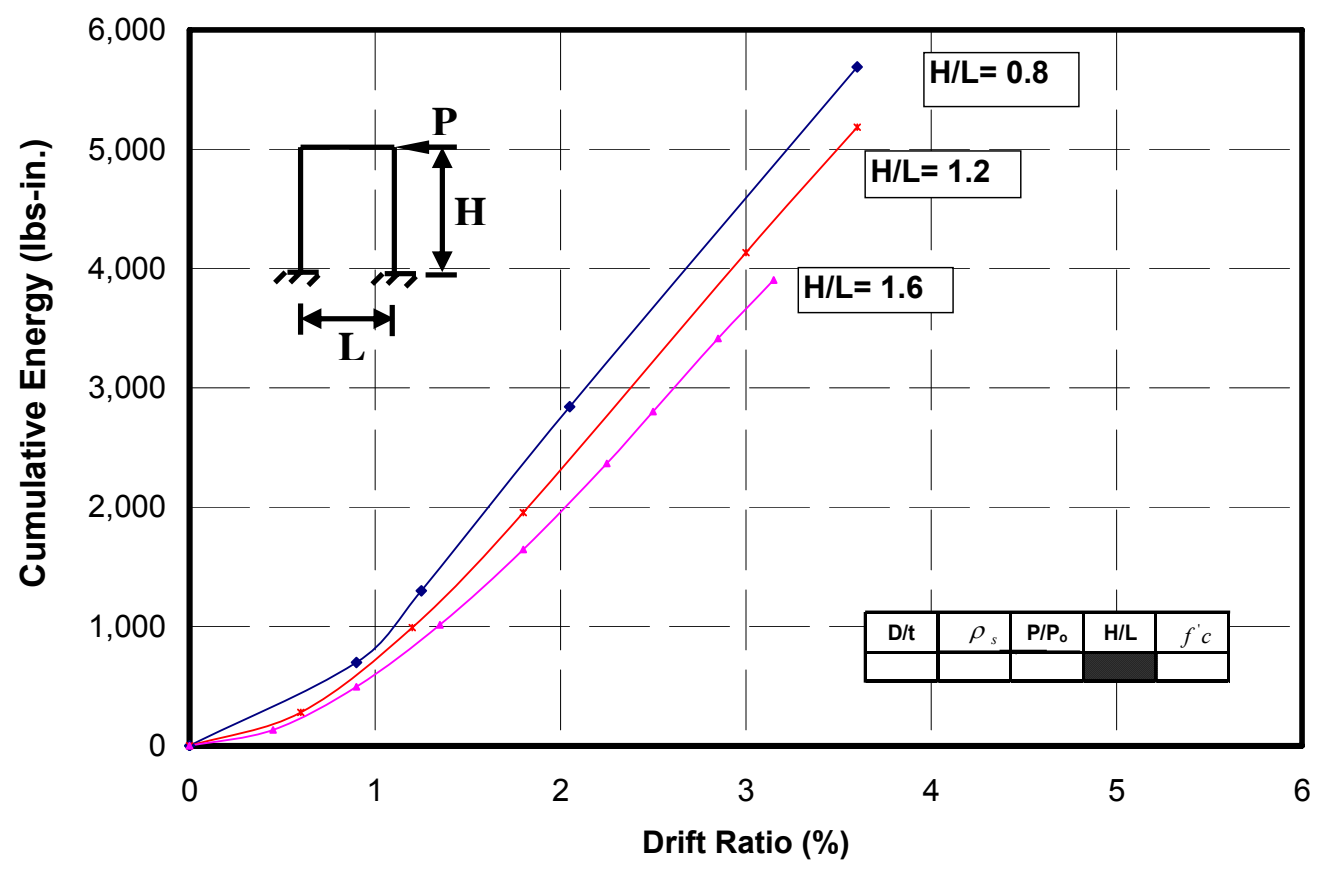

Fig. 4.54 Effect of H/L Ratio on Cumulative Energy of Frame CFF 


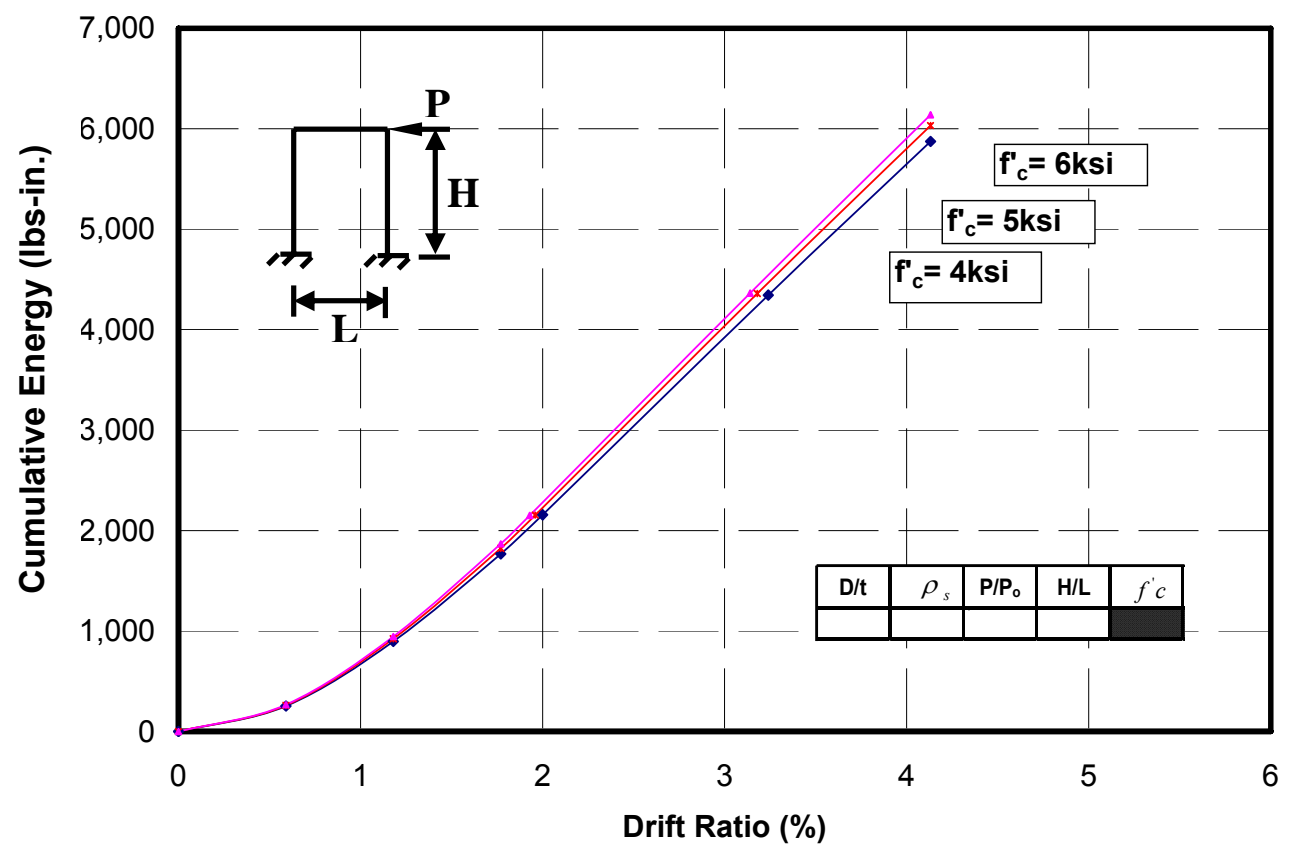

Fig. 4.55 Effect of Concrete Compressive Strength on Cumulative Energy of Frame $\mathrm{CFF}$

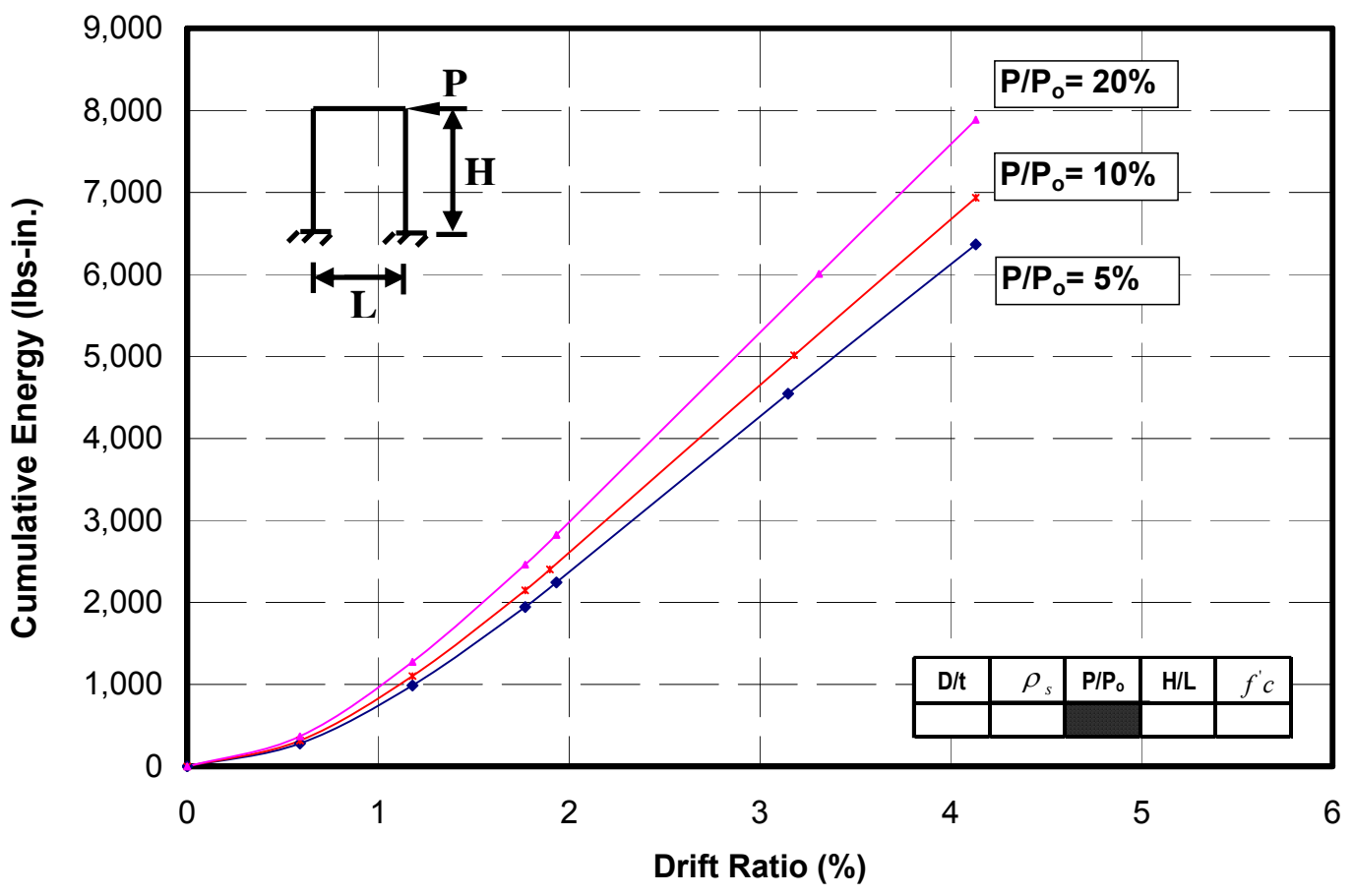

Fig. 4.56 Effect of Axial Load Ratio on Cumulative Energy of Frame CFF 


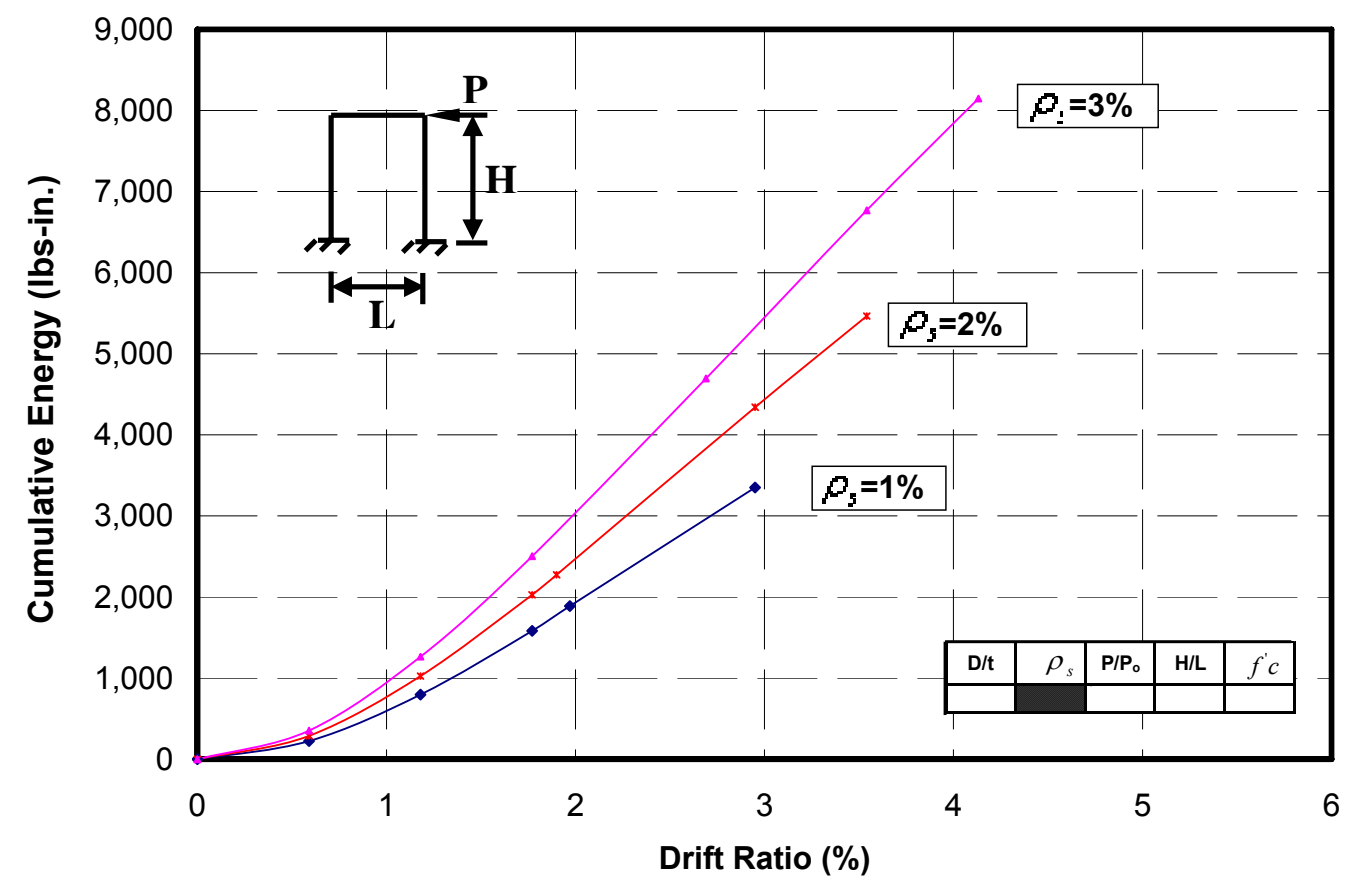

Fig. 4.57 Effect of Steel Reinforcement Ratio on Cumulative Energy of Frame CFF

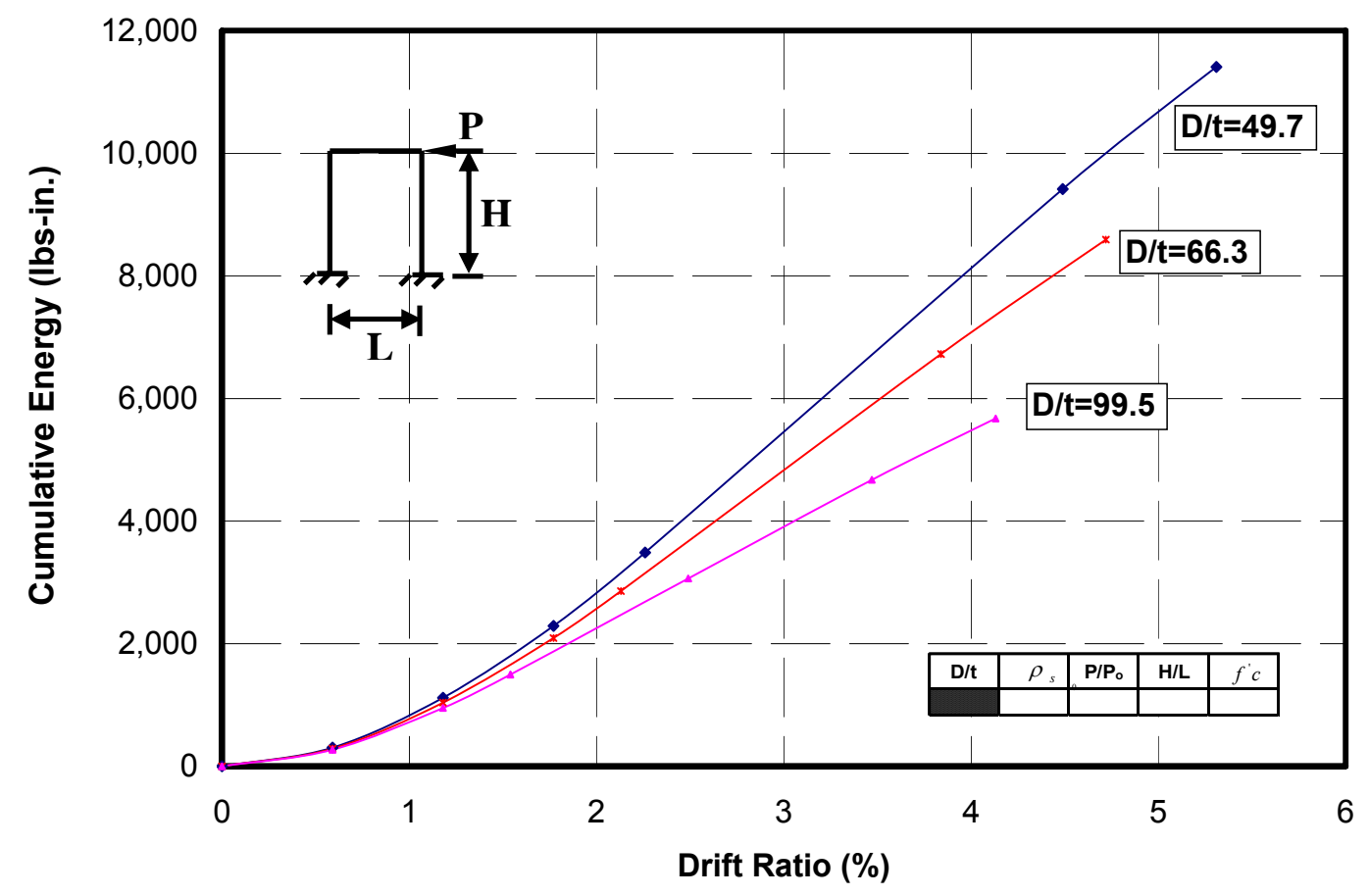

Fig. 4.58 Effect of D/t Ratio on Cumulative Energy of Frame CFF 


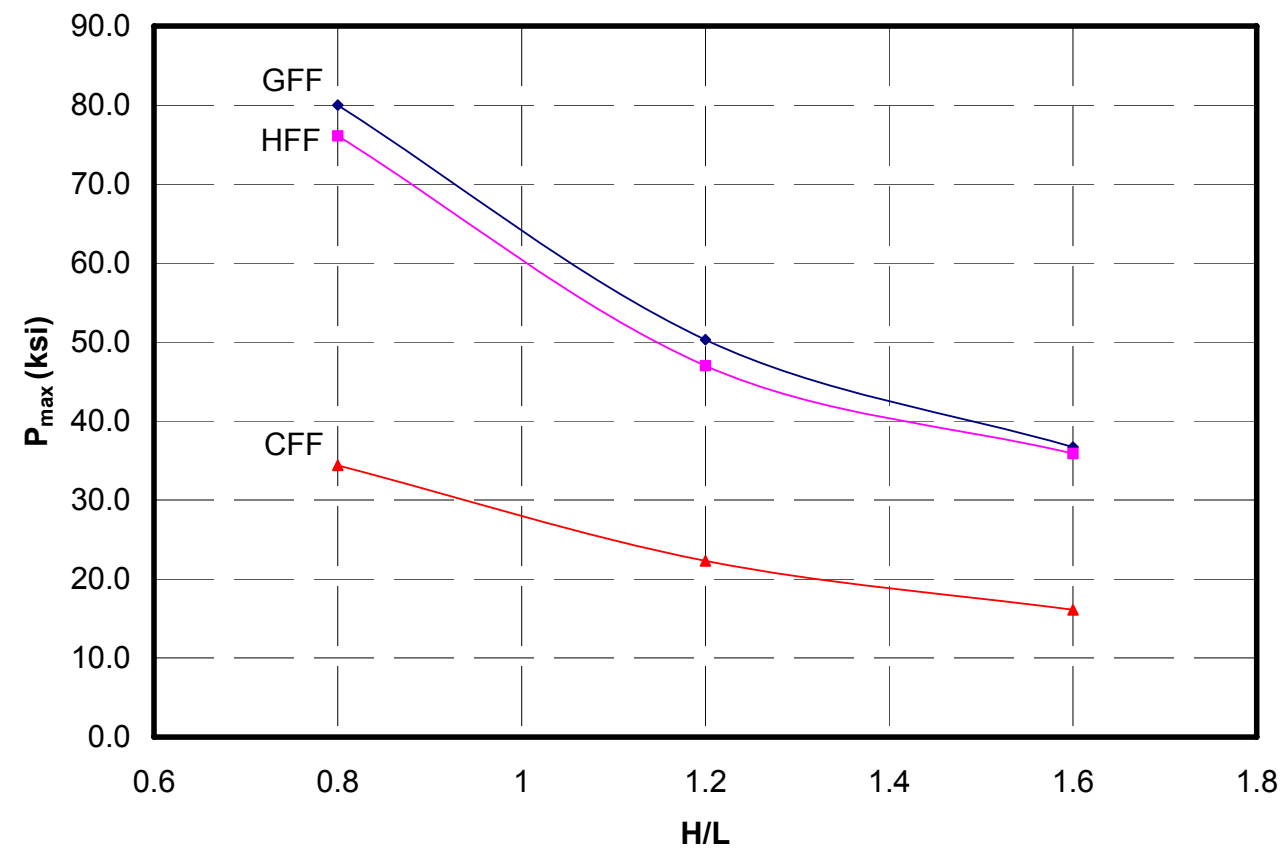

Fig. 4.59 Effect of H/L Ratio on Maximum Load of All Frames

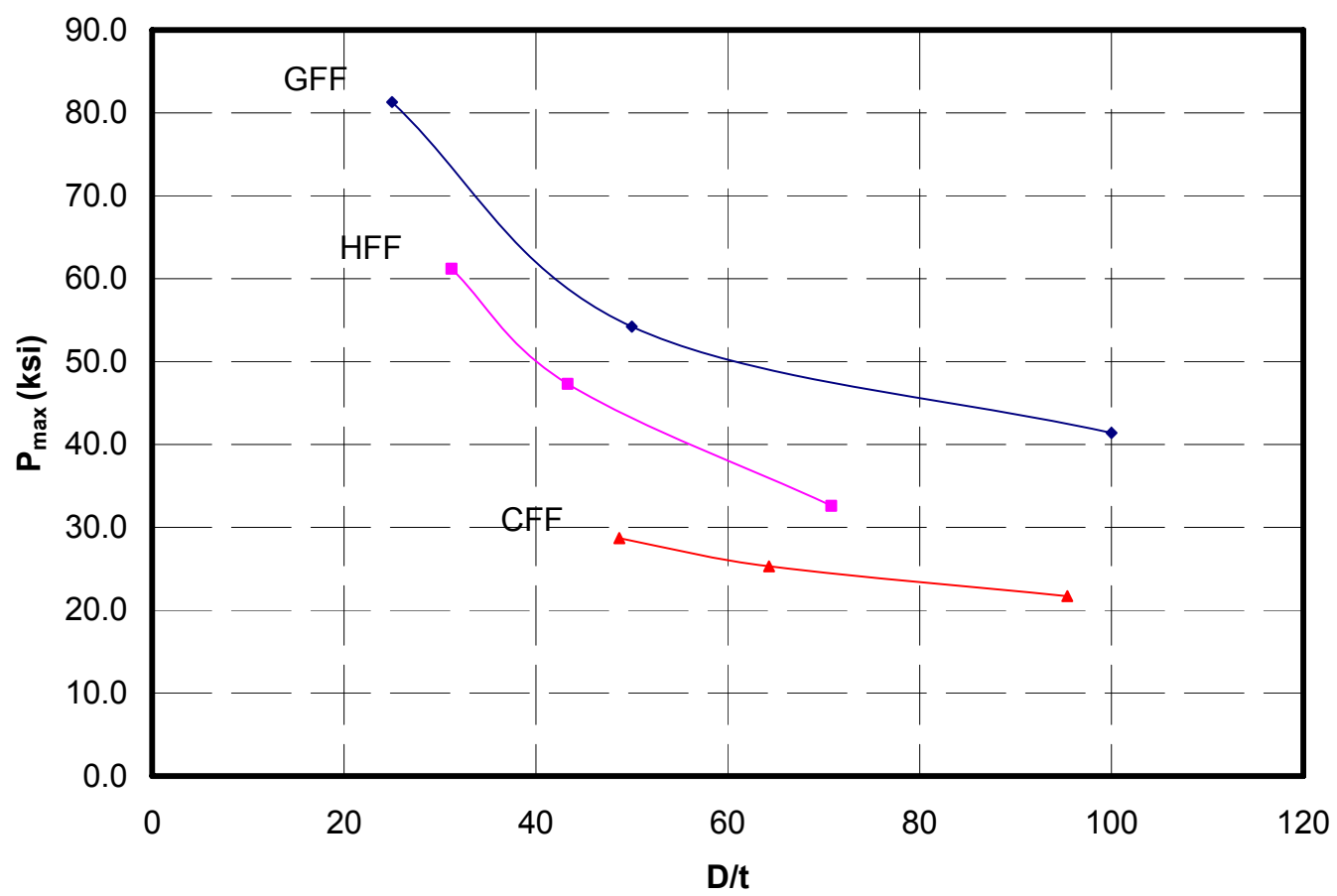

Fig. 4.60 Effect of D/t Ratio on Maximum Load of All Frames 


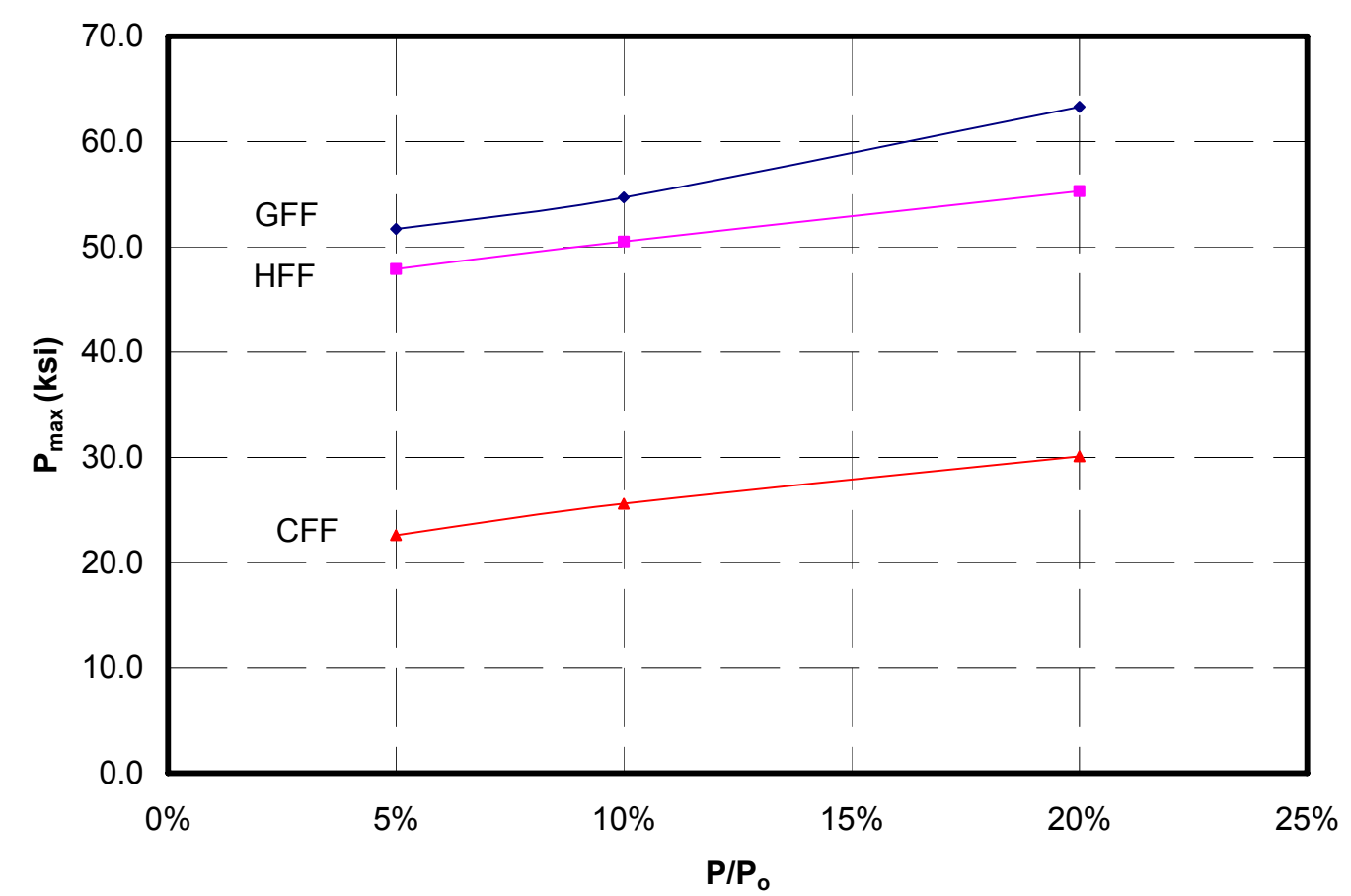

Fig. 4.61 Effect of Axial Load Ratio on Maximum Load of All Frames

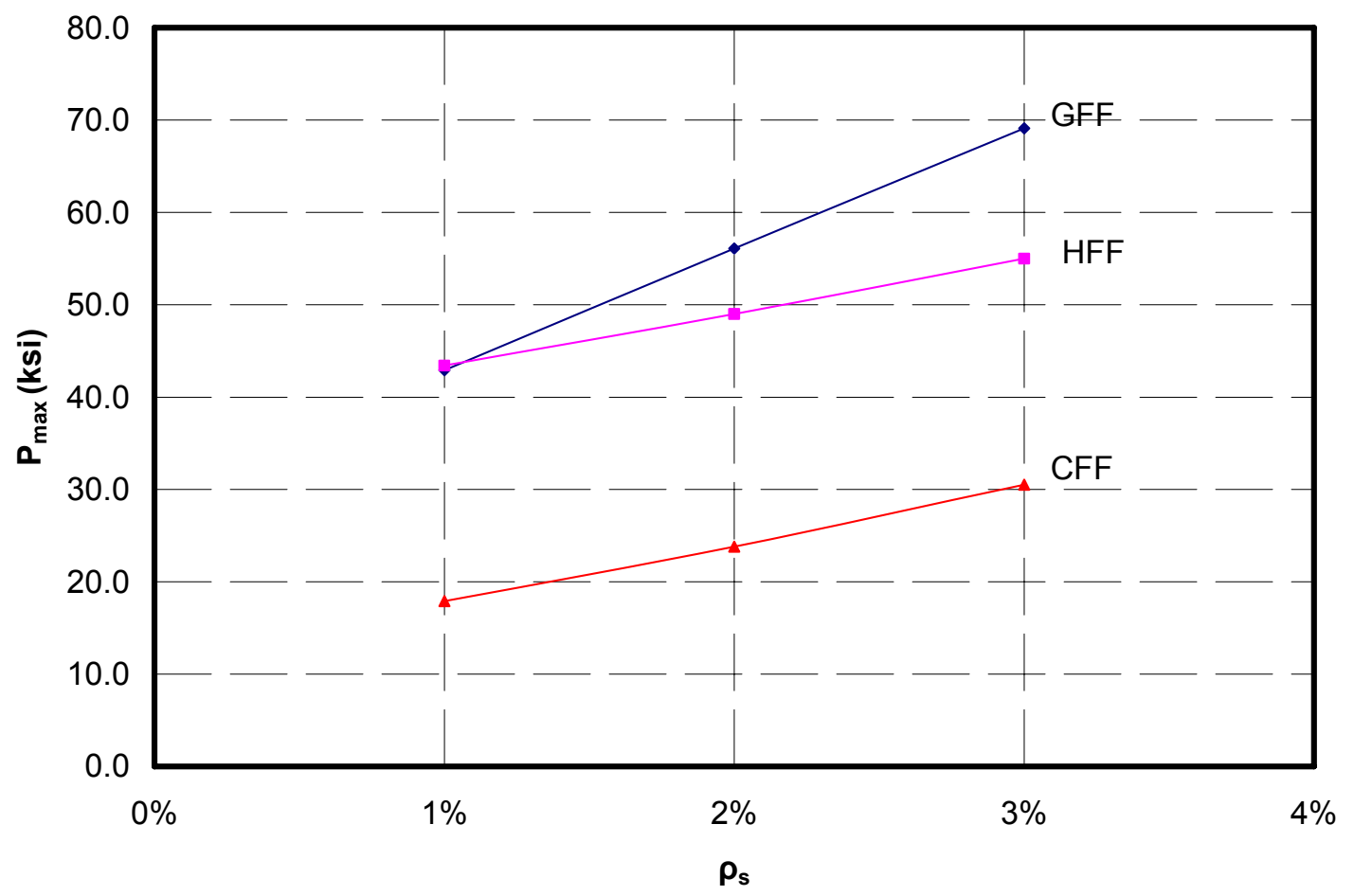

Fig. 4.62 Effect of Steel Reinforcement Ratio on Maximum Load of All Frames 


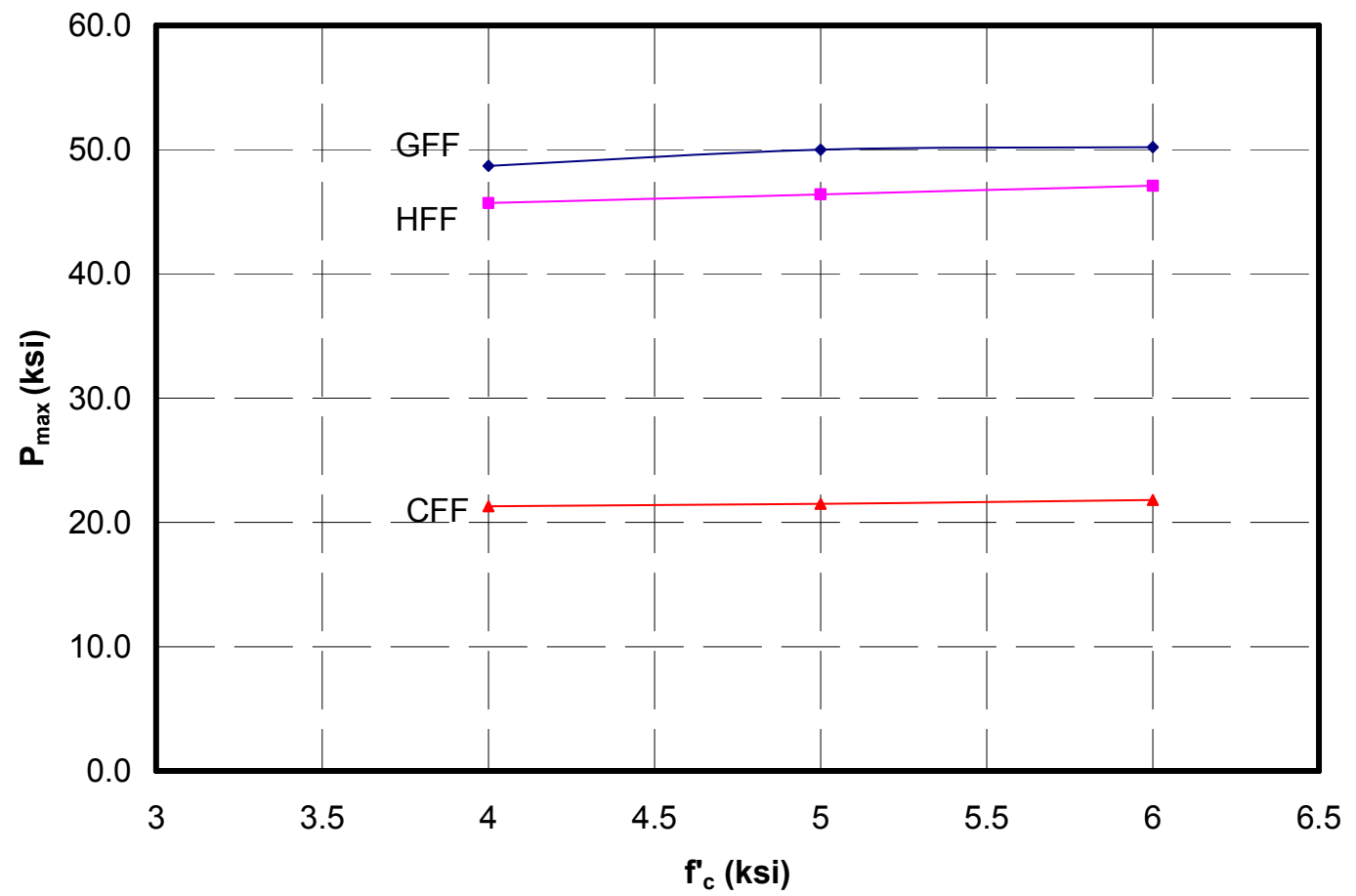

Fig. 4.63 Effect of Concrete Compressive Strength on Maximum Load of All Frames

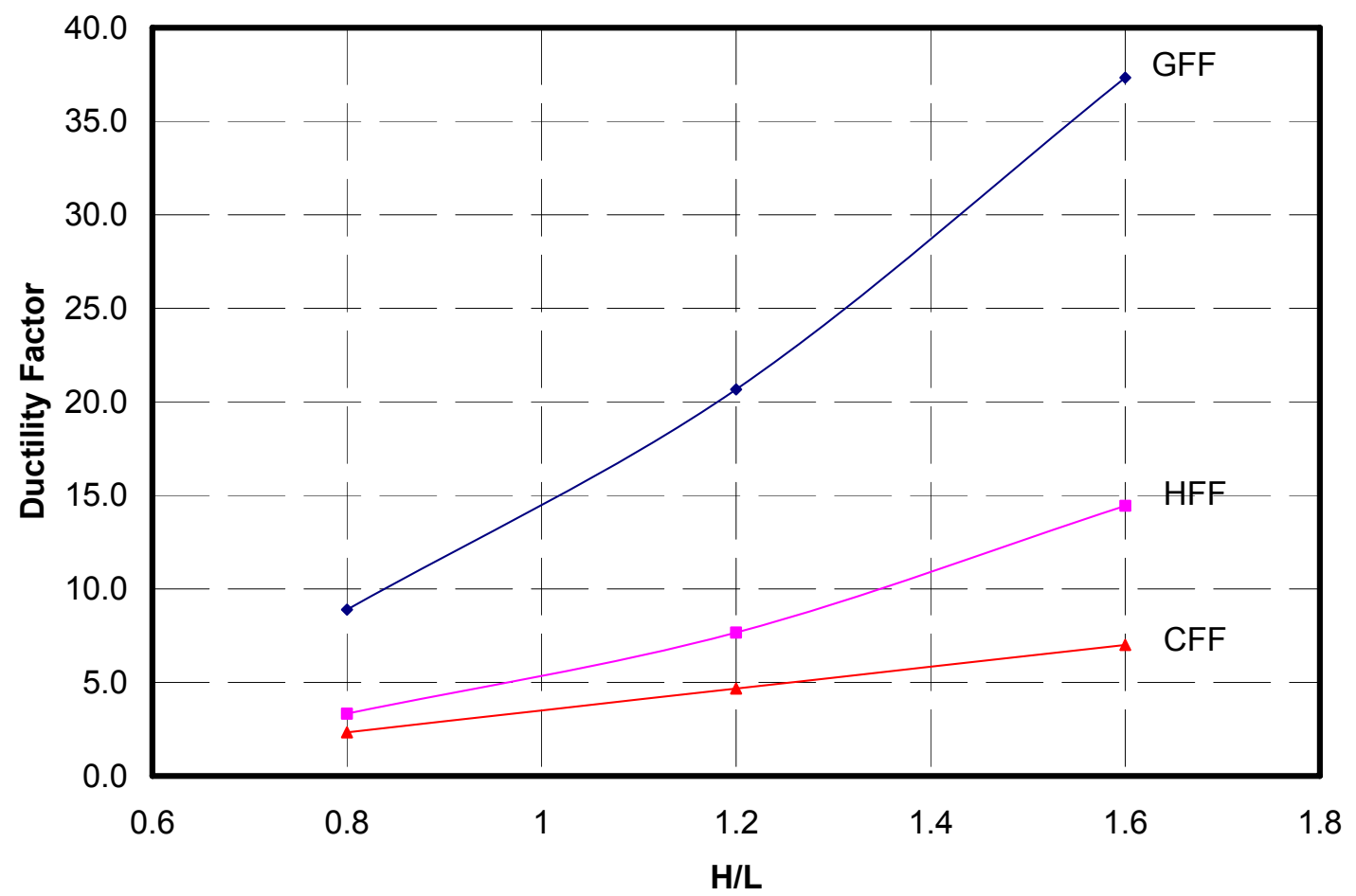

Fig. 4.64 Effect of H/L Ratio on Ductility Factor of All Frames 


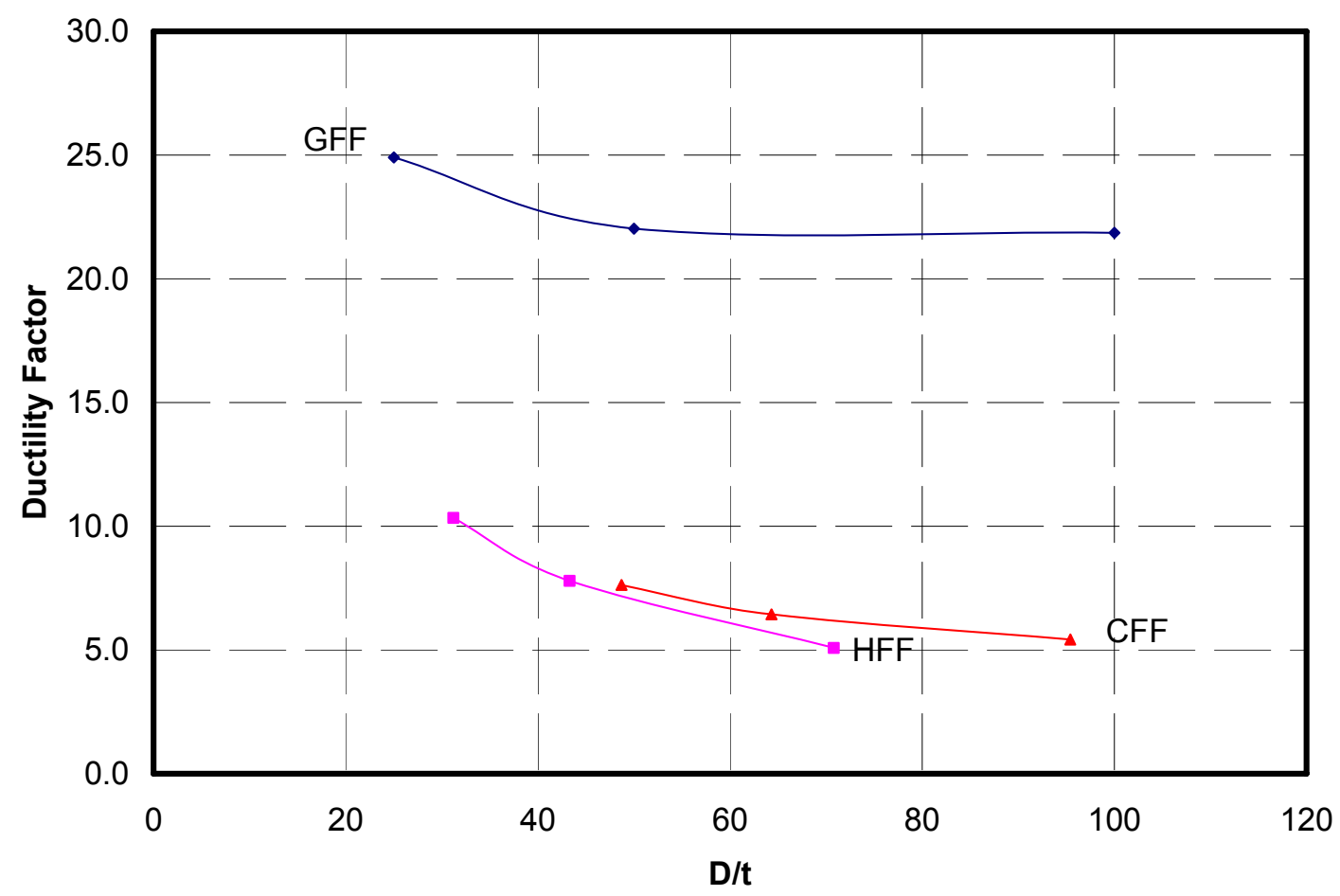

Fig. 4.65 Effect of D/t Ratio on Ductility Factor of All Frames

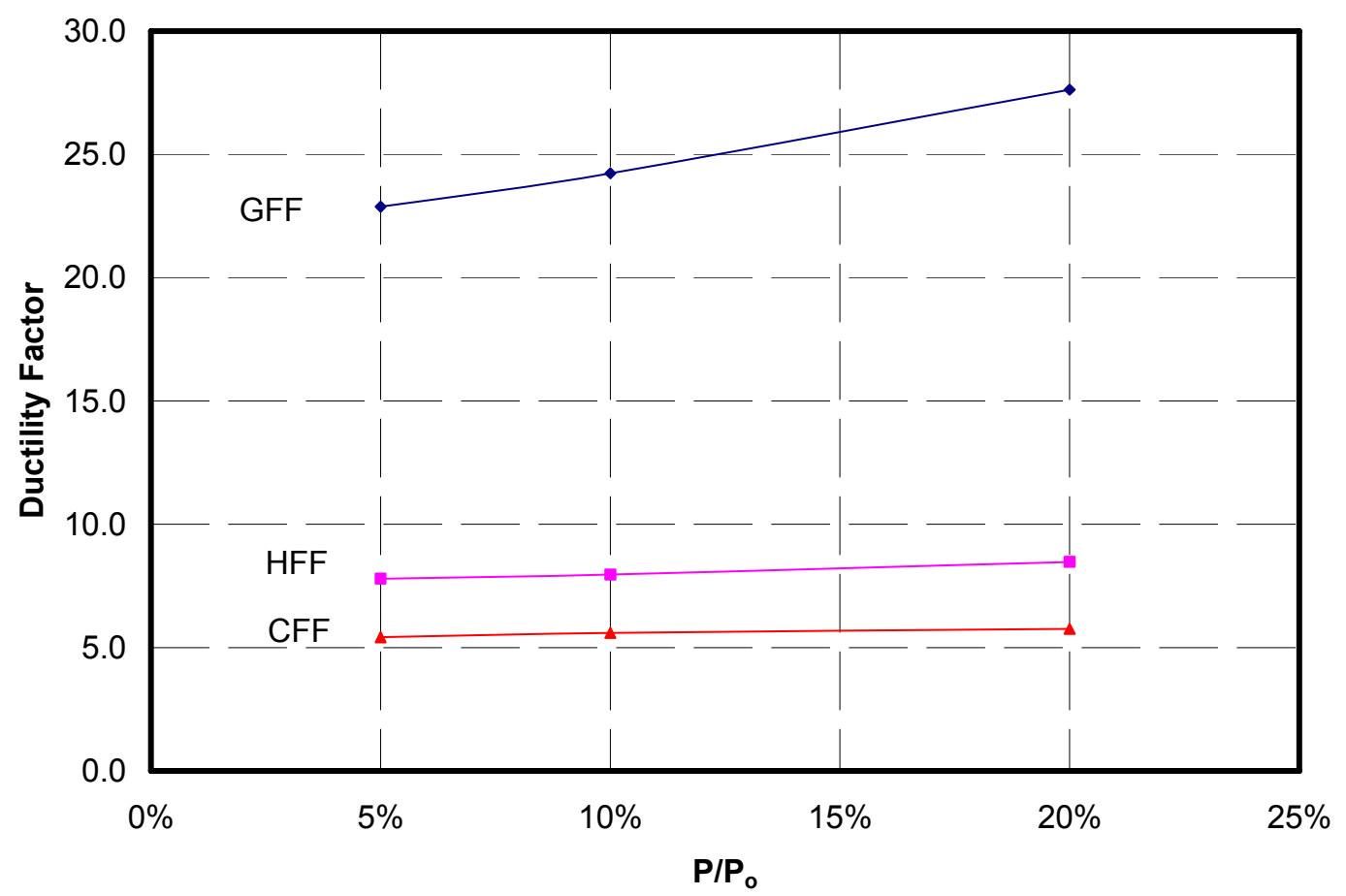

Fig. 4.66 Effect of Axial Load Ratio on Ductility Factor of All Frames 


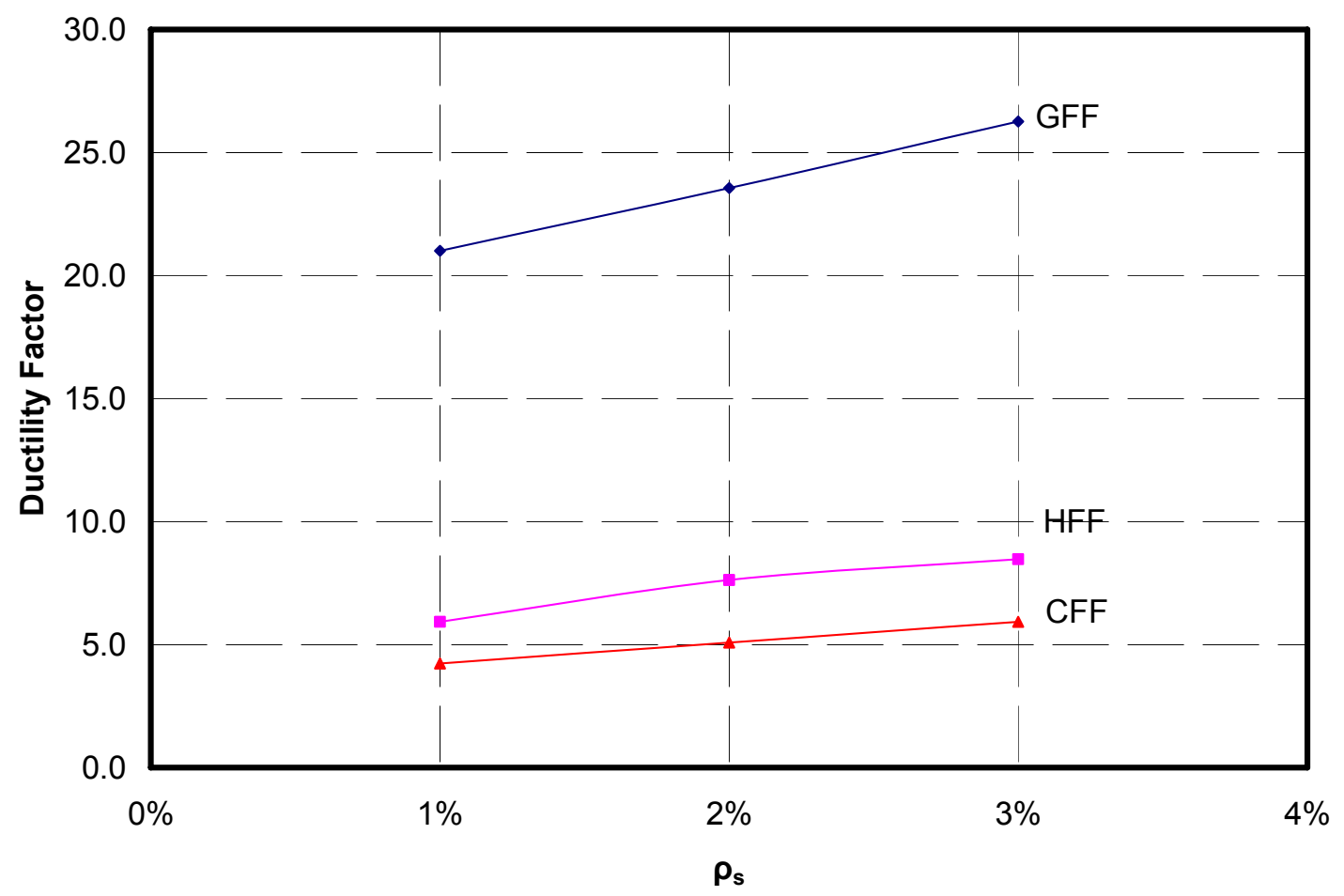

Fig. 4.67 Effect of Steel Reinforcement Ratio on Ductility Factor of All Frames

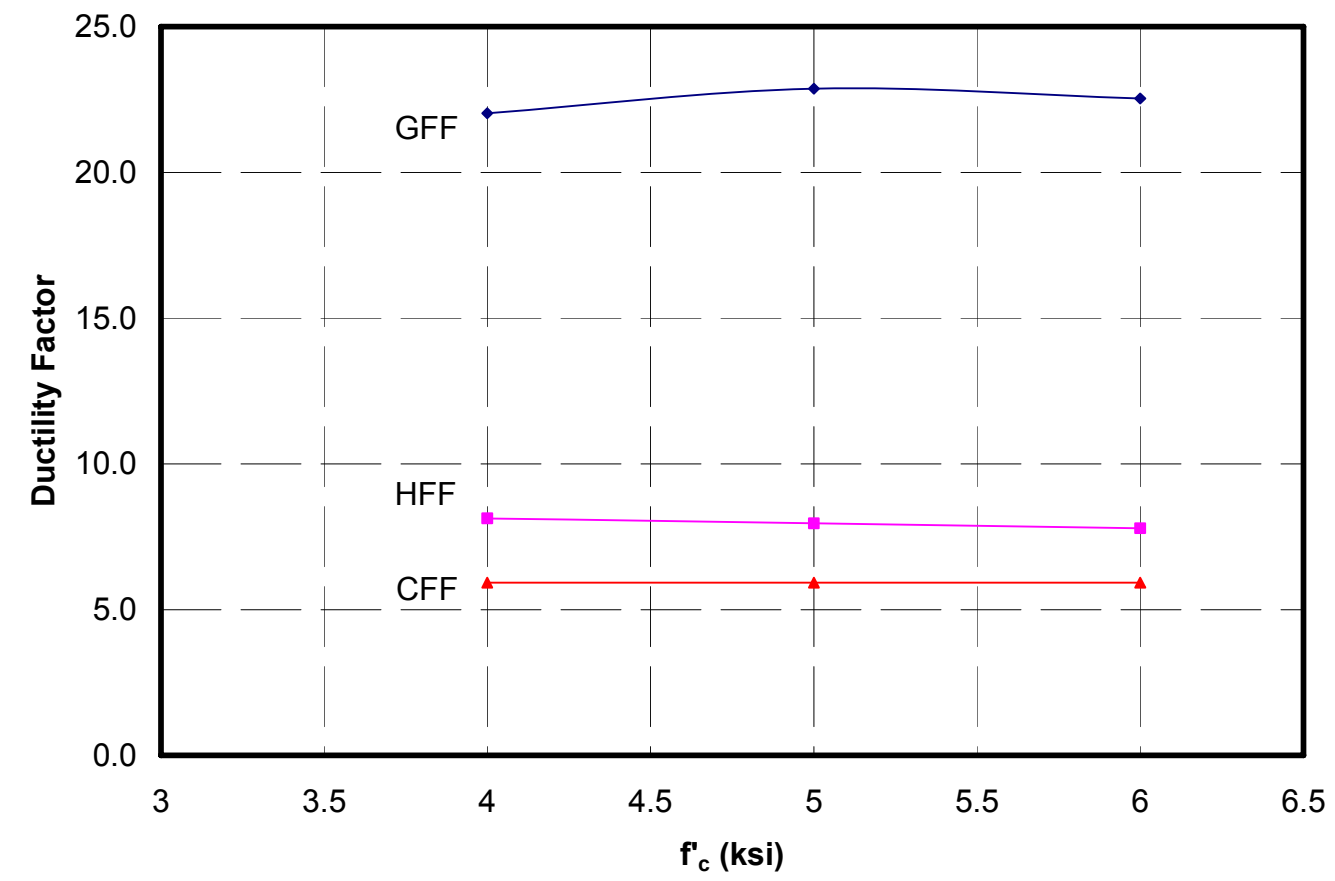

Fig. 4.68 Effect of Concrete Compressive Strength on Ductility Factor of All Frames 


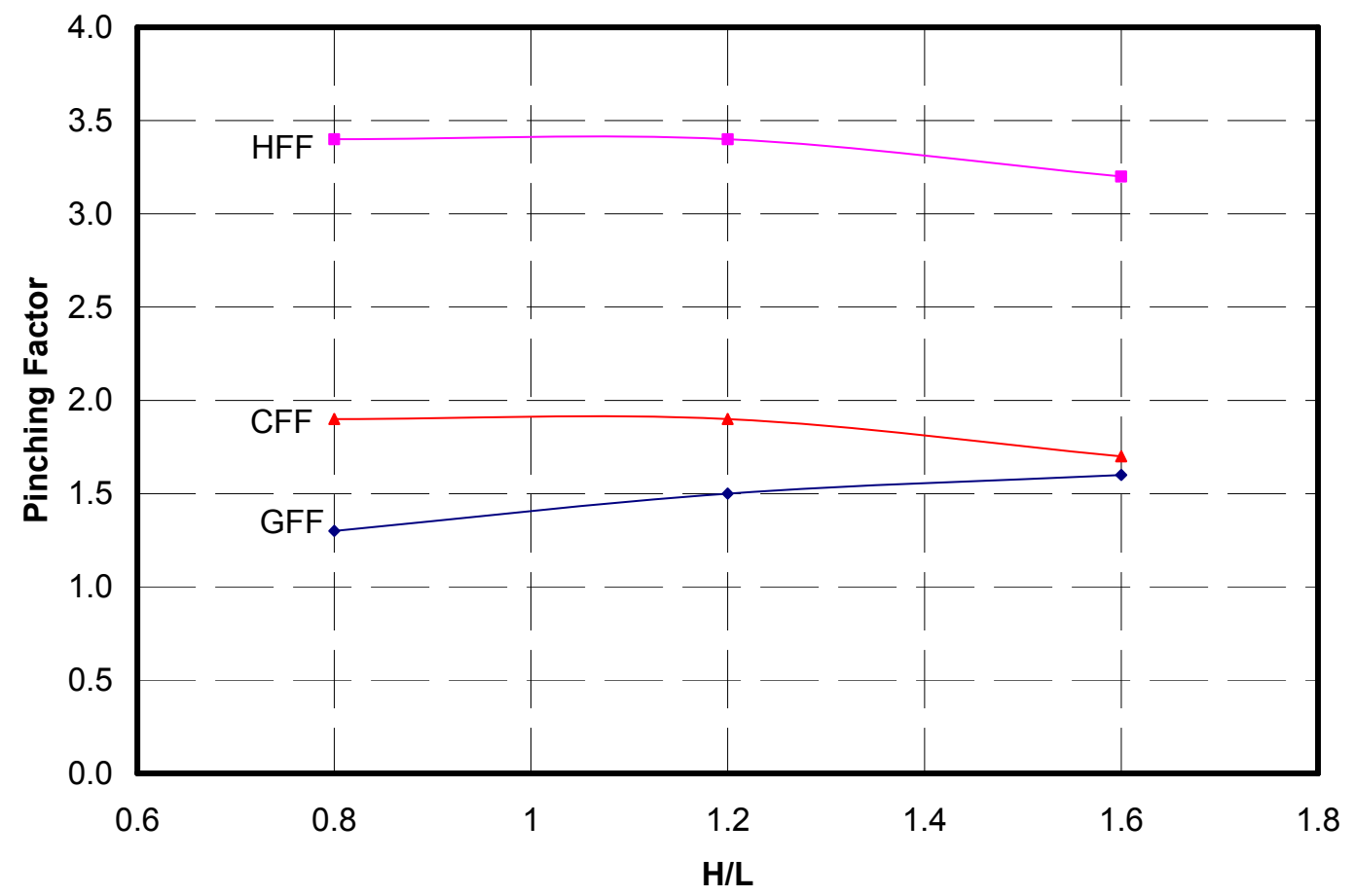

Fig. 4.69 Effect of H/L Ratio on Pinching Factor of All Frames

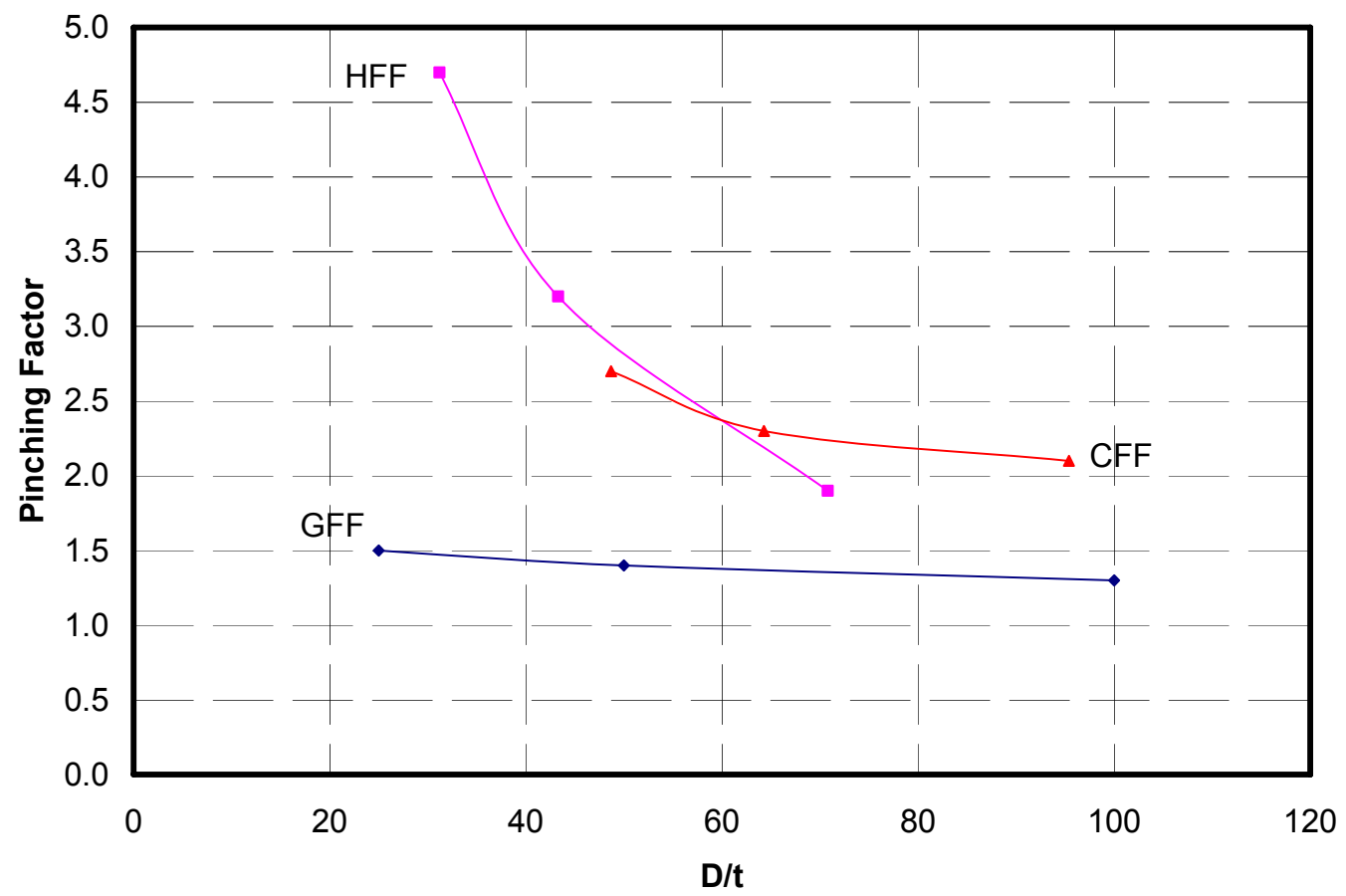

Fig. 4.70 Effect of D/t Ratio on Pinching Factor of All Frames 


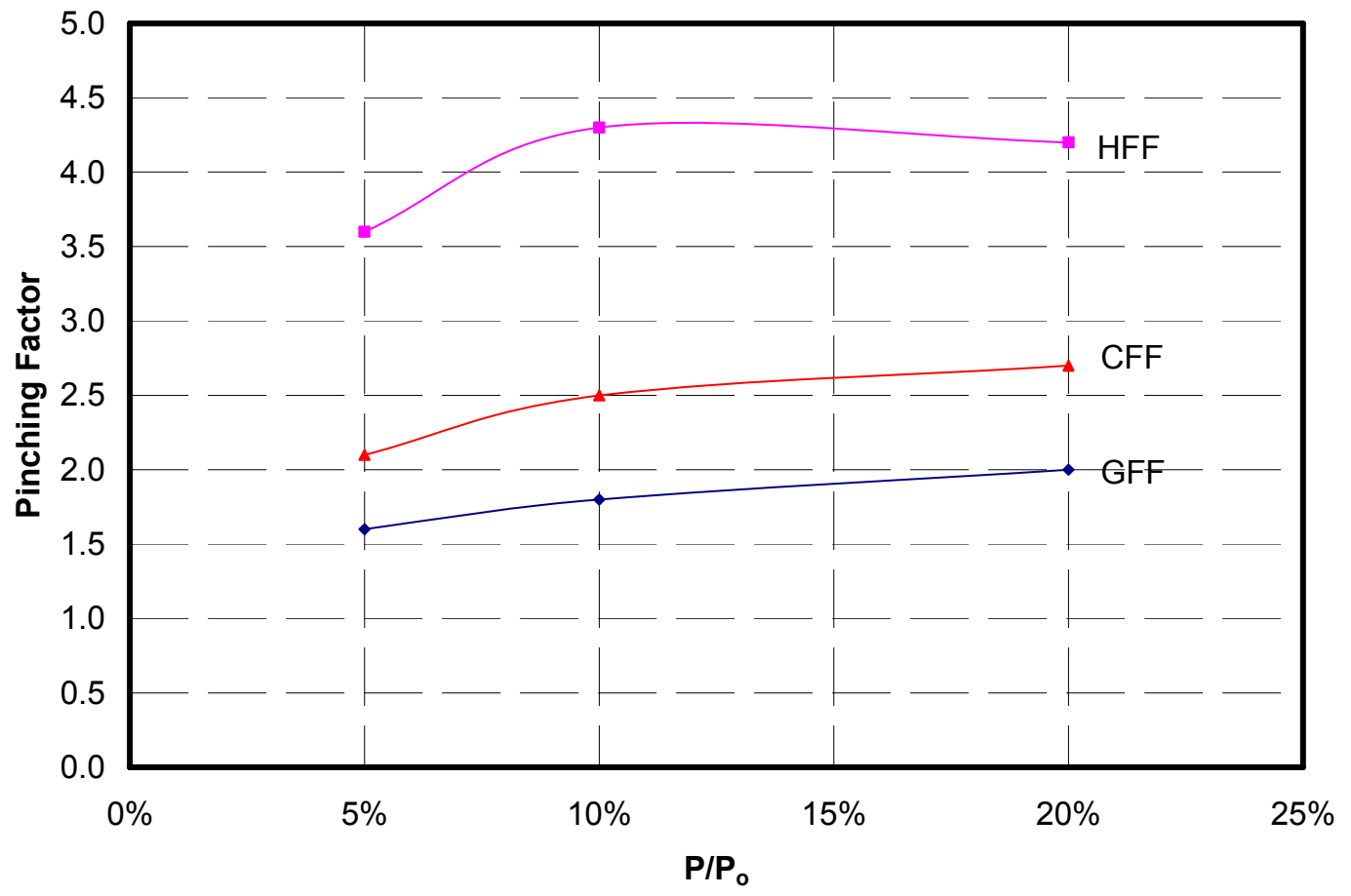

Fig. 4.71 Effect of Axial Load Ratio on Pinching Factor of All Frames

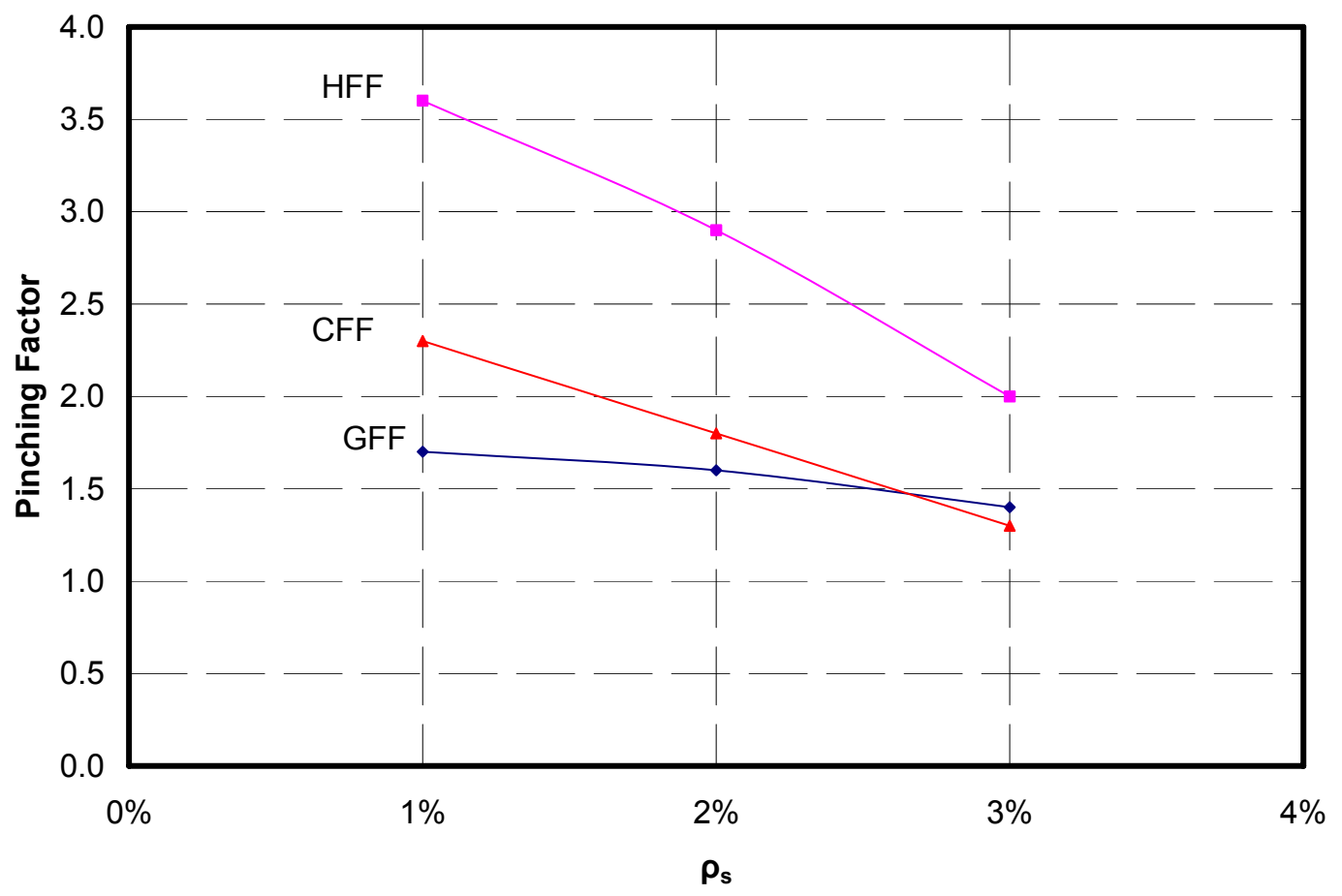

Fig. 4.72 Effect of Steel Reinforcement Ratio on Pinching Factor of All Frames 


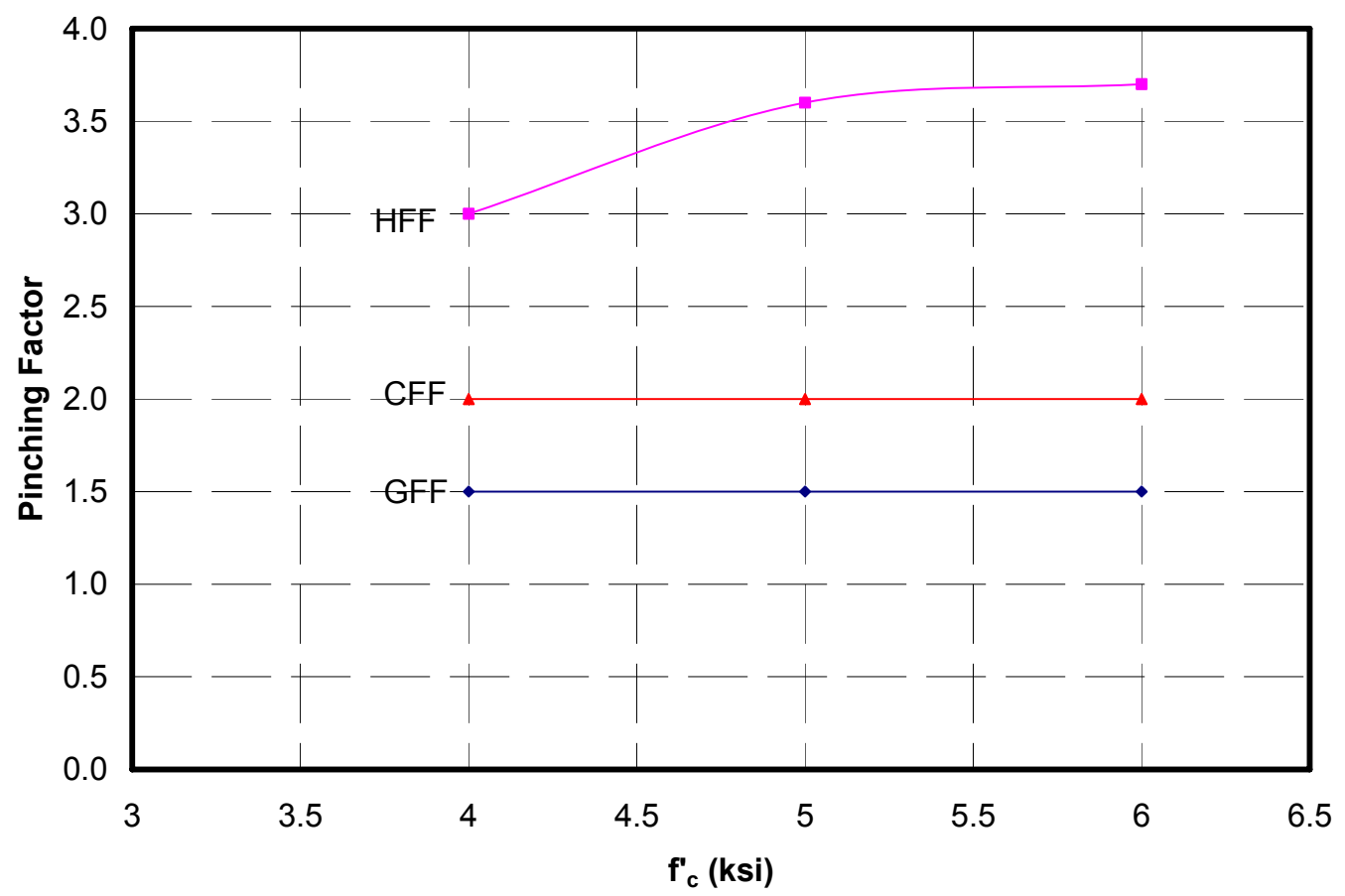

Fig. 4.73 Effect of Concrete Compressive Strength on Pinching Factor of All Frames

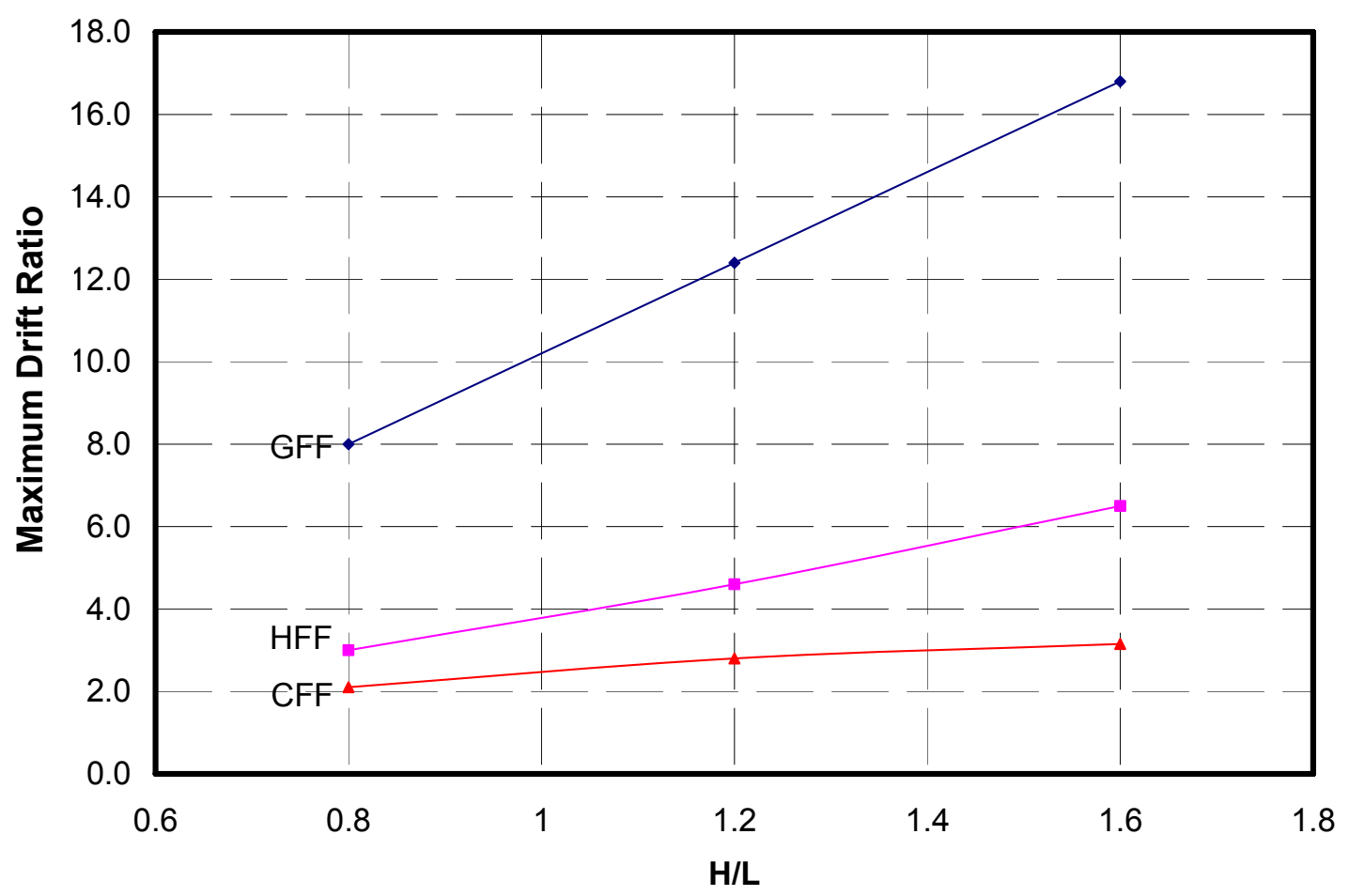

Fig. 4.74 Effect of H/L Ratio on Maximum Drift Ratio of All Frames 


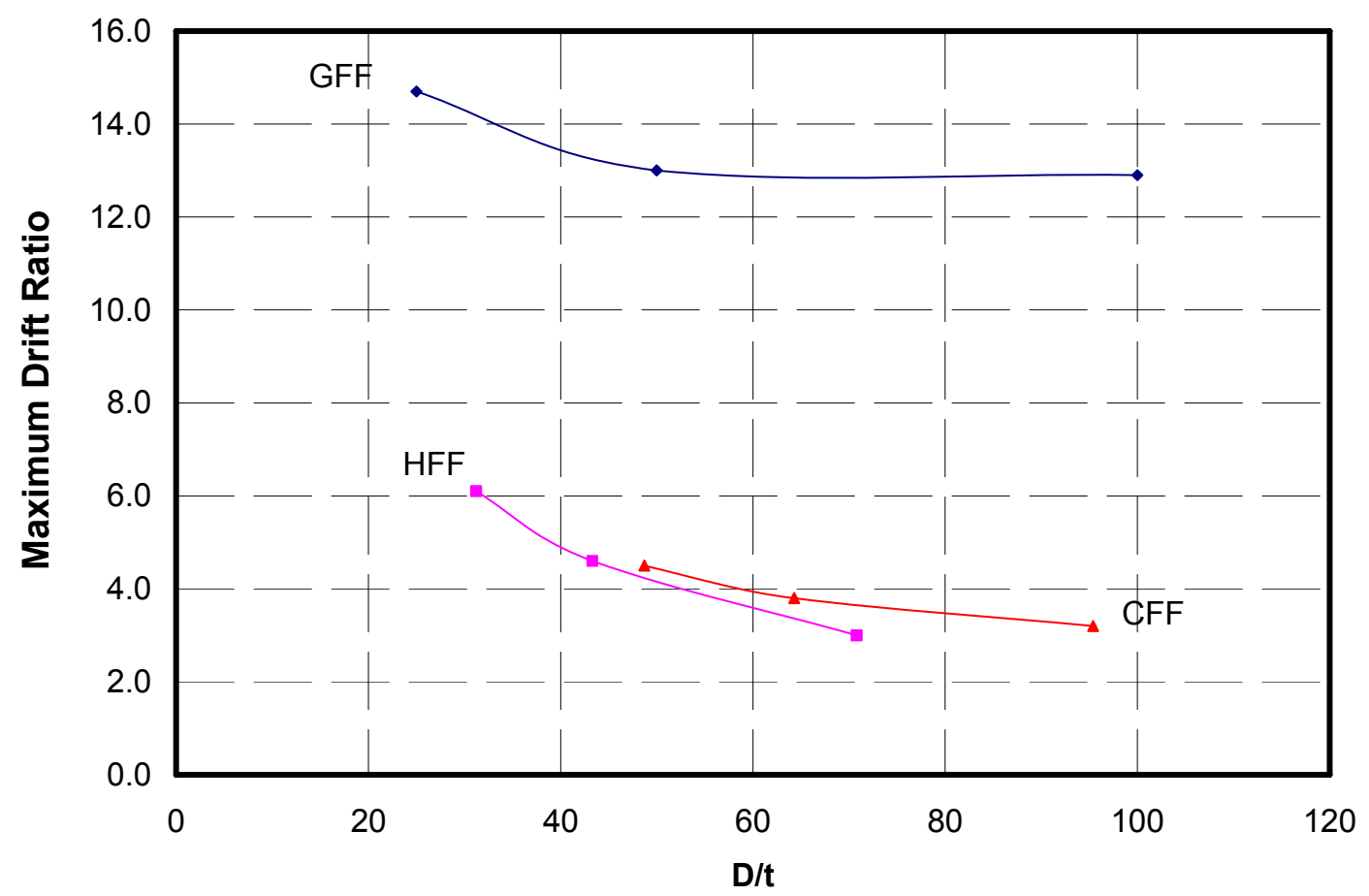

Fig. 4.75 Effect of D/t Ratio on Maximum Drift Ratio of All Frames

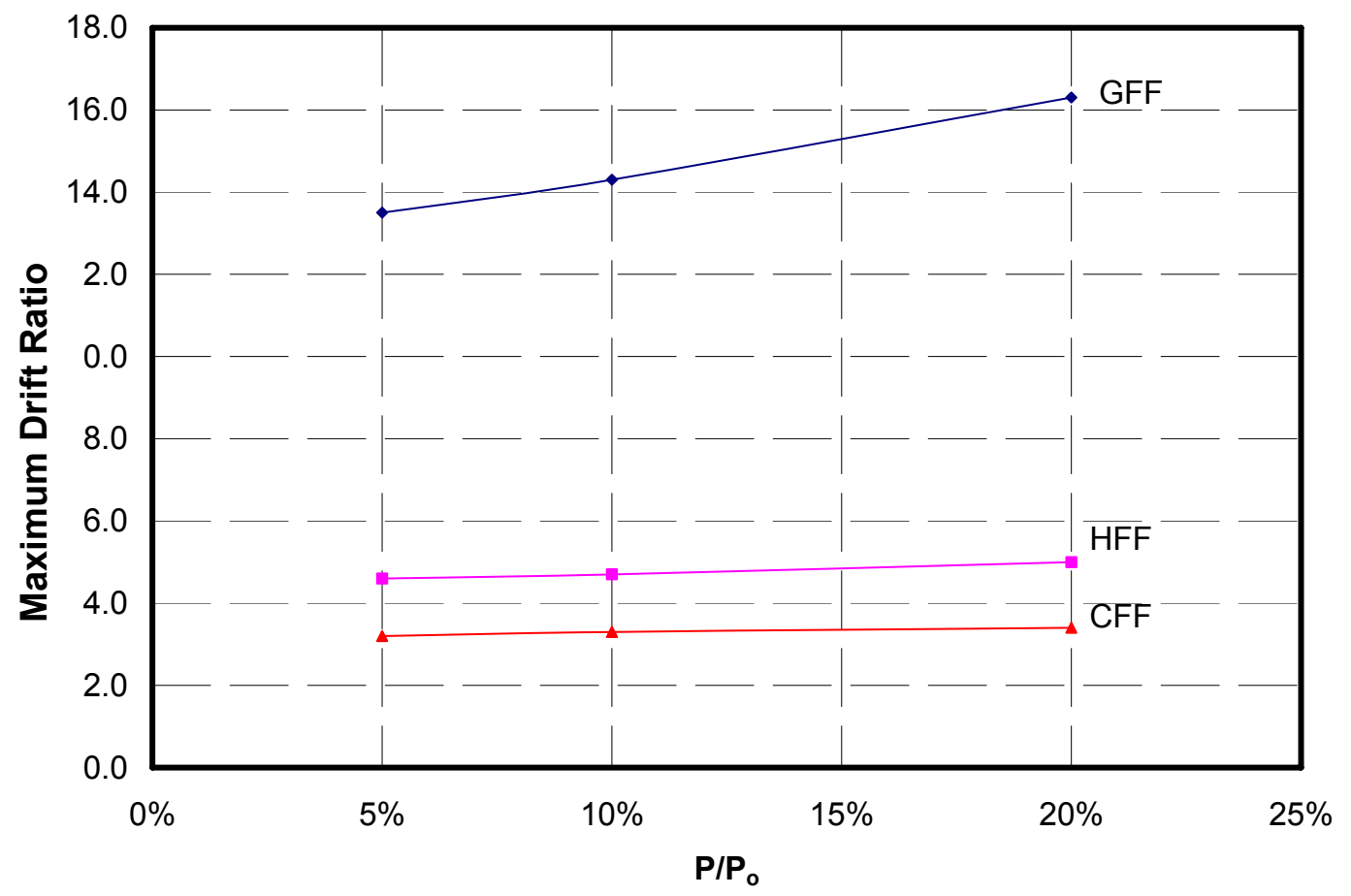

Fig. 4.76 Effect of Axial Load Ratio on Maximum Drift Ratio of All Frames 


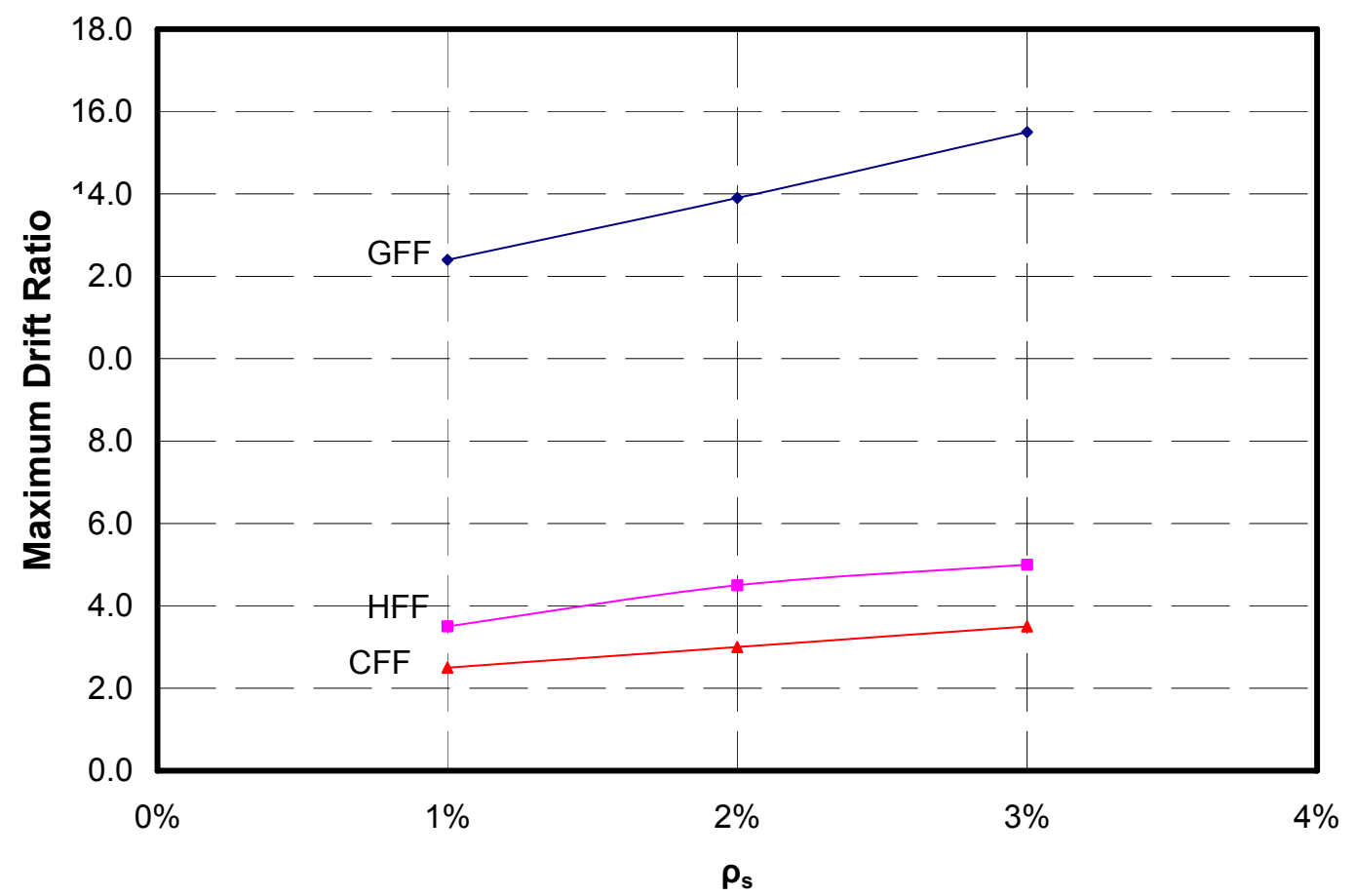

Fig. 4.77 Effect of Steel Reinforcement Ratio on Maximum Drift Ratio of All Frames

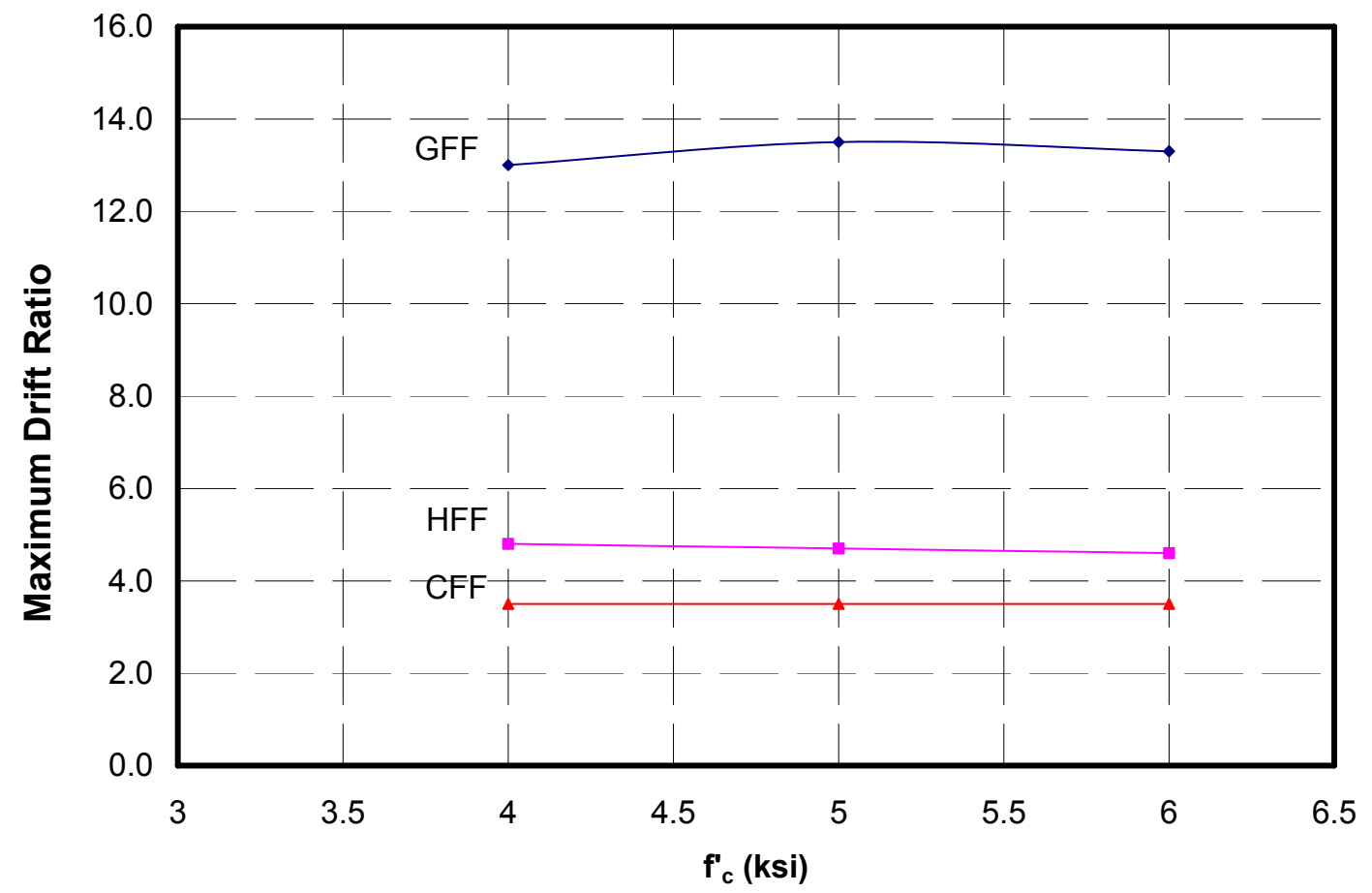

Fig. 4.78 Effect of Concrete Compressive Strength on Maximum Drift Ratio of All Frames 


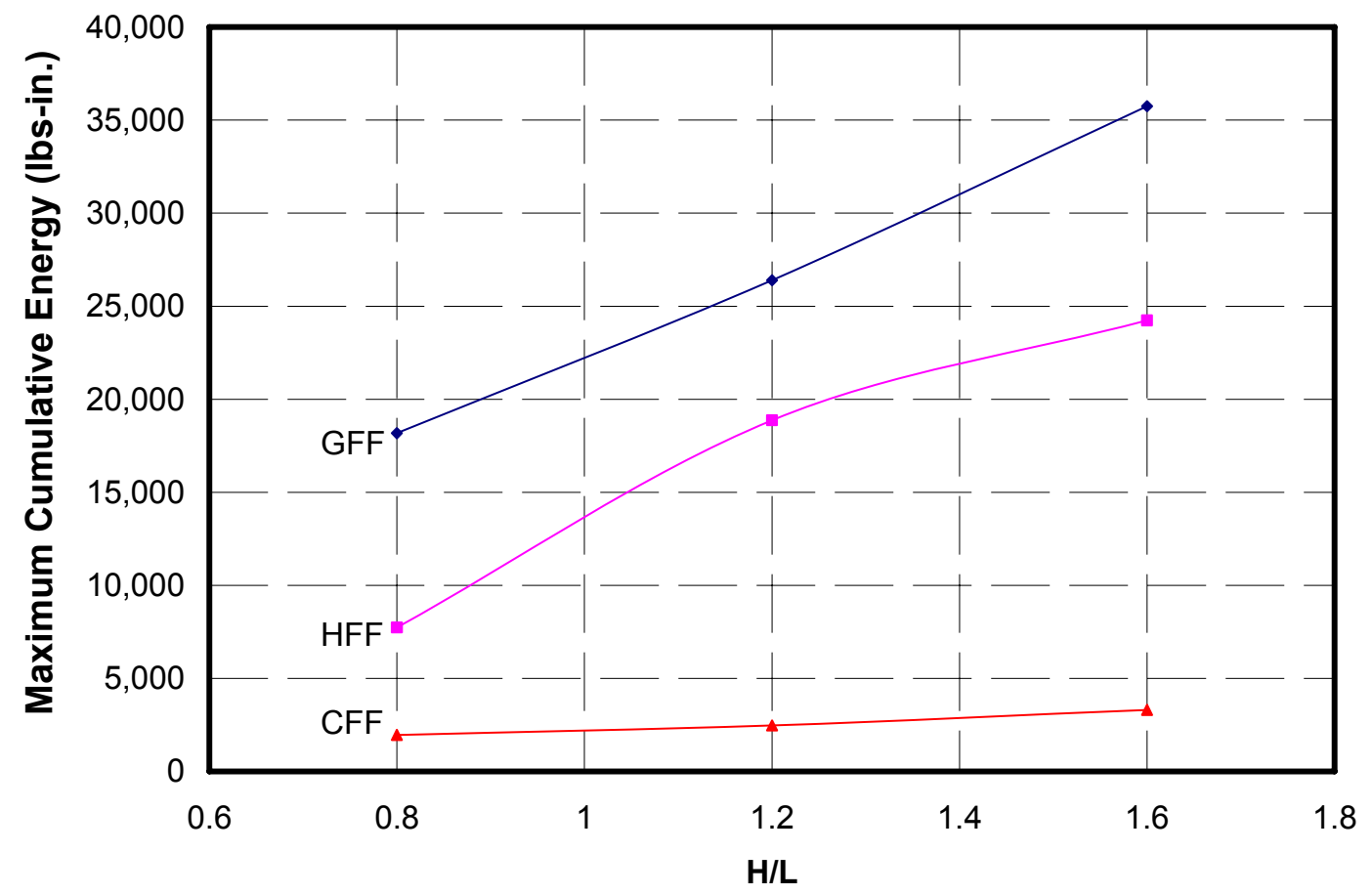

Fig. 4.79 Effect of H/L Ratio on Maximum Cumulative Energy of All Frames

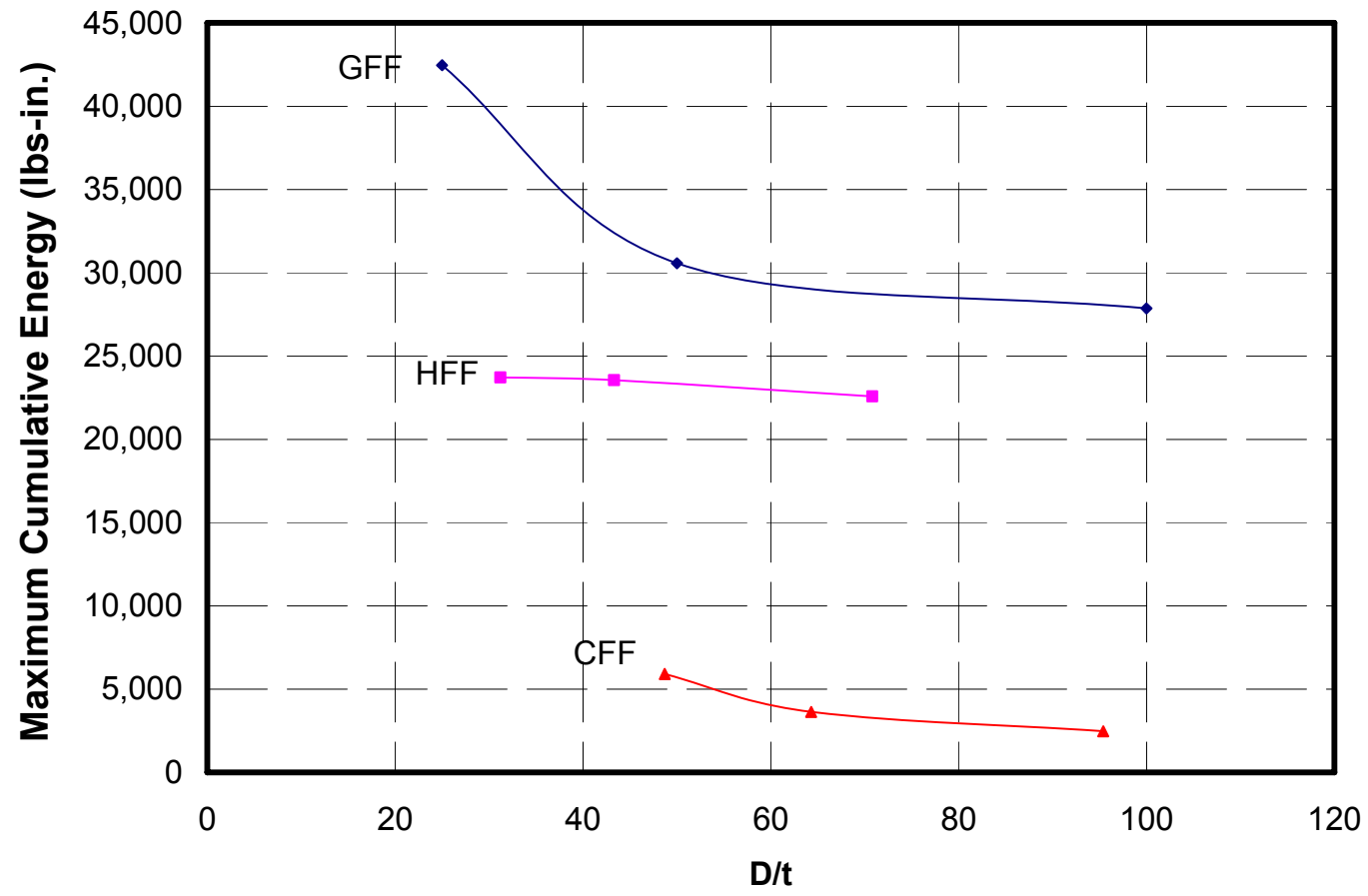

Fig. 4.80 Effect of D/t Ratio on Maximum Cumulative Energy of All Frames 


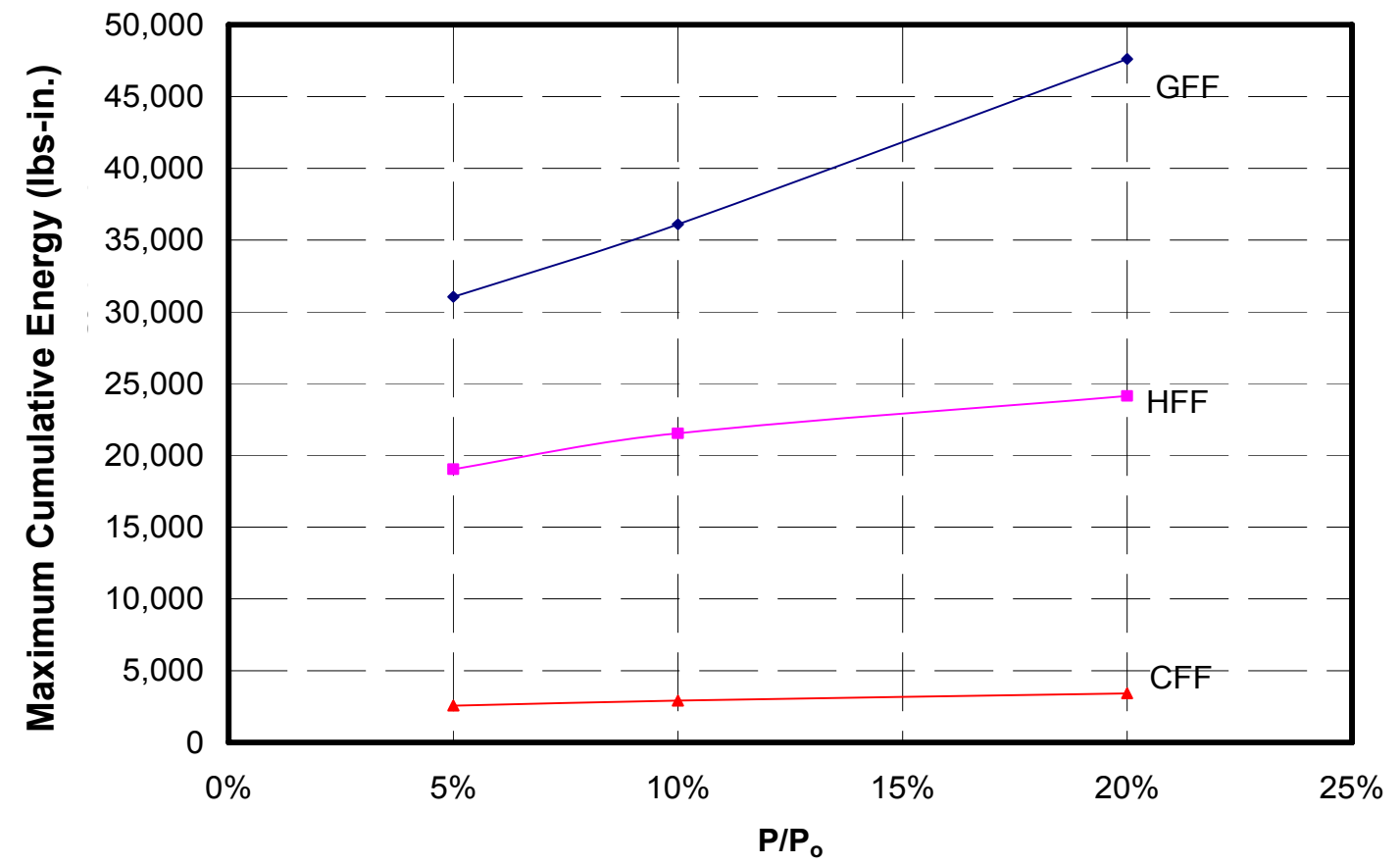

Fig. 4.81 Effect of Axial Load Ratio on Maximum Cumulative Energy of All Frames

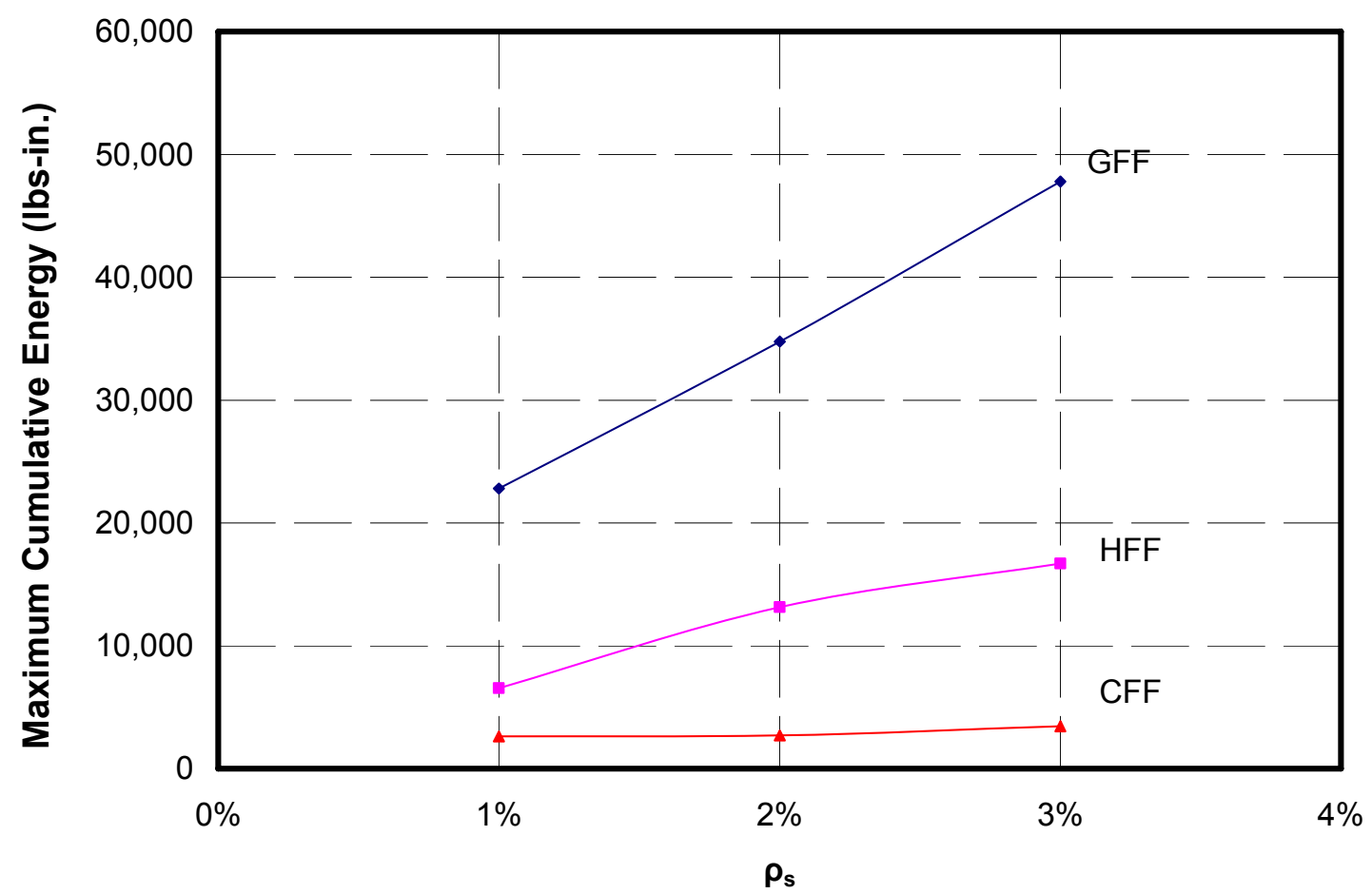

Fig. 4.82 Effect of Steel Reinforcement Ratio on Maximum Cumulative Energy of All Frames 


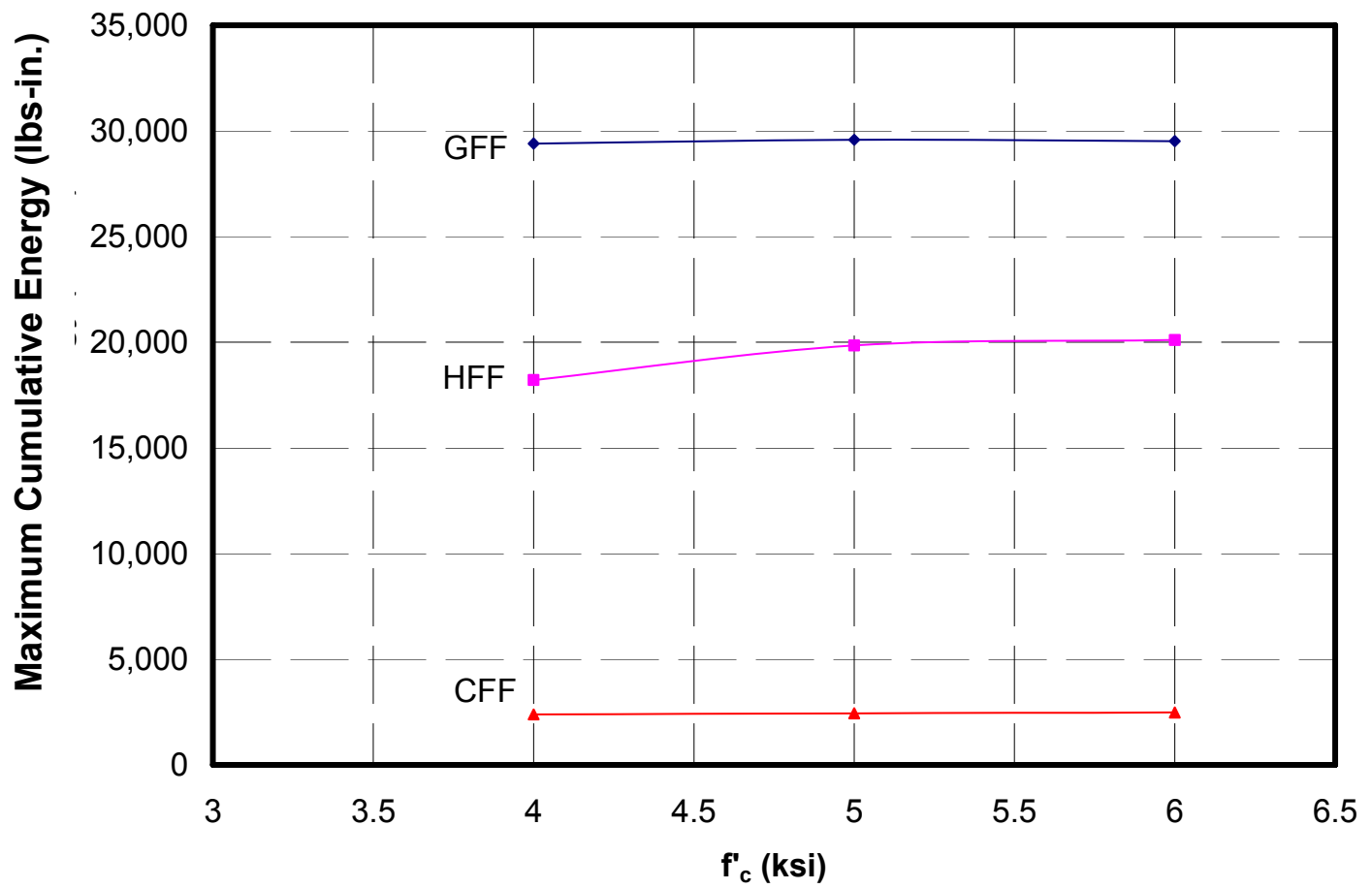

Fig. 4.83 Effect of Compressive Concrete Strength on Maximum Cumulative Energy of All Frames

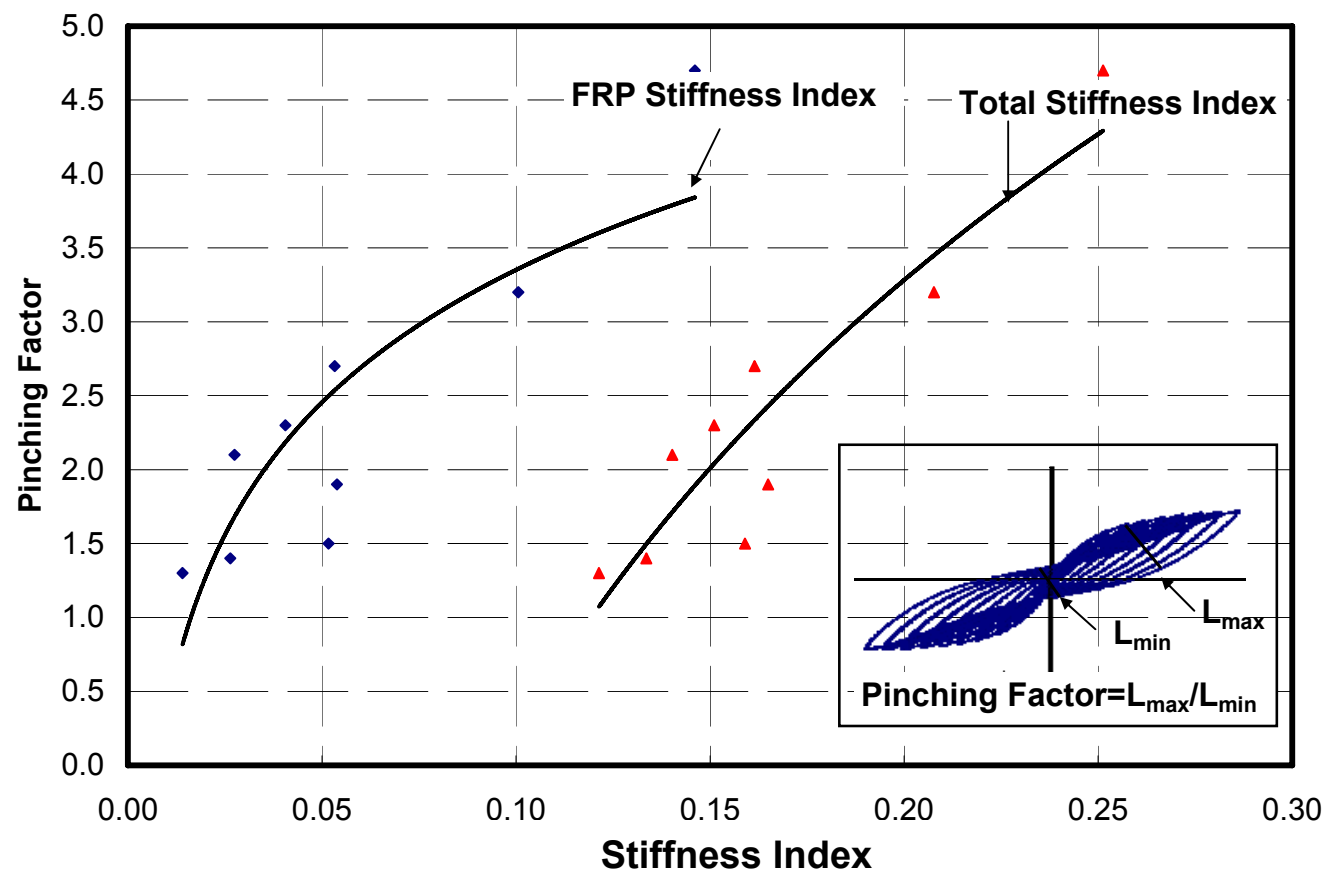

Fig. 4.84 Pinching Factor Versus FRP and Total Stiffness Index 


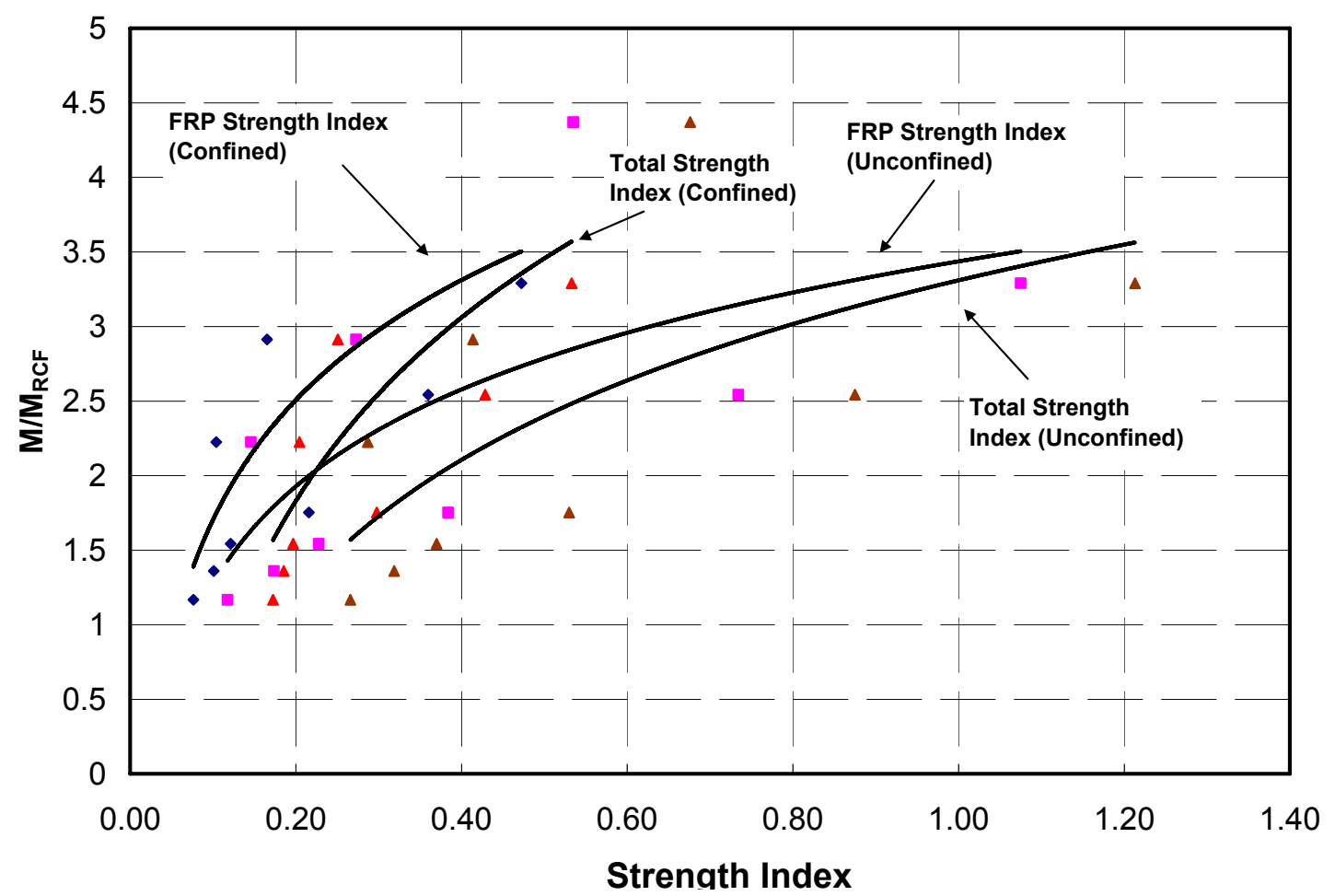

Fig. 4.85 Normalized Moment Versus FRP and Total Strength Index

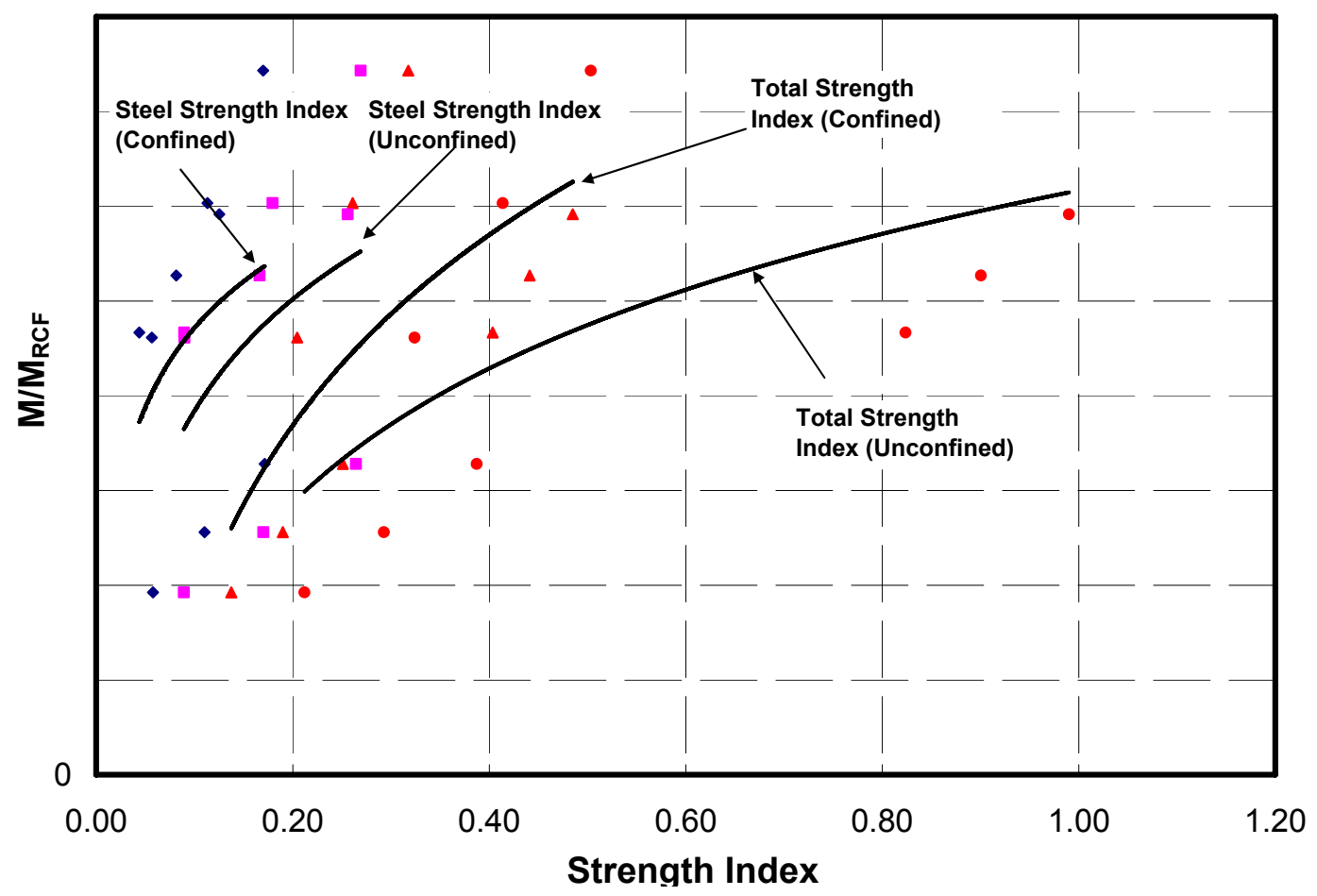

Fig. 4.86 Normalized Moment Versus Steel and Total Strength Index 


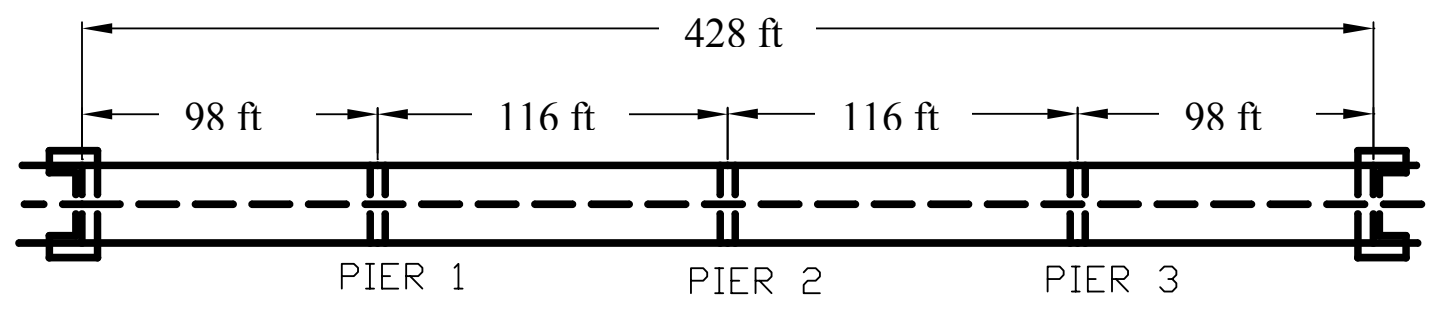

(a) Plan View

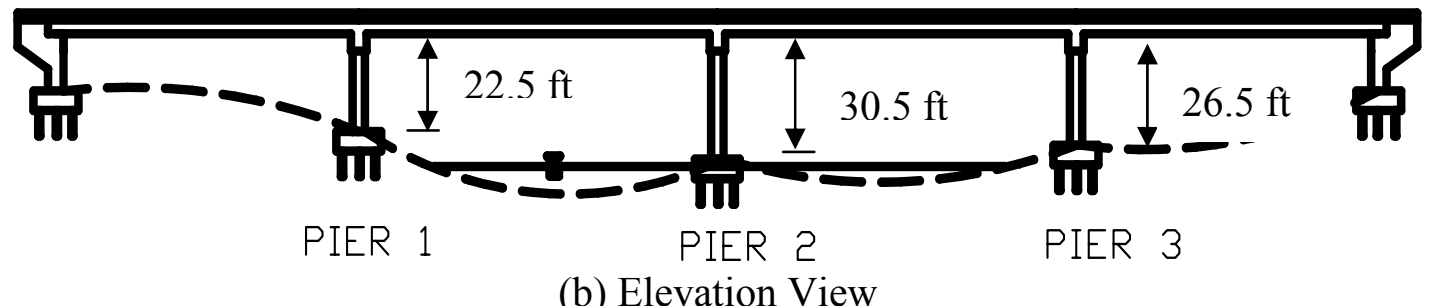

Fig. 4.87 Schematics of Bridge Case Study (a) Plan View, (b) Elevation

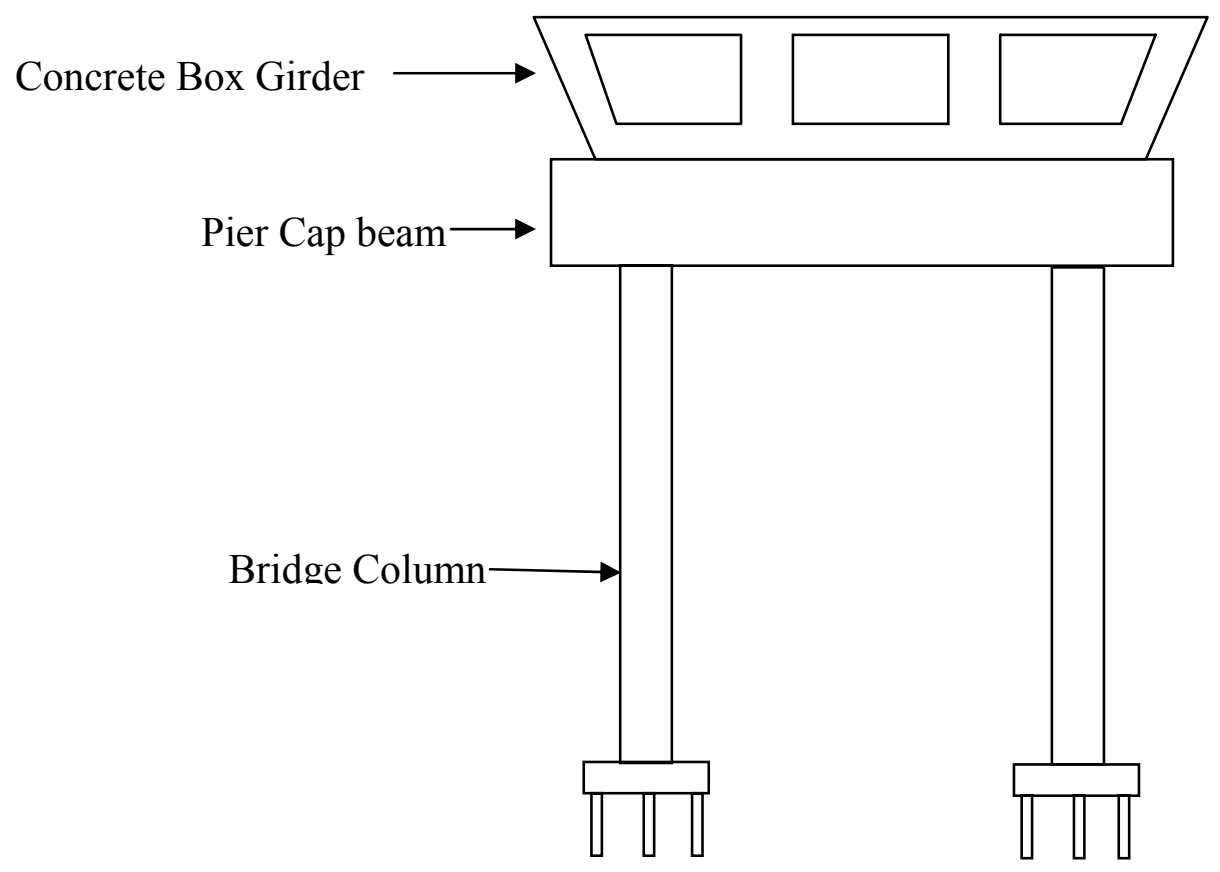

Fig. 4.88 Cross Section of Bridge Superstructure 


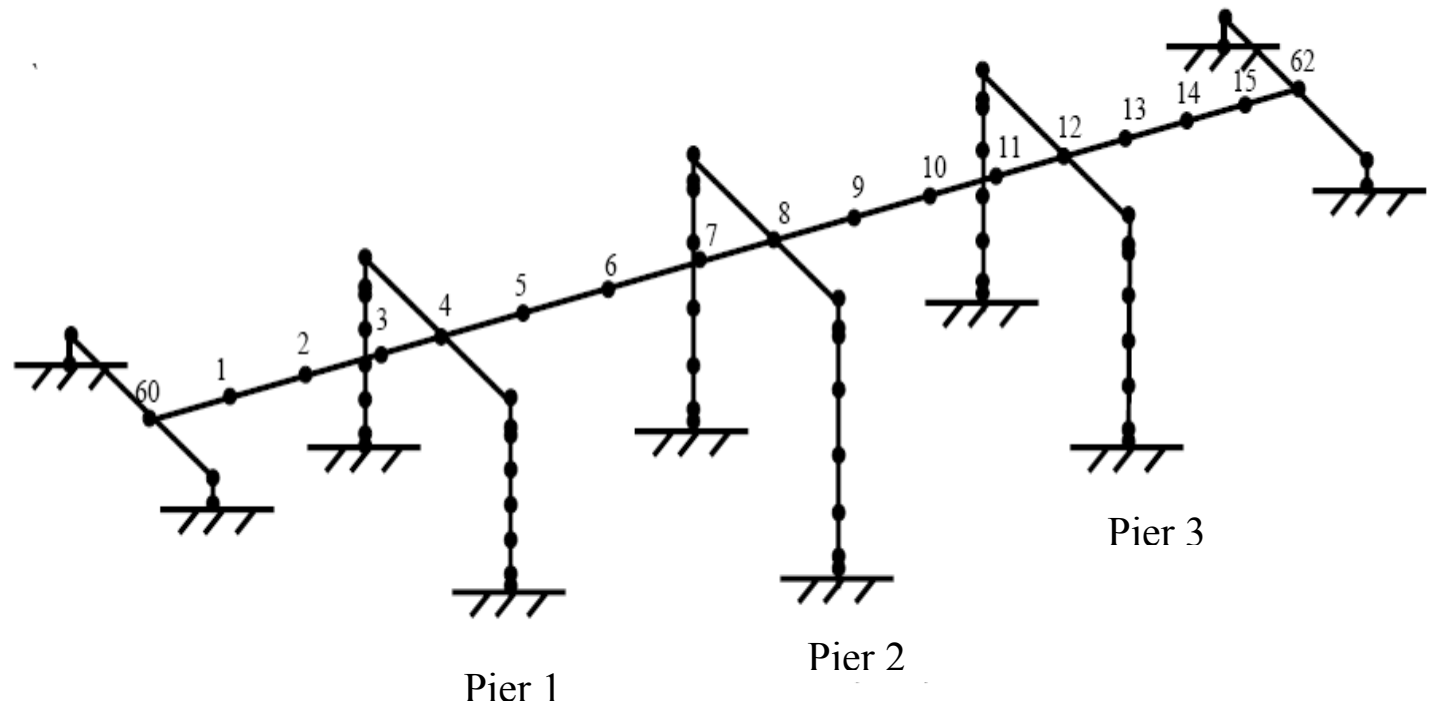

Fig. 4.89 Finite Element Model for Entire Bridge Structure

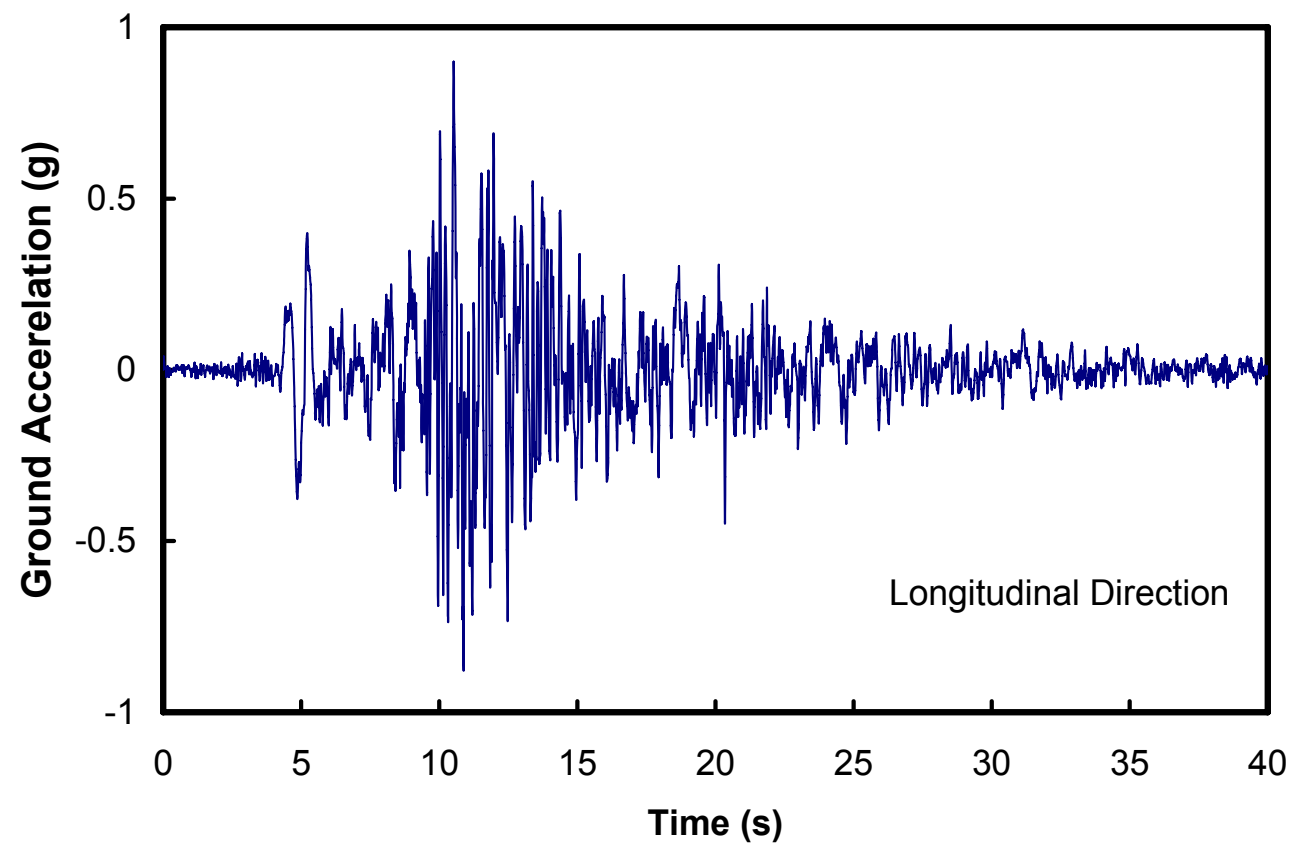

Fig. 4.90 Tabas (1978) Longitudinal Ground Acceleration Record 


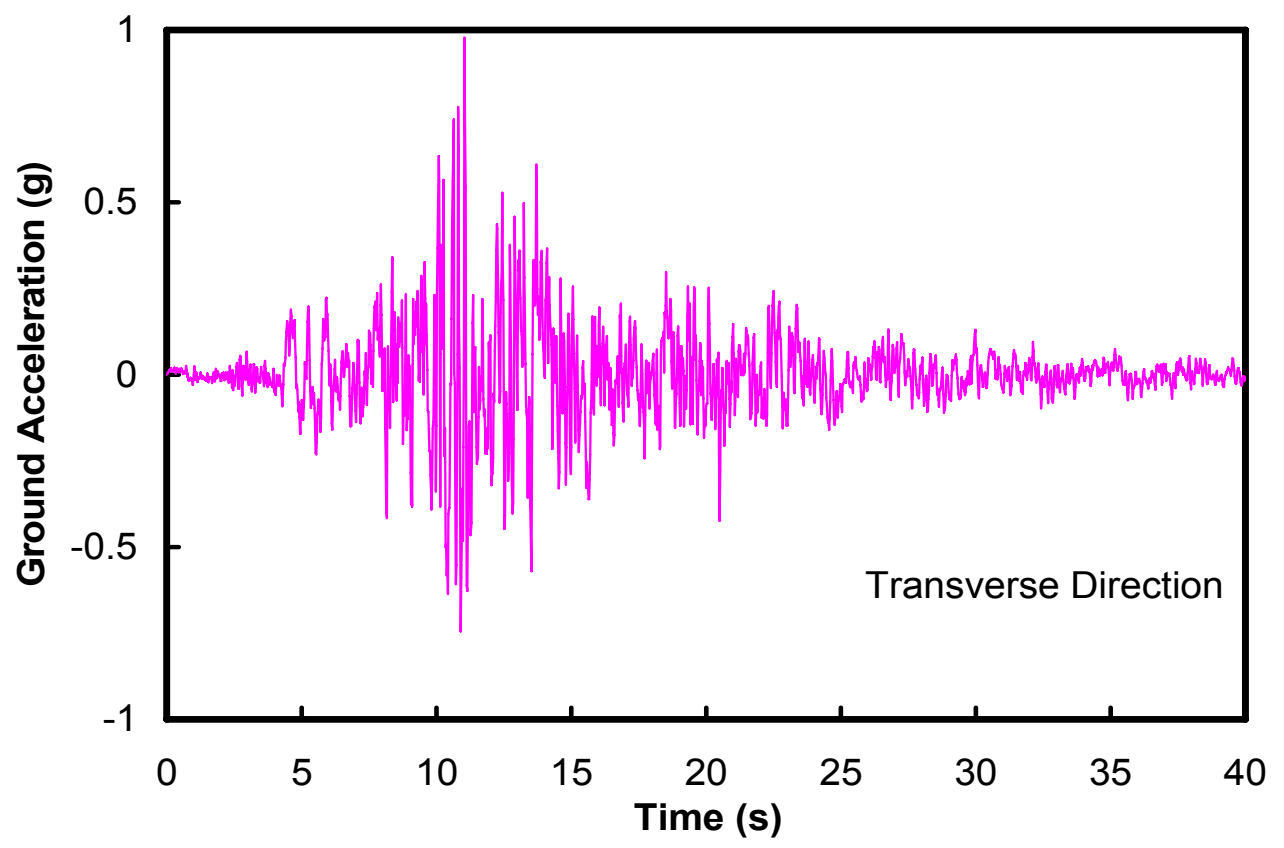

Fig. 4.91 Tabas (1978) Transverse Ground Acceleration Record

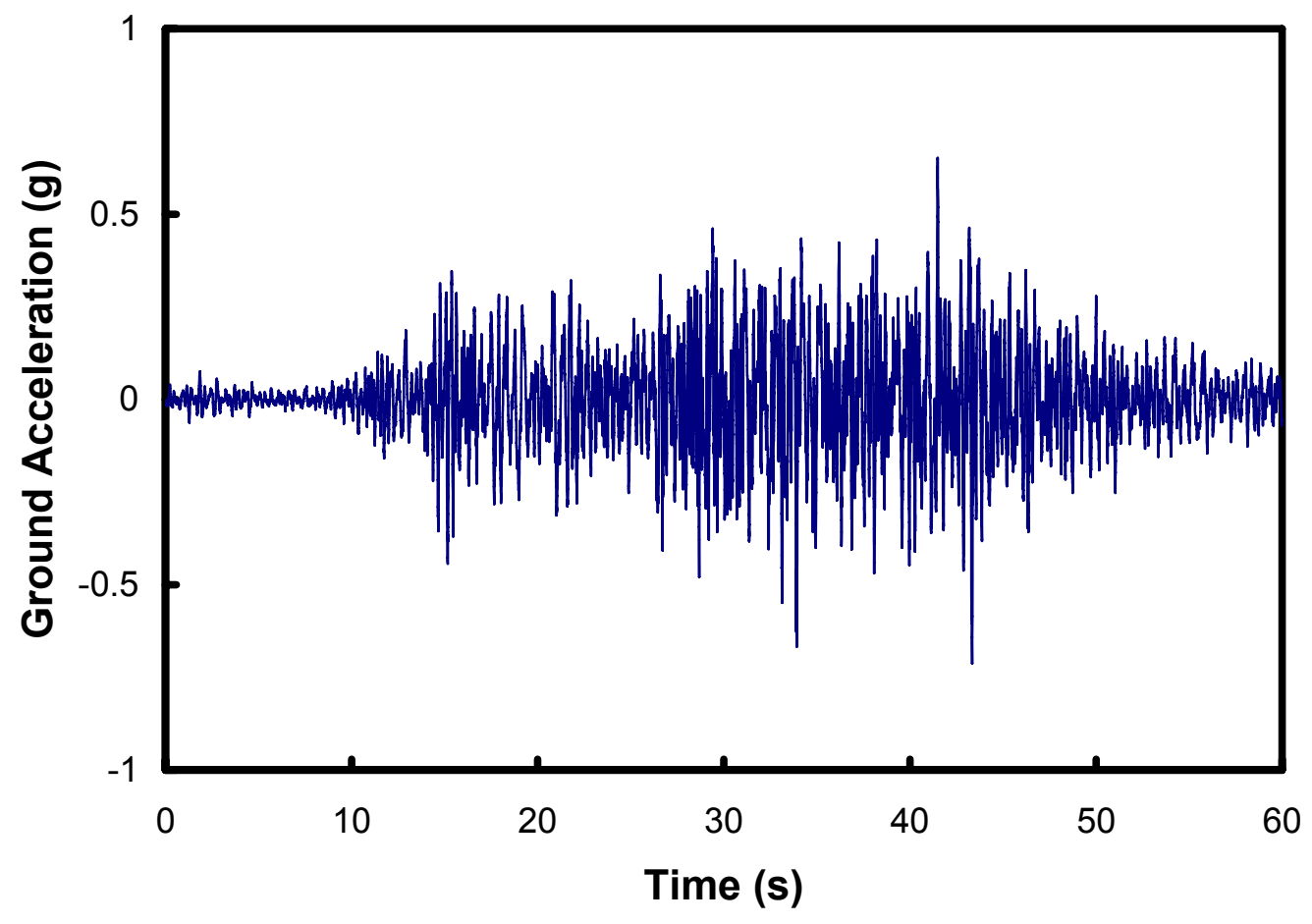

Fig. 4.92 Llollelo (1985) Ground Acceleration Record 


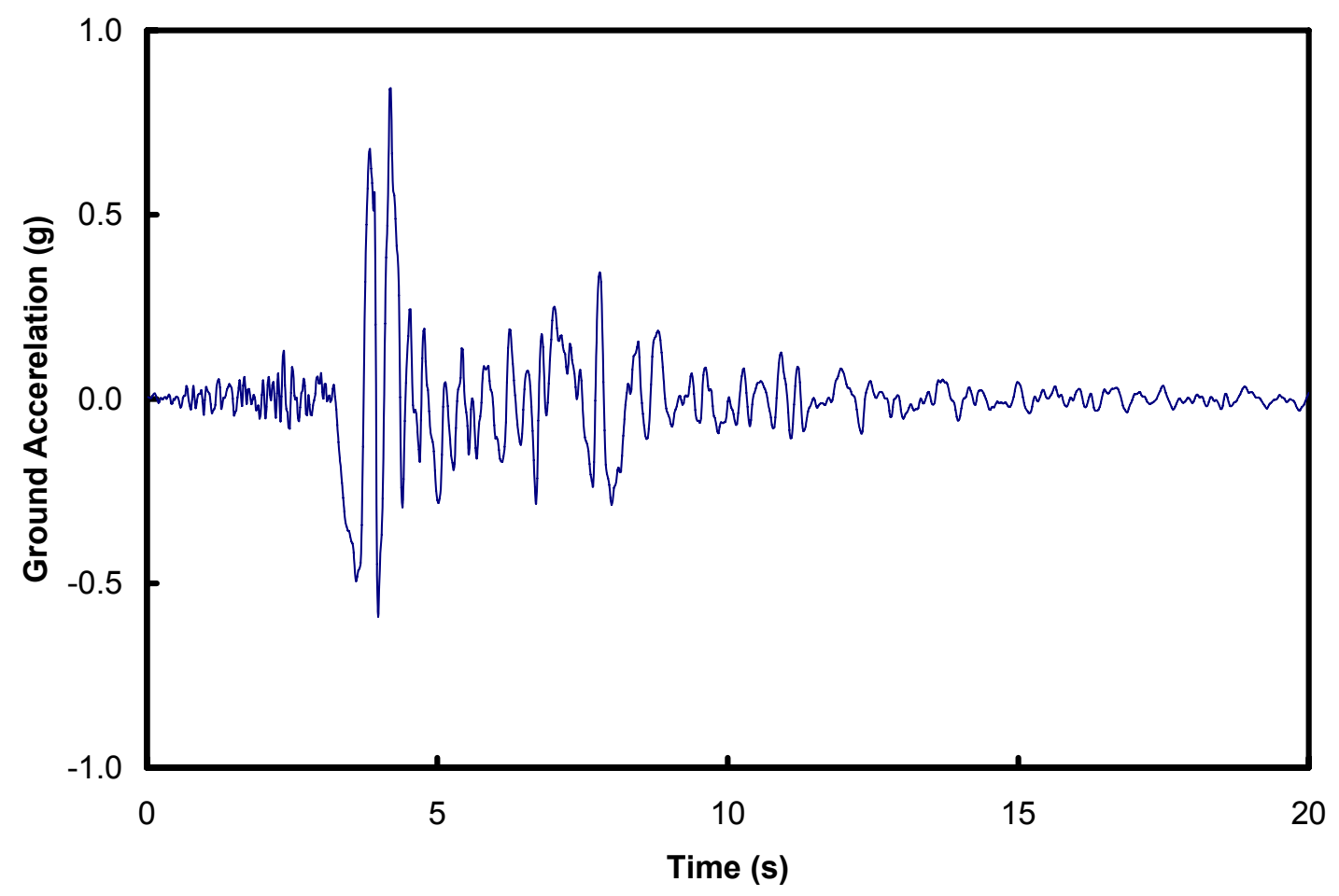

Fig. 4.93 Sylmar (1971) Ground Acceleration Record

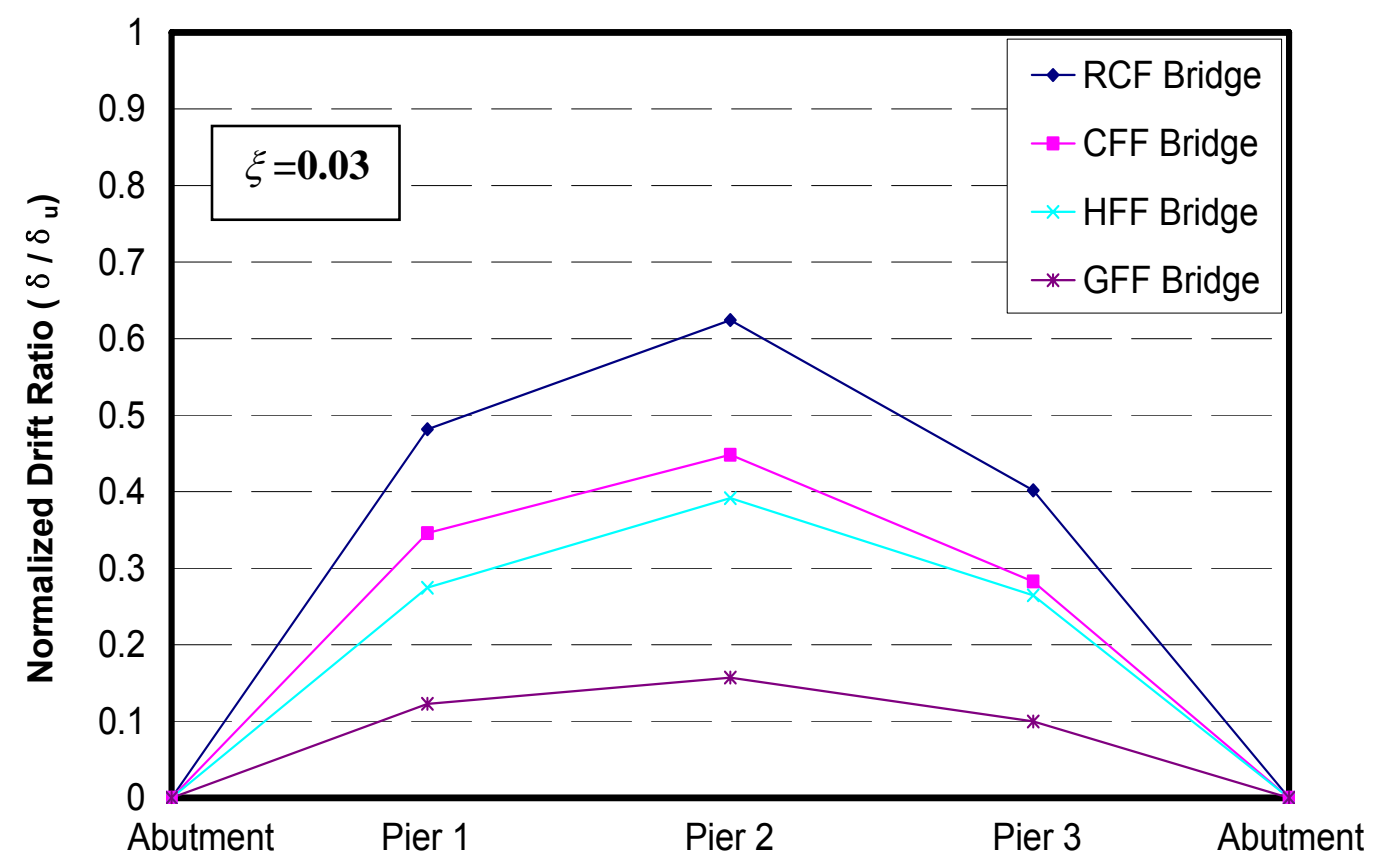

Fig. 4.94 Normalized Drift Ratio at Each Pier under Sylmar Earthquake at $\xi=0.03$ 


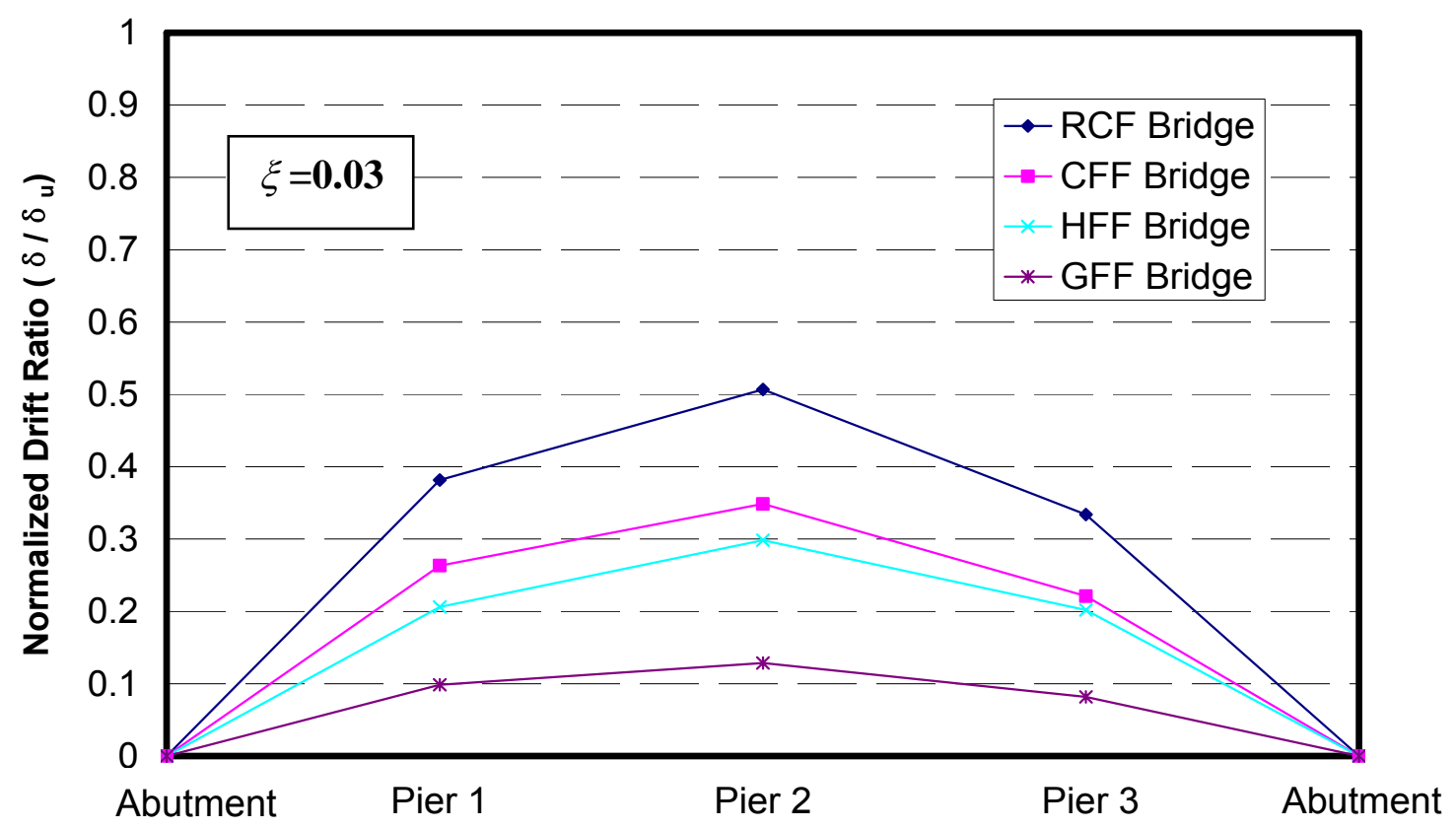

Fig. 4.95 Normalized Drift Ratio at Each Pier under Tabas Earthquake at $\xi=0.03$

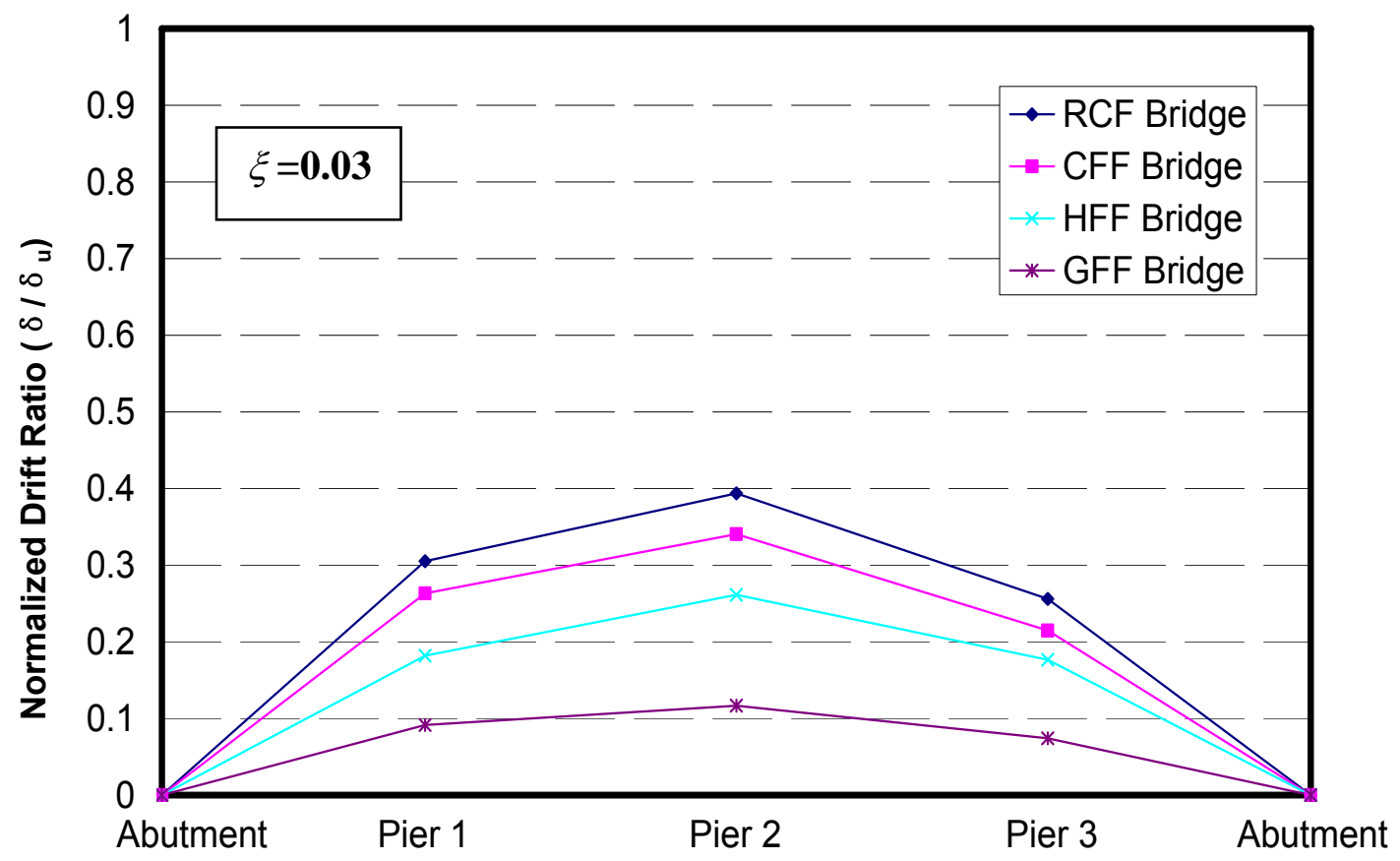

Fig. 4.96 Normalized Drift Ratio at Each Pier under Llolleo Earthquake at $\xi=0.03$ 


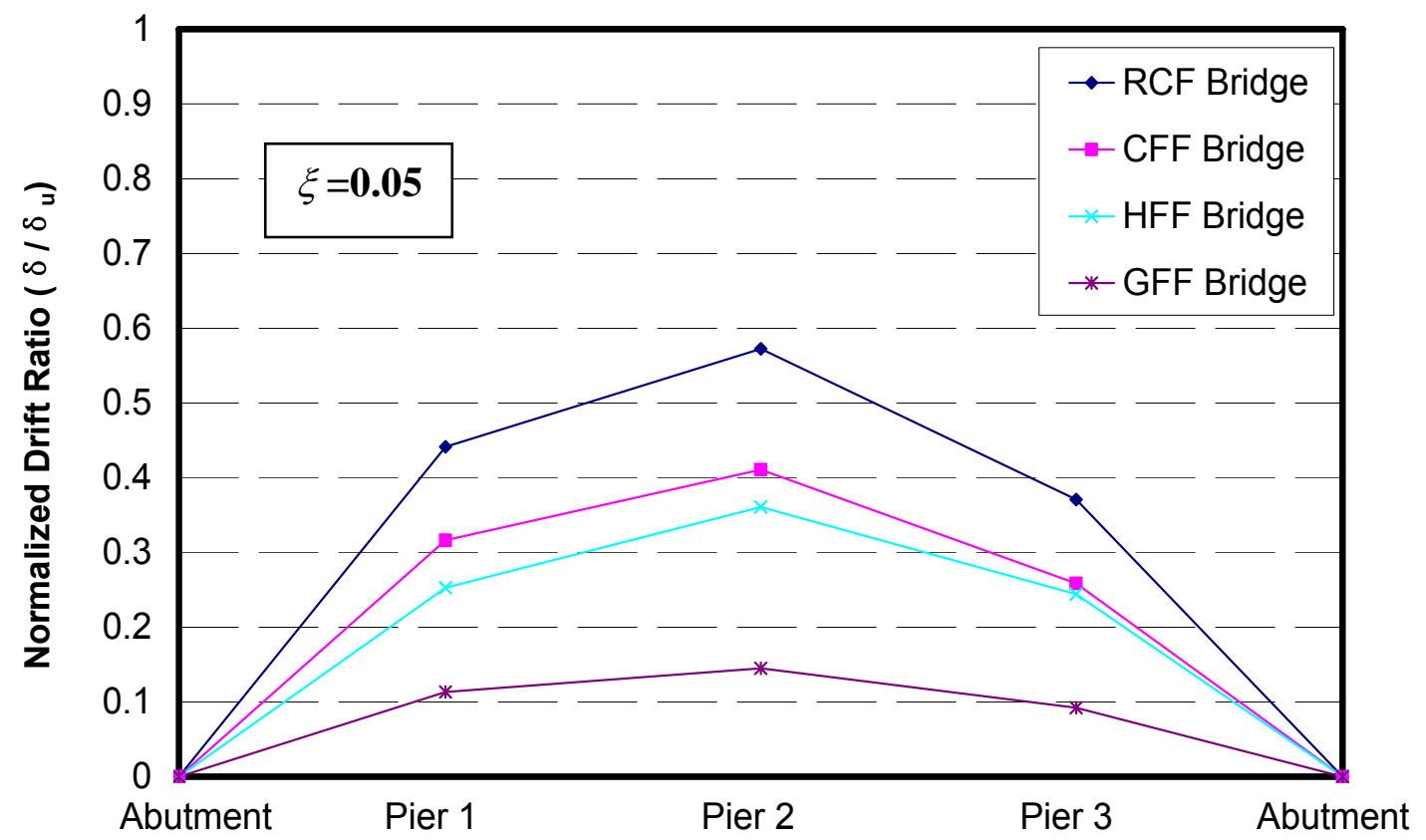

Fig. 4.97 Normalized Drift Ratio at Each Pier under Sylmar Earthquake at $\xi=0.05$

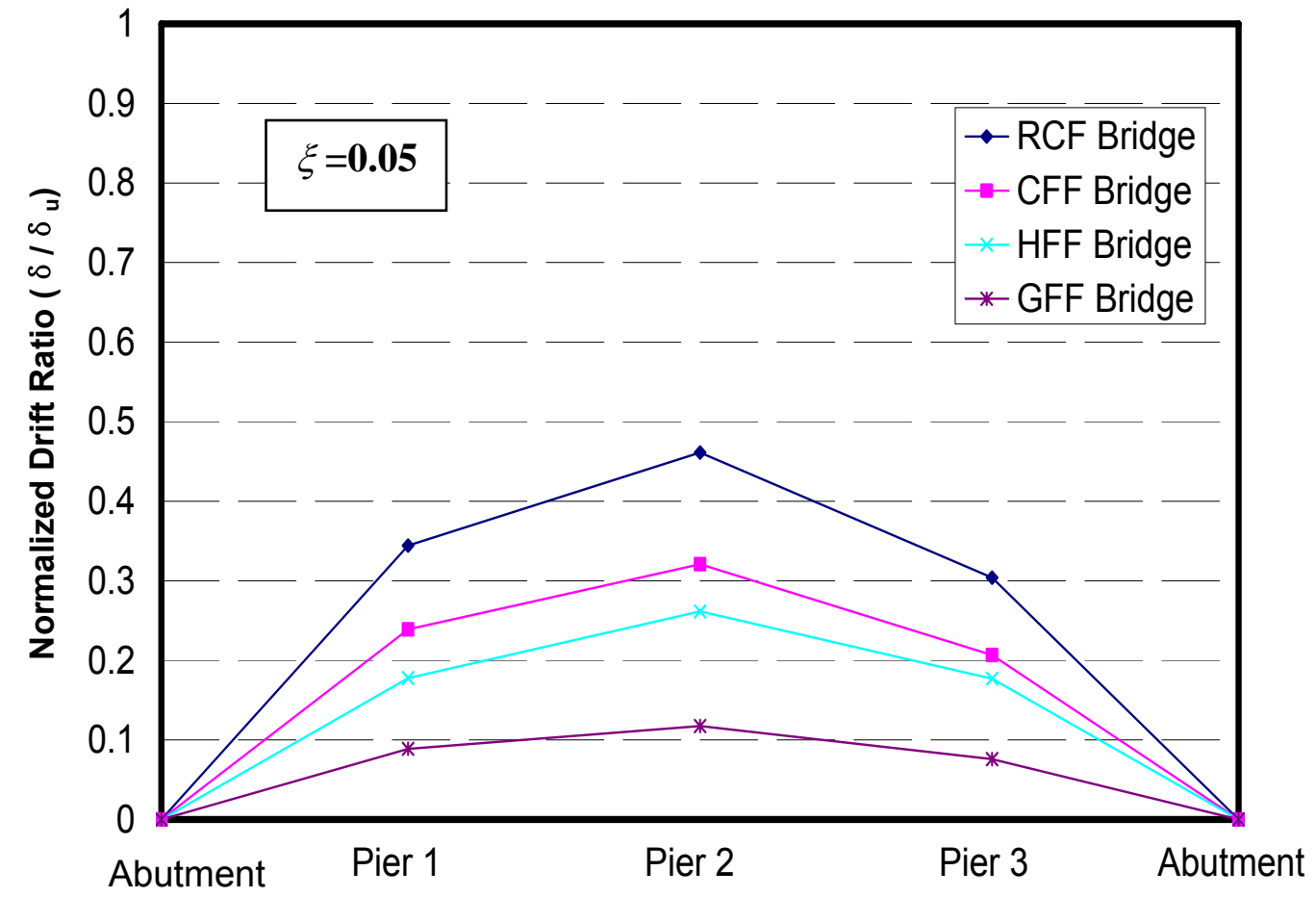

Fig. 4.98 Normalized Drift Ratio at Each Pier under Tabas Earthquake at $\xi=0.05$ 


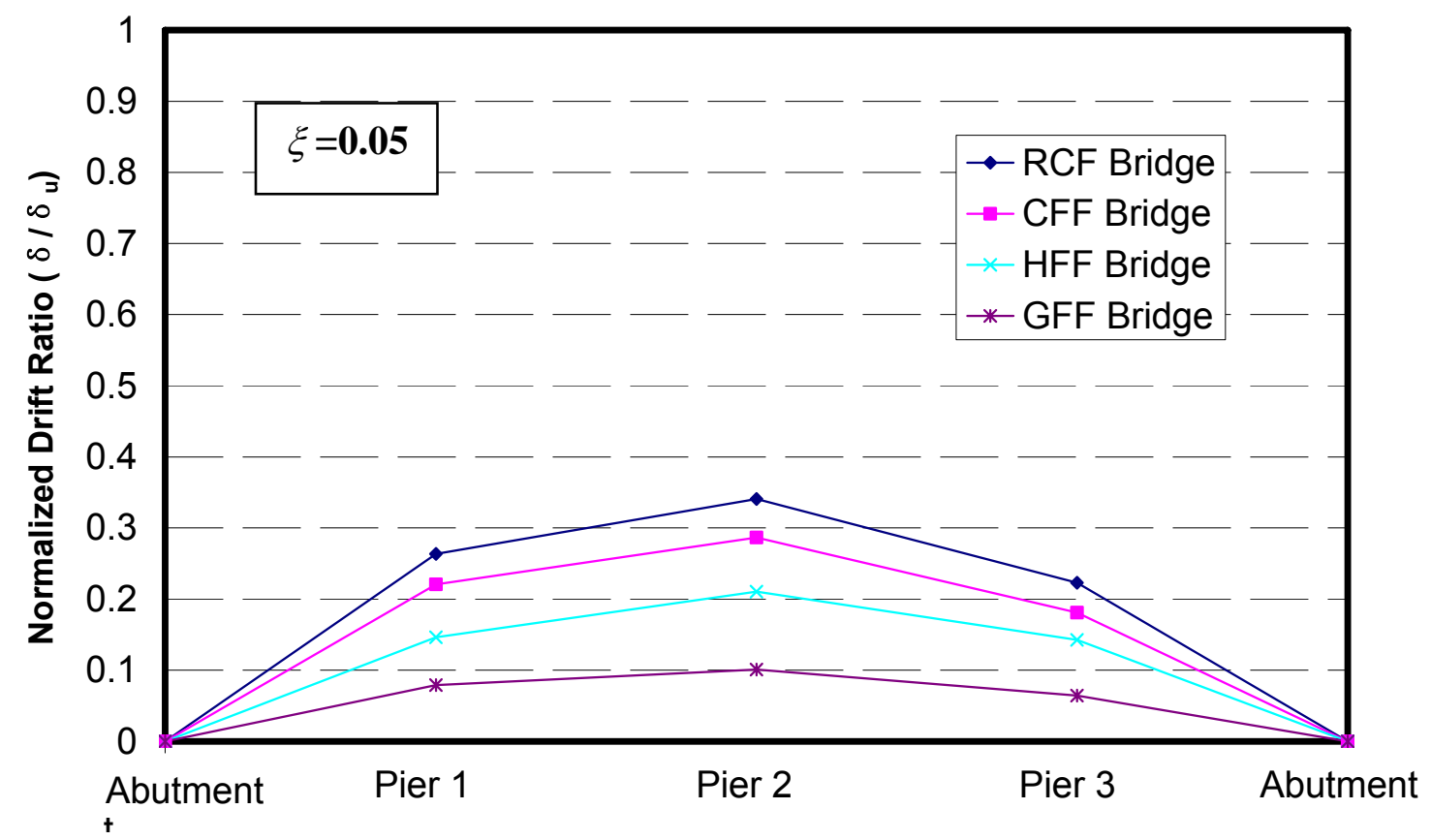

Fig. 4.99 Normalized Drift Ratio at Each Pier under Llolleo Earthquake at $\xi=0.05$

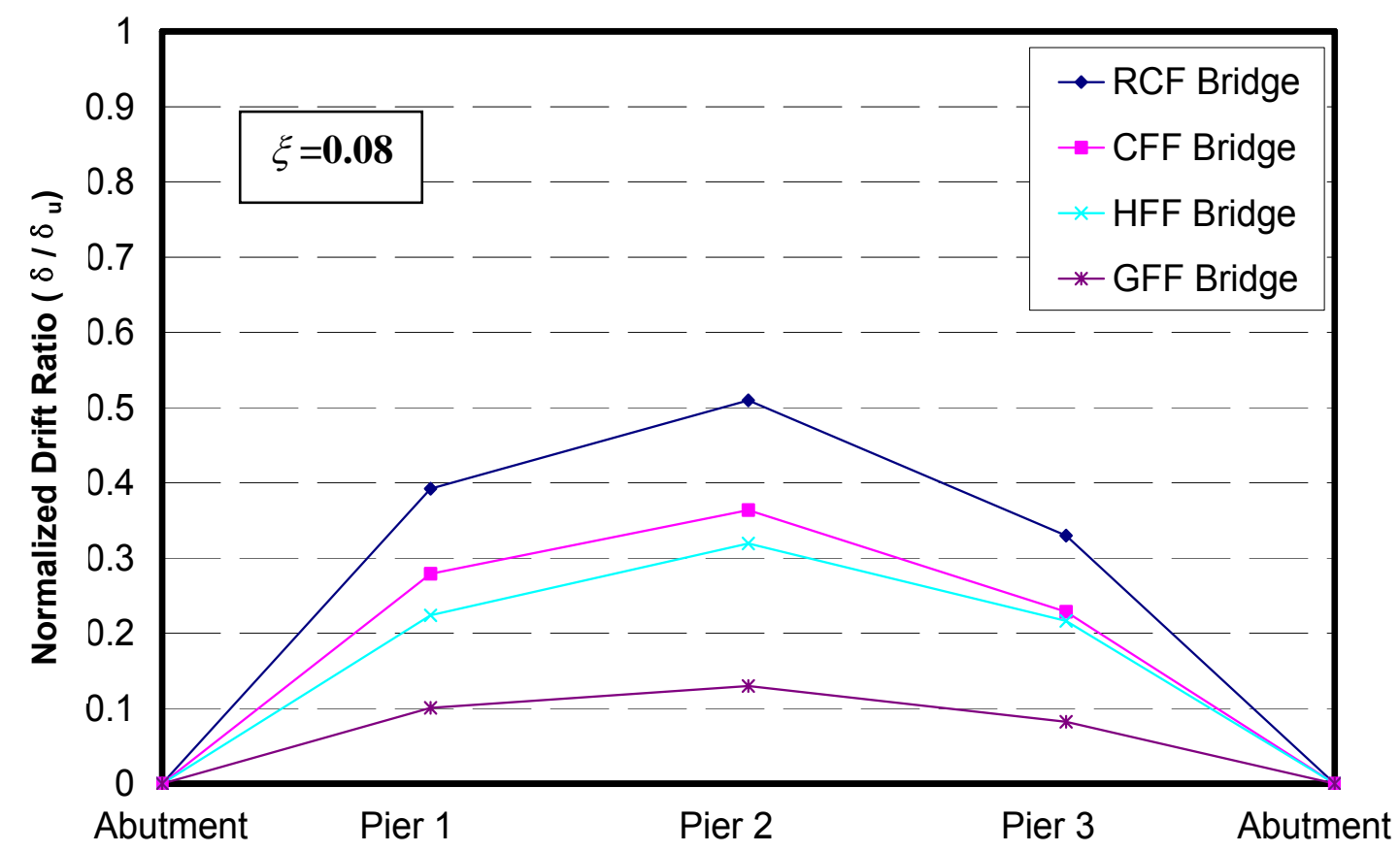

Fig. 4.100 Normalized Drift Ratio at Each Pier under Sylmar Earthquake at $\xi=0.08$ 


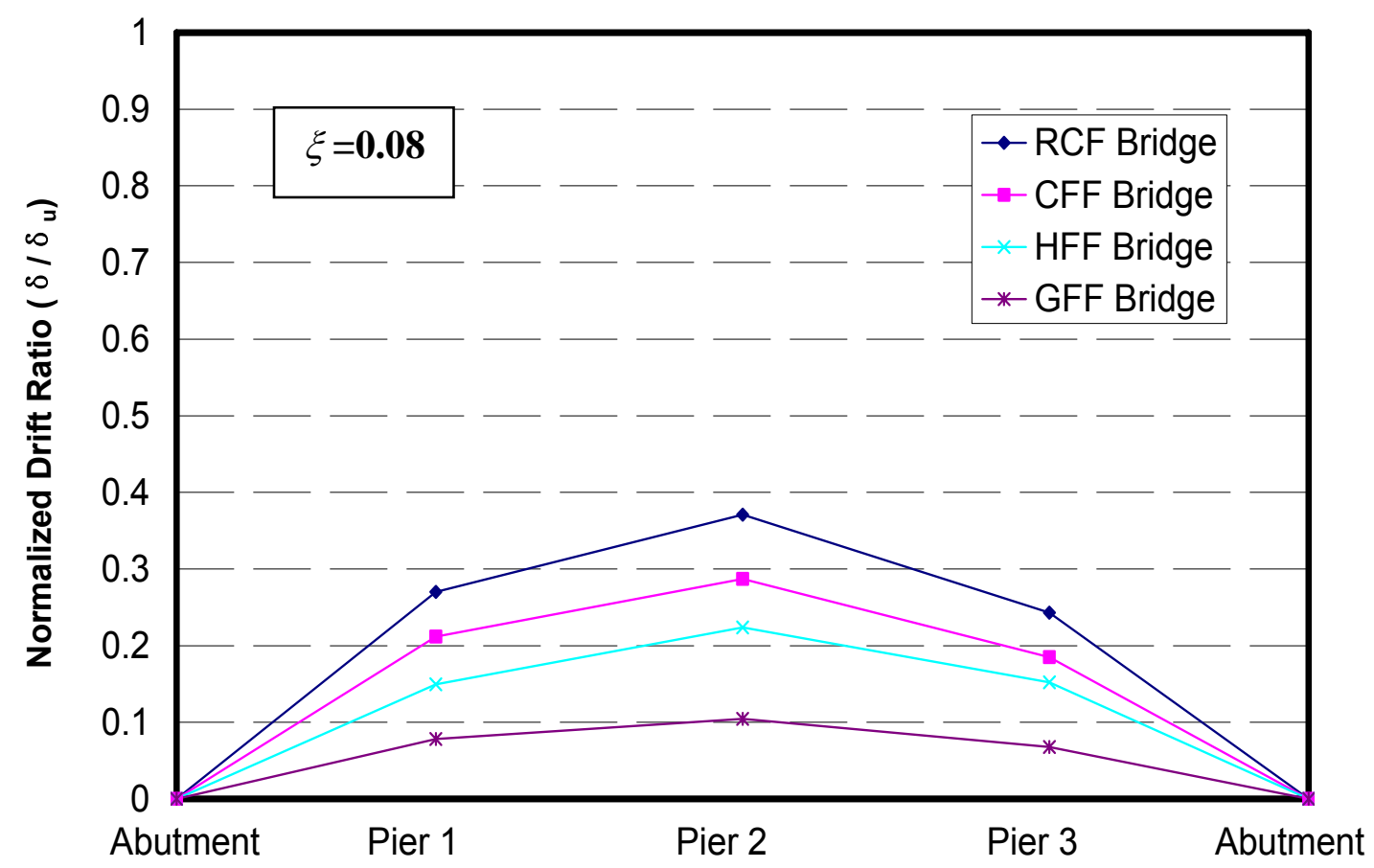

Fig. 4.101 Normalized Drift Ratio at Each Pier under Tabas Earthquake at $\xi=0.08$

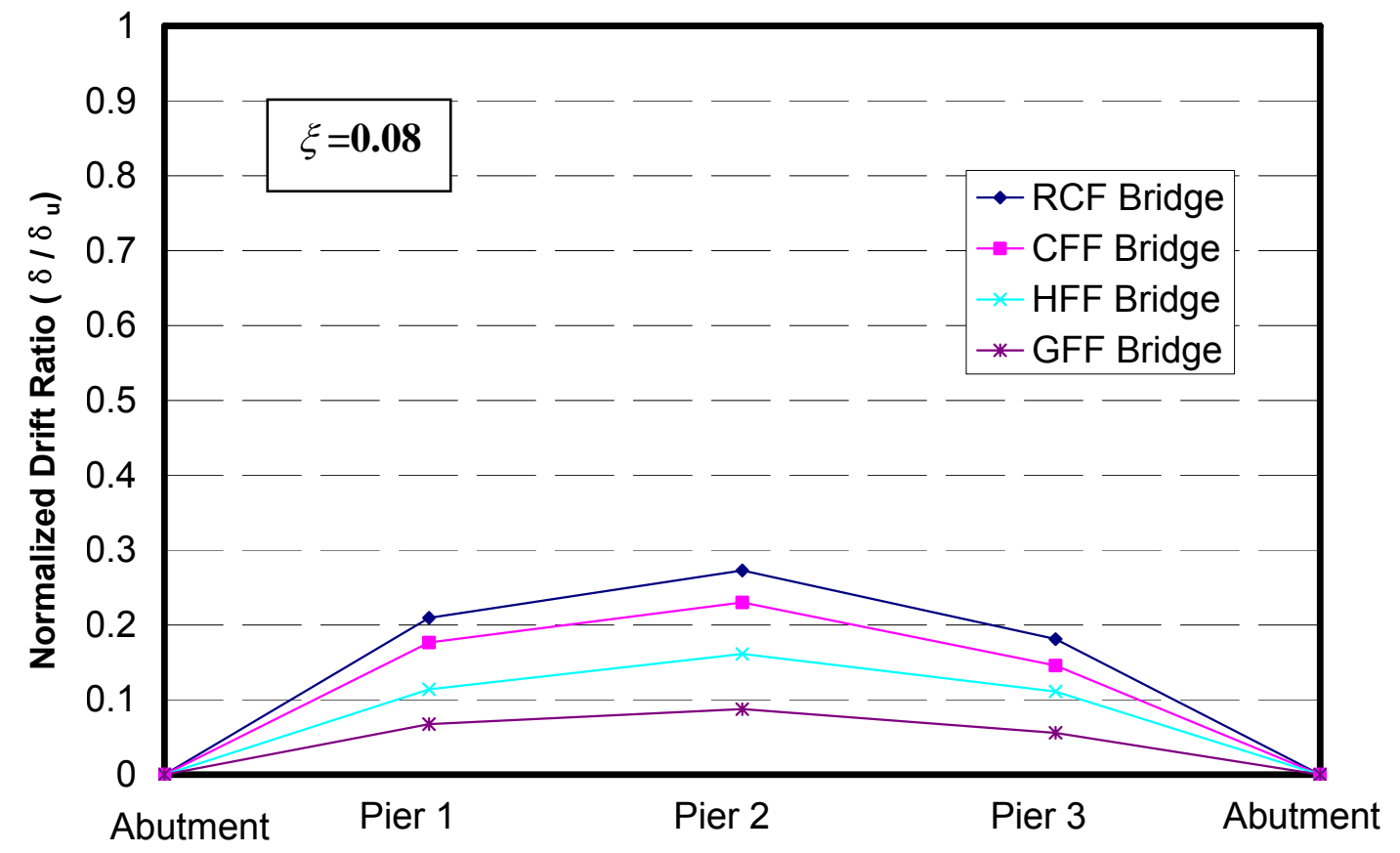

Fig. 4.102 Normalized Drift Ratio at Each Pier under Llolleo Earthquake at $\xi=0.08$ 


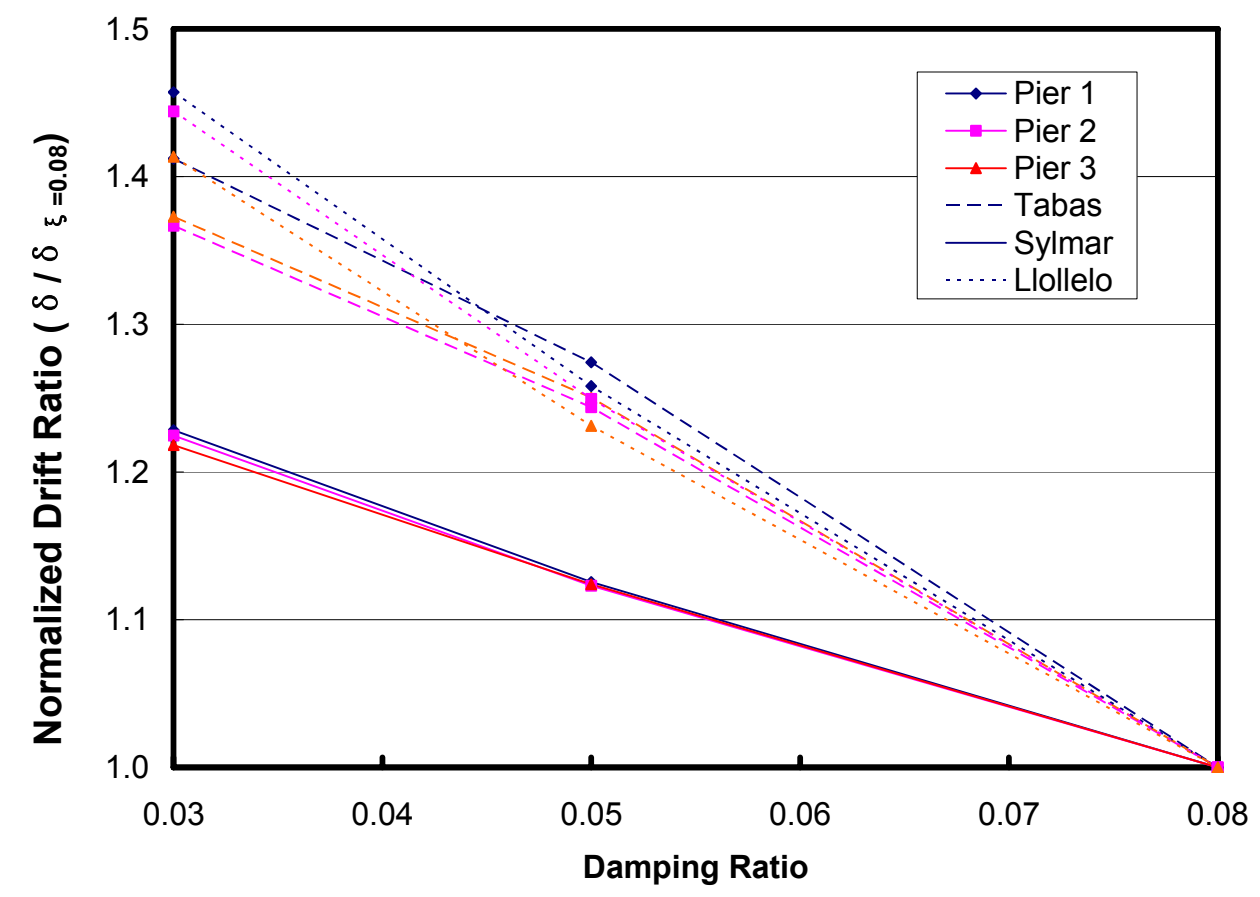

Fig. 4.103 Effect of Damping Ratio on Performance of RCF Bridge

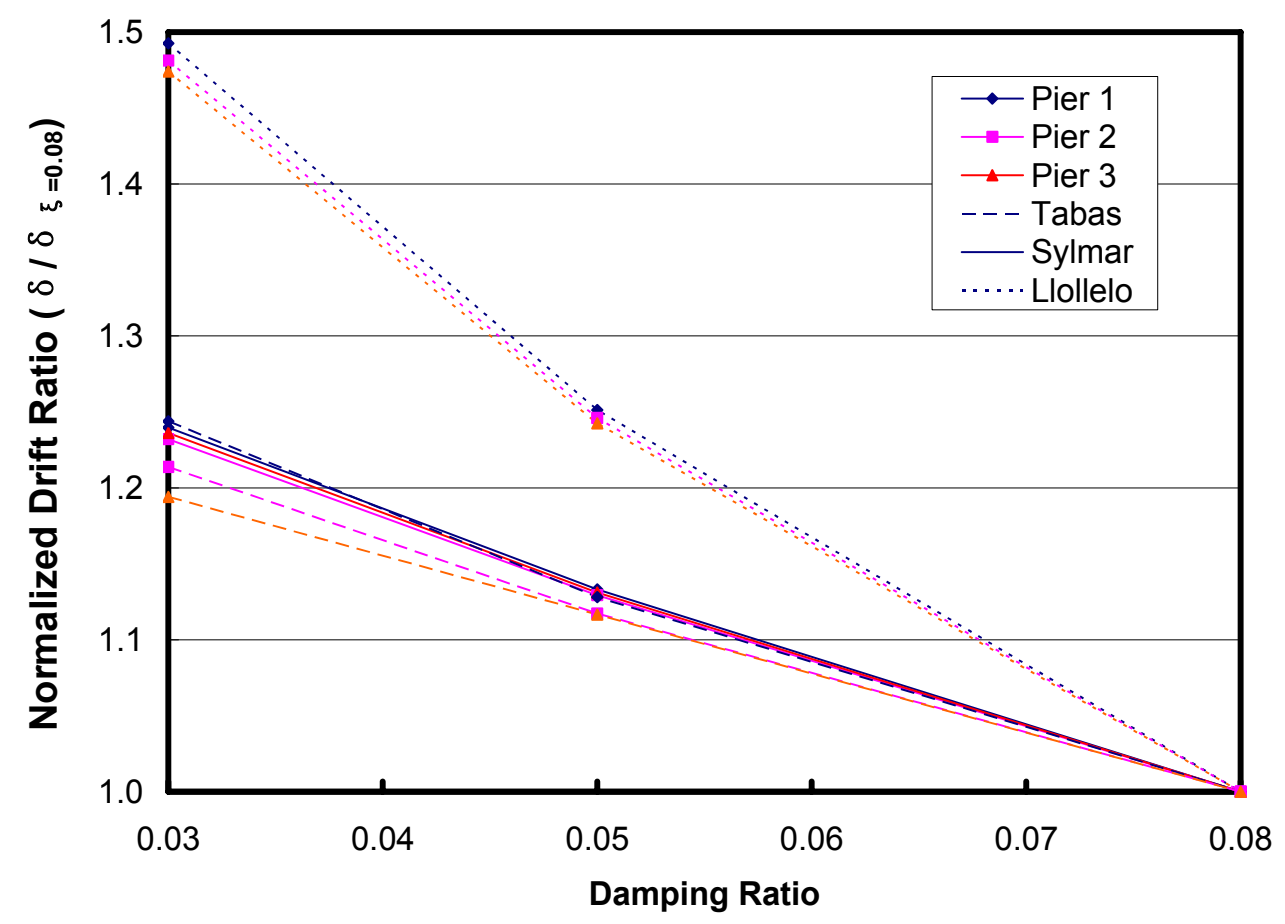

Fig. 4.104 Effect of Damping Ratio on Performance of CFF Bridge 


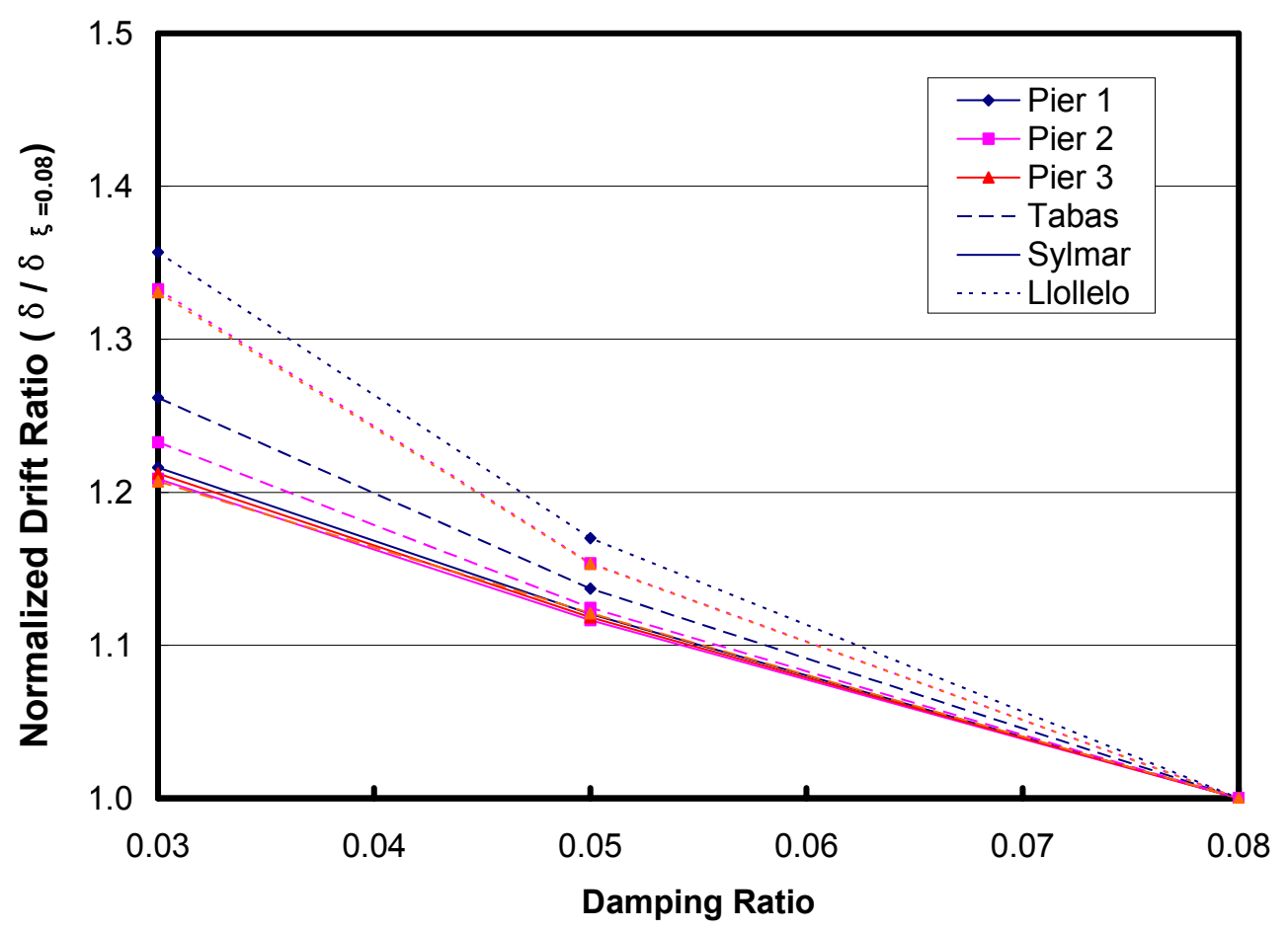

Fig. 4.105 Effect of Damping Ratio on Performance of GFF Bridge

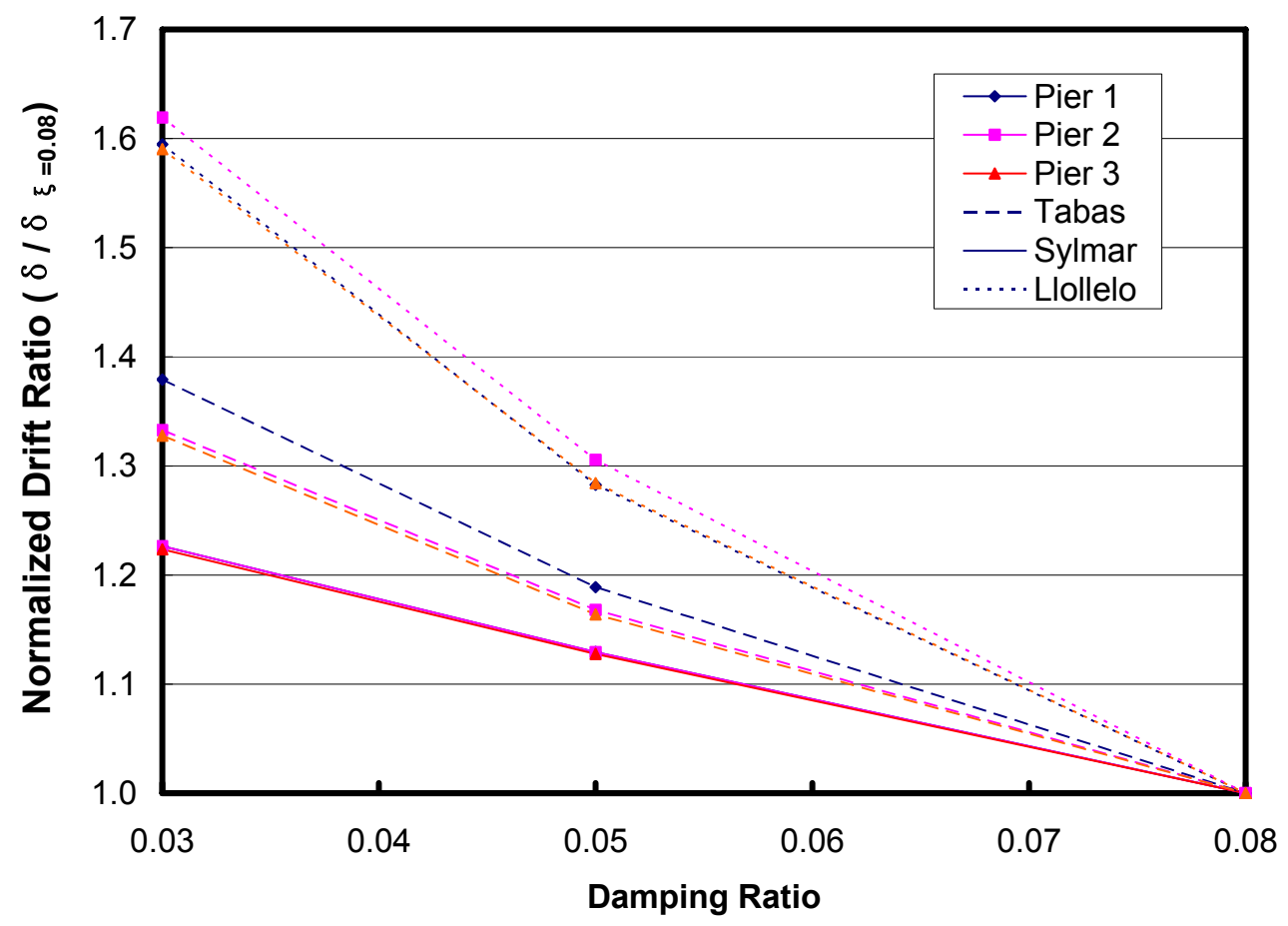

Fig. 4.106 Effect of Damping Ratio on Performance of HFF Bridge 


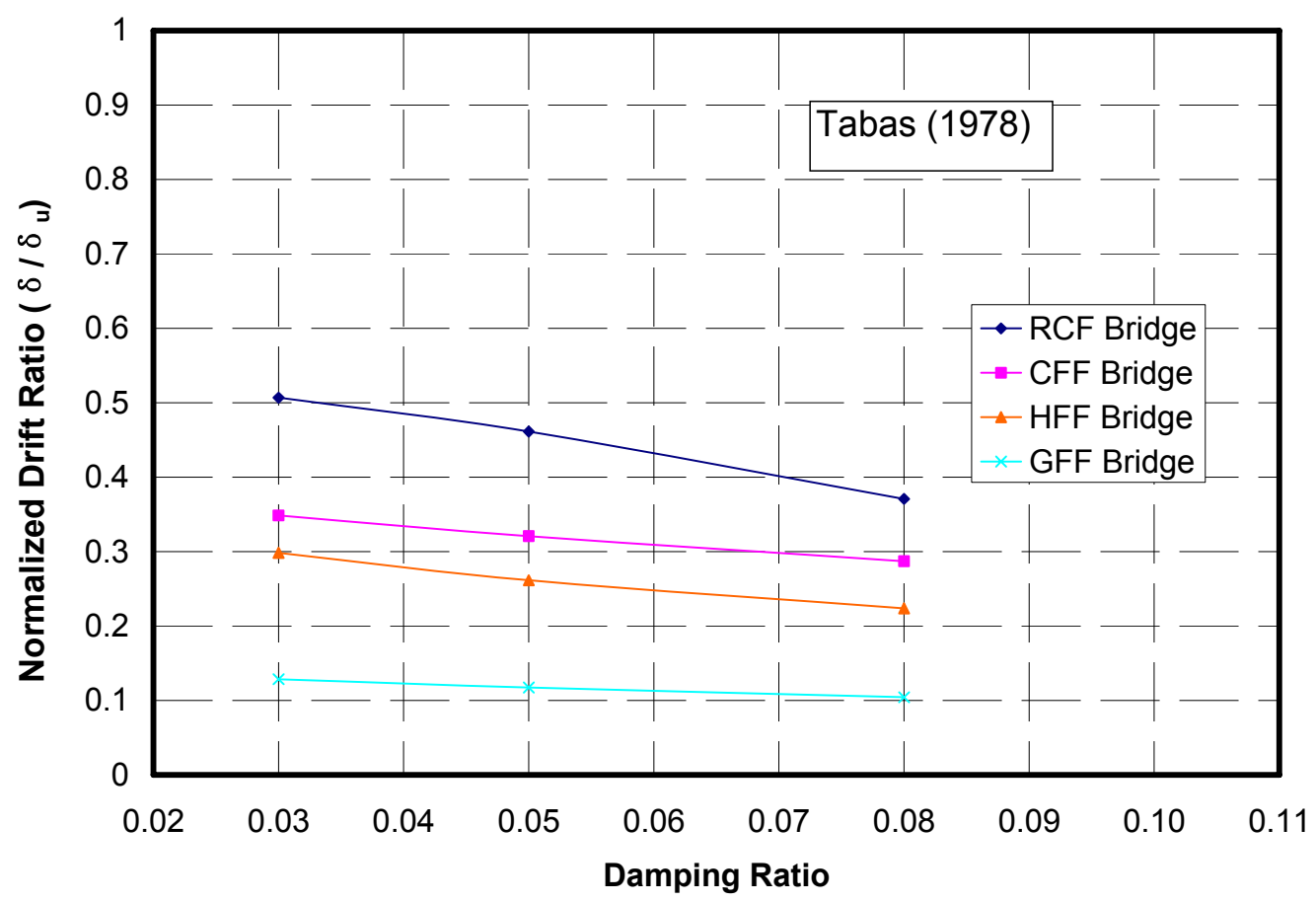

Fig. 4.107 Effect of Damping Ratio on Normalized Drift Ratio of Pier 2 for All Bridges under Tabas (1978) Earthquake

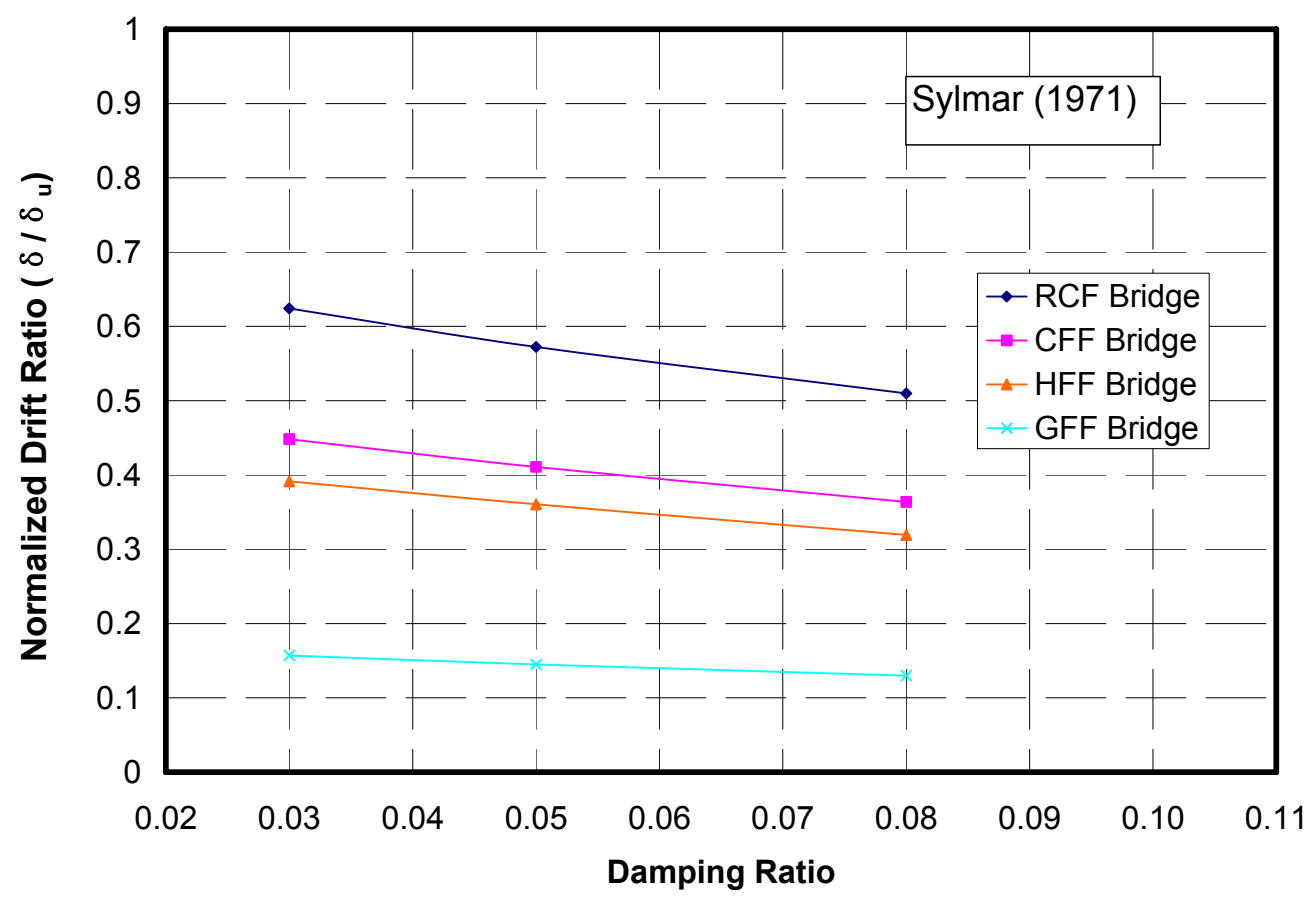

Fig. 4.108 Effect of Damping Ratio on Normalized Drift Ratio of Pier 2 for All Bridges under Sylmar (1971) Earthquake 


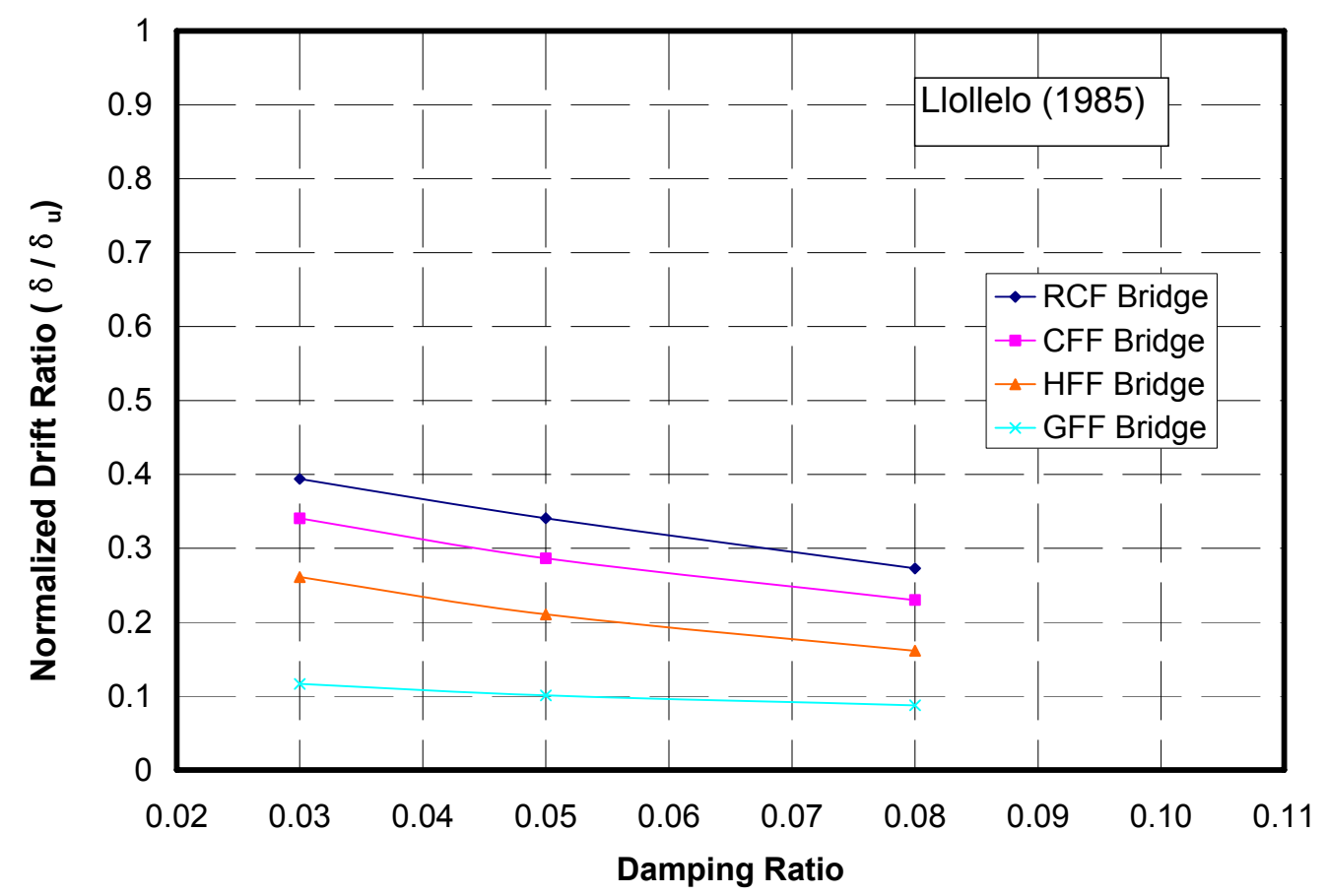

Fig. 4.109 Effect of Damping Ratio on Normalized Drift Ratio of Pier 2 for All Bridges under Llollello (1985) Earthquake

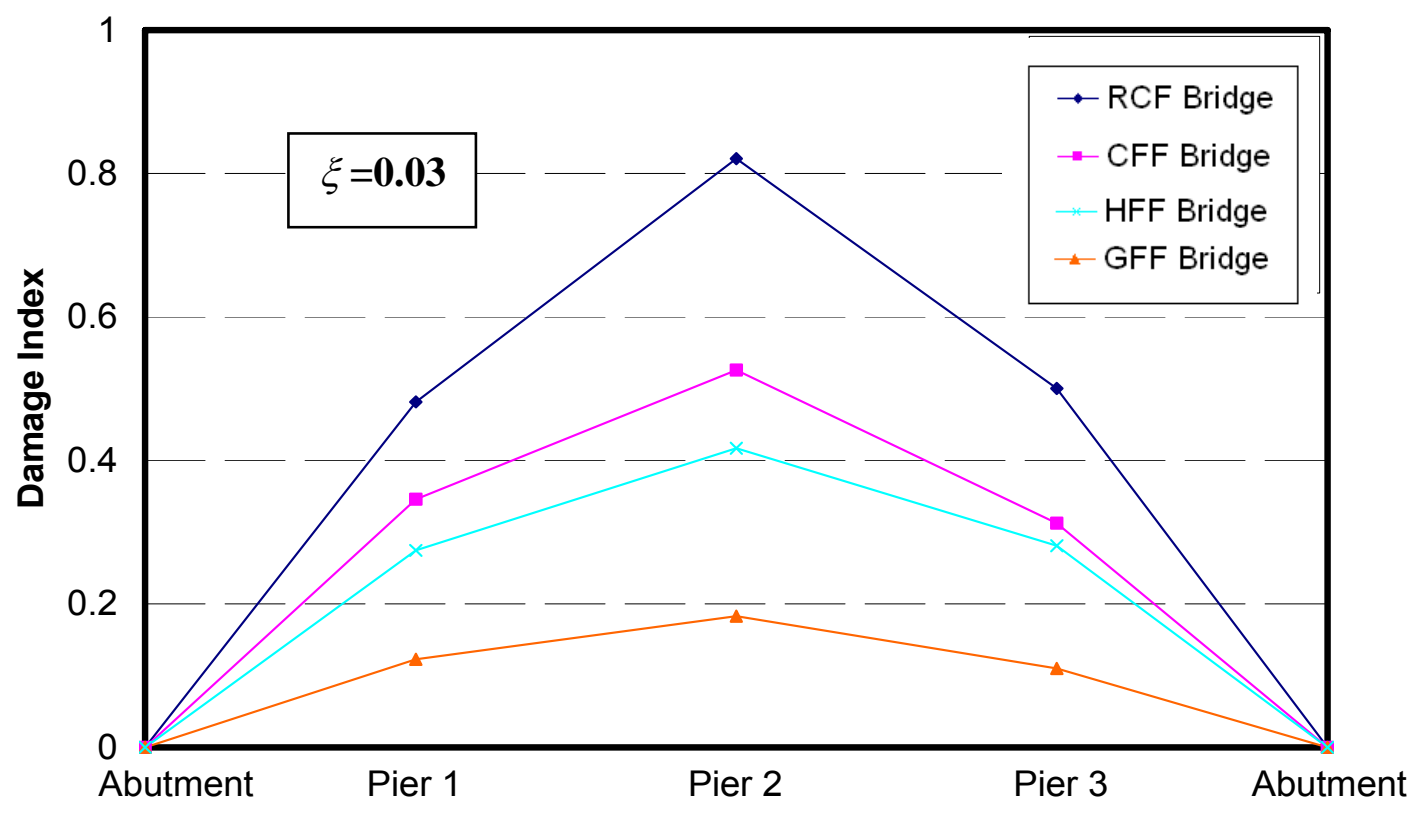

Fig. 4.110 Damage Index at Each Pier under Sylmar Earthquake at $\xi=0.03$ 


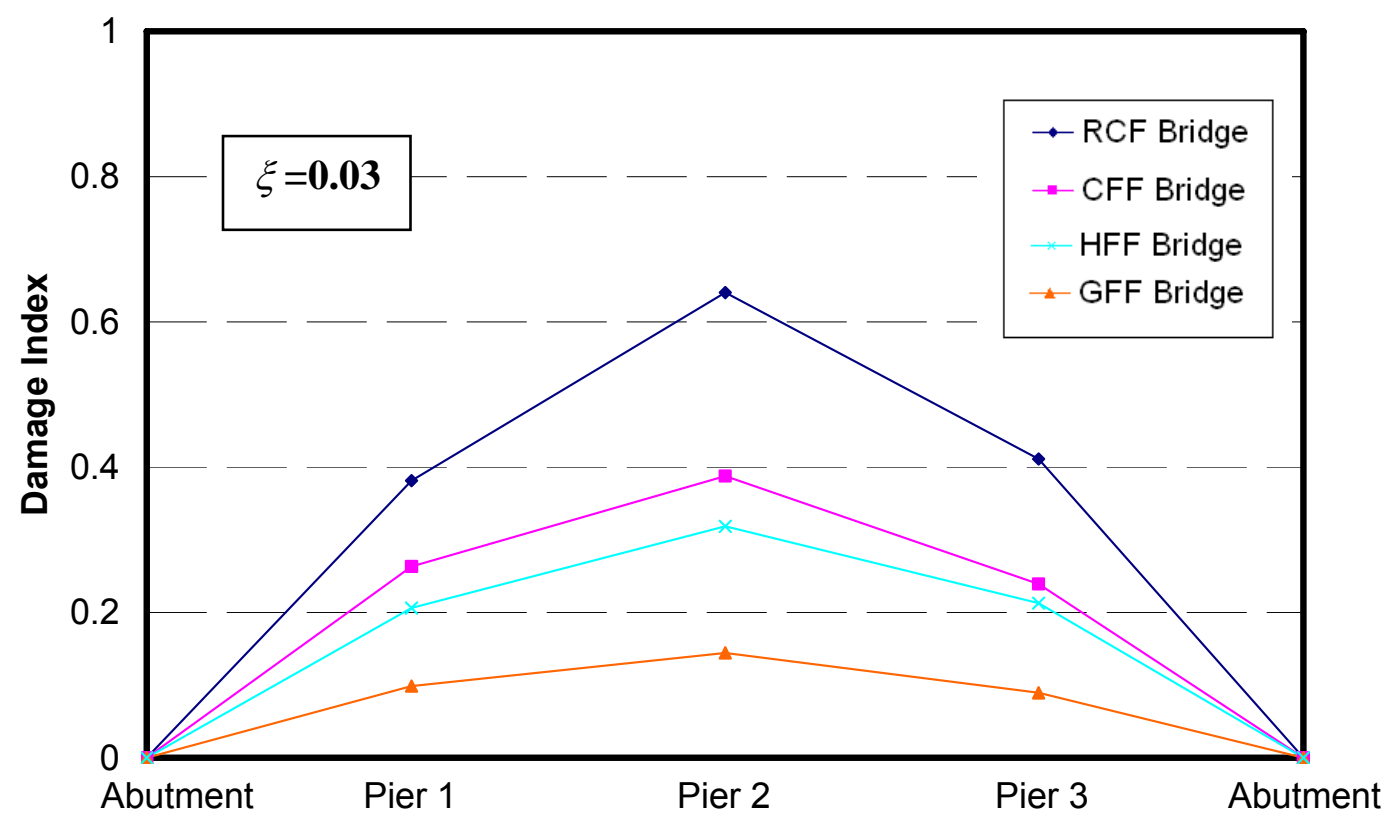

Fig. 4.111 Damage Index at Each Pier under Tabas Earthquake at $\xi=0.03$

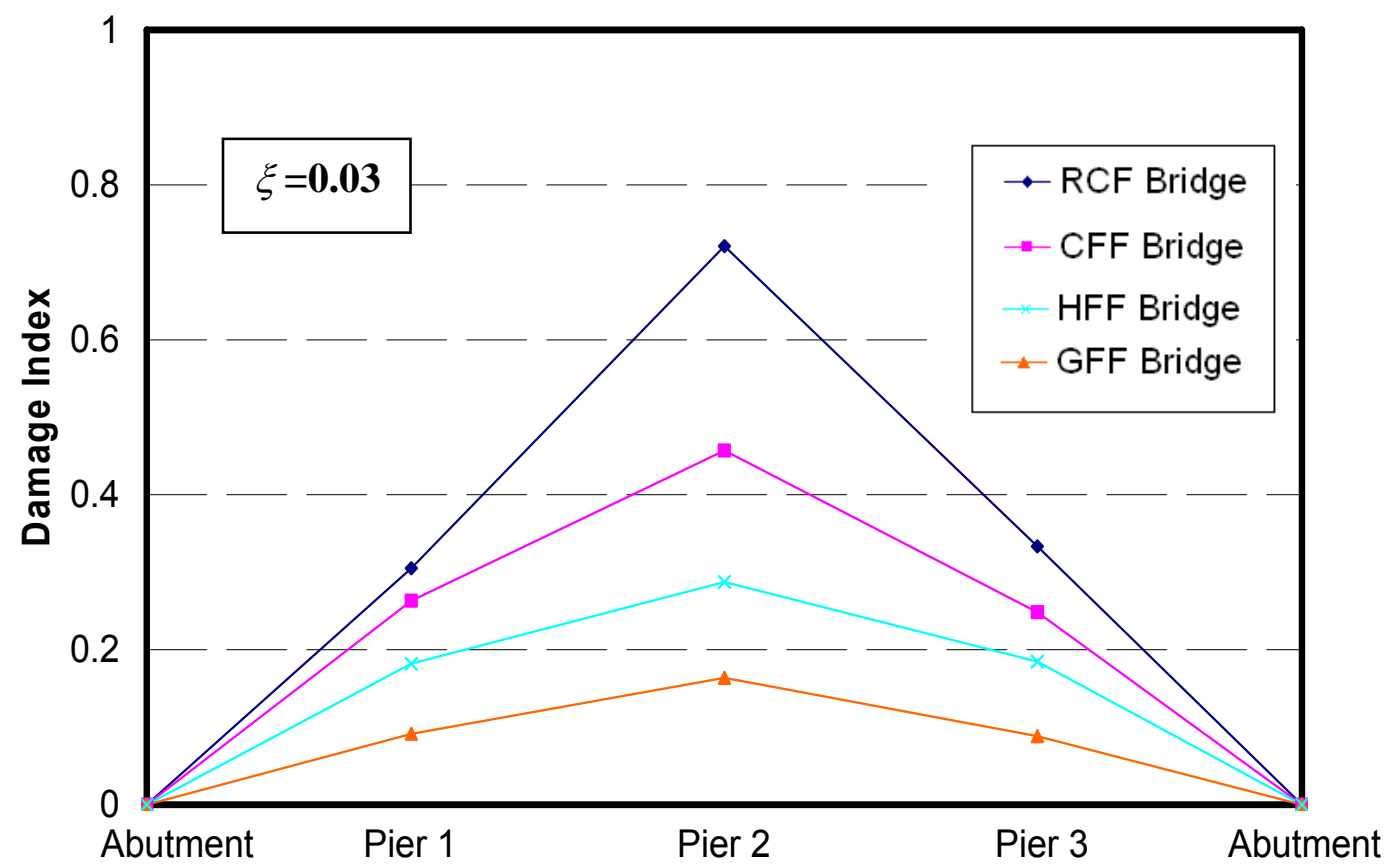

Fig. 4.112 Damage Index at Each Pier under Llollelo Earthquake at $\xi=0.03$ 


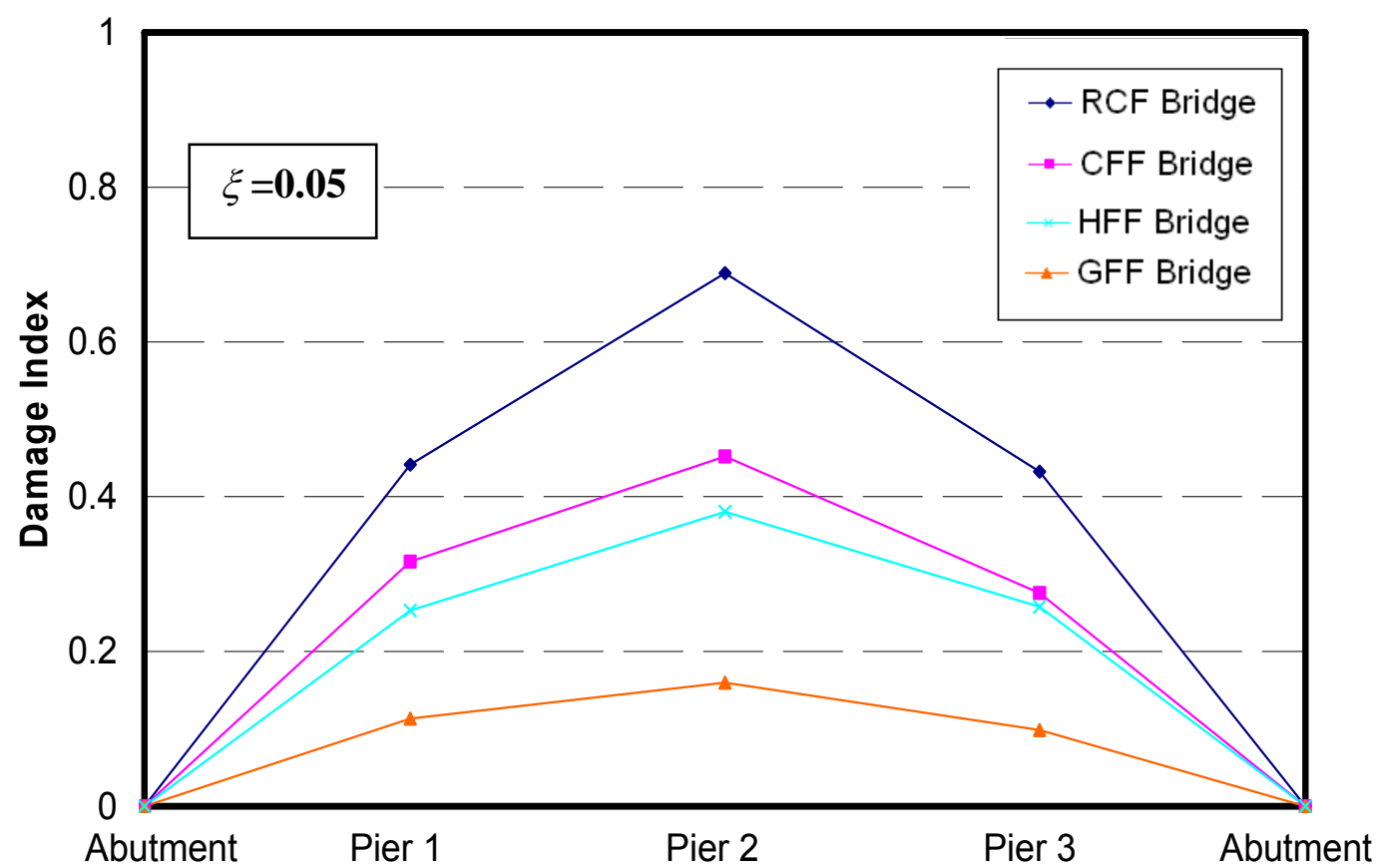

Fig. 4.113 Damage Index at Each Pier under Sylmar Earthquake at $\xi=0.05$

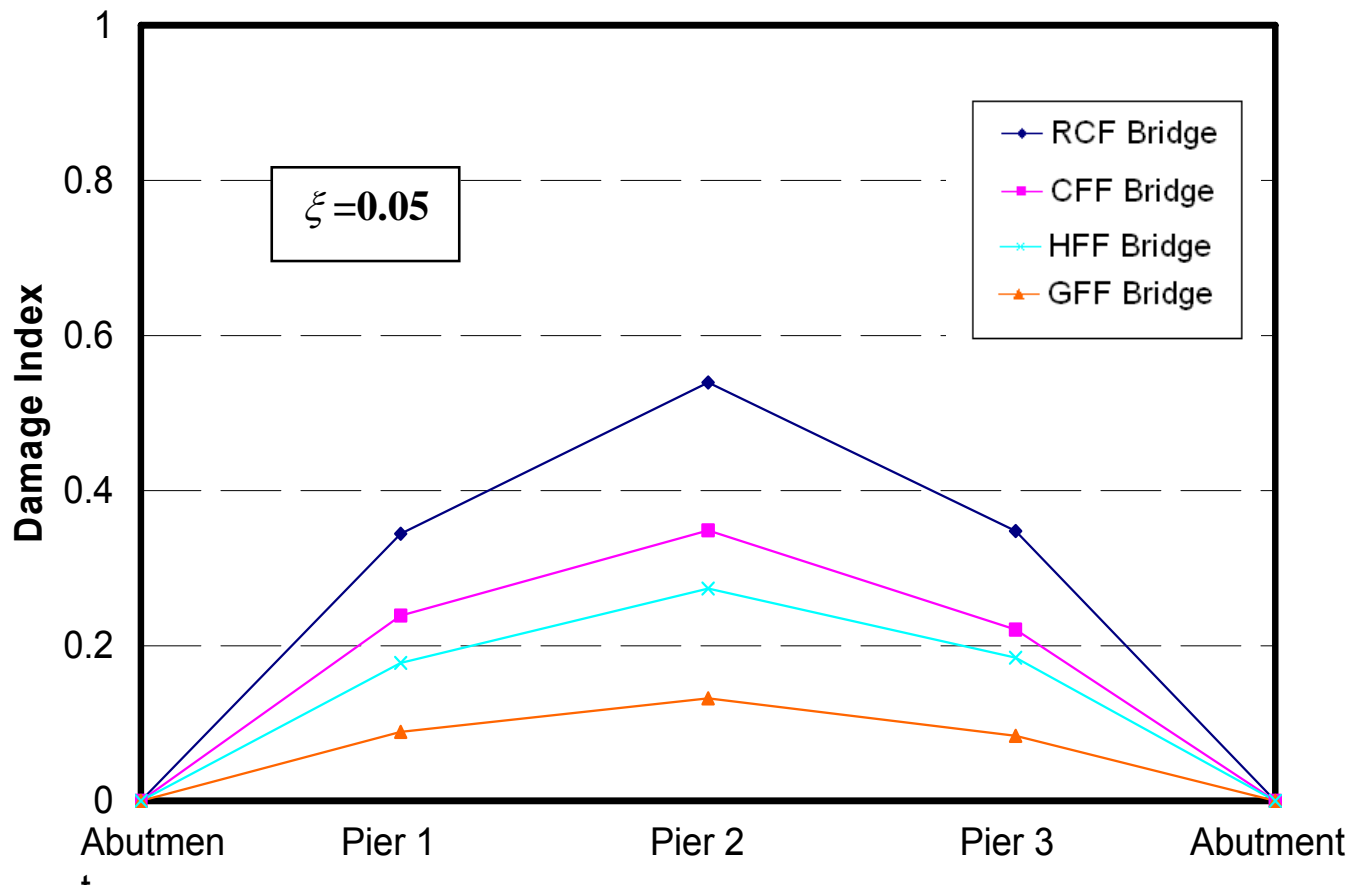

Fig. 4.114 Damage Index at Each Pier under Tabas Earthquake at $\xi=0.05$ 


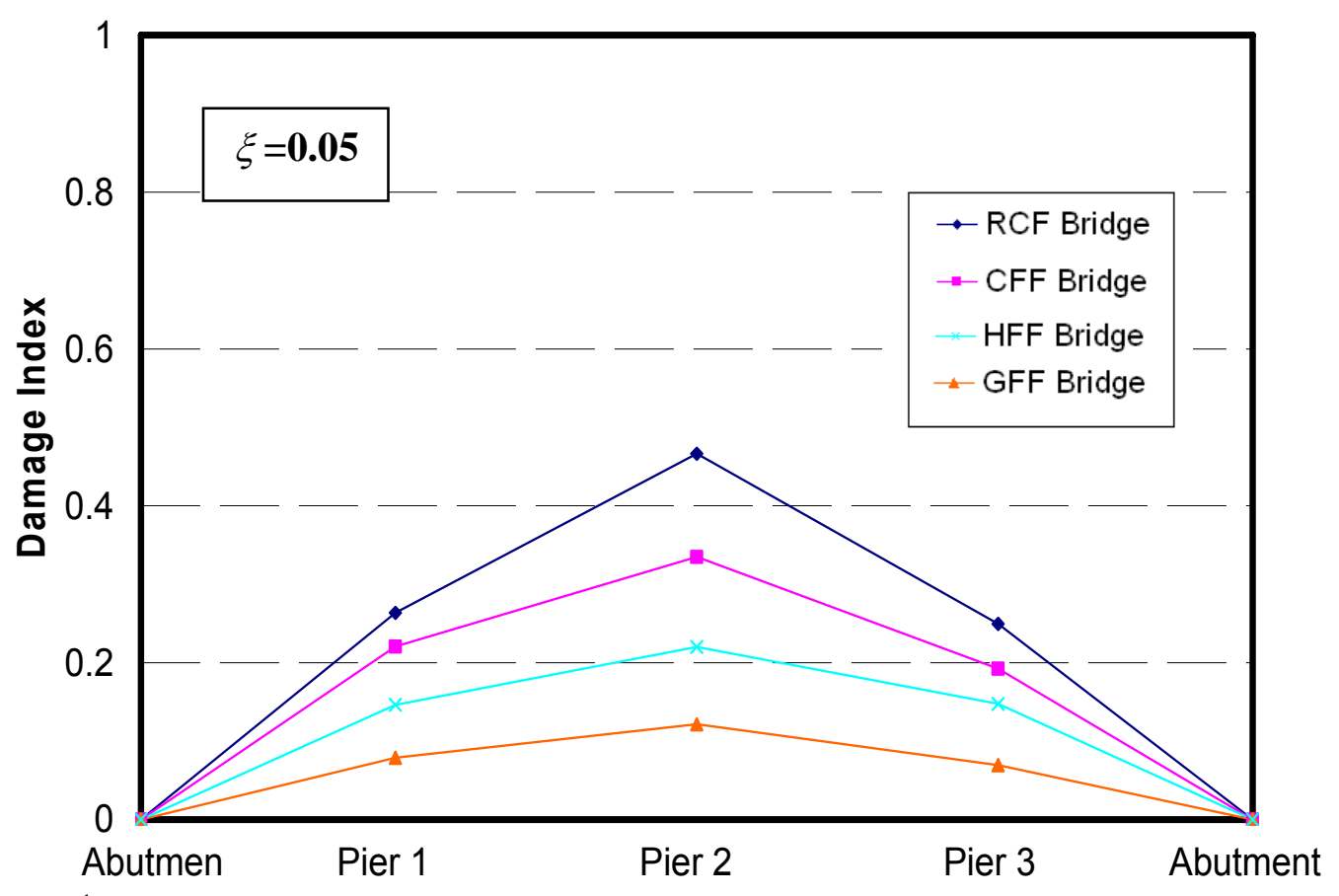

Fig. 4.115 Damage Index at Each Pier under Llolleo Earthquake at $\xi=0.05$

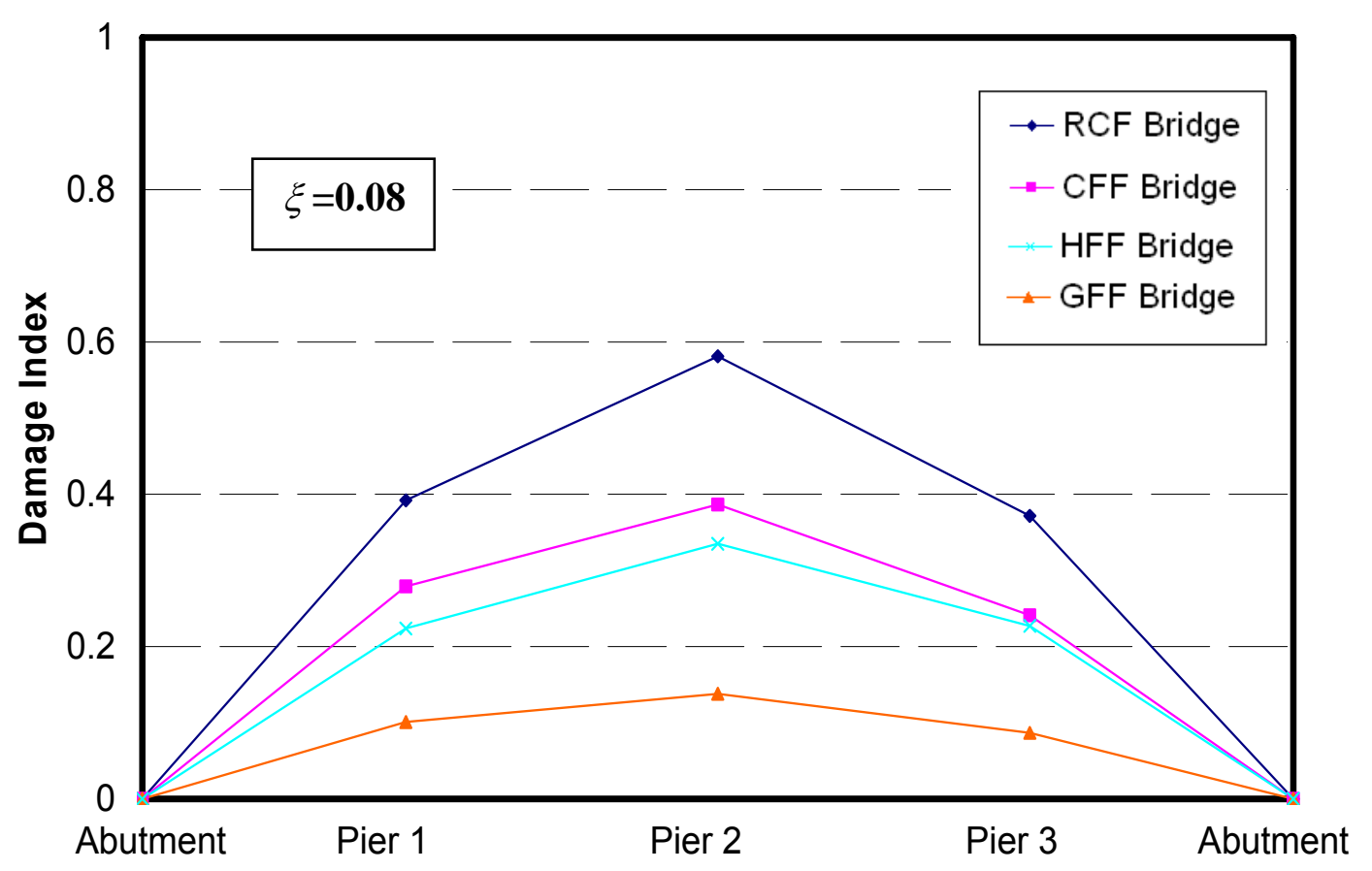

Fig. 4.116 Damage Index at Each Pier under Sylmar Earthquake at $\xi=0.08$ 


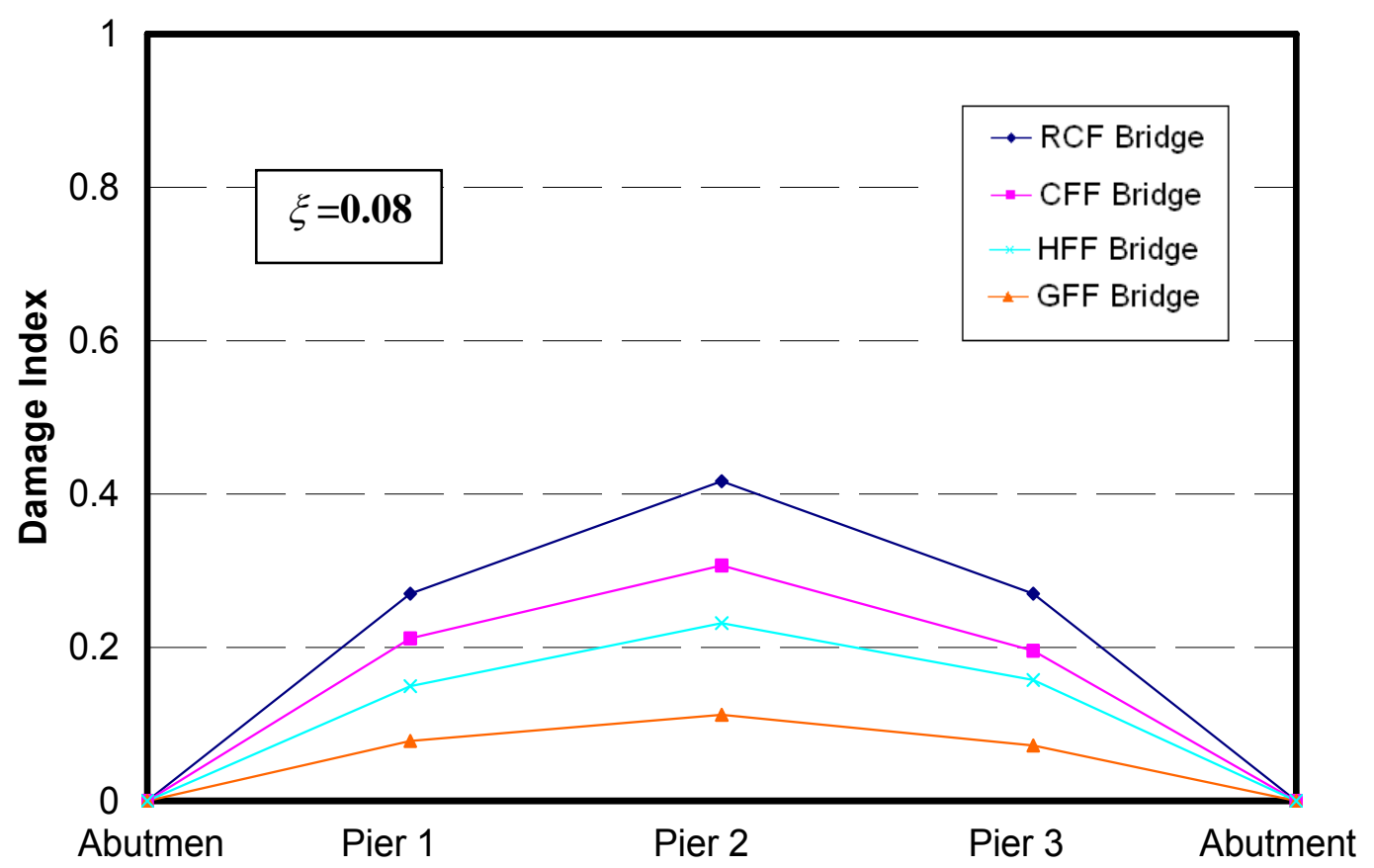

Fig. 4.117 Damage Index at Each Pier under Tabas Earthquake at $\xi=0.08$

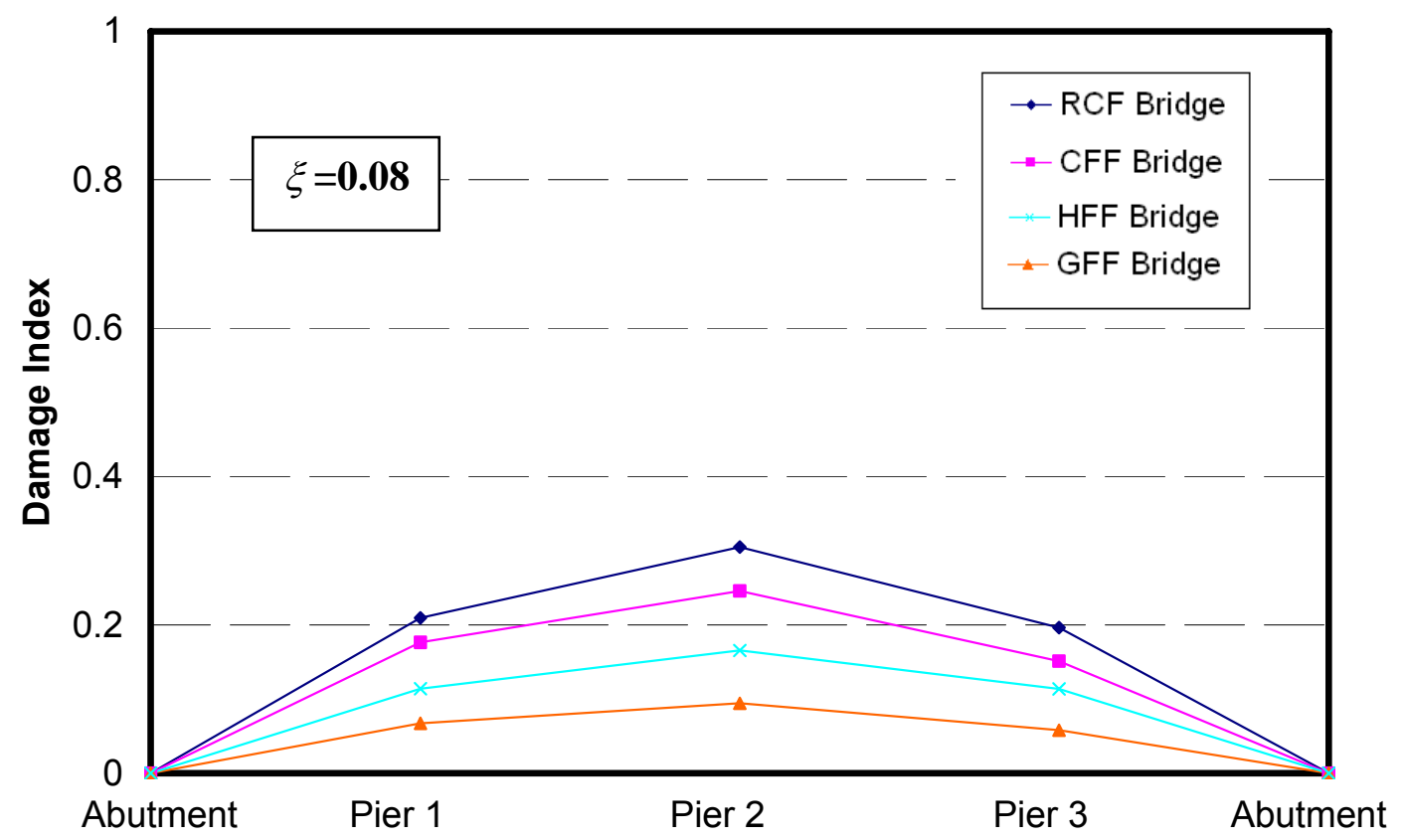

Fig. 4.118 Damage Index at Each Pier under Llolleo Earthquake at $\xi=0.08$ 


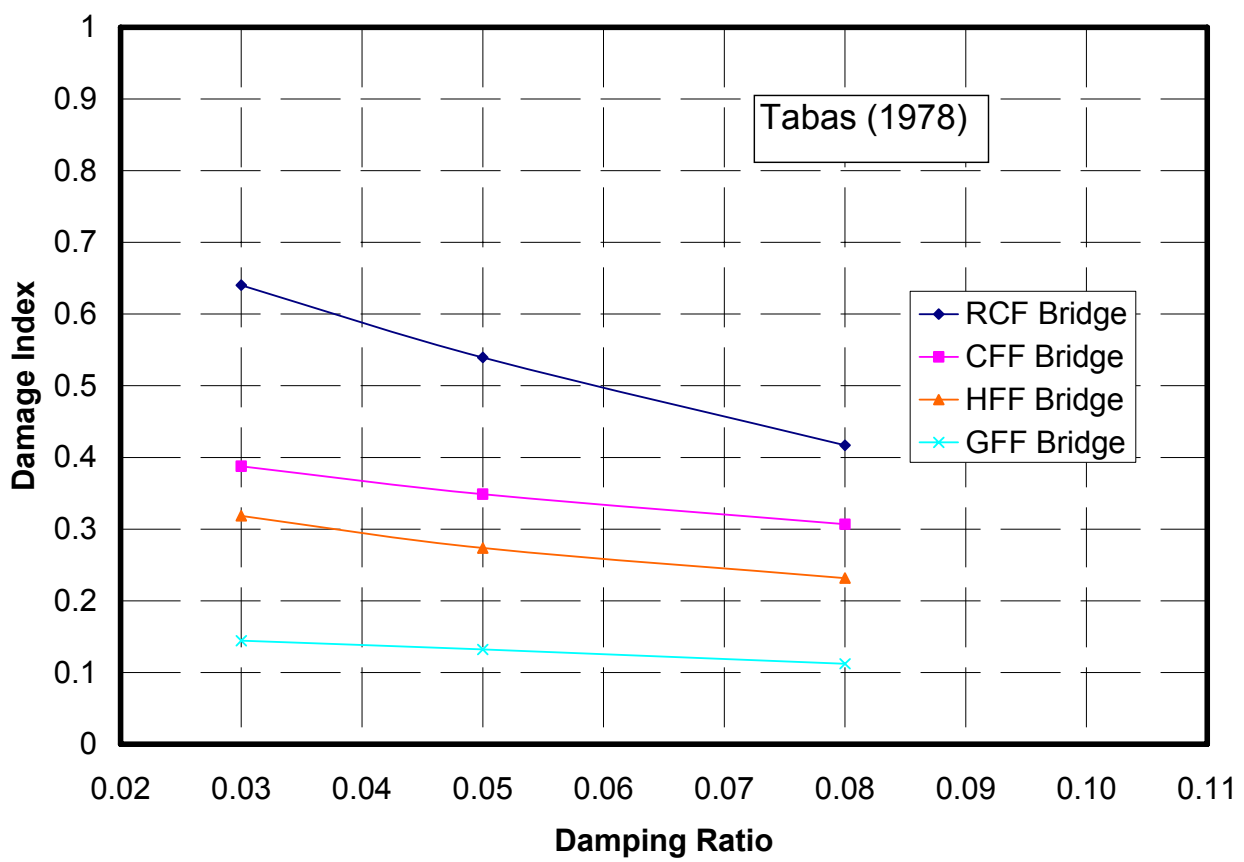

Fig. 4.119 Effect of Damping Ratio on Damage Index of Pier 2 in All Bridges under Tabas (1978) Earthquake

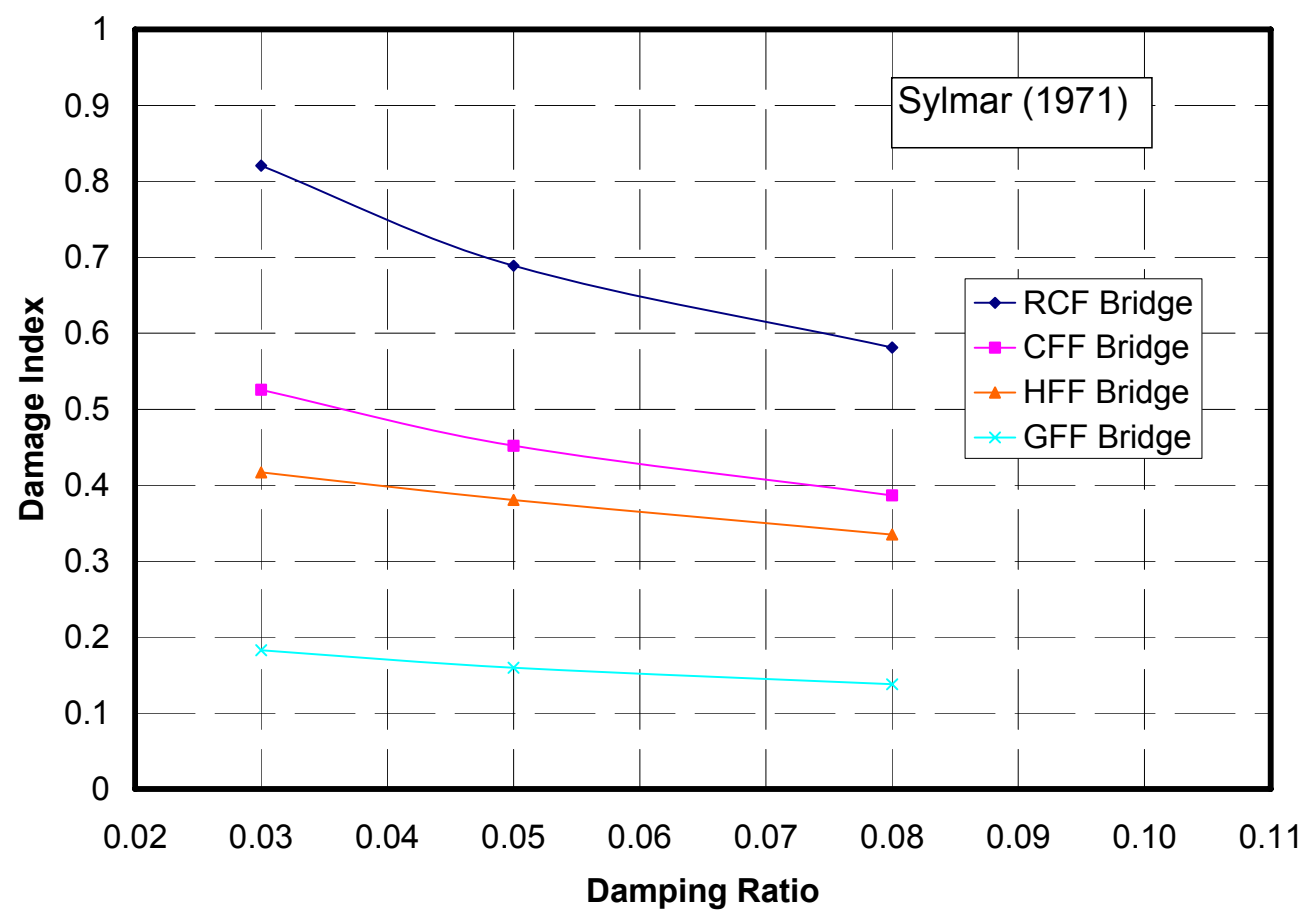

Fig. 4.120 Effect of Damping Ratio on Damage Index of Pier 2 in All Bridges under Sylmar (1971) Earthquake 


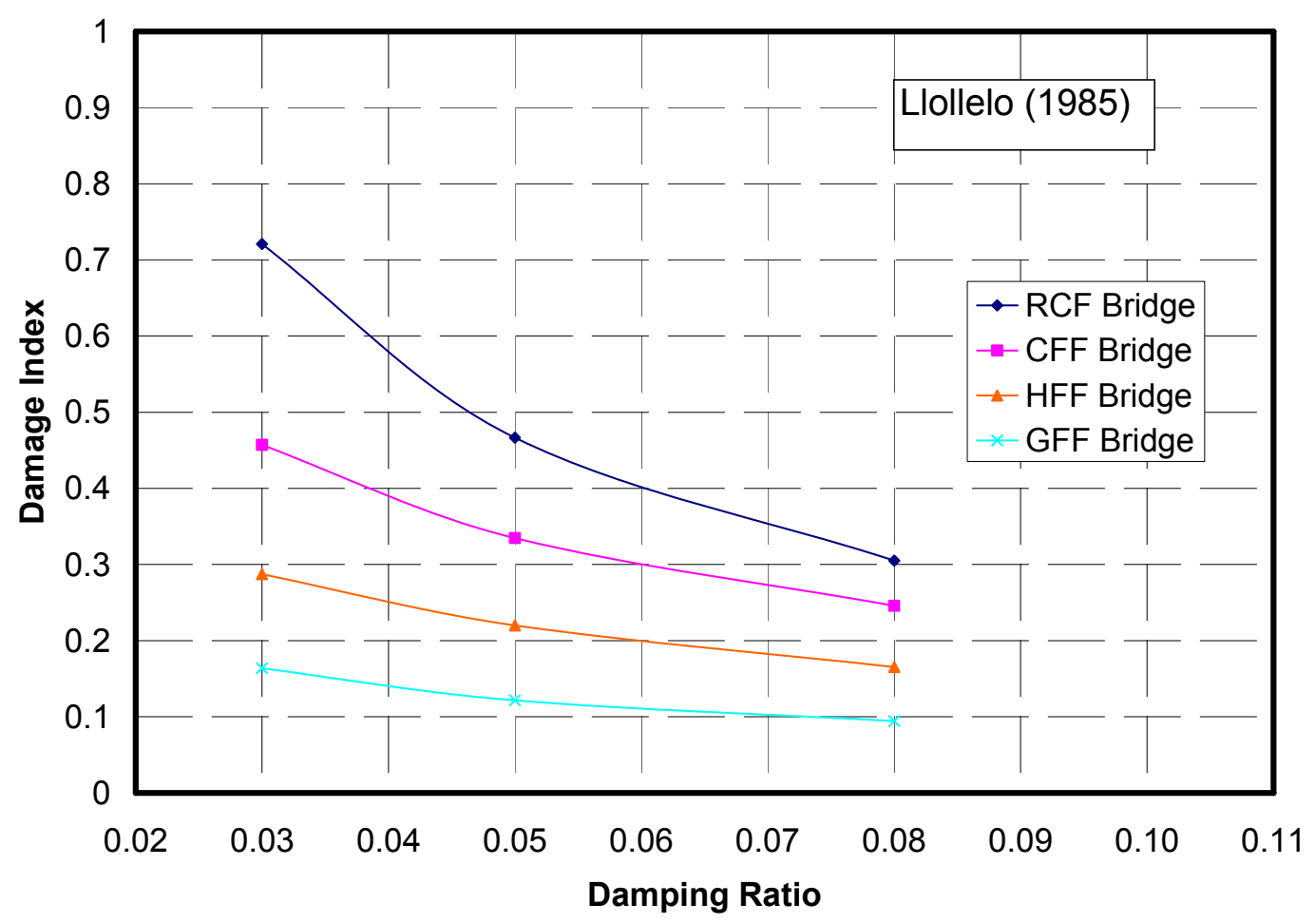

Fig. 4.121 Effect of Damping Ratio on Damage Index of Pier 2 in All Bridges under Llolleo (1985) Earthquake 


\section{CHAPTER 5}

\section{CONCLUSIONS AND DESIGN RECOMMENDATIONS}

As an alternative to transverse spiral or hoop steel reinforcement, fiber reinforced polymers (FRPs) were introduced to the construction industry in the 1980's. The concept of concrete-filled FRP tube (CFFT) has raised great interest amongst researchers in the last decade. FRP tube can act as a pour form, protective jacket, and shear and flexural reinforcement for concrete. However, seismic performance of CFFT bridge substructure has not yet been fully investigated.

The experimental work consisted of two-column bent tests, component tests, and coupon tests, all carried out in the Structures and Constructions Laboratory at Florida International University. Four 1/6-scale bridge pier frames, consisting of control reinforced concrete pier frame $(\mathrm{RCF})$, glass FRP-concrete pier frame (GFF), carbon FRPconcrete pier frame (CFF), and hybrid FRP-concrete pier frame (HFF) were tested under reverse cyclic lateral loading with constant axial loads. Specimen GFF had the most impressive deformation capability with a drift ratio of $15 \%$ under considerable lateral load with no cracks, whereas Specimen CFF showed that lowest ductility at a similar load level.

FRP-concrete columns and pier cap beams were then cut from the tested pier frame specimens, and were tested again in three point flexure under monotonic loading with no axial load. The tests indicated that bonding between FRP and concrete significantly affects the flexural response of the components, and yielding of steel plays 
an important role on the flexural strength and ductility of each component. The coupon tests were also carried out for FRP tubes, to establish their material constitutive models.

Analytical modeling of the hybrid FRP-concrete pier frame system was developed using an open source object-oriented nonlinear structural analysis software, namely, Open System Earthquake Engineering Simulation (OpenSees), developed by the University of California, Berkeley. Upon validating the model, a comprehensive parametric study was performed for the four different types of pier frames. Subsequently, a typical bridge was analyzed in its entirety with three different earthquake loadings and three different damping ratios.

The following conclusions may be drawn from the aforementioned experimental and analytical work:

- FRP tubes significantly increased the load capacity of pier frames, as compared to the conventional RC pier frame. Hybrid glass-carbon FRP tubes demonstrated the highest load capacity and initial stiffness among the four specimens. Glass FRP tubes showed the highest ductility with considerable load capacity, and as well the largest hoop strains at the column base among the four specimens. Moreover, the glass FRP specimen did not show any sign of crack even at a drift ratio of $15 \%$. The carbon FRP specimen had the least ductility among the FRP-concrete pier frames with a vertical crack developing after transverse cracks had enlarged.

- Total reinforcement strength index using the compressive strength of confined concrete showed a very good correlation with the normalized flexural strength 
of the pier frames. The pinching behavior in pier frames is seemingly affected in large by the internal steel reinforcement as well as the type of FRP tube.

- Pier cap beams performed as rigid beams with small rotations and a mid-span inflection point. The tests showed that the $1.5 \mathrm{D}$ and $0.625 \mathrm{D}$ embedments of the FRP tubes in the footing and the pier cap beam, respectively, were quite adequate, as no apparent slippage was noticed. D in the above relation is the diameter of the column. The slippage and separation of FRP tube at column base was the largest in the glass FRP specimen, as compared with those of the other FRP-concrete pier frame specimens.

- The interface bond between FRP and concrete affects the flexural behavior of pier cap beams. When concrete is cast against FRP with no epoxy, yielding of steel would play an important role in the flexural strength and ductility of the beam, and therefore, the response follows that of steel reinforcement. On the other hand, when concrete is strengthened with bonded FRP, the response is nonlinear.

- Glass FRP columns, when cut and tested again columns performed very well with quite large deflections and tensile strains at mid-span, as compared with the other two types of FRP-concrete columns. Hybrid FRP-concrete columns have the largest load capacity and a bilinear load-deflection response. Carbon FRP-concrete columns had the least load capacity, tensile strain, mid-span deflection, and ductility; perhaps due to a large horizontal crack that was developed earlier in the pier frame tests. On the other hand, larger slippage was 
noted in the glass and hybrid FRP-concrete columns, in comparison with the carbon FRP concrete columns.

- Modeling with zero-length element to account for the bond-slip effects leads to favorable agreement with the test results.

- The parametric study showed that the frame height to span ratio, FRP diameter to thickness ratio, and steel reinforcement ratio are the three parameters that affect the performance of pier frames the most. On the other hand, compressive strength of concrete, within the range studied, has almost no influence on performance of the pier frames.

- Bridges with FRP-concrete columns sustain much lower damage under severe earthquakes, as compared with conventional RC substructures. Damping ratio has a pronounced effect on bridge performance. Therefore, adding a dynamic damping device may improve the seismic performance of the bridge quite significantly.

\section{Future Research}

In this study, no damping ratio was included in the pier frame analysis. The damping ratios of $0.03,0.05$, and 0.08 used in the dynamic analysis of the bridge case study were verified experimentally. In order to measure the damping effects and to observe the performance of the FRP-concrete bridge under simulated ground acceleration, a large-scale 4-span bridge shake table test will be carried out at the University of Nevada, Reno in late 2009. The bridge columns will be built with FRP tubes, and will be tested on three bi-directional shake tables. This type of test will provide 
researchers with better understanding of the performance of the FRP-concrete substructure in seismic regions.

\section{Design Recommendations}

FRP tubes can replace the hoop or spiral steels to provide the same level of confinement effect. The stress-strain responses of steel-confined concrete based on KentPark (see Taucel et al. 1991) model and the FRP-confined concrete based on Samaan model ( Samaan et al.1998) are shown in Figures 5.1 and 5.2, respectively.

Equations (4-4) and (4-5) are used to obtain the peak stress and corresponding strain in Kent-Park model. The FRP tube design philosophy suggested here is to achieve the same confined concrete stress, when the strain in FRP-confined concrete reaches the same strain in steel-confined concrete, as calculated by Equation (4-4).

Equation (5-1) was derived from the Kent-Park and Samaan models to illustrate the relationship between the FRP tube thickness and volumetric reinforcement ratio of steel spiral or hoop based on this design philosophy:

$$
\frac{t}{D}=\frac{\left(\alpha \rho_{v}-\beta\right)}{\gamma+\zeta \rho_{v}}
$$

where

$$
\begin{aligned}
& \alpha=\left(f_{c}^{\prime}-0.105 f_{c}^{\prime}{ }_{c}^{0.2}\right) f_{y} \\
& \beta=0.105 f_{c}^{\prime}{ }_{c}^{0.2}-0.128 f^{\prime}{ }_{c}+0.908 \\
& \gamma=0.742 f_{t} f^{\prime}{ }_{c}+0.0027 f_{t} f^{\prime}{ }_{c} / \varepsilon_{u} \\
& \zeta=0.0027 f_{y} f_{t} / \varepsilon_{u}
\end{aligned}
$$


and $\mathrm{t}$ is the FRP tube thickness, $\mathrm{D}$ is the outside diameter of the column, $\rho_{v}$ is the volumetric reinforcement ratio of the hoop or spiral steel reinforcement, $\mathrm{f}_{\mathrm{y}}$ is the yielding strength of the steel hoop or spiral, $\mathrm{f}_{\mathrm{t}}$ is the FRP ultimate strength in the hoop direction and $\varepsilon_{u}$ is the ultimate strain of the FRP in the hoop direction.

Figure 5.3 plots Equation (5-1) for all FRP tube types in this study. The tested specimens are also shown for comparison. The FRP tube thickness of Specimen HFF matches the proposed thickness by this design method perfectly, whereas the FRP tube thickness of Specimens GFF and CFF are below the proposed thickness. However, the test results have already shown the superior effect of FRP tube in all specimens, as compared with Specimen RCF. Therefore, the proposed design method seems to be quite reasonable. 


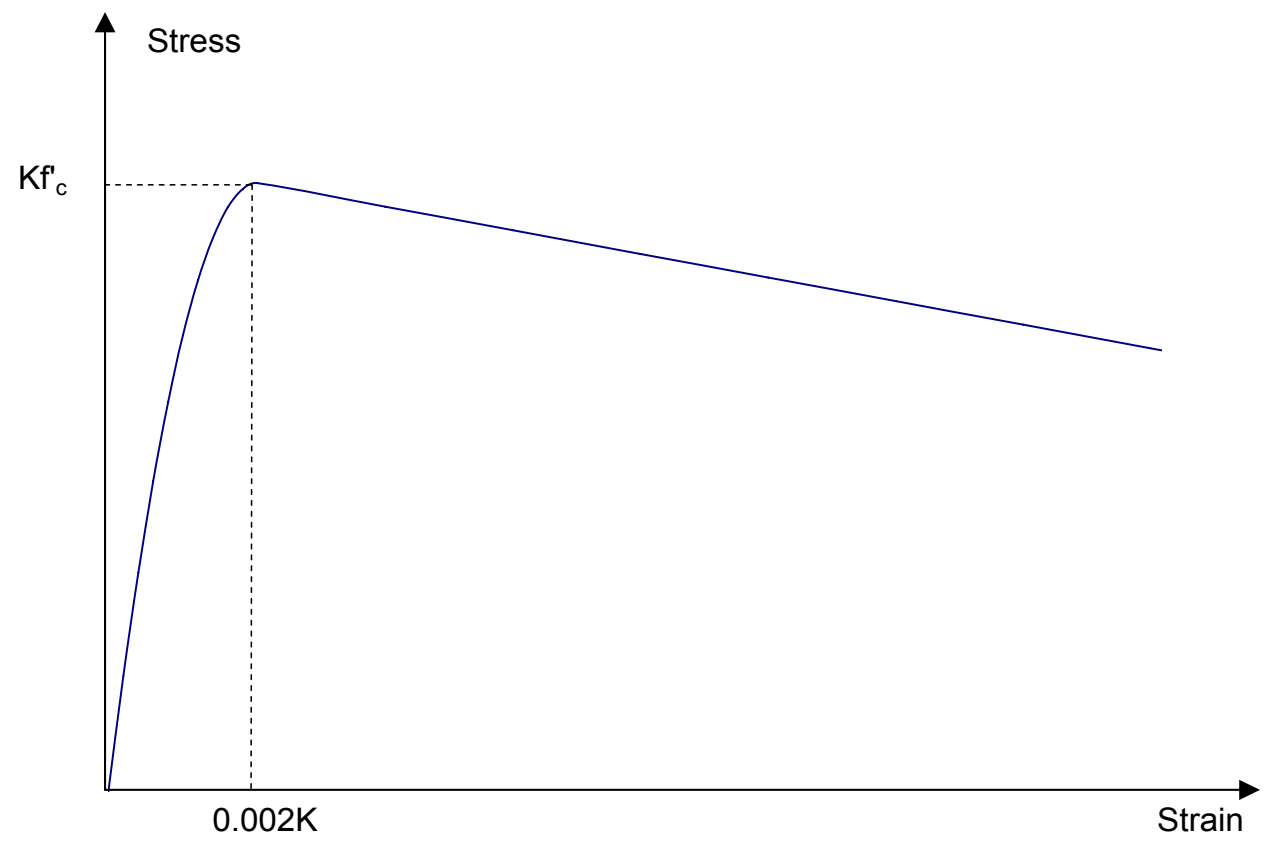

Fig. 5.1 Stress-Strain Response of Steel Hoop or Spiral Confined Concrete Based on Kent-Park Model

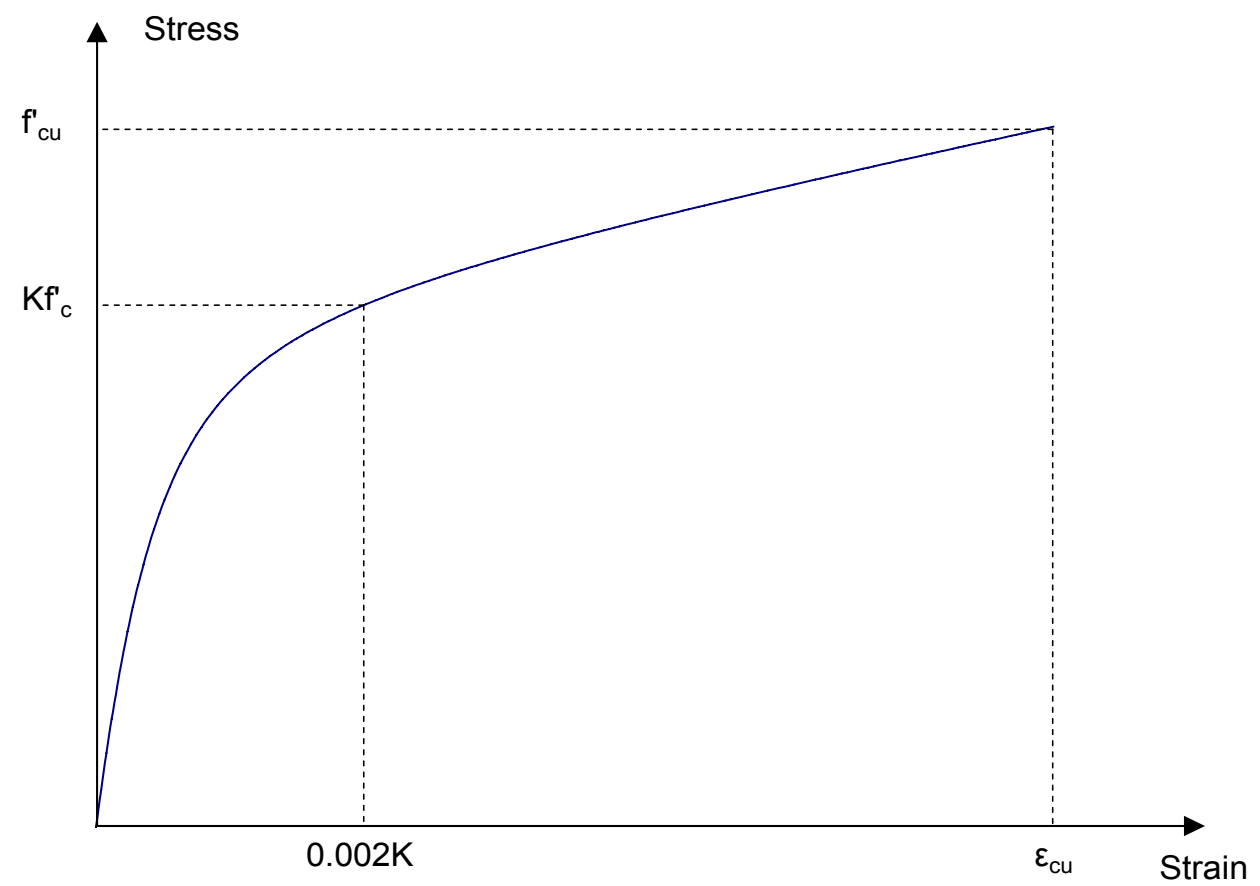

Fig. 5.2 Stress-Strain Response of FRP Confined Concrete Based on Samaan Model 


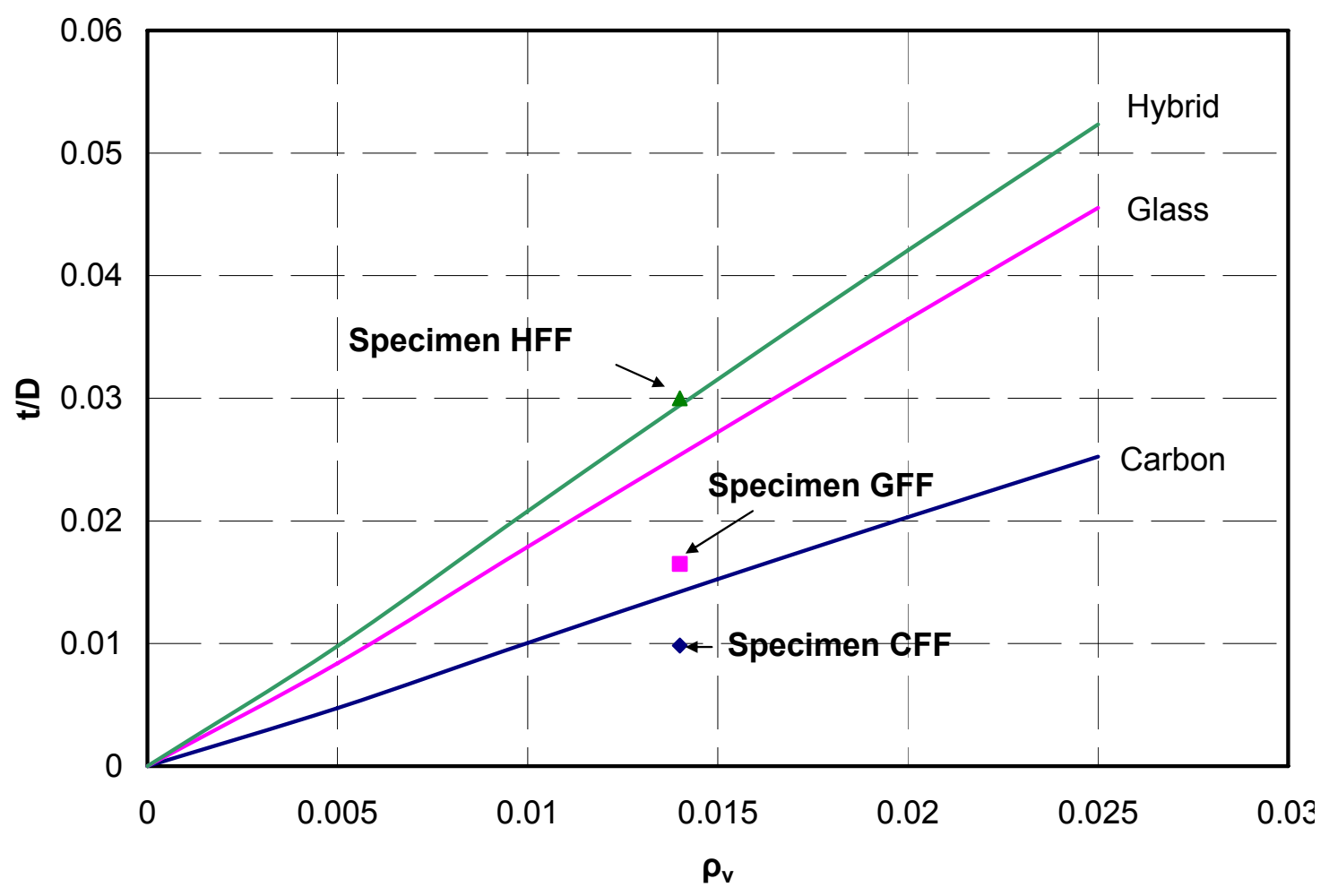

Fig. 5.3 t/D Versus $\rho_{v}$ of Proposed Design Method for All Types of FRP Tubes 


\section{REFERENCES}

Cole, B., and Fam, A. (2006). "Flexural Load Testing of Concrete-Filled FRP Tubes with Longitudinal Steel and FRP Rebar." Journal of Composites for Construction, $A S C E$, Vol. 10, No. 2, pp. 161-171.

Davol, A., Burgueño, R., and Seible, F. (2001). "Flexural Behavior of Circular Concrete Filled FRP Shells.” Journal of Structural Engineering, ASCE, Vol. 127, No. 7, pp. 810-817.

Fam, A., Pando, M., Filz, G., and Rizkalla, S. (2003). "Precasted Piles for Route 40 bridge in Virginia using Concrete-Filled FRP Tubes.” PCI Journal, Vol. 48, No. 3, pp. 32-45.

Fam, A., Bart, F., and Rizkalla, S. (2003). "Experimental and Analytical Modeling of Concrete-Filled Fiber-Reinforced Polymer Tubes Subjected to Combined Bending and Axial Loads.” ACI Structural Journal, Vol. 100, No. 4, pp. 499-509.

Fan, L., Zhou, W., and Xue, Y. (2000). "Preliminary Study of Seismic Performance of FRP Tube Confined Concrete Short Columns." Proc., 1st Chinese National Conf. of Application Technology of FRP Materials in Civil Engineering, National Diagnosis and Rehabilitation of Industrial Building Research Center, Beijing, pp. 113-117 (in Chinese).

Kovacs, T. (1998). "Dynamic Investigation on Reinforced Concrete Bridges." 2nd Int. PhD Symposium in Civil Engineering, Budapest, pp. 1-8.

Lam, L., and Teng, J.G. (2002). "Strength Models for Fiber-Reinforced Plastic-Confined Concrete." Journal of Structural Engineering, ASCE, Vol. 128, No. 5, pp. 612623.

Mirmiran, A., Naguib, W., and Shahawy, M. (2000). "Large Beam-Column Tests on Concrete-Filled Composite Tubes.” ACI Structural Journal, Vol. 97, No. 2, pp. 268-276.

Mirmiran, A., and Shahawy, M. (2003). "Composite Pile: A Successful Drive." Concrete International, ACI, Vol. 25, No. 3, pp. 89-94.

Naguib, W., and Mirmiran, A. (2002). "Time-Dependent Behavior of FRP-Confined Concrete Columns.” ACI Structural Journal, Vol. 99, No. 2, pp. 142-148.

OpenSees Manual (2006). University of California, Berkeley, CA. http://opensees.berkeley.edu/OpenSees/manuals/usermanual/index.html

OpenSees Software (2008). University of California, Berkeley, CA. http://opensees.berkeley.edu/ 
Ozbakkaloglu, T., and Saatcioglu, M. (2006). "Seismic Behavior of High-Strength Concrete Columns Confined by Fiber-Reinforced Polymer Tubes." Journal of Composites for Construction, ASCE, Vol. 10, No. 6, pp. 538-549.

Park Y., Ang A. (1985). "Seismic Damage Analysis of Reinforced Concrete Buildings,"

Journal of Structural Engineering, ASCE, Vol. 111, No. 4, 1985, pp. 722-739.

Park, Y. J., Ang, A.H-S and Wen, Y. K. (1987). "Damage-Limiting A Seismic Design of Buildings.” Earthquake Spectra, Vol.3, No.1, pp. 1-26

Popovics, S. (1973). "Analytical Approach to Complete Stress-Strain Curves." Cem. Concr. Res., Vol 3, No.5, pp.583-599

Pulido, C., Saiidi, S., Sanders, D., Itani, A., and EI-Azazy, S. (2004) "Seismic Performance of Two-Column Bent-Part I: Retrofit with Carbon Fiber-Reinforced Polymer Fabrics.” ACI Structural Journal, Vol. 101, No. 4, pp. 558-568.

Ravi. S., and Saatcioglu, M. (1999). "Confinement Model for High-Strength Concrete." Journal of Structral Engineering, ASCE, Vol. 125, No.3, pp 281-289

Saafi, M., Toutanji, H. A., and Li, Z. (1999). "Behavior of Concrete Columns Confined with Fiber Reinforced Polymer Tubes." Material Journal, ACI, Vol.96, No.4, pp. 500-509.

Samaan, M., Mirmiran, A., and Shahawy, M. (1998). "Model of Concrete Confined by Fiber Composite." Journal of Structural Engineering, ASCE, Vol. 124, No. 9, pp. 1025-1031.

Seible, F., Davol, A., Burgueño, R., Nuismer, R.J., and Abdallah, M.G. (1996). "Structural Behavior of Concrete Filled Carbon Fiber Composite Tubular Columns." International SAMPE Technical Conference, Vol. 28, pp. 1258-1269.

Scott, B.D., Park, R. and Priestley, M. (1982). "Stress-strain Behavior of Concrete Confined by Overlapping Hoops at Low and High Strain Rates." ACI Journal, Vol. 79, No. 1, pp.13-27.

Shao Y. (2003). "Seismic Performance of FRP-Concrete Beam-Column." Ph.D. Dissertation, North Carolina State University, Raleigh, NC.

Shao, Y., and Mirmiran, A. (2004). "Nonlinear Cyclic Response of Laminated Glass FRP Tubes Filled with Concrete." Composite Structures, Elsevier Science Ltd., Vol. 65, No.1, pp. 91-101

Shao, Y.,and Mirmiran, A. (2005). "Experimental Investigation of Cyclic Behavior of Concrete-Filled FRP Tubes." Journal of Composites for Construction, ASCE, Vol. 9, No.3, pp. 263-273 
Shi, Y., Li, B., and Mirmiran, A. (2007). Report: "Seismic Performance of Hybrid FRPConcrete Pier Columns." Final Report SCL-071201, Network for Earthquake Engineering Simulation Research-Small Group (NEESR-SG), National Science Foundation (NSF) Grant No. CMS-0420347, Miami, FL.

Tanimoto, T. (2007). "A New Vibration Damping CFRP Material with Interlayers of Dispersed Piezoelectric Ceramic Particles." Composites Science and Technology vol.67, pp. 213-221

Taucer, F., Spaone, E., and Filip F. (1991). "A Fiber Beam-Column Element for Seismic Response Analysis of Reinforced Concrete Structures." Report No. UCB/EERC91/17, Earthquake Engineering Research Center, University of California, Berkeley, CA.

Williams, M. and Sexsmith, R. (1995). "Seismic Damage Indices for Concrete Structures: A State-of-the-Art review." Earthquake Spectra, Vol.11, No. 2, May, pp.319-349.

Wu. G., Wu, Z.S., Lv, Z.T., and Gu, D.S. (2007). "Seismic Retrofit of Large Scale Circular RC Columns Wrapped with CFRP Sheets." 3rd International Conference on Composites in Civil Engineering (CICE), pp. 547-550, Miami, FL, USA

Yamakawa, T., Komesu, K., Zhong, P., and Satoh, H. (2001). "Seismic Performance of Aramid Fiber Square Tubed Concrete Columns with Nonmetallic Reinforcement." Proceeding of Inernational Conference on FRP Composites in Civil Engineerin , Vol. II, pp. 1329-1336.

Yuan, H., Xue, Y., Li, X., and Zhang, M. (2002). "Study on A Novel Hybrid GFRP/CFRP Composite Beam." Proc., 2nd Chinese National Conference of Application Technology of FRP Materials in Civil Engineering, Tsinghua University Publication, Beijing, pp. 296-305 (in Chinese).

Zhao, J. and Sritharan, S., (2007). "Modeling of Strain Penetration Effect in Fiber-Based Analysis of Reinforced Concrete Structures." ACI Structural Journal, Vol. 104, No. 2, pp. 133-141.

Zhuo, W., Fan, L., and Xue, Y. (2001). "Shaking Table Testing of Simply Supported Bridges with Prefabricated GFRP Tube Jacketed RC Columns." Proc., Int. Conf. on FRP Composites in Civil Engineering, Vol. II, pp.1337-1344.

Zhu, Z., Mirmiran, A., and Shahawy, M. (2004). "Stay-in-Place FRP Forms for Precast Modular Bridge Pier System." Journal of Composites for Construction, ASCE, Vol. 8, No. 8, pp. 560-568.

Zhu, Z. (2004). "Joint Construction and Seismic Performance of Concrete-Filled Fiber Reinforced Polymer Tubes." Ph.D. Dissertation, North Carolina State University, Raleigh, NC. 
Zhu, Z., Ahmad, I., and Mirmiran, A. (2006). "Splicing of Precast Concrete-Filled FRP Tubes." Journal of Composites for Construction, ASCE, Vol. 10, No. 4, pp. 345356.

Zhu, Z., Mirmiran, A., and Saiidi, M.S. (2006). "Seismic Performance of Fiber Composite Tubed Reinforced Concrete Bridge Substructure." Transportation Research Record No. 1976, Transportation Research Board, National Research Council, Washington, D.C., pp. 197-206.

Zheng, R., (2007). "Performance of FRP-concrete bridges under blast loading." Ph.D. Dissertaion, Florida Internatinal University, Miami. 


\section{APPENDICES}

The input files for CFFT column analysis in OpenSees were written in Tool Command Language (TCL). The common files which might be referred by various analysis programs are listed first, as follows:
A. Build File for Reinforced Concrete Circular Cross Section
B. Build File for Concrete-filled FRP Tube Circular Cross Section
C. Earthquake Record Data Reading File

Due to the space limit of the dissertation, only representative analysis input files are listed here. The TCL files for all analysis in this research program can be essentially modified from the following sample files, only by changing few parameters. The sample files are appended in the following sequence:
D. Input File for Specimen RCF Static Analysis
E. Input File for Specimen GFF Static Analysis
F. Input File for RCF Bridge Seismic Analysis
G. Input File for GFF Bridge Seismic Analysis 


\section{Appendix A. Build File for Reinforced Concrete Circular Cross Section}

\section{File Name: RCcirSection.tcl}

proc RCcircSection \{id ri ro cover coreID coverID steelID numBars barArea nfCoreR nfCoreT nfCoverR nfCoverT $\}\{$

\# Define the fiber section section fiberSec \$id \{

\section{\# Core radius}

set rc [expr \$ro-\$cover]

\# Define the core patch

patch circ \$coreID \$nfCoreT \$nfCoreR 00 \$ri \$rc 0360

\# Define the cover patch

patch circ \$coverID \$nfCoverT \$nfCoverR 00 \$rc \$ro 0360

if $\{$ numBars $<=0\}\{$ return

\}

\# Determine angle increment between bars

set theta [expr 360.0/\$numBars]

\# Define the reinforcing layer

layer circ \$steelID \$numBars \$barArea 00 \$rc \$theta 360

\}

\}

\section{Appendix B. Build File for Concrete-Filled FRP Tube Circular Cross Section}

\section{File Name: CFFTcirSection.tcl}

proc RCcircSection \{id ri ro $t$ rs coreID coverID steelID numBars barArea nfCoreR nfCoreT nfCoverR nfCoverT $\}\{$

\# Define the fiber section

section fiberSec $\$$ id \{

\# Core radius

set rc [expr \$ro-\$t]

\# Define the core patch

patch circ \$coreID \$nfCoreT \$nfCoreR 00 \$ri \$rc 0360

\# Define the cover patch

patch circ \$coverID \$nfCoverT \$nfCoverR 00 \$rc \$ro 0360

if $\{$ \$numBars $<=0\}\{$

return 


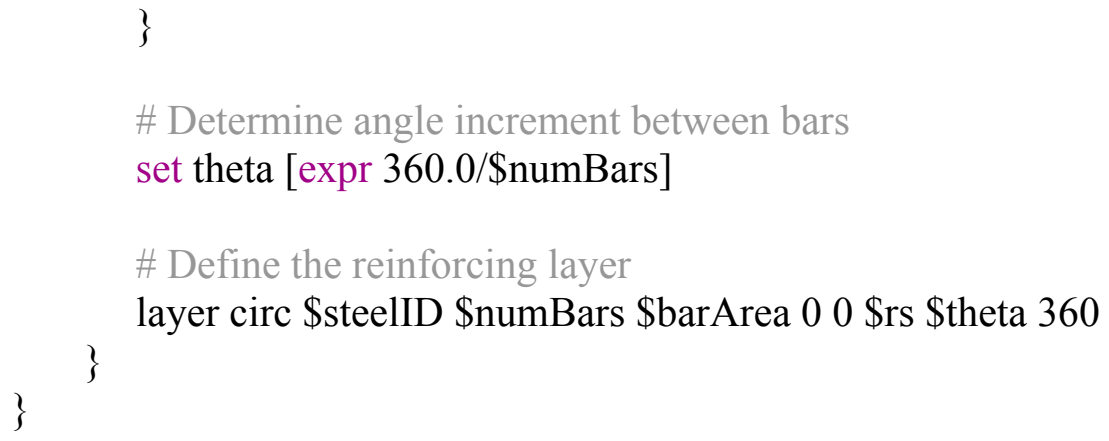

\section{Appendix C. Earthquake Record Data Reading File}

File Name: ReadSMDFile.tcl

proc ReadSMDFile $\{$ inFilename outFilename $\mathrm{dt}\}\{$

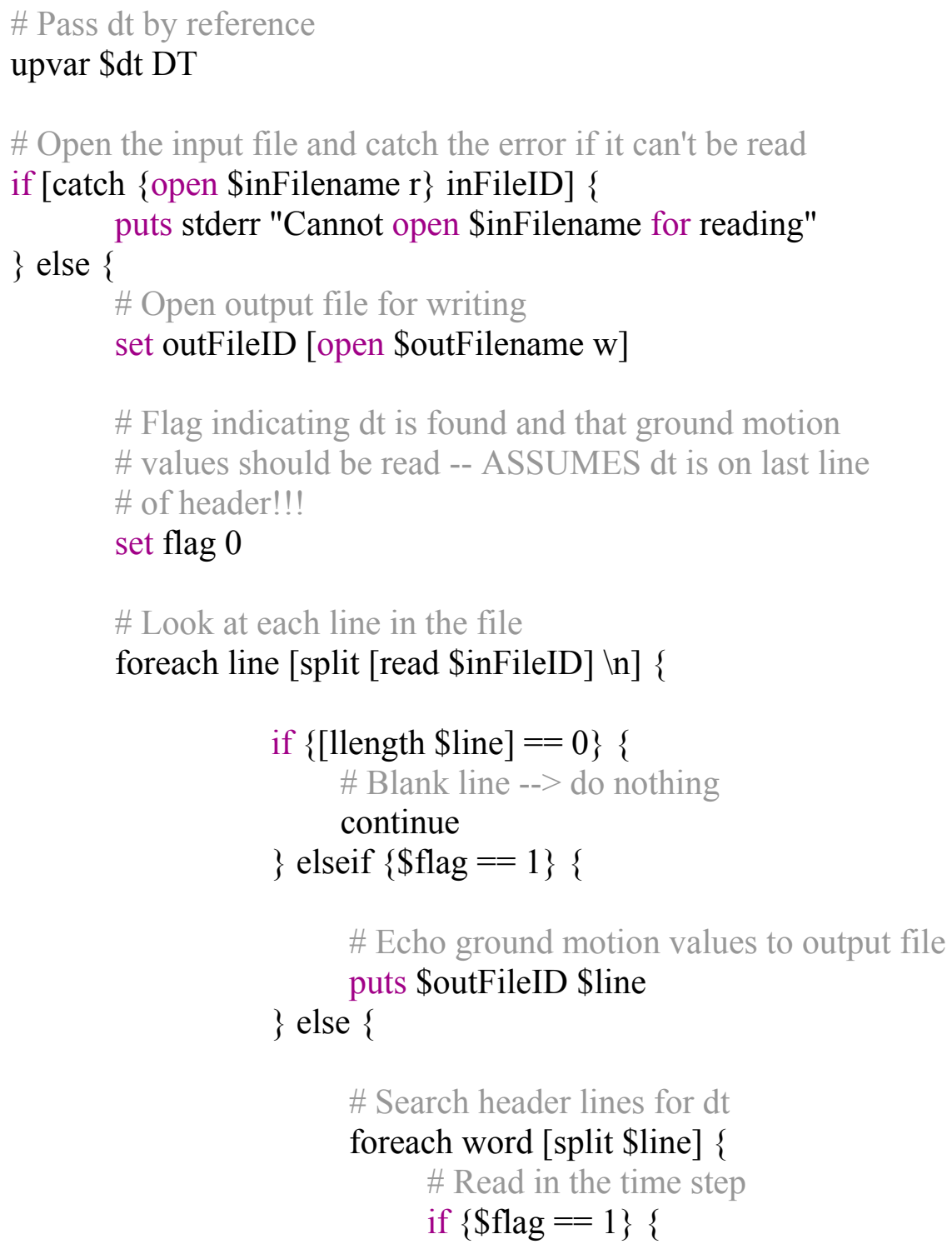




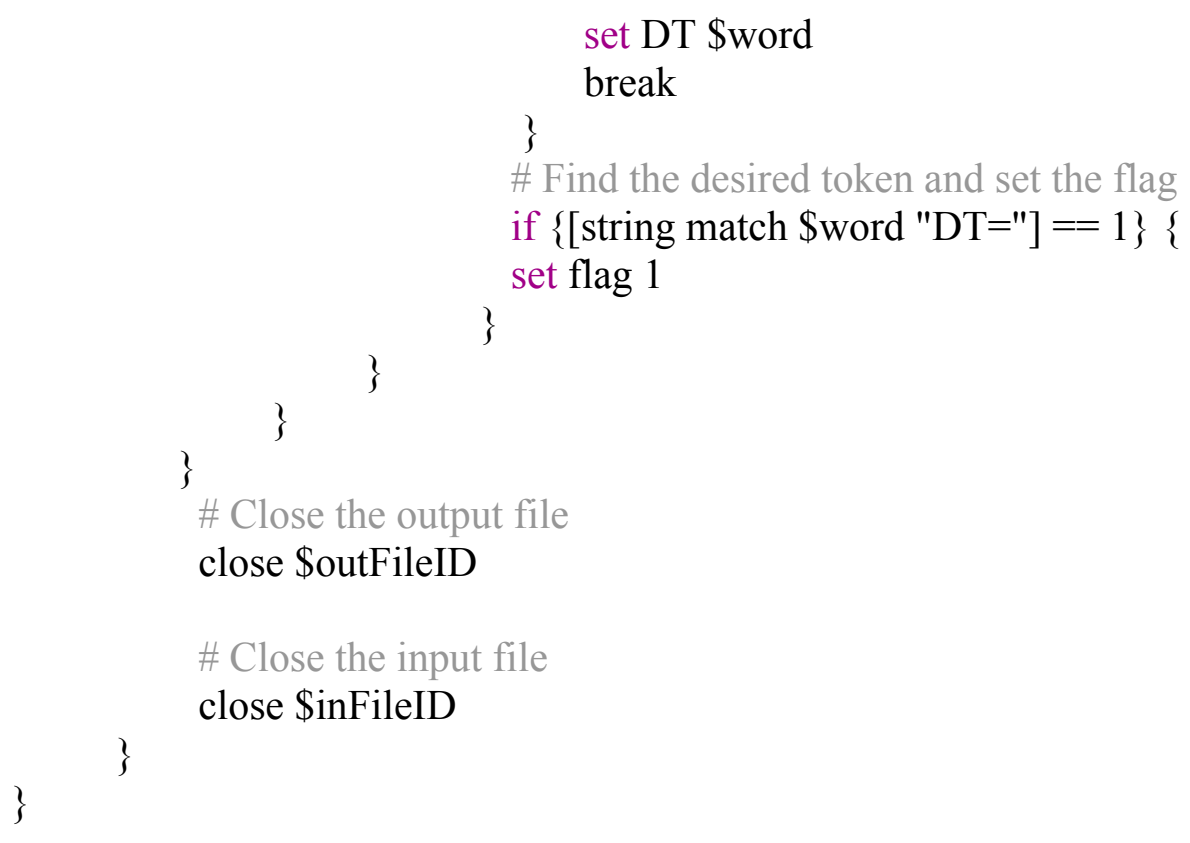

Appendix D. Input File for Specimen RCF Static Analysis

File Name: Specimen RCF

wipe;

model BasicBuilder -ndm 3 -ndf 6

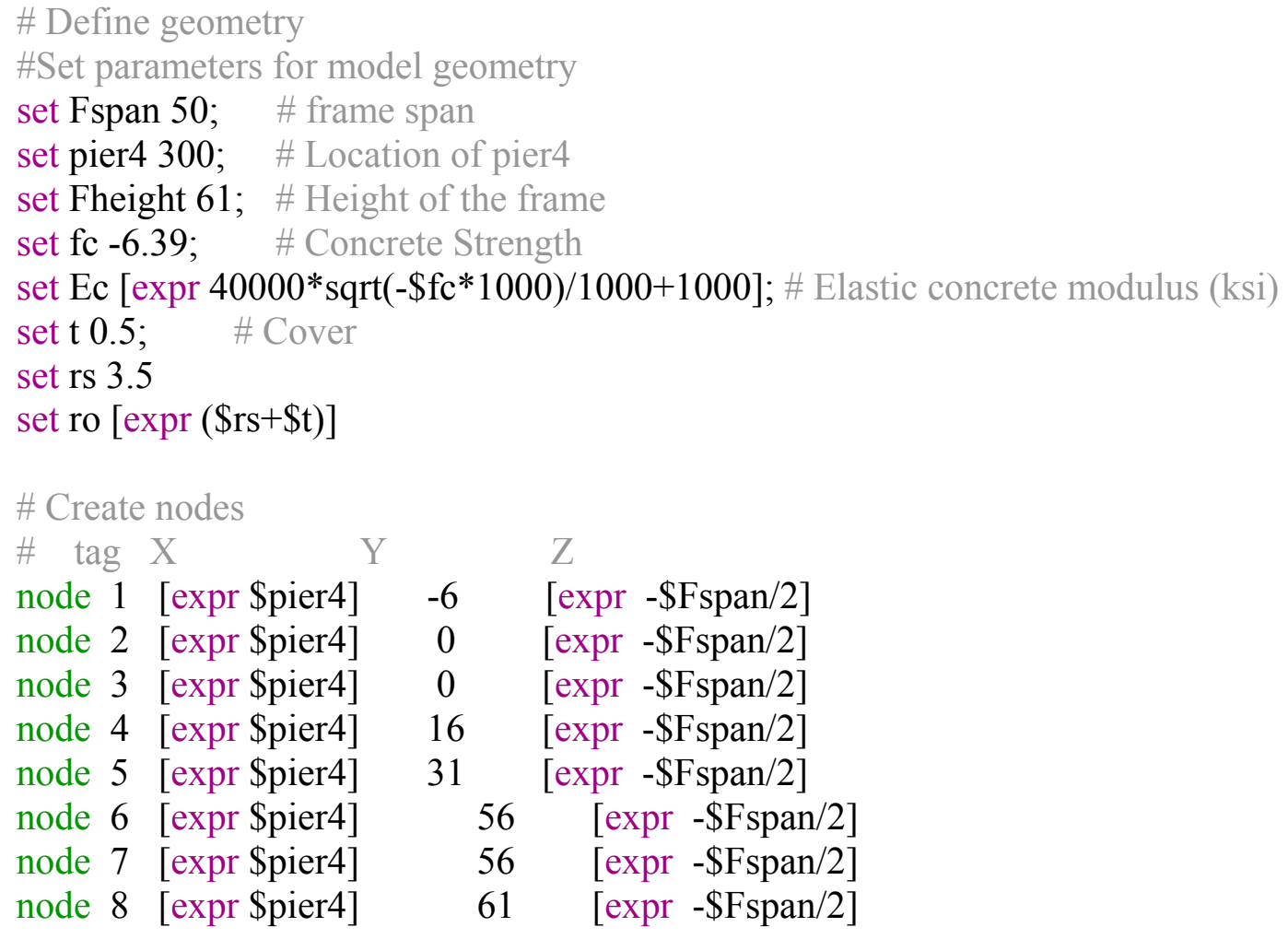




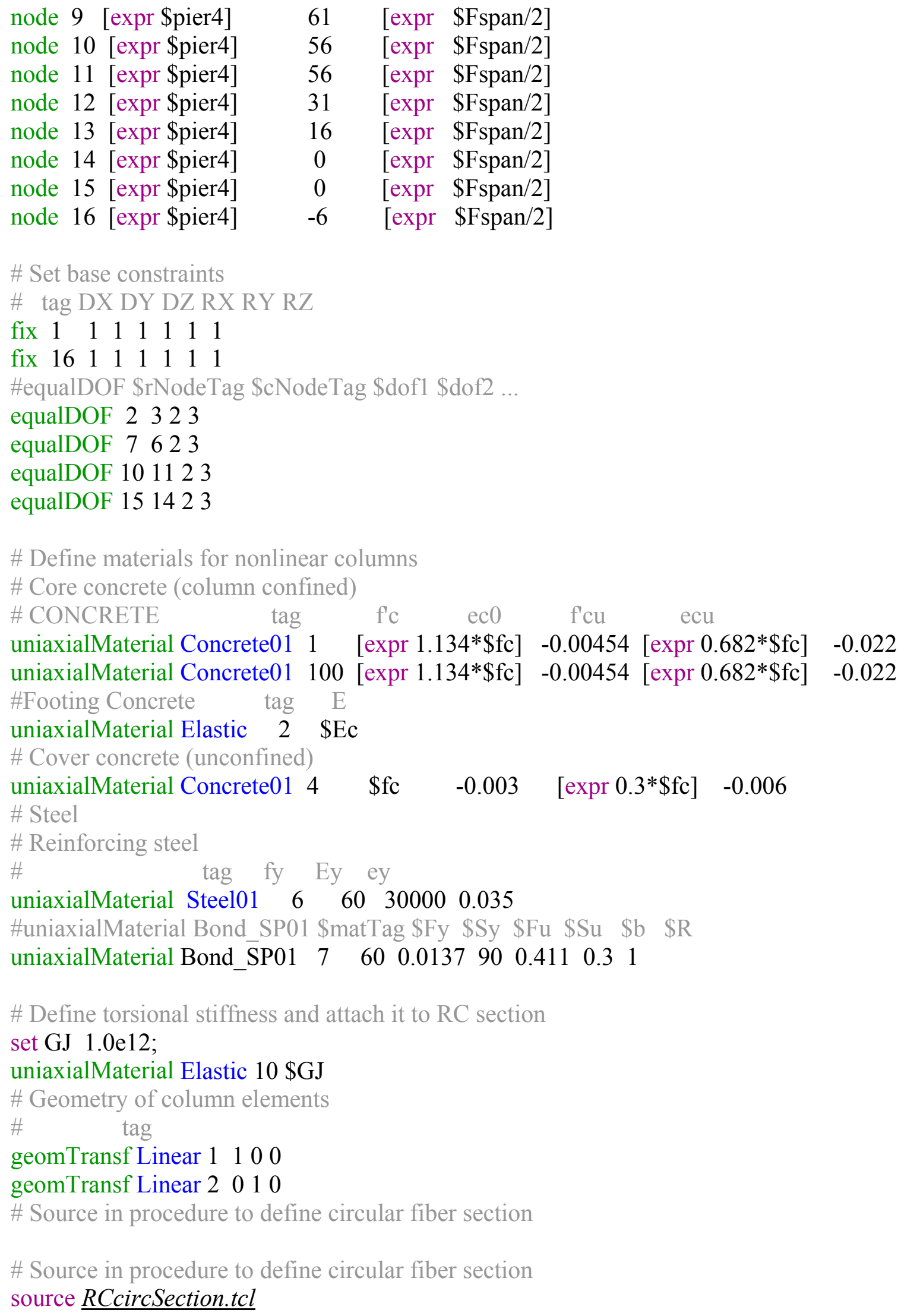




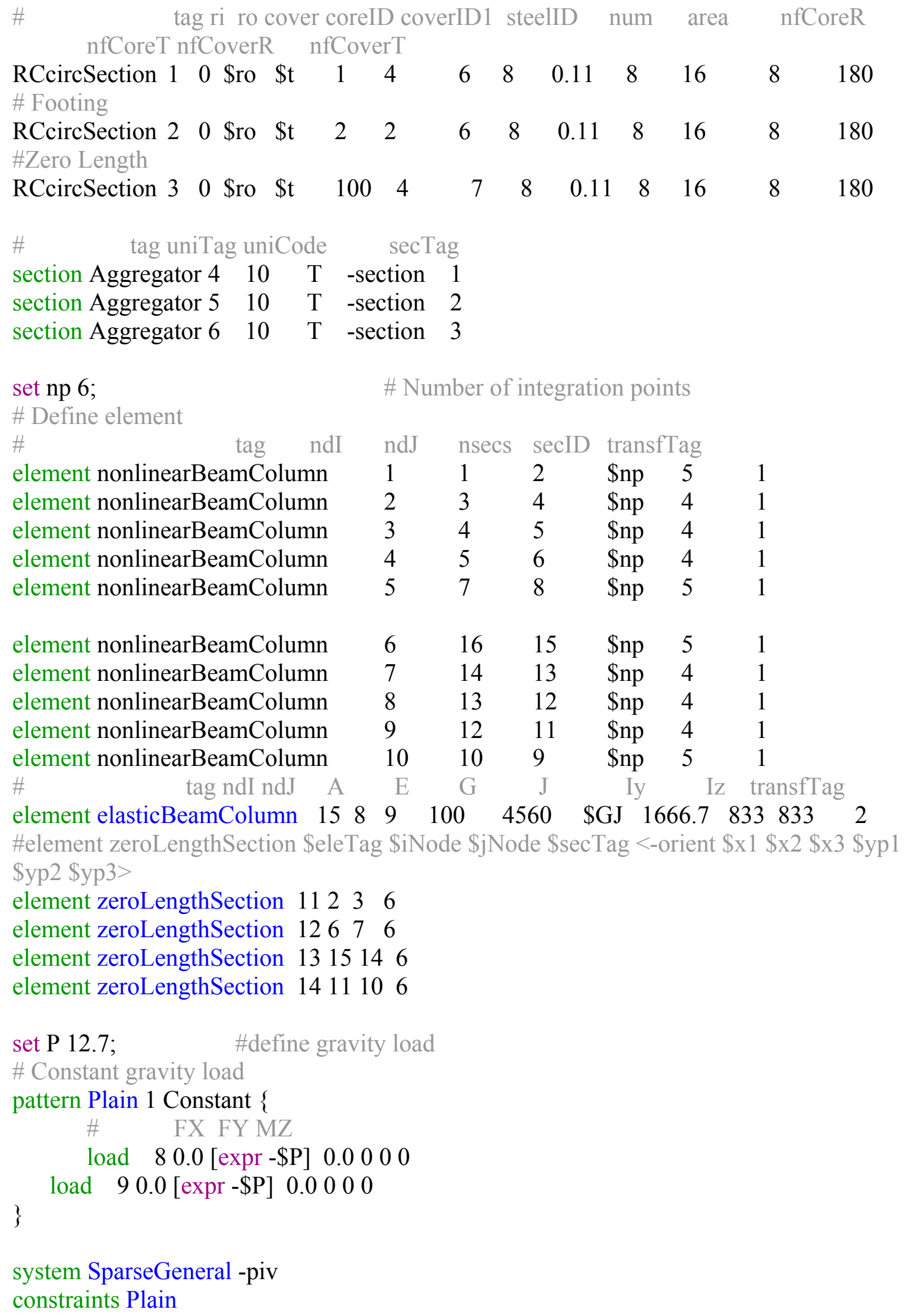


numberer Plain

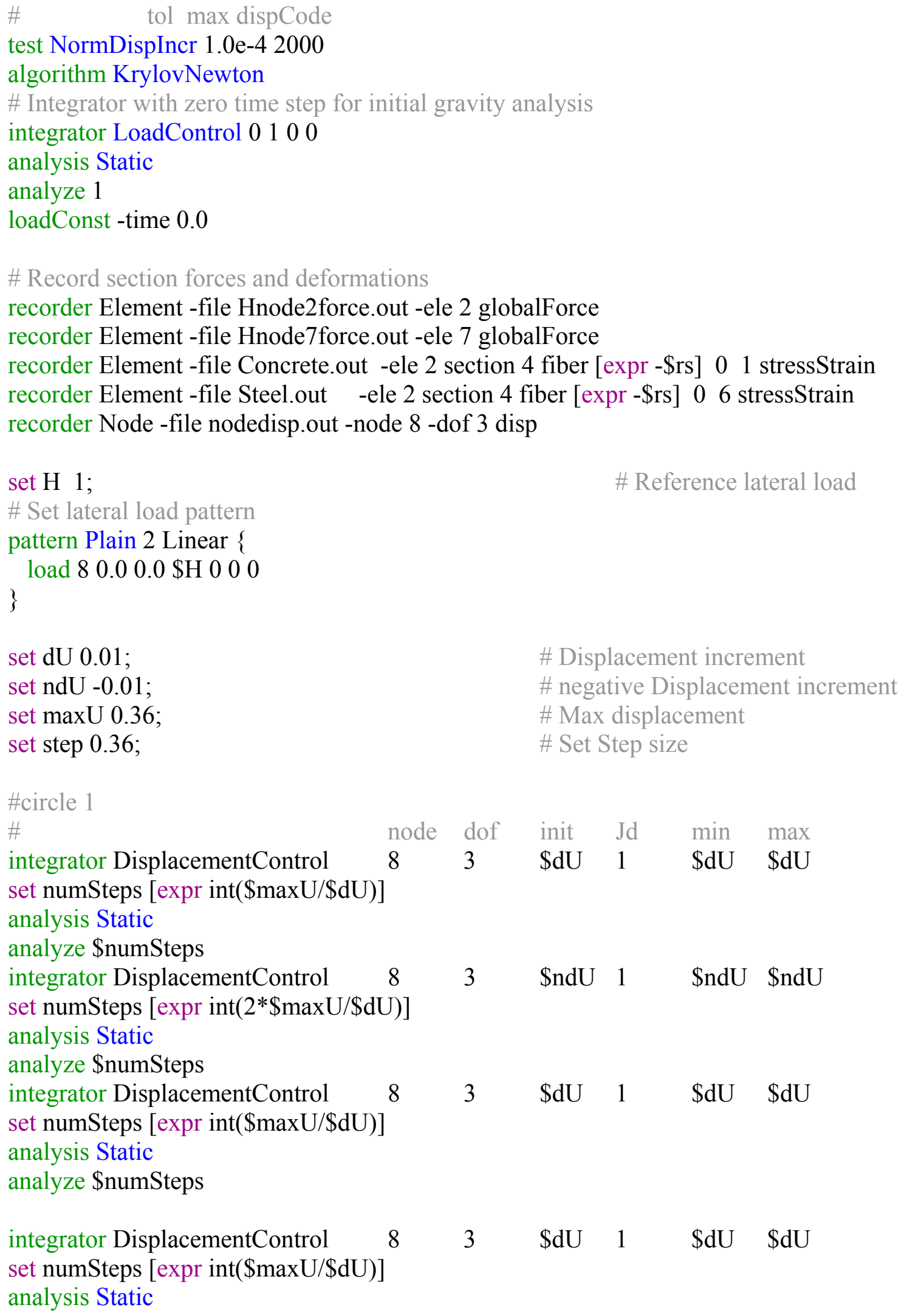


analyze \$numSteps

integrator DisplacementControl $\quad 8 \quad 3 \quad$ \$ndU $1 \quad$ \$ndU $\quad$ \$ndU

set numSteps [expr int $(2 * \$ \max U / \$ d U)]$

analysis Static

analyze \$numSteps

integrator DisplacementControl $\quad \begin{array}{lllllll}8 & 3 & \$ d U & 1 & \$ d U & \$ d U\end{array}$

set numSteps [expr int $(\$ \max U / \$ d U)]$

analysis Static

analyze \$numSteps

puts "Done, Cycle 1!"

\section{\#circle 2}

set maxU [expr \$maxU+\$step]

$\begin{array}{lllllll}\text { integrator DisplacementControl } & 8 & 3 & \$ d U & 1 & \$ d U & \$ d U\end{array}$

set numSteps [expr int $(\$ \max U / \$ d U)]$

analysis Static

analyze \$numSteps

integrator DisplacementControl $\quad 8 \quad 3 \quad$ \$ndU $1 \quad$ \$ndU $\quad$ SndU

set numSteps [expr int $(2 * \$ \max U / \$ d U)]$

analysis Static

analyze \$numSteps

$\begin{array}{lllllll}\text { integrator DisplacementControl } & 8 & 3 & \text { SdU } & 1 & \$ d U & \$ d U\end{array}$

set numSteps [expr int $(\$ \max U / \$ d U)]$

analysis Static

analyze \$numSteps

$\begin{array}{lllllll}\text { integrator DisplacementControl } & 8 & 3 & \$ d U & 1 & \$ d U & \$ d U\end{array}$

set numSteps [expr int $(\$ \max U / \$ d U)]$

analysis Static

analyze \$numSteps

integrator DisplacementControl $\quad 8 \quad 3 \quad$ \$ndU $1 \quad$ \$ndU $\quad$ SndU

set numSteps [expr int $(2 * \$ \max U / \$ d U)]$

analysis Static

analyze \$numSteps

$\begin{array}{lllllll}\text { integrator DisplacementControl } & 8 & 3 & \$ d U & 1 & \$ d U & \$ d U\end{array}$

set numSteps [expr int $(\$ \max U / \$ d U)]$

analysis Static

analyze \$numSteps

puts "Done, Cycle 2!"

\#circle 3

set maxU [expr \$maxU+\$step]

$\begin{array}{lllllll}\text { integrator DisplacementControl } & 8 & 3 & \$ d U & 1 & \$ d U & \$ d U\end{array}$

set numSteps [expr int $(\$ \max U / \$ d U)]$

analysis Static

analyze \$numSteps 


\begin{tabular}{|c|c|c|c|c|c|}
\hline $\begin{array}{l}\text { integrator DisplacementControl } 8 \\
\text { set numSteps [expr int }(2 * \text { maxU } / \$ \mathrm{dU})] \\
\text { analysis Static } \\
\text { analyze \$numSteps } \\
\text { integrator DisplacementControl } 8 \\
\text { set numSteps [expr int }(\$ \operatorname{maxU} / \$ \mathrm{dU})] \\
\text { analysis Static } \\
\text { analyze \$numSteps }\end{array}$ & 3 & $\$ n d U$ & 1 & \$ndU & \$ndU \\
\hline $\begin{array}{l}\text { integrator DisplacementControl } 8 \\
\text { set numSteps [expr int }(\$ \operatorname{maxU} / \$ \mathrm{dU})] \\
\text { analysis Static } \\
\text { analyze \$numSteps }\end{array}$ & 3 & $\$ \mathrm{dU}$ & 1 & $\$ \mathrm{dU}$ & $\$ d U$ \\
\hline $\begin{array}{l}\text { integrator DisplacementControl } 8 \\
\text { set numSteps [expr int }(2 * \$ \operatorname{maxU} / \$ \mathrm{dU}) \text { ] } \\
\text { analysis Static } \\
\text { analyze \$numSteps }\end{array}$ & 3 & \$ndU & 1 & \$ndU & \$ndU \\
\hline $\begin{array}{l}\text { integrator DisplacementControl } 8 \\
\text { set numSteps [expr int(\$maxU/\$dU)] } \\
\text { analysis Static } \\
\text { analyze \$numSteps } \\
\text { puts "Done, Cycle 3!" }\end{array}$ & 3 & $\$ d U$ & 1 & $\$ d U$ & $\$ d U$ \\
\hline $\begin{array}{l}\text { \#circle } 4 \\
\text { set maxU [expr \$maxU+\$step] } \\
\text { integrator DisplacementControl } 8 \\
\text { set numSteps [expr int }(\$ \operatorname{maxU} / \$ \mathrm{dU})] \\
\text { analysis Static } \\
\text { analyze \$numSteps }\end{array}$ & 3 & $\$ \mathrm{~d} U$ & 1 & $\$ \mathrm{dU}$ & $\$ \mathrm{dU}$ \\
\hline $\begin{array}{l}\text { integrator DisplacementControl } 8 \\
\text { set numSteps [expr int }(2 * \$ \operatorname{maxU} / \$ \mathrm{dU}) \text { ] } \\
\text { analysis Static } \\
\text { analyze \$numSteps }\end{array}$ & 3 & \$ndU & 1 & \$ndU & \$ndU \\
\hline $\begin{array}{l}\text { integrator DisplacementControl } 8 \\
\text { set numSteps [expr int }(\$ \operatorname{maxU} / \$ \mathrm{dU})] \\
\text { analysis Static } \\
\text { analyze \$numSteps }\end{array}$ & 3 & $\$ \mathrm{dU}$ & 1 & $\$ d U$ & $\$ d U$ \\
\hline $\begin{array}{l}\text { integrator DisplacementControl } 8 \\
\text { set numSteps [expr int }(\$ \operatorname{maxU} / \$ \mathrm{dU})] \\
\text { analysis Static } \\
\text { analyze \$numSteps }\end{array}$ & 3 & $\$ \mathrm{dU}$ & 1 & $\$ \mathrm{dU}$ & $\$ d U$ \\
\hline $\begin{array}{l}\text { integrator DisplacementControl } 8 \\
\text { set numSteps [expr int }(2 * \$ \operatorname{maxU} / \$ \mathrm{dU}) \text { ] } \\
\text { analysis Static } \\
\text { analyze \$numSteps }\end{array}$ & 3 & \$ndU & 1 & \$ndU & \$ndU \\
\hline
\end{tabular}




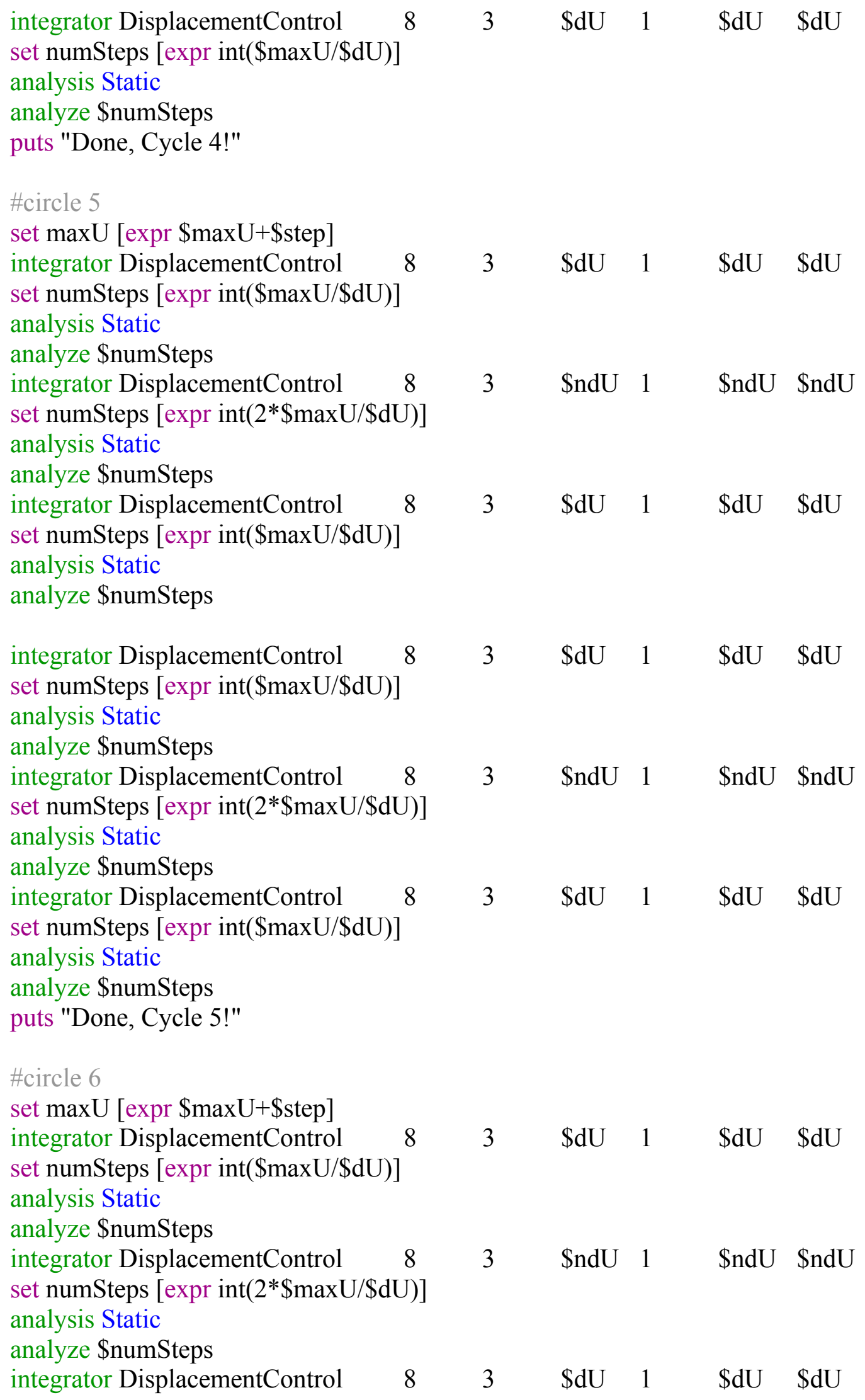


set numSteps [expr int $(\$ \max U / \$ d U)]$

analysis Static

analyze \$numSteps

$\begin{array}{lllllll}\text { integrator DisplacementControl } & 8 & 3 & \$ d U & 1 & \$ d U & \$ d U\end{array}$

set numSteps [expr int $(\$ \max U / \$ d U)]$

analysis Static

analyze \$numSteps

integrator DisplacementControl $\quad 8 \quad 3 \quad$ \$ndU $1 \quad$ \$ndU $\quad$ SndU

set numSteps [expr int $(2 * \$ \max U / \$ d U)]$

analysis Static

analyze \$numSteps

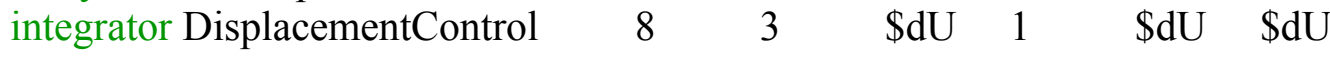

set numSteps [expr int $(\$ \max U / \$ d U)]$

analysis Static

analyze \$numSteps

puts "Done, Cycle 6!"

puts "Done, Great Job!"

\section{Appendix E. Input File Specimen GFF Static Analysis}

\section{File Name: Specimen GFF}

wipe;

model BasicBuilder -ndm 3 -ndf 6

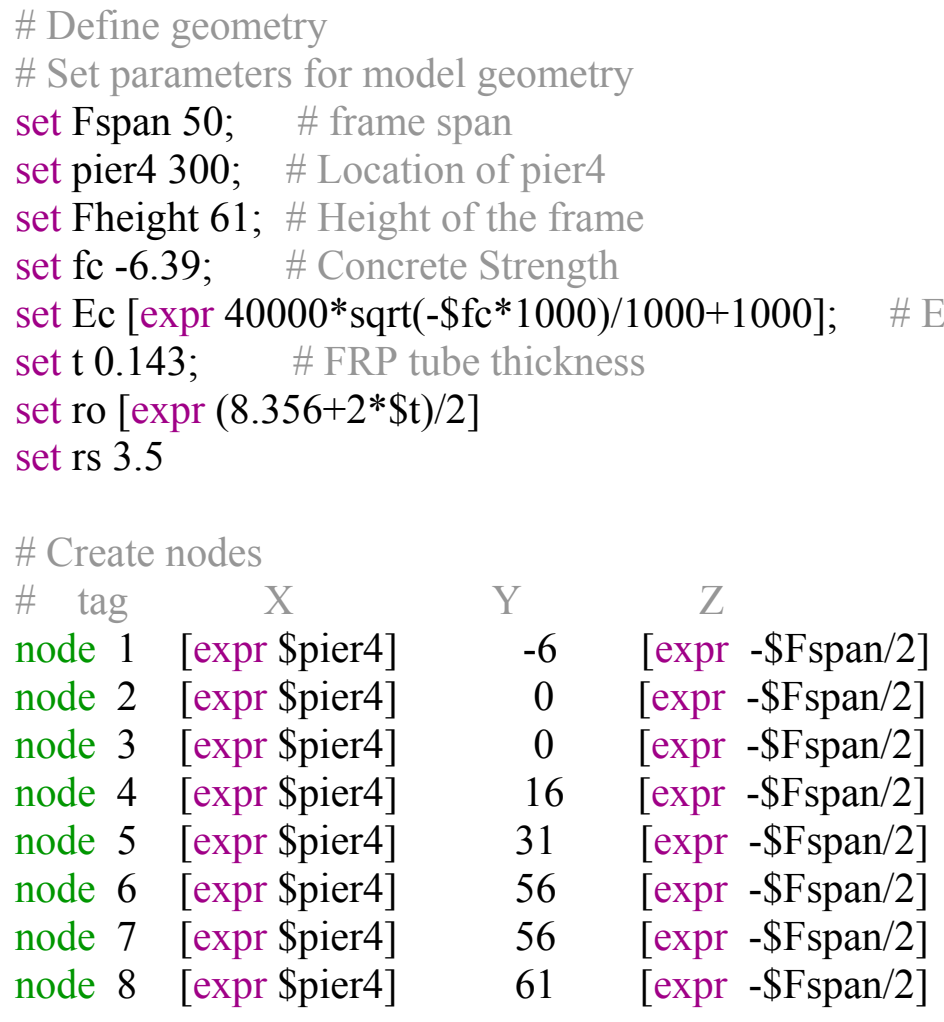




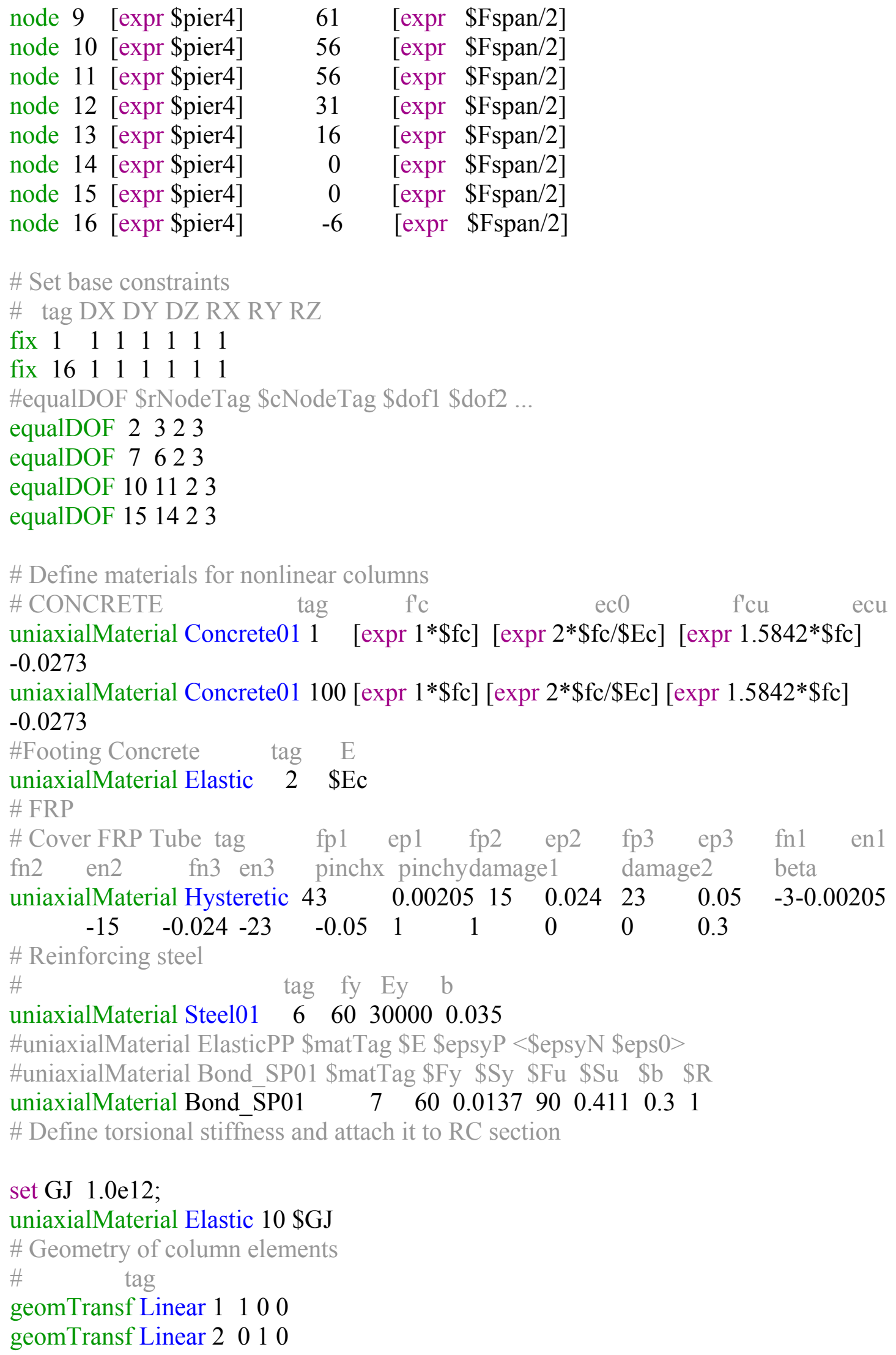




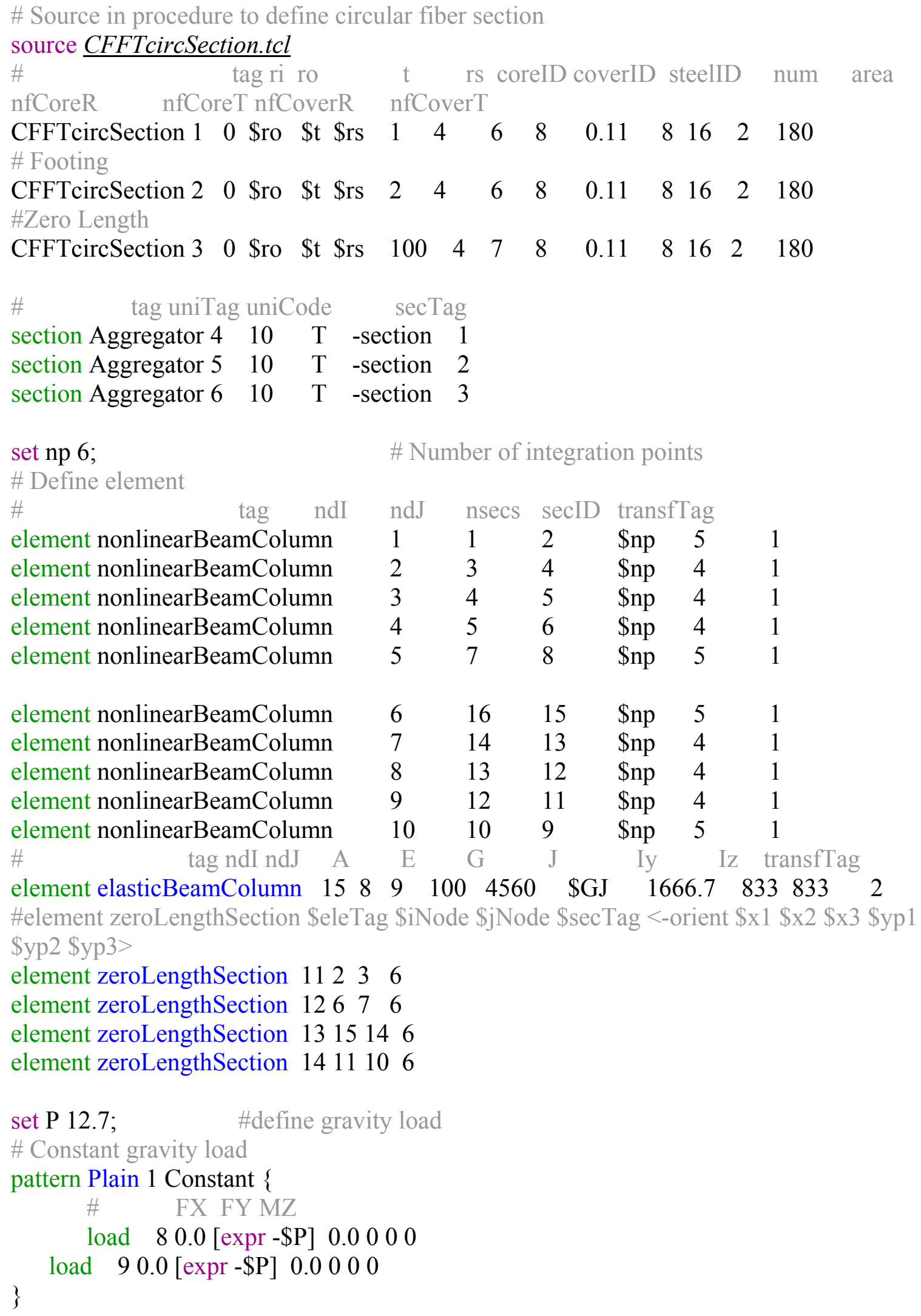


set numSteps [expr int $(\$ \max U / \$ d U)]$

analysis Static

analyze \$numSteps

integrator DisplacementControl $\quad \begin{array}{lllllll}8 & 3 & \$ d U & 1 & \$ d U & \$ d U\end{array}$

set numSteps [expr int $(\$ \max U / \$ d U)]$

analysis Static

analyze \$numSteps

integrator DisplacementControl $\quad 8 \quad 3 \quad$ \$ndU $1 \quad$ \$ndU $\quad$ SndU

set numSteps [expr int $(2 * \$ \max U / \$ d U)]$

analysis Static

analyze \$numSteps

$\begin{array}{lllllll}\text { integrator DisplacementControl } & 8 & 3 & \$ d U & 1 & \$ d U & \$ d U\end{array}$

set numSteps [expr int $(\$ \max U / \$ d U)]$

analysis Static

analyze \$numSteps

puts "Done, Cycle 1!"

\# circle 2

set maxU [expr \$maxU+\$step]

$\begin{array}{lllllll}\text { integrator DisplacementControl } & 8 & 3 & \$ d U & 1 & \$ d U & \$ d U\end{array}$

set numSteps [expr int $(\$ \max U / \$ d U)]$

analysis Static

analyze \$numSteps

integrator DisplacementControl $\quad 8 \quad 3 \quad$ \$ndU $1 \quad$ \$ndU $\quad$ \$ndU

set numSteps [expr int $(2 * \$ \max U / \$ d U)]$

analysis Static

analyze \$numSteps

integrator DisplacementControl $\quad \begin{array}{lllllll}8 & 3 & \$ d U & 1 & \$ d U & \$ d U\end{array}$

set numSteps [expr int $(\$ \operatorname{maxU} / \$ d U)]$

analysis Static

analyze \$numSteps

integrator DisplacementControl $\quad \begin{array}{lllllll}8 & 3 & \$ \mathrm{dU} & 1 & \$ \mathrm{dU} & \$ \mathrm{dU}\end{array}$

set numSteps [expr int $(\$ \max U / \$ d U)]$

analysis Static

analyze \$numSteps

integrator DisplacementControl $\quad 8 \quad 3 \quad$ \$ndU $1 \quad$ \$ndU $\quad$ SndU

set numSteps [expr int $(2 * \$ \max U / \$ d U)]$

analysis Static

analyze \$numSteps

integrator DisplacementControl $\quad \begin{array}{lllllll}8 & 3 & \$ d U & 1 & \$ d U & \$ d U\end{array}$

set numSteps [expr int $(\$ \max U / \$ d U)]$

analysis Static

analyze \$numSteps

puts "Done, Cycle 2!" 


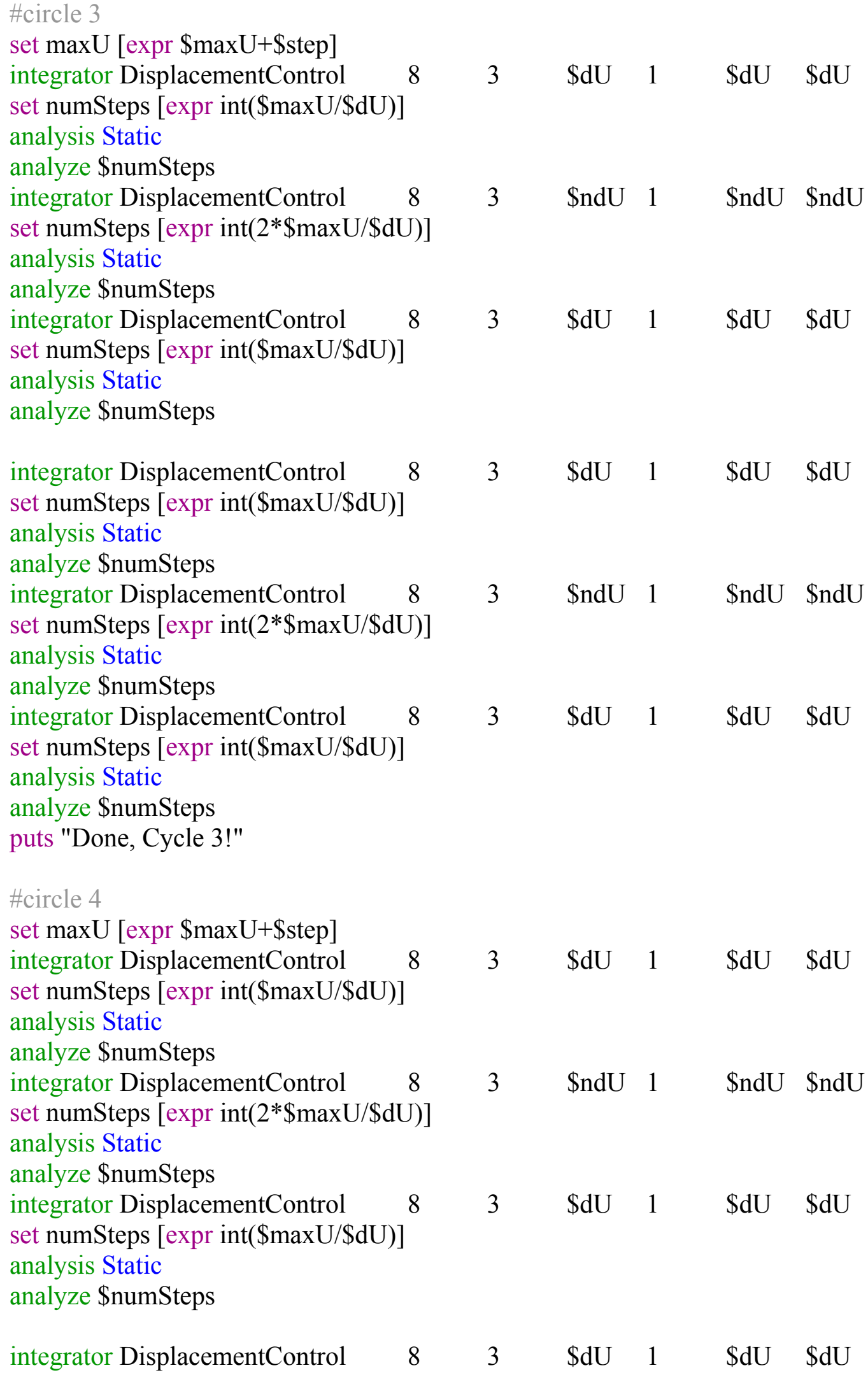




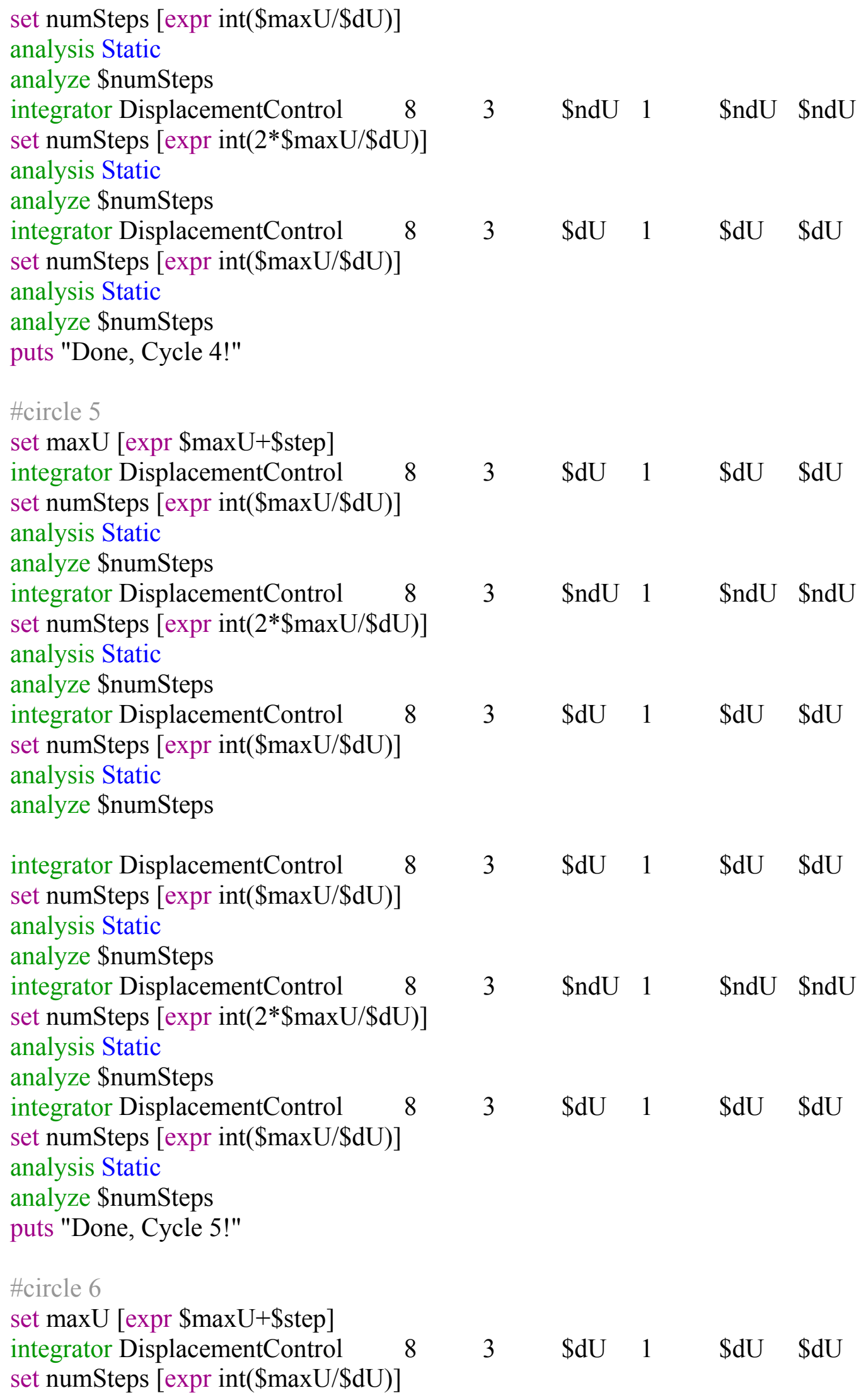


analysis Static

analyze \$numSteps

integrator DisplacementControl $\quad 8 \quad 3 \quad$ \$ndU $1 \quad$ \$ndU $\quad$ SndU

set numSteps [expr int $(2 * \$ \max U / \$ d U)]$

analysis Static

analyze \$numSteps

integrator DisplacementControl $\quad \begin{array}{llllll}8 & 3 & \$ d U & 1 & \$ d U & \$ d U\end{array}$

set numSteps [expr int $(\$ \max U / \$ d U)]$

analysis Static

analyze \$numSteps

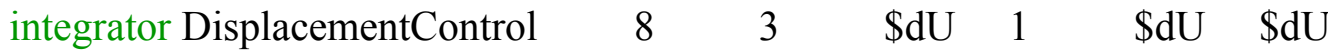

set numSteps [expr int $(\$ \max U / \$ d U)]$

analysis Static

analyze \$numSteps

integrator DisplacementControl $\quad 8 \quad 3 \quad$ \$ndU $1 \quad$ \$ndU $\quad$ SndU

set numSteps [expr int $(2 * \$ \operatorname{maxU} / \$ \mathrm{dU})]$

analysis Static

analyze \$numSteps

integrator DisplacementControl $\quad \begin{array}{lllllll}8 & 3 & \$ d U & 1 & \$ d U & \$ d U\end{array}$

set numSteps [expr int $(\$ \max U / \$ d U)]$

analysis Static

analyze \$numSteps

puts "Done, Cycle 6!"

\section{\#circle 7}

set $\max U$ [expr \$maxU+\$step]

integrator DisplacementControl $\quad \begin{array}{lllllll}8 & 3 & \$ d U & 1 & \$ d U & \$ d U\end{array}$

set numSteps [expr int $(\$ \max U / \$ d U)]$

analysis Static

analyze \$numSteps

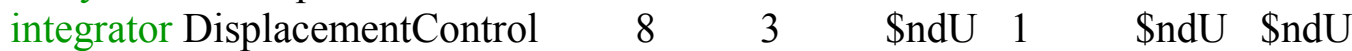

set numSteps [expr int $(2 * \$ \max U / \$ d U)]$

analysis Static

analyze \$numSteps

$\begin{array}{lllllll}\text { integrator DisplacementControl } & 8 & 3 & \$ d U & 1 & \$ d U & \$ d U\end{array}$

set numSteps [expr int $(\$ \max U / \$ d U)]$

analysis Static

analyze \$numSteps

$\begin{array}{lllllll}\text { integrator DisplacementControl } & 8 & 3 & \$ d U & 1 & \$ d U & \$ d U\end{array}$

set numSteps [expr int $(\$ \max U / \$ d U)]$

analysis Static

analyze \$numSteps

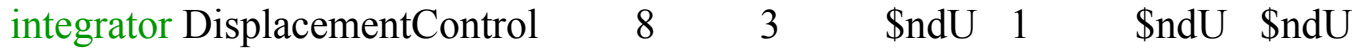

set numSteps [expr int $(2 * \$ \max U / \$ d U)]$ 
analysis Static

analyze \$numSteps

integrator DisplacementControl $\quad \begin{array}{lllllll}8 & 3 & \$ d U & 1 & \$ d U & \$ d U\end{array}$

set numSteps [expr int $(\$ \max U / \$ d U)]$

analysis Static

analyze \$numSteps

puts "Done, Cycle 7!"

\section{\#circle 8}

set maxU [expr \$maxU+\$step]

integrator DisplacementControl $\quad \begin{array}{lllllll}8 & 3 & \$ \mathrm{dU} & 1 & \$ \mathrm{dU} & \$ \mathrm{dU}\end{array}$

set numSteps [expr int $(\$ \max U / \$ d U)]$

analysis Static

analyze \$numSteps

integrator DisplacementControl $\quad 8 \quad 3 \quad$ \$ndU $1 \quad$ \$ndU $\quad$ \$ndU

set numSteps [expr int $(2 * \$ \operatorname{maxU} / \$ d U)]$

analysis Static

analyze \$numSteps

integrator DisplacementControl $\quad \begin{array}{llllll}8 & 3 & \$ \mathrm{dU} & 1 & \$ \mathrm{dU} & \$ \mathrm{dU}\end{array}$

set numSteps [expr int $(\$ \max U / \$ d U)]$

analysis Static

analyze \$numSteps

$\begin{array}{lllllll}\text { integrator DisplacementControl } & 8 & 3 & \$ d U & 1 & \$ d U & \$ d U\end{array}$

set numSteps [expr int $(\$ \max U / \$ d U)]$

analysis Static

analyze \$numSteps

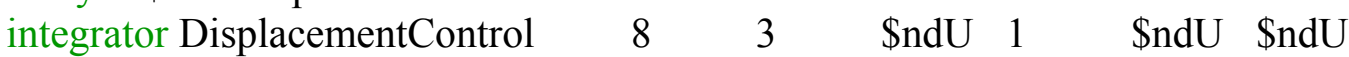

set numSteps [expr int $(2 * \$ \max U / \$ d U)]$

analysis Static

analyze \$numSteps

$\begin{array}{lllllll}\text { integrator DisplacementControl } & 8 & 3 & \text { SdU } & 1 & \$ d U & \$ d U\end{array}$

set numSteps [expr int $(\$ \max U / \$ d U)]$

analysis Static

analyze \$numSteps

puts "Done, Cycle 8!"

\section{\#circle 9}

set maxU [expr \$maxU+\$step]

integrator DisplacementControl $\quad \begin{array}{lllllll}8 & 3 & \$ d U & 1 & \$ d U & \$ d U\end{array}$

set numSteps [expr int $(\$ \max U / \$ d U)]$

analysis Static

analyze \$numSteps

integrator DisplacementControl $\quad 8 \quad 3 \quad$ \$ndU $1 \quad$ \$ndU $\quad$ \$ndU

set numSteps [expr int $(2 * \$ \max U / \$ d U)]$

analysis Static 
analyze \$numSteps

integrator DisplacementControl $\quad \begin{array}{lllllll}8 & 3 & \$ d U & 1 & \$ d U & \$ d U\end{array}$

set numSteps [expr int $(\$ \max U / \$ d U)]$

analysis Static

analyze \$numSteps

$\begin{array}{lllllll}\text { integrator DisplacementControl } & 8 & 3 & \text { \$dU } & 1 & \$ d U & \$ d U\end{array}$

set numSteps [expr int $(\$ \max U / \$ d U)]$

analysis Static

analyze \$numSteps

integrator DisplacementControl $\quad 8 \quad 3 \quad$ \$ndU $1 \quad$ \$ndU $\quad$ \$ndU

set numSteps [expr int $(2 * \$ \max U / \$ d U)]$

analysis Static

analyze \$numSteps

integrator DisplacementControl $\quad \begin{array}{lllllll}8 & 3 & \$ d U & 1 & \$ d U & \$ d U\end{array}$

set numSteps [expr int $(\$ \max U / \$ d U)]$

analysis Static

analyze \$numSteps

puts "Done, Cycle 9!"

\section{\#circle 10}

set maxU [expr \$maxU+\$step]

integrator DisplacementControl $\quad \begin{array}{lllllll}8 & 3 & \$ d U & 1 & \$ d U & \$ d U\end{array}$

set numSteps [expr int $(\$ \max U / \$ d U)]$

analysis Static

analyze \$numSteps

integrator DisplacementControl $\quad 8 \quad 3 \quad$ \$ndU $1 \quad$ \$ndU $\quad$ SndU

set numSteps [expr int $(2 * \$ \max U / \$ d U)]$

analysis Static

analyze \$numSteps

$\begin{array}{lllllll}\text { integrator DisplacementControl } & 8 & 3 & \$ d U & 1 & \$ d U & \$ d U\end{array}$

set numSteps [expr int $(\$ \max U / \$ d U)]$

analysis Static

analyze \$numSteps

$\begin{array}{lllllll}\text { integrator DisplacementControl } & 8 & 3 & \$ d U & 1 & \$ d U & \$ d U\end{array}$

set numSteps [expr int $(\$ \max U / \$ d U)]$

analysis Static

analyze \$numSteps

integrator DisplacementControl $\quad 8 \quad 3 \quad$ \$ndU $1 \quad$ \$ndU $\quad$ \$ndU

set numSteps [expr int $(2 * \$ \max U / \$ d U)]$

analysis Static

analyze \$numSteps

$\begin{array}{lllllll}\text { integrator DisplacementControl } & 8 & 3 & \$ d U & 1 & \$ d U & \$ d U\end{array}$

set numSteps [expr int $(\$ \max U / \$ d U)]$

analysis Static 
analyze \$numSteps

puts "Done, Cycle 10!"

puts "Done, Great Job!"

\section{Appendix F. Input File RC Bridge Seismic Analysis}

\section{File Name: RC Bridge under Tabas Earthqukae Accelaration}

\# Units: kips, ft, sec

\# Start of model generation

\# Create ModelBuilder with 3 dimensions and 6 DOF/node

wipe;

model BasicBuilder -ndm 3 -ndf 6

\# Define geometry

\#Set parameters for model geometry

set $\mathrm{w}$ 12.5; \# Half of the superstructure width

set p11 98; \# Location of pier 1

set p21 214; \# Location of pier 2

set p31 330; \# Location of pier 3

set p1h 22.5; \# pier 1 column height

set $\mathrm{p} 2 \mathrm{~h} 30.5$; \# pier 2 column height

set p3h 26.5; \# pier 3 column height

set hb 2.5; \# half height of the pier cap beam

set ro 2; \# Radius of Column

set t 0.25 ; \# Cover

set rs 1.75; \# Radius of Steel

\# Create nodes

\# $\operatorname{tag} \mathrm{X}$ Y $\mathrm{Z}$

\#Span 1

node $\begin{array}{llll}60 & 0 & 33.8 & 0\end{array}$

$\begin{array}{llll}\text { node } 1 & 24.5 & 33.8 & 0\end{array}$

$\begin{array}{llll}\text { node } 2 & 49.0 & 33.8 & 0\end{array}$

$\begin{array}{llll}\text { node } 3 & 73.5 & 33.8 & 0\end{array}$

\#Span 2

node $\begin{array}{llll}4 & 98 & 33.8 & 0\end{array}$

$\begin{array}{llll}\text { node } 5 & 127 & 33.8 & 0\end{array}$

node $\begin{array}{llll}6 & 156 & 33.8 & 0\end{array}$

node $\begin{array}{llll}7 & 185 & 33.8 & 0\end{array}$

\#Span 3

node $8 \quad 214 \quad 33.8 \quad 0$

$\begin{array}{llll}\text { node } 9 & 243 & 33.8 & 0\end{array}$

node $\begin{array}{llll}10 & 272 & 33.8 & 0\end{array}$

node $\begin{array}{llll}11 & 301 & 33.8 & 0\end{array}$

\#Span 4

node $\begin{array}{llll}12 & 330 & 33.8 & 0\end{array}$ 
node $13 \quad 354.533 .8 \quad 0$

node $14 \quad 379 \quad 33.8 \quad 0$

node $\begin{array}{llll}15 & 403.5 & 33.8 & 0\end{array}$

node $\begin{array}{llll}62 & 428 & 33.8 & 0\end{array}$

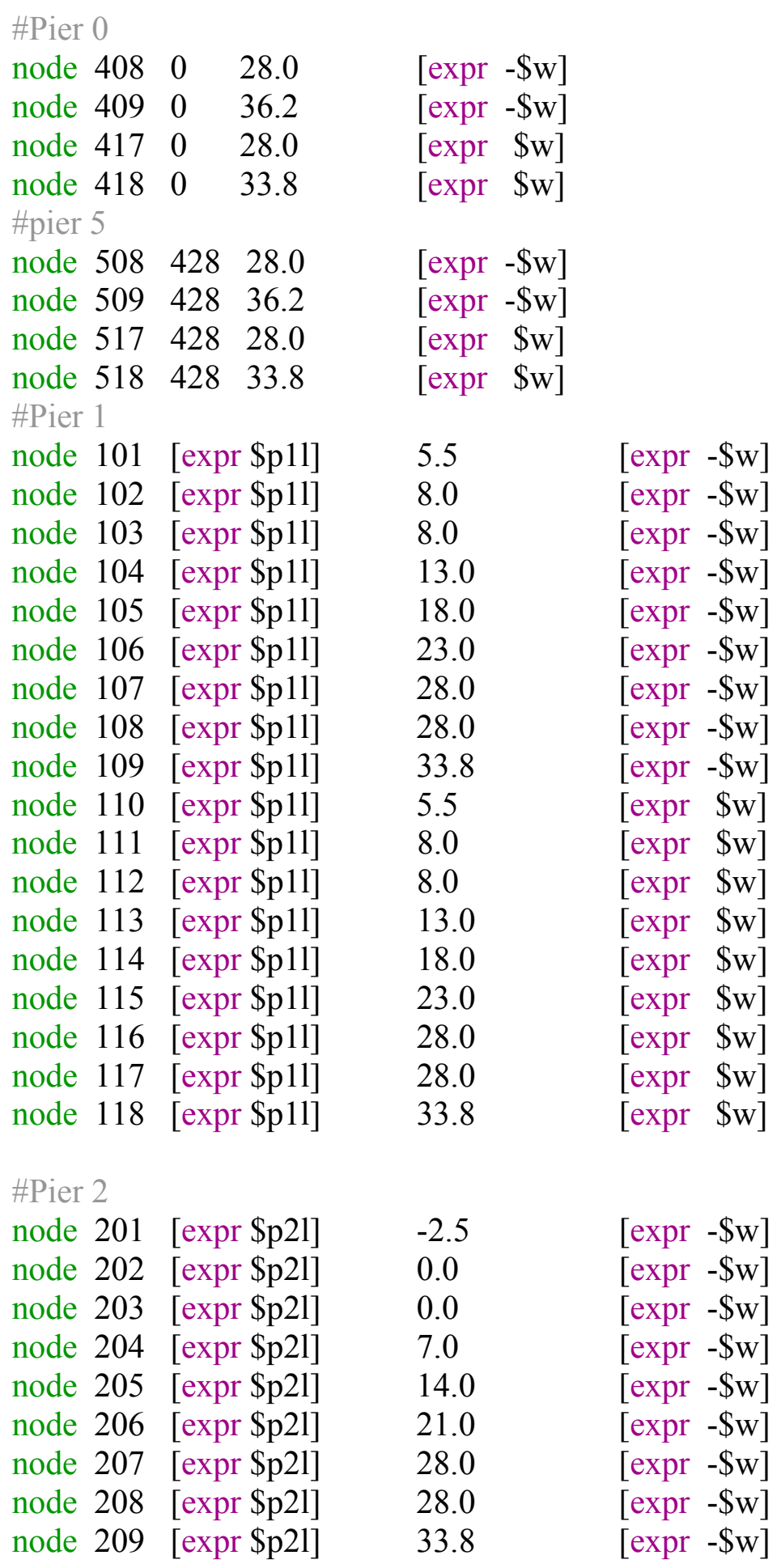




$\left.\begin{array}{llllll}\text { node } & 210 & \text { [expr } \$ \mathrm{p} 21] & -2.5 & {[\operatorname{expr}} & \$ \mathrm{w}\end{array}\right]$




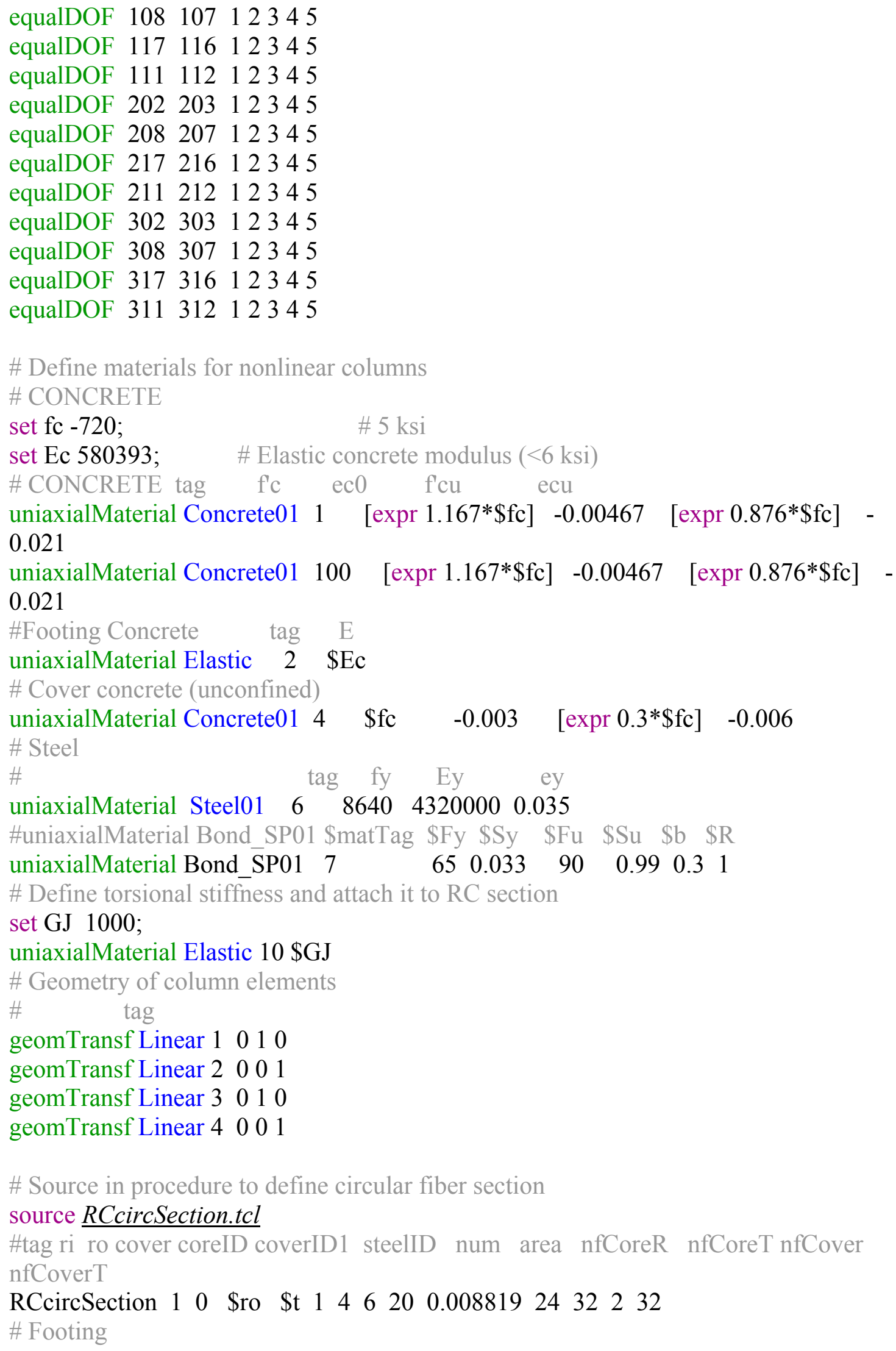


RCcircSection $20 \quad$ \$ro $\$$ \$t 2226200.0088192432232

\#Zero Length

RCcircSection $30 \quad$ \$ro $\$$ \$t $10047200.00881924 \quad 32 \quad 232$

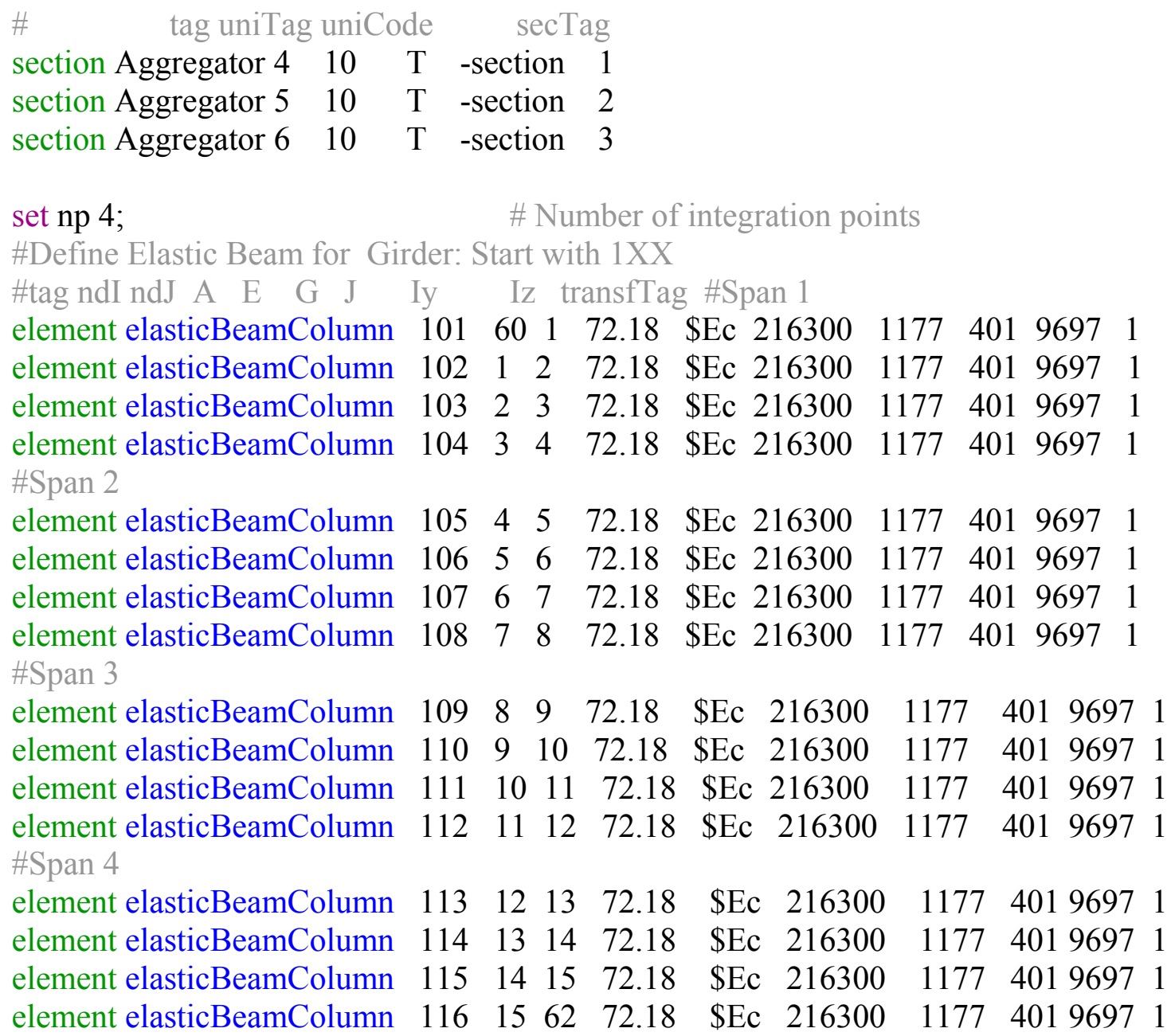

\#Define Elastic Pier Cap Beam: Start with 2xx

\# tag ndI ndJ A E G J Iy Iz transfTag

element elasticBeamColumn $197 \begin{array}{lllllll}1909 & 60 & 25 & \text { \$Ec } & 216300 & 10000 & 10000\end{array}$

$10000 \quad 3$

element elasticBeamColumn $\quad \begin{array}{llllllll}198 & 60 & 418 & 25 & \text { SEc } & 216300 & 10000 & 10000\end{array}$

10000

element elasticBeamColumn $\quad \begin{array}{llllllll}199 & 509 & 62 & 25 & \text { \$Ec } & 216300 & 10000 & 10000\end{array}$

$10000 \quad 3$

element elasticBeamColumn $\quad \begin{array}{llllllll}200 & 62 & 518 & 25 & \text { \$Ec } & 216300 & 10000 & 10000\end{array}$

$10000 \quad 3$

$\begin{array}{lllllllll}\text { element elasticBeamColumn } & 201 & 109 & 4 & 25 & \text { \$Ec } & 216300 & 10000 & 10000\end{array}$

$10000 \quad 3$

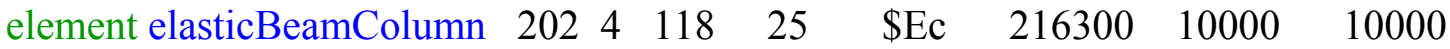

$10000 \quad 3$ 


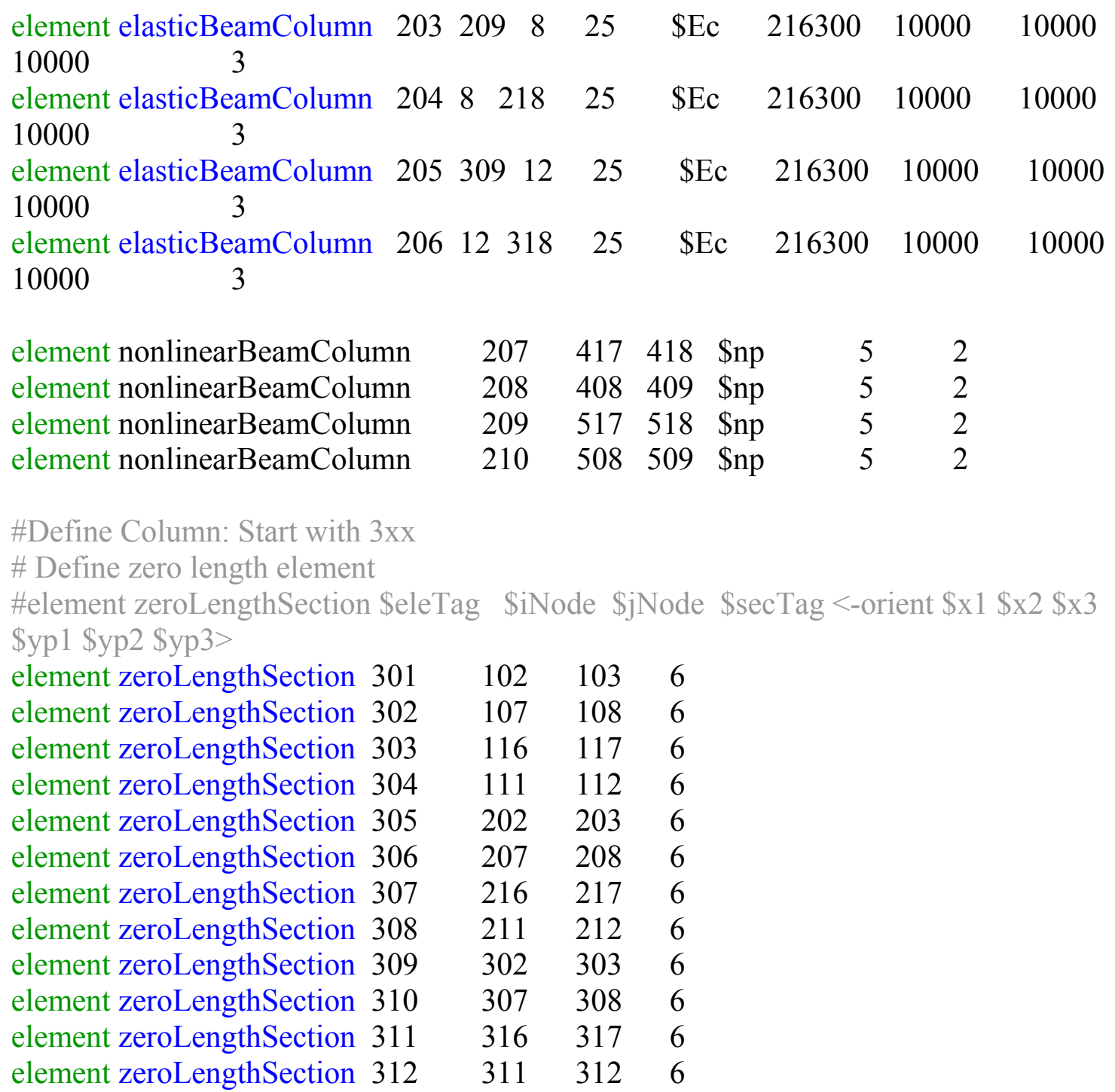

\begin{tabular}{|c|c|c|c|c|c|c|}
\hline \#Pier 1 & ndJ & \multicolumn{2}{|c|}{ nsecs secID } & \multicolumn{3}{|c|}{ transfTag } \\
\hline element nonlinearBeamColumn & 320 & 101 & 102 & $\$ n p$ & 5 & 2 \\
\hline element nonlinearBeamColumn & 321 & 103 & 104 & $\$ n p$ & 4 & 2 \\
\hline element nonlinearBeamColumn & 322 & 104 & 105 & $\$ n p$ & 4 & 2 \\
\hline element nonlinearBeamColumn & 323 & 105 & 106 & $\$ n p$ & 4 & 2 \\
\hline element nonlinearBeamColumn & 324 & 106 & 107 & $\$ n p$ & 4 & 2 \\
\hline element nonlinearBeamColumn & 325 & 108 & 109 & $\$ n p$ & 5 & 2 \\
\hline element nonlinearBeamColumn & 326 & 110 & 111 & $\$ n p$ & 5 & 2 \\
\hline element nonlinearBeamColumn & 327 & 112 & 113 & $\$ n p$ & 4 & 2 \\
\hline element nonlinearBeamColumn & 328 & 113 & 114 & $\$ n p$ & 4 & 2 \\
\hline element nonlinearBeamColumn & 329 & 114 & 115 & $\$ n p$ & 4 & 2 \\
\hline element nonlinearBeamColumn & 330 & 115 & 116 & \$np & 4 & 2 \\
\hline element nonlinearBeamColumn & 331 & 117 & 118 & $\$ n p$ & 5 & 2 \\
\hline
\end{tabular}




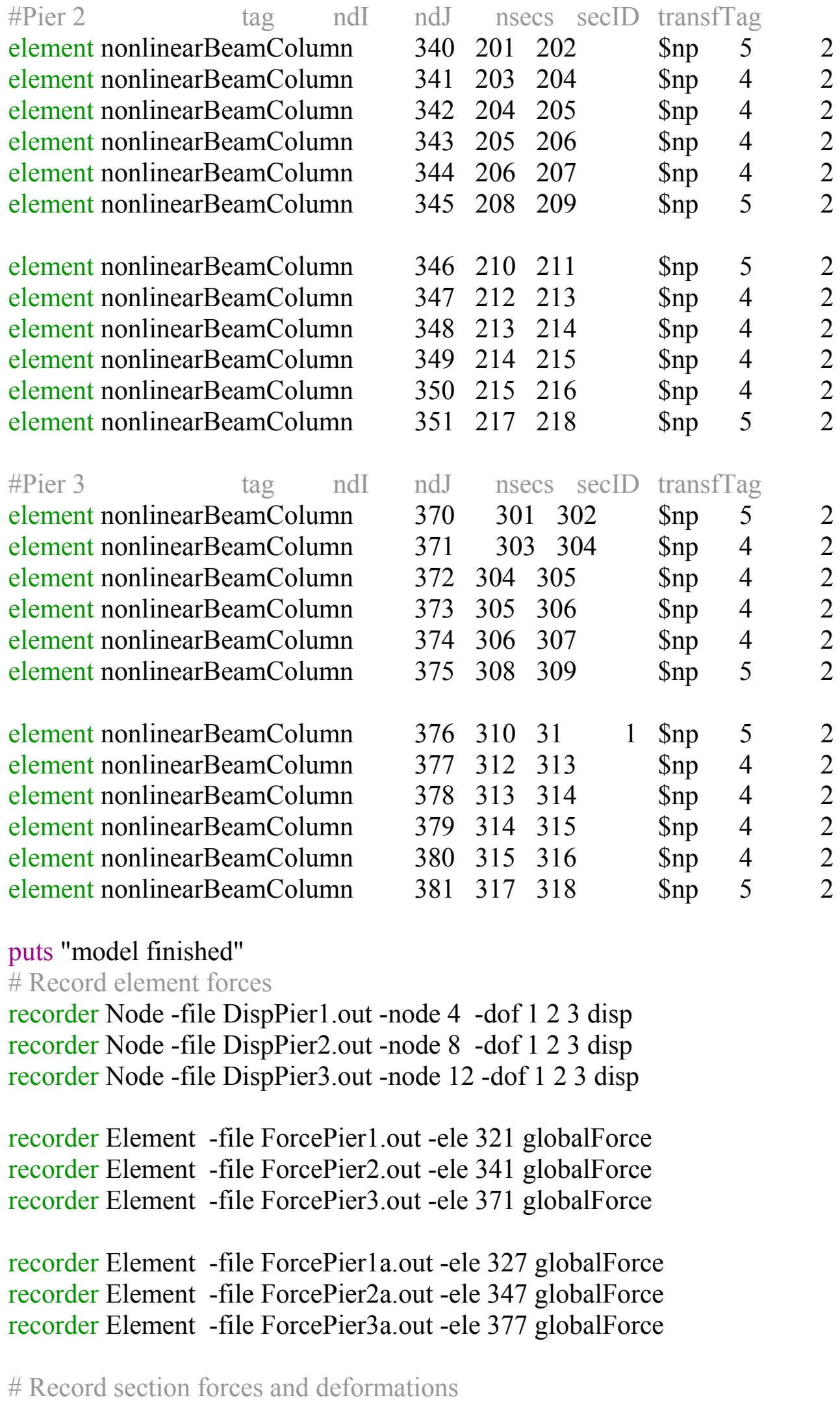


recorder Element -file Constress102.out -ele 102 section 4 fiber -1.75 0 stressStrain recorder Element -file Constress111.out -ele 111 section 4 fiber -1.75 0 stressStrain recorder Element -file Constress202.out -ele 202 section 4 fiber -1.750 stressStrain recorder Element -file Constress211.out -ele 211 section 4 fiber -1.750 stressStrain recorder Element -file Constress302.out -ele 302 section 4 fiber -1.750 stressStrain recorder Element -file Constress311.out -ele 311 section 4 fiber -1.750 stressStrain recorder Element -file Constress102a.out -ele 102 section 4 fiber 1.750 stressStrain recorder Element -file Constress111a.out -ele 111 section 4 fiber 1.750 stressStrain recorder Element -file Constress202a.out -ele 202 section 4 fiber 1.750 stressStrain recorder Element -file Constress211a.out -ele 211 section 4 fiber 1.750 stressStrain recorder Element -file Constress302a.out -ele 302 section 4 fiber 1.750 stressStrain recorder Element -file Constress311a.out -ele 311 section 4 fiber 1.750 stressStrain \# End of recorder generation

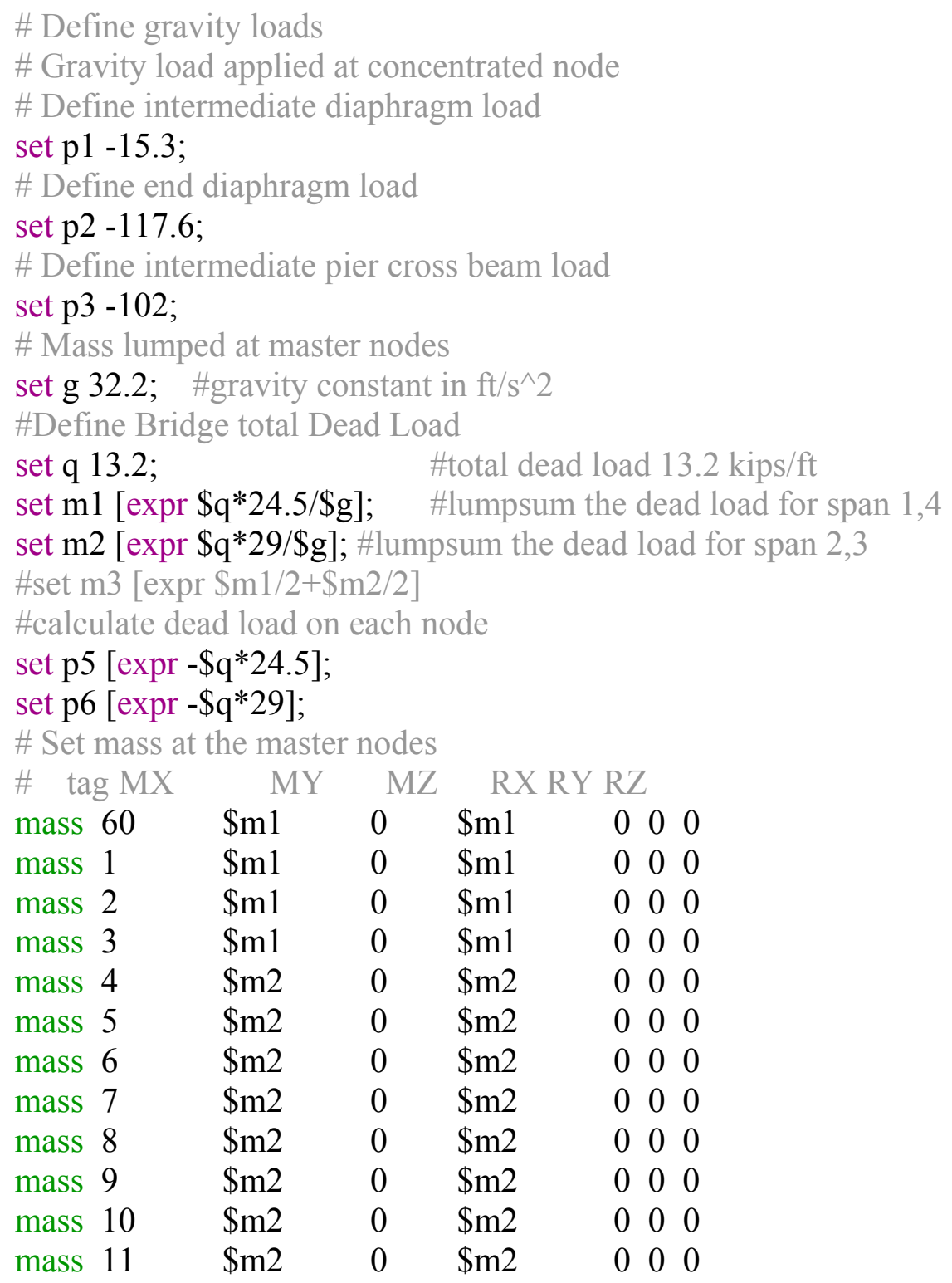




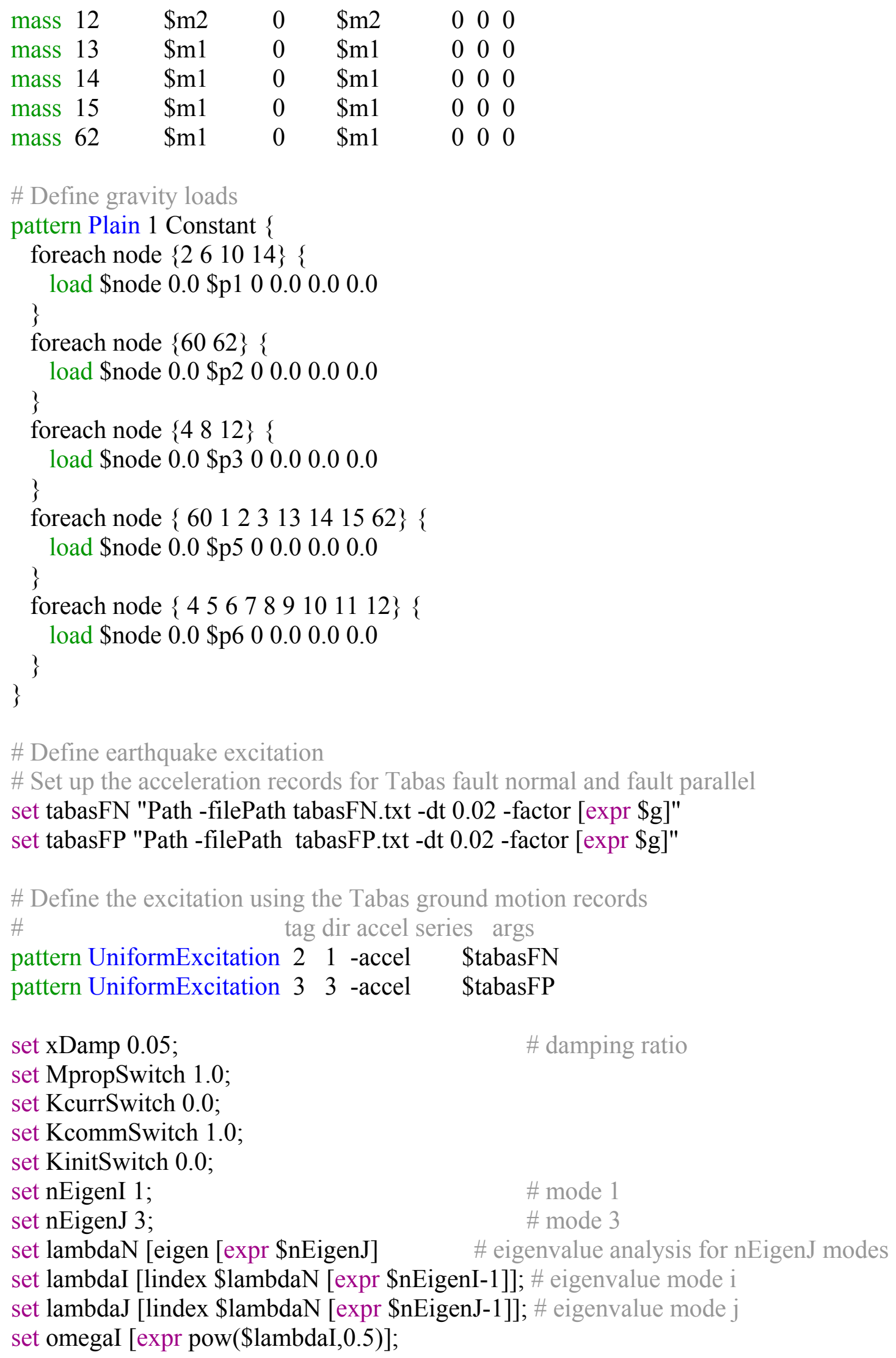


set omegaJ [expr pow(\$lambdaJ,0.5)];

set alphaM [expr \$MpropSwitch*\$xDamp*(2*\$omegaI*\$omegaJ)/(\$omegaI+\$omegaJ)];

\# M-prop. damping; $\mathrm{D}=$ alphaM*M

set betaKcurr [expr \$KcurrSwitch*2.*\$xDamp/(\$omegaI+\$omegaJ)];

\# current-K; +beatKcurr*KCurrent

set betaKcomm [expr \$KcommSwitch*2.*\$xDamp/(\$omegaI+\$omegaJ)];

\# last-committed K; +betaKcomm*KlastCommitt

set betaKinit [expr \$KinitSwitch*2.*\$xDamp/(\$omegaI+\$omegaJ)];

\# initial-K; +beatKinit*Kini

rayleigh \$alphaM \$betaKcurr \$betaKinit \$betaKcomm;

\# RAYLEIGH damping

\# End of model generation

\# Start of analysis generation

\# Create the convergence test

\# tol maxIter printFlag

test EnergyIncr 1.0e-6 $20 \quad 3$

\# Create the solution algorithm

algorithm Newton

\# Create the system of equation storage and solver

system ProfileSPD

\#system SparseGeneral -piv

\# Create the constraint handler

constraints Transformation

\# Create the time integration scheme

\# gamma beta

integrator Newmark $\quad 0.5 \quad 0.25$

\# Create the DOF numberer

numberer RCM

\# Create the transient analysis

analysis Transient

\# Perform the analysis

\# Analysis duration of 20 seconds

\# numSteps dt

analyze $2000 \quad 0.02$

puts "Done, Great Job!"

\section{Appendix G. Input File GFF Bridge Seismic Analysis}

\section{File Name: GFF Bridge under Llolleo}

\# Units: kips, ft, sec

\# Start of model generation

\# Create ModelBuilder with 3 dimensions and 6 DOF/node

wipe; 
model BasicBuilder -ndm 3 -ndf 6

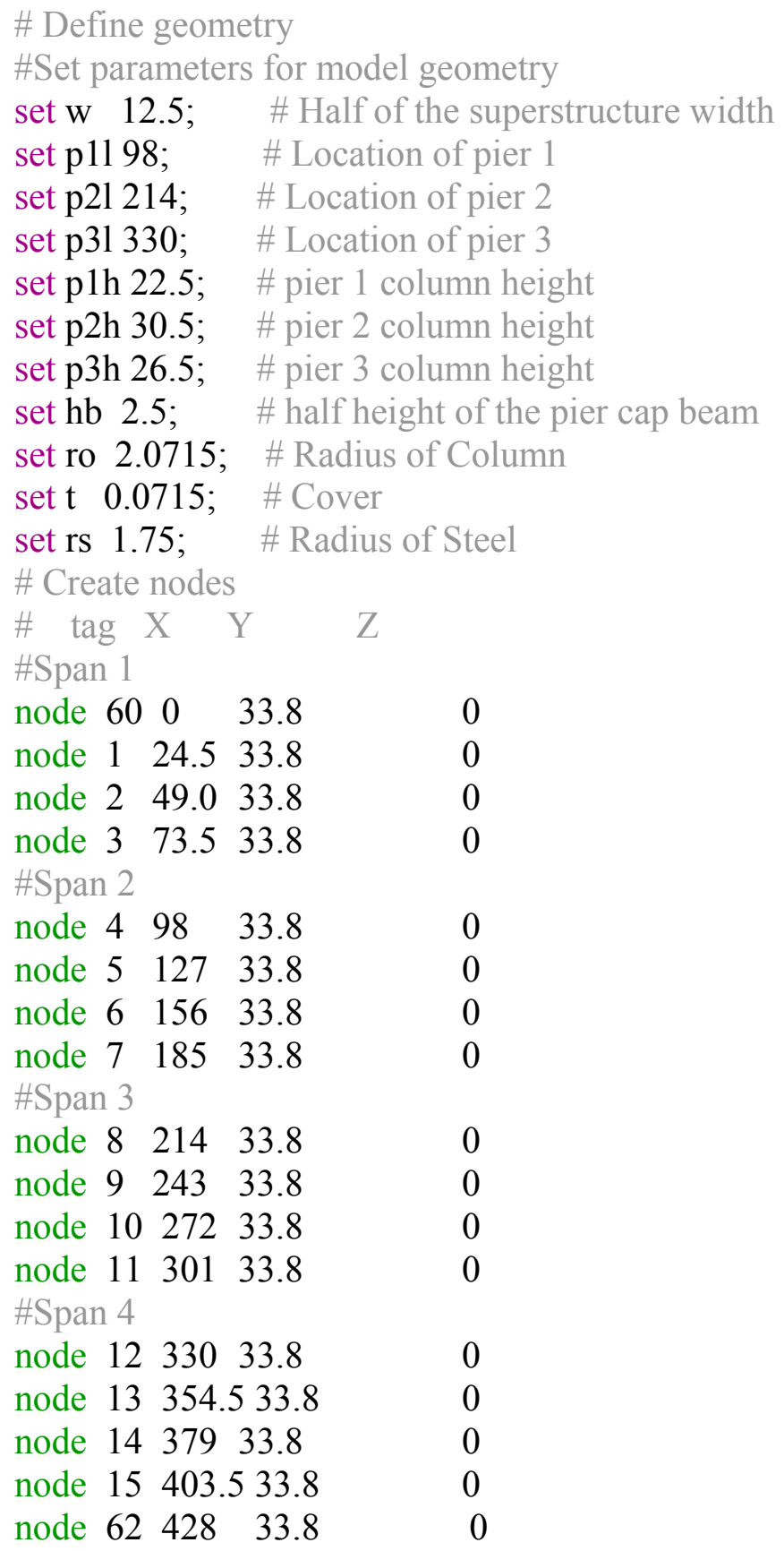

\#Pier 0

node $408 \quad 0$

28.0

node 4090

36.2

[expr $-\$ w]$

node $417 \quad 0$

[expr $-\$ w]$

node 4180

28.0

[expr \$w]

\#pier 5

33.8

$\left[\begin{array}{ll}\operatorname{expr} \$ \mathrm{~W}\end{array}\right]$

node $508 \quad 428$

28.0

node 509428

36.2

[expr $-\$ w]$

[expr -\$w] 


\begin{tabular}{|c|c|c|c|c|}
\hline de 517 & 428 & & [expr & $\$ w]$ \\
\hline node 518 & 428 & & expr & $\$ w]$ \\
\hline \#Pier 1 & & & & \\
\hline node 101 & [expr \$p11] & 5.5 & [expr & $-\$ w]$ \\
\hline node 102 & [expr \$p11] & 8.0 & [expr & $-\$ w]$ \\
\hline node 103 & [expr \$p11] & 8.0 & [expr & $-\$ w]$ \\
\hline node 104 & [expr \$p11] & 13.0 & [expr & $-\$ w]$ \\
\hline node 105 & [expr \$p11] & 18.0 & [expr & $-\$ w]$ \\
\hline node 106 & [expr \$p1l] & 23.0 & [expr & $-\$ w]$ \\
\hline node 107 & [expr \$p11] & 28.0 & {$[\mathrm{expr}$} & $-\$ w]$ \\
\hline de 108 & [expr \$p11] & 28.0 & [expr & $-\$ w]$ \\
\hline ode 109 & [expr \$p11] & 33.8 & expr & $-\$ w]$ \\
\hline de 110 & [expr \$p11] & 5.5 & [expr & $\$ w]$ \\
\hline de 111 & [expr \$p11] & 8.0 & [expr & $\$ w]$ \\
\hline de 112 & [expr \$p11] & 8.0 & [expr & $\$ w]$ \\
\hline ode 113 & [expr \$p11] & 13.0 & {$[$ expr } & \$w] \\
\hline de 114 & [expr \$p11] & 18.0 & [expr & $\$ w]$ \\
\hline de 115 & [expr \$p11] & 23.0 & [expr & $\$ w]$ \\
\hline de 116 & [expr \$p11] & 28.0 & [expr & $\$ w]$ \\
\hline node 117 & [expr \$p11] & 28.0 & [expr & $\$ w]$ \\
\hline node 118 & [expr \$p11] & 33.8 & [expr & \$w] \\
\hline $\mathrm{r} ?$ & & & & \\
\hline de 201 & [expr \$p21] & -2.5 & [expr & $-\$ w]$ \\
\hline de 202 & [expr \$p21] & 0.0 & [expr & $-\$ w]$ \\
\hline de 203 & [expr \$p21] & 0.0 & [expr & $-\$ w]$ \\
\hline de 204 & [expr \$p21] & 7.0 & expr & $-\$ w]$ \\
\hline de 205 & [expr \$p21] & 14.0 & [expr & $-\$ w]$ \\
\hline de 206 & [expr \$p21] & 21.0 & [expr & $-\$ w]$ \\
\hline de 207 & [expr \$p21] & 28.0 & [expr & $-\$ w]$ \\
\hline de 208 & [expr \$p21] & 28.0 & {$[\mathrm{expr}$} & $-\$ w]$ \\
\hline de 209 & [expr \$p21] & 33.8 & [expr & $-\$ w]$ \\
\hline de 210 & [expr \$p21] & -2.5 & [expr & \$w] \\
\hline de 211 & [expr \$p21] & 0.0 & [expr & $\$ w]$ \\
\hline de 212 & [expr \$p21] & 0.0 & expr & $\$ w]$ \\
\hline de 213 & [expr \$p21] & 7.0 & [expr & $\$ w]$ \\
\hline de 214 & [expr \$p21] & 14.0 & {$[$ expr } & $\$ w]$ \\
\hline 215 & [expr \$p21] & 21.0 & [expr & $\$ w]$ \\
\hline 216 & [expr \$p21] & 28.0 & [expr & $\$ w]$ \\
\hline 217 & [expr \$p21] & 28.0 & [expr & $\$ w]$ \\
\hline de 218 & [expr \$p21] & 33.8 & [expr & $\$ w]$ \\
\hline
\end{tabular}

\#Pier 3 


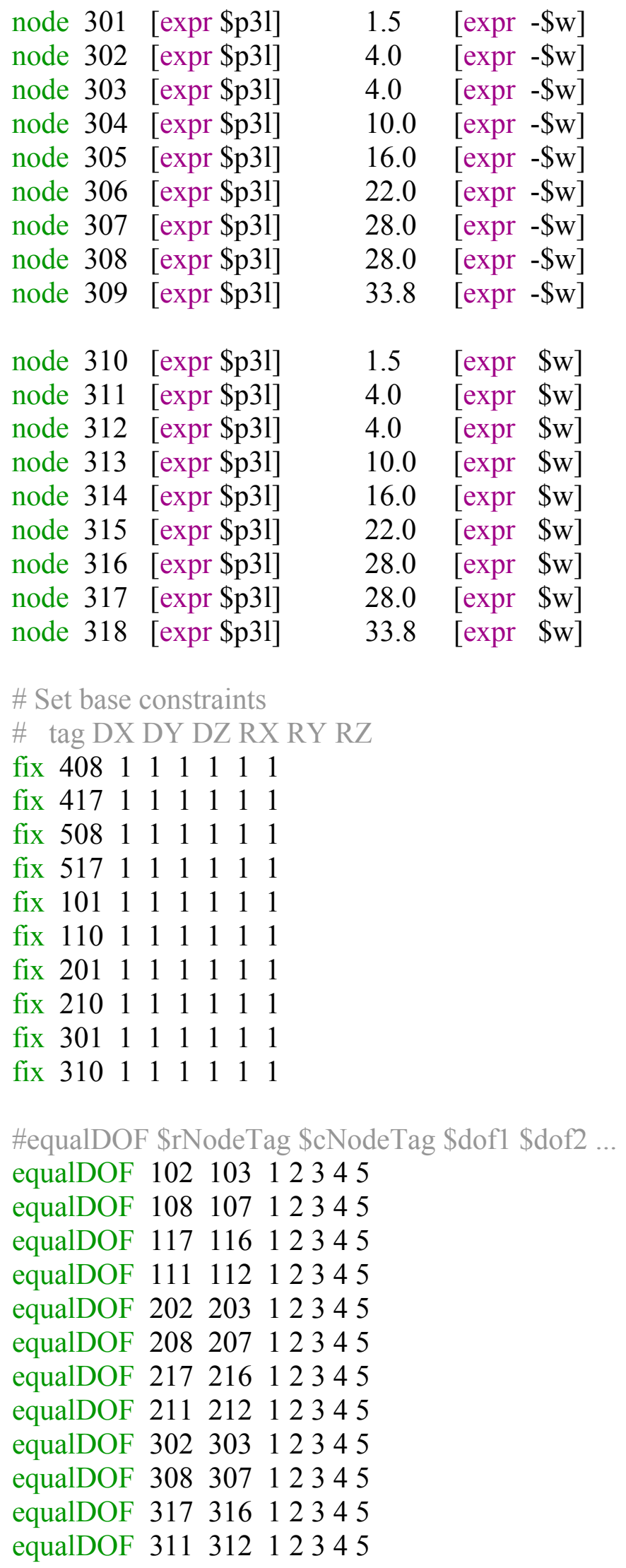




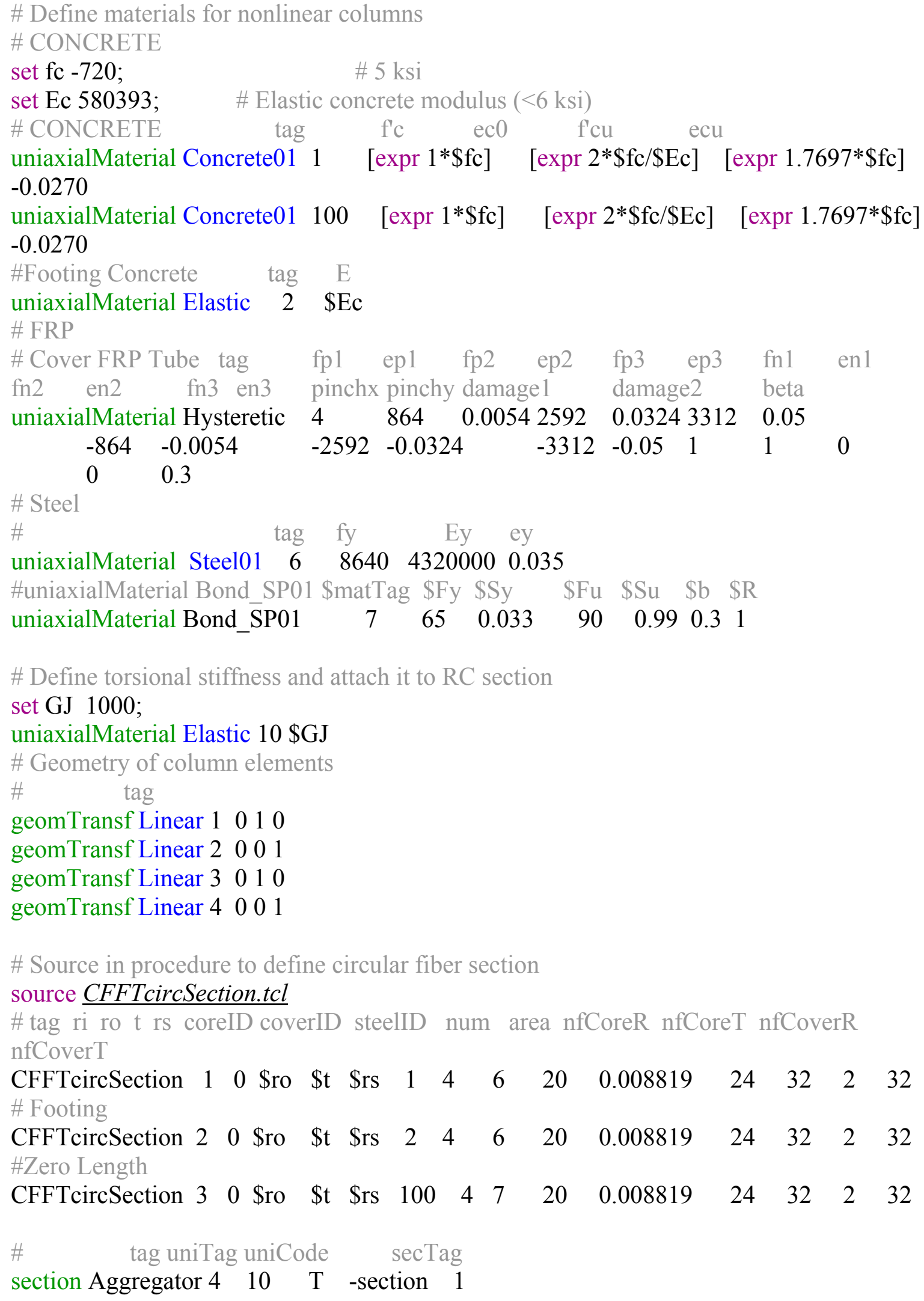




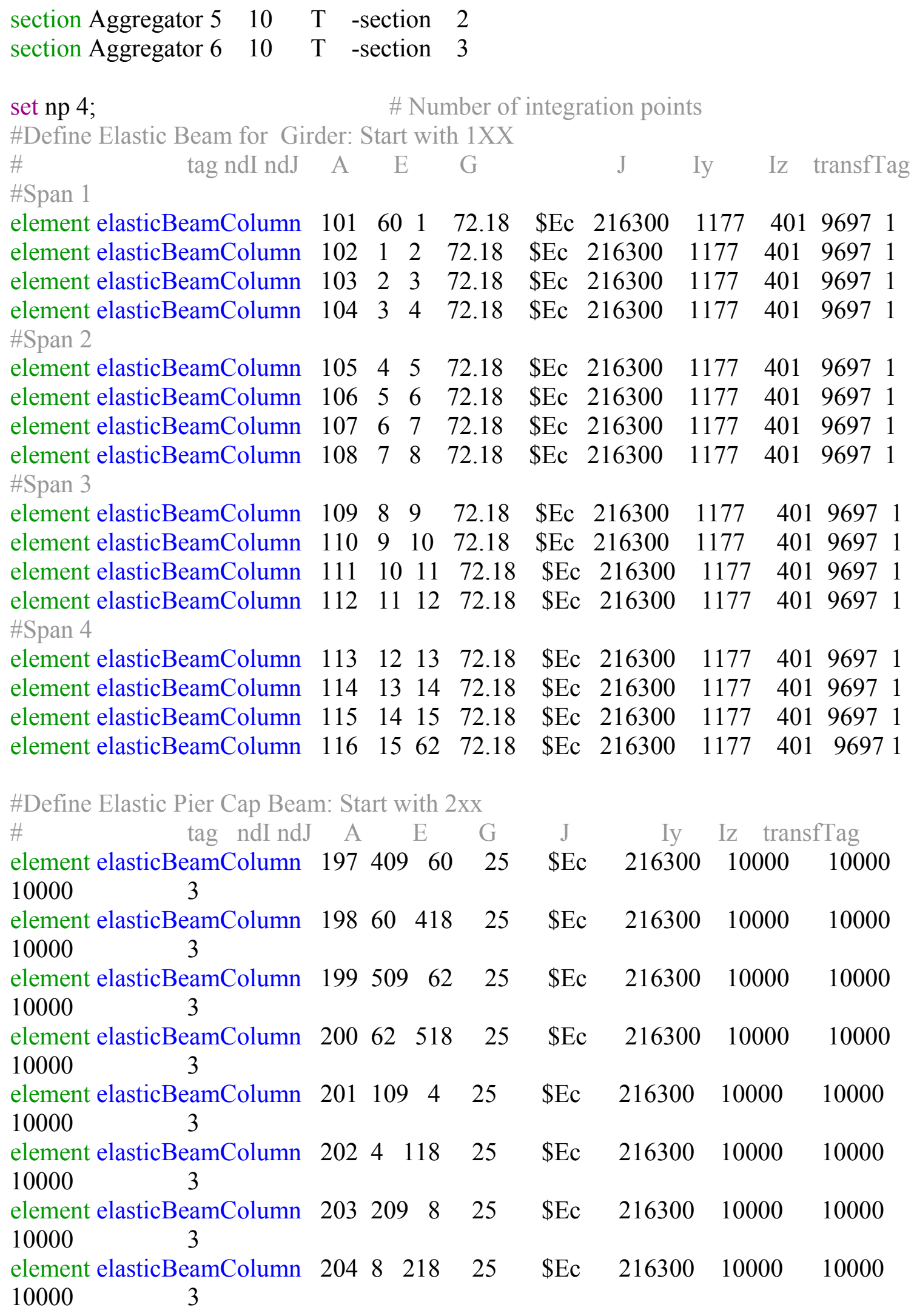




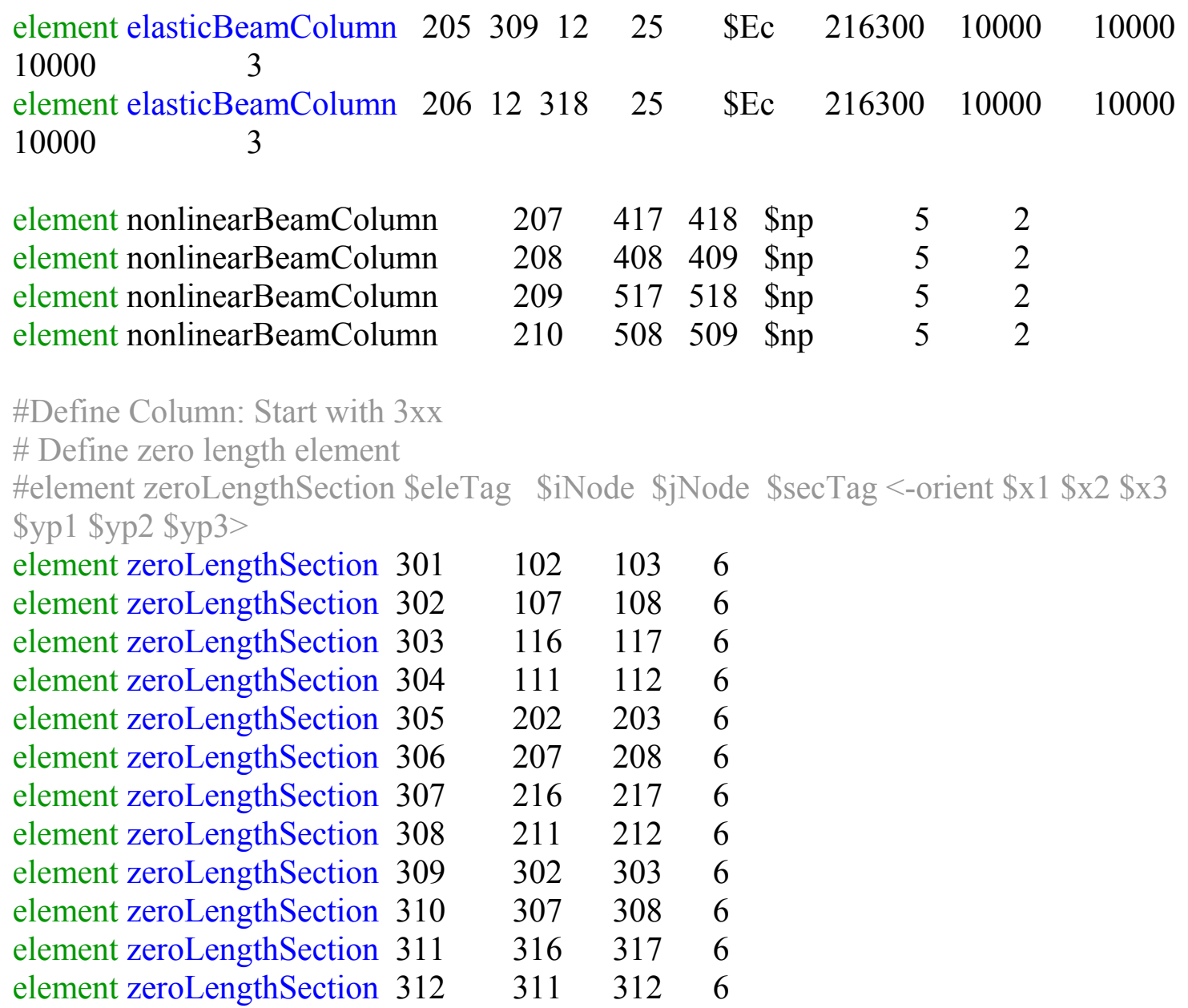

\#Define nonlinearBeamColumn element

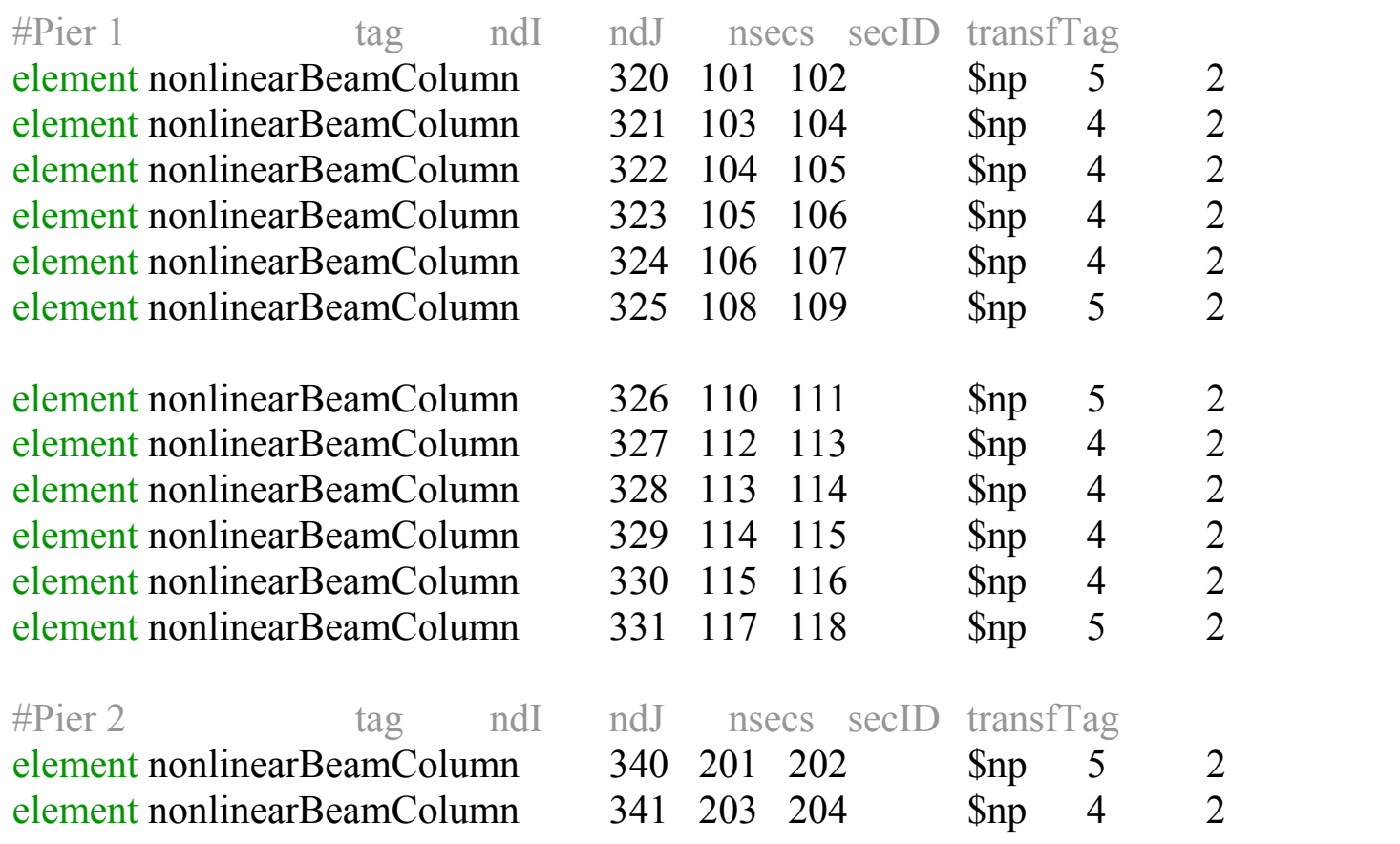




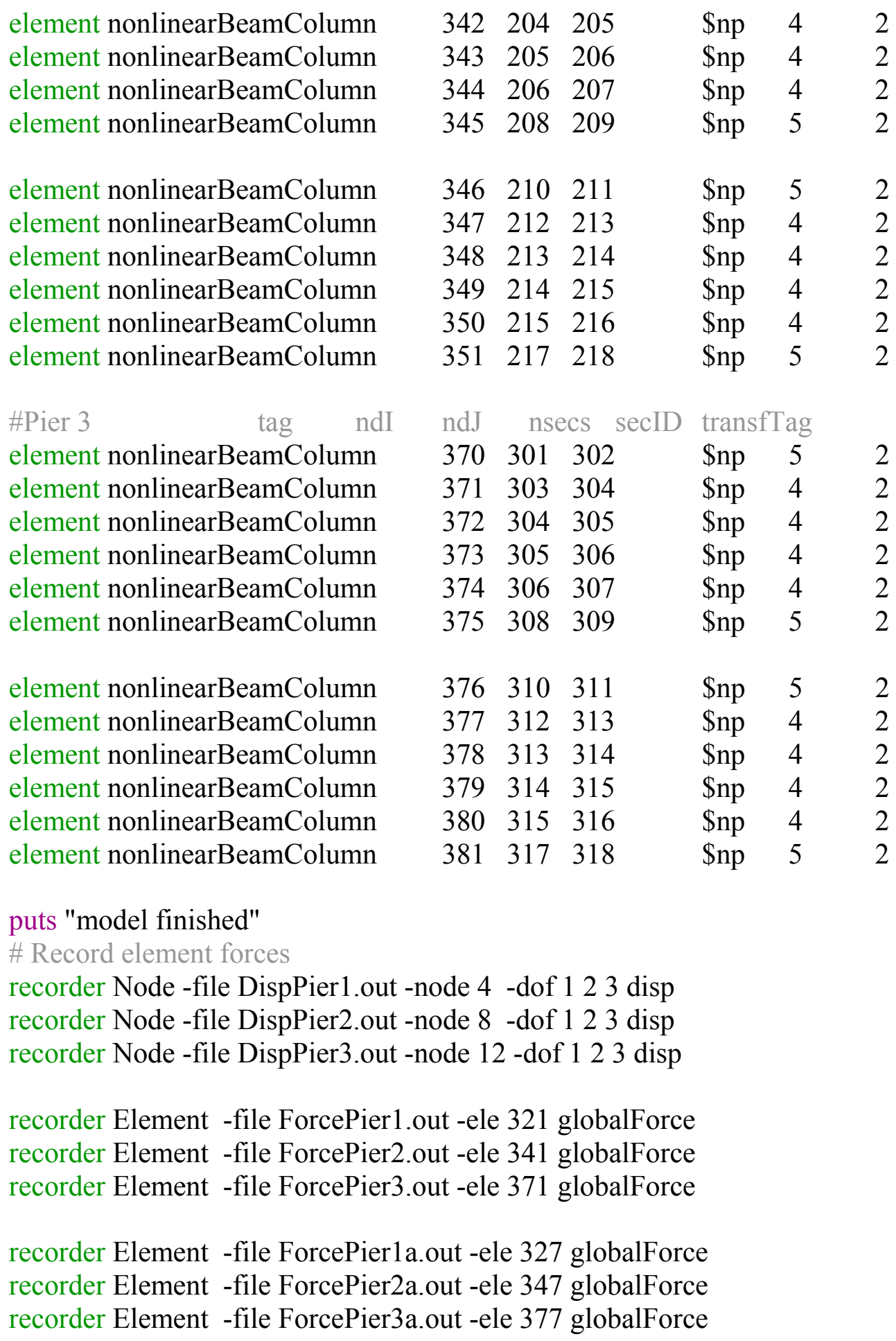

\# Record section forces and deformations

recorder Element -file Constress102.out -ele 102 section 4 fiber -1.75 0 stressStrain recorder Element -file Constress111.out -ele 111 section 4 fiber -1.750 stressStrain recorder Element -file Constress202.out -ele 202 section 4 fiber -1.75 0 stressStrain recorder Element -file Constress211.out -ele 211 section 4 fiber -1.750 stressStrain 
recorder Element -file Constress302.out -ele 302 section 4 fiber -1.750 stressStrain recorder Element -file Constress311.out -ele 311 section 4 fiber -1.750 stressStrain

recorder Element -file Constress102a.out -ele 102 section 4 fiber 1.750 stressStrain recorder Element -file Constress111a.out -ele 111 section 4 fiber 1.750 stressStrain recorder Element -file Constress202a.out -ele 202 section 4 fiber 1.750 stressStrain recorder Element -file Constress211a.out -ele 211 section 4 fiber 1.750 stressStrain recorder Element -file Constress302a.out -ele 302 section 4 fiber 1.750 stressStrain recorder Element -file Constress311a.out -ele 311 section 4 fiber 1.750 stressStrain

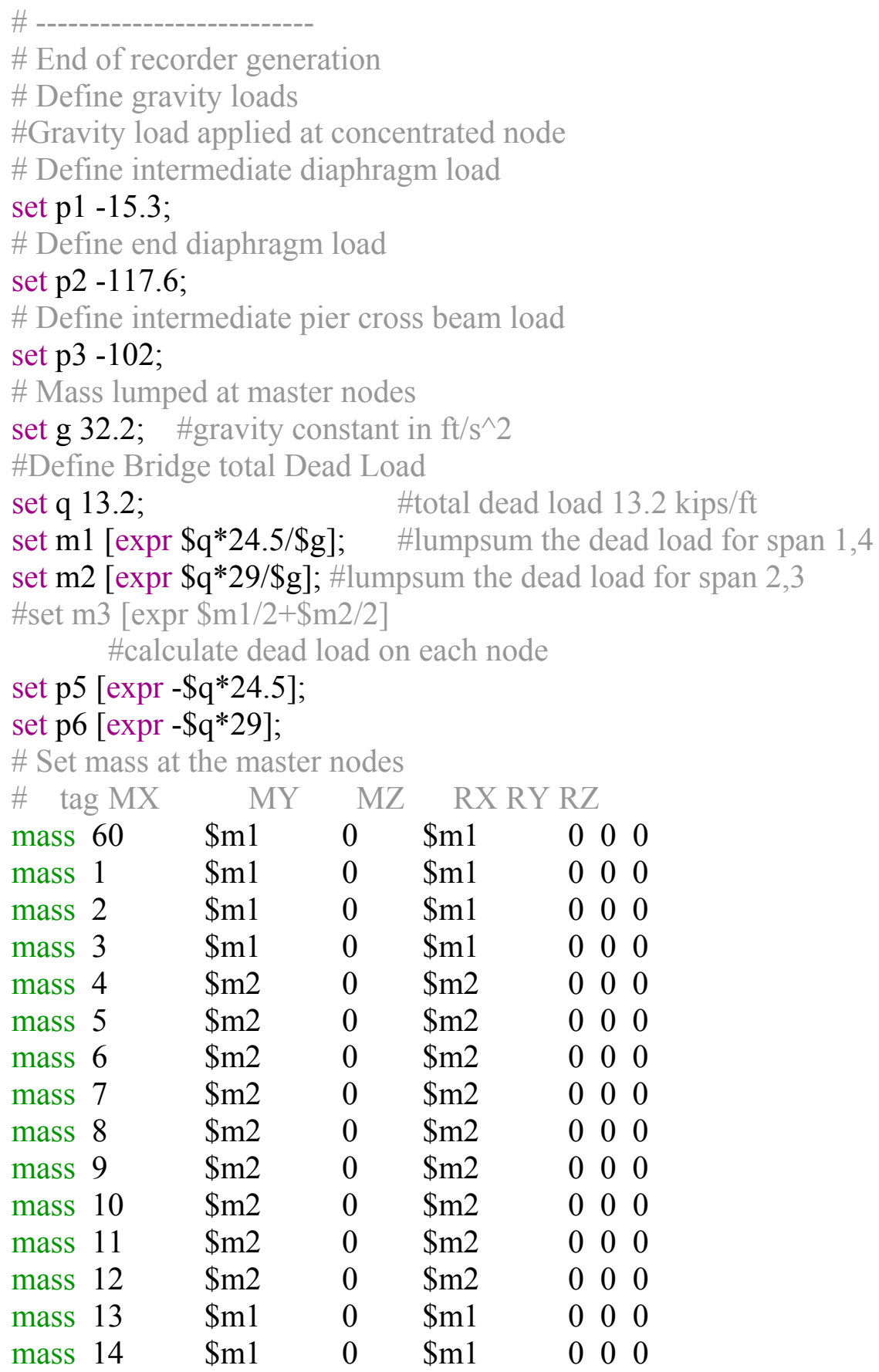




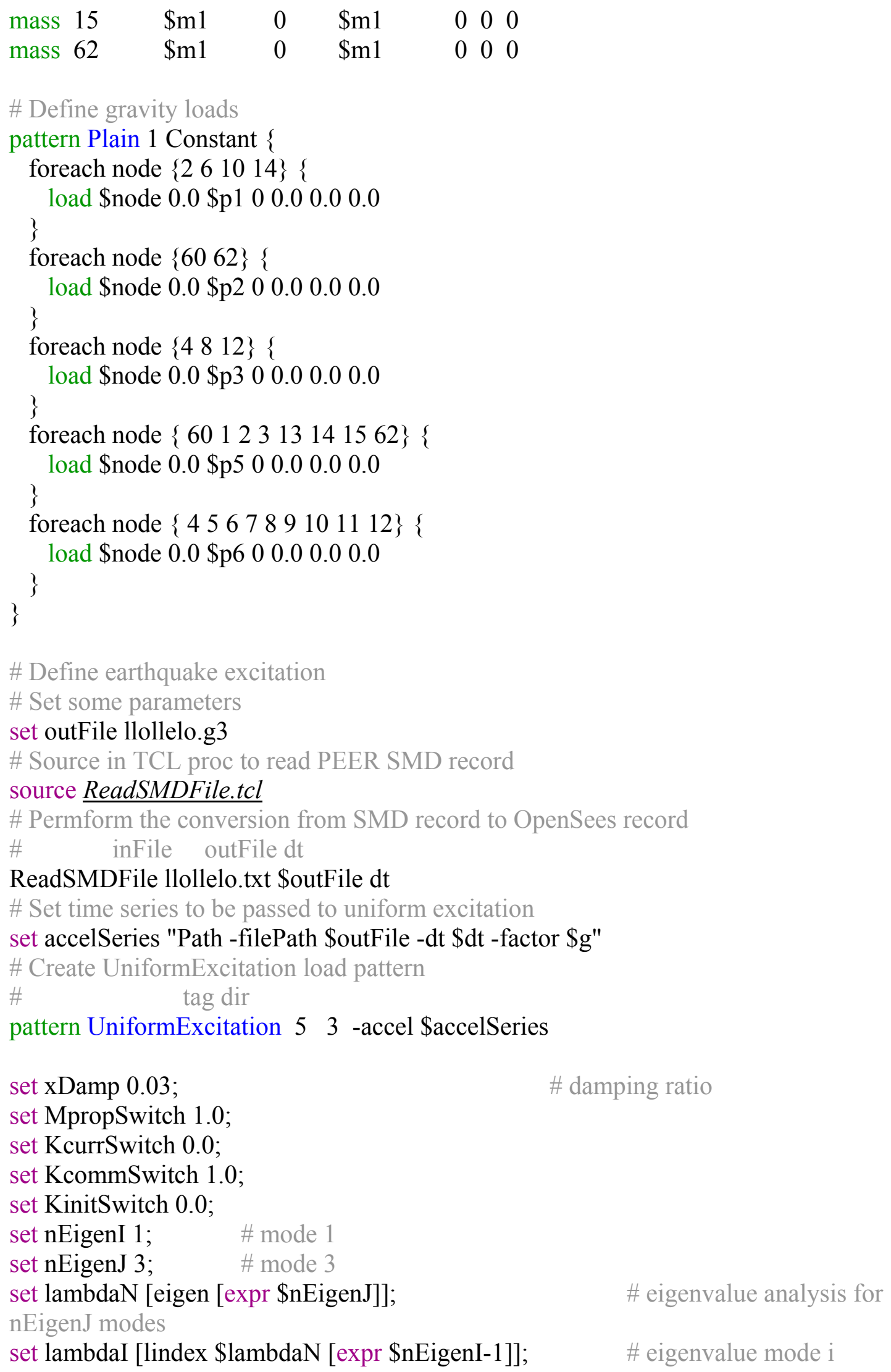


set lambdaJ [lindex \$lambdaN [expr \$nEigenJ-1]]; \# eigenvalue mode j

set omegaI [expr pow(\$lambdaI,0.5)];

set omegaJ [expr pow(\$lambdaJ,0.5)];

set alphaM [expr \$MpropSwitch*\$xDamp*(2*\$omegaI*\$omegaJ)/(\$omegaI+\$omegaJ)]; \# M-prop. damping; D = alphaM*M

set betaKcurr [expr \$KcurrSwitch*2.*\$xDamp/(\$omegaI+\$omegaJ)];

\# current-K; +beatKcurr*KCurrent

set betaKcomm [expr \$KcommSwitch*2.*\$xDamp/(\$omegaI+\$omegaJ)];

\# last-committed K; +betaKcomm*KlastCommitt

set betaKinit [expr \$KinitSwitch*2.*\$xDamp/(\$omegaI+\$omegaJ)];

\# initial-K; +beatKinit*Kini

rayleigh \$alphaM \$betaKcurr \$betaKinit \$betaKcomm;

RAYLEIGH damping

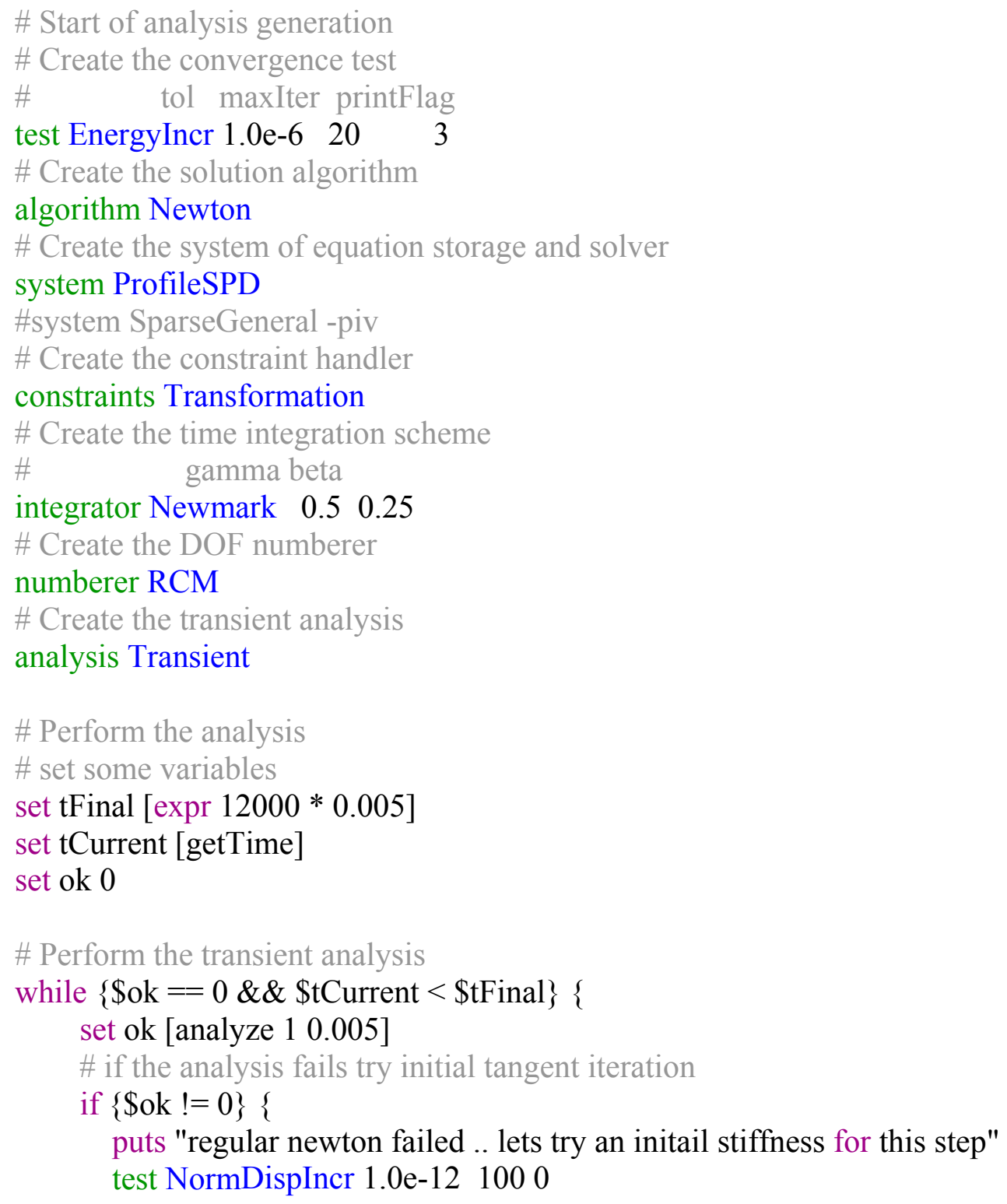




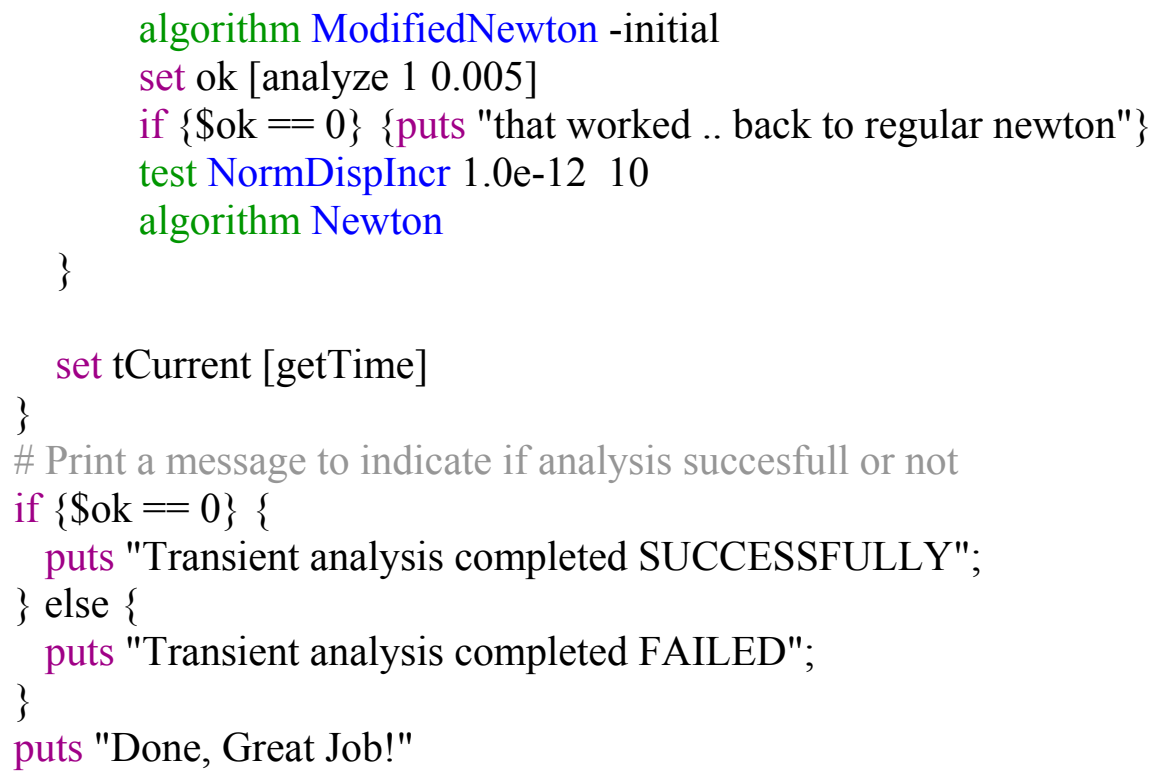


VITA

BIN LI

1995-1999

B.S., Highway and Bridge Engineering,

Zhejiang University

Hangzhou, China

1999-2002

M.S., Geotechnical Engineering

Zhejiang University

Hangzhou, China

2005-2008

$\mathrm{Ph}$. D. Candidate, Civil Engineering

Florida International University

Miami, Florida, U.S.A.

\section{PUBLICATIONS AND REPORTS}

Li, B., Shi, Y., and Mirmiran, A. (2008). "Rapid Construction of Modular Innovative Bridge Substructure." FHWA Accelerated Bridge Construction: Highway For Life Conference, Baltimore, MD.

Li, B., Shi, Y., and Mirmiran, A. (2008). "Flexure Tests of Beam-Column Components of Hybrid FRP-Concrete Pier Frame," Appendix to Final Report SCL-080201, Network for Earthquake Engineering Simulation Research, National Science Foundation (NSF) Grant No. CMS-0420347, Miami, FL.

Li, B., Shi, Y., and Mirmiran, A. (2008). "Seismic Performance of Hybrid FRP-Concrete Pier Frame System," Final Report SCL-080201, Network for Earthquake Engineering Simulation Research, National Science Foundation (NSF) Grant No. CMS-0420347, Miami, FL.

Shi, Y., Li, B., and Mirmiran, A. (2008). "Seismic Performance of Hybrid FRP-Concrete Pier Columns," Final Report SCL-071201, Network for Earthquake Engineering Simulation Research, National Science Foundation (NSF) Grant No. CMS0420347, Miami, FL.

Li, B., Shi, Y., Mirmiran, A. and Saiidi, M. (2007). "Seismic Performance of Hybrid FRP-Concrete Bridge Pier Columns and Bents." 9th Canadian Conference on Earthquake Engineering, Ottawa, ON, Canada.

Shi, Y., Li, B., and Mirmiran, A. (2006). "Seismic Performance of Hybrid FRP-Concrete Pier Columns." 3rd International Conference on Composites in Civil Engineering (CICE), Miami, FL. 
Mirmiran, A., Wang, T., Abishdid, C., Jiménez, D., Rodríguez, H., and Li, B. (2006). "Performance of Tile Roofs under Hurricane Impact - Phase I," Final Report SCL-060701, Florida Department of Community Affairs, through Florida International University International Hurricane Research Center, Miami, FL. 